Joseph Wilde-Ramsing

\title{
QUALITY KILOWATTS?
}

A Normative-Empirical Analysis of Corporate Responsibility for Sustainable Electricity Provision in the Global South
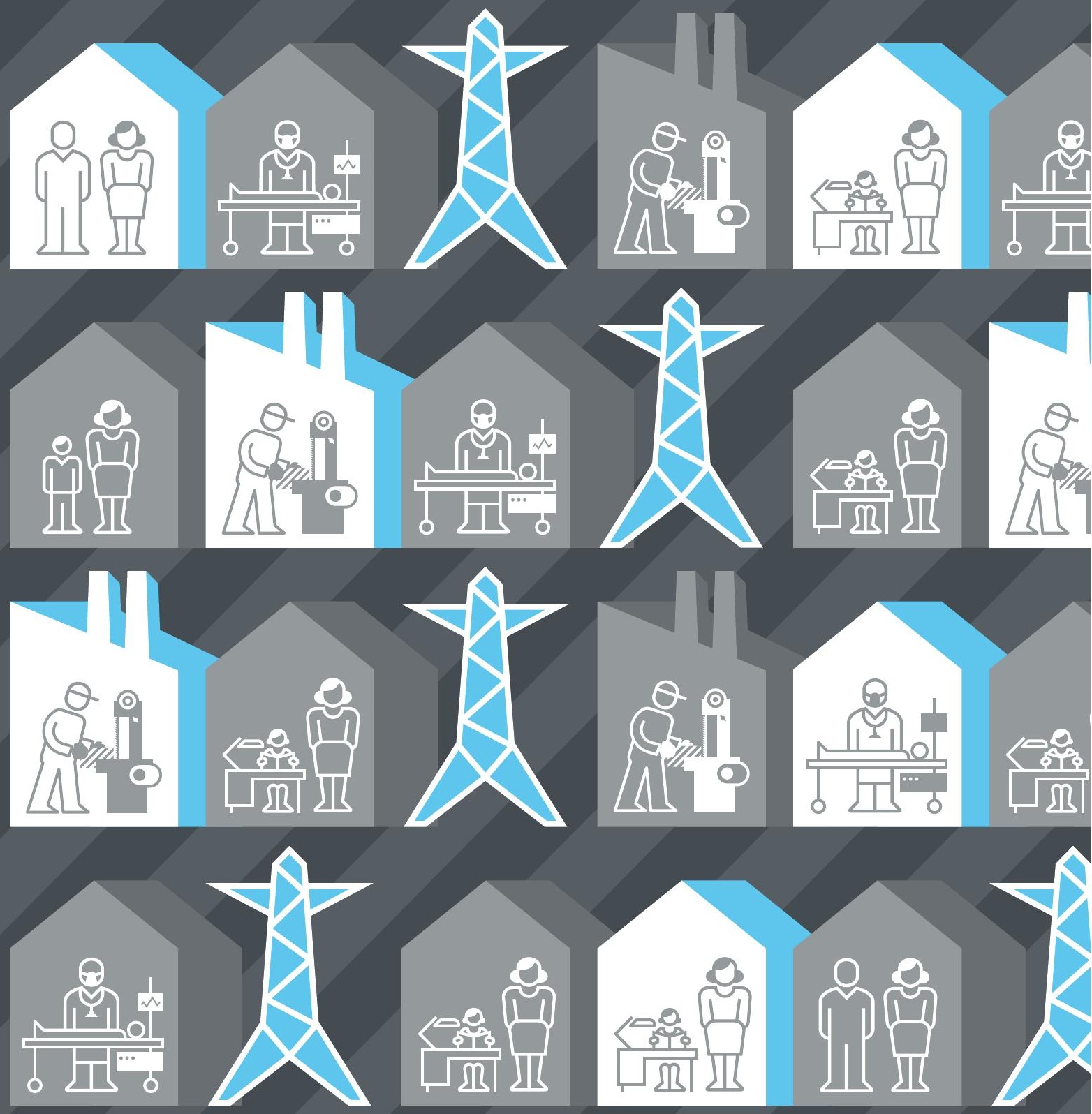
Joseph Wilde-Ramsing, University of Twente, MB/ CSTM

Quality Kilowatts? A Normative-Empirical Analysis of Corporate Responsibility for Sustainable Electricity Provision in the Global South.

Printing: proefschriftmaken.nl

FSC Mixed Sources: This book is printed on FSC certified paper.

Graphic design: Ed van Oosterhout, Leffe Goldstein Lab

ISBN: 978-90-365-1547-4

Production of this dissertation has benefitted from funding from SOMO and ProSUS.

\section{(C) $\$(0)$ CopyLeft2013, J.M.Wilde-Ramsing \\ BY NC SA All Wrongs Reserved}

This work is licensed under the Creative Commons Attribution-NonCommercial-ShareAlike 3.0 Unported license. You are free to copy, distribute, transmit, and adapt the work under certain conditions. For details, see http://creativecommons.org/licenses/by-nc-sa/3.0/

Designs and illustrations $\odot 2013$ Copyright Ed van Oosterhout all rights of the producer and the owner of the work reproduced reserved. Unauthorized copying in any form and or usage of the designs prohibited. The copyright of the designs and illustrations are owned by Ed van Oosterhout 


\title{
QUALITY KILOWATTS?
}

\section{A NORMATIVE-EMPIRICAL ANALYSIS OF CORPORATE RESPONSIBILITY FOR SUSTAINABLE ELECTRICITY PROVISION IN THE GLOBAL SOUTH}

\section{DISSERTATION}

\author{
to obtain \\ the degree of doctor at the University of Twente, \\ on the authority of the rector magnificus, \\ prof.dr. H. Brinksma, \\ on account of the decision of the graduation committee, \\ to be publicly defended \\ on Wednesday, the $8^{\text {th }}$ of May, 2013, at 14:45
}

by

Joseph Martin Wilde-Ramsing Born on the $18^{\text {th }}$ of November 1978, in Wilmington, North Carolina, USA 


\title{
This dissertation is approved by:
}

\author{
Promotor: $\quad$ Prof.dr. J.Th.A. Bressers \\ Promotor: $\quad$ Prof.dr. W.M. Lafferty \\ Assistant Promotor: Dr. M.J. Arentsen
}

\section{Members of the doctoral committee:}

$\begin{array}{ll}\text { Chair: } & \text { Prof.dr. R.A. Wessel } \\ \text { Secretary: } & \text { Prof.dr. R.A. Wessel } \\ \text { Promotor: } & \text { Prof.dr. J.Th.A. Bressers } \\ \text { Promotor: } & \text { Prof.dr. W.M. Lafferty } \\ \text { Assistant Promotor: } & \text { Dr. M.J. Arentsen } \\ \text { Member: } & \text { Dr. J.S. Clancy } \\ \text { Member: } & \text { Prof.dr. N.S. Groenendijk } \\ \text { Member: } & \text { Prof.dr. J. Meadowcroft } \\ \text { Member: } & \text { Dr. H.A.C Runhaar }\end{array}$

Chair:

Prof dr. R.A. Wessel

Prof.dr. R.A. Wessel

University of Twente / MB

University of Twente / MB

University of Twente / MB-CSTM

University of Twente / MB-CSTM

University of Twente / MB-CSTM

University of Twente / MB-CSTM

University of Twente / MB-PA

Carleton University, Ottawa, Canada

Utrecht University / Copernicus Institute 
Various parts of this dissertation have been published in one peer-reviewed article and three reports:

Wilde-Ramsing, J. (2013). "Quality Kilowatts: A normative-empirical framework for assessing TNC performance on sustainable electricity provision in the Global South". Progress in Development Studies. Forthcoming.

Wilde-Ramsing, J., Koen, M., Agbazue, T., Tchamba, Y., Liu Zhi, Qin Hui, and Racz, K. (2013). "Emerging Power(s): The impact of multinational electricity corporations from China and South Africa on sustainable electricity provision in least developed countries". Centre for Research on Multinational Corporations (SOMO). Forthcoming.

Wilde-Ramsing, J. (2009). "Quality Kilowatts: A normative-empirical approach to the challenge of defining and providing sustainable electricity in the Global South". SINTEF Energy Research and Centre for Research on Multinational Corporations (SOMO). Oslo and Amsterdam. Available at http://www.sintef.no/upload/ENERGI/Energisystemer/Politikk\%20og\%20styring/TR\%20A6837.pdf.

Wilde-Ramsing, J. and Steinweg, T. (2009). "Down the Wire: The Impact of Transnational Corporations on Sustainable Electricity Provision in the Global South: Case studies in Argentina and Peru". Centre for Research on Multinational Corporations (SOMO), Amsterdam. Available at http:// somo.nl/publications-nl/Publication_3088-nl. 


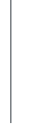


For Birka - my inspiration And for Mom, Dad, and Kee-my foundation 


\section{Table of contents}

$\begin{array}{ll}\text { List of figures } & 10\end{array}$

List of tables $\quad 11$

Symbols, abbreviations, and terminology 12

$\begin{array}{lr}\text { Chapter 1: Introduction } & 14\end{array}$

1.1. Relevance and point of departure 15

$\begin{array}{ll}\text { 1.2. Context and background } & 17\end{array}$

1.3. Aim, objectives, and research questions 27

1.4. Navigating the dissertation 29

Chapter 2: Approach and methods 30

2.1. Analytic approach 31

2.2. Evaluation methodology and normative-empirical analysis 35

Chapter 3: Defining "quality kilowatts" 48

3.1. General approach issues 50

3.2. Social issues 51

3.3. Environmental issues 62

3.4. Economic issues $\quad 72$

3.5. Cross-cutting issues 80

3.6. The SEP benchmarks for TNCS 88

Chapter 4: Modes of home-country business case study TNCs 98

4.1. The US mode 101

4.2. The European mode 103

4.3. The Nordic mode 106

4.4. The Chinese mode 108

4.5. The South African mode 112

4.6. Summary of modal projections for corporate approach to SEP 117

4.7. Selection of a TNC "representative" for each mode 118

Chapter 5: Case study-AES 124

5.1. AES background and operations $\quad 125$

$\begin{array}{ll}\text { 5.2. } & \text { AES's performance on the SEP benchmarks }\end{array}$ 
6.1. Endesa background and operations 151

6.2. Endesa's performance on the SEP benchmarks 153

Chapter 7: Case study - SN Power 180

7.1. SN Power background and operations 181

7.2. SN Power's performance on the SEP benchmarks 183

Chapter 8: Case study - Datang 204

8.1. Datang background and operations 205

$\begin{array}{ll}\text { 8.2. Datang's performance on the SEP benchmarks } & 207\end{array}$

Chapter 9: Case study - Eskom 230

9.1. Eskom background and operations 231

9.2. Eskom's performance on the SEP benchmarks 235

Chapter 10: Comparison and discussion of case study results 268

10.1. Comparison of company performance against the individual SEP benchmarks 269

10.2. Overall comparison of company policy and practice relative to the SEP standards 299

10.3. Relevance of home-country business culture 305

10.4. Discussion of limitations of the analytical framework and research methods and their potential impact on results 307

Chapter 11: Summary and conclusions 312

11.1. Summary of the main contributions 313

11.2. Major conclusions on the research questions 315

11.3. Reflections on the status quo and suggestions for further research 333

Samenvatting in het Nederlands 338

$\begin{array}{ll}\text { References } & 344\end{array}$

Acknowledgements 370

$\begin{array}{ll}\text { Curriculum vitae } & 372\end{array}$ 


\section{List of figures}

Figure 2.1 Analytic model

Figure 3.1 The SEP benchmarks for TNCs 89

$\begin{array}{lll}\text { Figure 4.1 Analytic model with variance } & 128\end{array}$

Figure 5.1 AES's global presence, 2011

Figure 5.2 AES's electricity generation capacity in Argentina by fuel source in MW, 2011

Figure 6.1 Endesa's global presence, 2009

Figure 6.2 Endesa's installed capacity in Argentina by fuel type, 2010. Based on: Endesa 2011c 172

Figure 7.1 SN Power's global presence, 2012

Figure 8.1 Datang's global presence, 2010206

Figure 9.1 Eskom's global presence, 2010232

Figure 9.1 New electricity connections made by Eskom in South Africa, 1991-2011 246

Figure 10.1 Comparison of company performance on the SEP benchmark "Endorse international normative standards for CR".

Figure 10.2 Comparison of company performance on the SEP benchmark "Adopt a commitment to CR in core-business activities and decision-making"

Figure 10.3 Comparison of company performance on the SEP benchmark "Increase access to affordable electricity" 276

Figure 10.4 Comparison of company performance on the SEP benchmark "Respect labor rights" 280

Figure 10.5 Comparison of company performance on SEP benchmark "Minimize environmental impact, including contribution to climate change"

Figure 10.6 Comparison of company performance on the SEP benchmark "Prioritize renewable sources of energy for electricity"

Figure 10.7 Comparison of company performance on the SEP benchmark "Contribute to local economic development"

Figure 10.8 Comparison of company performance on the SEP benchmark "Ensure reliable supply"

Figure 10.9 Comparison of company performance on the SEP benchmark "Engage in meaningful stakeholder consultation and participatory decision-making"

Figure 10.10 Comparison of company performance on the SEP benchmark "Assume responsibility for impacts throughout all business relationships"

Figure 10.11 Comparison of company performance on the SEP benchmark "Maximize transparency and provision of information"

Figure 10.12 Aggregated company performance across all 11 SEP benchmarks 


\section{List of tables}

Table 1.1 Benefits of electricity in relation to the Millennium Development Goals 20

Table 2.1 Overview of field work in host countries 46

Table 3.1 Overview of "critical issues" in sustainable electricity provision 50

Table 3.2 SEP benchmarks, guiding norms, and indicators for operationalization 90

Table 4.1 Approach to SEP benchmarks expected by each mode 119

Table 4.2 Key global operational aspects of selected case study companies, 2010122

Table 5.1 AES's installed generation capacity in Argentina, 2011

Table 5.2 Overview of AES's performance on SEP benchmarks in policy and in practice 128

Table 5.3 Degree to which AES's performance on SEP benchmarks reflects the US mode of business culture 149

Table 6.1 Endesa installed generation capacity in Argentina, 2010152

Table 6.3 Summary of Endesa's performance on SEP benchmarks in policy and in practice 154

Table 6.4 Endesa's global, Argentine, and Peruvian CO, emissions, 2010170

Table 6.5 Degree to which Endesa's performance on SEP benchmarks reflects the European mode of business culture $\quad 179$

Table 7.1 SN Power's electricity generation units in Peru, 2012

Table 7.2 Summary of SN Power's performance on SEP benchmarks in policy and in practice 184

Table 7.3 Degree to which SN Power's performance on SEP benchmarks reflects the Nordic mode of business culture $\quad 203$

Table 8.1 Datang power plants outside China that are operational or under construction, 2010206

Table 8.2 Summary of Datang's performance on SEP benchmarks in policy and in practice 208

Table 8.3 Datang's global fuel mix for electricity generation capacity and production, 2010221

Table 8.4 Datang's build program as of 2010, by fuel type 222

Table 8.5 Degree to which Datang's performance on SEP benchmarks reflects the Chinese mode of business culture $\quad 228$

Table 9.1 Overview of Eskom's key operational indicators, 2011

Table 9.2 Summary of Eskom's performance on SEP benchmarks in policy and in practice 236

Table 9.3 Degree to which Eskom's performance on SEP benchmarks reflects the South African mode of business culture $\quad 266$

Table 10.1 Company performance on all 11 SEP benchmarks in policy and practice 300

Table 10.2 Degree to which company performance on SEP benchmarks reflects the mode of home-country business culture 


\section{Symbols, abbreviations, and terminology}

ADB Asian Development Bank

ANC African National Congress (South African political party and movement)

b billion

BBBEE Broad-based black economic empowerment

BEE Black economic empowerment

BRICSAM Brazil, Russia, India, China, South Africa, and Mexico, largely recognized as the world's most important emerging economies

Capacity factor the ratio of the actual output of a power plant over a period of time and its potential output if it had operated at full nameplate capacity the entire time

CASS Chinese Academy of Social Sciences

CBA Collective bargaining agreement

CBD Convention on Biological Diversity

CCGT Combined cycle gas turbine

cCS Carbon capture and storage

CDM Clean Development Mechanism

CDP Carbon Disclosure Project

CEER Council of European Energy Regulators

CEO Chief executive officer

$\mathrm{CO}_{2}$ Carbon dioxide

$\mathrm{CO}_{2} \mathrm{e}$ Carbon dioxide equivalent (a unit of measure for greenhouse gas emissions)

CR Corporate responsibility

CSD Commission on Sustainable Development (UN body)

CSI Corporate social investment

CSI Corporate social investment

CSO Civil society organization

CSP Concentrated solar power

CSR Corporate social responsibility

DES Dominant electricity system

DRC Democratic Republic of Congo

EC European Commission

EIA Environmental impact assessment

EITI Extractive Industries Transparency Initiative

EMAS Eco-Management and Audit Scheme (an EU-developed certification scheme)
EMCEF European Mine, Chemical and Energy Workers' Federation

EMS Environmental Management System

ENRE Ente Nacional Regulador de la Electricidad (Argentina's electricity regulatory body)

EPSU European Public Service Unions

EU European Union

EUSS Electric Utilities Sector Supplement (a specific set of sustainability reporting guidelines for electricity companies developed by the GRI)

FCPA Foreign Corrupt Practices Act (US law)

FDI Foreign direct investment

GHG(s) Greenhouse gas(ses)

Global North Refers collectively to nations that have relatively higher per capita incomes and are relatively more industrialized. Not to be confused with the geographic North.

Global South Refers collectively to nations that have relatively lower per capita incomes and are relatively less industrialized. Not to be confused with the geographic South

GRI Global Reporting Initiative

GW(h) Gigawatt (hour) (one billion watts (per hour))

H\&S Health and safety

ha hectare ( $1 \mathrm{ha}=10,000 \mathrm{m2}=2.47$ acres)

Home country The country from which a TNC originates

Host country A country - usually in the Global South - "hosting" the operations of TNCS

HSE Health, safety and environment

IAEA International Atomic Energy Agency

ICE Costa Rican Institute of Electricity

IEA International Energy Agency

IFC International Finance Corporation (arm of the World Bank)

IHA International Hydropower Association

ILO International Labor Organization (UN body)

IPCC Intergovernmental Panel on Climate Change (UN body)

IPP Independent power producers

ISO International Organization for Standardization

kV Kilovolts

kW(h) Kilowatt (hour) (one thousand kilowatts (per hour))

LCA Life cycle assessment or life cycle analysis 
LDCs Less developed countries

Load shedding A rolling, or planned, blackout

MDGs Millennium Development Goals

m million

MNE Multinational enterprise

MoU Memorandum of understanding

MSA Minimum Services Agreement

Mt Megaton (one million metric tons)

Mvo Platform Dutch CSR platform

MW(h) Megawatt (hour) (one million watts (per hour))

NEPAD New Partnership for Africa's Development

NOSA National Occupational Health and Safety Association

(South Africa)

$\mathrm{NO}_{\mathbf{x}}$ Nitrous oxide

OECD Organization for Economic Cooperation and

Development

OHS Occupational health and safety

OHSAS Occupational Health and Safety Assessment Series

PDR (Lao) People's Democratic Republic

PM Particulate matter

PSI Public Services International

Quality Kilowatts A conceptual construction of the present study used synonymously with "sustainable electricity provision" to mean electricity provision that is in line with international normative standards for sustainable electricity provision (SEP norms)

RES-E Renewable energy sources for electricity

SA South Africa

SADC Southern African Development Community

SAIFI System Average Interruption Frequency Index

SAPP Southern African Power Pool

SASAC State-owned Assets Supervision and Administration Commission of the State Council (SOE regulatory authority in (hina)

SD Sustainable development

SEA Strategic environmental assessment

SEC Securities and Exchange Commission (US American corporate regulatory authority)
SEP Sustainable electricity provision

SHE Safety, Health and Environment (an Eskom term)

SIA Social impact assessment

SMEs Small and medium-sized enterprises

SNPP SN Power Peru (SN Power subsidiary)

$\mathrm{SO}_{2}$ Sulfur dioxide

SOE State-owned enterprise

SRI Socially responsible investment

TNC Transnational corporation

UN United Nations

UNCSD United Nations Commission on Sustainable

Development

UNCTAD United Nations Conference on Trade and Development UNDP United Nations Development Programme

UNEP United Nations Environment Programme

UNGC United Nations Global Compact

UNHRC United Nations Human Rights Council

UNRISD United Nations Research Institute for Social

Development

US United States

VP Vice-president

WBCSD World Business Council for Sustainable Development WCD World Commission on Dams

WCED World Commission on Environment and Development

WRI World Resources Institute

\section{Currency abbreviations and exchange rates (as per 7 April 2012)}

$\$$ United Stated dollar: $\$ 1=€ 0.76=4.4$ Argentine pesos $(A R S)=$ $¥ 6.30$ Chinese Yuan Renminbi (CNY) = 7.89 South African Rand $(Z A R)=501$ Central African Francs $(X A F)=2,480$ Ugandan Shillings $(U G X)=3,977$ Cambodian Riel $(K H R)=7,998$ Lao Kip (LAK)

$€$ Euro: $€ 1=\$ 1.31=5.74$ Argentine pesos (ARS) = $¥ 8.29$ Chinese Yuan Renminbi $(C N Y)=10.33$ South African Rand $(Z A R)=656$ Central African Francs $(X A F)=3,249$ Ugandan Shillings (UGX) = 5,264 Cambodian Riel (KHR) = 10,508 Lao Kip (LAK) 


\section{Chapter 1}

Introduction 


\subsection{Relevance and point of departure}

The issue of sustainable development in electricity provision has never been more relevant. Electricity is central to nearly every major challenge and opportunity the world faces today. It is vital for eradicating poverty, improving living standards, and achieving global development goals (Ha and Porcaro 2005). The United Nations (UN) has underscored that "The provision of adequate and reliable electricity at an affordable cost, in a secure and environmentally benign manner and in conformity with social and economic development needs is an essential element of sustainable development" (UN/ IAEA 2007:5). In recognition of the importance of electricity for sustainable development and achieving the Millennium Development Goals (MDGs), the UN designated 2012 the "International Year of Sustainable Energy for All" (UN 2012). Country delegations surveyed following the 2012 UN "Rio+20" Conference on Sustainable Development named "sustainable energy / access to energy" to be the "top priority area" for a new international effort do define and meet sustainable development goals (UNDESA 2013).

Despite the urgent and widely-recognized need for sustainable electricity provision (SEP), the International Energy Agency (IEA 2010a) has noted that most current patterns of electricity provision and consumption around the world continue to be gravely unsustainable. On the one hand, approximately one-third of the world's population has no access to adequate and affordable electricity. At the same time, the environmental degradation and emissions associated with electricity production and use in other areas inhibits sustainable development. For example, the electricity industry is a major source of air and water pollution. Due to its continued heavy reliance on fossil fuels, the industry is currently responsible for nearly half of global anthropogenic greenhouse gas (GHG) emissions (IEA 2009). In fact, hardly any other industrial sector has such potential to contribute to economic development, poverty alleviation, and to the improvement of people's living standards around the world at the same time as accounting for such significant negative impacts for both people and the planet. Given its critical importance for social and economic development and environmental sustainability, why are global efforts to "steer" electricity provision towards sustainable development not bearing fruit?

By improving understanding of how transnational electricity companies take up and implement normative standards for SEP - and by drawing lessons that can improve normative and regulatory frameworks - the present dissertation aims to improve the quality and contribution to sustainable development of electricity provision in the Global South. Doing so will involve the development of a framework of robust, internationally-accepted normative standards for SEP that can be used to benchmark and analyze the performance of transnational electricity companies - in other words, whether and how "quality kilowatts" are being conceived and implemented in the Global South. Since the wave of electricity sector privatizations in the 1980s and 1990s, transnational corporations (TNCS) based in the 
Global North - primarily Europe and the United States (US) - have played an important role in providing electricity in the Global South (Haar and Jones 2008). However, TNCs' record of providing electricity in a way that is consistent with sustainable development is mixed, at best (Thomas 2007, Ruggie 2008). Although investment from TNCs has helped to expand access to electricity in some countries, in many cases a focus on short-term profits has led to investment decisions and pricing policies that exclude those in greatest need and contribute to negative impacts on local economies, workforces, and infrastructure (Hall 2005, Yamin and Sinkovics 2008). In addition, the majority of private investment in the electricity sector of countries in the Global South has been in fossil fuel-based power plants, resulting in significant negative consequences for public health, the climate, and sustainable development (UK Committee on Climate Change 2009).

The international normative framework aimed at ensuring that electricity provision contributes to sustainable development is anchored in multilateral agreements, research, and decision-making at the UN-level (e.g. WCED 1987, UN 1992, UNCSD 2001, UN 2010, UNCSD 2010). This framework is supplemented by a range of other stakeholder groups:

- Intergovernmental: e.g. UN/IAEA 2007, IEA 2010b, IFC 2011, OECD 201

- Industry: e.g. WBCSD 2002, IHA 2004

- Academic: e.g. Hirschberg et al. 2004, Voß et al. 2005, Tully 2006, Fthenakis and Kim 2008

- Civil society: e.g. Public Services International (PSI/Pillinger 2009), Greenpeace (Teske 2010)
- Multi-stakeholder: e.g. World Commission on Dams (WCD 2000), Global Reporting Initiative (GRI 2008)

Despite the existence of this framework, ensuring that electricity provision contributes to sustainable development remains an unfulfilled and urgent task (WCD 2000). There is a clear need to devise lessons from current practice as to how the process of developing, promulgating, and implementing internationally recognized norms for sustainable electricity provision can be improved.

There has been an increase in activity around corporate responsibility (CR) in recent years in the electricity industry. However, there is little empirical knowledge regarding how electricity TNCs incorporate international norms for SEP into their CR policies and how they make the difficult trade-offs among the various social, environmental, and economic issues associated with SEP. Corporate scandals and failures of public-private partnerships involving electricity TNCs in the Global South have raised questions about the intentions of private utilities and their ability and willingness to provide high-quality services to their clients in the South (Palast et al. 2000). It is crucial to investigate the variation in electricity TNCs' approach to CR, as well as the associated impact on communities, workers, economies, and the environment in the Global South. Such knowledge is highly relevant for both governments and stakeholders in efforts to improve the quality of electric services in the Global South, a task that is particularly necessary during times of global economic crisis (Pillinger 
2009). The issue is becoming increasingly acute given that governmental protection for electricity consumers and workers in the Global South remains weak and poorly enforced (OECD 2006).

\subsection{Context and background}

In order to begin to address the multiple, complex, and interconnected challenges related to CR for sustainable electricity provision, a number of relevant contextual fields require further elaboration. These include the UN-anchored framework for sustainable development, the UN framework and SEP, the role of TNCs in electricity provision, CR for sustainable development, CR for SEP in the Global South, and the notion of homecountry business culture. An analysis of these contextual elements provides the basis for the dissertation's key research questions.

\subsubsection{The UN-anchored framework for sustainable development}

Before a successful evaluation of performance on sustainable development can take place, it is necessary to establish a common and scientifically sound understanding of what needs to be evaluated. As applied here, the concept of sustainable development is directly anchored in a "political" understanding of the term as put forth and developed within the UN framework. The locus classicus of the UN framework for sustainable development is the 1987 report Our Common Future by the Brundtland Commission.
Brundtland coined the most commonlycited definition of sustainable development: "Development that meets the needs of the present without compromising the ability of future generations to meet their own needs" (WCED 1987). This foundation was subsequently built upon by the accords from the 1992 Rio Summit, particularly Agenda 21 (UN 1992); the MDGs adopted by the UN Johannesburg Millennium Summit in 2000 (UN 2010); and the ongoing work of the UN Commission on Sustainable Development (UNCSD 2010). Virtually all members of the UN, as well as a wide range of other stakeholder groups, have committed to the UN institutional framework for sustainable development, making it a crucial normative anchoring point for research and analysis.

Building on Our Common Future and the Rio documents, the UN Commission on Sustainable Development (CED 2001) has developed a set of sustainable development indicators. Similar efforts have been undertaken by other political bodies such as the Organisation for Economic Co-operation and Development (OECD 2000a), the World Bank's International Finance Corporation (IFC 2006), and the European Commission (EC 2001a). Firmly anchored in decision-making at the UN and other intergovernmental bodies, sustainable development thus represents "a normative standard that serves a meta-objective for policy" (Meadowcroft 2007), and one that "deals with a goal-related and value-laden programme for change" (Lafferty 2004). Sustainable development is generally divided into three pillars. The social pillar of sustainable development is focused on satisfying basic 
human needs, as well as addressing protection of human and labor rights. Environmental issues include nature conservation, environmental protection, and ecological balance, while the economic component comprises local economic development, efficient value creation, and sustainable consumption. In this context, sustainable development implies integrating social welfare, environmental protection, and economic development to ensure equity across and within generations and countries. The key normative challenge, however, is addressing these three "pillars" of sustainable development in a balanced way.

The UN framework for sustainable development aims to simultaneously address the implicitly contradictory environmental, social, and economic issues and concerns and to find the balance between these three pillars. The framework recognizes the interdependency between modes of production, satisfying basic needs, and impacts on natural life support systems (Lafferty 2002a). For example, an economic impact (e.g. jobs created through a new electricity project) will inevitably have a social dimension (e.g. local incomes increase). Similarly, a social impact (e.g. human health compromised through coal power plant emissions) often has an environmental dimension (e.g. atmospheric ecosystem also damaged) (Voß et al. 2005).

In making the trade-off, it is important to note that the relative importance given to the different pillars of sustainable development is likely to differ between the Global North and the Global South (Lafferty 2002b). In the
Global North, the environmental or ecological component of sustainable development, i.e. the link to "sustainability", is often seen as the most fundamental dimension of sustainable development, as it anchors the concept logically and distinguishes it from other normative concepts and programs that are more devoted to socio-economic welfare or justice. In the Global South, however, the social and economic elements of sustainable development, in particular poverty reduction and meeting basic needs, have a stronger entitlement than in industrialized countries (Visser 2008). This perspective has a firm footing in traditional sustainable development discourse, reflected by the prominence given to "needs" in Our Common Future: "The concept of 'needs', in particular the essential needs of the world's poor, [should be given] overriding priority" (WCED 1987). Developing economies are thus vitally and avidly interested in the goods, such as sustainable jobs, functioning economic infrastructure, and tax revenues, that can be provided by business activity. This implies that each of the three pillars is important in its own right, as well as being profiled and balanced with reference to the other pillars and overall sustainable development demands.

\subsubsection{Specifying "quality kilowatts": The UN-anchored framework and sustainable electricity provision} Within the electricity industry, the "quality" of electricity is generally understood in terms of technical quality and reliability of supply 
(Marzinotto et al. 2011, Baggini 2008, CEER 2005). For the purposes of the present analysis, however, quality is understood more broadly in terms of sustainable development as put forth and developed within the UN framework. Electricity provision figures prominently in the UN framework for sustainable development (UN Energy 2005, Modi et al. 2005, Cherni et al. 2007, McDonald 2009b). Energy issues are richly integrated into the Rio Summit's action plan, in particular Chapters 7 to 9 and 30 of Agenda 21 (UN 1992). In this context, the notion of SEP, or "quality kilowatts", implies the generation, transmission, distribution, and supply of electricity in a manner that "contributes to poverty reduction and the satisfaction of basic needs, without damaging the natural environment or compromising the ability of future generations to meet their own needs" (WCED 1987). This logic implies a best-possible integration of social, economic, and environmental concerns in specific electricity projects in given societal settings. However, such an understanding is still very general. Specific benchmarks regarding what constitutes "quality kilowatts" in practice must be developed. Indeed, the World Commission on Dams (WCD 2000) has highlighted the need to achieve "a broad consensus...on the norms that guide development choices and the criteria that should define the process of negotiation and decision-making" in order to resolve underlying conflicts about the environmental, social, and economic benefits and impacts of electricity projects. The notion of "quality" electricity provision is directly related to such sustainable development norms.
Given that the MDGs were formulated as a set of sustainable development benchmarks, they provide a logical place to start for guidance in developing specific benchmarks for SEP. Expanded access to affordable electric power is crucial for meeting all of the goals (Modi et al. 2005). Table 1.1 summarizes some of the benefits of electricity for achieving the MDGs.

Beyond the MDGs and the UN basis, there is an array of initiatives and literature from academic, (inter-) governmental, trade union, civil society organizations (CSOs), industry, and multi-stakeholder sources that could inform the development of benchmarks for SEP in the Global South. For example:

- Voß et al. (2005) develop a conceptual framework for sustainable electricity supply employing a lifecycle assessment approach to evaluate various generation technologies.

- Tully (2006) assesses the normative and legal standards associated with access to electricity as a human right.

- Fthenakis and Kim (2008) explore the social and environmental impacts of land use change associated with the extraction of electricity generation fuels.

- The IAEA (2007) has led an effort to establish a set of national-level energy indicators for sustainable development.

- The OECD (2000) has attempted to develop sustainable development indicators for nuclear power.

" Another OECD initiative, the IEA's "Task 29 " program, uses an integrated approach to economic, environmental, and social components of bioenergy systems (IEA 2010b). 
Table 1.1: Benefits of electricity in relation to the Millennium Development Goals

\begin{tabular}{|c|c|}
\hline MDG & Benefits of electricity \\
\hline $\begin{array}{l}\text { 1: Eradicate extreme poverty and } \\
\text { hunger }\end{array}$ & $\begin{array}{l}\text { " Job creation } \\
\text { " Better cooking methods } \\
\text { " Reduced time spent on fuel gathering } \\
\text { " Improved irrigation (through electric pumps) } \\
\text { " Refrigeration }\end{array}$ \\
\hline $\begin{array}{l}\text { 2: Achieve universal primary } \\
\text { education }\end{array}$ & $\begin{array}{l}\text { " Frees up time for school } \\
\text { " Allows access to new media outlets } \\
\text { " Attracts teachers to rural areas } \\
\text { " Allows for study after dark }\end{array}$ \\
\hline $\begin{array}{l}\text { 3: Promote gender equality and } \\
\text { empower women }\end{array}$ & $\begin{array}{l}\text { " Reduces indoor air pollution } \\
\text { " Frees up time for education and other economic activity } \\
\text { " Illuminated streets are safer for travel } \\
\text { " Traditional women's domestic burdens are made easier }\end{array}$ \\
\hline 4: Reduce child mortality & $\begin{array}{l}\text { "Improved water quality through better treatment } \\
\text { " Parents have more time to spend with children } \\
\text { " Reduces indoor air pollution } \\
\text { " Improved health services and hospitals }\end{array}$ \\
\hline 5: Improve maternal health & $\begin{array}{l}\text { " Reduces indoor air pollution } \\
\text { " Improved pre- and post-natal health services and hospitals } \\
\text { " Improved access to medication, vaccines and tests (through refrigeration) } \\
\text { " Better illumination for nighttime deliveries }\end{array}$ \\
\hline $\begin{array}{l}\text { 6: Combat HIV/AIDS, malaria, and } \\
\text { other diseases }\end{array}$ & $\begin{array}{l}\text { " Improved access to medication, vaccines and tests (through refrigeration) } \\
\text { " Improved health services and hospitals } \\
\text { " Improved communication of public health information }\end{array}$ \\
\hline $\begin{array}{l}\text { 7: Ensure environmental } \\
\text { sustainability }\end{array}$ & $\begin{array}{l}\text { "Renewable sources of electricity have huge potential to reduce emissions } \\
\text { " Reduced deforestation, acidification, soil erosion, and climate change }\end{array}$ \\
\hline $\begin{array}{l}\text { 8: Develop a global partnership for } \\
\text { development }\end{array}$ & $\begin{array}{l}\text { The World Summit for Sustainable Development called for partnerships between } \\
\text { public entities, development agencies, civil society and the private sector to } \\
\text { support sustainable development, including the delivery of affordable, reliable and } \\
\text { environmentally sustainable energy services. }\end{array}$ \\
\hline
\end{tabular}

Based on: UN Energy 2005 and McDonald 2009b. There is a plethora of literature on the subject (cf. Modi et al. 2005 and Cherni et al. 2007).

- The WCD (2000) focuses on the core values of equity, efficiency, participatory decisionmaking, sustainability, and accountability for sustainable hydroelectric projects

" Public Service International's "Quality Public Services" campaign elaborates norms for providing electricity based on equality, social justice, and respect for electricity industry workers in order to ensure electricity provision contributes to poverty alleviation (Pillinger 2009).

- Jacobson and Delucchi (2011) and Greenpeace's Teske (2010) thoroughly flesh out the technical, environmental, and economic feasibility of various SEP technologies. 
Three of the most significant international normative initiatives specifically directed at defining "quality kilowatts" for companies engaged in electricity provision are:

- the World Business Council for Sustainable Development's (WBCSD 2002) "Sustainability in the Electricity Utility Sector" project

- the International Hydropower Association's (IHA 2004) "Sustainability Guidelines"

- the Global Reporting Initiative's (GRI 2008) "Electric Utilities Sector Supplement" (EUSS) to its G3 Sustainability Reporting Guidelines

The WBCSD's project identifies key social, environmental, and economic elements of sustainable development relevant to the electricity industry. The project then lays out principles, objectives, and strategies that companies should follow to ensure they contribute to sustainable development. The IHA Sustainability Guidelines provide a framework of standards, issues, and industry best practices that companies involved in hydropower provision are encouraged to follow. Though issues related to hydroelectricity are the focus of the guidelines, many of the recommendations could be applied to electricity provision based on other technologies or fuels. Finally, the GRI's EUSS is a reporting framework that covers a wide range of social, environmental, and economic topics. It is a tool for identifying the critical electricityrelated sustainable development issues and encouraging electricity providers to be aware of and report on such issues.

Although the UN-anchored framework for sustainable development provides a solid foundation, the electricity-specific initiatives and indicators are institutionally and thematically diverse. The standards and norms they propose remain diffuse and fragmented. Despite a "general consensus that promotion of sustainable development within the electric sector calls for the integration of economic, ecological and social dimensions" by electricity providers (Hirschberg et al. 2004:13), a set of universally accepted normative standards for SEP in the Global South remains elusive. The development of an operational set of SEP benchmarks in Chapter 3 of the present dissertation represents in this regard a unique contribution to the field.

\subsubsection{The role of TNCs in electricity provision}

Transnational corporations are playing an increasingly important role in the electricity systems of countries around the globe. Until the 1980s, the electricity sector of most countries in the Global South was dominated by large state-owned enterprises (SOEs). However, the liberalization and subsequent privatization of many electricity markets during the 1980 s and 1990s allowed transnational electricity companies, generally based in Europe and the US, to extend their operations into the Global South. They did this by buying out formerly state-owned electricity enterprises, or by developing greenfield projects as independent power producers (Haar and Jones 2008). As a result, the early 1990s saw an explosion of private investment in electricity generation, transmission, distribution and supply in 
the Global South, reaching US\$50 billion in 1997 (Woodhouse 2006). Although a series of economic crises and the failure of several private electricity projects in the Global South caused private investment in the sector to dip in the late 1990s, by 2009 private investment commitments to electricity projects had soared to over US\$67 billion (World Bank 2010a).

Recent years have also seen TNCs based in emerging economies like South Africa and China playing an increasingly important role in electricity provision in the Global South. The South African company Eskom, for example, is a dominant player in regional electricity markets in sub-Saharan Africa. Similarly, under the Chinese government's "Going out" strategy, Chinese electricity and dam construction companies have expanded their operations beyond Chinese borders into South-East Asia and Africa. Between 2002 and 2010, Chinese outward foreign direct investment increased by an annual growth rate of $49.9 \%$ (World Bank 2011). The emergence of these new players and the implications of South-South relationships both add an interesting new dynamic to the understanding of the role of TNCs in electricity provision.

Initially a great deal of optimism abounded about the unlimited positive impact of foreign direct investment (FDI) from TNCs as "an engine of development" (UNCTAD 1992) in the 1990s. However, TNCs' record of providing electricity in a manner that is consistent with sustainable development has been mixed, at best (Thomas 2007, Ruggie 2008). Although investment from
TNCs has helped expand access to electricity in some countries, in many cases a focus on short-term profits has led to investment decisions and pricing policies that exclude those in greatest need and contribute to negative impacts on local economies, workforces, and infrastructure (Hall 2005, Yamin and Sinkovics 2008). It is now generally recognized that the positive developmental impacts of FDI are not automatic, particularly when it comes to investment in infrastructure, and that some current TNC strategies are actually having a "negative effect on the development of infrastructure in LDCs" (Yamin and Sinkovics 2008). In addition, the majority of private investment in the electricity sector of countries in the Global South has been in fossil fuel-based power plants, resulting in significant negative consequences for public health and the climate (UK Committee on Climate Change 2009).

It is not the purpose of the present dissertation to debate whether privatization of electricity systems in the Global South is good or bad, although it should be noted that that debate is ongoing and is very relevant (see, for example, Heller et al. 2003, Mun 2003, McGuigan 2007, Thomas 2007, McDonald 2009a, Chavez 2012, Hathaway 2012, McDonald and Ruiters 2012). What is of primary concern here is what the WBCSD (2002) calls the increasing expectation by governments, labor unions, civil society, and other stakeholders that private enterprises should assume greater responsibility and accountability for ensuring sustainable and high-quality electric service as they take on a greater role as producers and suppliers 
of electricity in the Global South. In fact, the electricity industry is an ideal forum for testing how TNCs interpret and implement standards and norms for sustainable development (Burke 2010). Electricity provision epitomizes the need to make the difficult trade-offs and choices between economic, social, and environmental interests that lie at the crux of sustainable development. Adopting and implementing international standards related to SEP can help businesses make the appropriate trade-offs and find a balance that contributes to sustainable development in the Global South (Utting 2002).

\subsubsection{Corporate responsibility for sustainable development}

Recent years have seen goverments and civil society increas pressure on TNCs to adopt and abide by international standards for CR such as:

- OECD (2011) Guidelines for Multinational Enterprises

- UN (2011) Guiding Principles for Business and Human Rights

- IFC (2011) Sustainability Framework

- UN Human Rights Council's (UNHRC 2011) Guiding Principles for Business and Human Rights

- UN Global Compact (UNGC 2008)

- International Organization for Standardization (ISO) (2011) 26000 Guidance on Social Responsibility, and

- International Labor Organization (ILO) (2001) Tripartite Declaration of Principles concerning Multinational Enterprises and Social Policy.
This pressure has largely come to bear in the form of growing demands for corporations to assume responsibility for the social and environmental, as well as economic, impacts of their operations (Welford 2005). There is ample precedent and increasing support among both academics and practitioners for setting CR within the context of sustainable development. In 2005, the UN Research Institute for Social Development (UNRISD 2005) confirmed the "business responsibility for sustainable development". Debroux (2009:25) argues that the notion of CR has been close to that of sustainable development "from the start". Welford (2010) defines CR as "the private sector's commitment to the broader concept of sustainable development". From the business side, Holmes and Watts (2000) define CR for sustainable development as "the continuing commitment by business to behave ethically and contribute to economic development while improving the quality of life of the workforce and their families as well as of the local community and society at large". The Dutch MVO Platform (2012), a coalition of civil society organizations working on CR and accountability, similarly invokes the three pillars of sustainable development in its definition of CR - "a process whereby a company assumes responsibility, across its entire supply chain, for the social, ecological and economic consequences of the company's activities, reports on these consequences, and constructively engages with stakeholders". Although these definitions differ in their emphasis, each expresses a conviction that, rather than maximize shareholder value at any cost, firms must take broader social objectives 
and the demands of a wide range of other stakeholders into account. The concept of CR for sustainable development thus applies a distinct normative content (Sedlacko and Martinuzzi 2012). According to internationallyagreed standards such as the OECD (2011) Guidelines, companies must balance the social, environmental, and economic pillars of sustainable development in all of their activities. Commitment to and implementation of sustainable development within core business activities and across the supply chain is thus taken as the starting point for CR as conceptualized here. This conceptualization recognizes that there are limits to win-win solutions as painful trade-offs must be made. Gauging and interpreting how electricity TNCs that operate in the Global South approach and carry out this responsibility, both in policy and practice, is the empirical focal point of the dissertation research.

The study of CR has become progressively more complex as the increasingly globalized nature of business implies that corporations must compete and interact with competitors and stakeholders from different countries and with different values (Burton et al. 2000). In this increasingly multicultural globalized business environment, examining CR for sustainable development in the Global South is particularly interesting for a number of reasons. Reimann et al. (2011) have pointed out that there is little empirical knowledge regarding how the CR policies of TNCs are implemented in the Global South nor the impact they are having there. Utting (2002) notes that "if sustainable development is to become a reality, then transnational corporations must take their [social and environmental] responsibilities seriously. In the industrialized countries, some positive steps have been taken. The rhetoric of CR is also extending to the Global South, but very little is known about the extent of concrete changes there in the performance of big business". Visser (2008:474) provides additional rationale for focusing on $\mathrm{CR}$ in the Global South:

1. "Developing countries represent the most rapidly expanding economies, and hence the most lucrative growth markets for business

2. Developing countries are where the social and environmental crises are usually most acutely felt in the world

3. Developing countries are where globalization, economic growth, investment, and business activity are likely to have the most dramatic social and environmental impacts (both positive and negative)

4. Developing countries present a distinctive set of CSR agenda challenges which are collectively quite different to those faced in the developed world"

\subsubsection{Corporate responsibility in electricity provision}

Applying the above understanding of $\mathrm{CR}$ in the context of the UN-anchored framework for sustainable development and electricity provision means that private corporate actors that provide electricity are responsible for doing so in a manner that contributes to poverty reduction and the satisfaction of basic needs without damaging the natural environment or compromising the 
ability of future generations to meet their own needs. This is the essence of "SEP" and "quality kilowatts" as conceptualized here. As identified more generally above, the increasing pressure on companies to abide by international standards for sustainable development is also evident in the electricity sector.

The work of the WBCSD's (2002:2) "Sustainability in the Electricity Utility Sector" project, particularly the identification of principles, objectives, and strategies that companies should follow to ensure they contribute to sustainable development, is based on the underlying assumption that businesses have a responsibility to "meet electricity needs in a sustainable manner". Similarly, the GRI's (2008) EUSS encourages electricity companies to be aware of SEP issues and report to their stakeholders on them. As a result of initiatives like these, recent years have seen a significant increase in CR interest and activity among electricity companies (ECOTEC 2007). In 2004, in a move unprecedented in the industry, the European electricity sector labor unions and employers' representatives released a joint statement outlining their vision of CR (EPSU et al. 2004).

Despite this increase in activity related to $\mathrm{CR}$ in the electricity industry, a common understanding of exactly what CR for SEP entails remains elusive. For example, there is currently no electricity sector code of conduct, as exists in other sectors. This creates a situation in which companies can define their responsibility as they see fit, leading to great variations in approach and impact (Welford
2004). While some electricity TNCs appear to have made CR a part of their long-term strategy, others have simply produced a one-off report lacking a clear strategy for systematizing and further developing the concept (ECOTEC 2007). Palast et al. (2000) note that a number of recent corporate scandals and failed publicprivate partnerships involving electricity TNCs operating in the Global South have raised questions about the intentions of private utilities and their ability and willingness to provide high-quality services to their clients in the South. Even among the electricity industry's leading CR initiatives, participation by companies is limited. The WBCSD's (2002) project counts only nine core members, and a recent study by Kerckhoffs and Wilde-Ramsing (2010) revealed that less than half of the electricity companies surveyed were reporting according to the GRI's sustainability guidelines.

It is essential to investigate how variation in electricity TNCs' approach to and performance on SEP in the Global South comes about. The implications are highly relevant for the international and regional providers of overarching SEP norms (e.g. the UN, OECD, EU), as well as communities, customers, workers, and other stakeholders in the Global South affected by electricity TNCs' behavior. The results also have important implications for Southern governments, as they decide whether and which TNCs should be prioritized for electricity provision to their citizens and businesses. 


\subsubsection{Home-country business culture}

Literature from the fields of institutional sociology and economics offers some insights into variation in firms' incorporation of certain normative standards related to CR for sustainable development into their policies and practices. These studies generally emphasize factors external to the individual firm that drive companies beyond simple profit maximization and economic efficiency (Hoffman and Ventresca 2002) and towards adoption of international standards (Delmas and Toffel 2004). Such external factors include regulation (Dean and Brown 1995, Russo and Fouts 1997, Delmas 2003), competitive forces (Nehrt 1996, Aragón-Correa 1998, Sharma and Vredenburg 1998, Christmann 2000), and pressure from CSOs (Lawrence and Morell 1995, van Huijstee and Glasbergen 2010). Institutional theory underscores the importance of cultural and social pressures in influencing organizational practices and structures (Scott 1992). DiMaggio and Powell (1983) argue that coercive (e.g. regulatory), mimetic (e.g. through competition), and normative institutional mechanisms influence managerial decisions by creating and diffusing a common set of values, norms, and rules. In this way, institutional theory predicts that similar practices and structures will be reflected across companies that share a common organizational field. For example, Jennings and Zandbergen (1995) argue that regulations and regulatory enforcement (i.e. coercive forces) are the primary driver of firms' environmental management practices and that such practices vary by industry. Similarly, Milstein et al. (2002) assert that different industries are exposed to varying levels of regulatory pressure, leading to distinct, industry-specific environmental strategies. Runhaar and Lafferty (2009) have found that companies are often more attuned to industry-specific CR issues, and are thus more responsive to industry-specific CR codes and networks addressing these issues.

Other studies have investigated idiosyncratic factors (Egri and Herman 2000) and analyzed managerial attitudes (Cordano and Irene Hanson 2000). A few have included both internal and external pressures driving companies to go beyond regulatory compliance, but these have largely been confined to one particular industry (e.g. the pulp and paper industry in Gunningham et al. 2003).

Literature from the fields of (cross-cultural) management and business ethics sheds additional light on the problem. Although it is generally assumed that all TNCs are primarily focused on maximizing profit, numerous studies (Hofstede 1983, Orpen 1987, Hofstede et al. 1990, Pinkston and Carroll 1994, Vogel 1996, Stajkovic and Luthans 1997, Burton et al. 2000, Hofstede 2001, Hall and Soskice 2001, Levy and Rothenberg 2002, Delmas and Toffel 2004, Welford 2005, Dimitratos et al. 2010, Barron 2010 ) indicate that variation in adoption of international normative standards by TNCs is likely to come from the business culture in which the firm "grew up". International competition for electricity provision means that the uptake of international norms at the firm level and the prioritization of certain aspects of 
sustainable development over others is affected by differing business cultures (Burton et al. 2000, Lyons and Deutz 2010).

Hofstede (1983) hypothesizes that the home nation of internationalized firms "is distinguished by specific value systems that can account for divergences in the types of strategies implemented by enterprises originating from different countries" (Dimitratos et al. 2010:1). For example, Hofstede et al. (1990:313) found that "organizational cultures are partly pre-determined by nationality". Lyons and Deutz (2010) note that various regions around the world prioritize certain elements of sustainable development over others, while Vogel (1996) identified differences in behavior of TNCs from distinct groups of countries with varying cultures and traditions for business regulation. Examples of regional variation in regulatory style/culture are the US pattern (ubiquitous regulation, shareholder capitalism, and privately-owned companies), the European pattern (stakeholder capitalism, clear publicinterest focused performance), and the Nordic pattern (European-style stakeholder capitalism further informed by the Nordic social welfare tradition, many state-owned enterprises). Lyons and Deutz (2010) and Swilling (2010) observe particular differences in sustainable development priorities between the countries of the Global North - which tend to address environmental issues and natural resource limits above other issues - and those in the Global South, which often place economic development and poverty alleviation above all else. Visser (2008) concurs, noting that social challenges related to $\mathrm{CR}$ tend to be given more political, economic, and even scholarly attention in the Global South. This is in stark contrast to the Global North, where the focus is often on environmental or stakeholder issues. In addition, many countries in the Global South exhibit a tradition of corporate philanthropic efforts, often focused on community development (Reimann et al. 2011).

Based on this literature, it is expected that a framework based on TNCs' geographic-cultural origins can serve to generate variance on the dissertation's crucial variables (i.e. uptake and operationalization of SEP norms by electricity TN(s), and structure a testing of the variables through comparative case analysis.

\subsection{Aim, objectives, and research questions}

Situated within the applied science realm and normatively anchored in the UN-based framework for sustainable development, the present study's overall aim is to improve the quality and the contribution to sustainable development of electricity provision in the Global South. In order to achieve this aim, the dissertation has the following specific objectives: - To contribute to the development of a framework of robust, consensual normative standards for SEP by deriving a set of SEP-related norms that can be used to benchmark TNCs' performance and by devising lessons from current practice as to 
how the process of developing, promulgating, and implementing internationallyestablished norms for SEP can be improved

- To generate empirical findings that contribute to an improved understanding of how electricity TNCs prioritize and implement normative standards for SEP in their operations in the South by exploring the possible impacts of modes of homecountry business culture. Such an improved understanding should also contribute to the academic debate on cross-cultural management

- Through comparative analysis of specific implementation situations, to generate insights and draw lessons that can improve the performance of electricity TNCs in integrating and implementing normative standards for SEP

- To contribute to the further development of the normative-empirical methodological approach

Framed by these objectives, the central problematic of the dissertation involves determining how individual transnational electricity companies conceptualize, operationalize, and implement norms for SEP. In other words, the dissertation seeks to determine how "quality kilowatts" are being conceived and implemented. This formulation of the problematic can be further broken down into the following research questions:

1. Are there standards for sustainable electricity provision available within the UN-anchored sustainable development framework and other international normative discourses that can be systematized into a common set of benchmarks for evaluating the performance of TNCs involved in electricity provision in the Global South?

2. Given the prospect that a TNC's homecountry business culture conditions its behavior - and assuming that differences in such "modes" of home-country business culture are increasingly important in an era of growing competitive globalization of electricity provision - how are SEP standards generally taken up, interpreted, and valued within various national modes? Can these different modes of business culture be used to structure and analyze variation in TNC uptake of SEP standards?

3. Using an applied-science, "normativeempirical" approach to guide a systematic gathering of information about the operations of electricity TNCs operating in the Global South, how do selected TNCs perform - in both policy and practice vis-à-vis normative benchmarks for SEP and relative to each other?

4. Does an empirical analysis of the performance of electricity TNCs from varying home-country "modes" of business culture on the SEP benchmarks contribute to theoretical notions of international business strategy?

5. What general lessons can be drawn from a comparative analysis of TNC performance on SEP benchmarks that are of direct practical relevance for improving the effectiveness of normative frameworks, particularly with regard to increasing uptake 
and implementation of SEP norms by TNCs and creating a more "level playing field" for sustainable development?

\subsection{Navigating the dissertation}

The remainder of the dissertation is structured as follows:

Chapter 2 outlines the analytical approach taken - including a visualization of the approach in Figure 2.1 - and the methods employed.

Chapter 3 represents a broad survey of the literature on various critical issues related to CR for SD in the field of electricity provision and concludes with the presentation of the framework of SEP benchmarks, which are used to measure and evaluate the performance of the case study TNCs. The crucial overview and operationalization of the SEP benchmarks can be found in Table 3.2, along with a visualization in Figure 3.1.

Chapter 4 delves into further detail on the notion of "modes of home-country business culture" and describes how each of the five selected modes - US, European, Nordic, Chinese, and South African - is likely to condition how companies take up and interpret the SEP norms. Chapter 4 concludes with the selection of the five case study TNCs, a further specification of the analytic approach in Figure 4.1, and an overview of their operational aspects of the selected TNCs.
Chapters 5- 9 comprise the five TNC case studies. Each case study begins with a brief operational introduction to the company, providing additional detail on the company's activities in those host countries in which field research was conducted. Each chapter then contains a table summarizing the company's performance on the SEP benchmarks in policy and practice, followed by a detailed presentation of the findings. Each concludes with a reflection on the degree to which the company's homecountry mode of business culture conditions its performance on the benchmarks.

Chapter 10 analyzes, compares, and discusses the results of the case studies on each SEP benchmark, including an analysis of company performance across all SEP indicators. Table 10.1 and Figure 10.12 at the end of the chapter provide an overview. The chapter concludes with a consideration of the limitations of the research design and methods and the impact they may have had on the results.

Finally, Chapter 11 summarizes the dissertation's main contributions, draws a number of conclusions and lessons based on the analysis, reflects on the status quo for SEP, and makes suggestions for future research. 


\section{Chapter 2}

Approach and methods
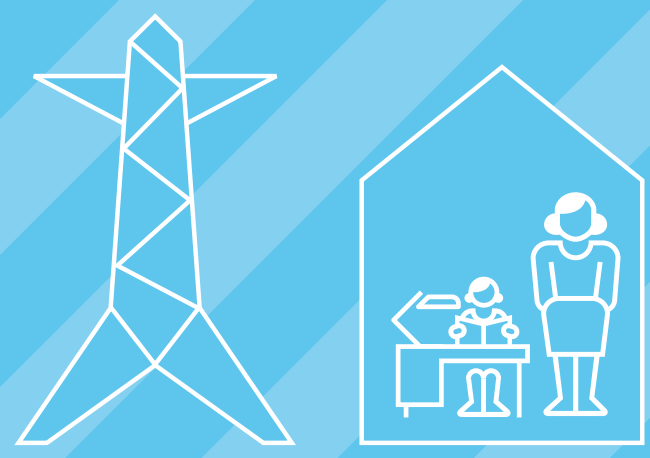


\subsection{Analytic approach}

The confluence of increasingly interrelated, complex, and urgent socio-economic developmental issues and environmental concerns involved in sustainable development and SEP implies a distinct need to integrate knowledge with action (Patavalis and Aravossis 2004, Runhaar et al. 2006). The present dissertation employs an evaluative "normativeempirical" approach, through which strategic research builds knowledge that can be used to promote sustainable and equitable energy systems. Such an approach is necessarily situated within the applied-science realm. The knowledge produced by this type of actionoriented research is relevant for multiple target audiences. It contributes to the academic discourse and is in tune with the "Realpolitik of policy formation", giving it direct relevance for practitioners in the field (Pawson and Tilley 1995:20). Such knowledge is directly relevant for norm-setters at the international and regional levels seeking to affect a transition to sustainable development (Meadowcroft 2011) and stakeholders at the local level in efforts to improve the generally poor quality of electric services in the Global South. By enhancing understanding of how electricity TNCs conceive of and incorporate international standards for CR in their operations in the South, the study aims to contribute to the development of more effective strategies for ensuring that electricity provision contributes to sustainable development.

The analytic approach to the research questions involves four key elements:
1. International normative standards for $\mathrm{CR}$ in electricity provision

2. Modes of home-country business culture

3. Adoption and eventual integration of the normative standards by transnational electricity companies in their policies and procedures

4. The practice of TNCs "on the ground" in the Global South vis-à-vis the normative standards

There is currently no sector-wide code of conduct for the electricity industry. Nevertheless, there are a number of international normative standards for CR that are applicable to corporations involved in electricity provision. The norms have been developed and promulgated within the UN-anchored framework for sustainable development and supplemented by a wide range of intergovernmental, business, civil society, and academic organizations. Although the normative standards are generally not currently binding on companies (i.e. there is generally no sanction mechanism attached to them), they are internationally recognized, with full democratic commitments from the member states of the intergovernmental organizations that have developed them. As such, the norms provide a source of moral pressure on electricity TNCs around the world to align their policies and practices with the principles of sustainable development and responsible business conduct. Chapter 3 systematizes the relevant normative standards and establishes benchmarks that allow for an objective evaluation and analysis of electricity TNC performance. This 
systematization also provides a potential basis for the development of a more rigorous sectorspecific code of conduct for the electricity industry.

Although the international normative standards for CR can be expected to have some influence on the policies and practice of all electricity TNCs, the degree to which the norms are taken up and implemented by TNCs varies widely (Welford 2004, Hall 2005, Thomas 2007, Yamin and Sinkovics 2008). In order to systematize variance within the analysis of TNC performance, the analytical approach introduces an intervening variable - the mode of homecountry business culture. This approach is firmly grounded in the literature on institutional economics, (cross-cultural) management, and business strategy and ethics (Hofstede 1983, Hofstede et al. 1990, Vogel 1996, Stajkovic and Luthans 1997, Hall and Soskice 2001, Levy and Rothenberg 2002, Delmas and Toffel 2004, Dimitratos et al. 2010). The increasingly international competition for electricity provision highlights the fact that the uptake of international norms is affected by differing business cultures. Not only do different business cultures prioritize different values and norms, but the overall value and relevance given to voluntary international normative standards also varies between regions. Based on existing literature, the present approach thus assumes that a TNC's adoption and implementation of normative standards is conditioned by the business culture of the country or region in which it "grew up". "Mode of home-country business culture" is thus considered to be an intervening variable between the international normative standards for SEP and the uptake of SEP norms by electricity companies into their own policies and procedures.

Filtered by a TNC's home-country business culture, adoption of the normative standards for CR is reflected in an electricity company's perceptions of and reference to the norms in its policy documents as well as the specific policies and procedures related to SEP issues (Stajkovic and Luthans 1997). Of crucial importance is how electricity TNCs make the trade-offs between the social, environmental and economic aspects of SEP. Through an empirical analysis of the adoption of the norms by electricity TNCs from different corporate cultural backgrounds, the dissertation aims to generate lessons and implications that are directly relevant for the work of normsetters and academics at the international level. Are the SEP norms equally interpreted and applied by electricity firms from different corporate cultures? Or are the SEP norms understood differently by companies from different backgrounds? Do certain corporate cultures prioritize some aspects of SEP over others? What can norm-setting bodies do to increase the uptake of SEP norms by corporate representatives of different cultures?

TNC practice in providing "quality kilowatts" at the local level in host countries is expected to largely follow on from the CR policies and procedures adopted by the company at the headquarters level (Delmas and Toffel 2004, IHA 2004, Ruud 2002, Ramus and Steger 2000, 
Sharma 2000, Marcus and Nichols 1999, Arias and Guillen 1998). The exact translation of corporate policies into practice by managers at the plant or ground level in local settings is, however, not expected to be a one-to-one conversion (Reimann et al. 2011, Ruud 2002, Khanna and Vidovic 2001). Stajkovic and Luthans (1997:19) emphasize the need to evaluate actual corporate conduct, alongside corporate policies and perceptions, in order to fully operationalize corporate "responsibility". Determining whether and how $\mathrm{CR}$ policies related to normative standards for SEP are implemented on the ground in host countries is one of the dissertation's key objectives and contributions. It is recognized that internal corporate processes are not solely responsible for the translation of policy into practice. Institutional and cultural factors in different local settings have an impact on how local TNC managers implement normative standards adopted at the headquarters level and can limit the "room for maneuver" that companies have to put their policies into practice. These contextual factors are likely to exhibit significant variation in the different local settings in which electricity TNCs operate, and they must be acknowledged to the greatest extent possible in the analysis.

Through an empirical analysis of TNCs' practice with regard to the norms and their own corporate policies, the study aims to elaborate and extend the lessons and implications for $\mathrm{CR}$ norm-setting within the UN intergovernmental framework. The analysis of the TNC performance on the ground provides the crucial empirical basis for drawing conclusions on the effect (or lack thereof) of the modes of homecountry business culture on whether and how "quality kilowatts" are actually being realized and how they can be realized more effectively. The lessons learned from the analysis of TNC practice on the ground will also have direct and concrete implications for corporate managers seeking to improve policies and implementation mechanisms at the headquarters level. In other words, the analysis aims to produce lessons and implications regarding "what works (and what doesn't work) for whom, where, when, and how" with respect to improving overall TNC performance in line with the international norms.

The analytic model depicted in Figure 2.1 illustrates the research problematic and analytic approach. International normative standards for SEP apply, in theory, equally to all electricity TNCs operating around the world. However, the mode of business culture in a TNC's home country or region serves as a filter to the SEP norms and thus "intervenes" to condition the degree to which the TNC adopts the norms and how it translates them into its own CR policies. TNC performance on the ground in host countries is largely derived from the company's corporate policies, but is also influenced by institutional and cultural factors in the hostcountry context. The empirical analysis, which involves a broad comparison of the policy and practice of multiple TNCs across different developmental settings, has an exceptional potential to inductively generate lessons and implications that are relevant for both corporate 


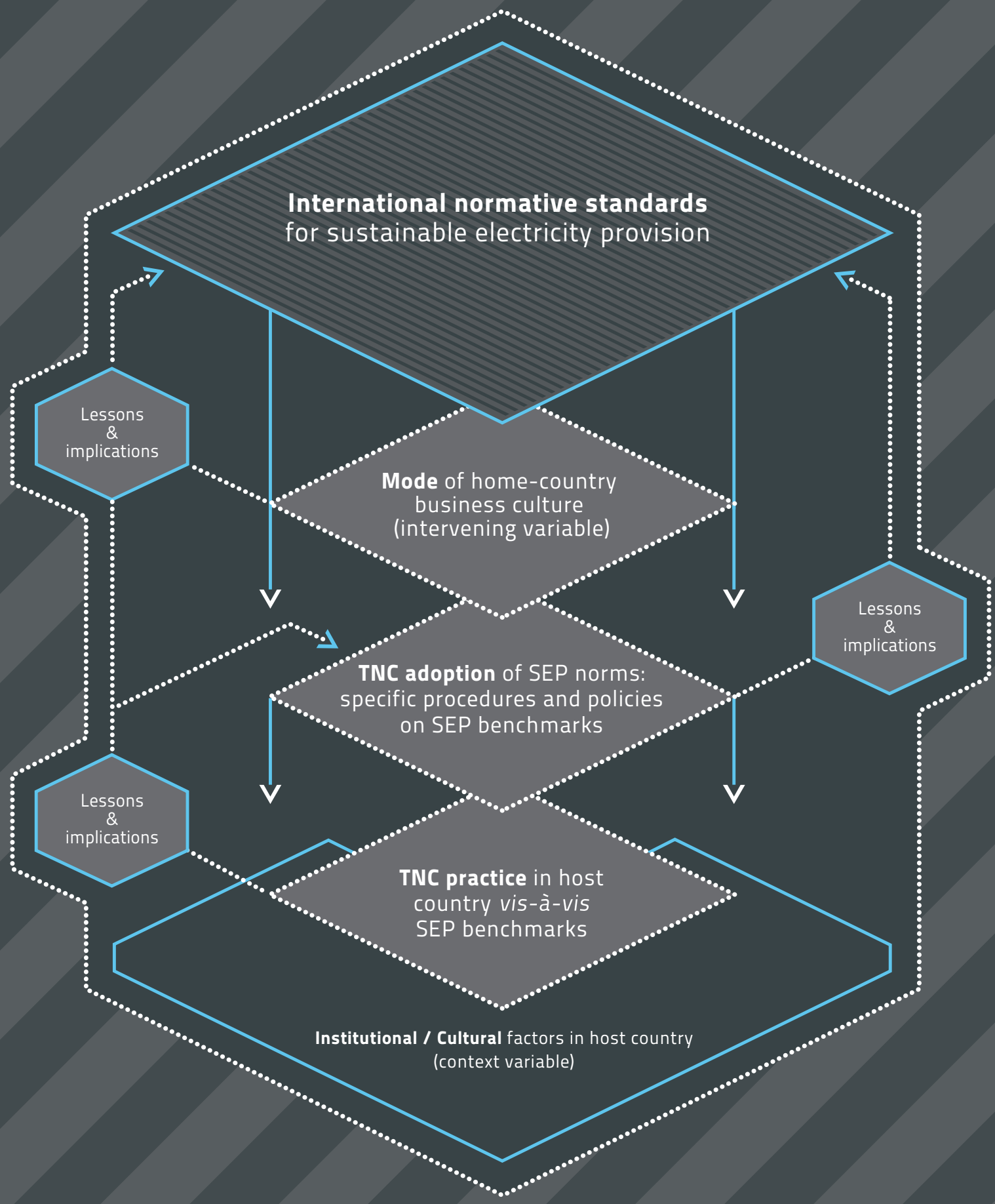

Figure 2.1: Analytic model 
decision-making as well as international norm-setting. In addition to the feedback loops for improving SEP norm-setting and TNC performance on the norms, the dissertation also seeks to provide a valuable contribution to the development of the normative-empirical methodology. The comparative method can be employed here to generate insights and findings that do not generally come forth through single-case evaluative studies.

\subsection{Evaluation methodology and normative-empirical analysis}

Addressing the specific analytic problematic of the dissertation requires a methodology that is at once inductively empirical, questioning, evaluative, and practically relevant. The evaluative methods employed by the field of policy analysis within political science are particularly well-suited to these demands. The general approach and its different variations are well-documented in, for example, BemelmansVidec et al. (2003) and Alkin et al. (2012).

A basic premise of this research approach is the use of established normative standards to make an assessment that can play "a pragmatic role in helping to inform policy decisions" (Stufflebeam 1980). Evaluation serves to create (and transform) knowledge in a manner that "has meaning for agents" (Sedlacko and Martinuzzi 2012). In short, evaluation methods facilitate "improvement through understanding"
(Patavalis and Aravossis 2004), which is a primary aim of the present dissertation. Evaluation methodology is well-suited to adress complex research problems. The evaluation approach embraces the contextual complexity found in social interactions and relationships by attempting to answer the question "What works for whom in what circumstances?" (Patavalis and Aravossis 2004). This, according to Pawson and Tilley (1995), "is what is needed for policy and practice improvements" in relation to problems based on normative goal achievement, such as the promotion of sustainable development.

More specifically, the approach employed here is "normative-empirical analysis", where the overall goal is an empirically-based assessment of the $\mathrm{CR}$ policies and practices of transnational electricity companies. The method has been most explicitly outlined by Lafferty (2002b) in terms of six basic steps for normative-empirical research.

1. Identification of the specific practical discourse where questions of norms and standards are at issue. In this case, the problematic involves questions of sustainable development in electricity provision.

2. Connection of the specific issue in question to one or more academic discourses related to the problematic.

3. "Translation" of the practical discourse problem into a normative-theoretical discourse problem, clarifying the implications of the problem within a normativetheoretical context 
4. Formulation of empirical indicators of the normative standards identified in the practical discourse by which the problematic can be addressed and clarified.

5. Determination of the relevant empirical methodology necessary for an objective analysis of the normative problematic.

6. Execution of the empirical analysis, with conclusions for both the practical discourse and the normative-theoretical discourse.

Each of these six steps can be elaborated in greater detail for the context of the present dissertation's analytic problematic and approach.

\subsubsection{Identification of the practical discourse}

As a point of departure for identifying the relevant practical discourse for assessing SEP in the Global South, a broad survey of the literature related to the problematic has been conducted. The survey focused on literature from inter-governmental bodies such as the UN and OECD, as well as academic, industry, trade union, non-governmental and multistakeholder sources identifying and elaborating different aspects of the current normative discourse on CR and SEP.

With TNCs increasingly involved in electricity provision (World Bank 2010a), there are growing demands from a wide range of stakeholders (from government to civil society to the business community itself) for electricity companies to take greater responsibility and be held accountable for the social, economic and environmental impacts of their activities (WBCSD 2002). A robust discussion within the business school literature documents how these societal demands are being translated into an increasingly standardized norm set for TNC operations (Moon 2002, Welford 2004). Such normative standards, and the societal demands they represent, form the source of the moral pressure on electricity TNCs from different corporate backgrounds to align their policies and practices with the principles of sustainable development. At the heart of the debate is the need to provide affordable access to electricity to the two billion people around the world who currently lack it (UN/ IAEA 2007, OECD/IEA 2010, Ha and Porcaro 2005). However, the devastating environmental and human rights impacts associated with some forms of electricity generation mean that electricity provision cannot come at any cost. Balancing the social, environmental and economic interests and impacts of electricity provision implies that corporate actors involved in electricity provision must make difficult tradeoffs (Debroux 2009). The crux of the practical debate involves exploring how companies choose to move within the normative and regulatory space they are provided in host countries to find their own balance between providing energy to large numbers of people for development, respecting the environment, and generating a return on investment.

The literature survey served to map that normative space by identifying the critical issues that exist within the practical discourse, 
clarifying the problematic, and enabling the formulation of a normative benchmark for assessing CR with regard to SEP. This approach is explicitly operational. Because it is anchored in the practical discourse on internationallyrecognized normative standards, the framework of evaluative benchmarks developed and systematized in the present dissertation can be used as objective benchmarks for analyzing electricity TNC policy and practice. In so doing, the dissertation's findings should have direct relevance for the practical discourse.

\subsubsection{Connection to the academic discourse}

There is currently a robust academic debate with regard to the variation in electricity TNCs' incorporation of certain normative standards into their policies and practices. This debate connects the practical discourse surrounding CR and SEP to the academic discourse related to the problematic. The term "corporate responsibility" continues to be hotly debated in both political science and business school discourses (Moon 2002, Welford 2004). For the purposes of the present analysis "corporate responsibility" is seen as a more analytically correct term than the frequently used "corporate social responsibility" (CSR) since the latter predisposes the necessary trade-off between social, economic, and environmental concerns, which the author views as the essence of the sustainable development normative perspective in an operational context. Carroll's (1979) classic construction of CR envisioned four defining components: economic, legal, ethical, and discretionary responsibilities of business:

- Economic responsibilities reflect the belief that business has an obligation to be productive and profitable and meet the consumer needs of society.

- Legal responsibilities indicate a concern that economic activities must be carried out within the confines of written law.

- Ethical responsibilities include unwritten codes, norms, and values implicitly derived from society - these responsibilities go beyond mere legal frameworks and are often ambiguously defined.

- Finally, discretionary responsibilities refer to volitional or philanthropic endeavors (Aupperle et al. 1985).

Recently however, the applicability and relevance of Carroll's (1979) conceptualization of CR has been questioned, especially as understood and practiced in the Global South (Visser 2007). Indeed, CR is still interpreted by some to consist primarily of corporate philanthropy or charity. Welford (cited in AEDAS 2010), on the other hand, notes that "Philanthropy is about what you do with your profits. Corporate responsibility is about how you make those profits in a sustainable, ethical, socially responsible way that benefits people and the planet". Others, such as Sverjensky (2010), caution that "continuing to frame corporate efforts on sustainable development as 'CSR' also keeps those efforts in the CSR department, instead of driving their integration into core-business operations". 
Various sources in the literature (Dimitratos et al. 2010, Barron 2010, Kirca et al. 2009, Welford 2005, Delmas and Toffel 2004, Levy and Rothenberg 2002, Hofstede 2001, Hall and Soskice 2001, Burton et al. 2000, Stajkovic and Luthans 1997, Vogel 1996, Pinkston and Carroll 1994, Hofstede et al. 1990, Orpen 1987, Hofstede 1983) suggest that companies will have different approaches toward CR and sustainable development and will incorporate the associated international normative standards into their overall business strategy to varying degrees depending on the regulatory framework and culture of the country in which they "grew up". Hofstede et al. (1990: 313) emphasize, "Organizational cultures are partly pre-determined by nationality". Although it is assumed that all TNCs are primarily focused on maximizing profit rather than necessarily contributing to sustainable development, Vogel (1996) has identified differences in behavior of TNCs from distinct groups of countries with varying cultures and traditions for business regulation. In particular, national cultures affect how corporations internalize values and norms such as responsibility, equality, innovativeness, flexibility and the need to protect the environment (Kirca et al. 2009).

\subsubsection{Translation of the practical problem into a normative-theoretical problem}

The initial step toward systematizing a normative-analytic framework and operationalizing what is variably referred to in the literature as a national or regionalbased "cultural filter" (Levy and Rothenberg
2002), "cognitive frame" (Delmas and Toffel 2004) or "historically deposited culture" (Kim and Benson 2007) that conditions the way firms behave is the construction of different national/regional "modes" of home-country business culture. "Business culture" is employed here as an heuristic term, and "mode" is used in what Kaplan (1964) refers to as the "academic cognitive style". The goal is to identify systematic, problem-relevant (i.e. related to CR for SEP) differences within the general category of TNCs providing electricity in the Global South. The systematic aspect is "introduced by way of great 'principles', applied over and over to specific cases, which illustrate the generalization rather than serve as proofs for it" (Kaplan 1964: 260). In the present case, the "great principles" are the internationally recognized normative standards related to CR for SEP, and the normativeempirical assessment focuses on the policies and practices of electricity-providing TNCs. The approach is, therefore, an empiricallybased variant on Kaplan in which the general "model" is the sustainable development-based normative model for CR in the electricity sector. The "modes" are posited regional-cultural variations on this model and are analyzed here to improve the pragmatic effectiveness of applying the "model".

Five specific regional modes of home-country business culture have been selected for study: United States (US), European, Nordic, Chinese, and South African. The US, European, and Nordic modes were chosen because they are home to the vast majority of electricity TNCs active 
in the Global South. TNCs from these three regions accounted for $66 \%$ of all foreign direct investment (FDI) in electricity infrastructure in the Global South in 2008 (World Bank 2008). In addition to these three "Northern" modes, TNCs based in emerging economies are playing an increasingly important role in electricity provision in the Global South through so-called "South-South relationships". The South African company Eskom, for example, is active in several countries in sub-Saharan Africa and is the dominant player in the regional market. Similarly, Chinese power companies have expanded their operations beyond Chinese borders into South-East Asia, particularly the Mekong Delta region, and some have begun to explore investment opportunities in Africa. For these reasons, the "Chinese" and the "South African" modes have also been included in the study. Combined, TNCs from these five modes represented $76 \%$ of all FDI in the electricity sector in countries in the Global South in 2008 (World Bank 2008). These five "diverse case" modes are expected to produce a significant degree of variation on the study's primary dependent variables: uptake and implementation of SEP norms (Seawright and John 2008).

Each of these five modes of home-country business culture will be described and analyzed as part of the inductive comparative analysis. The analysis will attempt to determine where there are national or regional cultural differences in the way governments, businesses and civil society relate to the normative standards related to CR for SEP. Elements of each mode that may elucidate these differences include evidence such as formal declarations of shared values related to sustainable corporate practice within the country or region, formalization of the country/region's interpretation and definition of the concepts of sustainable development and $\mathrm{CR}$, regulatory style in the area of sustainable corporate practice, degree of demonstrable interest of the region's business and civil society in sustainable corporate practice, and evidence of prioritization of some of the SEP issue areas over others.

\subsubsection{Systematization of the normative standards}

The first step toward an empirically-based assessment of company performance on SEP is to document and condense the available SEP-related standards. Although no readily available "code of conduct" for the electricity industry exists, the UN-anchored framework for sustainable development and supplemental intergovernmental, business, and civil society initiatives provides a diverse range of normative standards, as well as concerns, problems, issues, and broad criteria for CR with respect to SEP. Chapter 3 of the dissertation is dedicated to detailing these standards and critical issues, which can be roughly categorized according to the three sustainable development pillars social, environmental, and economic - as well as a number of "cross cutting" and "general approach" issues. Balancing the numerous sustainable development issues represents an essential challenge for electricity companies operating in the Global South. This is the 
normative endpoint of the exercise of defining "quality kilowatts", providing a well-anchored set of norms for analyzing and assessing how trade-offs are made at both the policy level within TNCs, as well as their followthrough with practical electricity provision in developmental settings. The normative benchmarks arrived at through the process of documenting and condensing the range of relevant norms will thus represent the essential standards for "quality kilowatts".

The task of systematizing the normative standards into a manageable set of benchmarks will be aligned with the emphasis by the World Commission on Dams' (WCD 2000) on equity, efficiency, participatory decisionmaking, sustainability, and accountability in responsible electricity provision. The benchmarks will be anchored in a wide range of UN and other intergovernmental and multistakeholder agreements and declarations. In determining the appropriate operational indicators for the condensed indicator set, it will be important to recall that the overall aim here is to produce knowledge that contributes to improving the quality and contribution to sustainable development of electricity provision in the Global South. Emphasis must thus be placed on both the TNCs' policies and approach at the headquarters level, as well as the practical implementation of those policies and standards on the ground in host countries.

The condensed SEP benchmarks will be operationalized through a series of specific indicators - also drawn from the literature
- for corporate policy and practice. The SEP benchmarks and operational indicators are presented in Table 3.2 in Chapter 3. Company performance in both policy and practice will be measured against the operationalized benchmarks using a modified Likert (1932) scale to roughly classify a company's performance as "largely in line", "moderately in line", "moderately out of line", or "largely out of line" with each operationalized SEP benchmark. The "largely in line" category will be assigned if the company's performance is deemed to be in line with the corporate behavior suggested by the SEP norms on $76-100 \%$ of the indicators, "moderately in line" on $51-75 \%$ of indicators, "moderately out of line" on $26-50 \%$ of indicators, and "largely out of line" on $0-25 \%$ of indicators. A similar method will be used to measure the degree to which each company reflects its mode of home-country business culture.

\subsubsection{Empirical methodology - comparative case study analysis}

The study employs a comparative case study method for analyzing the policy and practice of five electricity TNCs from different backgrounds of business culture. The comparative method is one of the basic methods for scientific analysis, one that works well with a reduced number of cases and thus allows for the identification of complex and non-linear interactions (Lijphart 1971, George and Bennet 2004). Nissen (1998:399) has emphasized that "in-depth analysis of a limited number of cases is the core of comparative political science with a good ratio between methodological input 
and analytical results". The comparative case study approach based on inductive, narrative analysis is recognized as an important research method in the fields of energy analysis and energy policy (Rochlin and Meier 1994, Sovacool and Bulan 2011, Bambawale et al. 2011). Furthermore, Yin (2003) argues that the case study is a particularly appropriate approach to empirical enquiry when the phenomenon under investigation is not easily separated from the organizational context, as is the case with TNC approach to SEP. Such an approach enables an examination of the "what", "why" and "how" questions related to the phenomenon (Yin 2003). This includes questions like, "Why do companies that are subject to the same levels of normative/moral and institutional pressure pursue different strategies with regard to SEP?" and "How do electricity TNCs with corporate bases in different national-regional settings conceptualize and operationalize CR for sustainable development with respect to the nature and overall impact of electricity provision in the Global South?"

The small-n, qualitative, comparative case study approach is justifiably criticized because the ratio of variables to cases is unfavorable. Lijphart (1971:685) summarizes: "The principal problem facing the comparative method can be succinctly stated as: many variables, small number of cases". Indeed, the range of issues addressed in the present dissertation is broad, with the full spectrum of social, environmental and economic aspects of sustainable development at play, condensed down to the 11 SEP benchmarks. However, the comparative case study method is qualitatively different from the statistical method in its ability to address complex, multivariate cases (Ragin 1987, Yin 2003). Taras et al. (2009) contend that the qualitative approach has been the most successful mode of enquiry in crosscultural studies. Nissen (1998:404) goes further, noting, "what is often seen as a problem of the comparative method, namely the generally small number of cases, is actually its virtue. Just because the advantage of the comparative method lies in its detailed analysis of the single case as an entity with all its particularities, it is only possible to analyze a strongly restricted number of cases". Furthermore, it is often not possible to use quantitative methods and statistical-mathematical procedures to control for the multitude of intervening variables found in real-life social relationships (Lijphart 1971). Nissen (1998:415) believes that "the complex, intensive analysis of each case solves the problem of 'many variables'" and that "comparative analysis of a very small number of cases can provide a great increase in findings." This is because in-depth case analysis "remain[s] sensitive to the details of particular [cases] and policies while retaining some ability to form, and test, explanations" (Hague et al. 1992:40).

The five cases analyzed in the present dissertation fulfill the basic rule of comparability that " $n$ " (the number of cases) should be greater than one (i.e. $n>1$ ), thus addressing the "systematic" criticism commonly leveled at single case study analyses. Furthermore, the combination of multiple cases with the 
development of the normative benchmarks that are part of the normative-empirical analysis applied here allows for an additional element of comparison as each case study will not only be compared with the other case studies, but also measured against the benchmarks provided by the international normative standards. This method should serve to increase the validity of the results of the analysis and provides a clear basis for generalization (Nissen 1998).

The fact that all of the cases come from a single industry - electricity provision - may potentially limit the generalizability of the results, but it is a decision that has been made consciously. The enormous economic, social, and environmental implications of electricity provision uniquely situate the industry at the crux of sustainable development. While crucial for development, the fact that the generation of electricity is also the world's largest source of greenhouse gas emissions, yet at the same time offers some of the most cost-effective opportunities to reduce such emissions, means that the sector warrants particular research attention (Stern 2006). The electricity provision industry is thus ideal for examining corporate approaches to sustainable development. Furthermore, concentrating the case studies within a single industry has methodological advantages. Sharma and Vredenburg (1998:731) note, "Comparative case studies of organizations within the same industrial context facilitate comparison through replication of results, either literally (when similar responses emerge) or theoretically (when contrary results emerge for predictable reasons), to enable 'analytic generalization'.
A common industrial context also facilitates control for relevant external influences such as the degree of environmental regulation, the degree of scrutiny by media and special interest groups, and industry-wide standards and practices." Although the term "electricity provision", which comprises electricity generation, transmission, and distribution, is used frequently throughout the analysis, the electricity generation segment is considered the most crucial segment for a normativeempirical analysis of SEP by TNCs. This is because it is the segment with by far the most private investment (World Bank 2011) and the segment with the greatest potential to impact sustainable development (both positively and negatively) (OECD 2000a). The case study TNCs have thus been selected and will be evaluated based primarily on their electricity-generation operations, although some are also active in the transmission and distribution segments.

Following the presentation of the empirical research and analysis of each of the five case study companies (Chapters 5 through 9), Chapter 10 contains a crucial synthesis, comparison, and discussion of the results. For each of the 11 SEP benchmarks, the synthesized data from all five companies are analyzed and presented in both narrative and tabular format. The presentation of the data in (extensive) tables is regarded as an essential tool for comparative case-study analysis and one that is particularly suited (and necessary) for the type of structured qualitative analysis upon which the present dissertation builds (Yin 2003). 


\subsubsection{Specific methods for the empirical analysis of TNC policy and practice}

The dissertation employs a case study, narrative, inductive approach as the empirical method to gather information on TNC policy and practice. The empirical method relies on what Nissen (1998:414) calls "intrusive" measures aimed at "data collection directly for the particular research interest". In the present case, the data collection measures include semi-structured, open-ended, grounded research interviews and site visits, supplemented by an extensive review of the academic, industry, and civil society literature. This approach enables and facilitates an in-depth exploration of the drivers behind corporate policy and practice that results in a deeper, more nuanced understanding of why certain actors, in this case electricity TNCs, behave the way they do (Yin 2003, Baxter and Jack 2008, Sovacool and Bulan 2011). By providing what George and Bennett (2004) call a "detailed examination of an aspect of a historical episode to develop or test explanations that may be generalizable to other events", the study has exceptional inductive potential to generate lessons for corporate decision-making and international norm-setting (Caspary 2008, Sovacool and Bulan 2011).

The analysis of TNC policy and practice is grounded in 11 SEP benchmarks identified in and condensed from the literature and operationalized according to the international normative standards associated with each benchmark. However, it must be acknowledged that the varied host country contexts in which the companies are operating affect the degree to which the same data can be gathered for all companies on all indicators. Nevertheless, these "imperfect" benchmarks are necessary for analyzing performance and effectiveness using the evaluative method (Campbell 1969). According to Nissen (1998:414), using such "relatively equal" benchmarks and indicators "means that the data to be compared is gathered from the particular cases respectively, in order to allow similar statements in the research context. This recourse to relative categories can be especially important when the analysis deals with qualitative data". The 11 benchmarks for measuring the policy and practice of the TNCs can be understood as "relatively equal" between cases. There is a clear understanding that the specific contexts in which the TNCs operate influence the conceptual space that TNCs have to implement their CR policies in the host country setting, and thus the way in which TNC practice can be measured and compared. In fact, information gathered empirically on the various contexts in which the electricity TNCs are operating is crucial for inductively drawing conclusions as to what works when, where, and how with regard to the implementation of normative standards for SEP.

Information about how the various companies integrate the international standards into their policies and their approach to providing sustainable electricity was collected and documented primarily through use of a standardized questionnaire and telephone interviews with management and board members in each of the five companies. 
Interviews were sought with managers at the headquarters level as well as the local level in host countries. Information was also gathered through analysis of company materials such as websites and policy documents related to CR for SEP. One month prior to sending the questionnaire, a letter describing the research and announcing the forthcoming questionnaire was sent by e-mail and regular mail to the companies. The letter requested that each company identify the correct contact person for answering questions related to the company's policies on CR and SEP. The announcement letter was sent to a contact address (e-mail and postal) in the company that had been identified on the company's website as a CR or sustainability manager. If no individual address could be identified, the announcement letter was sent to a general contact address for the company. After the contact person was confirmed, two weeks prior to sending the questionnaire, the contact person was informed about the exact date of the arrival of the questionnaire and asked to reserve time in his or her schedule to answer the questionnaire. This method follows the practice employed by the Centre for Research on Multinational Corporations (SOMO) and other academic studies on cross-cultural CR management (e.g. Welford 2004, Welford 2005).

The questionnaire, designed for qualitative analysis, included 30 open-ended items addressing the critical issues identified in the literature. The questionnaire and all communications with corporate headquarters were conducted in English (though communications with local managers and other stakeholders in the host countries (see below) were conducted in local languages). After the questionnaire was sent, each company was given three weeks to respond with their answers. Follow-up telephone interviews served to clarify, confirm, and deepen the responses provided in the questionnaires. Finally, news sources, civil society and union reports, academic articles, and company information databases were used to give additional insight into the approach taken by the companies.

In order to evaluate performance of the five companies in practice on the ground in host countries, information about the their activities and practices in the host countries was documented by field informants in professional research organizations that are long-term SOMO partners. All of the field informants have undergone SOMO-led training in research and documentation methods and conducted their documentation according to the SOMO research manual (SOMO 2010). In addition, the author met with each of the field informants to discuss the set-up of the study and the research; the benchmarks to be employed; documentation methods; selection of questions to be used in interviews; and methods for selecting interviewees, interviewing techniques, and ethical questions related to working with human subjects.

While information directly from the companies and their representatives is crucial for forming an accurate picture of how TNCs approach SEP, Russo and Fouts (1997) criticize those studies 
on CR policy and performance that rely solely on self-reported data from corporations. They argue that these fail to take into account the views and opinions of other stakeholders, which can provide a more nuanced take on corporate behavior. The present study avoids this pitfall by including a wide range of stakeholders in the empirical research, especially in the documentation of TNC practice on the ground in host countries. Field informants in Argentina, Cambodia, Laos, Mali, Peru, and Uganda visited power plants and adjacent communities and conducted semi-structured, open-ended interviews with individuals from a range of stakeholder groups that can be classified in the following nine categories: corporate managers; business association representatives; workers; trade union representatives; community residents; national, regional, and municipal elected officials; national and local electricity sector regulators; environmental and consumer civil society organizations (CSOs) and academics and independent researchers. In total, 217 individuals in six different host countries in the Global South were interviewed about the SEP-related performance of the respective case-study company. Though the focus of the interviews was the company's performance on the 11 SEP benchmarks, the interviews were intentionally conducted in a semi-structured and open-ended manner in order to allow for developing unforeseen lines of inquiry that arose during the interview. Many interviewees raised issues not anticipated by the author and field informants.

The interviews undertaken in the host countries were "grounded" in the sense that there was no pre-conceived hypothesis built into the questioning, and the responses and data provided by the interviewees were taken at face value. The wide range of stakeholder types interviewed in each country enabled a "crosschecking" of responses from interviewees and thus allowed the author to minimize potential bias from any one interviewee. All interviews and communications with local managers and other stakeholders in the host countries were conducted in local languages (either through the field informants themselves or with the help of interpreters). This empirical methodological approach is similar to that frequently employed by SOMO and taken in other cross-cultural energy project studies such as those by Rochlin and Meier (1994) and Sovacool and Bulan (2011).

Special care was taken in interviews with workers and representatives of affected communities. These stakeholders were interviewed individually or in small "focus groups" of three to nine workers. Interviews took place in locations where the interviewees felt safe to speak freely, often away from the grounds of the company's operations. They were offered the option of remaining anonymous in order to encourage candor. Many of the workers and community members interviewed requested anonymity for fear of reprisals from company management. In addition, a number of company managers in host countries spoke to the field informants on condition of anonymity because they were not authorized by higher management to speak on behalf of the company. The identities of all individuals who requested anonymity have 
been kept confidential. Table 2.1 presents an overview of the field work in the six host countries, including dates, number and types of interviews, and names of field informants (who are also co-authors on the respective company case study chapters).

\section{Another crucial element of the research} methodology was the "company review". Each of the five companies was given the opportunity to review a draft of the company profile and to provide comments, feedback, and corrections before the profile was incorporated into the analysis. Both headquarter-level and local-level management representatives of each of the five companies were given the opportunity to review all of the information related to their company. The draft sent to each company for review included information about that company only, not about the other companies in the study. Two months prior to sending the draft, a letter announcing the research and review process was sent to the companies requesting that each identify the appropriate manager in

Table 2.1: Overview of field work in host countries

\begin{tabular}{|c|c|c|c|c|}
\hline $\begin{array}{l}\text { Host country } \\
\text { (relevant TNC) }\end{array}$ & $\begin{array}{l}\text { Date of } \\
\text { interviews }\end{array}$ & $\begin{array}{l}\text { Number of } \\
\text { interviews }\end{array}$ & $\begin{array}{l}\text { Type of interviewee (number interviewed } \\
\text { per type) }\end{array}$ & $\begin{array}{l}\text { Field informants / co- } \\
\text { authors on case studies } \\
\text { (organization) }\end{array}$ \\
\hline $\begin{array}{l}\text { Argentina (AES } \\
\text { and Endesa) }\end{array}$ & $\begin{array}{l}\text { July/Sept } 2008 \\
\text { and } \\
\text { Dec } 2011\end{array}$ & 37 & $\begin{array}{l}\text { Corporate manager (1), workers (14), union } \\
\text { representatives (3), community residents } \\
\text { (19), CSO representative (1) }\end{array}$ & $\begin{array}{l}\text { Belén Esteves and María } \\
\text { Marta di Paola (FARN) }\end{array}$ \\
\hline $\begin{array}{l}\text { Cambodia } \\
\text { (Datang) }\end{array}$ & Jan 2010 & 46 & $\begin{array}{l}\text { Corporate managers (6), business } \\
\text { association representatives (2), workers } \\
\text { (7), community residents (15), government } \\
\text { officials (3), CSO representatives (8), } \\
\text { academics (5) }\end{array}$ & $\begin{array}{l}\text { Prof. Dr. Qin Hui and Liu } \\
\text { Zhi (Transition Institute) }\end{array}$ \\
\hline $\begin{array}{l}\text { Lao PDR } \\
\text { (Datang) }\end{array}$ & Aug/Sept 2009 & 21 & $\begin{array}{l}\text { Company managers (4), government } \\
\text { officials (4), workers (5), CSO } \\
\text { representatives (5), academic s (3) }\end{array}$ & $\begin{array}{l}\text { Prof. Dr. Qin Hui and } \\
\text { Wu Aoqi (Transition } \\
\text { Institute) }\end{array}$ \\
\hline Mali (Eskom) & $\begin{array}{l}\text { Nov/Dec } 2010 \\
\text { and Mar } 2012\end{array}$ & 47 & $\begin{array}{l}\text { Corporate managers (12), workers (2), } \\
\text { community residents (7), government } \\
\text { officials/ regulators (19), CSO } \\
\text { representatives (7) }\end{array}$ & $\begin{array}{l}\text { Yanick Tchamba } \\
\text { and Tagbo Agbazue } \\
\text { (Influence Africa), } \\
\text { Michael Koen (CSRSC) }\end{array}$ \\
\hline $\begin{array}{l}\text { Peru (Endesa } \\
\text { and SN Power) }\end{array}$ & $\begin{array}{l}\text { July/Sept } 2008 \\
\text { and April } 2012\end{array}$ & 20 & $\begin{array}{l}\text { Corporate manager (1), worker (1), union } \\
\text { representatives ( } 2 \text { ), community residents } \\
\text { (8), government officials (4), electricity } \\
\text { sector regulators (4) }\end{array}$ & $\begin{array}{l}\text { Themis Castellanos } \\
\text { (PLADES) }\end{array}$ \\
\hline Uganda (Eskom) & $\begin{array}{l}\text { Nov/Dec } 2010 \\
\text { and } \\
\text { Oct } 2011\end{array}$ & 46 & $\begin{array}{l}\text { Corporate managers (10), union leader } \\
\text { (1), community residents ( } 2 \text { ), government } \\
\text { officials (3), regulators (4), CSO } \\
\text { representatives (26) }\end{array}$ & $\begin{array}{l}\text { Dr. Victor Madziakapita } \\
\text { and Tagbo Agbazue } \\
\text { (Influence Africa), } \\
\text { Michael Koen (CSRSC) }\end{array}$ \\
\hline
\end{tabular}


the company to be the contact person for the review. Two weeks prior to sending the draft company profile for review, the contact person was informed about the exact date of the arrival of the draft and asked to reserve time in his or her schedule to answer the questionnaire. When the draft was sent, the company representatives that had been identified in the pre-review process were given two weeks to provide comments on and corrections to the draft. Follow-up phone calls served to verify and clarify the comments. At the request of Eskom managers, a follow-up interview was held with three Eskom managers at the Eskom headquarters in Johannesburg, South Africa, on 11 September 2012.

Comments from the companies were incorporated into the drafts wherever relevant, and factual inaccuracies identified by the companies were corrected. Findings that the companies objected to were not necessarily removed, but the company's objection and reasons were included with the findings. The company review and feedback process is a critical part of the methodology, not only because it increases the accuracy and quality of the research findings and the information about each company, but also because it provides an additional mechanism for gauging the TNCs' openness to and transparency in responding to inquiries about CR for SEP. Three companies (Endesa, Eskom, and SN Power) responded to the review. Two companies (AES and Datang) did not respond. Whether or not a company responds to the questionnaire and review process is an indication of how important the concepts of CR and stakeholder engagement are to a company (Welford 2004). This element is thus included as an indicator in the operationalization of the normative benchmarks used in the empirical analysis.

Chapters 5 through 9 present the empirical research results and associated analysis of the performance of each of the five companies on the 11 SEP benchmarks. At the start of each case study chapter, the results are synthesized systematically in an (extensive) table reflecting the SEP benchmarks and the company's performance in policy and practice. Again, the presentation of the data in table format is regarded as an essential tool for comparative case-study analysis and one that is conducive to the type of structured qualitative analysis employed by the present dissertation (Yin 2003). 


\section{Chapter 3}

Defining "quality kilowatts": Normative standards for sustainable electricity provision in the global south
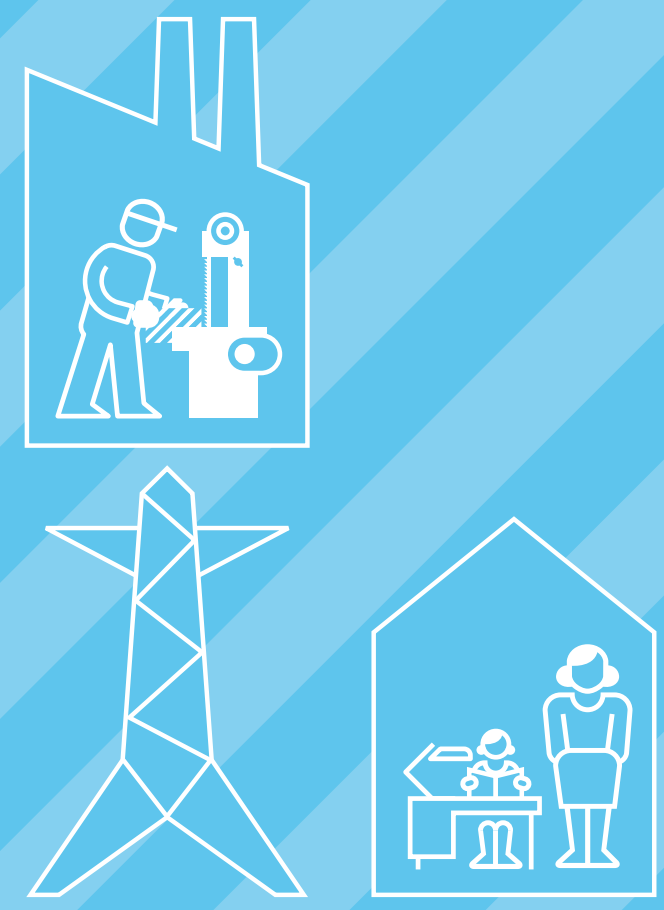
Despite the increase in activity related to sustainable development and CR in the electricity sector in recent years, there was still no single set of internationally-endorsed normative standards for SEP available at the conclusion of documentation. This chapter provides an overview of critical issues for SEP found in the literature and in the various normative standards related to CR for sustainable development that currently exist. The primary sources of the standards are international agreements and conventions, codes of conduct, civil society positions, and industry best practice. The chapter concludes by condensing the diverse standards and norms into a single set of SEP benchmarks that can be used to analyze the performance of the case study companies. The purpose of the exercise is not to impose a universal understanding of "sustainable electricity provision", but rather to provide a means for empirically assessing specific attempts to provide electricity in a manner consistent with the principles of sustainable development.

The "critical issues" for SEP outlined in Table 3.1 represent a comprehensive list of concerns, problems and criteria identified in the literature. These issue areas provide general guidance as to internationally recognized normative standards related to SEP in the Global South. The critical issues can be roughly categorized according to the three sustainable development "pillars": social, environmental, and economic. A number of "cross-cutting" critical issues cut through all three pillars. How companies balance the various concerns and interests within these three pillars and the crosscutting issues indicates how they perceive and implement "quality kilowatts" in a sustainable development context. For example, providing electricity can alleviate poverty and provide much-needed jobs and infrastructure to local communities. This must be balanced, however, with the impacts on biodiversity, the climate, and the local environment associated with electricity provision.

It is also necessary that the normative issues in question should be practically applicable within the contexts of individual "host countries" (the settings for electricity provision), including underlying conditions such as the state of economic development, the nature of the economy, its geography, natural (energy) resource availability, and the state of the electricity system. The standards and recommendations are oriented directly toward corporations active in electricity provision. It is assumed that international human rights, labor, and environmental conventions apply not only to states, but also to other actors, including corporations, that operate within the domains covered by such agreements (ICRC 2006). The Special Representative of the UN Secretary-General on the issue of business and human rights (Ruggie 2008) has emphasized the responsibility of individual companies to respect all human rights. The recommendations expressed in the normative standards are intentionally general because they will need to be adapted to fit individual energy systems, situations and projects. While the responsibility of corporations to address sustainability issues 
Table 3.1: Overview of "critical issues" in sustainable electricity provision

\begin{tabular}{|c|c|c|c|}
\hline Social issues & Environmental issues & Economic issues & Cross-cutting issues \\
\hline Respect for human rights & $\begin{array}{l}\text { Climate change and GHG } \\
\text { emissions }\end{array}$ & Local economic development & Compliance with the law \\
\hline Access to electricity & $\begin{array}{l}\text { Waste, pollution and } \\
\text { ecosystem impact }\end{array}$ & Reliability of supply & $\begin{array}{l}\text { Poverty reduction and } \\
\text { satisfying basic needs }\end{array}$ \\
\hline Affordability & $\begin{array}{l}\text { Natural resource depletion } \\
\text { and renewable sources of } \\
\text { energy for electricity }\end{array}$ & Operational efficiency & $\begin{array}{l}\text { Precautionary principle } \\
\text { and evaluation of risks and } \\
\text { alternatives }\end{array}$ \\
\hline Respect for labor rights & Biodiversity & Demand-side management & $\begin{array}{l}\text { Transparency and provision } \\
\text { of information }\end{array}$ \\
\hline Displacement & $\begin{array}{l}\text { Energy and resource } \\
\text { efficiency (eco-efficiency) }\end{array}$ & Corruption & $\begin{array}{l}\text { Meaningful stakeholder } \\
\text { consultation and public } \\
\text { participation in decision- } \\
\text { making processes }\end{array}$ \\
\hline Community lifestyle impact & & $\begin{array}{l}\text { Competition and local } \\
\text { market structure }\end{array}$ & $\begin{array}{l}\text { Responsibility for impacts } \\
\text { throughout the value chain } \\
\text { and business relationships }\end{array}$ \\
\hline Public health and safety & & Taxation & \\
\hline Gender equality & & Research and development & \\
\hline Indigenous People's rights & & Due diligence & \\
\hline Consumer rights & & & \\
\hline
\end{tabular}

is highlighted here, it should also be recognized that other crucial actors - governments, regulators, unions and CSOs - also have roles to play in improving the quality and sustainability of electricity provision.

The bulk of this chapter is dedicated to an elaboration of these issues with the aim of "condensing" the various norms, interests, and concerns into a concise set of operational SEP standards that can be used as benchmarks for the analysis and comparison of companies in the present study. The framework of SEP benchmarks that will be used to evaluate the case study TNCs' performance is presented in the final section of this chapter.

\subsection{General approach issues}

Before delving into the social, environmental, economic, and cross-cutting issues that form the basis of the SEP framework, it is important 
to recognize two issues related to a company's general management approach to CR that are at the forefront of determining and understanding how TNCs "take up" and implement normative standards for SEP. These two overarching issues, which are found frequently throughout the literature, are of crucial importance in laying the foundation for a corporation's development and adoption of policies in specific SEP areas, as well as its implementation of those policies on the ground at the operational level.

\subsubsection{Endorsement of international normative standards for CR}

Electric utilities should publicly endorse and align their policies with internationally recognized and verified standards for $C R$ (WBCSD 2002, UNGC 2008). Leading international normative standards for CR include:

- The ILO (ILO 2001) Tripartite Declaration of Principles concerning Multinational Enterprises and Social Policy

- The OECD (2011) Guidelines for Multinational Enterprises

- The UN Global Compact (2008)

- The IFC (2011) Sustainability Framework

- The UNHRC's (2011) Guiding Principles for Business and Human Rights

- The ISO (2011) 26000 Guidance on Social Responsibility

In addition to endorsing these normative standards, companies should make use of standardized management procedures and measurements in implementing their $\mathrm{CR}$ policies (OECD 2011).

\subsubsection{Commitment to CR in core-business activities and decision-making}

Electricity companies should adopt a holistic and integrated view of the role and impacts of electricity provision, considering and balancing the essential elements of economic development, environmental quality and social equity in utility operations. This entails a policy commitment to CR throughout all corebusiness activities, as opposed to a primary focus on philanthropic activities (WBCSD 2002, MVO Platform 2012). Electric utilities should seek to introduce environmental and social factors and procedures into high-level corporate planning and decision-making and should aim to have a board-level position responsible for CR issues (WBCSD 2002).

\subsection{Social issues}

Social issues related to SEP refer to the impact that an electricity company's operations have on the social well-being of a country or community, and they are of particular interest in the Global South (Visser 2008). It is clear that electricity provision has tremendous implications for poverty, human rights, and development. With this in mind, electricity companies must be conscious of issues such as affordability of and access to the services they provide. In addition, due to the large scale of power plants and electricity infrastructure, electric utilities' assets and operations can have far-reaching impacts on the communities in which they operate, as well as in neighboring 
areas. Furthermore, in order to provide highquality service, electricity companies rely on a highly skilled workforce that is often exposed to difficult and dangerous working conditions. Electricity companies have a responsibility to provide electricity in a manner that is socially equitable and safe, contributes to poverty alleviation and is respectful of employees and of the communities impacted.

\subsubsection{Respect for human rights}

There is considerable support for viewing human rights as a fundamental normative reference point in any debate on CR in electricity provision projects (World Commission on Dams 2000, Welford 2004, Tully 2006, UNGC 2008, OECD 2011, UNHRC 2011). The Special Representative of the Secretary-General on the issue of human rights and transnational corporations and other business enterprises has affirmed the responsibility of TNCs to respect all human rights and to develop concrete programs and actions for discharging this responsibility (Ruggie 2008). As defined by the UNHRC (2011) and for the purposes of the present study, "human rights" comprise all economic, social, cultural, civil, and political rights.

Transnational electricity companies should endorse, observe and promote internationally recognized human rights, based on the following international standards:

- The UN Universal Declaration of Human Rights and its Protocols

- The International Covenant on Civil and Political Rights
- The International Covenant on Economic, Social, and Cultural Rights

- The UN Declaration on the Rights of Indigenous Peoples

- ILO Convention 169 on Indigenous and Tribal Peoples

- The UN Guiding Principles on Business and Human Rights

- The UN General Assembly Declaration on the Right to Development

- The Vienna Declaration and Programme of Action

- The OECD Guidelines for Multinational Enterprises

- The Rio Principles.

Human rights are more vulnerable in the Global South than in the industrialized world due to weak governance or enforcement (OECD 2006). It is thus crucial that electricity TNCs conduct risk-based due diligence to identify, prevent, and mitigate adverse human rights impacts of their own activities and those of their business relationships (e.g. in the supply chain) (OECD 2011, UNHRC 2011). Transnational electricity companies operating in the Global South should take extra care in ensuring that international standards for human rights are complied with and see to it that investment agreements include clauses protecting human rights or have agreements screened to make sure there are no risks of human rights violations. The human rights situation in a potential host country should be evaluated before a decision to invest is made in order to ensure that no human rights violations were perpetrated to promote electricity projects in that country. 
Companies also need to screen suppliers and (sub)contractors for human rights compliance. Employees should be trained in human rights aspects relevant to their work, in particular those employees or contractors responsible for security and protection of the TNC's property and staff.

In addition to the rights outlined in these international standards, electricity TNCs operating in the Global South should refrain from activities that obstruct individuals' rights to health, food, education, housing, and cultural affairs. They should also ensure that their activities do not directly or indirectly contribute to war or war crimes, particularly when operating in conflict areas.

Given the significance of rights-related issues for all parties concerned in electricity provision projects, TNCs should clarify the rights context as an essential step early in a proposed electricity project. As the first part of the "rights and risks approach" to electricity projects promoted by the World Commission on Dams (2000), such an exercise should identify the legitimate entitlements and claims that the proposed project might affect.

\subsubsection{Access to electricity}

In addition to ensuring that internationally proclaimed human rights are not violated, there is growing support among governments and intergovernmental organizations for viewing access to electricity itself as a human right that electricity providers must respect and support. Tully (2006: 30) notes, "Access to electricity is well established within the UN-based framework of human rights, either as an implicit attribute of a pre-existing right (such as non-discrimination or sustainable development) or explicitly in the context of eliminating discrimination against women. There is also broad acknowledgement by states of the desirability of eliminating energy poverty - for all, but particularly for the rural poor, and women."

Approximately 1.6 billion people - nearly one-third of the Earth's population - lack access to electric services or have insufficient access to provide for basic needs. Lack of access to modern energy services such as electricity is particularly acute in rural areas, but impoverished urban areas such as inner-city slums also often have little or no access to electricity. While countries in the Global South need not adopt unsustainable Western standards and patterns of electricity consumption, access to electricity is increasingly recognized as a basic human right.

Access to reliable electricity is a crucial factor for meeting the first Millennium Development Goal of halving the proportion of people living in poverty by 2015, as well as meeting other MDGs such as achieving universal primary education and reducing child mortality. Access to electricity can improve standards of living in numerous ways, for example, by helping spread literacy and education, contributing to improved heath through refrigeration of medicines, and increasing communication and awareness (SEW 
2006). In areas without access to electricity, up to six hours a day is spent (usually by women) collecting wood, dung, charcoal or whatever other fuel is available for heating and cooking. These fuels are then burned in the home, often without proper equipment or ventilation, creating a serious health hazard. When access to electricity is extended to rural areas, it raises the quality of life there and can have the indirect effect of stemming migration flows from rural regions to overpopulated and marginal urban areas (Legisa and Bohorquez 2007).

Additional references to electricity in international law and international normative standards/frameworks include:

- State parties to the 1979 Convention on the Elimination of All Forms of Discrimination Against Women (CEDAW) are obligated under Article 14(2)(h) to "take all appropriate measures to eliminate discrimination against women in rural areas...and, in particular, shall ensure to such women the right...to enjoy adequate living conditions, particularly in relation to...electricity".

- Article 11(1) of the 1966 International Covenant on Economic, Social and Cultural Rights recognizes "the right of everyone to an adequate standard of living...including... housing, and to the continuous improvement of living conditions". Electricity access is accordingly addressed in the context of the human right to adequate housing. For example, the mandate of the UN Special Rapporteur on adequate housing has been interpreted as such that the right to housing includes access to essential civic services such as electricity.

- The WBCSD (2002) insists that electric utilities should contribute to poverty alleviation through promoting access to electricity, particularly for services such as health and education. Electric utilities should contribute to alleviating fuel poverty through pricing power at affordable levels and should seek pricing and market reforms that are fair and affordable and noting the need to sustain electricity supply to disadvantaged communities.

- PSI asserts that electricity providers must contribute to achieving the MDGs by making electricity equally available for all, irrespective of employment levels, income levels, geographic location, age, gender, religion, national identity, race or ethnicity, disability, and sexual orientation (Pillinger 2009).

Several individual states have recognized or are recognizing that electricity access qualifies as a human right. For example, Section 10(1) of the South African Electricity Act (No 41) 1987 imposes duties upon electricity service providers to supply electricity "to every applicant who is in a position to make satisfactory arrangements for payment". This obligation was interpreted by the South African High Court during 2003 in the matter of Hendrick Frederick Meyer v. Moqhaka Local Municipality to the effect that applicants enjoy a prima facie right entitling them to demand electricity once they have satisfied this supply condition (Tully 2006). However, delivering electricity to rural 
populations is a challenging task because it involves remote areas with dispersed customers whose consumption is low. This means it is generally more expensive (costs of connecting new customers increase exponentially with the customer's distance from a power station), while at the same time the customer base is generally poorer and less able to pay the full cost of service (Zerriffi 2007). Private utilities that expand into unserved areas tend to limit their expansion to strategic demand points to minimize investment costs and increase profitability, but this strategy can have serious negative impacts for long-term electricity system planning and future expansion of services (Zvoleff et al. 2009).

One of the primary indicators of access that companies should report on is the percentage of households (or population) in the company's licensed areas of operations (broken down into rural and urban) without electricity (UN/IAEA 2007, GRI 2008). In order to increase access to electricity, companies should undertake initiatives to extend electricity services to unserved and underserved communities, particularly in rural or remote areas, but also to the poor in urban and peri-urban areas (Modi et al. 2005). Such initiatives are often undertaken in partnership with local municipal, state and national governments and should be carried out in collaboration with local community organizations. Companies should also address language, cultural, low literacy and disability related barriers to accessing and using electricity services. Rural electrification schemes often need to utilize and stimulate small-scale and decentralized technologies such as small-scale hydro plants, wind turbines, photovoltaic panels. Such decentralized technologies are not only more sustainable, but are often more cost and energy efficient (Bambawale et al. 2011). Companies should invest in these technologies and stimulate their use in rural areas.

\subsubsection{Affordability}

Even in places where the power grid is accessible, electricity prices are often beyond the financial means of large portions of the population in many, primarily developing, countries. In fact, consumers in the Global South sometimes pay more for electricity than their counterparts in industrialized countries, despite the fact that the costs of labor and other imports are generally cheaper in the the Global South. For example, residents of São Paulo, Brazil, and Santiago, Chile, pay twice the average rate residents pay in the US, despite the fact that the US lacks the abundant hydro resources found in the Latin American nations (Palast et al. 2000). Poor customers who cannot pay their electricity bill are then often cut off by electricity companies, depriving them of a life-essential service, and reconnection fees imposed by companies after a temporary disconnection can often be prohibitively expensive. PSI (Pillinger 2009) insists that electric utilities must contribute to achieving the MDGs by making electricity equally available for all, irrespective of employment levels, income levels, geographic location, age, gender, religion, national identity, race or ethnicity, disability, and 
sexual orientation. The WBCSD (2002) concurs, noting that fuel poverty particularly affects vulnerable communities in the Global South, and that electricity providers should contribute to alleviating this through pricing power at affordable levels.

Indicators that can be used to measure the affordability of electricity include the end-use price of electricity with and without tax/subsidy and the ratio of daily disposable income of the poorest $20 \%$ of the population to the price of electricity (UN/IAEA 2007). Companies should also report to stakeholders on the number of debt recovery actions and disconnections for non-payment as well as the duration of disconnection and average reconnection time for customers disconnected for non-payment (GRI 2008).

Companies should aim to provide electricity at prices that are just, reasonable, and payable for local incomes (Palast et al. 2000). This means that efforts to extend access to electricity services to the wider population should be complemented by social tariff schemes to ensure that new users and those of genuinely limited resources have access to enough electric power to satisfy basic needs. In situations where the local government or regulator sets the prices, companies should advocate pricing reforms that are fair and affordable and noting the need to sustain electricity supply to disadvantaged communities (WBCSD 2002). At a minimum, electric utilities should advocate openness and transparency in the price setting process. In addition, companies should have programs in place to enable underprivileged, low-income or vulnerable customers to afford electricity connection and consumption. Such programs include lower tariffs for small users, flexible billing arrangements, timely reconnection and other practices to assist customers in managing debt and avoiding electricity disconnection (GRI 2008). These types of programs are often undertaken in collaboration with local government and generally require the cooperation of the local regulator. Companies should also acknowledge that the introduction of prepaid metering systems, a practice frequently used by utilities in low-income and hard-to-collect areas, can be prohibitively expensive and cause customers to disconnect themselves.

\subsubsection{Respect for labor rights}

There are numerous international normative standards on labor issues that must provide the basis for any normative benchmark regarding SEP. The most relevant internationally recognized labor standards include:

- The UN Universal Declaration of Human Rights and its Protocols

- The International Covenant on Civil and Political Rights

- The International Covenant on Economic, Social, and Cultural Rights

- The ILO Declaration on Fundamental Principles and Rights at Work of 1998 (particularly the Core Conventions of the ILO)

- The OECD Guidelines for Multinational Enterprises, Chapter IV on Employment and 
Industrial Relations

- The ILO Tripartite Declaration Concerning Multinational Enterprises and Social Policy, and

- The Vienna Declaration and Program of Action.

Electricity TNCs should base their labor policies on international standards and should respect workers' full rights and provide workers with fair pay, job security, and decent working conditions, including high levels of health and safety standards in all aspects of operations (WBCSD 2002, Pillinger 2009, OECD 2011). Companies should pay special attention to issues in the following areas.

\section{Forced and child labor}

Both forced labor and child labor should be strictly prohibited. ILO Conventions 138 and 182 address the elimination of child labor, while ILO Conventions 29 and 105 deal with the prevention of forced and compulsory labor. Principles 4 and 5 of the UN Global Compact (UNGC 2008) instruct companies to work toward the elimination of all forms of forced and compulsory labor and the effective abolition of child labor.

\section{Occupational health and safety}

Work in the electricity industry can be highly dangerous. Laborers often have to work in confined spaces or at high altitudes, and projects often involve close contact with high voltage wires and a significant risk of electrocution. ILO Convention 155 covers workers' right to occupational health and safety
(OHS). Electric utilities should strive towards high levels of health and safety standards in all aspects of their operations (WBCSD 2002). In addition to providing adequate healthcare for their employees, electricity TNCs should ensure the presence of a health and safety committee with worker representation to evaluate and recommend occupational health and safety programs. OHS topics should also be covered in formal agreements with trade unions. Furthermore, companies should report to their stakeholders on rates of injury, occupational diseases, company spending on healthcare, lost days and absenteeism, and number of workrelated fatalities by region (GRI 2008).

Contractors in the electricity industry also engage in high-risk activities, and thus employees of contractors should also be given health and safety training and information. In addition, companies should monitor the health and safety performance of suppliers and contractors.

\section{Dialogue with workers}

Companies should actively encourage dialogue between employees and managers and employee participation in high-level decisionmaking within the company. Group-wide meetings enable employees to voice their opinions and concerns (ECOTEC 2007). Workers should have input on things like the quality and type of training they receive and on recreational and leisure activities. Companies should conduct regular, periodic employee satisfaction surveys and have in place a complaint mechanism allowing workers to (anonymously) 
express grievances. Companies should ensure that CR polices are developed through meaningful consultation and negotiation with workers and unions.

\section{Freedom of association, collective bargaining and right to strike}

ILO Conventions 87 and 98, expanded by Convention 135, assert the right of workers to organize and to collective bargaining. Companies should uphold and publicly recognize these rights (UNGC 2008), and strive to have as high a percentage of employees covered by collective bargaining agreements as possible. Furthermore, due to the electricity industry's need to ensure continuous provision of services, electricity workers' right and ability to strike may be at greater risk than in other industries. Electricity companies should have management mechanisms in place to address the right to strike or instances of lock out. Where workers do not have the right to strike, companies should have in place remedial measures, e.g. binding arbitration (GRI 2008).

\section{Skills training and lifelong learning}

Electricity workers must be highly skilled in order to perform the technical and specialized tasks required of the industry. A highly skilled workforce is essential in order to ensure a highquality electric service as well as the safety of the operations, both for the workers themselves and the community in which they operate. Companies should encourage lifelong learning among employees, including through ongoing higher education and continuous skills training in apprenticeship programs and leadership training. Encouragement and recognition of innovation among employees can stimulate continuous employee development. Companies should also seek to facilitate skills transfer to the local population and address the issue of "brain drain", which affects many countries in the Global South, by offering educated and skilled locals the opportunity to apply their knowledge locally.

\section{Working hours and wages}

ILO Convention 1 relates to the requirement to observe a limit on the maximum number of working hours, and Article 34 of the ILO Tripartite Declaration addresses the rights of workers to a living wage. Companies should observe these standards based on the local "living wage" calculations of the countries in which they operate.

\section{Diversity and opportunity}

ILO Conventions 100 and 111 ban discrimination. Electricity TNCs should ensure equal opportunity for all employees regardless of gender, race, age, other minority groups, both in general employment and in management. Companies should also make sure that women and men receive equal pay for equal work (UNGC 2008).

\section{Job security}

Maintaining a highly-skilled workforce is essential for ensuring the quality and safety of the electricity system for the society it serves. Articles 24-28 of the ILO Tripartite Declaration address security of employment. Companies should report to their stakeholders on staff turnover and layoffs and should take into 
account the impact that exiting or transferring operations has on workers, and to put into place retraining programs as necessary.

\section{Rights of workers at business relationships}

The use of contractors and contracted labor by electricity companies at various stages of the product chain is extensive in the electricity industry and is only increasing with time. Contractors and contracted labor can be working onsite or offsite on behalf of a company, and the performance of contractors can have a significant impact on the quality of electricity services. Contracted and temporary workers, however, are often not provided the same benefits as full-time employees and are often not covered by collective bargaining agreements. It is thus crucial for power companies to monitor the performance of contractors in order to make sure working conditions meet the same standards as those expected for workers employed directly by an electricity company. Companies should ensure that full workers' rights - including fair pay, job security, and decent working conditions, and also including high levels of OHS standards - are respected not only among their own employees, but also among suppliers, contractors, and other business relationships (ISO 2011, OECD 2011, UNHRC 2011).

\subsubsection{Displacement}

Large electricity infrastructure projects frequently require the physical and/or economic displacement of large numbers of people. The GRI (2008) notes, "Physical displacement can be defined as relocation or loss of shelter. Economic displacement means loss of assets or access to assets which results in loss of means of livelihood." Both types of displacement can affect the quality of life of the displaced.

Companies should first and foremost prioritize alternatives that avoid or minimize population displacement (IHA 2004). If displacement appears necessary, companies should ensure that consultations take place in which affected people have a formal and meaningful role (OECD 2011). Those affected should be provided with a draft resettlement plan with a budget and timeline. Companies should ensure that any and all people who are to be displaced have given their free, prior informed consent to the resettlement plan (IFC 2011). Compensation, such as landfor-land arrangements, and improved living standards, where such improved living standards are derived through decision-making with the displaced, should be offered to the displaced, and grievance mechanisms for complaints about resettlement and compensation should be in place. There should be periodic evaluation of progress, and programs to help displaced indigenous people ensure social and cultural identity (IHA 2004, IFC 2006, IFC 2011).

Displacement can also occur in an electricity company's supply chain as an indirect result of the power company's operations - for example, when a coal mine begins or expands production to feed a power plant. For more on supply chain issues, see Section 3.4.6 on corporate responsibility for impacts among business relationships. 


\subsubsection{Community lifestyle impact}

Electricity projects can have significant impacts on the lifestyle of communities near electricity infrastructure, even if those communities are not necessarily displaced by the project. For example, construction of a large power plant or transmission line that results in an influx of workers into neighboring communities can cause changes to local social structures and culture. Such projects can also change traditional land-use patterns, including access to land, natural resources, and heritage and the loss of global commons. Electricity projects can impact existing infrastructure such as roads and housing, as well as access to services like education, utilities, and healthcare. Finally, electricity infrastructure often changes the aesthetics of a local landscape, which can have physical and psychological impacts on adjacent communities (GRI 2008). Companies should evaluate and address all such community lifestyle impacts when planning projects and consult with the affected communities before, during, and after construction of installations (IHA 2004, IFC 2011, OECD 2011).

Changes in existing electricity projects and infrastructure can also have an impact on communities. For example, the decision to shut down or abandon an electricity project can have a significant impact on local communities if large numbers of jobs are lost. Companies should also be aware of and address such impacts by consulting with communities before making any decisions about altering existing infrastructure.

\subsubsection{Public health and safety}

A top priority of electricity TNCs operating in the Global South should be the safety of their electricity infrastructure and assets, including generation plants, transmission lines, distribution stations, and individual hook-ups. This is particularly relevant for nuclear and large hydroelectric plant operators, where reactor or dam failure could have catastrophic consequences for life, property, and environment. Low-quality power lines can also present a significant threat to public health and safety. Sub-standard distribution lines are frequently used in the Global South, with utility companies often arguing that low cost power lines are necessary to reduce costs so that more households can be connected. Additional community health risks can include issues such as electro-magnetic fields, emissions, noise, and diseases. Furthermore, power outages and other supply disruptions can endanger public health and safety if, for example, hospitals, transportation infrastructure or security systems are left without power, or through paraffin fires or generator accidents during load shedding.

Companies should factor safety into all phases of an electricity project, from planning and design to construction and operation, and invest appropriately in improving the safety of electricity infrastructure. Comprehensive risk assessments should be carried out and safety requirements should be identified and defined. Companies should develop processes for assessing community health risks including monitoring, prevention measures and, if applicable, long-term health-related 
studies. Safety management programs should include emergency response plans, mitigation procedures, and training of local emergency response personnel to ensure that nearby communities are not exposed to unacceptable risks from potential disasters and accidents. These plans should be developed in collaboration with relevant local stakeholders and authorities (IHA 2004, OECD 2011). All accidental injuries and fatalities related to electricity infrastructure among the public should be reported.

\subsubsection{Gender equality}

MDG 3 calls for the promotion of gender equality and the empowerment of women. Companies should ensure that electricity provision activities directly benefit women and should take into account how the availability and provision of electricity impacts men and women both economically and socially. Women and women's issues should be incorporated into all phases of electricity projects and should be specifically addressed in company policy (Modi et al. 2005). PSI notes that companies should enable women and men to participate equally as workers and electricity users (Pillinger 2009).

\subsubsection{Indigenous Peoples' rights}

Electricity infrastructure can have an impact on land that is traditionally inhabited by Indigenous Peoples. For example, large hydroelectric dams can flood Indigenous Peoples' lands, and power lines often cut through swathes of jungle occupied by Indigenous Peoples.
Companies should ensure that Indigenous Peoples' rights are respected, including rights to land, water, other natural resources, culture and a traditional way of life. These rights are enshrined in the UN (2008) Declaration on the Rights of Indigenous Peoples (UNDRIP) and ILO (1989) Convention 169 on Indigenous and Tribal Peoples. Companies should first and foremost prioritize alternatives that avoid or minimize impacts on Indigenous Peoples. In cases in which Indigenous Peoples' traditional lands, natural resources or cultural heritage are impacted by an electricity project, or if the project requires displacement of Indigenous Peoples, electricity companies must obtain the free, prior, and informed consent (FPIC) of all affected peoples (World Commission on Dams 2000, UN 2008, IFC 2011). If Indigenous Peoples give consent and are displaced, companies must meaningfully involve all affected people in any decision-making related to the resettlement process and have in place programs to help displaced Indigenous Peoples' maintain their social and cultural identity and improve livelihoods (IFC 2011, OECD 2011).

\subsubsection{Consumer rights}

Consumers of electricity depend on the service for many aspects of their daily lives, economic activity and their general health and well-being. The 1999 UN Guidelines for Consumer Protection outlines eight basic principles that companies should observe in their dealing with consumers: consumers' right to access necessary goods and services, to safety, to information, to choice, to be heard, to appeal 
and lodge a complaint, to consumer education and to sustainability. PSI further outlines that the provision of quality public services implies ensuring consumer consultation and participation in the planning/designing, developing, and monitoring of electricity provision (Pillinger 2009).

\subsection{Environmental issues}

The production, transmission, distribution and use of electricity all create pressure on environments and ecosystems in the household, the workplace, the community, the city, and the natural surroundings at the national, regional, and global levels. It is therefore imperative that electricity companies should strive to minimize the environmental impact over the full life cycle of their product, from inputs such as fuels, water, and materials, to waste products such as emissions and effluents. The electric power industry is among the world's largest consumers of fossil fuels and, as a result, the largest anthropogenic source of carbon dioxide emissions, making fuel use and mix a critical concern. If not properly addressed by the industry and companies, power generation can result in significant negative environmental impacts such as diminishing soil, water, and air quality; climate change; loss of biodiversity, in which Southern countries are particularly rich; production of radioactive waste; and acid rain. In the Global South, where large numbers of people live in precarious situations, environmental problems, climate change, and pollution affect a greater number of people and have a more direct and more acute impact on people.

Furthermore, electricity infrastructure (such as hydroelectric dams and high-voltage transmission grids) is often located in ecologically sensitive areas that are rich in biodiversity. Electricity companies thus have a responsibility to ensure the environmental sustainability of their operations by using renewable fuels, developing alternative technologies, installing pollution control systems, seeking to minimize environmental impacts, and reducing demand by promoting efficiency and other demand-side management.

Some of the most relevant international environmental standards that apply to electricity companies providing services in the Global South include: the 1992 Rio Declaration on Environment and Development, the 1992 Convention on Biological Diversity, the 1997 Kyoto Protocol, and Chapter V on Environment of the OECD Guidelines for Multinational Enterprises (2000). In addition, the role of private companies in sustainable development was emphasized at the 2002 Johannesburg UN World Summit on Sustainable Development. There are also a wide range of conventions and treaties addressing the responsibilities of corporations with regard to their impact on natural ecosystems, air, water, soil, climate, health, and biodiversity. In general, companies should minimize the negative environmental impacts of their activities and should at least follow the most important principles for 
environmental sustainability expressed in the above-mentioned standards, including:

- The precautionary principle (Principle 15 of the Rio Declaration)

- The principle of preventative action

- Addressing environmental damage at its source

- The polluter pays principle (Principle 16 of the Rio Declaration)

- Stakeholders' right to information about environmental impacts.

One major benchmark for evaluating the environmental sustainability of corporations is the International Organization for Standardization's ISO 14001 certification, which audits the environmental management processes of companies in all sectors. The ISO has steadily won adherents over the past decade, and ISO 14001 certification now covers companies employing at least 20 million people. However, one drawback of ISO 14001 is that it measures environmental management policies and processes rather than the results of company policies and their impact.

As part of a responsible environmental management system, companies should conduct appropriate and thorough environmental impact assessments (EIA) for each electricity project in order to determine the relevant environmental issues that should be addressed, the potential positive and negative impacts of a project, and the mitigation measures necessary. ElAs should be based on factual information, should be appropriate to the size and nature of the project, and should involve consultations with local stakeholders and regulatory authorities as early as possible in the process. The International Hydropower Association (IHA 2004) identifies six crucial steps in an EIA: initial screening, scoping, environmental studies, appraisal, implementation, and monitoring.

While some of the specific environmental issues that need to be addressed will vary per project and per country, the literature identifies a number of environmental sustainability issues that are particularly relevant for all companies involved in electricity provision in the Global South. These issues are discussed in detail below.

\subsubsection{Climate change and GHG emissions}

According to the United Nations

Intergovernmental Panel on Climate Change (IPCC), anthropogenic emissions of greenhouse gasses (GHGs) such as carbon dioxide, methane, and nitrous oxide are causing the Earth's atmosphere to warm, which is altering climates in irreversible ways. Kovats and Haines (2005) estimate that climate change is already causing approximately 150,000 additional deaths a year and is disrupting ecosystems around the world. As a result, climate change has become the preeminent global environmental concern, and the enormous GHG emissions by the power industry is currently the most frequently cited negative environmental impact of electricity provision. Electricity generation is the primary source of $\mathrm{CO}_{2}$ emissions from 
fossil fuels, currently comprising over $41 \%$ of global emissions and on track to contribute over half of such emissions by 2030 (IEA 2009). This means that electricity provision companies are among the world's largest single emitters of GHGs. It also means, however, that improvements in the sources of fuel in the electricity supply mix, in particular a decrease in fossil fuel use, can potentially yield enormous reductions in GHG emissions. The WBCSD (2002) asserts that, "The risks raised by the climate change issue put a responsibility on electric utilities as emitters of greenhouse gases to develop GHG strategies".

The combustion of fossil fuels such as coal, diesel, and gas in electricity generation is the primary source of the electricity industry's GHG emissions. Replacing fossil fuels with renewable energy sources for electricity (RES-E) can significantly reduce GHG emissions.

Electricity generation companies should therefore develop RES-E and include as high a percentage of these as possible in their fuel mix (see also Section 3.3.3). However, in addition to fossil fuel combustion, hydroelectricity can be a significant source of GHG emissions, especially when it involves large reservoirs in tropical climates. $\mathrm{CO}_{2}$ is released by decomposing vegetation and soils trapped under water when the reservoir was first filled. It should be noted that reservoirs also absorb atmospheric $\mathrm{CO}_{2}$ through photosynthesis by plankton and aquatic plants and that this uptake can occasionally exceed $\mathrm{CO}_{2}$ emissions. However, recent studies suggest that reservoirs also release other $\mathrm{GHGs}$, such as methane $\left(\mathrm{CH}_{4}\right)$ and nitrous oxide $\left(\mathrm{N}_{2} \mathrm{O}\right)$, which are much more potent than $\mathrm{CO}_{2}$ (2008). Furthermore, while it has generally been assumed that small-scale runof-river projects (which usually have only very small reservoirs) do not cause significant GHG emissions, a 2008 study by the Swiss Federal Institute of Aquatic Science and Technology revealed that a run-of-river facility was emitting significant amounts of methane. Switzerland is a temperate climate, in which methane emissions from run-of-river reservoirs in the tropics are likely to be even higher (Eawag 2008).

Electricity companies should have in place initiatives and strategies to reduce GHG emissions in both the short and long term. Current GHG emissions (in tons of $\mathrm{CO}_{2}$ equivalent) from electricity production, both in overall amount of GHG emissions and per kWh of electricity generated (i.e. emissions intensity), as well as GHG emissions reduction targets and progress toward the targets should be clearly communicated to stakeholders (UN/IAEA 2007). Although more and more electricity companies are disclosing information about their GHG emissions, a recent study (Procter 2008) found that the type and scale of information provided by differing companies varied so widely that it made it extremely difficult for a layperson to understand what the information means and to compare the performance of the companies.

There are currently a number of initiatives attempting to standardize the reporting of GHG emissions by companies. Going beyond the ISO's 14001 standard, which says nothing about corporate contribution to climate change 
because it audits policies rather than practice/ results, ISO 14064 (2006) and ISO 14065 (2007) aim to standardize the methodologies for measuring GHG emissions. Unlike ISO 14001, ISO 14064 is not a certification standard, but rather a tool/checklist of essential accounting elements in verifying emissions. ISO 14064's companion, ISO 14065, accredits verification organizations. The uptake by companies and thus impact of these new schemes is still very limited (Procter 2007). In addition to the ISO, the World Resources Institute (WRI) and the World Business Council for Sustainable Development's (WBCSD) 2005 Greenhouse Gas Protocol for Project Accounting also attempts to standardize corporate efforts to reduce emissions. Currently, however, although the WRI/WBCSD protocol and ISO 14064 provide indications of essential elements and critical issues, neither can be considered a standard for accurately determining emission reductions.

Since its formation in 2000, the Carbon Disclosure Project (CDP 2011) "has become the gold standard for carbon disclosure methodology and process, providing primary climate change data to the global market place" and aims to help organizations and countries to "measure and disclose their greenhouse gas emissions and climate change strategies, in order that they can set reduction targets and make performance improvements. The CDP is an independent, not-for-profit organization holding the largest database of corporate climate change information in the world". The data is obtained from responses to CDP's annual "Information Requests", issued on behalf of institutional investors, purchasing organizations and government bodies. Electricity companies should disclose climate change data to CDP.

Emissions trading schemes in Europe and some states in the US provide carbon offset credits for renewable energy projects in the Global South through the Kyoto Protocol's Clean Development Mechanism (CDM). However, companies often use offset projects in the Global South to balance out increased carbon emissions in industrialized countries. Companies should report on their net carbon reduction (or lack thereof) and include alternate net emissions scenarios where the utility simply reduces emissions as opposed to using an offset project. Opportunity costs associated with a carbon trade entered into by the utility should also be estimated. Offset projects can generate significant additional revenues for companies. For example, SN Power's La Higuera project in Chile, which is CDM certified, is expected to generate approximately US\$9.4 million in revenue per year.

Companies should also take responsibility for the fact that their electricity provision activities create indirect GHG emissions related to the extractive and productive processes throughout their supply chains. There is growing recognition that indirect GHG emissions from the supply chain are the responsibility of the electricity provision companies. In early 2009, CDP began asking all companies listed within London's FTSE 350, New York's Standard \& Poor's 500 and other global stock exchanges not only to 
report on their own emissions, but also to send carbon disclosure questionnaires out to their suppliers.

\subsubsection{Waste, pollution, and ecosystem impact}

Waste products from electricity provision take the form of emissions or discharges to land, water, and air, and represent a major sustainable development challenge for companies. This waste, combined with the use of natural resources for electricity provision, creates an ecological footprint on atmospheric, terrestrial, and aquatic ecosystems. Electric utilities should contribute to the protection of the environment and ensure that electricity provision is environmentally sustainable (WCD 2000, UNGC 2008, Pillinger 2009). In practice, doing so implies implementing policies and practices aimed at the continual reduction of emissions, developing and implementing technologies that are low-pollution and environmentally friendly (WBCSD 2002). In cases where proposed activities may have a significant impact on the ecosystem, electric utilities should prepare an appropriate environmental impact assessment (OECD 2011).

\section{Atmospheric impact}

The combustion of fossil fuels to produce electricity can result in the emission of a range of different types of air pollutants such as $\mathrm{SO}_{2^{\prime}} \mathrm{NO}_{\mathrm{X}^{\prime}}$ mercury, particulates such as ash and dust, CO and VOC. The release of these waste products into the air can have a negative impact on the health of humans, animals, and the ecosystem. Companies should minimize air pollutant emissions from their operations, both in overall amounts as well as per $\mathrm{kWh}$ of electricity generated (UN/IAEA 2007).

\section{Terrestrial impact}

Electricity provision and the waste it generates can have an impact on terrestrial ecosystems and landscapes in myriad ways. For example, electricity generation produces solid waste which, if improperly disposed of, can decrease the quality of the soil and harm the health of humans, animals, and plants. Waste storage in tailings dams can leach harmful chemicals into soil and water and, without permanent treatment solutions, can transfer environmental costs and liabilities to future generations. Acid rain caused by air pollution can cause soil acidification to exceed its critical load. Construction of power plants and transmission lines can lead to deforestation and erosion.

Companies should minimize the solid waste produced by their activities, both overall and per kWh of electricity generated. Fossil fuel-based electricity generation has a much higher ratio of waste to electricity generated than generation based on renewable sources of energy. Waste that is produced must be properly disposed of, according to environmental management plans that have been developed in consultation with the community.

Electricity generation from nuclear fuel produces various types of solid radioactive waste with high, medium, and low-level radioactivity (UN/IAEA 1994). As a result 
of the lack of a technological solution for permanently treating nuclear waste to rid it of dangerous radioactivity, generation of electricity from nuclear fuel represents a transfer of costs and environmental liability to future generations and is therefore considered unsustainable. Those electricity companies that do generate power from nuclear fuel, despite its non-sustainable nature, should have a comprehensive management strategy for mitigating the environmental and health and safety risks to surrounding communities, ecosystems, and workers; storing the various types of radioactive waste generated; and should have in place plans for phasing out nuclear power and replacing it with sustainable sources of energy.

In addition to the waste produced, electricity provision can affect land in other ways. Large hydro facilities using dams and reservoirs, for example, have a large terrestrial footprint, whereas the impact of wind farms on other land uses is limited (IHA 2004). At the same time, wind farms, as well as other electricity infrastructure such as transmission lines, can have an undesirable impact on the visual landscape. Companies should seek to minimize the land area affected by their operations and keep in mind that their production and extractive activities in their supply chain can also have impacts for which they are responsible. For example, coal mining destroys the soil of the land it is mined on, permanently transforms the landscape, and removes ground vegetation. Coal mining also produces pollutants and effluents that can cause deterioration of water, soil and air quality (Fthenakis and Kim 2008). See Section 3.5.6 for more on the indirect impacts of electricity provision and value chain responsibility.

\section{Aquatic impact}

Electricity companies use water and impact aquatic ecosystems during various power generation processes. The most obvious is water's use in hydroelectric power plants that convert the kinetic energy of falling or rushing water into electric energy. Electricity companies also draw water to cool reactors and turbines in thermal and nuclear power plants, as well as for consumption and processing, such as in the treatment of ash generated in thermoelectric plants. Furthermore, electricity transmission and distribution companies frequently use pesticides and herbicides to control pests and vegetation along power line corridors. If not properly managed, these often toxic chemicals can run off into bodies of surface water and leach into groundwater tables.

The International Hydropower Association (IHA 2004) provides a list of water-related sustainability issues that must be taken into consideration for companies planning, constructing, and operating hydropower schemes. Some of the water-related sustainability issues listed by the IHA are water quality (temperature changes, reduced oxygenation, and increased potential for algal bloom), sediment transport and erosion, changes to river hydrology, and downstream flows, passage of fish species, and pest species (flora and fauna) in reservoirs. 
A brief additional explanation of sustainability with regard to the distinction between largeand small-scale hydroelectric facilities is pertinent here, especially given the fact that many countries in the Global South rely heavily on hydropower for electricity generation. The UN Environment Programme (UNEP 2009) sets the upper limit of "small-scale" hydro at $10 \mathrm{MW}$, mini-hydro at $<1 \mathrm{MW}$. Everything larger than $10 \mathrm{MW}$ is thus considered to be "large scale". Although water is a renewable source of energy, large-scale hydro is generally not considered sustainable because of the significant negative environmental impacts of large dams and reservoirs. The World Commission on Dams (2000) asserts that "Where other options offer better solutions, they should be favored over large dams." The IHA (2004) emphasizes that small-scale run-of-river and mini-hydro projects generally have less impact on aquatic ecosystems and resources than larger projects requiring dams and reservoirs. However, while small-scale hydro is generally considered more sustainable, it is not necessarily free of negative impacts. Small-scale run-of-river projects can cause negative effects for aquatic ecosystems by diverting water to feed turbines and thus removing water from long stretches of river.

Hydropower, both large and small-scale, can be part of sustainable energy solutions if they are undertaken as one of a range of options that have been considered on the basis of a proper needs assessment and stakeholder consultations identified needs, and with a thorough review of likely impacts. Governments and electricity TNCs must address issues of integrating hydropower into overall energy and water planning, applying the precautionary principle in evaluating risks and alternatives, and prioritizing impact avoidance over impact minimization.

The IHA (2004) also includes recommendations for how companies can address these issues in a sustainable way and mitigate the negative impacts of their activities on aquatic ecosystems. It is essential that companies conduct adequate data collection and an EIA that identifies potential problems early in the planning stage. Since watersheds and reservoirs are resources that provide multiple public goods to society - such as drinking water, biodiversity, and ecosystem conservation, and irrigation for cropland, in addition to electricity generation - it is important that electricity companies develop integrated management plans regarding how to use water resources sustainably and share them with other uses. Companies should have long-term plans for how to preserve aquatic ecosystems, maintain adequate flows of surface water (both maximum and minimum), and ground water volume, and minimize contaminant discharges in liquid effluents and from pest/vegetation management. Furthermore, electricity companies should recycle and reuse as much water as possible in order to reduce their consumption from watersheds. 


\subsubsection{Natural resource depletion and renewable sources of energy for electricity}

Many countries in the Global South are rich in renewable natural resources such as wind, water, sun, geothermal energy, biomass, and ocean power, giving such countries a high exploitable renewable energy potential. Yet this potential is often left unexploited in favor of developing nonrenewable electricity projects. Electricity projects based on traditional, non-renewable sources such as fossil fuels consume finite natural resources and thus transfer costs to future generations. In addition to having the potential to significantly reduce GHG emissions, renewable energy options for electricity do not directly consume finite natural resources. Renewable energy technologies vary significantly in their degree of economic and technical maturity, but many are already available and economically competitive. A study by Jacobson and Delucchi (2011) reveals that a complete shift away from fossil fuels and nuclear power to electricity from renewable sources such as wind, water, and sun is entirely technologically and economically possible by 2030 . Such an electricity system would require new infrastructure, but would not have any significant disadvantages with regard to cost or reliability compared to the current dominant system. According to Delucchi and Jacobson (2011), the current barriers to such a transition to renewable energy are primarily political, not technological or economic. The economic advantages of renewable sources of energy will only continue to improve as fossil fuels, and the GHG emissions they entail, become more expensive (Teske 2010).
Companies need to have in place initiatives to stimulate the increase of renewable energy in their fuel mix (WBCSD 2002, UNGC 2008) and a medium- and long-term strategy for phasing out fossil fuels and completely switching to renewables (Pillinger 2009). Companies should report to stakeholders on their use of renewable sources by indicating the share of renewable energy in their electricity fuel mix, both in terms of generation capacity (MW) and electricity generated (GWh). They should also give these figures over time to indicate their progress, and provide information on their future targets and investment plans in renewable vs. nonrenewable sources, both in planned new generation capacity (MW) as well as financial investments. Electricity TNCs should also indicate the fraction of technically exploitable renewable energy potential currently not in use in the countries in which they operate (UN/IAEA 2007).

When evaluating potential projects, companies should consider the resource depletion implications of the various options. Preference should be given to projects based on renewable natural resources over projects that imply the consumption of non-renewable resources, particularly in countries that have a high potential for technically exploitable renewable energy. However, it should also be noted that some renewable technologies involve high levels of non-energetic natural resource consumption, primarily in the construction phase (see Section 3.5.6). 


\subsubsection{Biodiversity}

Electricity infrastructure and assets are often located in or near sensitive ecological areas that are high in biodiversity. This is especially true in the Global South, where the vast majority of the world's biodiversity is located. Hydroelectric dams are frequently located in remote river valleys, and electricity transmission lines can slice through forests of high biodiversity value, reducing crown density and dividing contiguous ecosystems. Although bio-diverse areas are sometimes protected by local and national governments in reserves or parks, often such areas are left unprotected.

Large hydroelectric dams present the most acute threat of biodiversity loss given that they often require the flooding of large areas of land, but, as noted above, even small-scale run-ofriver hydro projects can have negative impacts on migratory fish and other species by diverting water to feed turbines and removing water from long stretches of river. Companies that build large dams often compensate for the loss of biodiversity in the flooded area by purchasing, protecting or creating replacement habitats. However, the replacement habitats may not have the same biodiversity value. Electricity infrastructure that alters natural habitats can alter the migration and breeding habits of animals. Large-scale wind farms may impact the migration patterns of flocks of migratory birds. In addition to impacting terrestrial biodiversity, electricity-related activities also have consequences for biodiversity in aquatic ecosystems. Changes in downstream water quality due to increased turbidity, siltation, sedimentation, and temperature change from thermal discharge of cooling effluents may affect the breeding habits of fish.

There are also a number of indirect impacts of electricity infrastructure on biodiversity. For example, if a company decides to build a power plant in a pristine area, new roads and infrastructure will also have to be constructed, which increases the chance that other companies and developers will come and further impact the ecosystem and biodiversity. This is the case in Endesa's HidroAysén project in Chilean Patagonia, where a wide range of stakeholder groups claim that, in addition to the project's direct impacts on biodiversity, the dams and accompanying transmission line will open up Chilean Patagonia to further industrialization as other companies and industries use the roads and infrastructure constructed for the project (Witte 2008).

As a matter of priority, companies should seek to avoid impacts on biodiversity and ecosystem services (WCD 2000). If negative impacts are unavoidable, companies should have plans and take efforts to minimize and mitigate those impacts during the design phase (IHA 2004). The IFC (2011) insists that the companies it supports should not implement any project activities in areas of critical habitat with high biodiversity value unless all of the following are demonstrated:

- "No other viable alternatives within the region exist for the project on modified or natural habitats that are not critical 
- The project does not lead to measurable adverse impacts on those biodiversity values for which the critical habitat was designated, and on the ecological processes supporting those biodiversity values

- The project does not lead to a net reduction in the global and/or national/regional population of any Critically Endangered or Endangered species over a reasonable period of time

- A robust, appropriately designed, and long-term biodiversity monitoring and evaluation program is integrated into the client's management program."

Biodiversity offsets should only be considered after all appropriate avoidance, minimization, and restoration measures have been exhausted and must adhere to the "like-for-like or better" principle (WCD 2000, IFC 2011).

\subsubsection{Energy and resource efficiency (eco- efficiency)}

Eco-efficiency refers to reducing the impact on natural resources for producing goods and services. Although the long-term aim should remain on replacing all fossil fuels with renewable sources of electricity, improving the efficiency of fossil fuel-based electricity generation can reduce overall resource consumption and emissions. Efficiency of energy conversion in electricity generation is measured by comparing the gross energy going into the plant to the net energy leaving the plant. Improving efficiency is largely dependent on the choice of technology for power plants.
For example, combined cycle gas turbines (CCGT) that use natural gas as a fuel and combine heat and power (CHP) generation can reach much higher energy conversion efficiency levels than traditional coal and diesel-fired plants. Electric utility companies should report to their stakeholders on the energy conversion efficiency of their plants and have in place initiatives to stimulate the increase of more efficient technologies in their portfolio and a long-term strategy for increasing the efficiency of their overall generation activities.

Another element of eco-efficiency that is crucial for the sustainability of an electricity generation project is the energy payback ratio. Determining the ratio of energy payback consists of dividing the total energy produced during the normal lifespan of a project by the energy required to build, maintain, and fuel the plant. Whereas projects based on renewable resources generally have most of their energy input up front during the construction phase, fossil fuel-based systems continue to consume energy throughout their entire lifespan since fuel must be continuously extracted, transported, processed, and combusted. Electricity generation companies should assess the sustainability of all options in terms of both energy conversion efficiency and energy payback ratio before deciding to build (IHA 2004).

The WBCSD (2002) insists that "Electric utilities should seek to promote cost-effective energy and resource efficiency in their own operations, among their customers, and in the broader economy". 


\subsection{Economic issues}

The economic pillar of SEP refers to issues that affect progress in economic development and the sustainability of the development.

Economic aspects of corporate activity are being increasingly debated from a CR perspective (MVO Platform 2012). Power companies are expected to contribute to sustainable economic development in host countries by improving electricity infrastructure, researching and developing sustainable new technologies that can be used by the host country in the future, ensuring a reliable supply of electricity for local homes and businesses, managing demand, and conducting their operations in an efficient and transparent manner.

The subsections below primarily address the normative aspects of the relevant economic critical issues for SEP. It is recognized, however, that commercially operating electricity corporations must add to shareholder value to survive. The WBCSD (2002) emphasizes that "electricity assets will only be maintained, and likewise a supply of sustainable electricity, if a minimum return can be achieved in the long term" (WBCSD 2002). Companies should realize, however, that expanding access to electricity in the Global South is rarely a profitable venture in the short term. Investments in electricity infrastructure have a low rate of return and require a long time horizon and long-term planning (Bambawale et al. 2011).

It must also be noted that governments and communities in the Global South place high value on the basic "economic engine" activities such as job creation, rapid electrification and infrastructure development, and (tax) revenues, as well as improving the competitive environment, that are provided by companies and are necessary for development (Visser 2008). Indeed, it is unreasonable to expect countries in the Global South to accept standards for and conditions on their own economic development that Northern countries never had to accept. Electricity TNCs operating in the Global South are thus frequently confronted with demands from host country governments and populations, as well as unprincipled competition from other firms, that may be at odds with some of the other social, environmental, and economic standards found in this chapter. Given this reality, such purely economic contributions should not be viewed per se viewed in a negative light, but it is how TNCs choose to balance these purely economic interests with the often contradictory interests of the other sustainable development pillars that is relevant for measuring a company's commitment to SEP.

\subsubsection{Local economic development}

Chapter II of the OECD Guidelines stipulates that companies operating in the Global South should contribute to local economic development, and indeed, electricity provision projects carried out by TNCs can be a source of sustainable livelihoods and an engine for economic development for the host country and community. Electricity provision can be a source of jobs for adjacent communities that 
can contribute to local economic development, both through direct and indirect employment in construction, operating, and maintenance. Improved physical and institutional electricity infrastructure and access to electricity can improve lives and alleviate poverty. These "economic engine" activities are often the core of what host-country governments and communities want from TNCs in their areas.

However, although the 1990s saw a wave of optimism about the unlimited positive impact of FDI from TNCs in the Global South and about FDI as "an engine of development" (UNCTAD 1992), it is now generally recognized that the positive developmental impacts of FDI are not automatic, particularly when it comes to investment in infrastructure, and that some current TNC strategies are actually having a "negative effect on the development of infrastructure in LDCs" (Yamin and Sinkovics 2008: 2). Recent research indicates that the strategies and investment approaches of some TNCs leave little potential for development and may even hinder recipient countries' development by forcing them to pay ever increasing costs (in terms of lowering environmental and labor standards) to attract "increasingly footloose" investment (Dunning and Narula 2004).

TNCs' investment strategies are becoming increasingly linked to incentives, such as tax breaks and reduced administrative burdens offered by host countries (Easson 2001, Mutti and Grubert 2004). This "incentive elasticity" of TNC investment forces host countries in the
South to compete with each other, and often poorer regions of developed countries, to attract investment. In addition to paying out the tax incentives if they are successful in attracting employment, host countries must spend limited public resources in an attempt to attract investment, even if they don't actually receive the investment in the end. The establishment of government agencies to attract investment and employment of skilled personnel to assist and guide potential investors through the administrative processes both deprive governments of resources that could be spent directly on improving infrastructure (Yamin and Sinkovics 2008).

SEP implies that electricity companies should first assess the local needs and determine whether new generation capacity is truly necessary or whether the demand could be met through efficiency measures and other demand-side initiatives rather than additional supply. This may often be contradictory to a company's desire to initiate a project to generate revenue. It should be noted that there is a difference between the need for electricity, which is a social aspect related to healthcare, education, and poverty reduction, and demand for electricity, which is a pure market aspect and includes demand from aluminum smelters and wasteful consumption. Improving local efficiency of demand and supply will contribute to the long-term development of a community and make development more sustainable. If it is determined that new capacity is required, attention should be paid to local needs in determining the appropriateness of various 
types of capacity and technologies. For example, some countries in the Global South need greater peak load capacity while others are looking for stable base load generation (IHA 2004).

With relation to job creation, in order to ensure that employment of locals truly contributes to sustainable livelihoods and economic development, the jobs created must be high-quality jobs (see the critical labor issues discussed above in Section 3.2.4). Locals should also be hired into management positions at operations in the Global South (OECD 2011). Ensuring that they pay their fair share of taxes in host countries is another way electricity companies can contribute to local economic development (see also Section 3.4.7).

Furthermore, Yamin and Sinkovics (2008: 3-4) observe that "The distinctive contribution of FDI to economic development revolves around its potential to generate positive spillovers". In general, "the greater the degree of an MNE's resource commitment to the host economy, through linkages and sourcing of intermediate inputs, the greater the degree of positive spillovers are likely to occur". It is thus crucial for transnational electricity providers to, at a minimum, comply with the investment agreements they have signed with host country governments and invest in developing the host country's electricity infrastructure. However, there are numerous cases where, for a variety of reasons, electricity TNCs have failed to live up to their investment agreements (Woodhouse 2006). For example, Nicaragua's electricity regulator, the INE, recently (23 March
2007) initiated an arbitration process against the Spanish electricity TNC Unión Fenosa at the Nicaraguan Chamber of Commerce. INE's complaint, which was based on an INE audit report, alleged that Unión Fenosa failed to invest in improving the country's electricity infrastructure as it had agreed in its concession contract with the Nicaraguan government. The lack of investment led to several fatalities among the public due to poorly-maintained infrastructure and to widespread blackouts that caused an economic and humanitarian crisis (McGuigan 2007).

Another way TNCs can create linkages and spillovers is by transferring knowledge and technology to local firms. Chapter VIII of the OECD Guidelines for Multinational Enterprises stipulates that enterprises should "contribute to the long term development prospects of the host country...[through the] transfer and rapid diffusion of technologies and know-how" (OECD 2000b). Because electricity systems in many Southern countries continue to employ outdated technology, the introduction of technologies already available in industrialized countries can significantly reduce emissions and improve efficiency. Companies should employ such technologies whenever possible in the Global South and develop local expertise to use, maintain and spread the technology. In order to facilitate knowledge transfer and to further create backward linkages that contribute to local economic development, local (small and medium-sized) suppliers should be given priority in sourcing and procurement decisions (WBCSD 2002). 


\subsubsection{Reliability of supply}

The degree of reliability of electricity supply is a major concern in the Global South, particularly with regard to rural electrification, given that the benefits of access to electricity are significantly diminished when supply is subject to frequent and/or lengthy disruptions (Pereira et al. 2011). Electricity blackouts and losses of power are a daily occurrence in many Southern countries. Reliability of electricity supply refers to the ability of an electricity system to provide an adequate, secure and uninterrupted supply of electrical energy at any point in time. Continuity of supply is measured by the number and duration of electricity supply interruptions. Power outage frequency is measured by System Average Interruption Frequency Index (SAIFI), which is the average number of interruptions that a customer experiences. SAIFI is commonly used as a reliability of supply indicator by electric power utilities and is generally measured over the course of a year (GRI 2008). Continuous electricity supply is important to both large and small electricity customers for a number of reasons. For large industrial users, even relatively short interruptions can lead to production shutdowns and substantial financial losses, while for smaller domestic users, power losses can leave people in the cold and dark without heating, lighting and cooking facilities. Outages can also endanger public health and safety if, for example, hospitals or security systems are left without electricity (CEER 2005).

In order to avoid shortages, companies should align both short- and long-term planned capacity development with predictions for demand over the same time periods. Planning should also include concerns such as cost, maintenance, asset life, regulatory requirements, climate change implications, and potential changes in population demographics. Some types of plants are not always available to generate electricity. Companies should undertake and communicate assessments of the percentage of the year during which the plant is available to generate electricity, both in terms of planned generation stoppages and the change of unplanned stoppages (GRI 2008). In case of interruptions, companies need contingency plans for restoring supply in a timely manner, as well as for the development and deployment of backup power (WBCSD 2002).

In addition to continuous supply, other aspects that are important to the reliability of a power system are the speed with which supply interruptions are restored and the timeliness and dependability of information that is provided to the public when an outage occurs (CEER 2005:3).

\subsubsection{Operational efficiency}

Electric utilities should seek to improve productivity by seeking to increase operational efficiency (WBCSD 2002At hydroelectric facilities, for example, operational efficiency can be improved by decreasing seepage losses from the reservoir and intake diversion systems, decreasing head loss and increasing the potential capacity of water-supply conduits and structures, consideration of the variation in the performance curves of units with time 
and asymmetry of the water supply to turbines in problems dealing with optimum scheduling of power plants operation, particularly for short-term regulation (Autse 1976).

Efficiency is also an issue in the transmission and distribution of electricity, where excessive losses can contribute to electricity system inefficiency. Transmission and distribution losses are generally categorized in two types of losses: technical and non-technical.

Technical losses occur as a portion of energy is lost when electricity is transmitted from one place to another through power lines. Even given the best technology available today, some technical losses are unavoidable in the transmission of electricity. However, electricity transmission companies can reduce technical losses by employing state of the art transmission technology, which is often not the case in the Global South, and by shifting generation capacity to smaller, decentralized facilities that are located closer to end users and therefore require the electricity to be transmitted over smaller distances.

Non-technical losses generally refer to electricity theft and makeshift grid connections through which unauthorized users pilfer electricity from the system. This often occurs in poor, inner-city urban areas where customers cannot afford to pay their electricity bills and have been cut off by the electricity company. Companies can reduce non-technical losses by implementing programs that enable low-income customers to pay electricity bills (see Section 3.2.3) and encourage them to establish a regular connection rather than an informal hook-up. Furthermore, by relying on decentralized energy systems based on local renewable energy sources rather than large central power stations, power companies can avoid wasting energy during conversion and distribution (Teske 2010).

\subsubsection{Demand-side management}

There is enormous potential for reducing consumption of electricity through efficiency measures on the demand side. Energy experts insist that more than a third of current GHG emissions could be saved through conservation and efficiency improvements in homes, businesses, and industry (Teske 2010). However, because electricity companies are generally concerned with increasing sales of electricity to raise profits, they often encourage increased, rather than reduced, consumption. Nevertheless, companies have a responsibility to make customers aware of the negative impacts of wasting electricity and the positive consequences of energy conservation and to encourage them to use energy rationally and prudently. When considering constructing a new plant, companies should assess whether the need is genuinely from the supply side, or whether efficiency measures and other demand-side initiatives could cover the demand (Pillinger 2009). In addition to promoting conservation and a reduction in electricity use, another demand-side mechanisms that companies can use is to encourage consumers to shift their consumption to off-peak hours. This will allow the base load supply to cover 
more of the demand and avoid the use of expensive and often very environmentally unfriendly peak production.

\subsubsection{Corruption}

Transparency International's Global Corruption Barometer reveals that the risk of corruption is higher in the Global South than in the industrialized world (TI 2007). Within the electricity industry of the Global South, "Influencing the bidding process, the price of the public property to be sold and relaxed regulation are occasions where corruption can occur by both enterprises and government officials. Examples around the globe have shown that opening up the [electricity] sector to competition can lead to corruption and the manipulation of markets" (ILO 2006).

The UN Global Compact (2008) insists that "Businesses should work against corruption in all its forms, including extortion and bribery". Companies should have in place a policy on corruption that references the 1992 OECD Convention on Combating Bribery of Foreign Public Officials in International Business Transactions and Chapter VI of the OECD Guidelines for Multinational Enterprises, which deals with Combating Bribery. In implementing these standards, electricity companies need to analyze corruption-related risks in various business units and train their employees in the company's anti-corruption policies, especially those responsible for operations in the Global South. Beyond bribery, electricity TNCs should abstain from improper involvement in local political processes, as is stipulated in Chapter II of the OECD Guidelines for Multinational Enterprises.

\subsubsection{Competition and local market structure}

According to the MVO Platform (2012: 12), "Profits and margins are not divided equitably across market and value chain players if dominant market positions are abused and corporations enter into agreements that undermine free trade and competition." This observation is particularly relevant for the electricity industry, since some characteristics of electricity provision, such as the natural monopoly properties of power transmission, as well as the high technical expertise and large capital expenditures necessary, can have the effect of limiting competition and increasing market concentration. This can have undesirable economic consequences for local consumers, governments and suppliers by reducing their bargaining power vis-à-vis electricity providers.

The WBCSD (2002) insists that "Electric utilities should seek pricing and market reforms that are fair". International standards related to fair competition include the OECD Guidelines for Multinational Enterprises Chapter IX on Competition, the 2004 UNCTAD Model Law on Competition, UNCTAD's 1980 Multilaterally Agreed Equitable Principles and Rules for the Control of Restrictive Business Practices. International competitive bidding should be required on all electricity provision projects. 
Electricity companies seeking to increase their market share in the Global South should prevent market consolidation and domination and avoid engaging in business practices that restrict competition or are disadvantageous for local consumers, governments and suppliers.

\subsubsection{Taxation}

Many host countries in the Global South rely on tax payments by TNCs operating on their territory as a crucial source of income. Yet the host countries are often deprived of substantial amounts of revenue due to corporations' internal trading practices and tax exemptions. The OECD (2009) estimates tax dodging probably costs poor countries more than the roughly US $\$ 120$ billion they get in aid every year, suggesting that if TNCs were to end the practice of tax dodging and pay the fair taxes they owe to host country governments, the MDGs could easily be achieved (McCaughey 2010).

The MVO Platform (2012: 13) stresses that TNCs operating in the Global South should observe the following responsibilities:

- "Tax should be paid in the country where business activities have actually taken place and profits have actually been made

- No transfer pricing, in other words, no price manipulation at borders with the aim of avoiding taxes or trading tariffs

- No thin capitalization, i.e. hiving off profits from subsidiaries by having them pay interest to other subsidiaries elsewhere

- No undue use or abuse of tax benefits linked to specific conditions."
In addition, corporate transparency and accountability for the payment of tax is crucial. Companies are provided with the privilege of incorporation within a country by the legislative system within that country. In return, corporations generate many benefits including jobs, infrastructure, and revenue payment. It is clear from developments in China and the US that there is a growing interest in corporate transparency for tax purposes. With major governments now supportive and businesses recognizing the new reality of the world in which they operate, it is likely that pressure to increase tax transparency will continue to grow.

A significant development in this respect is the 2010 passage of the Dodd-Frank Financial Reform Bill in the US, which is hugely significant because it contains a transparency amendment that requires extractive companies listed on the Securities and Exchange Commission (SEC) to report profits and tax payments on a country-by-country basis. Similarly, the Hong Kong stock exchange now requires mineral companies applying for listing to disclose important information about their exploration and extraction activities, including payments to host governments (McCaughey 2010).

\subsubsection{Research and development}

Heller et al. (2003) have observed that, even in the industrialized world, market-oriented restructuring has led to a focus on the short-term and a neglect of investment in innovation for the future. Despite the fact that technological development has historically been 
a key driver in the energy sector (Musango and Brent 2011), total spending on research and development in the electricity industry has declined sharply over the past two decades. Companies' research and development (R\&D) activities should be aimed at providing sustainable, high-quality electricity. New technologies can improve efficiency, reduce emissions and pollution, facilitate greater use of renewable fuels and make electricity infrastructure safer for workers and the public. Companies should publish information on their expenditure on and investment in renewables exploration and development compared to expenditure/investment in exploration and development of non-renewable technologies (i.e. hydrocarbons and nuclear). Electric utilities should vigorously pursue R\&D and training programs (WBCSD 2002) and ensure that adequate resources are made available for innovation and development of sustainable electricity technologies (Pillinger 2009).

\subsubsection{Due diligence}

Over the past decade, a number of electricity projects involving foreign investment in the Global South have failed due to the inability of foreign investors, generally large TNCs, to adequately assess the economic feasibility and viability of electricity projects, given the risks involved and the long lifespan and slow rate of return on investment (Haar and Jones 2008). Provision of electricity is an essential service for the well-being of populations. Citizens and governments in the Global South have rebelled against sharp electricity tariff increases or a reduction in the quality of service by companies seeking to make their investment economically viable. Projects that fail in this way can have disastrous consequences for the quality and sustainability of electricity provision (Woodhouse 2006).

There is an inherent difficulty in balancing the sustainable provision of an essential service such as electricity and the profit-making prerequisite of private companies. It is essential that companies conduct an appropriate and thorough due diligence study in which they assess the options in terms of economic feasibility and viability over a facility's entire projected life, which, in the case of some hydroelectricity plants, can be up to 40 years or longer.

An appropriate due diligence review also includes consideration of the cost and availability of resources and fuels needed to run and maintain the plant over its lifetime. As supplies are depleted and demand continues to rise, the price of fossil fuels, particularly oil and gas, is likely to increase over the life of an electricity plant. Renewable technologies have a clear advantage over fossil fuels in this regard, but natural events like drought and shifting wind patterns due to climate change can affect the availability of resources.

Electricity companies should also undertake an analysis of the legal risks involved with electricity infrastructure development and ensure that they will be in compliance with all host country laws and regulations, including local legislation with regard to the relevant critical issues mentioned here, in particular 
environment, cultural heritage and indigenous rights, waste management, reporting, and occupational health and safety (WBCSD 2002).

In addition to fulfilling their fiduciary duty to shareholders by conducting due diligence on issues that may impact the company's financial position, leading international normative standards (OECD 2011, UNHRC 2011) insist TNCs also conduct risk-based human rights due diligence to identify, prevent, and mitigate adverse social and environmental impacts of their own activities and those of their business relationships (e.g. in the supply chain).

\subsection{Cross-cutting issues}

\subsubsection{Compliance with the law}

It almost goes without saying, but a basic requirement for SEP is that electricity TNCs comply with all regulations and laws issued by central, regional, and local governments of the countries in which they operate (WBCSD 2002). The OECD (2011) Guidelines put it succinctly: "Obeying domestic laws is the first obligation of enterprises".

\subsubsection{Poverty reduction and satisfying basic needs}

Eradicating poverty and hunger is the first MDG and is the top sustainable development priority for many the Global South. The Johannesburg Plan of Implementation agreed upon at the
World Summit on Sustainable Development explicitly identified the link between energy services and poverty reduction, noting that "access to energy facilitates the eradication of poverty" (ESMAP 2005).

Electricity TNCs operating in the Global South should therefore ensure that their activities are clearly and concretely leading to poverty reduction. This will require addressing many of the social and economic critical issues for SEP mentioned above. For example, social issues such as access to electricity (see Section 3.2.2) and affordability (3.2.3), as well as the labor issues of living wages, job security, and skills training (3.2.4), affect the degree to which electricity provision contributes to poverty reduction. Similarly, electricity TNCs must address critical economic issues like taxation (3.4.7), local economic development (3.4.1), and corruption (3.4.5) to ensure that their activities are contributing to poverty reduction.

Companies should approach the issue of poverty reduction in a broad and comprehensive manner, as the exact relationship between any one of these critical issues and poverty reduction may not be clear cut. For example, while it is generally accepted that there is a link between increased access to electricity and a reduction in poverty (Modi et al. 2005), Kooijman-van Dijk (2008) and Pereira et al. (2011) warn that simply improving access to electricity in a given area does not necessarily lead to increased incomes for local individuals and businesses. Complementary inputs such as payment of fair and appropriate tax revenues 
and creation of decent jobs, as well as making backward linkages into the local economy, are crucial if increased energy supply is to lead to poverty reduction.

In addition to social and economic issues, for electricity provision to lead to sustainable poverty reduction, meeting the basic needs of current generations cannot compromise future generations' ability to meet their own basic needs by polluting the environment or damaging natural life support systems. The World Commission on Dams (2000) notes that an electricity project can only contribute to sustainable poverty reduction and human development if it is "economically viable, socially equitable and environmentally sustainable". This implies that, in their efforts to reduce poverty through electricity provision, power companies must also take into account critical environmental issues like GHG emissions and climate change (see Section 3.3.1), emissions and waste (3.3.2), natural resource depletion and renewable sources of energy (3.3.3), and biodiversity (3.3.4). If companies fail to address these environmental concerns, efforts to reduce poverty now could lead to even greater poverty and greater disparities between rich and poor among future generations.

\subsubsection{Precautionary principle and evaluation of risks and alternatives}

The precautionary principle states that, if an action or policy might cause severe or irreversible harm to the public or to the environment, in the absence of a scientific consensus that harm would not ensue, the burden of proof falls on those who would advocate taking the action (OECD 2000b). The precautionary principle is identified by the 1992 Rio Declaration, the UN Global Compact and the OECD Guidelines for Multinational Enterprises as one of the key principles for sustainable development. The WBCSD (2002) insists that companies should adopt a precautionary attitude and modify electric utility operations in order to prevent serious or irreversible environmental degradation. Taking a precautionary approach to scientific uncertainties in both new and existing projects is thus crucial for SEP.

Before a decision is made on whether to undertake an electricity project, a comprehensive and participatory evaluation of all feasible policy, institutional, and technical alternatives and the potential risks of each alternative should be made. Traditional practice is to restrict the definition of risk to the capital invested and expected returns of voluntary risk-takers, such as the companies developing and financing an electricity project. However, a much larger group of stakeholders often have risks involuntarily imposed on them. These involuntary risk-takers often have little or no say in how the risks are managed and mitigated, despite the fact that the risks can have a direct impact on their livelihoods, well-being, quality of life, and even survival. Transnational electricity companies should therefore identify, articulate, and thoroughly and explicitly address the risks of each potential project and alternative, rather than simply 
using mathematical formulas or actuarial tables. In evaluating alternatives, avoidance of environmental impacts and displacement should always be given preference, followed by minimization and mitigation of such impacts (World Commission on Dams 2000). The WCD (2000) provides further detailed guidance to electricity companies, noting that companies should ensure that:

- "Planning approaches take into account the full range of development objectives are used to assess all policy, institutional, management, and technical options before the decision is made to proceed with any programme or project.

- Social and environmental aspects are given the same significance as technical, economic and financial factors in assessing options.

- Increasing the effectiveness and sustainability of existing energy systems are given priority in the options assessment process.

- Decisions value ecosystems, social and health issues as an integral part of project and river basin development and prioritise avoidance of impacts in accordance with a precautionary approach."

Largely based on the WCD's (2000) guidance, the International Hydropower Association (IHA 2004) has formulated a list of key standards that should be used in evaluating and comparing potential hydroelectric project alternatives. Primary among the standards is that social and environmental factors should be given the same significance as economic and financial aspects, and that projects that avoid impacts should always be prioritized.
Companies should also evaluate the human rights situation in a potential host country before a decision to invest is made in order to ensure that no human rights violations have been, are being, or will be perpetrated to promote electricity projects in that country (see Sections 3.2.1 and 3.4.9 on the importance of comprehensive human rights due diligence).

\subsubsection{Transparency and provision of information}

Modi et al. (2005) cite insufficient transparency in the management and operations of electric utilities as one of the primary challenges for sustainable development in the Global South. Proactively providing timely access to all relevant information to stakeholders, particularly indigenous and tribal peoples, women and other vulnerable groups, is a crucial element of SEP because it enables the informed participation of stakeholders in decision-making processes (World Commission on Dams 2000, UNGC 2008).

The UNECE (1998) Århus Convention stipulates citizens' rights to access to information, and the OECD (2011) Guidelines' Chapter III on Disclosure specifies TNCs' responsibility to provide stakeholders with accurate and timely information on their activities. According to the UN Global Compact (2008), enterprises should "establish two-way communication with stakeholders, in a pro-active, early stage and transparent manner, to ensure effective communication of information". Electricity companies should thus report regularly and 
systematically on their activities and the impact of their operations using the GRI G3 Guidelines and the GRI EUSS (GRI 2008). Reporting should include all wholly and partly owned subsidiaries and joint ventures, whether in generation, transmission or distribution. Reporting should have year-on-year comparisons from the commencement of reporting and should include measurement and interactions with government and the public. Reports should be externally verified and checked to ensure credibility and transparency (WBCSD 2002).

Principled sustainability reporting should also include an assessment of externalities (i.e. offthe-balance-sheet social and environmental costs) of production processes, supply chain practices, operational measures and approach to electricity generation. Externalized costs are born by the society as a whole and are therefore key to sustainable reporting and for presenting a balanced view of the real impact of electricity provision. The South Africa Energy Policy, for example, calls for a reflection of quantifiable externalities. Quantifiable externalities are not easy to calculate, and many assumptions need to be made. Attributing a monetary figure to environmental impacts, resource use, human health, etc. must be situation/context specific and allow for margins. Hence, a range of approaches is suggested, e.g. low, medium and high estimates of externalized costs, with the weighting of and values assigned to the various factors clearly stated. In addition to external costs, calculations should also include externalized benefits, such as health improvements through electrification and clean air through reduction of fuels burned in the home. The reporting should include discussion of opportunity costs associated with the various choices companies make in their approach to electricity provision.

Critical operational and managerial information that electricity TNCs must provide to customers and other stakeholders cuts across the various social, environmental, and economic critical issues mentioned above.

In order to provide accurate information in an adequate and timely fashion, electricity companies operating in the Global South should be aware of language and cultural barriers to communication of information. Furthermore, low literacy rates in the Global South may prove to be an additional barrier to communication that companies must overcome (GRI 2008).

Furthermore, transparency and adequate provision of information are particularly important during the process of fixing electricity tariffs. Palast et al. (2000) note that the key principle that must be adhered to in such processes is "the observance of 'due process' rights of participation and transparency where anyone with an interest in utility rates can participate in the process (e.g. those interested in environmental protection, protection of poor people, economic development and employment and labor issues, for example)".

Important initiatives aimed at improving transparency in supply chains are also underway at the European and international 
levels. Various sets of recently-released normative guidelines and standards have furthered the debate and have specified in more detail what is expected of companies with respect to providing transparency about their business relationships. Such initiatives include the 2011 Communication on CSR by the European Commission (EC 2011), the 2011 update of the OECD's (2011) Guidelines, and the UNHRC's (2011) Guiding Principles on Business and Human Rights. These internationallyrecognized standards and recommendations provide guidance for companies and can be used as a benchmark for their performance on supply chain transparency. The standards insist that companies should identify, prevent, and mitigate negative impacts among their business relationships and encourage companies to disclose information about their relations with business partners such as suppliers, contractors, and customers.

\subsubsection{Meaningful stakeholder consultation and public participation in decision-making processes}

There is broad agreement throughout the literature (e.g. UNECE 1998, World Commission on Dams 2000, WBCSD 2002, IHA 2004, IFC 2007, UNGC 2008, IFC 2011, OECD 2011, Reimann et al. 2011) that meaningful and open dialogue with local stakeholders is essential for the sustainability of electricity provision projects. The OECD (2011) Guidelines insist that "Enterprises should engage with relevant stakeholders in order to provide meaningful opportunities for their views to be taken into account in relation to planning and decisionmaking for projects or other activities that may significantly impact local communities". The WCD (2000) asserts that "Public acceptance of key decisions is essential for equitable and sustainable energy resources development". Stakeholders' views and concerns should be reflected in policy and operational decisions so that those decisions are consistent with community goals, values, and needs. The identification and recognition of the rights and risks associated with a proposed electricity provision project should provide the basis for identifying stakeholders that must be included in decision-making (see Section 3.5.3).

Electricity TNCs should consult and enable informed, meaningful participation by surrounding and affected communities, unions, end users, and other stakeholders in decisionmaking processes throughout the entire cycle of a project including the initial planning phase, the environmental impact assessment, decisions on siting of plants and power lines, as well as construction and operation (WCD 2000, IFC 2007, Pillinger 2009). There should be demonstrable public acceptance of all key decisions. Affected and potentially affected stakeholders' roles and rights should be clearly documented and communicated in the stakeholders' language of preference (ISO 2011). The quality of information provided to stakeholders during these processes is a key determinant of their sustainability, as is the timing of engagement in projects, the level and nature of stakeholder engagement, and the provision of resources for vulnerable stakeholders. In the event that the 
project in question affects, or will potentially affect, Indigenous Peoples, all project decisions should be guided by the principle of free, prior, and informed consent (FPIC) as established in the UN Declaration on the Rights of Indigenous Peoples and ILO Convention 169 on Indigenous and Tribal Peoples.

Sinclair et al. (2009) provide a best practice example of a community-based approach to strategic environmental assessment (SEA) taken by the Costa Rican Institute of Electricity (ICE). ICE's approach involved organizing four highly interactive workshops that used visioning, brainstorming, and critical reflection exercises. The communities assessed the environmental, social and economic impacts of a proposed hydroelectric power plant. According to Sinclair et al. (2009), the process revealed the importance of having community participants understand the process both conceptually and methodologically. The interactive workshops were also effective in reducing the power differentials among program participants (proponent, communities, government agencies) and, crucially, "the logistical importance of notice, timing and location for meaningful participation".

\section{Electricity TNCs should strive to reach} negotiated, legally enforceable agreements with communities affected by proposed electricity provision projects. The World Commission on Dams (2000) notes that "Demonstrable public acceptance of all key decisions is achieved through agreements negotiated in an open and transparent process conducted in good faith and with the informed participation of all stakeholders. Development needs and objectives are clearly formulated through an open and participatory process before the identification and assessment of options for water and energy resource development."

\subsubsection{Responsibility for impacts throughout the value chain and business relationships}

Life cycle analysis (LCA) is a systematic method for assessing the environmental, economic and social impacts associated with the entire lifetime of all relevant processes upstream and downstream in the electricity production chain (Voß 2005). An LCA of the electricity chain reveals that there are a multitude of potential direct and indirect impacts not only from the construction, operation, and decommissioning of power plants, but also from the extraction, processing, transport, and storage of fuel and non-energy inputs such as manufacturing materials and chemicals, as well as the treatment, transport, and storage of waste (Hirschberg et al. 2004).

Product chain responsibility implies that a company does all it can to enable, promote, and implement responsible business conduct throughout its supply chain. Electricity companies should seek to prevent, avoid, and mitigate negative impacts throughout their entire range of business relationships, including suppliers and contractors (ISO 2011, OECD 2011, UNHRC 2011). This also implies that companies have the responsibility to know 
their own supply chain. For large companies, finding sustainable suppliers that are still able to provide them with necessary products and services can be a major challenge. However, the fact that electricity supply chains can be very complicated as a result of the outsourcing of construction, technical maintenance, and resource extraction does not excuse companies from this responsibility. By adopting a "systems approach", the WBCSD (2002) suggests electricity companies take a holistic and integrated view of the role and impacts of electric utility operations.

In essence, this critical issue entails the responsibility electricity companies have for the entire range of social, environmental, and economic impacts of electricity provision. Identifying impacts on the climate, natural environments and biodiversity, and communities, as well as effectively monitoring these impacts and promoting positive change, is crucial for SEP. Responsible business behavior implies moving beyond intentions and policy "outputs" and ensuring that there are effective procedures for monitoring and regulating the "outcomes", i.e. impacts, of their operations. Companies are also expected to identify the links in their supply chain that cause significant environmental, social and economic impacts, and ensure that their suppliers are aware of and are managing their own impacts. When problems arise with contractors and suppliers, companies should actively engage them to improve performance. The WBCSD suggests that electricity companies should seek to leverage their buying power to improve conditions among business relations and monitor performance using third-party audits.

Upstream supply chain issues primarily involve the exploration, extraction, processing, storage, and transport of fuels and other inputs. Negative environmental and social impacts, such as displacement, can also occur in an electricity company's supply chain as an indirect result of the power company's operations, for example, when a coal or uranium mine begins or expands production to feed a power plant, especially since electric utilities are frequently the sole or a majority consumer of an energy source such as a coal or uranium mine.

It is beyond the scope of this report to fully examine the sustainability standards for all electricity generation fuels and inputs, but it is worthwhile listing a few indicative examples:

- Oil extraction is associated with human rights and environmental problems around the world. The pollution and armed conflict in the Niger Delta is an example (UNEP 2011).

- Coal mining destroys the soil of the land it is mined on, permanently transforms the landscape, removes ground vegetation, and often displaces people. Coal mining also produces pollutants and effluents that can cause deterioration of water, soil, and air quality (Fthenakis and Kim 2008).

- Mining of uranium is taking place increasingly in African countries such as Niger, Namibia, and Malawi where health and environmental protection is at best weakly-enforced and at worst non-existent (Shinondola-Mote 2008). 
- The planting and harvesting of biomass to be used for electricity generation can often have environmental and social impacts that reduce or nullify the positive $\mathrm{CO}_{2}$ balance. Some of the factors that determine if biomass for electricity is truly sustainable include the carbon balance for the entire value chain and lifecycle of the biomass (e.g. transport, emissions due to land use change - see, for example, Wicke et al. 2008), loss of biodiversity due to changes in land use, decent labor standards on plantations and in processing chains, indigenous land rights issues, etc. The Dutch Cramer Commission (Cramer Commission 2007) has developed sustainability criteria for biomass, but these are still being hotly debated.

- The construction of power plants and other electricity infrastructure requires a great deal of energy, as well as nonenergetic raw materials such as iron, copper, bauxite, and concrete, which can have negative environmental and social impacts throughout their life cycle. Voß (2005) has indicated that the material costs of wind and solar generation technologies are much higher than those of other non-renewable technologies. Companies should attempt to increase the use of recycled material for inputs in infrastructure construction in order to reduce their exposure to and responsibility for such impacts.

This list clearly indicates that, according to the norms developed within the relevant field of enterprise, electricity TNCs operating in the Global South are expected to assess their suppliers in terms of performance, environmental and social criteria (not just price) in purchasing and contracting activities, as well as requiring suppliers to report on these issues. Companies are also expected to implement a pricing system that incorporates the social and environmental costs and quality of supplied goods and services, and suppliers should not be squeezed to supply goods at such low prices that they are forced to compromise on the social and environmental sustainability of their operations. This can happen when market concentration creates an imbalance in bargaining powers between various players in the supply chain (see also Section 3.4.6). Furthermore, companies should provide suppliers with reasonable supply lead times so that workers are not excessively pressured to meet production targets. Companies should seek continuity in trading relations and try to build long-term relationships with suppliers in order to contribute to economic security for suppliers. They should give small-scale and local suppliers preferential treatment. Suppliers should be encouraged to report on environmental and social criteria and should be monitored in order to ensure that the quality of electricity is high.

Companies are also expected to take into account downstream impacts on quality. Waste treatment and storage and decommissioning of power plants are two of the major downstream processes in the electricity product chain.

The use of contractors and subcontractors at various downstream stages is extensive in the electricity industry, and the performance of contractors can have a significant impact on the quality of electricity service provided. Like 
suppliers, contractors should be selected and monitored based on a range of environmental, social, labor, and economic criteria. Another downstream product chain issue is the sale of electricity to large (industrial) users such as smelters and mines. The high electricity demands of these industrial users can have a direct impact on supply constraints and affordability for the majority of the population. Electricity companies should be transparent about the price and amounts of electricity they provide to such consumers.

\subsection{The SEP benchmarks for TNCS}

The previous sections have identified and elaborated on the wide range of social, environmental, economic, and cross-cutting issues that experts from government, academia, business, and civil society find critical for electricity provision to be consistent with sustainable development. These 31 issues lay the foundation for the moral pressure on transnational corporations to provide electricity in a sustainable manner. However, these critical issues and associated standards represent an impractically wide range of concerns, and there is a need to condense them further into a more concise and potentially "operational" set of norms that can be used as benchmarks for evaluating the performance of TNCs.

Based on a thorough analysis of the most relevant and widely-accepted norms related to SEP (e.g. ILO 1998, UNECE 1998, Palast et al. 2000, WCD 2000, ILO 2001, WBCSD 2002, IHA 2004, CEER 2005, Modi et al. 2005, ILO 2006, BSI 2007, IFC 2007, OECD 2007, UN/IAEA 2007, UN Global Compact 2008, Pillinger 2009, Teske 2010, IFC 2011, OECD 2011, UNHRC 2011, GRI 2012, MVO Platform 2012, UN 2012), a condensed set of 11 "essential standards" can be organized into a framework of benchmarks for SEP. The framework of SEP benchmarks is depicted in Figure 3.1. Table 3.2 presents the framework of SEP benchmarks along with a summary of the international normative standards that were considered to guide each benchmark, as well as an operationalization of each benchmark in terms of company policy at the headquarters level and practice on the ground in the host country setting. Taken together, these SEP benchmarks reflect the values inherent in the broader sustainable development perspective and encompass social, environmental, economic, and cross-cutting issues. They thus represent basic standards for "quality kilowatts" - that is, electricity provision that contributes to the eradication of poverty, promotes sustainable production and consumption, respects the full range of human rights, and aims to protect the natural resource base and ecosystems that sustain life. As outlined in Chapter 2, company performance in both policy and practice will be measured against the operationalized benchmarks using a modified Likert (1932) scale to roughly classify a company's performance as "largely in line", "moderately in line", "moderately out of line", or "largely out of line" with each operationalized SEP benchmark. 


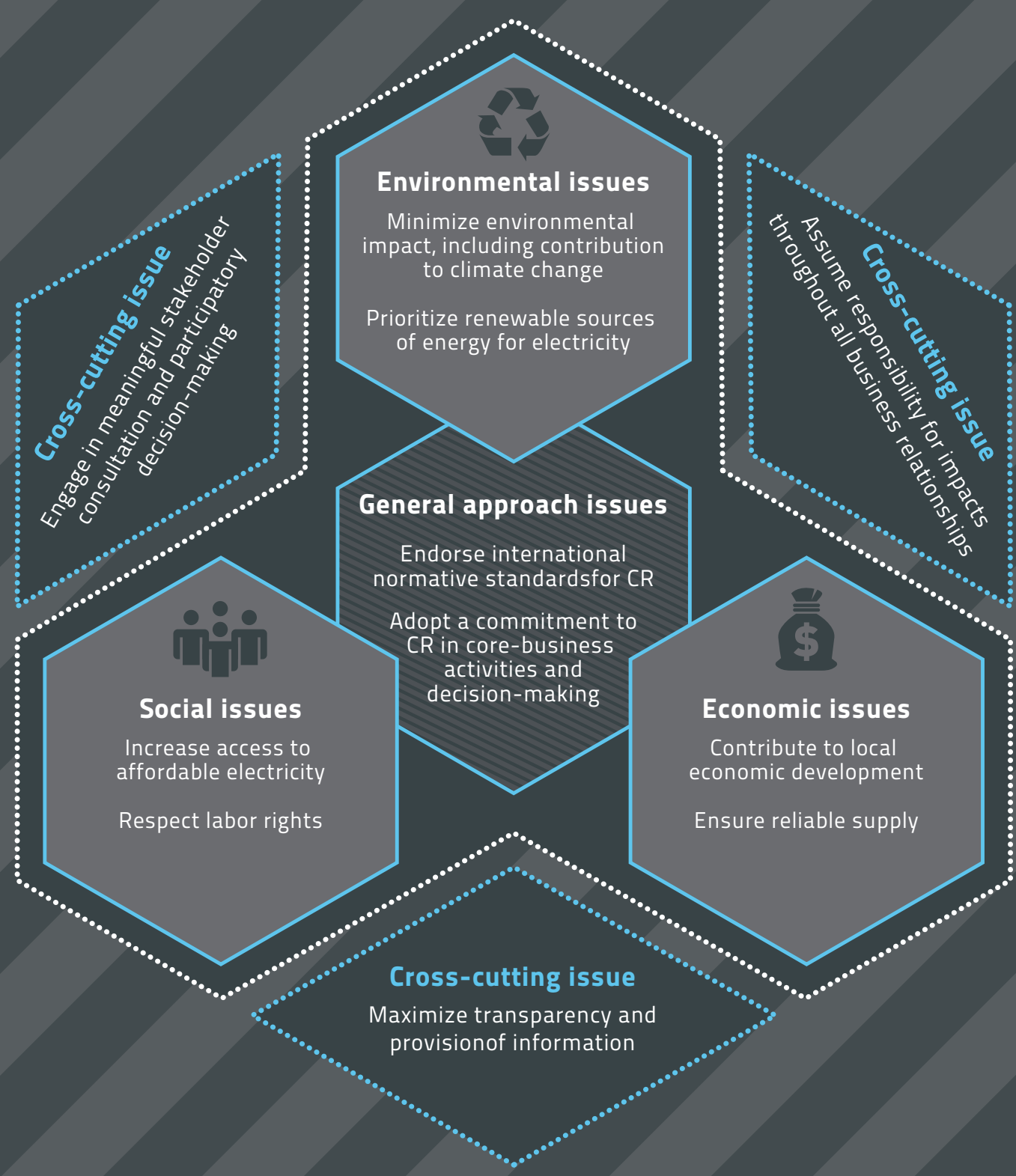

Figure 3.1: The SEP benchmarks for TNCs 
Table 3.2: SEP benchmarks, guiding norms, and indicators for operationalization

\section{SEP benchmark}

\section{General approach to corporate responsibility}

Endorse international normative standards for CR

Adopt a commitment to CR in core-business activities and decisionmaking

\section{Guiding international normative SEP standards}

Electric utilities should seek to bring their policies in line with internationally recognized and verified standards for CR (WBCSD 2002, UNGC 2008). Leading international normative standards for CR include:

- The ILO (ILO 2001) Tripartite Declaration of Principles concerning Multinational Enterprises and Social Policy

- The OECD (2011) Guidelines for Multinational Enterprises

- The UN Global Compact (2008)

- The IFC (2011) Sustainability Framework

- The UNHRC (2011) Guiding Principles for Business and Human Rights

- The ISO (2011) 26000 Guidance on Social Responsibility

Electricity companies should adopt a holistic and integrated view of the role and impacts of electricity provision, considering and balancing the essential elements of economic development, environmental quality, and social equity in utility operations. This entails a policy commitment to CR throughout all core-business activities, as opposed to a primary focus on philanthropic activities (WBCSD 2002, MVO Platform 2012). Electric utilities should seek to introduce environmental and social factors and procedures into highlevel corporate planning and decision-making and should aim to have a board-level position responsible for $\mathrm{CR}$ issues (WBCSD 2002)

\section{Social issues}

Increase access to affordable electricity
Electric utilities should develop policies that promote access to electricity priced at affordable levels for all, paying special attention to disadvantaged communities (ILO/Palast et al. 2000, WBCSD 2002, PSI/Pillinger 2009). In order to do so, companies should undertake initiatives to extend electricity services to unserved and underserved communities, particularly in rural or remote areas, but also to the poor in urban and peri-urban areas (UNDP/Modi et al. 2005, WBCSD 2002) 


\section{Operationalization of company policy on SEP}

- Does the company endorse internationally recognized and verified standards for CR such as the ILO Tripartite Declaration, the OECD Guidelines for MNEs, the UNGC, and the IFC Performance Standards?

- Which other international standards on CR and sustainable development does the company publicly endorse/use?

- Does the company endorse/use other national/regional normative standards?

- Does the company make use of standardized procedures and measurements such as the ISO, OHSAS, SA at the corporate headquarters level?

- What is the nature and focus of the company's CR policy in terms of core-business activities (i.e. directly related to electricity provision) versus charitable/philanthropic projects?

- What is the highest level of direct responsibility for CR in the company?

-What management structures are in place to implement CR policy and foster high performance on CR issues?

\section{Operationalization of company practice on SEP}

Does host country management promote the same international (or national/regional) standards for CR as the headquarters?

- Do the company's power plants in the host country have standardization certifications (e.g. ISO, OHSAS, SA)?

n What is the nature and focus of local level CR programs in terms of core-business activities (i.e. directly related to electricity provision) versus charitable/philanthropic projects?

" How do local stakeholders (e.g. communities, workers, civil society organizations, local government officials) perceive the nature and focus of the company's CR programs in terms of core-business activities (electricity provision, environmental impact) vs charity projects?
- Does the company have a public policy that promotes access to electricity for all?

- Does the company have a public policy that seeks to make electricity affordable for all?

- How much priority does the company place on providing access to poor communities surrounding its generation facilities compared to large population centers or industrial uses?

- Does the company promote off-grid, decentralized electricity provision projects as well as large grid connections?

- How much electricity generation capacity does the company have in countries without universal electrification and what percentage is this of the company's total installed capacity?
How much electricity does the company produce in the host country?

" What is the electrification rate of the host country as a whole and specifically in the areas in which the company has electricity generation facilities?

- What are electricity prices in the areas in which the company has electricity generation facilities?

" Does the company have programs in place in the host country that promote access to affordable electricity for all?

- Does the company support off-grid, decentralized access as well as grid connections?

" How do local stakeholders (e.g. communities, workers, civil society organizations, local government officials) perceive the company's efforts on access and affordability?

- How much priority does the company place on providing access to poor communities surrounding its generation facilities compared to large population centers or industrial uses in the host country? 


\section{Respect labor rights}

\section{Environmental issues}

Minimize environmental impact, including contribution to climate change
Electric utilities should respect workers' full rights and provide workers with fair pay, job security, and decent working conditions, including high levels of health and safety standards in all aspects of operations (WBCSD 2002, PSI/ Pillinger 2009, OECD 2011). Furthermore, electric utilities should uphold freedom of association and the right to collective bargaining and pay particular attention to the right to strike (ILO 1998, ILO 2001, UNGC 2008, OECD 2011). Companies should ensure that these rights are respected not only among their own employees, but also among suppliers, contractors and other business relationships (ISO 2011, OECD 2011, UNHRC 2011)

\section{Guiding international normative SEP standards}

Electric utilities should continually seek to minimize emissions and discharges to air and water and prioritize low-environmental impact technologies (WBCSD 2002, UNGC 2008, Pillinger 2009, Teske 2010, OECD 2011). Companies should manage their environmental impact in line with internationally recognized and verified standards such as the ISO 14000 series and should always undertake rigorous and verifiable environmental impact assessments (WCD 2000, WBCSD 2002, IHA 2004, OECD 2011). Electric utilities should minimize their contribution to climate change and develop strategies for reducing GHG emissions (WBCSD 2002, OECD 2011). As a matter of priority, electric utilities should seek to avoid impacts on biodiversity and ecosystem services, refraining from any activities in areas of critical habitat with high biodiversity value while at the same time initiating or supporting conservation and biodiversity efforts related to impacts on natural habitats from utility operations (WBCSD 2002, IHA 2004, IFC 2011) 
- Does the company endorse the ILO Core Conventions?

- What is the company's policy on freedom of association and collective bargaining?

- What is the company's policy on workers' right to strike?

- Does the company have policies and structures in place to engage \& consult workers on key operational decisions?

- What is the company's policy on occupational health and safety?

- Does the company have a policy aimed at ensuring that the rights of workers at the company's business relationships (e.g. contractors and suppliers) are also respected?
- Is a union present at the company's facilities in the host country? What percentage of the employees are members?

- What is the nature of the relationship between the union and the management in the host country?

- Is a collective bargaining agreement in place at the company's facilities in the host country? What percentage of the workers is covered by the CBA?

- Have there been strikes by the company's employees in the host country in recent years?

- What is the lowest wage paid to employees of the company in the host country and how does this compare to the national minimum?

" What OHS standards and programs are implemented by management in the host country?

- What is the number of work-related deaths/injuries in recent years at the company's facilities in the host country?

- What is the degree of outsourcing and job security for the company's employees in the host country?

" What are the working conditions of workers contracted by the company like?

\section{Operationalization of company policy on SEP}

- Does the company have a policy on continually improving environmental performance by reducing waste \& pollution?

- Does the company have a company-wide EMS (with a standardized certification) in place?

- Does the company have a policy to always conduct EIAs, even when not required by law?

- How much $\mathrm{CO}_{2}$ does the company emit each year? What is the $\mathrm{CO}_{2}$ emission intensity?

- What is the company's policy on $\mathrm{CO}_{2}$ emissions \& combating climate change? Does it make commitments to reduce emissions?

"What is the company's policy on protecting biodiversity?

- What is the total capacity (MW) of the company's large hydroelectric facilities, the average capacity of those facilities, and the size of the largest hydroelectric facility?

\section{Operationalization of company practice on SEP}

- Do the company's power plants in the host country have an EMS (with a standardized certification) in place?

- Has the company conducted an appropriate and thorough EIA for all power plants in the host country?

- Have there been any instances of serious environmental pollution in the last five years?

- What are the $\mathrm{CO}_{2}$ emissions and emission intensity of the company's power plants in the host country?

- Are there any plant-level commitments to reducing $\mathrm{CO}_{2}$ emissions?

- What are the other plant-level emissions $\left(\mathrm{NO}_{x^{\prime}} \mathrm{SO}_{2^{\prime}} \mathrm{PM}\right)$ ?

- What has the company's impact on biodiversity in the host country been?

- What measures does the company have in place to avoid and mitigate its negative impact on biodiversity?

- What is the total capacity (MW) of the company's large hydroelectric facilities in the host country, the average capacity of those facilities, and the size of the largest hydroelectric facility? 
Prioritize renewable sources of energy for electricity

Economic issues

Contribute to local economic development

\section{Ensure reliable supply}

Electric utilities should evaluate the renewable energy potential of the host country and prioritize development and exploitation of renewable options over fossil fuelbased technologies (WBCSD 2002, UN/IAEA 2007, Pillinger 2009, Teske 2010). Electric utilities should also prioritize renewables in their research and development programs and should support the diffusion of renewable energy technologies into the local economy (UNGC 2008, WBCSD 2002).

\section{Guiding international normative SEP standards}

Electric utilities prioritizing the development of local energy-related infrastructure and employing local workers to the greatest extent practicable and providing those workers with sustainable jobs and training to improve skills levels (WBCSD 2002, PSI/Pillinger 2009, OECD 2011). In order to maximize local economic development and job creation, electric utilities should support local small and medium-sized enterprises in their procurement practices and contribute to the long-term development prospects of the host country through the transfer and rapid diffusion of technologies, skills and knowledge (WBCSD 2002, OECD 2011). Fair payment of due taxes to local, regional, and national governments in the host country is a crucial element of contributing to local economic development (OECD 2011, MVO Platform 2012)

Electric utilities should ensure reliable supply by minimizing generation interruptions, maintaining contingency plans, responding to interruptions in a timely manner, and providing timely information to the public about planned and unplanned interruptions (WBCSD 2002, CEER 2005) 
- What is the company's global installed capacity or electricity produced by technology?

- Does the company have a policy prioritizing renewable sources of energy in favor of fossil fuel?

" Is there any evidence of the company prioritizing renewables or more efficient fossil fuels (e.g. natural gas) at the company-wide level (e.g. recent trend, build program, investment in $\mathrm{R} \& \mathrm{D})$ ?

\section{Operationalization of company policy on SEP}

- How does the company propose to contribute to local economic development?

- Does the company have a policy to prioritize local workers?

- Does the company have a policy to prioritize SME suppliers in host countries?

"What is the company's policy on ensuring reliability of supply?

- Does the company have a policy for responding to interruptions in a timely manner, and providing timely information to the public about planned and unplanned interruptions?

- How much does the company invest in R\&D for improving reliability of supply?
- What is the installed capacity \& electricity produced in the host country by technology type?

- Is there any evidence of the company prioritizing renewables or more efficient fossil fuels (e.g. natural gas) in the host country?

\section{Operationalization of company practice on SEP}

- How many employees does the company employ in the host country?

- What percentage of employees are nationals of the host country? What percentage comes from communities in the region where the company's power plants are located?

" Does the company prioritize SME suppliers?

" How much has the company invested in the (electricity infrastructure) of the host country?

- What taxes (in tax rate or absolute amount) does the company pay to the local/national governments of the host country?

- What is the average capacity factor of the company's power plants in the host country? What is the number of power cuts/ failures for which the company was responsible in recent years?

- Does the company properly maintain electricity infrastructure in the host country?

- Has the company been fined by host country regulators for inadequacies related to reliability of supply?

- Has the company responded to interruptions in a timely manner, and provided timely information to the public about planned and unplanned interruptions? 


\section{Cross-cutting issues}

\section{Engage in meaningful stakeholder consultation and participatory decision-making}

\section{Assume responsibility for impacts throughout all business} relationships

\section{Guiding international normative SEP standards}

Electric utilities should adopt a participatory approach to the planning/designing, developing, operating, and monitoring/ evaluation of electricity projects, and consult and engage stakeholders throughout the entire process in order to provide meaningful opportunities for their views to be taken into account in decision- making (WCD 2000, WBCSD 2002, IHA 2004, IFC 2007, UNGC 2008, Pillinger 2009, OECD 2011). The consultation process should be tailored to local decision-making processes and involve the timely provision of all relevant information about activities, impacts and potential impacts translated into local languages (WBCSD 2002, ISO 2011). The process should take into account the needs of disadvantaged or vulnerable groups, and if Indigenous Peoples are affected by a potential project, electric utilities must gain the free, prior, and informed consent of those peoples before proceeding with any part of the project cycle (IFC 2011)

Electric utilities should seek to prevent, avoid, and mitigate negative impacts throughout their entire range of business relationships, including suppliers and contractors (ISO 2011, OECD 2011, UNHRC 2011). Companies should seek to leverage their buying power to improve social and environmental conditions among business relations, and monitor performance using third party audits (WBCSD 2002)
Maximize transparency and provision of information
Electric utilities should apply a high degree of transparency in operations, including reporting on activities and progress, measurement, business partners and relationships, and interactions with government and the public (UNECE 1998, WBCSD 2002, UNGC 2008, OECD 2011, UNHRC 2011). Electric utilities should regularly report on their activities, progress, and performance against "the triple bottom line" using internationally accepted sustainability reporting guidelines such as the GRI's G3 guidelines and the Electric Utilities Sector Supplement (GRI 2008), have an external party verify the data, and disseminate the report in an appropriate manner (WBCSD 2002, OECD 2011) 


\section{Operationalization of company policy on SEP}

- Does the company adopt a participatory approach in all phases of electricity projects, consulting, and engaging stakeholders throughout the entire process in order to provide meaningful opportunities for their views to be taken into account in decision-making?

- Does the company reference international standards on stakeholder engagement?

- Does the company endorse the principle of free, prior, and informed consent (FPIC)?

- Is the company willing to engage with the author in research related to the present study?

\section{Operationalization of company practice on SEP}

- Have stakeholders in the host country been involved/engaged in decision-making related to the company's operations in that country?

" How do host country stakeholders (e.g. communities, workers, civil society organizations, local government officials, local government officials) perceive the company's performance on stakeholder engagement?

" Did the company engage with field informants in their research related to the present study?

" Does the company monitor suppliers and contractors in the host country to prevent, avoid, and mitigate negative impacts throughout the value chain?

- Does the company seek to leverage its buying power in the host country to improve conditions in the supply chain?

- Does the company provide sufficient and timely information to stakeholders in the host country about its local operations and its CR policies?

- Does the company translate such information into local languages?

- What are the primary channels of communication used by the company to communicate with stakeholders in the host country? 


\section{Chapter 4}

Modes of home-country business

culture and selection of case study TNCS

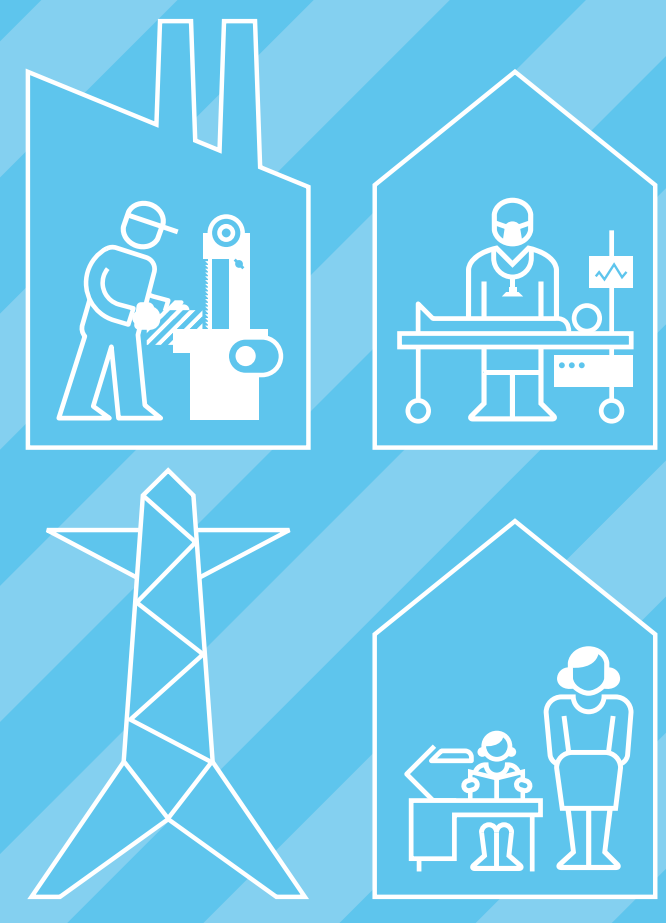


The international normative standards for SEP identified in the previous chapter are expected to influence the policies and practice of all electricity TNCs. However, the way in which international standards are interpreted and implemented by individual TNCs varies (Ruud 2002, Welford 2004, Hall 2005, Thomas 2007, Yamin and Sinkovics 2008). In order to systematize and structure variance within the analysis of electricity TNC performance on the SEP standards, the present study introduces an intervening variable - mode of home-country business culture.

The literature on institutional economics, (crosscultural) management, and business strategy and ethics suggests that individual TNCs often behave in ways that reflect their geographical corporate cultural background when it comes to prioritization of certain values and norms related to CR for sustainable development, as well as the overall relevance given to voluntary international normative standards (Hofstede 1983, Orpen 1987, Hofstede et al. 1990, Pinkston and Carroll 1994, Vogel 1996, Stajkovic and Luthans 1997, Burton et al. 2000, Hall and Soskice 2001, Hofstede 2001, Levy and Rothenberg 2002, Delmas and Toffel 2004, Welford 2005, Barron 2010, Dimitratos et al. 2010). For example, Graus et al. (2004) revealed strong regional differences between major electricity companies from the European, Nordic, and US regions in terms of transparency, fuel mix, and other sustainability indicators.

A recent examination of internationallyoperating firms from four different countries by Dimitratos et al. (2010:1) found that "the national culture of the focal internationalized enterprise has significant impact on its strategic posture and activity". Similarly, Stajkovic and Luthans (1997:19) consider adoption of certain normative standards to be a key part of business strategy that is influenced by national culture, noting that, "Ethical standards and resulting ethical behavioral conduct is grounded in the unique characteristics of each national culture". In particular, Kirca et al. (2009) found that national cultures affect how corporations internalize values and norms such as responsibility, equality, innovation, flexibility, and the need to protect the environment. Welford (2005:33) contends that "many CSR policies are based on cultural traditions" and that "companies often reflect what is important among stakeholders in their own home country" (Welford 2004:45).

As an intervening variable, the function of the "modes" within the overall analytic design is to capture and systematize what is a common understanding of the variation across countries and regions as to how TNCS operate with respect to SEP norms. The framework of modes of home-country business culture employed here serves to structure the dissertation's crucial variables (i.e. uptake and operationalization of SEP norms by electricity TNCs) and generate variance in the problematic that can be addressed through comparative case analysis. It is important to reemphasize that the modes framework is a tool used to analyze the behavior of different firms, not a research question in itself nor an 
empirical focus of the research. The literature points to the probable importance of the regional/national differences in a context of globalization, but the framework employed in the present study is not intended to provide definitive conclusions about the impact of home-country business culture. Rather, the "intervening" nature of the variable is understood as a "conditioning" effect. The mode "intervenes" between the SEP norms and the TNC to "condition" the degree to which the TNC adopts the norms and how it translates them into its own CR policy and practice.

Five specific regional modes of home-country business culture have been selected for study. The US, European, and Nordic modes were chosen because they are home to the vast majority of electricity TNCs active in the Global South. TNCs from these three regions accounted for $66 \%$ of all FDI in electricity infrastructure in the Global South in 2008 (World Bank 2008). In addition to these three "Northern" modes, TNCs based in emerging economies are playing an increasingly important role in electricity provision, and in global politics and economics in general. It is thus particularly timely and relevant to include the Chinese and the South African modes in the present study. Combined, TNCs from these five modes represented $76 \%$ of all FDI in the electricity sector in the Global South in 2008 (World Bank 2008).

This chapter elaborates on each of the five selected modes: US, European, Nordic, Chinese, and South African. The description of each mode of business culture is based on the literature.
No empirical research to define or determine the characteristics of the modes was conducted. Nor does the present study purport to present a rigorous or detailed analysis of culture. Culture, including business culture related to CR for sustainable development, is a multi-level, multifaceted concept (Trompenaars 1993, Hofstede 2001, Taras et al. 2009). For the purposes of the present study, culture is broadly understood to be the values, norms, beliefs, and behavior that are shared by - and socially transmitted within - a given society (Barron 2010). The five modes of home-country business culture in question here are described using general qualitative indicators such as:

- Evidence of shared values related to CR for SEP within the country, including formal declarations of these common values

" Formalization of the country's interpretation/definition of the concepts of sustainable development and CR

- Regulatory style in the areas of CR and SEP

- Degree of demonstrable interest of the country's business and civil society in CR

- Evidence of prioritization of certain SEP issue areas over others

Although the analytical concept related to the intervening "modes" variable is termed "homecountry business culture", note that two of the modes (the Nordic and the European) refer to regions rather than specific countries. The decision to use the regional rather than the national modes in these two cases is based on the suggestion in the literature that countries within both the Nordic and the European regions share a strong "regional" culture with 
regard to CR for sustainable development (Maanavilja 2010, Hoel 2011). For example, the European Union has developed joint positions on CR for all EU member countries. A similar exercise has been conducted by the Nordic Council for the Nordic countries. While maintaining the analytical and conceptual focus on a TNC's cultural-geographical background, this approach also parries some of the criticism of the interpretation of "culture" by Hofstede $(1983,1990)$ and followers for being too focused on national borders in defining culture when, in reality, culture often extends beyond borders into a larger region (Hall and Soskice 2001, Søderberg and Holden 2002).

\subsection{The US mode}

Through their voluntary philanthropic endeavors in the early 20th century, US business leaders like Rockefeller, Carnegie, and Ford may have been the first to popularize the notion of CR in the United States. Since then, however, CR in the US has evolved along a strict legal path, with corporate social and environmental responsibility being driven by legislative initiatives rather than proactive corporate policies. American economist and professor at the University of Chicago, Milton Friedman (1962:135), famously wrote in his book Capitalism and Freedom that:

\section{"Few trends could so thoroughly undermine} the very foundation of our free society as the acceptance by corporate officials of a social responsibility other than to make as much money for their stockholders as possible. There is one and only one social responsibility of business - to use its resources and engage in activities designed to increase its profits so long as it stays within the rules of the game, which is to say, engages in open and free competition without deception or fraud".

While it would be neither fair nor correct to characterize all US corporations as embodying Friedman's narrow interpretation of $\mathrm{CR}$, the economist's thinking did go far in both reflecting and influencing US business culture in this regard. Vogel (1996) characterizes the US pattern of regulatory style and corporate culture as one based on a fierce respect for private property and ownership, and a culture of shareholder capitalism that leaves little or no room for CR among companies. This leads to a focus on generating return on investment in the short term, what McSweeney (2002) calls the "shorttermism of the US capital market".

Friedman's reference to staying "within the rules of the game" alludes to the distinct legal and legislative (as opposed to morally or ethically driven) nature of the conception of CR within US business culture. Jones (2010:437) confirms this observation, noting, "Beginning in the late 1960s and early 1970s, the US government established regulatory agencies that shaped much of the CSR benchmarks guiding business operations". For example, Stajkovic and Luthans (1997) highlight how ethics legislation in the US, specifically the Foreign Corrupt Practices Act (FCPA) of 1977, which prohibits bribery of foreign officials by 
US businesses, has influenced the behavior of US companies. They cite a survey in which 124 anonymous US business managers were asked how they would respond to a request for a bribe to obtain a contract in a foreign country. The majority of the US managers responded that they would not pay the bribe, not necessarily because of their own personal values, but because paying the bribe would be illegal under the FCPA and therefore unethical (i.e. contrary to their personal values against illegality). Their European counterparts responded in the converse. Europeans would either pay the bribe because they felt it was not unethical, or they would not pay the bribe because they felt it was unethical, not because it was illegal.

More recently, the 2002 Sarbanes-Oxley act, passed in the wake of the Enron and WorldCom corporate financial scandals to improve the accountability of the private sector, is another example of the US tendency to legislate elements of CR and impose stiff legal penalties for noncompliance (Daugherty and Georgieva 2011).

Similarly, Ciulla (1991) believes that US companies develop and comply with strict codes of ethics, which are much more common in the US than in other regions of the world, primarily as a defense against legal suits. This notion is corroborated by Langlois and Schlegelmilch (1990) who, after analyzing a wide range of ethical codes of both US and European corporations, concluded that the vast majority of US company codes are "concerned about the company's legal impact" and "focused on specific issues of procedural and distributive justice".
In contrast, the European corporate ethical codes "mostly emphasize codetermination and a sense of belonging and responsibility to overall society", and are more focused on "macro ethical issues" (Langlois and Schlegelmilch 1990). Stajkovic and Luthans (1997: 28) explain that "most US managers appear to be primarily focused on upholding the legal requirements relative to ethical conduct". A study by Burton et al. (2000) found that US business school students placed a higher value on fulfilling firms' legal responsibilities and lower priority on economic responsibilities than their Chinese counterparts.

Taka and Foglia (1994) emphasize another characteristic of business practice in the US - that of treating the decisions about which relevant ethical standards and conduct a company should adopt as the responsibility of individual managers rather than a multilateral process involving various other stakeholder groups, as is the general practice in Europe. A study by Welford (2005) found that, on average, US firms place lower value on "two-way stakeholder dialogue" than companies from Europe and Asia.

Instead of a culture of CR based on (voluntary) international standards for sustainable development, as exists in Europe and the Nordic region, the US mode thus exhibits a high degree of legislative control. To give just one illustration, subsequent to the initial voluntary endeavors of the early 20th century philanthropists, even corporate philanthropy in the US has been guided by legislation, with tax breaks for corporations that make charitable 
contributions to non-profit organizations being written into the legal code (Jones 2010). The strict legalistic mode of CR also plays out in the US electricity industry. Palast et al. (2000: 2) note that, in the electricity sector, "Despite a few new areas of deregulation, the United States holds to the strictest, most elaborate system of regulation anywhere".

A possible consequence of the strict legislative focus of CR within US business culture is that in those areas of business activity that are covered by legislation, or in jurisdictions where legislative measures are weak or unenforced, American businesses are left with little guidance as to responsible behavior. Where European businesses would be constrained by the interests and opinions of other stakeholder groups or by international normative standards and soft law, for US businesses, if something is not explicitly legally prohibited, the economic interests of shareholders are given priority. This is relevant because many elements of CR remain unregulated: "In the American approach, CSR is considered as a process that is not consistently controlled" (Debroux 2009).

With regard to sustainable development priority issues, the US culture of shareholder capitalism (Vogel 1996) generally prioritizes economic issues such as job creation and return on investment over environmental and social concerns. Visser (2008: 489) observes a "narrow focus on profitability" among US businesses and a lower priority for ethical responsibilities in the US approach to CR compared to that in Europe. Indeed, Graus et al. (2004) found that
North American electricity companies scored the lowest on their scale of commitment to renewable energy (an average of just 0.7 out of $10)$ and had by far the lowest response rate $(3 \%)$ to their questionnaire.

In sum, instead of a culture of CR based on international standards for sustainable development, as exists in Europe and the Nordic region, the US mode exhibits a high degree of legal regulation and individualism. As a result, US corporations are expected to have less well developed CR policies than their European and Nordic counterparts. The approach of US electricity TNCs to SEP is expected to be more focused on economic issues such as job creation and generating short-term return on investment than on social or environmental issues, especially in host countries where strict American-style legal regulation is often lacking or weak.

\subsection{The European mode}

Although the economic, political, and cultural diversity of the European continent makes it difficult to define a single mode of business culture with regard to CR for sustainable development, Maanavilja (2010: 27) affirms that "the idea that companies can contribute to societal well-being beyond their legal obligations has a long tradition in many parts of the region." Vogel (1996) describes the European business culture as one of stakeholder capitalism and clear public-interest focused performance. 
Maanavilja (2010: 27) contends that, in Europe, "growing attention is being paid to the voluntary action that companies take as part of their CSR strategies to manage their economic, social and environmental impacts and to contribute to wider sustainable development" (Maanavilja 2010: 27). This cultural trend is reflected in the fact that the European Commission (EC) has recently sought to formalize its interpretation of the concept and to promote a common vision of CR throughout the continent. The EC's work on CR dates back to 2000 when, during the setting of strategic goals during Lisbon Council, it encouraged businesses to embrace CR (EC 2001b). When the EC, with the aim of developing a common European approach to CR, issued its first communication on CR in 2001, it received more than 250 responses to the document, half of which were from European companies (EC 2002). Since then, the Commission has issued three more communications on CR, in 2002, 2006, and 2012. In its communications, the EC (2006) stresses that the notion of CR and the importance given to it rests on common values found throughout the European region. The EC (2006) goes on to state that CR can contribute to addressing a number of the critical sustainable development issues, such as more rational use of natural resources, poverty reduction, respect for human rights, skills development, and better innovation performance.

The European culture of CR extends beyond government to a wide range of European stakeholder groups that have demanded a more prominent and clearer role for $\mathrm{CR}$. The European Coalition for Corporate Justice (ECCJ) is a non- profit umbrella group that brings together national platforms of civil society organizations (CSOs) from around Europe promoting CR and corporate accountability. The ECCJ represents more than 250 CSOs from 16 different countries in Europe. One of the ECCJ's members, the Dutch MVO Platform, has produced a frame of reference that defines CR more sharply than the EC does: "CSR is a process whereby a company assumes responsibility, across its entire supply chain, for the social, ecological and economic consequences of the company's activities, reports on these consequences, and constructively engages with stakeholders" (MVO Platform 2012).

European businesses have also shown interest in CR. As mentioned above, more than 100 European businesses provided input on the EC's 2001 communications. The European Alliance for CSR and CSR Europe are networks of European businesses that provide a forum for businesses to exchange best practices on CR in a number of issue areas, such as fostering innovation and entrepreneurship in sustainable technologies, products, and services; assisting enterprises to integrate social and environmental considerations in their business operations, especially those in the supply chain; improving and developing skills for employability; improving working conditions; and, importantly, operating outside the borders of the European Union in a socially and environmentally responsible way. CSR Europe counts more than 70 corporate members, including a number of electricity companies.

Graus et al. (2004) found that European 
companies generally scored highest (compared to North American and Asian companies) in their ranking of commitment to renewable energy and that Europe was the region with the highest response rate to the group's request for collaboration in the research. It should be noted, however, that European businesses have fought and lobbied hard to keep the elements and critical issues raised in the CR debate voluntary and to prevent any normative standards for sustainable development from becoming law. For example, the European Federation of Energy Traders, an industry lobby group, has fought efforts to improve the EC's counting scheme for renewable energy (CEO 2008).

Although Europe has far-reaching social and environmental regulations, the European mode of approaching CR for sustainable development is not as dominated and defined by governmental regulation and the legal system as is, for example, the US mode. Stajkovic and Luthans (1997: 23) noted that the legal impact of ethics legislation in defining corporate conduct "does not seem to be as great in European countries as it is in the US" and that "legislation might not be as directly influential in affecting ethical business standards" in Europe as in the US. The European business culture surrounding CR and sustainable development is both more oriented toward voluntarism and more toward a multi-stakeholder approach than is the case in the US. Many CR concepts are influenced by Europe's traditional tripartite model, which institutionalizes the relationships between public authorities, unions, and businesses (Debroux 2009).
Langlois and Schlegelmilch (1990) emphasize that, in contrast to the American focus on issues of legality and procedural correctness, European businesses' codes of conduct concentrate more on macro-level ethical issues and greater responsibility to overall society. Individual managers of European firms are expected to engage with other stakeholder groups such as unions in determining ethical business standards, whereas a go-it-alone attitude among American managers characterizes the US business culture. Stajkovic and Luthans (1997: 29) call the European mode of recognizing the interests of other stakeholders an "ethics of consensus" or "communicative ethics". They claim that this "trans-individual level of analysis" may be the reason that the European approach to CR for sustainable development is less legalistic and regulatory than the US: "European managers may focus more on vicarious self-regulation than US managers" (Stajkovic and Luthans 1997: 29). This leads to a distinct tendency in the European business culture to regard international standards as important and to rely heavily on cooperative initiatives such as the UNGC and "soft law" such as the EC "communications" on CR (Welzel et al. 2007).

In terms of priority issues within the realm of sustainable development, Maanavilja (2010: 28) notes that "as a relatively wealthy, stable region with a developed economic and societal structure, the current CSR issues and challenges in Europe naturally differ to some extent from those faced by the world's less developed regions". She notes that environmental issues 
such as climate change, biodiversity, pollution, and use of natural resources are currently given high priority in Europe. For example, in the 2010 Yale University Environmental Performance Index, which compares the environmental performance of 163 countries worldwide, 16 of the top 25 countries are European (Yale University 2010). Transparency and reporting on CR and sustainable development issues are also increasingly becoming an integral part of European business culture. Denmark, France, Norway, Sweden, and the UK have all recently introduced requirements for corporate reporting on social and environmental performance (Maanavilja 2010).

\section{Compared to environmental and economic} issues, a study by ECOTEC (2007) found that the social pillar of $C R$ is often the dimension most neglected by European electricity companies. This may be due to the fact that European companies have a longer history of reporting on economic and environmental issues than on the social impacts of their operations. Social issues are often overshadowed by the dominance of the current debate on global warming, which has focused the region's CR concerns on the environmental aspects of sustainable development.

\subsection{The Nordic mode}

According to Hoel (2011), "The Nordic region is often referred to as an area where businesses share a number of strong common trends".
The Nordic countries have much in common with mainland Europe, but maintain a few key differences with regard to firms' approach to CR for sustainable development (Welford 2005). It was the Norwegian Gro Harlem Brundtland - then-Chair of the UN World Commission on Environment and Development and author of the 1983 Our Common Future report - who first brought the term "sustainable development" to the world stage. More recently a "white paper" published by the Norwegian Ministry of Foreign Affairs (2009) firmly sets CR in the context of sustainable development, highlighting the importance of ethical frameworks, international standards, and transparency. In its 2011-2013 "Nordic Co-operation Programme for Innovation and Business Policy", the Nordic Council (2010) outlines its plan to develop a "Joint Nordic CSR strategy" to distinguish its values and vision on CR from the rest of Europe. The joint strategy is to be based on a number of core values that define the Nordic business culture (Nordic Council 2011).

The Nordic region is considered a global leader in implementing ethical standards (Welford 2005, Nordic Council 2011). Hohnen (2009) believes that the "Scandinavian appetite for pushing the corporate responsibility agenda to the fore" is in fact based on the "[Nordic] region's support for high ethical standards". For example, the Norwegian government has also established ethical guidelines for its "Government Pension Fund -Global" that are based on international standards and are the strictest in the world for pension fund investments (Norwegian Finance Ministry 2008). Many Nordic companies 
claim that the concepts of CR and sustainable development are "deeply ingrained" and a "natural part" of their business culture (SN Power 2008b, Nordic Council 2011).

The Nordic mode thus goes beyond the European CR tradition by combining it with the Scandinavian welfare culture that incorporates added importance for such aspects as universal coverage for services including social insurance, an advanced level of gender equality, a long tradition of respect for human rights, and a high degree of equality in income distribution embedded in the Norwegian welfare state (Welford 2005, Aarhus 2010, Hoel 2011). There is also a Nordic tradition of transparency and social dialogue with companies that dates back to the beginning of the industrial period (Kuhnle and Ervik 1996). In 2007 the Swedish government made sustainability reporting by state-owned companies mandatory. The Danes have gone even further with their "Social Responsibility for Large Businesses law", which came into effect on 1 January 2009. In order to encourage Danish businesses "to work actively on ways they can contribute to solving social challenges", the new law requires over 1,000 of the country's largest companies, both listed and state owned, to include information on their $\mathrm{CR}$ policies and practices in their annual financial reports. This may be the reason that ECOTEC (2007) found that, among European electricity companies, enterprises based in Nordic countries publish the most information about their CR policies.

Nordic countries like Norway and Iceland have a long history of using renewable sources of energy for electricity, primarily the countries' abundant hydraulic and geothermal resources, and Denmark is a global leader in wind energy.

The Nordic business culture surrounding CR for sustainable development is characterized by a high degree of commitment to international standards, as is indicated by the Norwegian government's CR "white paper", which expresses strong support for international standards such as the OECD Guidelines for Multinational Enterprises, the GRI, and the UNGC (Norwegian Ministry of Foreign Affairs 2009). Such standards can thus be expected to drive CR and the approach to sustainable development in Nordic companies. In addition, rather than being seen as an external charitable project, CR is generally understood to be an integral part of core business (Nordic Council 2011). Environmental problems such as climate change are likely to have a prominent place in corporate policies, along with human rights, and transparency issues. As in the European mode, engagement with civil society and other stakeholder groups is expected to characterize the approach of Nordic businesses. In addition, Aarhus (2010) identifies supply chain management as another CR issue that is gaining importance in the Nordic region, noting that the Norwegian Research Council is currently investigating the responsibility of Norwegian companies in global value chains. 


\subsection{The Chinese mode}

"The Chinese are doing more than the G8 to make poverty history. If a G8 country had wanted to rebuild the stadium, we'd still be holding meetings! The Chinese just come and do it. They don't hold meetings about environmental impact assessments, human rights, bad governance and good governance. Chinese investment is succeeding because they don't set high benchmarks."

- Sahr Johnny, Ambassador of Sierra Leone to China, 2005 (cited in Brewer 2008)

"China's approach to our needs is simply better adapted than the slow and sometimes patronizing postcolonial approach of European investors, donor organizations and nongovernmental organizations. In fact, the Chinese model for stimulating rapid economic development has much to teach Africa."

\section{- Abdoulaye Wade, President of Senegal, 2008 (cited in Brewer 2008)}

The vastness and diversity of China's geography and demography make it difficult to pin down any one dominant Chinese business "culture", but a number of key elements and overarching characteristics of the Chinese approach to CR for sustainable development can be identified. In addition to thousands of years of history, China's unique combination of a Communist political system and a more recent shift to a (largely) free-market economy operating in an increasingly globalized world has an impact on how Chinese businesses approach their responsibility to society, the environment, and economic development.

The Chinese notion of CR is rooted in and continues to be largely influenced by the country's cultural traditions and historic value systems such as placing the interests of family and society at large over those of the individual (Lee and Wickerham 2010). Alfonso et al. (2010) believe that the practice of CR in China is influenced by Confucianism, which includes an ethical mandate for individuals to work toward the greater good of the community or collective. It may be for this reason that the Chinese approach to CR retains a distinctive paternalistic overtone, in which "CSR emanates from the personal responsibility of organizational leaders (often also owners) rather than organizational responsibility" (Alfonso et al. 2010: 16). This notion is corroborated by Debroux (2009), who notes that, "In China, CSR is imposed from the top" and suggests that the result is often a focus on large-scale, national issues over more localized or individual concerns.

Olson et al. (2007: 37) characterize the Chinese business culture as "collectivist" because of the high value it places on unity and cohesion, loyalty within groups, traditional vertical hierarchical authority, and its discouragement of disharmony. Indeed, the concept of a "harmonious society" (xiaokang) weighs heavily in the CR policies of many Chinese businesses. The Chinese mode also rests on a long tradition of charity and philanthropy, and the current social responsibility practices of Chinese companies continue to be largely focused on gift-giving and donations (Visser 2008). 
Such charitable giving, however, is often done through unstructured, ad hoc cash donations to charities (such as after natural disasters) rather than through a coherent strategic corporate policy to address clearly identified social and sustainable development issues (APEC 2005).

Following Chairman Mao Zedong's banning of private enterprise in 1949, the country's state-owned enterprises were largely expected to exercise a broad responsibility to society. Under the socialist notion of "companies as society", corporations were idealized as economic engines providing for society (Chang 2010). Although many Chinese enterprises, including the majority of the country's largest TNCs, remain in state hands, the re-emergence of private enterprise in the 1980s under Chairman Deng Xiaoping - who famously proclaimed that, "To get rich is glorious" - has seen profit-driven goals rematerialize and displace social responsibilities (Lee and Wickerham 2010).

A study by Chen (1995) found that employees of Chinese firms placed more value on the economic success of their company and gave lower priority to humanistic goals than did employees of US firms. These findings were confirmed in a follow-up study by Burton et al. (2000), which found that Chinese business school students place much more value on economic responsibilities and much less value on legal and ethical responsibilities than their US counterparts. When the Chinese "sweatshop" scandals of the late 1990s and early 2000s covered the media and damaged the Chinese image, the Chinese government began viewing
"Western-style CSR" and the normative standards associated with it as a de facto trade barrier and a strategy to prevent Chinese businesses from "going out, going global". Lee and Wickerham (2010: 123) noted that, "During this stage, government attitudes toward CSR ranged from skeptical to hostile". In response to the spread of such international standards, the Chinese government sought to gain control over the situation by developing their own standards, such as the "China Social Compliance 9000 for the Textile \& Apparel Industry", a social responsibility management system based on China's laws and regulations, international conventions and standards, and China's particular situation (Chang 2010, CSC9000 2010).

More recently, however, the Chinese government and many Chinese businesses have begun to embrace, at least publicly, the notion of corporations' responsibility to contribute to broader sustainable development aims. The Chinese government held the first national CSR Summit in the Great Hall of the People in 2006, and "CSR" was first mentioned in a 2006 company law. In 2008, the Taida Environmental Index for the Shenzhen Stock Exchange was developed, and China's state regulatory authority and majority owner of many of China's SOEs, the State-owned Assets Supervision and Administration Commission of the State Council (SASAC 2008), published its "Guidelines for state-owned enterprises directly under the Central Government on fulfilling corporate social responsibilities". These guidelines provide an indication as to why the government feels businesses should adopt 
CR policies and practices. The reasons given include businesses' responsibility to contribute to a "harmonious society", a reference to the traditional Confucian values mentioned above; the fact that CR can contribute to "modernizing" firms and improve their competitiveness; an acknowledgement of the importance being placed on CR by the international community, and the need for China to establish a "responsible public image" internationally; and, notably, CR's potential to help Chinese firms "realize sustainable development", going even so far as to call firms' fulfillment of their CR a "necessary condition" for sustainable development (SASAC 2008). In order to do so, the SASAC encourages Chinese state-owned TNCs to adopt international CSR standards and best practices. Even more recently, the Chinese government actively participated in developing and officially endorsing the ISO 26000 social responsibility guidance (ISO 2011).

This shift in approach to CR has been reflected in the policies and practices of Chinese businesses. In 2006, the state-owned electricity company State Grid Corporation of China became the first Chinese company to issue a CR report, with over 100 doing so in 2008 and more than 600 in 2009 (Lee and Wickerham 2010). It should be noted, however, that Sutherland and Whelan (2009) found that less than half of the Chinese TNCs' CR reports were easy to find and access, and that reporting was more common among Chinese state-owned enterprises (SOEs) than private businesses. A 2008 IBM "Global CEO" study reported that Asia-Pacific businesses are increasing their spending on CR faster than any other region of the world (IBM 2008), and the 2008 Grant Thornton International Business Report found that Chinese firms led the Asia-Pacific region in adopting CSR as a business strategy (cited in Enterprise Innovation 2008). This trend and the changing business culture in China was clearly reflected in a 23 June 2010 speech by Wang Zhongyu, President of the China Enterprise Confederation, who proclaimed, "Today, the [Chinese] business philosophy has changed. ... Cost, quality, and availability have become minimum requirements. Business ethics and social responsibility have become the key factors in enhancing competitiveness and sustainable development" (cited in Chang 2010).

With reference to the Chinese TNCs' adoption of international standards, as encouraged by the SASAC, a recent study by Sutherland and Whelan (2009) found that the largest Chinese TNCs are only selectively adopting and participating in global CR standards and norms. They are fully engaging in some - such as the UNGC, the ISO 14000 series (China ranks among the top ten countries worldwide in the number of ISO certifications issued (Debroux 2009)), and the GRI - while completely ignoring others, such as the Extractive Industries Transparency Initiative (EITI) and the UN Voluntary Principles on Security and Human Rights. Chan et al. (2008) suggested that this may be because the latter norms and initiatives are based on historically Western values, such as liberalism, democracy, and accountability, which may not be in line with Chinese values. Sutherland and Whelan (2009) expect Chinese 
TNCs to be favorable toward the more "technical" international CR initiatives and to avoid the more "political" standards that are often related to human rights. Although China has endorsed many of the ILO Conventions and has many progressive labor laws on the books, labor rights remain a notoriously contentious issue. China does not allow independent labor unions, thus restricting workers' rights to freedom of association and collective bargaining, and Chinese firms' implementation of labor laws on working hours, wages, and OHS standards is weak (Welford 2005).

Therefore, despite an obviously increasing interest in CR in China, the degree to which Chinese TNCs are genuinely implementing CR practices is questionable. Alfonso et al. (2010: 20) assert that Chinese businesses "are adopting CSR policies not so much to 'save the planet' as to survive and prosper". IBM (2008) found that Chinese CEOs had high expectations that their "investment" in CR would provide financial returns $-84 \%$ of Chinese CEOs felt this way, compared to a global average of $69 \%$. Sutherland and Whelan (2009:13) find an uncanny similarity between the language in the SASAC Guidelines and the CR policies and reports of many Chinese TNCs. Almost all Chinese CR reports mention the company's desire to contribute to a "harmonious society", suggesting that the policies may simply be a way for company executives to gain or maintain favor with central policy-makers by "clearly signifying their adherence to the political ideology of the Chinese Communist Party". The Chinese mode for CR thus entails a significant degree of simply using CR as a marketing tool and window dressing to please party officials and uncritical investors, with little genuine attention to critical CR issues (Visser 2008).

Therefore, although the Chinese mode is experiencing a transition brought on by globalization and increasing international scrutiny, many of the changes have thus far been cosmetic, and the core of the Chinese mode remains a focus on economic growth. Human rights and the environment are often sacrificed to drive the economic development of the country as a whole. Qin Hui (2010) notes, "The continuing rapid development of [the] Chinese economy, or the so-called 'China Model', is mainly attributed to China's 'low human rights advantage' in the context of globalization. Without independent trade unions, there is no collective bargaining right in China; without legal protection to the property right of land and house, the government can acquire or use any land it wants; the prices of certain productive elements such as resources are suppressed; the environment is neglected; public participation is repressed; independent thoughts and social justice are sacrificed to fulfill material desires... these are all reflections of 'the China Model'."

The hydropower sector provides an instructive example illustrating, and to a large degree encapsulating, the Chinese approach to development and CR. Over the past 60 years China has built half of the world's large dams. It boasts a higher hydropower capacity than any other country and with the Three Gorges Dam, has built the world's biggest hydropower 
project. China is also a major player in the hydropower sector of many countries in the Global South. Chinese companies are currently involved in more than 250 dam projects in 68 countries worldwide (Bosshard 2011).

As Bosshard (2011) explains, however, Chinese hydropower development has spawned a wide range of technical, social and environmental problems. "In international comparison, Chinese dams have a bad safety record. In many projects, thousands of local people have protested against insufficient compensation for their lands and abuses by corrupt local officials. In May 2011, China's highest government body acknowledged that the Three Gorges Dam, which had served as a symbol of the country's engineering prowess, was causing 'urgent problems' in terms of environmental protection, geological hazards, and relocated communities".

As Chinese hydropower companies have embraced the "Going out" policy and expanded their operations overseas, their approach has been met with outspoken approval by many host governments, who are pleased with the rapid pace at which Chinese hydropower companies assess and implement projects. The quotes from African government officials at the start of this section are telling, attributing the Chinese mode's competitive economic advantage to its low standards for human rights and environmental protection. As at home, the approach taken by Chinese hydropower companies to "prioritize technical and economic aspects at the cost of the environment, local communities, and human rights" has had severe negative impacts on people and the planet (Bosshard 2011). Qin Hui (2008) describes the Chinese power companies as having enjoyed a "competitive advantage based on no political conditions attached, engagement primarily with governments rather than communities, little concern for human rights, labor rights, or environmental protection, and a certain level of corruption - i.e. completely profit driven economic behavior."

In response, Chinese government officials have recently indicated that they want hydropower companies, often acting as the face of China abroad, to be socially and environmentally responsible actors, by respecting the rights of local communities, workers, and the environment (SASAC 2008). Chinese power companies can thus be expected to attempt (at least publicly) to balance these social and environmental interests against concerns, though in a way that continues to prioritize Chairman Deng Xiaoping's imperative of setting "economic development as the central task".

\subsection{The South African mode}

Carapinha and de Jongh (2008) contend that any consideration of the South African culture for CR cannot be made without regard for the historical distortions at both a social and economic level resulting from the institutionalized practice of racist policy and legislation during the apartheid period. During apartheid, South Africa was defined by a system of accumulation best described as 
"racial capitalism" (Fig 2005). In this system, economic exploitation of a working class was compounded by racially oppressive laws and institutions that ensured a cheap supply of exploitable labor resources from forced pools of rural labor. Corporate profits were essentially subsidized by rural subsistence, which was later formalized into the Bantustan system. A system of super exploitation thus dominated business culture in a transition from a colonial to a racially defined republic (Wolpe 1972). A strong and involved state is necessary for the functioning of such a system, and class and cultural accommodations are necessary to support such a state (Fig 2005). Much industrial development was aligned with ambitions of uplifting the white component of the working class and involved the state-facilitated provision of cheap energy and labor.

During apartheid, many South African and foreign businesses were directly involved in racially-based exploitation of black workers through the twin system of class and racial exploitation. Poor environmental practices and dismal occupational health and safety conditions were commonplace. A recent decision by the South African Constitutional Court confirmed this, acknowledging the widespread occupational diseases suffered by ex-mineworkers and finding that companies are indeed liable for this. In turn, this has given rise to a massive pending class action suit in South Africa (McKay and Vuuren 2011).

However, even before the official end of apartheid in 1994, a shift in corporate conduct began to take place in South Africa. A significant factor in reshaping corporate conduct in South Africa during the apartheid period was the rise of the new progressive labor movement in the mid-1970s (Webster 1985, Friedman 1987). Part of the opposition to the apartheid regime saw the rise of militant and well-organized workers' organizations in the labor market. A strong, non-racial trade union movement increasingly pushed back the control frontier of racial capitalism in the workplace. This forced companies to begin to re-evaluate their discriminatory employment practices and at the same time developed a culture of engagement and negotiation. It is also argued that some South African businesses, perhaps feeling the pressure of international sanctions, undertook voluntary initiatives aimed at improving the condition of black workers and changing government policy (King et al. 2010). At the same time, initiatives such as the Sullivan Code, which prescribed non-racial employment practices, served as a means to justify the continued presence of foreign companies in what was effectively a pariah state. Companies argued that their presence could have a transformative effect while profiting from the racist system (Fig 2005). Some unions used such codes, but the labor movement was largely opposed to this political accommodation (Fig 2002).

It was commonplace for trade unions to make overtly political demands in collective bargaining processes. These demands sometimes also extended to community issues and were often backed by community actions such as consumer boycotts. Through these interactions, 
businesses were forced to recognize the linkages between workplace and community, linkages that the apartheid apparatus had gone to great efforts to deny. The strength of trade unions meant that corporate entities were often forced to make accommodations to labor that exceeded what was required by law at the time. As a result, recognition agreements between unions and businesses became the de facto replacements to national labor market regulation (Friedman 1987).

This process of engagement with workers and communities, albeit one that was forced on business by the labor and community movements themselves, laid the foundation for a South African culture of stakeholder engagement that predates the transition away from apartheid. The result of this is that the South African business culture of engagement can be characterized as reactive and adversarial rather than proactive and integrative in nature. While resistance forced the emergence of an "enlightened self interest" accommodation in the social aspects of $C R$, environmental responsibility emerged much later, a feature of the post-apartheid period. This has resulted in a sometimes tense alliance between environmental CSOs, which are generally dominated by whites, and broader community and social movement organizations (Fig 2002).

In response to apartheid South Africa's isolation and pariah status on the international stage and the strength of domestic labor unions, South African businesses began a practice of making corporate social concessions in the interest of sustaining their social license to operate and maintain profitability. At the same time, South African businesses became adept at polishing their image through reporting processes. This has particularly been the case with corporations with high-impact activities, such as those in the mining sector. Mining corporations comprised the largest source of foreign earnings and were central to the minerals-energy complex and industrial development in South Africa. These skills have been put to effective use in the post-apartheid period, which has seen the rapid expansion of South African multinationals throughout Africa.

The post-apartheid transition period and the reintegration of South Africa into the global economy also carried important consequences for the culture of corporate governance and $\mathrm{CR}$. The changing nature and roles of boards of directors and the introduction of notions of triple-bottom-line reporting were a part of efforts to become internationally comparable, both at an institutional and corporate level. The post-apartheid relisting of a number of South Africa's largest corporations on foreign stock exchanges has also played a role in shaping corporate reporting and responsibility initiatives to be more in line with global trends in the developed world. There is also evidence that suggests a general convergence of the manner of $\mathrm{CR}$ engagement between corporations from OECD countries and those from emerging markets (Baskin 2005). This suggests that, while there are certainly unique aspects to the South African mode, these have become situated in a broader global CR framework. 
The transition away from the apartheid regime saw a shift, but not necessarily a decrease, in the role that the South African state plays in defining the social role of companies. Indeed, the government in South Africa continues to have a strong influence on business culture, more so than in many other parts of the world. This is based on the policy reform program undertaken since the inception of a democratic government in 1994. Since the fall of the apartheid regime, black economic empowerment (BEE) has been a major focus of government and has been widely adopted by South African business. More recently, the need to develop deeper levels of economic empowerment led to the emergence of the notion of broad-based black economic empowerment (BBBEE).

At the same time, starting in the mid-1990s, the South African state followed a distinctly neo-liberal macroeconomic agenda, creating a flexible labor market and removing capital controls in an effort to attract foreign direct investment. In this framework, the externalized costs of business, most notably the costs associated with the provision of cheap energy for the country's mining sector and TNCs, continue to be borne by society (Fine and Rustomjee 1996).

It is also argued that the South African approach is rooted in a number of traditional African communal values, such as the notion of Ubuntu, which encourages a harmonious relationship between an individual or organization and the community and a respect for community culture (Nussbaum 2003). Putting this principle into practice should thus imply a high degree of interaction and engagement between businesses and the communities they impact. Although this was clearly not the case during the apartheid era, Carapinha and de Jongh (2008: 62) examined the relationship between South African businesses and their non-financial stakeholders over the past two decades and believe that the nature of the relationship has evolved from noninvolvement during apartheid, through a sort of ad hoc involvement, to a present relationship of "mutualism" or "partnership" that mutually benefits both parties. However, the alignment between what is important to the corporation and the real needs of the community is often tenuous (King et al. 2010, (Rossouw et al. 2002).

In addition to the history of apartheid and traditionally held values, the influence of South Africa's socioeconomic context on its business culture cannot be underestimated. South Africa is a fast-growing, middle-income country with more than $50 \%$ of the population living below the poverty line and a high degree of income inequality (Swilling 2010). Poverty and lack of access to all manner of services and opportunities continues to define South Africa. Indeed, the 2002 World Summit on Sustainable Development in Johannesburg placed poverty eradication at the center of efforts to achieve sustainable development. The South African government has since issued numerous public policy documents calling on business to contribute to development. Legislation on other social issues, such as working conditions, 
and environmental issues is less extensive and/or poorly enforced (King et al. 2010). The South African mode thus suggests clear prioritization of economic development issues over environmental concerns. The South African Department of Environmental Affairs (2013) has made no secret of its plan to continue to increase greenhouse gas emissions in the near and even medium-term.

Given the huge social backlogs and extreme wealth differences in the country, South African companies often find themselves engaged in the provision of social services that would be seen as government's responsibility in developed countries (Reimann et al. 2011). Examples include investment in roads, schools, hospitals, and housing. Indeed, Visser (2007) argues that Caroll's (1979) classic CR pyramid - which orders corporate responsibilities as firstly economic, then legal, then ethical, and finally philanthropic - should be reorganized for the African context. Visser (2007) contends that economic and philanthropic responsibilities are placed first, with less importance for ethical and legal responsibilities. Given weak implementation and enforcement of South Africa's laws and regulations, Hamann (2009) believes that even compliance with the law should be seen as an issue of "voluntarism".

Many South African companies view their efforts in the area of CR primarily as a type of strategic, "positive payback philanthropy with public relations benefits" (Klins et al. 2010:3). King et al. (2010:364) obeserves that, "CSR in $\mathrm{SA}$ is generally seen and treated as a business investment...on which a return on investment is expected". Fig (2005:601) explains that "business in South Africa generally eschews the notion of 'corporate social responsibility' in favor of the term 'corporate social investment' (CSI) or 'corporate citizenship', concepts that ask no questions about legacy, memory, history, justice, or moral and ethical responsibilities... Firms' social responsibility spending is therefore often aimed at deflecting criticism of their unsustainable practices". Klins et al. (2010:3) concur, noting that, "Given the relative wealth of companies in comparison to the poverty of the societies in which they operate, CR can be a way to counteract negative perceptions of business", meaning that $\mathrm{CR}$ is often undertaken with the ultimate aim of creating a positive image. Fig (2005:604) concludes that, "The construction of reputation, whether to evade historical accounting from the apartheid era or to disguise contemporary violations, such as severe environmental pollution from coal-fired power generation, favors the notion of 'corporate social investment"'.

While these corporate social "investments" have, in some cases, contributed to development in the communities where they are made, they often serve the interests of the corporation rather than the real needs of the community, and they "are generally not integrated or aligned to core businesses" (King et al. 2010:364). In fact, the ad hoc nature of such engagements potentially undermines broader development practice at local levels and the key role that governments should play in this. A report by the South African Department 
of Mineral Resources (2009:10) evaluating the practice of CSI among South African mining companies noted that, "There is no evidence of a direct link between the proposed and implemented community development projects and the needs of affected communities. This is a result of inefficient consultation process, poor or lack of collaboration with communities and lack of alignment to established local economic development frameworks. The disjuncture between consultation and collaboration with affected communities minimizes the developmental impact of the mining industry on communities".

South Africa does have a long history of using and relying on international standards and voluntary codes related to $C R$, such as the Sullivan Principles, which advanced labor rights during apartheid (Sullivan Principles 2010); the King Code, which itself endorses the GRI, the UNGC, and the AA1000 standard (IODSA 2009); and the Social Responsibility Index of the Johannesburg Securities Exchange (Fig 2005). The King Code also encourages external (international) verification of CR reports, and the ISO 14001 certification standard is widely used in South Africa (King et al. 2010, Klins et al. 2010). CR reporting is in its formative years in South Africa and the number of companies that are reporting is growing rapidly. In 2002, only one South African company issued a sustainability report. By $2008,86 \%$ of listed companies included some level of sustainability reporting in their annual report or issued a standalone report (KPMG 2008).
The King Code of Corporate Governance (King II), one of the most respected and cited sources of guidance on CR in South Africa, recommends reporting on sustainability, and is a requirement for listing on the South African stock exchange (Rossouw et al. 2002). Reporting tends to focus on issues such as BEE, occupational health and safety, and employment equity. However, this does not generally apply to corporations' operations outside South Africa.

\subsection{Summary of modal projections for corporate approach to SEP}

Based on the broad portraits of the five modes presented above, a general picture can be sketched as to how a company from a given cultural mode might be expected to take up and interpret the SEP standards. Employing the type of modified Likert (1932) scale used to roughly classify company performance on the SEP benchmarks, Table 4.1 provides an overview of projections regarding how likely (ranging from "very likely" to "very unlikely") companies from the various modes are to adopt an approach in line with each SEP standard. It should be noted that this broad sketch of the implications of each mode for SEP does not purport to represent a comprehensive or blanket description of the modes that will apply equally to every company within the cultural mode. Rather, the overview comprises a summary of the essential features, common patterns of values and behavior, and 
hypothetical expectations for each mode. The expectations are generalized from the literature on each mode and the author's own "educated interpretation" as to what the basic features imply for the uptake of international normative standards related to SEP.

\subsection{Selection of a TNC "representative" for each mode}

One case study TNC for each of the five modes of home-country business culture was chosen for analysis in the present study. The case study companies were selected based on a wide survey of potential companies from each national or regional mode. The primary criterion for selecting the five cases was that the company had to be a TNC headquartered in one of the five national/regional modes with significant electricity provision operations in more than one country in the Global South. In order to make use of the modes framework, the selected TNCs also needed to be considered a representative "conduit" of their respective home-country mode. The initial survey of potential case study companies involved a review of CR and sustainable developmentrelated operational aspects of potential candidates.

The case study companies were thus not selected randomly. They were selected with a view to maximizing the variation on the study's key variables of TNC policy and performance.
This is method suggested by Gomm et al. (2000). Based on the average pattern of values and behavior identified in each mode, it is reasonable to assume that each case study TNC provides a credible reflection of its respective mode of home-country business culture. The non-random, educated selection of a single case study from each mode is necessary for the level of generalization sought (i.e. with relevance for the design and implementation of international normative standards) by the present study (Halkier 2011). However, the "fit" between the selected case study companies and the modes is not intended nor expected to be a one-to-one match-up.

The selection of the five case studies for the present analysis does not make any claims of statistical representativeness, nor is such representativeness necessary for the type of exploratory study undertaken here (Seawright and John 2008). Though the findings of the present study may be of interest to and have relevance for scholars of cross-cultural management, the problematic and analytical approaches were not designed to test the relationship between cultural mode and TNC. The modes framework is based on the literature and is simply being utilized in the present analytical approach primarily to generate variance on TNC performance on the SEP benchmarks. Any "probing" of the relationship between mode and TNC is purely exploratory at this point (Seawright and John 2008). It is thus possible, if not probable, that the empirical evaluation of TNC performance against the SEP benchmarks will reveal interesting anomalies 
between the cultural mode and actual policy and practice of the selected TNCs. A brief section at the end of each of the five case study chapters will reflect on the degree to which the company's performance on the SEP benchmarks reflects its mode of home-country

Table 4.1: Approach to SEP benchmarks expected by each mode

\begin{tabular}{|c|c|c|c|c|c|}
\hline SEP benchmark & $\begin{array}{l}\text { Approach to be } \\
\text { expected by the } \\
\text { US mode }\end{array}$ & $\begin{array}{l}\text { Approach to } \\
\text { be expected by } \\
\text { the European } \\
\text { mode }\end{array}$ & $\begin{array}{l}\text { Approach to be } \\
\text { expected by the } \\
\text { Nordic mode }\end{array}$ & $\begin{array}{l}\text { Approach to be } \\
\text { expected by the } \\
\text { Chinese mode }\end{array}$ & $\begin{array}{l}\text { Approach to be } \\
\text { expected by the } \\
\text { South African } \\
\text { mode }\end{array}$ \\
\hline $\begin{array}{l}\text { Endorse international } \\
\text { normative standards for CR }\end{array}$ & $\begin{array}{l}\text { Moderately } \\
\text { unlikely }\end{array}$ & Very likely & $\begin{array}{l}\text { Moderately } \\
\text { likely }\end{array}$ & $\begin{array}{l}\text { Moderately } \\
\text { unlikely }\end{array}$ & $\begin{array}{l}\text { Moderately } \\
\text { likely }\end{array}$ \\
\hline $\begin{array}{l}\text { Adopt a commitment to CR in } \\
\text { core-business activities and } \\
\text { decision-making }\end{array}$ & $\begin{array}{l}\text { Moderately } \\
\text { unlikely }\end{array}$ & $\begin{array}{l}\text { Moderately } \\
\text { likely }\end{array}$ & Very likely & $\begin{array}{l}\text { Moderately } \\
\text { unlikely }\end{array}$ & $\begin{array}{l}\text { Moderately } \\
\text { unlikely }\end{array}$ \\
\hline $\begin{array}{l}\text { Increase access to affordable } \\
\text { electricity }\end{array}$ & $\begin{array}{l}\text { Moderately } \\
\text { unlikely }\end{array}$ & $\begin{array}{l}\text { Moderately } \\
\text { unlikely }\end{array}$ & $\begin{array}{l}\text { Moderately } \\
\text { likely }\end{array}$ & $\begin{array}{l}\text { Moderately } \\
\text { likely }\end{array}$ & $\begin{array}{l}\text { Moderately } \\
\text { likely }\end{array}$ \\
\hline Respect labor rights & $\begin{array}{l}\text { Moderately } \\
\text { unlikely }\end{array}$ & $\begin{array}{l}\text { Moderately } \\
\text { likely }\end{array}$ & Very likely & Very unlikely & $\begin{array}{l}\text { Moderately } \\
\text { likely }\end{array}$ \\
\hline $\begin{array}{l}\text { Minimize environmental } \\
\text { impact, including contribution } \\
\text { to climate change }\end{array}$ & $\begin{array}{l}\text { Moderately } \\
\text { unlikely }\end{array}$ & $\begin{array}{l}\text { Moderately } \\
\text { likely }\end{array}$ & Very likely & Very unlikely & Very unlikely \\
\hline $\begin{array}{l}\text { Prioritize renewable sources } \\
\text { of energy for electricity }\end{array}$ & $\begin{array}{l}\text { Moderately } \\
\text { unlikely }\end{array}$ & $\begin{array}{l}\text { Moderately } \\
\text { likely }\end{array}$ & Very likely & $\begin{array}{l}\text { Moderately } \\
\text { unlikely }\end{array}$ & $\begin{array}{l}\text { Moderately } \\
\text { unlikely }\end{array}$ \\
\hline $\begin{array}{l}\text { Contribute to local economic } \\
\text { development }\end{array}$ & $\begin{array}{l}\text { Moderately } \\
\text { likely }\end{array}$ & $\begin{array}{l}\text { No clear } \\
\text { prediction }\end{array}$ & $\begin{array}{l}\text { Moderately } \\
\text { likely }\end{array}$ & $\begin{array}{l}\text { Moderately } \\
\text { likely }\end{array}$ & $\begin{array}{l}\text { Moderately } \\
\text { likely }\end{array}$ \\
\hline Ensure reliable supply & $\begin{array}{l}\text { No clear } \\
\text { prediction }\end{array}$ & $\begin{array}{l}\text { Moderately } \\
\text { likely }\end{array}$ & $\begin{array}{l}\text { Moderately } \\
\text { likely }\end{array}$ & $\begin{array}{l}\text { Moderately } \\
\text { likely }\end{array}$ & $\begin{array}{l}\text { Moderately } \\
\text { likely }\end{array}$ \\
\hline $\begin{array}{l}\text { Engage in meaningful } \\
\text { stakeholder consultation and } \\
\text { participatory decision-making }\end{array}$ & $\begin{array}{l}\text { Moderately } \\
\text { unlikely }\end{array}$ & $\begin{array}{l}\text { Moderately } \\
\text { likely }\end{array}$ & Very likely & Very unlikely & $\begin{array}{l}\text { Moderately } \\
\text { likely }\end{array}$ \\
\hline $\begin{array}{l}\text { Assume responsibility for } \\
\text { impacts throughout all } \\
\text { business relationships }\end{array}$ & $\begin{array}{l}\text { Moderately } \\
\text { unlikely }\end{array}$ & $\begin{array}{l}\text { Moderately } \\
\text { likely }\end{array}$ & $\begin{array}{l}\text { Moderately } \\
\text { likely }\end{array}$ & $\begin{array}{l}\text { Moderately } \\
\text { unlikely }\end{array}$ & $\begin{array}{l}\text { Moderately } \\
\text { unlikely }\end{array}$ \\
\hline $\begin{array}{l}\text { Maximize transparency and } \\
\text { provision of information }\end{array}$ & $\begin{array}{l}\text { Moderately } \\
\text { unlikely }\end{array}$ & $\begin{array}{l}\text { Moderately } \\
\text { likely }\end{array}$ & Very likely & $\begin{array}{l}\text { Moderately } \\
\text { unlikely }\end{array}$ & $\begin{array}{l}\text { Moderately } \\
\text { likely }\end{array}$ \\
\hline
\end{tabular}


business culture. A modified Likert (1932) scale is used to roughly classify a company's performance as "largely reflective", "moderately reflective", "moderately unreflective", or "largely reflective" of its home-country mode, as summarized in section 4.6 above.

The five selected TNC case studies chosen for the empirical analysis are:

" AES Corporation (hereinafter "AES") for the US mode. With headquarters in Washington, D.C., USA, AES provides electricity in 17 host countries in Asia, Africa, and Latin America.

- Endesa for the European mode. Based in Spain, Endesa is one of the world's largest providers of electricity with significant electricity provision activities in Latin America.

- SN Power for the Nordic mode. Based in Oslo, Norway, SN Power has electricity generation operations in Latin America and Asia.

- China Datang Corporation (hereinafter "Datang") for the Chinese mode. Headquartered in Beijing, China, Datang has electricity provision operations in Burma, Cambodia, Laos, and Kazakhstan.

- Eskom for the South African mode. Based in Sandton, South Africa, Eskom has electricity provision operations in several sub-Saharan African countries, the most significant of which are in Mali and Uganda.

The variance introduced by the five case studies further specifies and refines the analytic model presented in Chapter 2. Figure 4.1 illustrates the dissertation's analytic approach with variance, following the same logic as in Figure 2.1, but with each of the five different modes specified. Table 4.2 lists the selected TNCs and details about their operations that are relevant to the present analysis.

The five modes of home-country business culture identified and described above are employed as an analytical tool for generating data on how different TNCs "take up", interpret, and translate the SEP norms into policy and practice. As increasingly globalized competition for electricity provision contracts forces electricity TNCs to compete with each other through ever more complex supply chains and cross-border business relationships (Burton et al. 2000, Lyons and Deutz 2010, Hoel 2011), using the modes to structure the normativeempirical analysis of TNC performance on the SEP standards can shed crucial light on the issue of trade-offs and contribute to the overall improvement of efforts to develop and implement standards for sustainable and responsible corporate practice. The "mode" variable is thus particularly important for the overall practical, applied-science goals of the dissertation. Should the empirical analysis in the subsequent company-case chapters reveal a relationship between the uptake and application of SEP norms and the modal business culture of electricity-providing TNCs, this will imply important lessons for the international and regional providers of overarching SEP norms (e.g. the UN, OECD, $E U$, etc), as well as communities and other stakeholders affected by electricity TNCs in host countries. 


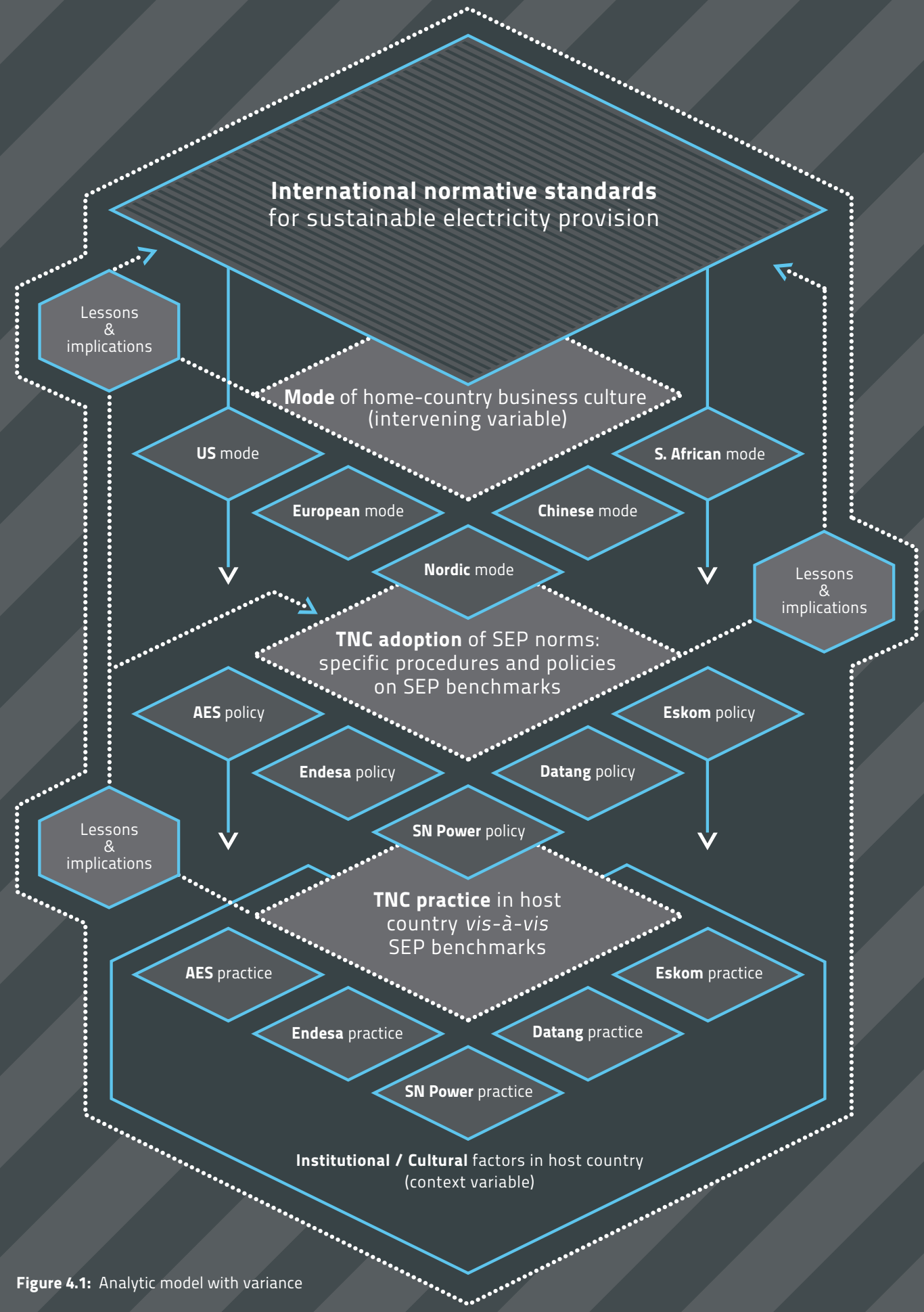


Table 4.2: Key global operational aspects of selected case study companies, 2010

\begin{tabular}{|c|c|c|}
\hline Operational aspect & AES & Endesa \\
\hline Headquarters & USA & Spain \\
\hline Ownership & Shareholder-owned & Shareholder-owned \\
\hline Revenue from electricity & $\$ 16.6$ billion (€12.2b) & $\$ 9.9$ billion (€7.3b) \\
\hline Net profit from electricity & $\$ 9$ million (€6.8m) & $\$ 5.4$ billion (€4.1b) \\
\hline $\begin{array}{l}\text { Countries in which the company has } \\
\text { electricity provision operations }\end{array}$ & $\begin{array}{l}29 \text { countries on five continents, largest } \\
\text { activity in Latin America }\end{array}$ & $\begin{array}{l}\text { Argentina, Brazil, Chile, Colombia, } \\
\text { Greece, Peru, Portugal, Morocco, Spain }\end{array}$ \\
\hline Employees & 29,000 & 24,732 \\
\hline Installed capacity (MW) & 40,500 & 40,141 \\
\hline $\begin{array}{l}\text { Current capacity (MW) in countries without } \\
\text { universal access (and \% of total) }\end{array}$ & $17,354(43 \%)$ & $14,707(37 \%)$ \\
\hline Annual production (GWh) & 85,000 & 130,484 \\
\hline Average capacity factor & $24 \%$ & $37 \%$ \\
\hline $\begin{array}{l}\text { Production (GWh) in countries without } \\
\text { universal access (and \% of total) }\end{array}$ & $36,426^{g}(43 \%)$ & $47,813^{i}(37 \%)$ \\
\hline Fuel mix for electricity generation (\%) & $\begin{array}{l}78 \% \text { non-renewable } \\
22 \% \text { renewable ( } 18 \% \text { large hydro, } 4 \% \text { wind) }\end{array}$ & $\begin{array}{l}66 \% \text { non-renewable } \\
34 \% \text { renewable ( } 29 \% \text { large hydro, } \\
4 \% \text { wind, } 1 \% \text { small hydro) }\end{array}$ \\
\hline $\begin{array}{l}\text { Global production (GWh) based on } \\
\text { renewables, including large hydro (excluding } \\
\text { large hydro) }\end{array}$ & $18,702^{\mathrm{g}}(3,400 \mathrm{~g})$ & $\mathrm{n} / \mathrm{a}$ \\
\hline Annual coal consumption(Mt) & $\mathrm{n} / \mathrm{a}$ & $\mathrm{n} / \mathrm{a}$ \\
\hline GHG emissions $\left(\mathrm{Mt} \mathrm{CO}_{2}\right)$ & $74^{d}$ & $39^{f}$ \\
\hline GHG intensity ( $\mathrm{tCO}_{2} / \mathrm{MWh}$ ) & $0.93^{d}$ & 0.28 \\
\hline New capacity under construction & $\begin{array}{l}2,000 \mathrm{MW} \text { total } \\
85 \% \text { non-renewable }(74 \% \text { coal, } 11 \% \text { gas) } \\
15 \% \text { renewables ( } 10 \% \text { wind, } 5 \% \text { solar) }\end{array}$ & $\mathrm{n} / \mathrm{a}$ \\
\hline
\end{tabular}

$\mathrm{a}=2011$ (includes all Eskom figures); $\mathrm{b}=2008 ; \mathrm{d}=2009 ; \mathrm{f}=$ including in the supply chain; $\mathrm{g}=$ AES does not provide production figures, this assumes the company's average capacity factor of $24 \% ; \mathrm{h}=$ no figures provided, assumes capacity factor of $50 \%$; $\mathrm{i}$ assumes capacity factor of $37 \%$. Sources for all information can be found in the individual company chapters. 


\section{SN Power}

Norway

State-owned

$\$ 114$ million (€84m)

$\$ 53$ million (€39m)

Chile, India, Nepal, Peru (largest market), Philippines, Sri Lanka

427

1,481

$1,481(100 \%)$

5,954

$46 \%$

$5,954(100 \%)$

\section{$0 \%$ non-renewable}

$100 \%$ renewable (95\% large hydro, $3 \%$ small hydro, $2 \%$ wind)

\section{$5,954(305)$}

0

0

0.0

$1,258 \mathrm{MW}$ total

$100 \%$ large hydro

\section{Datang}

China

State-owned

$\$ 27.0$ billion (€19.8b)

- \$18 million (- €13m)

Burma, Cambodia, China, Lao PDR

98,082

105,896

Including China: 105,896 (100\%)

Outside China: 240 (0.2\%)

472,575

$51 \%$

Including China: 472,575 (100\%)

Outside China: $1,052^{\mathrm{h}}(<1 \%)$

$88 \%$ non-renewable (all coal)

$12 \%$ renewable ( $10 \%$ large hydro, $2 \%$ wind)

$53,545(6,321)$

\begin{tabular}{|l|l}
$103^{b}$ \\
$250^{b}$
\end{tabular}

$0.71^{\mathrm{b}}$

$109,641 \mathrm{MW}$ total

$58 \%$ non-renewable ( $26 \%$ coal, $31 \%$ nuclear) $33 \%$ renewable ( $27 \%$ large hydro, $16 \%$ wind)

\section{Eskom $^{\mathrm{a}}$}

South Africa

State-owned

$\$ 11.2$ billion (€8.6b)

$\$ 1,069$ million (€811m)

Mali, South Africa, Uganda

41,778

41,194

Including S. Africa: 41,194 (100\%)

Outside S. Africa: 580 (1.4\%)

232,812

$65 \%$

Including S. Africa: $232,812(100 \%)$

Outside S. Africa: 2,000 (1\%)

$99 \%$ non-renewable ( $93 \%$ coal, $5 \%$ nuclear,

$1 \%$ other)

$1 \%$ renewable (1\% large hydro, negligible wind)

$1,962(2)$

125

230

0.99

$12,812 \mathrm{MW}$ total (by 2018)

$100 \%$ non-renewable ( $78 \%$ coal, $12 \%$ gas) other) 


\section{Chapter 5}

Case study-AES
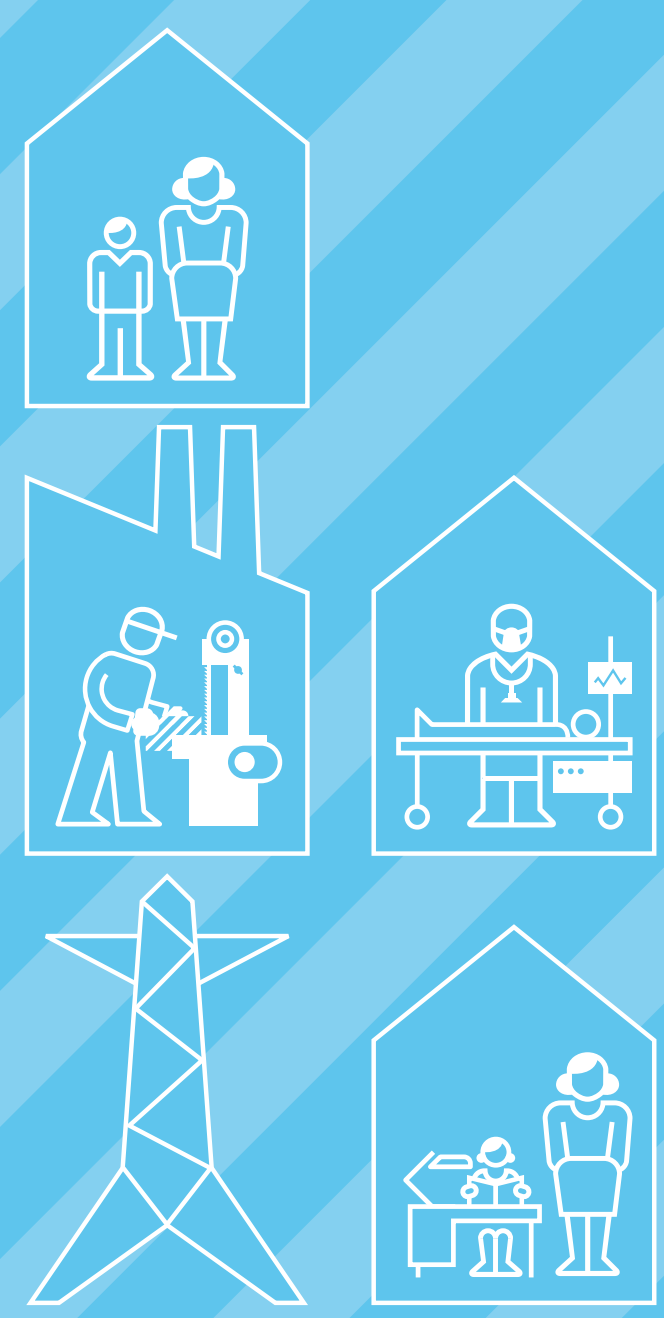


\subsection{AES background and operations}

\subsubsection{Basic company information}

AES was founded in 1981 and is headquartered in Arlington, Virginia, USA. The company built its first plant in Texas in 1985 and expanded when markets began to open worldwide in the early 1990s. Today, AES is one of the world's largest global power companies with electricity generation and distribution operations in 28 countries on five continents. In 2010, the company had an installed electricity generation capacity of over $40,500 \mathrm{MW}$ at 100 power plants and generated more than $85,000 \mathrm{GWh}$ of electricity for more than12 million customers. Approximately 78\% of AES electricity generation capacity is based on fossil fuels, while $22 \%$ uses renewable sources like water (18\% all large hydro) and wind $(4 \%)$ to generate electricity. AES also has a small solar generation business that had 37 MW of installed capacity in 2010. In 2010, the company employed 29,000 people globally and generated revenues of US\$16.6 billion (AES 2011a).

Two financial institutions hold relatively large percentages of AES's shares. Legg Mason Funds Management holds $17.89 \%$ of the shares, and Fidelity Investments owns $9.98 \%$ of the company (AES 2007c). AES Corporation is organized into four regional business units: North America, Latin America, Europe and Africa, and Asia and the Middle East.

\subsubsection{Operations and investments in the Global South}

AES has electricity provision operations in 27 countries, half of which are in the Global South. The company's Latin American operations are financially significant for AES. In 2007, four of the company's top ten (revenue earning) subsidiaries were Latin American. AES Gener S.A. is AES's primary subsidiary in Latin America. Gener is $80 \%$ owned by AES and is based in Chile, but employs 630 people in its activities in Colombia, Argentina and the Dominican Republic. Figure 5.1 maps the company's global presence.

\subsubsection{AES in Argentina}

AES has been active in Argentina since the country liberalized its electricity sector in the early 1990s. Responsible for 3,633 MW of installed electricity generation capacity, the company currently accounts for $12 \%$ of the total capacity in Argentina through a number of Argentine generation subsidiaries including AES Gener, Central Térmica San Nicolas S.A., Hidroeléctrica Río Juramento S.A., TermoAndes S.A., AES Caracoles S.R.L., and AES Parana. The fossil fuel-based TermoAndes plant is located in Argentina but produces electricity primarily for export to the Chilean market (AES 2006, 62). Table 5.1 lists the company's generation facilities in Argentina. 


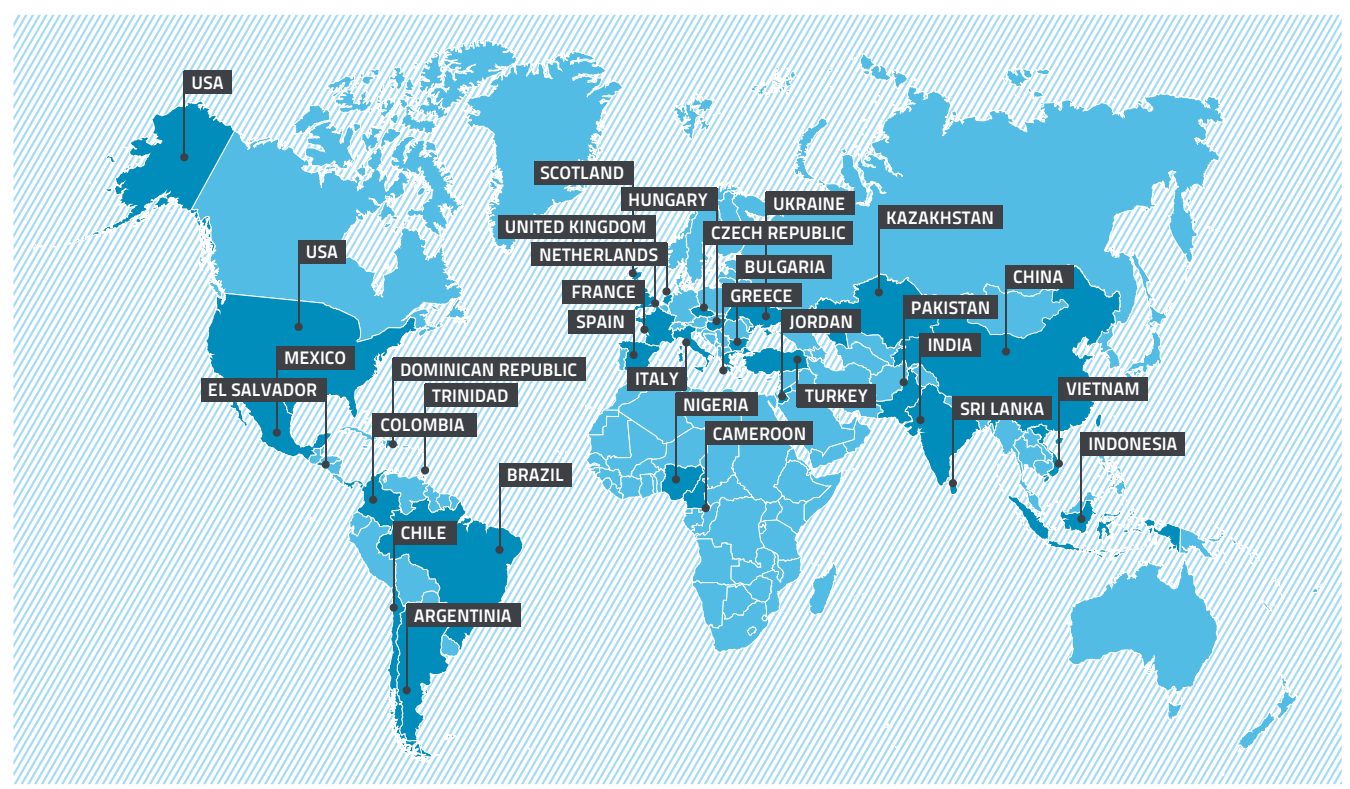

Figure 5.1: AES's global presence, 2011

Table 5.1: AES's installed generation capacity in Argentina, 2011

\begin{tabular}{|c|c|c|c|c|}
\hline $\begin{array}{l}\text { Facility name (and } \\
\text { location) }\end{array}$ & AES subsidiary & Fuel type & AES share (\%) & Capacity (MW) \\
\hline Paraná (Buenos Aires) & AES Paraná SA & Natural gas (CCGT) & 99 & 845 \\
\hline $\begin{array}{l}\text { San Nicolás (Buenos } \\
\text { Aires) }\end{array}$ & $\begin{array}{l}\text { San Nicolás Thermal } \\
\text { Station }\end{array}$ & Coal, natural gas \& oil & 99 & 675 \\
\hline Dique (Buenos Aires) & Central Dique & $\begin{array}{l}\text { Natural gas }(98 \%) \& \\
\text { diesel }(2 \%)\end{array}$ & 51 & 70 \\
\hline TermoAndes & & Natural gas \& diesel & 71 & 643 \\
\hline Sarmiento (San Juan) & AES Caracoles SRL & Natural gas & 99 & 33 \\
\hline Total fossil fuel & & & & 2,266 \\
\hline $\begin{array}{l}\text { Quebrada de Ullum (San } \\
\text { Juan) }\end{array}$ & AES Caracoles SRL & Hydro & Operate only & 45 \\
\hline Los Caracoles & AES Caracoles SRL & Hydro & Operate only & 125 \\
\hline Ullum (San Juan) & AES Caracoles SRL & Hydro & 99 & 45 \\
\hline Alícura (Río Negro) & AES Alícura SA & Hydro & 96 & 1,040 \\
\hline Cabra Coral (Salta) & AES Juramento SA & Hydro & 99 & 102 \\
\hline El Tunal (Salta) & AES Juramento SA & Hydro & 99 & 10 \\
\hline Total hydro & & & & 1,367 \\
\hline Total & & & & 3,633 \\
\hline
\end{tabular}

Based on: AES 2011c 
In addition to its generation activities, AES owns the distribution company Empresa Distribuidora La Plata (Edelap). In 2007, Edelap served 295,572 customers and sold 2,450 GWh of electricity in the Buenos Aires region, including La Plata, Berisso, Ensenada, Brandsen, Magdalena and Punta Indio. The company also owns the distribution company Edes, which serves 150,000 customers in the area south of Buenos Aires Province. Empresa Distribuidora Electrica Norte $\mathrm{SA}$, another AES distribution project, was sold in June 2007 (AES 2006). InterAndes is an AES transmission company that operates a $345 \mathrm{kV}$ transmission line from the TermoAndes plant to the Chilean border.

In the fourth quarter of 2011, AES sold its ownership in the distribution companies Edelap and Edes and the generation facility Central Dique. The company recorded a net loss of $\$ 346$ million ( $€ 263$ million) on the sale (AES 2011c). Because these operations were still under the ownership and control of AES in 2010, they are included in the analysis.

\subsection{AES's performance on the SEP benchmarks}

This section begins with an overview (in table format) of AES's performance, in both policy and practice, on the 11 SEP benchmarks. For each SEP benchmark, a reminder of the guiding international normative standards (taken from Chapter 3 ) is provided alongside key indicators of the company's performance and an evaluation of the degree to which the company's policy and practice are line with the SEP standards on that issue. Following the overview, the results of the empirical research on AES's policy and practice in Argentina are detailed for each SEP benchmark. These findings form the basis for the overview and evaluation provided in Table 5.2. The chapter concludes with a brief reflection on the relationship between the US mode of home-country business culture (as outlined in Chapter 4) and AES's performance on the SEP benchmarks. 
Table 5.2: Overview of AES's performance on SEP benchmarks in policy and in practice

\section{SEP benchmark}

General approach to corporate responsibility

Endorse international normative standards for CR

Adopt a commitment to CR in core-business activities and decisionmaking

\section{Guiding international normative SEP standards}

Electric utilities should seek to bring their policies in line with internationally recognized and verified standards for CR (ILO 2001, WBCSD 2002, IFC 2011, ISO 2011, OECD 2011 UNGC 2008, UNHRC 2011).

Electricity companies should adopt a holistic and integrated view of the role and impacts of electricity provision, considering and balancing the essential elements of economic development, environmental quality and social equity in utility operations. This entails a policy commitment to CR throughout all core-business activities, as opposed to a primary focus on philanthropic activities (WBCSD 2002, MVO Platform 2007). Electric utilities should seek to introduce environmental and social factors and procedures into high-level corporate planning and decision making and should aim to have a board-level position responsible for CR issues (WBCSD 2002).

\section{Social issues}

Increase access to affordable electricity

Electric utilities should develop policies that promote access to electricity priced at affordable levels for all, paying special attention to disadvantaged communities (WBCSD 2002, ILO/Palast et al. 2000, PSI/Pillinger 2009). In order to do so, companies should undertake initiatives to extend electricity services to unserved and underserved communities, particularly in rural or remote areas, but also to the poor in urban and peri-urban areas (UNDP/Modi et al. 2005, WBCSD 2002). 


\section{Indicators of AES policy}

- No reference to any international CR normative standards

- Relies on laws and regulations, most notably those set by the SEC, for normative guidance

- Recently achieved ISO 14001 certification for companywide EMS

- Largely out of line with SEP standards

- Overall nature of CR approach is legalistic, philanthropic, and profit-oriented

- Board-level committee chaired by a director ultimately responsible for $\mathrm{CR}$ issues

- Executive Office involved in some CR issues like climate change and renewable energy development

- No management incentives for attaining CR targets

- Mostly out of line with SEP standards

\section{Indicators of AES practice in Argentina}

Signed up to the UN Global Compact in 2004, but now no longer a member

- Some units and processes have ISO 14001, ISO 9001, and OHSAS 18001 certification

- Mostly out of line with SEP standards

" Largely focused on charity and philanthropy

- Local government officials have criticized AES "lack of commitment to corporate social responsibility" and "pursuit of profit over all else"

- Largely out of line with SEP standards
- Policies emphasize expanding access to electricity

- No publicly available policy or programs aimed at making electricity affordable for all

- Does not promote off-grid, decentralized electricity provision projects

- $17,354 \mathrm{MW}$ ( $43 \%$ of the company's total global capacity) located in countries without universal access to electricity

- Mostly in line with SEP standards
Operates $3,633 \mathrm{MW}$ of capacity (12\% of the country's total) and supplies 450,000 customers

- No information on electrification rates

- No programs in place to increase access

- Does not promote off-grid, decentralized electricity provision projects

- No information on electricity prices

- No programs in place to ensure affordability

- Has sued government to increase rates

- Accused by local city officials (mayors) of overcharging customers

- Largely out of line with SEP standards 


\section{Respect labor rights}

\section{Environmental issues}

Minimize environmental impact, including contribution to climate change
Electric utilities should respect workers' full rights and provide workers with fair pay, job security, and decent working conditions, including high levels of health and safety standards in all aspects of operations (WBCSD 2002, PSI/Pillinger 2009, OECD 2011). Furthermore, electric utilities should uphold freedom of association and the right to collective bargaining and pay particular attention to the right to strike (ILO 2001, ILO 1998, OECD 2011, UN Global Compact 2008). Companies should ensure that these rights are respected not only among their own employees, but also among suppliers, contractors and other business relationships (UNHRC 2011, OECD 2011, ISO 2011)

\section{Guiding international normative SEP standards}

Electric utilities should seek to minimize emissions and discharges to air and water and continually develop and implement low-pollution and low-environmental impact technologies (OECD 2011, WBCSD 2002, UN Global Compact 2008, Pillinger 2009). Companies should manage their environmental impact in line with internationally recognized and verified standards such as the ISO 14000 series and should always undertake rigorous and verifiable environmental impact assessments (IHA 2004, WBCSD 2002, OECD 2011, WCD 2000). Electric utilities should minimize their contribution to climate change and develop strategies for reducing GHG emissions (WBCSD 2002, OECD 2011). As a matter of priority, electric utilities should seek to avoid impacts on biodiversity and ecosystem services, refraining from any activities in areas of critical habitat with high biodiversity value while at the same time initiating or supporting conservation and biodiversity efforts related to impacts on natural habitats from utility operations (IHA 2004, IFC 2011, WBCSD 2002).

Electric utilities should evaluate the renewable energy potential of the host country and prioritize development and exploitation of renewable options over fossil fuel-based technologies (WBCSD 2002, UN/IAEA 2007, Pillinger 2009). Electric utilities should also prioritize renewables in their research and development programs and should support the diffusion of renewable energy technologies into the local economy (UN Global Compact 2008, WBCSD 2002). 
- No endorsement of the ILO Core Conventions

- No publicly available policy on freedom of association, collective bargaining or right to strike

- Very limited policies and structures in place to engage and consult workers on key operational decisions

- OHS is top corporate value and priority; OHS policy based on "international standards" that are not identified; goal of zero fatalities; conducts internal audits to monitor $\mathrm{OHS}$

- OHS policy extends to contractors but no information on monitoring and verification procedures

- Mostly out of line with SEP standards
- Majority of workers are unionized

- CBA in place for Edelap, covering $85 \%$ of workers; no CBA at Central Dique

- Union generally satisfied with terms of CBA, but recent strike by distractive workers and guards demanding equal treatment and pay, alleging AES breached CBA

- No information on wages

- Implemented worker training and special committee on OHS, but worker concern for safety remains high

- 4 worker deaths in 2007

- Collective agreement includes company commitment to reduce outsourcing

- Contract workers have complained about working hours and OHS conditions

- Mostly in line with SEP standards

\section{Indicators of AES policy}

- Claims to work toward improving environmental performance, but has recently been involved in several pollution scandals, primarily in developing countries

- Has a company-wide, ISO 14001-certified EMS

- No publicly available policy to always conduct an EIA

- Emitted $74 \mathrm{Mt}$ of $\mathrm{CO}_{2}$ in 2009; emission intensity of 0.93 $\mathrm{tCO}_{2} / \mathrm{MWh}$

- No targets to reduce $\mathrm{CO}_{2}$ emissions; climate change policy focused on generating carbon offsets to sell to other companies; but continuing to develop $\mathrm{CO}_{2}$-intensive power plants, primarily in developing countries

- No publicly available policy on protecting biodiversity

- Operates 23 hydroelectric dams globally; average size 359 MW; largest is the 2,659 MW plant on the Tietê River in Brazil

- Mostly out of line with SEP standards

\section{Indicators of AES practice in Argentina}

- Power plants and distribution company have an ISO 14001-certified EMS, but have received fines and censures from Argentine regulators and ISO bodies in recent years for environmental management failures

- AES Argentina among top four $\mathrm{CO}_{2}$ emitting companies in Latin America; Paraná 2.2 Mt (0.43 tCO $2 / \mathrm{MWh}$ ) in 2010; Dique 14,691 t (1.34 $\left.\mathrm{tCO}_{2} / \mathrm{MWh}\right)$ in 2008

" No publicly available plant-level commitments to reducing GHG emissions

- No publicly available biodiversity protection measures

- Operates 6 hydroelectric dams; average size 228 MW; largest is the 1,040 MW Alícura plant on the Río Negro

- Mostly out of line with SEP standards
- $78 \%$ of capacity based on fossil fuels; $22 \%$ on renewables (18\% large hydro, $4 \%$ wind)

- Aims to invest more in renewables if they present a business opportunity

- 2,000 MW build program consists of $85 \%$ fossil fuels $(74 \%$ coal, $11 \%$ gas) and $15 \%$ renewables ( $10 \%$ wind, $5 \%$ solar)

- Mostly out of line with SEP standards
- $63 \%$ of capacity based on fossil fuels ( $34 \%$ coal/diesel, $29 \%$ gas); $37 \%$ on renewables ( $36 \%$ large hydro, $1 \%$ small hydro)

- No evidence of prioritizing renewables on a large scale but has recently outfitted a thermal plant to combust a small (15 MW) amount of biodiesel

- Mostly out of line with SEP standards 
Economic issues

Contribute to local economic development

Ensure reliable supply

\section{Cross-cutting issues}

Engage in meaningful stakeholder consultation and participatory decision-making

\section{Guiding international normative SEP standards}

Electric utilities prioritizing the development of local energy-related infrastructure and employing local workers to the greatest extent practicable and providing those workers with sustainable jobs and training to improve skill levels (WBCSD 2002, OECD 2011, PSI/Pillinger 2009). In order to maximize local economic development and job creation, electric utilities should support local small and medium-sized enterprises in their procurement practices and contribute to the long-term development prospects of the host country through the transfer and rapid diffusion of technologies, skills and knowledge (WBCSD 2002, OECD 2011). Fair payment of due taxes to local, regional and national governments in the host country is a crucial element of contributing to local economic development (OECD 2011, MVO Platform 2007).

Electric utilities should ensure reliable supply by minimizing generation interruptions, maintaining contingency plans, responding to interruptions in a timely manner, and providing timely information to the public about planned and unplanned interruptions (CEER 2005; WBCSD 2002).
Electric utilities should adopt a participatory approach to the planning/designing, developing, operating, and monitoring/ evaluation of electricity projects and consult and engage stakeholders throughout the entire process in order to provide meaningful opportunities for their views to be taken into account in decision making (WBCSD 2002, UN Global Compact 2008, IFC 2007, OECD 2011, Pillinger 2009, WCD 2000). The consultation process should be tailored to local decision-making processes and involve the timely provision of all relevant information about activities, impacts and potential impacts translated into local languages (ISO 2011, WBCSD 2002). The process should take into account the needs of disadvantaged or vulnerable groups, and if Indigenous Peoples are affected by a potential project, electric utilities must gain the free, prior and informed consent of those peoples before proceeding with any part of the project cycle (IFC 2011). 


\section{Indicators of AES policy}

- Claims main contribution to local economic development is by "providing a safe and reliable supply of electricity, which is a prerequisite for economic development, security and public welfare"

- No public policy to prioritize local workers or SME suppliers

- Has been involved in some controversies related to fair payment of taxes

- Mostly in line with SEP standards

\section{Indicators of AES practice in Argentina}

- Employs 430, all Argentine nationals

- No public policy to prioritize local SME suppliers

- Invested $\$ 1$ billion ( $€ 762$ million) in electricity infrastructure, but acknowledges its commitment to investing in infrastructure "probably not met its clients' expectations"

- Has been accused by Argentine government of accounting fraud related to misrepresentation of investments and tax evasion

" Mostly out of line with SEP standards

- No information on average capacity factor, but one thermal plant achieved a $92 \%$ capacity factor in 2011

- Fined numerous times by national electricity regulator for inadequacies related to reliability of supply

- Numerous complaints from regulatory bodies about failure to properly maintain electricity infrastructure

- Numerous complaints from customers and regulatory bodies about failure to respond to interruptions in a timely manner

- Mostly out of line with SEP standards
- No indication of a willingness to engage in meaningful stakeholder consultation and participatory decision making

- No reference to international standards on stakeholder engagement

- Does not endorse the principle of FPIC

- Not willing to engage with the author in research related to the present study

- Largely out of line with SEP standards
- Primary mechanism for engaging stakeholders is a customer service hotline for reporting complaints

" Does not provide timely information to stakeholders in order to engage in meaningful consultation or participatory decision making

- One local manager agreed to engage with and be interviewed by researchers for the present study, but he insisted on anonymity

" Largely out of line with SEP standards 


\section{Cross-cutting issues}

Assume responsibility for impacts throughout all business relationships

\section{Guiding international normative SEP standards}

Electric utilities should seek to prevent, avoid and mitigate negative impacts throughout their entire range of business relationships, including suppliers and contractors (OECD 2011; UNHRC 2011; ISO 2011). Companies should seek to leverage their buying power to improve conditions among business relations, and monitor performance using third party audits (WBCSD 2002).

Electric utilities should apply a high degree of transparency in operations, including reporting on activities and progress, measurement, business partners and relationships, and interactions with government and the public (UNECE 1998, WBCSD 2002, UN Global Compact 2008, OECD 2011). Ewng internationally accepted sustainability reporting guidelines such as the GRI's G3 guidelines and the Electric Utilities Sector Supplement (GRI 2008). They should also have an external party verify the data, and disseminate the report in an appropriate manner (OECD 2011, WBCSD 2002). 


\section{Indicators of AES policy}

- AES Values, in particular on safety issues, also apply to business partners

- Purchasing policy focused on "price, quality, and performance" rather than social and environmental conditions

- No indication of monitoring business relations on social and environmental conditions other than inviting contractors to periodic meetings to discuss safety issues

- Mostly out of line with SEP standards

- Disclosure policy is to provide financial and operational information (social and environmental information not mentioned) to investors and government regulators (other stakeholder groups not mentioned)

- Does not publish a sustainability or CR report; website and annual report contain limited information on CR policies and impacts on SEP issue areas

- Annual report does contain financial and operational (but not social or environmental) information on developing country operations

- Does not report according to the GRI

- Provides some transparency about customers but not other business relationships

- Largely out of line with SEP standards

\section{Indicators of AES practice in Argentina}

- No publicly available policy to select or monitor suppliers and contractors based on social and environmental concerns

- Contracted workers not covered by the CBA; reports of extended working hours and a lack of proper OHS conditions among some contractors

- Mostly out of line with SEP standards

- Does not disseminate timely information about its project plans

- Has translated its CR material, including it Values document, code of conduct, and safety and environmental policies, into Spanish

- Primary channels of communication with stakeholders are website and customer service hotline

- Largely out of line with SEP standards 


\subsubsection{General approach to corporate responsibility}

\section{Endorsement of international normative standards for corporate responsibility}

AES does not make reference to any international normative CR standards in any of its CR or business ethics publications or on its website. Instead, AES largely refers to "local and international laws", as well as US regulatory and stock exchange (SEC) requirements, for normative guidance in its operations (AES 2012c). The only international instrument the company references is its recently implemented, company-wide IS014001consistent environmental management system (AES 2009).

In Argentina AES's Edelap signed up to the UNGC in 2004, but it is currently no longer listed as a Global Compact participant (Global Compact 2011). In addition to the ISO 14001:2004 certification for its EMS, AES Edelap has also achieved ISO 9001:2008 and OHSAS 18001 certification for some of its facilities and processes.

\section{Adopt a commitment to $C R$ in core-business activities and decision-making}

AES (2012e) maintains that "corporate responsibility is not a program", but is rather about how the company conducts business and "the overall impact [it has] on society and on the lives of the people [it] serves". AES recognizes that, "Providing electricity can radically improve the quality of life, especially in developing countries", where the company aims to provide electricity "reliably, safely and responsibly". The centerpiece of the AES $\mathrm{CR}$ approach is its code of conduct, in which it describes its principal "AES Values" such as safety, integrity, honor, excellence, and having fun at work (AES 2007b). The last page of the "Values" document provides guidance to employees on how to apply these general values in practice. The guidance is in the form of five questions that the company suggests its workers should ask themselves when trying to interpret whether they are acting in line with the values. The first two questions are, "Are my intended actions legal?" and "Would I want to see my actions reported in the media?" (AES 2007b: 12). This approach lends the values a distinctly legal overtone. AES's "Ethics and Compliance Program", which governs the company's operations around the world, maintains a strict focus on "compliance with local and international laws" (AES 2012c). The company also approaches some CR issues such as climate change primarily from a legal risk (as well as a business opportunity) perspective. A substantial portion of the company's 2011 Annual Report is dedicated to detailing the legal and regulatory risks related to climate change that the company faces, noting that it has formed a high-level unit to track developments in carbon regulation and legislation and to address compliance (AES 2012a).

Another characteristic of AES's general approach to CR is revealed on its "Corporate Responsibility" web page, where most of the examples the company lists as CR are philanthropic activities rather than actions 
targeted at critical issues in SEP. AES (2012e) claims that it undertakes philanthropic projects that "tie into our business", giving the impression of at least some relation to core business. However, the example it gives is building a medical center in Kazakhstan, for which the link to electricity provision is not immediately clear. The only two documents linked to the company's CR web page are its "Charitable Contribution and Political Donations Policy" and its annual "Political Contributions Report," reinforcing the impression of its philanthropic approach. One final salient characteristic of the company's general approach to CR is a basic profit-making orientation. One example the company provides of a CR activity is to turn "legacy infrastructure from the Soviet era into a profitable business" (AES 2012e). The company approaches environmental issues like climate change and the increasing demand for renewable energy primarily as a "business opportunity" to be exploited, rather than a responsibility to society (AES 2010, AES 2012d). The company does stress that "business results are never more important than conduct consistent with [AES] Values" (AES 2007b, 2).

The overall nature of AES's approach to CR can thus be characterized as legalistic, philanthropic, and profit-oriented. This is largely out of line with a more balanced approach suggested by international SEP-norms, which give equal weight to core-business social, environmental, and economic issues (WBCSD 2002, MVO Platform 2007).

The company's approach in Argentina is largely reflective of the headquarters' policies. AES Edelap has a brief CR section on its website that lists the company's environmental policy and has a link to a Spanish version of AES's code of conduct. However, there is little information available regarding how local AES management implements the company's CR policies. According to a local AES manager, this is because the company is against using CSR material for marketing purposes (Anonymous manager in Argentina 1 2008). Instead, AES Argentina indicates that the objective of its CSR programs is to contribute to the development of the communities where it operates, with particular focus on education, cultural development and satisfaction of unfulfilled basic needs, primarily among children. AES contributes labor, services, materials and monetary donations through CR programs that seek to, for example, improve nutrition among school-age children and contribute to the development of the arts. The company's interpretation of CR is thus clearly oriented toward philanthropic activities. Perhaps as a result, AES Argentina has not performed well on other core-business aspects of CR. In March 2008, the mayors of six Argentine municipalities supplied by AES presented a joint paper to the national energy regulator, in which they denounced the company for its terrible service, its "lack of commitment to corporate social responsibility" and its "pursuit of profit over all else" (Bruera et al. 2008).

As encouraged by SEP norms (WBCSD 2002), AES has a board-level Nominating, Governance and Corporate Responsibility Committee, the 
chair of which is a company director on the board of directors (AES 2008b, AES 2012b). Specifically on renewable energy development and climate change, "the Executive Office, which includes the CEO, the COO, the CFO, the General Counsel, and the Chief Risk Officer, has been involved with the development of the company's climate solutions business alternative energy business plans" (AES 2010). The company does not provide information regarding the management and implementation of $\mathrm{CR}$ policies in Argentina. There is also no indication that the company provides incentives for the management and attainment of targets on CR issues, as suggested by the SEP norms (WBCSD 2002).

\subsubsection{Social issues}

\section{Increase access to affordable electricity}

AES mentions on numerous occasions on its website and in its code of conduct that one of its primary aims is to bring reliable electric service to underserved customers. The company proclaims that, "Bringing electricity to places that never had it before" is often a "profound outcome" of its business (AES 2012e). Though AES does not have any projects promoting off-grid, as is encouraged by SEP norms, the company alludes to "bringing turbines on line in Sri Lanka or Chile", "restoring Soviet era infrastructure", and "extending power lines into a village in El Salvador or Cameroon" (AES 2012e). The company admits, however, that the main reason for undertaking all of these projects is that they are a "profitable business" (AES 2012e). AES operates 17,354 MW of electricity generation capacity $(43 \%$ of the company's total global capacity) in countries without universal access to electricity (AES 2012a). The company does not provide figures on actual production, but assuming a capacity factor of $24 \%$ (which is the average factor for AES's total production park), the $17,354 \mathrm{MW}$ is estimated to produce 36,426 GWh of electricity each year. Though it provides little detail on specific policies or projects, the company's general policy can be viewed as largely in line with international SEP norms, encouraging electric utilities to promote access to electricity in underserved areas (WBCSD 2002, Modi et al. 2005). In Argentina, AES operates 3,633 MW of electricity generation capacity $(12 \%$ of the country's total) and supplies 7,626 GWh of electricity to 450,000 customers yearly.

With regard to affordability, AES does not mention any policies or programs aimed at ensuring that the electricity it generates is affordable to the poor. In Argentina, the company has fought hard for increases in electricity rates. In 2001 and 2002, AES filed multiple lawsuits against the Argentine government with the Arbitration Court of the World Bank's International Centre for Settlement of Investment Disputes (ICSID) for the government's attempts to keep electricity affordable for its citizens in the wake of the 2001 economic crisis. AES claimed that the government's measures impacted its investments negatively. As part of a deal struck in 2004, AES was allowed to increase its average electricity tariff by $15 \%$ (Edelap no date). However, even with these 
increased rates, local city government officials have filed complaints against the company for allegedly overcharging customers (Bruera et al. 2008). AES's actions with regard to affordability of electricity in Argentina are thus out of line with SEP norms encouraging companies to seek to make electricity affordable for all.

\section{Respect labor rights}

AES does not endorse or mention the ILO Core Conventions anywhere in its policy documents. There is also no indication that the company has any policies or procedures in place to engage and consult workers on key operational or CR decisions. The charter of the Corporate Responsibility Committee states only that the committee "may request" that an employee provide input to the committee's decisionmaking processes (AES 2011b). The company does not mention workers' right to freedom of association or the right to strike. The only reference AES (2007b) makes to collective bargaining is in the context of punishing employees for violating laws or the AES code of conduct, noting that "Disciplinary measures and corrective action, up to and including termination, are subject to [...] the terms of any applicable collective bargaining agreement. The lack of policies designed to ensure the fundamental rights of workers is out of line with SEP norms (ILO 2001, ILO 1998, OECD 2011, UNGC 2008). In Argentina, a majority of workers are unionized in various electricity sector unions, and a national electricity sector collective bargaining agreement is in place. AES Edelap workers have negotiated an additional Edelapspecific collective agreement - Convenio
Colectivo de Trabajo (CCT) N 860/07 "E" which is applicable to approximately $85 \%$ of all Edelap workers. Senior staff, professionals with specific functions, legal representatives, and sub-contracted personnel are not covered (MEyOSP 2011). The agreement stipulates maximum working and extra payment for night shift workers. According to a union official, "We had raised the issue of working hours for years and never gotten a positive response [from AES]. After great pressure... we basically gained back what we had before privatization" (Fernández 2008). However, in November 2011، AES Edelap administrative workers and guards went on strike to demand better wages and working conditions, claiming that the company is violating the collective agreement with regard to equal treatment of all types of employees (Hoy 2011). Workers at AES's Central Dique currently lack a collective agreement. Though they are still covered by the National Labor Law, the lack of a company-specific agreement means that they enjoy fewer rights than their colleagues at other plants.

With regard to OHS, AES (2007b) claims that the foremost value and top priority is safety, both among its own employees and contractors as well as the communities in which it operates. The company has set itself a goal of "zero fatalities among AES people and contractors". AES (2012e) claims that it has developed and is implementing global safety standards based on "internationally recognized safety standards", but the company does not communicate about the content of its standards or on which international standards they are based, nor is 
there evidence that the principles are translated into concrete policies.

In order to monitor implementation of its OHS standards and policies, AES conducts periodic self-assessment safety audits at all of its subsidiaries, but no information is given about independent, third-party audits or the involvement of stakeholders in the audits. AES also holds annual Safety Action Forums at which personnel from various countries and levels of the company, including linemen, team leaders, dispatch operators, and contractors, discuss how to improve safety. In Argentina, AES Edelap has committed to providing ongoing training to ensure safety at work and to create a capacity-building, prevention, hygiene and safety commission to be comprised of representatives from both the trade union and the company. However, workers expressed concern about the significant number of workrelated accidents and deaths in recent years. Of the average 605 deaths at work reported in Argentina each year, $129(21.6 \%)$ occur in the electricity sector. According to Fernández (2008), four Edelap workers died in 2007.

In terms of job security and outsourcing, AES corporate policy provides little guidance. In Argentina, outsourcing increased markedly after privatization, but the unions have been fighting to reduce this practice. Fernández (2008) notes that "there was a shift from state-owned companies to privatized companies using a good deal of contracted labor. But the union pressured Edelap to stop this practice and focus again on having a company workforce made up of its own personnel, workers actually doing their own tasks directly. We have been successful in getting Edelap to backpedal on outsourcing and contract labor, because the model didn't work." The CBA with Edelap stipulates that the company should reduce the use of contractors and subcontractors. Contracted workers, who are not part of the CBA, have complained about having to work extended hours and a lack of safety and hygiene (Fernández 2008).

\subsubsection{Environmental issues}

\section{Minimize waste, pollution, emissions, and}

\section{contribution to climate change}

AES (2012f) recently implemented an ISO 14001-consistent EMS, which it claims will lead all AES businesses worldwide to implement internationally recognized environmental standards and management procedures. AES claims that it is working to reduce waste and pollution from its power plants around the world. However, most of the examples it gives of such reduction are at its plants in developed countries, primarily the USA (AES 2007a:24). The company has been involved in pollution scandals in the Dominican Republic (Xinhua 2007), Ireland (FoE UK 2008), and Cameroon (African Press Agency 2007). The company does not have a policy on EIAs, though this is strongly recommended by SEP norms.

In Argentina, AES Edelap and Central Dique have an ISO 14001-certified EMS. However, in 2006 ENRE fined AES's Central Dique for its failure to submit environmental management plans on time, despite being given a deadline 
extension. In addition, a May 2006 evaluation report by an ISO committee cited Central Dique for not anticipating certain potential accidents, such as those that could result from loading fuel in gas oil tanks. The report also mentioned the company's failure to establish and reprogram devices to test the methods used in case of emergencies. In addition, a random audit carried out by Argentine electricity regulator ENRE on 27 March 2008 indicated that, while Central Dique does have an EMS, it was not in full compliance with Argentine environmental regulations. ENRE has also fined AES's Edelap several times in recent years for non-compliance with environmental safety requirements in its Resolution 403/03, specifically for not presenting its environmental management plan within the allowed timeframe.

\section{AES (2010) reports on its GHG emissions} through the CDP questionnaire, but its most recent report (covering 2009 emissions) received a "Low" score (33 out of 100) from the CDP. AES's disclosure demonstrates a "limited ability to disclose known risks or potential opportunities related to climate change, a limited ability to measure and manage the company's carbon footprint, and a reluctance to disclose certain requested information due to commercial sensitivity" (CDP 2011). The leading ten electric utilities scored between 87 and 96 out of 100. In 2009, AES emitted 74 $\mathrm{Mt}$ of $\mathrm{CO}_{2}$ globally (AES 2010). That same year, it generated $79,000 \mathrm{GWh}$ of electricity, giving it an emission intensity of $0.93 \mathrm{tCO}_{2} / \mathrm{MWh}$. The company claims that it is "developing projects and technologies that reduce or offset greenhouse gas (GHG) emissions-primarily by capturing and destroying methane in a variety of forms before it reaches the atmospherecreating attractive opportunities for AES while improving the environment in the process" (AES 2012f).

AES views GHG emissions as one of today's most significant challenges, but a challenge that also represents "a potential US\$28 billion market" and a business "growth opportunity in projects and technologies to reduce or offset GHG emissions" (AES 2007a:11, AES 2008a). Rather than reducing the GHG emissions of its electricity provision activities, AES climate change strategy is focused on the creation of carbon offset credits. AES has developed a separate business unit, called Climate Solutions, to tackle climate change. The company's strategy for reducing greenhouse emissions is primarily focused on capturing and destroying methane gas from agricultural waste, landfills, and industrial processing plants.

AES has also undertaken a number of reforestation projects in Latin America in order to remove $\mathrm{CO}_{2}$ from the atmosphere and generate carbon credits it can sell to other companies. The company states twice on its short "Alternative Energy" web page, that activities like this will "create attractive opportunities for AES while improving the environment in the process" (AES 2012d).

At the same time, the company is rapidly expanding its coal and diesel-fired operations in Latin America, Asia, and Africa (AES 2012a). The company operates, or is planning, coal-fired 
power plants in China, the Dominican Republic, El Salvador, Vietnam, Chile - where it already has four coal plants in operation - and India, where the company is currently building a new coal-fired power plant with an accompanying coal mine (AES 2008c). According to Carney (2009), "AES [is] peddling greenhouse gas offsets while lobbying for policies to make those offsets valuable - the same buy-low, lobby-hard, sell-high strategy tried by Enron. AES's simultaneous expansion of coal-fired power in Asia, South America and Africa, however, highlights how environmental regulations can yield profit without necessarily yielding environmental gains".

In Argentina, two of the top four $\mathrm{CO}_{2}$ producing electricity companies in Latin America are AES subsidiaries: AES Argentina and AES Gener SA in Chile (CARMA 2007). There is no indication that AES Argentina has set any targets for reducing $\mathrm{CO}_{2}$ emissions. In 2010, AES's Central Paraná emitted 2.2 $\mathrm{Mt}$ of $\mathrm{CO}_{2}$ in order to generate 5,095 GWh of electricity (an emission intensity of 0.43 $\mathrm{tCO}_{2} / \mathrm{MWh}$ ). The last publicly available figures for AES's Central Dique are from 2008, when Dique emitted 14,691 tons of $\mathrm{CO}_{2}$ in order to generate $12.9 \mathrm{GWh}$ of electricity (an emission intensity of $1.34 \mathrm{tCO}_{2} / \mathrm{MWh}$ ) (Argentine Ministry of Energy 2010).

AES operates 23 dams around the world with an average capacity of $359 \mathrm{MW}$, the largest being the 2,659 MW plant on the Tietê River in Brazil (AES 2012a). AES has no publicly available policy on protection of biodiversity. The company has been involved in a large scandal surrounding significant negative impacts on biodiversity during the construction of hydroelectric dams in La Amistad Biosphere Reserve in Panama (Galvin 2009). AES Argentina also does not make its policies on biodiversity public nor does it report on its impacts on biodiversity at the six hydroelectric dams it operates in the country. The average size of its Argentine hydroelectric facilities is $228 \mathrm{MW}$, the largest being the 1,040 MW Alícura plant on the Río Negro.

\section{Prioritize renewable sources of energy for electricity}

Currently, approximately $78 \%$ of AES electricity generation capacity is based on fossil fuels, while $22 \%$ uses renewable sources like water (18\% large hydro) and wind $(4 \%)$ to generate electricity. This breakdown has remained relatively stable in recent years. AES's 1,750 MW of wind capacity is primarily located in the US, with only $196 \mathrm{MW}(11 \%)$ located in the Global South (all in China) (AES 2012a). AES also has a small solar generation business that had 37 MW of installed capacity in 2010, mostly in southern Europe (AES 2011a).

AES aims to increase its utilization of renewable sources of energy in the coming years, claiming in 2007 that it planned to invest up to $\$ 10$ billion ( $€ 7.6$ billion) in a newly launched renewables business over the next decade. As alternative sources of energy become more economically competitive with fossil fuels, AES views investment in renewables as a strategic business opportunity that, it mentions as an aside, "is also good for the environment" (AES 2007a: 17). AES's primary focus with regard 
to development of renewable sources is on wind, which it regards as "one of the lowest cost renewables per megawatt today" and a technology with "tremendous growth potential globally" (AES 2008a). However, despite such proclamations about intentions to shift toward renewable electricity generation, $A E S$ 's build program suggests that its fuel mix in the near future will look largely the same as it does today, if not even slightly more fossil-fuel dependent.

The company currently has approximately 2,000 MW of capacity under construction around the world, $85 \%$ of which is based on fossil fuels $(74 \%$ coal, $11 \%$ gas) and only $15 \%$ of which is based on renewables ( $10 \%$ wind, $5 \%$ solar). The new renewables capacity - which is broken down into 205 MW wind planned in India, China, US, UK, and France and $106 \mathrm{MW}$ solar - will mostly be located in the Global North. On the other hand, the company's new non-renewable capacity will be built in the Global South -1,200 MW coal in Vietnam, $270 \mathrm{MW}$ coal in Chile, and $216 \mathrm{MW}$ gas in Cameroon (AES 2012a). AES is thus clearly not prioritizing the development and exploitation of renewable options over fossil fuel technologies.

In Argentina, AES has a total attributable electricity generation capacity of 3,223. Despite Argentina's huge potential for renewable electricity, approximately two-thirds of AES Argentina's electricity generation capacity $(2,027 \mathrm{MW})$ is based on fossil fuels. Less than $1 \%$ of the company's capacity ( $10 \mathrm{MW})$ is based on sustainable sources of energy (small-scale hydro). The rest of its hydropower (1,186 MW, $36 \%$ of total capacity) is generated in large-scale hydroelectric facilities. Figure 5.2 illustrates AES's electricity generation capacity in Argentina based on fuel source.

While AES does have a global policy to increase investment in renewable energy, the company does not have an Argentina-specific plan or policy. When asked whether the company had plans to increase the share of sustainable energy in its fuel mix, a local AES manager responded, "The company does not disseminate information about its projects until they are firm" (Anonymous manager in Argentina 1 2008). The company has recently made a move to outfit one of its thermal stations to co-fire a small amount of biodiesel. In July 2010, AES Argentina initiated a new injection system for its San Nicolás plant that will give the facility 15 MW of soy-based biodiesel capacity.

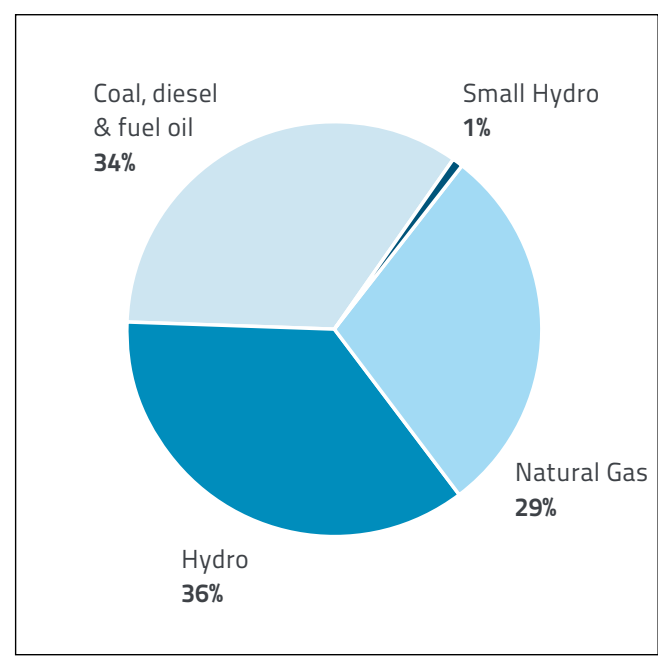

Figure 5.2: AES's electricity generation capacity in Argentina by fuel source in MW, 2011. Based on: AES 2011c 


\subsubsection{Economic issues}

\section{Contribute to sustainable local economic development}

AES (2012e) claims that its biggest contribution to the economic development of the countries it operates in is by "providing a safe and reliable supply of electricity, which is a prerequisite for economic development, security and public welfare". This element is largely in line with international SEP norms. However, the company has no publicly available policy to prioritize local workers or SME suppliers in host countries. AES's position on fair payment of taxes to support local economic development is also ambiguous. AES has touted its payment of taxes to the government of Kazakhstan, but it has sought to locate its operations in other countries in free-trade zones allowing it to avoid paying taxes and other royalties (Bayliss 2002). AES also has 81 subsidiaries listed in the Netherlands, including the Dutch Antilles, and six subsidiaries in Bermuda. This is noteworthy because these countries are generally known as "tax havens", and AES has few or no electricity provision operations in these countries. The majority of the subsidiaries and holding companies have no employees.

AES Argentina's (2010) website proclaims that, "The purpose of the company is to contribute to the development of the communities where its business units are located". AES employs 430 people in Argentina, all Argentine nationals. The company claims that, since it began operating in Argentina in 1993, it has invested a total of more than $\$ 1$ billion ( $€ 762$ million) in the Argentine power generation and distribution segments (AES Argentina 2010). The company also claims that, although its 2007 agreement with the Argentine energy regulator was to invest ARS 20 million $(\$ 4.6 / € 3.5 \mathrm{~m})$, the company has exceeded that to invest ARS 30 million (\$6.8/€5.2m) (Debesa 2006). A local AES manager explained that, apart from the $15 \%$ average increase in electricity tariffs in 2005, Argentine electricity rates have been largely frozen since 2002, but that the company has continued to invest in the country despite losses (Anonymous manager in Argentina 1 2008).

However, the company acknowledges that its investments in electricity infrastructure in Argentina have "probably not met its clients' expectations" (Anonymous manager in Argentina 1 2008). In December 2008, an investigation by the Argentine electricity regulator ENRE revealed "serious irregularities" in the accounting practices of AES's electricity distributor Edelap (Olivera 2008). ENRE alleged fraud in connection with Edelap's presumed losses of ARS 55.3 million (\$12.6/€9.6m). The alleged fraud comprised a debt restructuring process carried out by AES that left Edelap unfunded, Edelap's outsourcing of management services (to the company Luz del Plata S.A.), and alleged tax evasion.

According to the Argentine Planning Minister (cited in Olivera 2008), who raised the issue directly with the US ambassador to Argentina, the company's irregular accounting practices "directly benefit AES" but "significantly deteriorate" Edelap's ability to make "necessary investments" in distribution infrastructure 
in Argentina. Because the practices "put at risk the investment necessary to supply electricity,...ENRE and the Planning Ministry are evaluating corrective measures for this irregular practice, as well as civil and penal actions that might be appropriate". Edelap denies that its accounting practices are inappropriate and asserts that it has fulfilled "all its obligations under its concessionary agreement and with the regulator" (Olivera 2008). Nevertheless, Fernández (2008) attributes the difficulty AES has had in providing adequate levels of electricity in Argentina (see following section) to the company's "reluctance to invest in local electricity infrastructure".

\section{Ensure reliable supply}

AES (2012e) notes that it takes its responsibility for ensuring a reliable supply of electricity "seriously". However, the company does not have a publicly available policy for responding to interruptions in a timely manner, or for providing timely information to the public about planned and unplanned interruptions. In Argentina, AES has performed poorly on the reliability standards expressed in the SEP norms (CEER 2005, WBCSD 2002). Although its Central San Nicolás - one of the most versatile power plants in the country because it can generate electricity using gas, coal or fuel oil - set a record for high capacity factor of $92 \%$ in 2011, AES Argentina has had problems providing a reliable supply of electricity to its customers. The Argentine electricity regulator ENRE fined AES's Central Dique thermal generation station 20 times between 1999 and 2008 (ENRE 2011). 80\% of these fines were due to the station's inability to provide an adequate level of electricity required by the regulated wholesale electricity market, i.e. a lack of capacity. The remaining fines were due to AES's failure to report system disturbances, i.e. power cuts, to the relevant authority, or to their untimely reporting. ENRE has also issued 42 fines against AES's distribution company Edelap since the unit was purchased by AES in 1998. Of these fines, $45 \%$ were due to the company's inability to provide electricity to the national grid when required, a situation similar to that of the Central Dique. A further 14 fines were issued for breaches related to the technical quality of the company's commercial and residential service and for failing to meet requirements for the collection and processing of data concerning service quality (ENRE 2008).

AES's customers have also frequently complained about the poor quality of service provided by Edelap. Most complaints relate to the length of the planned and unplanned power cuts and the fact that both of these types of cuts continue to occur (El Día 2005). Other citizens have lamented being left without service for more than 30 hours, or experiencing power drops or surges that caused damage to electrical devices and merchandise. One small business owner (a butcher) in La Plata noted, "The explosion of the electricity transformer caused us to lose more in meat than what a new generator costs" (Rocío 2008). Citizens' complaints about the poor quality of Edelap's service were corroborated by complaints from the municipal authorities of La Plata about the company's failure to invest adequately in electricity infrastructure. A local official noted 
of AES's behavior, "There has been a lack of investment. The company committed to setting up three sub-stations to provide more energy to the system and improve the reliability. But they have not done this, and now we have street light blackouts" (Debesa 2006). The complaints about the company's poor quality of service became so frequent that ENRE opened an office in La Plata on 29 April 2008 to handle customer complaints. During its first three months of operation, the La Plata office received 574 complaints - 10 per day - related to the poor quality of Edelap's service (ENRE 2011). In a 2008 agreement with the Argentine Planning Ministry, AES acknowledged problems with the quality of its service and announced plans to invest ARS 300 million (\$68/€52m) in Edelap between 2009 and 2013. The government agreed to a revision of the distributor's rates in order to enable the company to make these investments (ENRE 2011).

\subsubsection{Cross-cutting issues}

Engage in meaningful stakeholder consultation and participatory decision-making

There is no indication that AES engages in meaningful stakeholder consultation and participatory decision-making. The only mention of stakeholder input in corporate decisionmaking processes is that the Corporate Responsibility Committee "may request" that an employee provides input to the committee's decision-making processes (AES 2011b). The company does not publicly reference international standards on stakeholder engagement or endorse the principle of free, prior, and informed consent. However, in response to criticism about its failure to respect the rights of local stakeholders, including indigenous peoples, at its hydroelectric dam site in Panama, AES (2009) issued a press statement claiming that it "fully respect[s] human rights in the development and operation of projects". Managers at AES headquarters did not respond to repeated requests by e-mail, written post, and telephone that someone from the company engage with researchers and provide information for the present study.

Researchers in Argentina were able to interview one local AES manager, however, who insisted on remaining anonymous. The primary mechanism that AES Argentina appears to have for engaging stakeholders is a customer service hotline for customers and other stakeholders to report complaints, to which AES promises to respond. However, there have been widespread complaints about the adequacy and timeliness of AES's response to complaints (ENRE 2011). Given that AES Argentina "does not disseminate information about its projects until they are firm", it is hard to see how it can consult stakeholders in a meaningful and timely manner on its plans and operations (Anonymous manager in Argentina 1 2008). This implies an unwillingness to engage stakeholders throughout the entire project cycle as is suggested by international SEP norms

\section{Assume responsibility for impacts throughout all business relationships \\ In addition to outlining the company's values and expectations of its own employees, AES's code of}


conduct also communicates its expectations for suppliers, consultants, agents, business partners, and others who perform work on behalf of the company (AES 2008a). The company claims that its "safety first" value applies to contractors, as well as its own employees. The company notes that it seeks to do business with contractors and suppliers that follow the highest standards of integrity and business conduct and that these must comply with AES policies. However, AES does not prioritize environmental and social concerns when selecting business partners. AES (2007b: 9) explains that, "We will make purchasing and procurement decisions that achieve the best value for AES, including price, quality, performance, and suitability". There is also no indication that AES monitors business relations for social and environmental conditions other than inviting contractors to periodic meetings to discuss safety issues (AES 2012e).

In Argentina, AES Argentina has no public policy to select or monitor suppliers and contractors based on social and environmental concerns. No information could be gathered on the working conditions of workers contracted by AES other than the fact that they are not covered by the CBA with AES and that there have been reports of extended working hours and a lack of proper OHS conditions among some contractors (Fernández 2008).

\section{Maximize transparency and provision of information}

AES (2007b, 9) pledges that it will "provide full, fair, accurate, timely, and understandable disclosures about financial and operational issues to investors and government agencies". Information on social and environmental issues, as well as other stakeholders besides investors and governments as key recipients of information, are notably not included in the AES disclosure policy. The company does not publish an annual sustainability or CR report, and its annual report does not comply with GRI reporting guidelines (GRI 2008). Its annual report and website contain limited information on the company's CR policies and impacts in SEP issue areas. AES's disclosure on climate change demonstrates "a reluctance to disclose certain requested information due to commercial sensitivity" (CDP 2011). The company makes no mention of any policy for providing information to the communities and other stakeholders affected by its operations in the Global South. The company promises not to engage in "manipulation, concealment, abuse of privileged information, misrepresentation of material facts, or any other unfair dealing practices" (AES 2007b: 6). However, the company has been accused of deliberately trying to conceal information from the public in Kazakhstan, where "Representatives from [AES] attempted to convince regulators not to reveal [anti-monopoly violations] to journalists, as it could affect their shares and cause problems for them" (KGN 2007). AES does provide some information on the identity of its customers, but often simply lists "various customers" (AES 2011c). Overall, AES's policies on disclosure and provision of information are largely designed to comply with legal obligations rather than following international normative guidance on sustainability reporting 
(UNECE 1998, WBCSD 2002, UN Global Compact 2008, OECD 2011).

In Argentina, AES's Spanish-language website serves as its primary channel of communication with stakeholders. The website explains AES's Code of Conduct, its social and environmental responsibility programs, and the ISO and OHSAS certifications that it has. The website also contains annual reports published by AES, and the dates and times of planned outages for repairs. However, the website does not contain detailed information about the company's plans or impacts on SEP issue areas. Not disseminating information on projects "until they are firm" cannot be considered timely provision of information (Anonymous manager in Argentina 12008 ).

\subsubsection{The US mode and AES's performance on the SEP benchmarks}

AES's performance on the SEP benchmarks indicates that the US mode of business culture plays a significant role in conditioning how AES takes up the internationally-derived standards for SEP and how it applies them in practice.

Table 5.3 provides an overview of the degree to which AES's performance on SEP benchmarks is deemed to reflect the US mode as described in Chapter 4.

Overall, AES's approach to CR can be characterized as legalistic, philanthropic, and profit-oriented. This approach is largely reflective of the US mode's legalistic focus and economic profit orientation. The company's lack of reference to international normative standards for CR is also reflective of the US mode, which tends to focus on legal and regulatory requirements over voluntary norms.

With regard to increasing access to affordable electricity, AES (2012e) does have policies aimed at increasing electrification in the Global South but admits that the main reason for undertaking such projects is that they are a "profitable business", a clear vestige of the US mode. In Argentina, AES fought against the government's efforts to keep electricity affordable for its citizens in the wake of the 2001 economic crisis, filing multiple lawsuits domestically and internationally. The legalistic approach and the clear prioritization of profits over ensuring affordability are hallmarks of the US mode. AES's lack of policies designed to ensure the fundamental rights of workers is indicative of the US mode's relative antipathy toward organized labor. AES's workers in Argentina are nevertheless largely unionized and, despite some complaints about health and safety and job security, the workers and union leaders interviewed for the present study indicated general satisfaction with the conditions and terms of their employment with AES.

In the environmental realm, despite the recent ISO 14001 certification of its EMS, AES has performed poorly in terms of policy and practice on the environmental standards expressed in SEP norms. The low priority set on achieving a high level of environmental performance, as well 
Table 5.3: Degree to which AES's performance on SEP benchmarks reflects the US mode of business culture

\begin{tabular}{|c|c|c|}
\hline $\begin{array}{l}\text { Is AES's policy/practice reflective of } \\
\text { the US mode on the following SEP } \\
\text { benchmarks? }\end{array}$ & Policy & Practice \\
\hline $\begin{array}{l}\text { Endorse international normative } \\
\text { standards for CR }\end{array}$ & Moderately reflective & Largely reflective \\
\hline $\begin{array}{l}\text { Adopt a commitment to CR in core- } \\
\text { business activities and decision- } \\
\text { making }\end{array}$ & Largely reflective & Moderately reflective \\
\hline Increase access to affordable electricity & Moderately unreflective & Moderately reflective \\
\hline Respect labor rights & Largely reflective & Moderately unreflective \\
\hline $\begin{array}{l}\text { Minimize environmental impact, } \\
\text { including contribution to climate } \\
\text { change }\end{array}$ & Largely reflective & Largely reflective \\
\hline $\begin{array}{l}\text { Prioritize renewable sources of energy } \\
\text { for electricity }\end{array}$ & Largely reflective & Largely reflective \\
\hline $\begin{array}{l}\text { Contribute to local economic } \\
\text { development }\end{array}$ & Largely reflective & Moderately unreflective \\
\hline Ensure reliable supply & $\mathrm{n} / \mathrm{a}$ & $\mathrm{n} / \mathrm{a}$ \\
\hline $\begin{array}{l}\text { Engage in meaningful stakeholder } \\
\text { consultation and participatory } \\
\text { decision-making }\end{array}$ & Moderately reflective & Moderately reflective \\
\hline $\begin{array}{l}\text { Assume responsibility for impacts } \\
\text { throughout all business relationships }\end{array}$ & Largely reflective & Largely reflective \\
\hline $\begin{array}{l}\text { Maximize transparency and provision } \\
\text { of information }\end{array}$ & Moderately reflective & Moderately reflective \\
\hline
\end{tabular}

as the company's tendency toward the continued development of economically-attractive fossil fuels, are reflective of the US mode.

Among the cross-cutting issues, though largely out of line with SEP norms, AES's failure to engage stakeholders in a meaningful way is reflective of the US mode, which tends to treat ethical and CR decisions as the responsibility of individual managers rather than a multilateral process involving various other stakeholder groups (Taka and Foglia 1994). An AES manager in Argentina indicated that his company follows the American parent company's tradition of not publishing a high degree of information about SEP issues (Anonymous manager in Argentina $12008)$. Finally, there is also no indication that AES monitors its suppliers or contractors for social and environmental conditions other than inviting contractors to periodic meetings to discuss safety issues (AES 2012e). The focus on economic concerns over social and environmental interests, in both its own operations and among its business relations, is largely indicative of the US mode. 


\section{Chapter 6}

Case study-Endesa
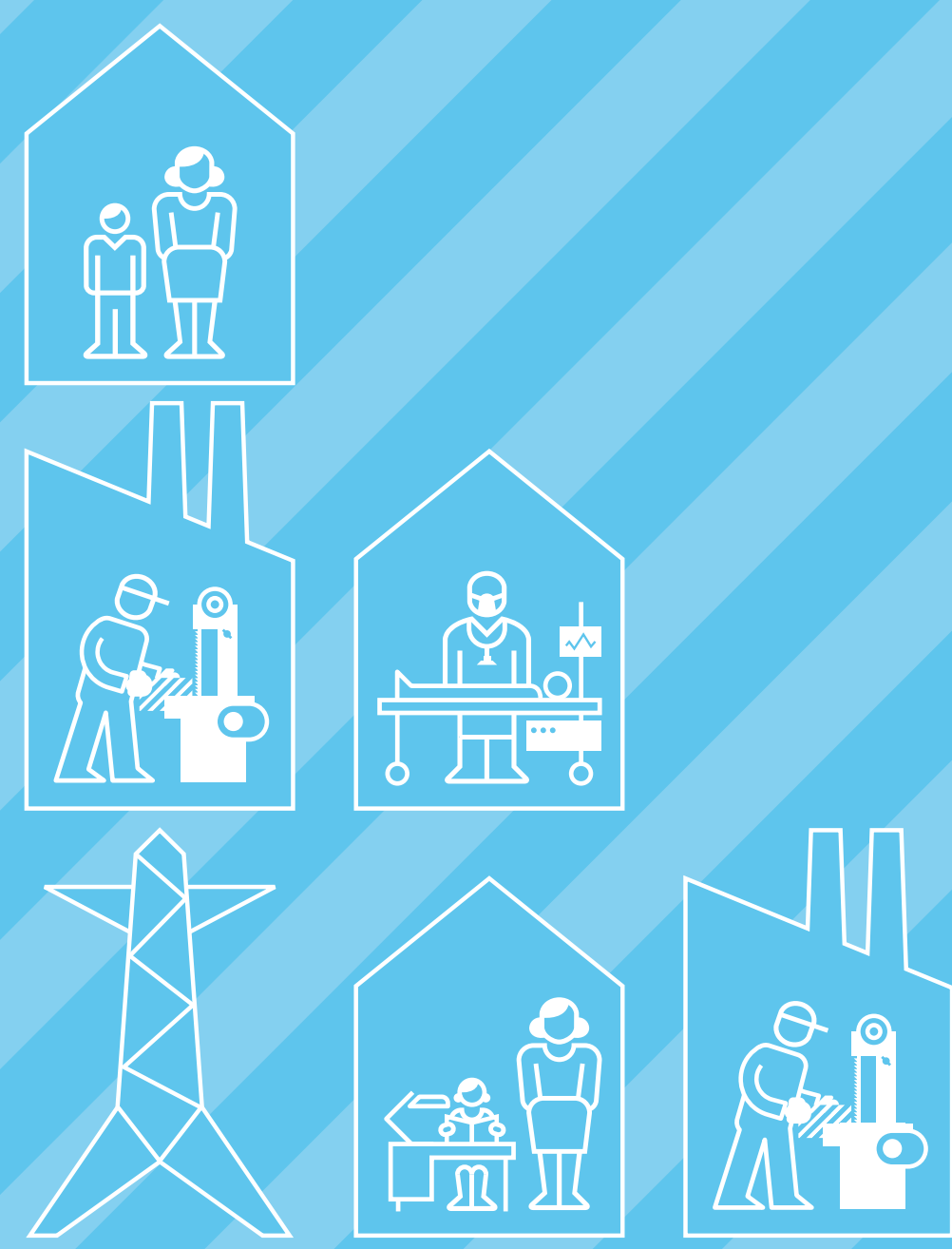


\subsection{Endesa background and operations}

\subsubsection{Basic company information}

With headquarters in Madrid, Endesa is Spain's largest electric utility company and has major operations in Latin America. In 2007, Acciona (Spain) and Enel, an Italian transnational electricity company partially (22\%) owned by the Italian Ministry of Economics and Finance, purchased $92.06 \%$ of the shares in Endesa. In February 2009, Acciona sold all of its Endesa shares to Enel, which now alone owns more than $90 \%$ of the company. Despite the takeover, Fraile (2008) confirms that the Endesa headquarters in Madrid is still responsible for CR issues and policies at assets formerly owned and/or operated by Endesa in the Global South. For this reason, this report considers Endesa as a separate entity from Enel, although readers should keep in mind that Enel is now the owner of Endesa and that the policies and approaches taken by the two companies are likely to converge.

As of December 2010, the company had a global installed electricity generation capacity of more than $40,141 \mathrm{MW}$, generating more than 130,484 GWh of electricity for approximately 25 million customers each year. Approximately $66 \%$ of Endesa's global electricity generation capacity is based on non-renewable sources of energy ( $57 \%$ fossil fuels, $9 \%$ nuclear), while $34 \%$ uses renewable sources like water (29\% large hydro, $4 \%$ small hydro) and wind ( $4 \%$ ) to generate electricity. In 2010, the company employed
24,732 and generated revenues of $\$ 9.9$ ( $€ 7.5$ ) billion (Endesa 2011e, Endesa 2011d).

\subsubsection{Operations and investments in the Global South}

In addition to its European operations in Spain, Portugal, and Greece, Endesa is active in electricity provision in a number of countries in the Global South, primarily in Latin America. Endesa currently has $14,707 \mathrm{MW}$ of electricity generation capacity in the Global South, which is nearly one-third of its total worldwide capacity. However, in March 2009 Endesa announced that it was slashing its investments in Latin America for the period 2009-2013. The company will invest $\$ 5.4$ billion ( $€ 4.1$ billion) over the five-year period, down more than $30 \%$ from what it had announced in 2008. The majority of Endesa's total $\$ 17.3$ (€13.1) billion worth of investments in the period will go to Spain and Portugal, with only a third going to its operations in the Global South (Business News America 2009).

Figure 6.1 indicates the geographical distribution of Endesa's electricity provision activities. The present study's evaluation of Endesa's practice vis-à-vis the SEP benchmarks is based on field research in Argentina and Peru.

\subsubsection{Endesa in Argentina}

Endesa's operations in Argentina began in 1996 when it acquired the Central Dock Sud thermal generation station, which had been recently privatized (in 1992) and which is located only four kilometres from the city of Buenos Aires. Endesa currently operates 4,522 MW of 
electricity generation capacity and employs a total of 3,264 people in Argentina. Table 6.1 lists all of Endesa's electricity generation facilities in Argentina. Endesa also owns a majority share (72.1\%) of Edesur, a distribution company that provides electricity to 2.3 million people south of the Buenos Aires area, and holds a minority stake $(22.2 \%)$ in the transmission company Yacylec (Endesa 2011c).

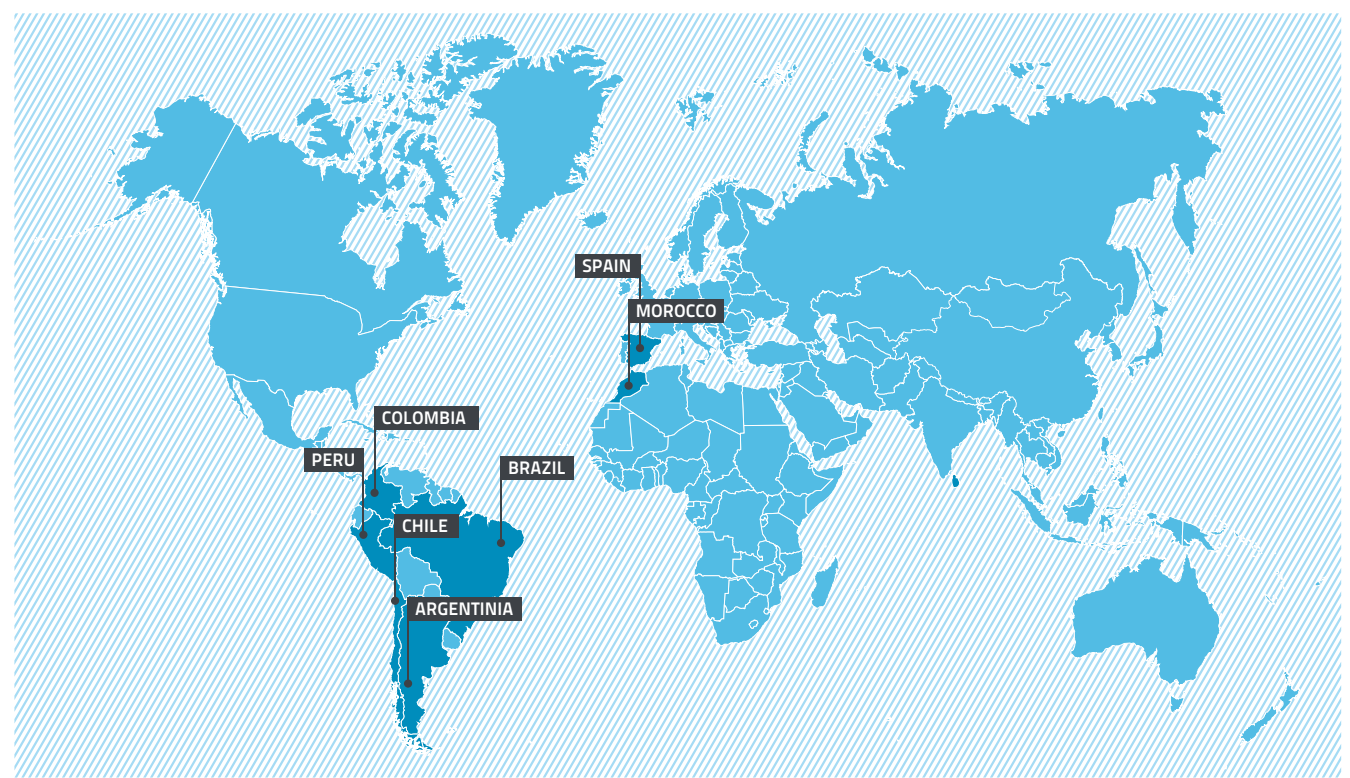

Figure 6.1: Endesa's global presence, 2009

Table 6.1: Endesa installed generation capacity in Argentina, 2010

\begin{tabular}{|l|l|l|l|}
\hline Facility name & Fuel type & Capacity (MW) & Endesa ownership (\%) \\
\hline Costanera turbine 1 & Gas oil & 1,138 & 70 \\
\hline Costanera turbine 2 & Natural gas (CCGT) & 859 & 70 \\
\hline Central Buenos Aires & Natural gas (CCGT) & 327 & 100 \\
\hline Dock Sud turbine 1 & Natural gas (CCGT) & 798 & 70 \\
\hline Dock Sud turbine 2 & Gas oil & 72 & 70 \\
\hline Total fossil fuel & & 3,194 & \\
\hline El Chocón & Hydro & 1,200 & 68 \\
\hline Arroyito & Hydro & 128 & 100 \\
\hline Total hydro & & 1,328 & \\
\hline Total Argentina & & 4,522 & \\
\hline
\end{tabular}

Based on: Endesa 2011c 


\subsubsection{Endesa in Peru}

Of Endesa's Latin American assets, 11\% are located in Peru. Endesa operates in Peru through three electricity distribution subsidiaries: Etevensa, Piura, and Edelnor, which supplies 986,000 customers in Lima's northern area. The company also controls a sizeable electricity generation capacity in Peru through its subsidiaries Edegel (in which Endesa owns $61.06 \%$ of shares) and Empresa Eléctrica de Piura (Eepsa, in which Endesa owns $60 \%$ ). In three thermal power plants and eight hydroelectric stations, the company controls $1,574 \mathrm{MW}$ of electricity generation capacity in Peru. Edegel S.A.A. is Peru's largest private electricity generation company. The results of the field research on Endesa's performance on SEP norms in Peru focus on the company's Huinco, Matucana, Chimay, and Ventanilla plants. Table 6.2 reveals all of Endesa's installed capacity for electricity generation in Peru.

\subsection{Endesa's performance on the SEP benchmarks}

This section begins with an overview (in table format) of Endesa's performance, in both policy and practice, on the 11 SEP benchmarks. For each SEP benchmark, a reminder of the guiding international normative standards (taken from Chapter 3) is provided alongside key indicators of the company's performance and an evaluation of the degree to which the company's policy and practice are line with the SEP standards on that issue. Following the overview, the results of the empirical research on Endesa's policy and practice in Argentina and Peru are detailed for each SEP benchmark. These findings form the basis for the overview and evaluation provided in Table 6.3. The chapter concludes with a brief reflection on the relationship between the European mode of home-country business culture (as outlined in Chapter 4) and Endesa's performance on the SEP benchmarks.

\subsubsection{General approach to corporate responsibility}

\section{Endorse international normative standards for} corporate responsibility

Endesa's CSR policies are based on a wide range of international standards and norms. Six sets of international norms and standards relevant to SEP are mentioned specifically in the company's 2008 Sustainability Report:

- UN Global Compact (and supporting implementation documents)

- UN Human Rights Norms for Business

- OECD Guidelines for Multinational Enterprises

- UN Millennium Development Goals

- ILO Standards

- UNICEF Child Labor Resource Guide

Endesa makes use of various standardized procedures and measurements such as ISO 9001 and 14001, OHSAS 18001, and SA8000 at the corporate headquarters level. The company also uses Europe-oriented certification systems such as the EU Eco-Management and Audit Scheme (EMAS). 
Table 6.3: Summary of Endesa's performance on SEP benchmarks in policy and in practice

\begin{tabular}{l} 
SEP benchmark \\
General approach to corporate responsibility \\
\hline Endorse international normative standards for CR \\
\hline
\end{tabular}

Adopt a commitment to CR in core-business activities and decisionmaking

\section{Guiding international normative SEP standards}

Electric utilities should seek to bring their policies in line with internationally recognized and verified standards for CR (ILO 2001, WBCSD 2002, IFC 2011, ISO 2011, OECD 2011، UNGC 2008, UNHRC 2011).

Electricity companies should adopt a holistic and integrated view of the role and impacts of electricity provision, considering and balancing the essential elements of economic development, environmental quality, and social equity in utility operations. This entails a policy commitment to CR throughout all core-business activities, as opposed to a primary focus on philanthropic activities (WBCSD 2002, MVO Platform 2012). Electric utilities should seek to introduce environmental and social factors and procedures into high-level corporate planning and decision-making and should aim to have a board-level position responsible for CR issues (WBCSD 2002).

\section{Social issues}

Increase access to affordable electricity
Electric utilities should develop policies that promote access to electricity priced at affordable levels for all, paying special attention to disadvantaged communities (ILO/Palast et al. 2000, WBCSD 2002, PSI/Pillinger 2009). In order to do so, companies should undertake initiatives to extend electricity services to unserved and underserved communities, particularly in rural or remote areas, but also to the poor in urban and peri-urban areas (WBCSD 2002, UNDP/Modi et al. 2005). 


\section{Indicators of Endesa's policy}

- CR policies highly reliant on international norms and standards

- Endorses six sets of norms: OECD Guidelines, UNGC, ILO Core Conventions, UN Human Rights Norms for Business, UN MDGs, UNICEF Child Labor Resource Guide

- Makes heavy use of standardized procedures and measurements such as ISO 9001 and 14001, OHSAS, SA8000, and EU EMAS.

- Largely in line with SEP standards

- Clear core business focus; vision on CR is to "supply customers with quality service responsibly and efficiently, while providing a return to shareholders, fostering employees' professional development, assisting with the development of the social environments where it operates and using the natural resources necessary for activities in a sustainable manner"

- Responsibility for CR issues at highest level of management in committee chaired by CEO

- Numerous management structures in place to implement CR policy and foster high performance on CR issues including strategic action plans and monetary incentives.

- Largely in line with SEP standards

- Has a policy promoting increasing access to electricity for disadvantaged and underserved communities

- Has set affordability of energy for low-income populations as a top priority

- Does not promote off-grid, decentralized electricity provision projects as well as large grid connections

- Operates 14,707 MW of capacity in countries without universal access to electricity ( $37 \%$ of total capacity).

- Largely in line with SEP standards

\section{Indicators of Endesa's practice in Argentina and Peru}

Not all standards endorsed by headquarters are named, but they are still relatively norm-oriented; GRI and UNGC mentioned prominently (though Edelnor in Peru listed as non-reporting member of UNGC)

- Certifications in ISO 9001 and 14001, OHSAS 18001, SA8000.

- Moderately in line with SEP standards

" Committed to "act beyond mere compliance with the law"; CR management structures similar to those at headquarters level

- But CR programs primarily focused on philanthropic and charitable projects

- Local communities and government officials claim their relationship with Endesa is based on charity rather than engagement on core business

" One felt Endesa's vision of CR is little more than "donating candy bars"

- Moderately out of line with SEP standards
Produced 16,047 GWh of electricity in Argentina and 9,160 GWh in Peru in 2010

- Electrification rates in Argentina and Peru are $97 \%$ and $85 \%$, respectively, but rates are as low as $22 \%$ in some areas where power plants are located

- Prices in areas of operation in Argentina range from $\$ 0.02 / \mathrm{kWh}$ (in Buenos Aires) to $\$ 0.18$ (in rural areas) for small domestic consumers; prices set to increase dramatically in 2013

- Majority of electricity generated in Argentina transported to large urban centers and to large industrial and commercial customers

- Only one project to expand access in Peru, none in Argentina; no programs to ensure affordability

- No support for off-grid, decentralized access.

Moderately out of line with SEP standards 


\section{Respect labor rights}

\section{Environmental issues}

Minimize environmental impact, including contribution to climate change
Electric utilities should respect workers' full rights and provide workers with fair pay, job security, and decent working conditions, including high levels of health and safety standards in all aspects of operations (WBCSD 2002, PSI/Pillinger 2009, OECD 2011). Furthermore, electric utilities should uphold freedom of association and the right to collective bargaining and pay particular attention to the right to strike (ILO 1998, ILO 2001, UNGC 2008, OECD 2011). Companies should ensure that these rights are respected not only among their own employees, but also among suppliers, contractors, and other business relationships (ISO 2011, OECD 2011, UNHRC 2011).

\section{Guiding international normative SEP standards}

Electric utilities should seek to minimize emissions and discharges to air and water and continually develop and implement low-pollution and low-environmental impact technologies (WBCSD 2002, UNGC 2008, PSI/Pillinger 2009, OECD 2011). Companies should manage their environmental impact in line with internationally recognized and verified standards, such as the ISO 14000 series and should always undertake rigorous and verifiable environmental impact assessments (WCD 2000, WBCSD 2002, IHA 2004, OECD 2011,). Electric utilities should minimize their contribution to climate change and develop strategies for reducing GHG emissions (WBCSD 2002, OECD 2011). As a matter of priority, electric utilities should seek to avoid impacts on biodiversity and ecosystem services, refraining from any activities in areas of critical habitat with high biodiversity value while at the same time initiating or supporting conservation and biodiversity efforts related to impacts on natural habitats from utility operations (WBCSD 2002, IHA 2004, IFC 2011).

Electric utilities should evaluate the renewable energy potential of the host country and prioritize development and exploitation of renewable options over fossil fuel-based technologies (WBCSD 2002, UN/IAEA 2007, PSI/Pillinger 2009). Electric utilities should also prioritize renewables in their research and development programs and should support the diffusion of renewable energy technologies into the local economy (WBCSD 2002, UNGC 2008). 
- Endorses the ILO Core Conventions

" Affirms workers' rights to freedom of association and collective bargaining

- Says right to strike may be restricted because electricity is a vital service

- Engages with workers during collective bargaining negotiations

- OHS is a top priority; has a detailed OHS policy and management systems that is OHSAS 18001 certified

- OHS policy also applies to contractors; communicates this policy directly to the workers of contractors

- Largely in line with SEP standards
- Unions present in both countries; approximately $66 \%$ of workers in Argentina are members

- Unions in both countries indicate relationship with company generally good but that management has pressured workers to prevent them from joining unions

- CBA in place in both countries covering $100 \%$ of employees in Peru

- No strikes reported in recent years

- Lowest wage paid in Argentina \$780/month, double national minimum

- Good OHS conditions in both countries; both have OHSAS 18001 certification

- Little specific information could be gathered on accidents/ injuries; at least 13 in Argentina in 2009

- Over $60 \%$ of workers are outsourced in Argentina and over $80 \%$ in Peru

- Contracted workers not unionized, not covered by CBA, and have higher rate of accidents/injuries despite company measures to ensure acceptable OHS conditions

- Moderately in line with SEP standards

\section{Indicators of Endesa's practice in Argentina and Peru}

- ISO certified EMS in both countries

- No information could be gathered on EIAs for all plants, but one plant in Argentina in conflict with local authorities over failure to submit EIA

- Several instances of serious environmental pollution in recent years in Argentina

- In Argentina, emitted $7 \mathrm{Mt} \mathrm{CO}_{2}$ with emission intensity of 0.42 $\mathrm{tCO}_{2} / \mathrm{MWh}$ in 2008; in Peru, emitted $2 \mathrm{Mt} \mathrm{CO}_{2}$ with emission intensity of $0.21 \mathrm{tCO}_{2} / \mathrm{MWh}$

- Has seen increases in $\mathrm{CO}_{2}$ emissions in both countries in recent years

- No information could be gathered on impact on biodiversity

- Only biodiversity protection measures mentioned in Argentina is keeping tourists away from dam site to protect native bird species

- In Argentina, operates 1,328 large hydro capacity, average capacity $664 \mathrm{MW}$, largest is 1,200 MW; in Peru, operates 739 MW large hydro capacity, average capacity is $93 \mathrm{MW}$, largest is $247 \mathrm{MW}$.

Moderately out of line with SEP standards

- Global fuel mix comprises $57 \%$ fossil fuels, $9 \%$ nuclear, $29 \%$ large hydro, $1 \%$ small hydro, $4 \%$ wind

- Policy to increase the use of renewable energy, primarily in Europe

- Little evidence of prioritizing renewables in current investments - only $9 \%$ of capital expenditures in home market in $\mathbf{2 0 1 0}$ were in renewable electricity.

- Fuel mix in Argentina comprises $44 \%$ natural gas, $27 \%$ gas oil, $29 \%$ large hydro

- Fuel mix in Peru comprises 53\% fossil fuels and $47 \%$ large hydro

- No evidence of prioritizing renewables or more efficient fossil fuels; continues to invest in thermal stations, and has shifted away from natural gas toward gas oil in recent years

- Moderately out of line with SEP standards

" Moderately out of line with SEP standards 


\section{Economic issues}

\section{Contribute to local economic development}

\section{Ensure reliable supply}

\section{Cross-cutting issues}

Engage in meaningful stakeholder consultation and participatory decision-making

Assume responsibility for impacts throughout all business relationships
Guiding international normative SEP standards

Electric utilities prioritizing the development of sustainable local energy-related infrastructure and employing local workers to the greatest extent practicable and providing those workers with sustainable jobs and training to improve skill levels (WBCSD 2002, PSI/Pillinger 2009, OECD 2011). In order to maximize local economic development and job creation, electric utilities should support local small and medium-sized enterprises in their procurement practices and contribute to the long-term development prospects of the host country through the transfer and rapid diffusion of technologies, skills and knowledge (WBCSD 2002, OECD 2011). Fair payment of due taxes to local, regional and national governments in the host country is a crucial element of contributing to local economic development (OECD 2011, MVO Platform 2012).

Electric utilities should ensure reliable supply by minimizing generation interruptions, maintaining contingency plans, responding to interruptions in a timely manner, and providing timely information to the public about planned and unplanned interruptions (WBCSD 2002, CEER 2005).
Electric utilities should adopt a participatory approach to the planning/designing, developing, operating, and monitoring/ evaluation of electricity projects and consult and engage stakeholders throughout the entire process in order to provide meaningful opportunities for their views to be taken into account in decision- making (WCD 2000, WBCSD 2002, IFC 2007, UNGC 2008, PSI/Pillinger 2009, OECD 2011). The consultation process should be tailored to local decisionmaking processes and involve the timely provision of all relevant information about activities, impacts, and potential impacts translated into local languages (WBCSD 2002, ISO 2011). The process should take into account the needs of disadvantaged or vulnerable groups, and if Indigenous Peoples are affected by a potential project, electric utilities must gain the free, prior and informed consent of those peoples before proceeding with any part of the project cycle (IFC 2011).

Electric utilities should seek to prevent, avoid and mitigate negative impacts throughout their entire range of business relationships, including suppliers and contractors (ISO 2011, OECD 2011, UNHRC 2011). Companies should seek to leverage their buying power to improve social and environmental conditions among business relations, and monitor performance using third party audits (WBCSD 2002). 


\section{Indicators of Endesa's policy}

- Proposes to contribute to local economic development through infrastructure development and prioritizing local suppliers

- No publicly available policy on prioritizing local workers

- Has a policy to prioritize SME suppliers in host countries.

- Moderately in line with SEP standards

\section{Indicators of Endesa's practice in Argentina and Peru}

- Employs 3,264 directly in Argentina

" Nearly all employees are nationals of Argentina and Peru, but in Peru few are hired from local communities where plants are located

- Prioritizes local suppliers in Argentina; local suppliers account for $89 \%$ of purchases

- Invested \$209 million in electricity infrastructure in Argentina in 2010, but has been criticized and threatened by regulators for failing to invest enough to ensure reliable service

- Pays no more than $1 \%$ of annual revenues in taxes in Peru.

" Moderately out of line with SEP standards

- Average capacity factor in Argentina is $40 \%$; average in Peru is $58 \%$

- Has been cited and threatened by regulators in Argentina for failing to properly maintain electricity infrastructure

- Has been fined frequently by regulators for inadequacies related to reliability of supply.

- Moderately out of line with SEP standards

" In Argentina, engages stakeholders through "surveys, dialogue with unions, lectures, seminars, and meetings"

- A major conflict with a community in Argentina involved involuntary resettlement and residents claimed they were not meaningfully consulted

- Stakeholders in Peru perceive engagement as primarily focused on philanthropic activities and lacking in meaningful consultation on key SEP issues

- One manager in Peru engaged with researchers; management in Argentina declined to do so.

- Moderately out of line with SEP standards

- Requires major business partners to have ISO and OHS certifications and uses audits of those involved in high-risk activities to monitor compliance

- Includes social and environmental criteria in supplier/contractor selection processes and in contracts with business partners

- Nevertheless, working conditions are poorer among contracted workers, who are not unionized, earn lower wages, have a higher rate of injury.

- Moderately in line with SEP standards 


\section{Cross-cutting issues}

Maximize transparency and provision of information
Guiding international normative SEP standards

Electric utilities should apply a high degree of transparency in operations, including reporting on activities and progress, measurement, business partners and relationships, and interactions with government and the public (UNECE 1998, WBCSD 2002, UNGC 2008, OECD 2011). Electric utilities should regularly report on their activities, progress, and performance against "the triple bottom line" using internationally accepted sustainability reporting guidelines such as the GRI's G3 guidelines and the Electric Utilities Sector Supplement (GRI 2008), have an external party verify the data, and disseminate the report in an appropriate manner (WBCSD 2002, OECD 2011).
In Argentina and Peru, Endesa also takes an approach to CSR that is highly reliant on international normative standards and certifications. Endesa Argentina specifically references the GRI reporting guidelines, the UNGC, the ILO's Core Conventions, and Endesa's SA8000 certification. Dock Sud has also obtained the ISO 9001 quality management certification, the ISO 14001 certification for its EMS, and the OHS 18001 certification of its OHS management system (Endesa Dock Sud 2007). Endesa Costanera (2010b) also has an extensive, ISO 14001-certified EMS. In Peru, Edegel has achieved certifications for the SA8000, ISO 9001, and OHSAS 18001. These achievements are displayed prominently on Edegel's (2010) website. Many of Endesa's local subsidiaries in Latin America, including Edegel, are signatories to the UN Global Compact. It should be noted, however, that Endesa's Peruvian Edelnor subsidiary is currently listed as a non-reporting member of the Global Compact (Global Compact 2011).

\section{Adopt a commitment to CR in core business activities and decision-making}

Endesa believes that, through CR, companies make their contribution to sustainable development (Fraile 2008). For Endesa, CR means responsible growth based on the integration of social and environmental elements into all aspects of the operational and management spheres of its business strategy. The company's core values include people, health and safety, innovation, customer orientation, community, and environment. Endesa's (2008b) "Sustainability Policy" seeks to balance the three sustainable development pillars by "supplying customers with quality service responsibly and efficiently, while providing a return to shareholders, fostering employees' professional development, assisting with the development of the social environments where [it] operate[s] and using the natural resources necessary for activities in a sustainable manner". According to Endesa, the company understands that its core business is related to an activity that 


\section{Indicators of Endesa's policy}

- High degree of accessibility and visibility of CR policies on website and in annual sustainability report

- Report includes information on operations in the Global South

- Reports according to the GRI G3.1 and EUSS; received A+ rating in 2010

Sustainability report is assured by external party

Does not provide transparency about business

relationships

- Largely in line with SEP standards
Indicators of Endesa's practice in Argentina and Peru

Produces annual sustainability reports along GRI standards, but many are outdated

All CR policies and reports translated into Spanish

Primary channels of communication with stakeholders are annual sustainability reports, "specific reports", and website; specific channels for employees include internal communication board, newsletter, and website.

Moderately in line with SEP standards is essential for society, and has therefore made social development a key aim in its Sustainability Strategic Plan (Endesa 2008a). At the same time, the issue of finding solutions to climate change is a core concern for the company. Responding appropriately to these two challenges is at the core of the company's CSR commitment to secure society's shortand long-term energy need with minimum environmental impact.

Endesa (2008a) believes that its own standards become more important in the Global South where the regulatory framework is weak, noting that "human rights and bribery issues need to be tackled more carefully in some host countries such as Peru and Brazil. Hence, the set of standards that Endesa's subsidiaries in those countries have to adhere to must be more demanding than, for instance, in Europe".

Similarly, Endesa believes that its standards and practices for reducing $\mathrm{CO}_{2}$ emissions must be more stringent in the Global South where, in contrast to Europe, regulatory structures to control emissions are not yet in place. Endesa believes that this makes business sense because by working to minimize emissions now, "Endesa will be better positioned when the pressure to regulate GHG emissions extends to those markets" (Fraile 2008).

Operational responsibility for SEP issues is found at the highest level of management at Endesa. The company has a top-level Environment and Sustainability Committee, which is composed of members of the Executive Management Committee and chaired by the CEO. This Committee approves plans, programs, and actions relating to sustainability, and is responsible for monitoring implementation of the Strategic Plan. Furthermore, the General Directors of each business unit are responsible for environment, social and human rights, and labor rights issues from an operational point of view. Endesa has also established a formal structure to coordinate all sustainability activities and a permanent working group 
incorporating managers from all of Endesa's operation areas to assure the implantation of CR throughout the company's management. Finally, each employee's evaluation is linked to sustainability performance (Endesa 2008a). For example, the company provides monetary incentives to management for attaining targets on developing renewable energy and reducing $\mathrm{CO}_{2}$ emissions (Endesa 2011b). The Carbon Disclosure Project (CDP) has indicated that Endesa is "building climate related risks and opportunities into core business" (CDP 2011).

In order to implement its CR goals and values, Endesa periodically develops "Sustainability Strategic Plans" that contain the general programs to be developed through "Action Plans" during the following five years. The action plans are developed by a multidepartmental group, and progress is evaluated at least twice a year. This structure is followed by every Endesa subsidiary, and global results are consolidated in the headquarters. Furthermore, Endesa has developed an internal tool to ensure the fulfillment of its commitment to the Global Compact principles. This tool sets a group of standards for Endesa and all its subsidiaries that have to be followed on all management areas, based on international standards. The company uses external certifications, such as ISO 14001 and EMAS, and has its sustainability policy evaluated by a third party.

Endesa (2008a) notes that the implementation of its standards is more difficult and requires more effort in the Global South than in industrialized nations. In order to determine strategies for implementing its policies and ensuring compliance by its subsidiaries in the Global South, the company has conducted an in-depth analysis of the ILO Core Conventions and UN Global Compact principles, taking into account the general situation in the host countries in which the company operates and evaluating which standards should be applied and which implementation measures should be used in each situation. Since all Endesa subsidiaries have the same CR structure as the headquarters, implementation of its standards is generally monitored in the same way in the South as it is in the North. The structures of a top executive-level Environment and Sustainable Development Committee and Sustainability Group are replicated in each country, and Endesa requires that all subsidiaries respect the international initiatives that the headquarters endorses, such as the UN Global Compact. In some critical issue areas, however, more specific or in-depth monitoring tools are used in the Global South than in the North (Fraile 2008).

In Argentina and Peru, many of Endesa's subsidiaries' policies also reflect a core business CR approach, with one subsidiary claiming that environmental management should be "integrated into the corporate strategy, using environmental criteria in planning and decision-making processes" and vowing to "act beyond strict compliance with the legislation in force, intensifying the necessary efforts and establishing adequate procedures to ensure both rational use of resources and minimization 
of residues, and contributing to the extent possible to the sustainable development expected by society" (Endesa Dock Sud 2011). Another subsidiary's approach to CR involves "a proactive attitude in terms of prevention and anticipation with regard to the protection of humans, nature and the environment and seeking continuous advice on ways to reduce or eliminate the environmental impacts they could generate, adopting the cleanest, most efficient and economically sustainable technology in order to prevent air, water or soil pollution" (Endesa Costanera 2010b). Despite these core business commitments, a hint of a tendency toward philanthropy is revealed in Endesa Dock Sud's (2007) three primary CR commitments:

- Educational development in the community where the company operates

- Social and cultural development of the community's families

- Protection of the environment in the area where the generation plant operates.

Indeed, beyond its policy commitments, Endesa's CR activities in Argentina are primarily focused on philanthropy and charitable projects. The company's CR activities include making donations to schools, sponsoring local sports teams, organizing fundraisers for the community, and supporting a number of social and environmental programs. However, local stakeholders claim that Endesa spends more money advertising its CSR programs to improve the company's image than it does on the programs itself (FeTRA 2007). One union leader noted bluntly that he felt Endesa's vision of CR is little more than "donating candy bars"
(Fernández 2008). According to residents living adjacent to Endesa's Dock Sud thermal plant, Endesa's CR programs have not contributed to improving the living conditions of the communities and have actually aggravated rather than improved the local conditions (Residents of Villa Inflamable 2008).

In Peru, the company's CR initiatives are also perceived by locals as being more focused on philanthropic activities and gift-giving in the communities rather than sustained engagement with communities and governments on local planning and development issues. Residents of a community adjacent to the Ventanilla power station, for example, told researchers that there is no engagement with their community other than a Christmas present offered by the company to the community's children (Ventanilla Resident 1 2008). Local government officials explain that their relationship with Endesa generally consists of financial support and other philanthropic activities toward the communities rather than a relationship based on critical issues such as meaningful engagement and participatory decision-making (Mungi 2008). For example, Edegel financially supports schools in the Ventanilla and Monobamba regions and promotes the development of productive activities, such as fish farming. However, communities in these areas lack access to basic levels of electric service, and local authorities complain that they are not consulted on the company's plans or operations (Ventanilla Resident 2 2008). 


\subsubsection{Social issues}

\section{Increase access to affordable electricity}

According to Endesa, extending electricity access is one of the company's main aims, especially considering that nearly half its business is located in Latin America, where access in rural and low-income urban areas is limited and a large portion of the population lives below the poverty threshold (Endesa 2007b). Endesa sees making electricity accessible and affordable as part of its contribution to the development of the societies in which it operates. The company claims that it "develops infrastructure through rural electrification programs aimed at making new connections and bringing access to new customers in certain rural parts of various Latin American countries, paying attention to more vulnerable communities or those with greater difficulties gaining access to supply" (Endesa 2007b). Endesa currently operates 14,707 MW of capacity in countries without universal access to electricity, which is approximately $37 \%$ of its total capacity worldwide.

\section{Endesa also claims to have set affordability} of energy for low-income populations as a top priority. For example, a program in Brazil consists of allowing low-income locals exchange their waste for discounts on their electricity bill. Between 2006 and 2008, the project saw 3,128 tons of waste exchanged for electricity worth $\$ 242,295$ (€183,284), with more than 40,000 low-income residents participating (Waste Management World 2008). Another project in Brazil helps low-income customers save money by improving energy efficiency through measures such as installing more efficient light bulbs and refrigerators (Fraile 2008). In the same year, however, Endesa was also criticized for overseeing a $500 \%$ increase in household electricity prices and a large number of disconnections of poor customers, while at the same time arranging discounts and facilitating payment plans for wealthier customers in Bogotá, Colombia (FoEl 2008).

In Argentina, Endesa operated 4,522 MW of capacity and generated 16,047 GWh of electricity in 2010. In Peru, the company operated 1,801 MW of capacity and generated 9,160 GWh in the same year (Endesa 2011b). Endesa Dock Sud provides energy to the larger Buenos Aires Province, where more than $96.7 \%$ of all households and industries have access to electricity, including the Avellaneda municipality where Endesa Dock Sud is located (INDEC 2002). Villa Inflamable, one of the poorest dwellings in the Avellaneda municipality, also has high levels of access to electricity from the public grid due to its proximity to Endesa Dock Sud. Many poor residents of Villa Inflamable do not pay for their electricity, not because of a policy or program of Endesa's, but because the complexity of the distribution system in the poor neighborhood makes it difficult for the company to determine exact usage (Villar 2008). The Federation of Energy Workers (FeTRA 2007) has documented how, in times of peak seasonal demand, for example, during extremely hot periods in the summer, poor areas of the city experience frequent blackouts - more frequent than in other areas of the city. Dock Sud's (2009) 
main customers are industrial (e.g. refineries, automotive factories) and commercial (e.g. supermarkets, shopping malls) users. Nearly $100 \%$ of the electricity produced by the El Chocón and Arroyito plants is transmitted to Buenos Aires (Endesa El Chocón 2010). With regard to affordability, electricity prices paid by small domestic users in Endesa's areas of operations in Argentina vary widely. In Neuquén, where the El Chocón plant is located, small domestic users paid ARS 0.803 (\$0.18/€0.14) per kWh plus a fixed charge of ARS 7.70 (\$1.75/€1.34) per month in 2011. In Misiones Province, where Yacyretá is located, prices were ARS 0.12 (\$0.03/€0.02) per kWh and a fixed charge of ARS 8.78 (\$2.00/€1.53) per month. In the more metropolitan Edesur concession, which includes the Costanera and Dock Sud plants, prices were ARS 0.08 (\$0.02/€0.01) per kWh plus ARS 4.44 (\$1.01/€0.77) per quarter fixed (EPEN 2011).

All of these prices are set to increase up to threefold when government subsidies are cut in 2012. There is no evidence that Endesa has any programs to ensure that the electricity it generates is affordable for poor customers, but the company does note that customers who are medically dependent on electricity (e.g. those on refrigerated medication) will be exempt from paying the increase in 2012 if they submit proof of their situation (Edesur 2012).

In Peru, access to electricity is extremely low in some of the districts in which Endesa's Edegel operates. For example, in the rural Monobama district of the Junin Province, where Edegel's Chimay power station is located, only $22 \%$ of households have access to electricity (INEIP
2005). The small number of households that do have electricity in this district are supplied by a municipal station rather than by Edegel. This situation has created an atmosphere of distrust and uneasiness towards the company among local government officials, who oppose the fact that a power plant established in the area does not supply electricity to the district. While Edegel is not legally required to supply electricity to the local community, municipal authorities feel that it is the company's responsibility to do so (Mungi 2008). A company representative did claim Edegel is arranging to transfer one of its transmission lines to Electrocentro, the public company that supplies electricity in the area, in order to serve a small village of 12 households (Prieto 2008). Aside from this initiative, there are no programs to increase access to electricity for the underserved in either Argentina or Peru.

In sum, Endesa's policies at the headquarters level are largely in line with SEP norms that encourage electric utilities to promote access to electricity priced at affordable levels for all, paying special attention to disadvantaged communities (Modi et al. 2005, WBCSD 2002, Palast et al. 2000, PSI/Pillinger 2009). However, there is little or no evidence of such programs or initiatives in Argentina or Peru.

\section{Respect labor rights}

Endesa cites ILO conventions as one of the guiding norms for its CR policies. The company notes that the implementation of these core labor policies is more difficult and requires more effort in the Global South than in industrialized 
nations. Endesa (2008a) expressly condemns child labor. In addition to implementing mechanisms to ensure that all of its employees are of legal age, part of the company's strategy to combat child labor includes participating in projects aimed at improving education and reducing extreme poverty to indirectly eradicate child labor. Endesa also condemns forced labor and recognizes its employees' right to freedom of association. In 2007, 49 collectively bargained agreements were in place, covering $80 \%$ of the company's employees. The most recent agreement was the III Collective Agreement, which was reached in April 2008. Coverage by the collective agreements does reveal some differences between the Global North and the South. In Europe, 92\% of the company's employees are covered by such agreements, while in Latin America only 65\% are covered (Endesa 2008a). With regard to workers' right to strike, Endesa maintains that, since electricity is such a crucial service, the right to strike is heavily regulated by most countries and the conditions determined by the local regulator.

In Argentina, approximately two-thirds of direct employees at Endesa's Costanera and Dock Sud plants are unionized (Endesa Costanera 2011, Endesa Dock Sud 2009). A collective bargaining agreement is in place, but no information could be gathered as to what percentage of the employees it covers. One interesting element of the agreement is a complaint procedure for workers, enabling them to contest management decisions and/or file claims about the company's interpretation of the agreement. However, the Federation of Energy Workers (FeTRA 2007) has complained that the company has worked to make it difficult for workers to unionize and engage in collective bargaining. In Peru, leaders of the Edegel workers' union noted that, while the company maintains good relations with the union and enables it to carry out its activities, company management sometimes pressures workers to prevent them from joining the union (Jica 2008). A collective agreement is currently in force for all permanent Edegel workers.

Endesa gives extensive coverage to the topic of $\mathrm{OHS}$ in its sustainability report, claiming that it is one of the company's primary values (Endesa 2007b, Endesa 2008a). Endesa relies on the OHSAS 18001 certification in its "Strategic Plan for Health and Safety" and its "Health and Safety Model". Due to increased OHS risks in the Global South, Endesa has implemented a policy of providing all subsidiaries with countryspecific OHS operating guidelines.

In Argentina, OHS conditions are covered by the collective agreement between the company and the workers. A key topic in this agreement is staff training and the issue of safety and hygiene at work. The agreement establishes a permanent OHS commission, comprised of both worker and management representatives. The company has OHSAS 18001 certification. Little information could be gathered about the exact frequency and severity of accidents. Costanera indicated that it had reduced the rate of accidents by $50 \%$ between 2008 and 2009 (Endesa Costanera 2010a), while the six 
accidents at El Chocón in 2009 represented a six-fold increase (Endesa El Chocón 2010). Dock Sud experienced 13 accidents and 208 lost work days in 2008, which the company itself attributed to the increasing use of contractors (Endesa Dock Sud 2009). In Peru, the company has OHSAS 18001 certification, and workers reported high health and safety standards (Jica 2008).

A major concern among Endesa workers in both Argentina and Peru is the lack of job security and increasing rate of outsourcing. In Argentina, more than $60 \%$ of the total number of those working on Edesur projects and at the El Chocón (Hidalgo 2009, El Sindical 2010, Endesa El Chocón 2010). In Peru, more than $80 \%$ of workers are outsourced contract laborers. According to Jica (2008), Endesa is constantly seeking to reduce the number of permanent employees and replace them with outsourced and contracted personnel. While permanent workers are generally assigned to administrative and operational tasks, almost all of the maintenance work done on Edegel's power plants is outsourced to contract workers.

In terms of working conditions among contracted workers, Endesa has a strategy for seeing that its OHS standards are observed by contractors. This policy includes communicating the company's OHS policy directly to the employees of contractors (Endesa 2008a:68). In Peru, Endesa claims that its contractors are required to comply with all applicable OHS legislation and has set certification under the SA8000 standard as a requirement for contractors in order to ensure their compliance with legally established labor conditions. The company also reported that the OHS management systems of the three contractor companies it works with are certified under OHSAS 18001 (Endesa Edegel 2008). In Argentina, Endesa Costanera (2010a) has also sought OHSAS 18001 certification from three of its main contractors. In 2010, Edesur (2011) conducted audits of 14 focus contractor companies, in order to ascertain how many were aligned with the OHSAS standard and insisted that all workers dealing directly with live electricity should be covered by the OHSAS certification. This resulted in the certification of contractors covering 1,400 workers.

Despite these measures, the rate of accidents and injuries remains higher among contracted workers than among the company's own employees (Endesa Dock Sud 2009). And aside from OHS, there is no evidence that Endesa seeks to ensure that other rights of Endesa contract workers, such as job security, wages, and working hours, are respected. The contracted workers are not unionized, nor do they enjoy the benefits under the collective agreement (Jica 2008).

The lowest wage paid to workers at Endesa Costanera (2011) is ARS 3,433 (\$780/€590) per month. This is nearly double the minimum wage in Argentina, which is ARS 1,440 (\$327/€247) per month.

In sum, Endesa's policies are largely in line with SEP norms on labor rights, insisting that 
electric utilities provide workers with fair pay, and decent working conditions, including high levels of health and safety standards in all aspects of operations and among suppliers and contractors (ILO 1998, WBCSD 2002, UN Global Compact 2008, Pillinger 2009, ILO 2001, OECD 2011). Two areas where Endesa's policies are not fully in line with the SEP norms are regarding the right to strike and job security, given the company's push to increase the ratio of contracted workers to those it directly employs. Job security is also the primary concern in Argentina and Peru, where the ratio of contracted workers to employees reaches 5:1.

\subsubsection{Environmental issues}

\section{Minimize waste, pollution, and emissions (including GHG emissions)}

Endesa's "Sustainability Strategic Plan 2009-2012" groups the environmental issues to be addressed into three main blocks: implementing advanced environmental management, fighting climate change, and deepening preservation of biodiversity.

Endesa employs an ISO 14001-certified EMS to manage the environmental impact of discharges, emissions, and waste. As part of its "Advanced Environmental Management" program, Endesa has set objectives such as the reduction of water consumption each year over the previous year, $100 \%$ processing of waste waters, $100 \%$ use of river beds (not leaving any stretch dry), $100 \%$ evaluation of facilities with environmental liabilities, and $100 \%$ of facilities
ISO 14001-certified. Endesa carries out an internal compulsory regulation related to the management and elimination of wastes in all its facilities. These guidelines establish criteria and specific procedures on the treatments that have to be done, as well as on the contracting of the proper waste operators. The company's approach to waste and pollution differs little between the Global South and industrialized nations. For example, in $2007,89.18 \%$ of the electricity Endesa produced in Spain and Portugal was ISO 14001 certified, while in Latin America 94.2\% received the certification (Endesa 2008a:140). It is not company policy to always conduct an EIA.

In Argentina, Endesa's Dock Sud and Costanera have both obtained ISO 14001 certification for environmental management. Despite these certifications, both plants have had problems with performance on environmental issues. Endesa Dock Sud was been fined by the Argentine electricity regulator ENRE three times between 2003 and 2008 for failing to submit its environmental management plans or due to the plans' untimely submission (ENRE 2008). In addition, Endesa Dock Sud is currently being sued for pollution of the Matanza Riachuelo River. Endesa maintains that its emissions and waste releases into the river are within the legally permitted amounts (FARN 2011a).

In another incident, in 2006 residents of several Buenos Aires neighborhoods complained to the city's Ombudsman about acid-like pollution from Endesa's Costanera plant. According to the Ombudsman of Buenos Aires (2007), Costanera's use of fuel oil with content levels 
of sulfur dioxide (SO2) exceeding national and international norms was the cause. The levels of sulfur in the plant's fuel oil exceeded the national regulatory standard, which sets the maximum relative content at $0.5 \%$ (Argentine Ministry of Environment 2001). The fuel oil had been imported from Venezuela, a result of the Argentine energy crisis and a subsequent agreement between the Argentine and Venezuelan governments regarding the import of Venezuelan fuel oil for use in Buenos Aires. The Argentine government has a clear responsibility for choosing to import fuel with high sulfur content and the consequent air pollution. The Ombudsman stated that the use of fuel oil with high sulfur content was partially due to the Argentine government's non-compliance with its own regulations. However, Endesa Costanera also had a responsibility to minimize the negative effects of its electricity generation operations. When asked by the Ombudsman whether Central Costanera used catalysts to reduce the sulfur content of its emissions, the company responded that they believed there was no need for such measures. They simply said that they are provided with fuel oil conditional to approval by the Secretary of Energy and the Secretary of Planning, and the company believes that it is acting within the regulatory limits (Alimena 2008). Such statements indicate that the company is not willing to make efforts beyond the legal requirements in order to reduce pollution and hazardous emissions.

Endesa Costanera has also had a conflict with the City of Buenos Aires regarding jurisdiction for regulation of emissions and EIAs. Endesa
Costanera has argued that, since it is located on national territory, it is only accountable to the national regulators, and not to the city of Buenos Aires (Alimena 2008). The company has failed to submit an EIA, as required by Law 123 of the city of Buenos Aires (Alimena 2008). The failure to fulfill this requirement constitutes a violation of local legislation, and the situation has been exacerbated by the fact that Central Costanera's activities are considered to have "significant environmental impact" due to air pollution caused by the use of high-sulfur fuel (as discussed above).

Thus, while Endesa's policies appear to be widely in line with international SEP norms, numerous incidents related to poor environmental management in Argentina reveal an inability or unwillingness to translate the norms and policies into practice on the ground.

According to Fraile (2008), a wide-ranging analysis of its stakeholders' expectations in the coming five years revealed climate change to be one of the two main challenges facing the company in the near future and is thus a top priority in its "Sustainability Strategic Plan 2009-2012". Endesa devotes a great deal of its sustainability reports to the issue of climate change and GHG emissions reduction (Endesa 2007b, Endesa 2008a). The company's strategy on climate change also involves participation in a number of international initiatives focused on research and development of solutions for climate change (Endesa 2008a). Endesa (2011b) reports extensively on its GHG emissions and reduction targets via the CDP. Its latest report 
received a "high" score, meaning that Endesa's

"Senior management understand the business issues related to climate change and are building climate related risks and opportunities into core business" (CDP 2011). Endesa is among the top-scoring electric utilities in the CDP ranking, and is also one of the few electric utilities that reports on emissions in its supply chain (Endesa 2011a). The company claims that it has reduced its specific emissions by $36.5 \%$ in the period 1990-2007, beating its target of $35 \%$ reduction, and that it aims to halve its GHG by 2020 (Endesa 2007b:84). Table 6.4 lists Endesa's $2010 \mathrm{CO}_{2}$ emissions at the global level, as well as specifically in Argentina and Peru.

Despite meeting its targets and achieving a further 15\% reduction in 2010 over 2009 at the company-wide level, its emissions in Argentina and Peru have been increasing in recent years. 2010 saw a 13\% increase over 2009 in Argentina and a 17\% increase in Peru.

Endesa Argentina's heavy reliance on fossil fuels for electricity generation (see Figure 6.2) results in a high level of $\mathrm{CO}_{2}$ emissions. Endesa's Dock Sud and Costanera thermal facilities are two of the country's three largest emitters of GHGs. Endesa has a considerable capacity
(1,978 MW) from relatively efficient combined cycle natural gas turbines, but at the same time much of its capacity relies on less efficient gas oil turbines. Similarly, in Peru Endesa's recent increase in generation from and investment in thermal power stations is at odds with the company's commitments to combat climate change. Endesa acknowledges that there is less regulatory pressure to reduce GHG emissions in the Global South than in Europe, but sees this as an opportunity for developed countries and companies to invest in renewable energy technologies in order to help reach their own GHG emissions reduction targets (Endesa 2008a).

An indicative example of the company's Argentine facilities, Endesa's Dock Sud thermal cogeneration plant is fuelled by natural gas and gas oil. In 2010, when natural gas was in short supply, the company began using more gas oil and diesel to compensate for the lack of natural gas. This had negative consequences for the environment in terms of $\mathrm{CO}_{2}$ and other emissions, as gas oil is dirtier and more carbon-intensive than natural gas. In 2010, the facility consumed approximately 783,742 cubic decametres of natural gas and nearly 191,000 cubic metres of gas oil. Endesa's Costanera

Table 6.4: Endesa's global, Argentine, and Peruvian $\mathrm{CO}_{2}$ emissions, 2010

\begin{tabular}{|l|l|l|l|l|l|}
\hline $\begin{array}{l}\text { Geographical } \\
\text { scope }\end{array}$ & Capacity (MW) & Production (GWh) & $\begin{array}{l}\text { Absolute } \\
\text { emissions (tCO })\end{array}$ & $\begin{array}{l}\text { Change } \\
\text { over 2009 }\end{array}$ & $\begin{array}{l}\text { Emission } \\
\text { intensity (tCO } / \\
\text { MWh) }\end{array}$ \\
\hline Global & 38,131 & 129,075 & $38,631,350$ & $-15 \%$ & 0.28 \\
\hline Argentina & 4,522 & 16,047 & $6,590,440$ & $+13 \%$ & 0.42 \\
\hline Peru & 1,801 & 9,160 & $1,958,720$ & $+17 \%$ & 0.21
\end{tabular}

Source: Endesa 2011b 
consumed 1,259,772 cubic decametres of natural gas, 212,539 cubic metres of gas oil, and 466,121 tons of fuel oil in 2010. Similarly, in Peru Endesa's dual-fuel thermal generation stations have been relying more heavily on oil-firing as a result of the energy crisis.

Endesa (2011f) has an extensive policy on protecting biodiversity. One of the basic principles in Endesa's environmental policy is to conserve the natural environment in the regions in which its facilities are located by adopting measures to protect flora and fauna and their habitats. The company has a specific "Biodiversity Conservation Strategic Program". In 2009, the program focused on two priority areas:

- Projects to restore and improve local habitats and areas affected by the company's facilities

- Projects and studies on conserving biodiversity with a socio-environmental component.

Overall the program is focused on achieving the following specific objectives:

- Adapting the physical environment to increase its ability to encourage biodiversity

- Managing environmental factors (processes) that help improve the habitats of certain species in particular or the biotopes they comprise

- Identifying natural ecosystems in order to identify their ability to respond to pressures caused through their use, or to unlock the value of the natural assets they represent or house

- Preserving indigenous species
- Controlling and eradicating invasive exotics with a severe ecological impact.

Endesa's hydroelectric power plants in Argentina and Peru impact biodiversity. In Argentina, the company operates 1,328 MW of large hydroelectric capacity. The average capacity of Endesa's two large hydro plants is $664 \mathrm{MW}$. The largest of these is the 1,200 MW El Chocón plant, which has a water surface of $816 \mathrm{~km}^{2}$, and is also the largest hydroelectric reservoir in all of Argentina. In Peru, the company operates $739 \mathrm{MW}$ of large hydroelectric capacity. The average capacity of these seven large hydro facilities is $93 \mathrm{MW}$. The largest is the $247 \mathrm{MW}$ Huinco plant. Endesa does not communicate any measures it is taking to preserve biodiversity in either country. The only mention Endesa Argentina made of biodiversity in its latest annual report was protecting the site of its $128 \mathrm{MW}$ Arroyito from tourists and other outsiders in order to reduce the impact on native bird species in the area (Endesa El Chocón 2010).

\section{Prioritize renewable sources of energy for electricity}

Approximately $66 \%$ of Endesa's 40,141 MW of global electricity generation capacity is based on non-renewable sources of energy, with $57 \%$ generated from fossil fuels and $9 \%$ from nuclear. $34 \%$ is based on renewable sources: $29 \%$ large hydro, 1\% small hydro, 4\% wind (Endesa 2011e).

Endesa's strategy on developing renewable sources of energy for electricity is outlined in its "Sustainability Strategic Plan", in which 
the company sets itself a target of installing $6,000 \mathrm{MW}$ of new renewable electricity generation capacity between 2008 and 2012, primarily in Europe. For Endesa, increasing the use of renewable sources of energy is a much more urgent issue in Europe than in the Global South because regulatory and social pressures to increase renewables are greater in Europe (Fraile 2008). While the company aims to develop $50 \mathrm{MW}$ of renewable electricity generation capacity in Latin America by the end of 2009, the amount to be developed in Europe is much higher (Fraile 2008). Even so, expenditure on renewable electricity development in Endesa's home market of Spain and Portugal in 2010 totaled less than $\$ 260$ million (€200 million), just $9 \%$ of the company's total expenditures (Endesa 2011b). Renewable electricity contributed only $4 \%$ to the company's earnings in the same year. This indicates that the company is not prioritizing renewable sources of energy as suggested by SEP norms.

\section{Endesa (2011f) claims that its research and} development (R\&D) activities are a part of the company's strategy to increase the exploitation of renewable sources of energy. In 2010, Endesa directly invested $\$ 42$ million ( $€ 32$ million) in R\&D activities, mobilizing a further $\$ 15$ million (€11 million) through technological partners participating in projects and consortia led by the company. It should be noted that Endesa includes CCS for coal plants, large hydro, and nuclear power in this budget.

In Argentina, Endesa's electricity generation capacity is also largely (71\%) based on fossil fuels, mostly natural gas. The company does have 1,320 MW of hydroelectric generation capacity, all from large-scale hydro facilities. Figure 6.2 reveals the fuel mix of Endesa's electricity generation capacity in Argentina.

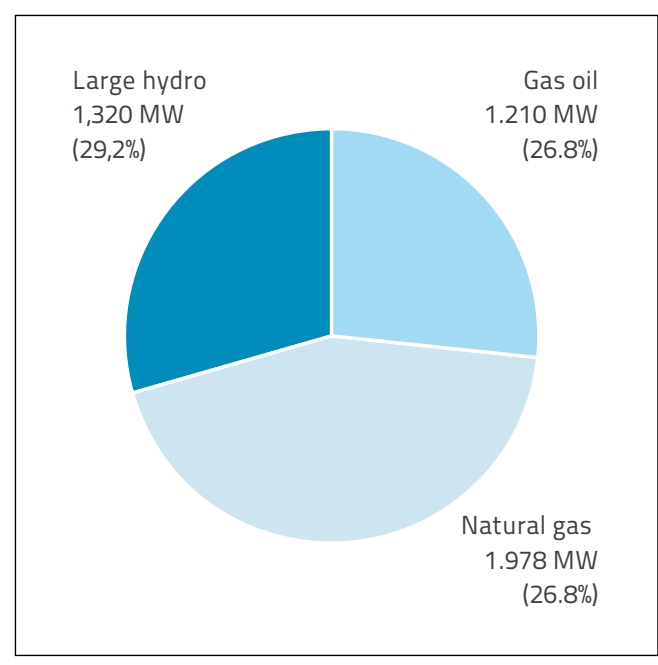

Figure 6.2: Endesa's installed capacity in Argentina by fuel type, 2010. Based on: Endesa 2011c

In Peru, approximately $47 \%$ of Edegel's generation capacity is based on hydraulic resources and $53 \%$ on fossil fuels, mainly natural gas. Nearly all of the company's hydroelectric capacity comes from large-scale hydro facilities, but it does have one small-scale $2.3 \mathrm{MW}$ hydro facility. Edegel was the first company in Peru to invest in electricity generation from natural gas. Its investment in the combinedcycle gas turbine in Ventanilla turned it into the country's most efficient and most potent power generation station. The company has also invested heavily in the enlargement of the Santa Rosa thermal station, which has also shifted from using natural gas to using 
more $\mathrm{CO}_{2}$-intensive fuel oil. This is at odds with SEP standards encouraging electricity companies to promote renewable sources of energy and contribute to long-term sustainable development.

\subsubsection{Economic issues}

\section{Local economic development}

Endesa (2007b:22) states that it "develops the infrastructures and actions necessary to cover the evolution of the demand for electricity in the communities where the company is present and reach as many customers as possible". The company further claims that increasing local capacity and human capital is one of the "strategic criteria" applied when selecting from potential business partners (Fraile 2008). In 2007, for example, Endesa's Latin American business unit purchased a total of $\$ 1.8$ billion (€1.4 billion) worth of material, equipment, and services from Latin American suppliers. Endesa's policy on taxation cites the tax chapter (Chapter XI) of the OECD Guidelines for Multinational Enterprises.

In Argentina, it is not known what percentage of the 3,264 people Endesa employs are Argentine nationals or how many employees come from communities adjacent to its power plants, but the company claims that $100 \%$ of its new hires were locals. The company is in line with SEP norms on its policy to prioritize local suppliers. In $2010,89 \%$ of purchases for goods and services were with local suppliers, though $65 \%$ of the largest contracts went to non-Argentine multinationals (Endesa 2011d). Endesa has more difficultly living up to SEP norms with regard to a commitment to develop local electricity infrastructure. Though the company claims it invested $\$ 209$ million (€158 million) in 2010, early 2011 saw the company criticized by the national electricity regulator ENRE (2011) for "deficiencies in service delivery" and threatened with a cancellation of its contract if it did not increase its level of investment in the electricity infrastructure of the country.

In Peru, the vast majority of the workers at Edegel power stations investigated are not from the local communities or even districts in which the stations are located. For example, residents and local authorities in Monobamba claim that the Chimay power station employs only two workers from the district (as guards). A Monobamba official observed, "[Edegel] has been laying off its local personnel and only keeping the minimum" (Mungi 2008). Furthermore, local government officials claim Edegel has done little to improve local infrastructure in districts where its power plants are located. Edegel's recent move to transfer one of its transmission lines to Electrocentro in order to serve a small village adjacent to one of its plants is a welcome initiative, but local authorities and community residents feel that more should be done (Mungi 2008). According to the Law on Electricity Concessions, Edegel is required to pay taxes and other obligatory duties, the total of which cannot exceed $1 \%$ of its annual revenues. It also pays an amount of up to $1 \%$ of the average electricity price at generation level, calculated 
in accordance with the Law on Electricity Concessions, as a one-time compensation for the use of water resources for power generation.

\section{Ensure reliable supply}

"Service Quality" is one of Endesa's seven Commitments for Sustainable Development, and the company alleges that "adequate, secure and uninterrupted supply of electrical energy to all customers, wherever they are, must be the main objective" (Endesa 2008b). That said, Fraile (2008) acknowledges that there are differences between its performance on reliability of supply in developed countries compared to the supply in the Global South. The main difference is that the electricity infrastructure in the Global South is usually in worse condition, but Endesa also notes that people's expectations as to the quality of service are lower there than in the North.

In Argentina, Endesa's 4,522 MW of capacity generated 16,047 GWh of electricity in 2010 for a relatively low average capacity factor of $40 \%$. Endesa Dock Sud was fined by electricity regulator ENRE four times between 2003 and 2007 in relation to the technical quality of electricity generation. Specifically, the sanctions were due to the company's failure to notify (either completely or within the required timeframe) the regulator about disturbances in power supply and power cuts (ENRE 2008). The Federation of Energy Workers (FeTRA 2007) has documented how, in times of peak seasonal demand, for example, during extremely hot periods in the summer, poor areas of the city experience frequent blackouts - more frequent than in other areas of the city. Between 2005-2010, EDESUR was fined a total of $\$ 19.8$ million (€17.5 million) by Argentina's electricity regulator ENRE for causing major interruptions to the electricity supply (ENRE 2010). Endesa (2011d) has argued that the recent increase in its supply interruptions was a result of a shortage of natural gas, as well as extreme weather conditions. This led to an average annual interruption time per customer in 2010 of 11.3 hours, $26 \%$ higher than the previous year.

In Peru, Endesa's 1,801 MW of capacity generated 9,160 GWh in 2010 for an average capacity factor of $58 \%$. Endesa Peru is regularly cited by the country's electricity regulator for its failure to ensure reliable supply. In 2006 alone, Edegel experienced 43 involuntary failures or forced power cuts. Of these, 26 were due to failures in the protection system (FEC), ten due to unidentified causes, and three due to human error (COES-SINAC 2007).

\subsubsection{Cross-cutting issues}

\section{Engage in adequate stakeholder consultation} and participatory decision-making

Endesa (2008a:27) claims that constant contact with stakeholders is a priority and offers "a wide variety of communication channels to stakeholders in order to facilitate a bidirectional communication and their participation in a fluid dialogue with the company". With regard to stakeholder expectations, Endesa's strategy for engagement consists of three 
elements: identification of stakeholders, dialogue and management of expectations, and transparency throughout the process (Endesa 2007b:11). Since 2008, the company has attempted to integrate stakeholder consultation systematically throughout its activities, beginning with the EIAs carried out when developing new projects (Endesa 2011f). One strategy that Endesa uses to engage stakeholders is to work with local Global Compact offices to organize "Square Tables" in which different stakeholder groups such as CSOs, regulators, competitors, and clients can engage in open dialogue over relevant issues (Fraile 2008). Endesa does not recognize stakeholders' right to free, prior, and informed consent.

In Argentina, the measures employed by Endesa for engaging with stakeholders are much more limited than those laid out in corporate headquarters policies. Endesa's Costanera (2010a) claims that its methods for consulting workers include "surveys, dialogue with unions, and a series of dialogue sessions and participation programs for workers". Methods for consulting suppliers and contractors comprise "lectures and seminars", and the only method listed for consulting social and environmental organizations is "meetings". Dock Sud (2009) claims that communication with stakeholders is essential and has designed communication and participation channels to establish a dialogue. The company lists the primary channel of communication as its annual sustainability report, which it consults stakeholders about through a questionnaire.
In addition to the sustainability report, the company lists "specific reports" and its website as its specific channels of communication with social and environmental organizations. For employees, specific channels of communication are the internal communication board, an internal newsletter, and the website.

However, a long-running conflict with a poor community near Buenos Aires reveals that Endesa has not always been willing or able to implement its stakeholder engagement policy in practice. In early 1999, Endesa decided to construct several transmission towers with high-voltage power lines to transmit electricity produced by a new generator at Dock Sud. Without consulting residents who would be affected, Endesa planned the cabling route to run directly through the densely-populated Villa Inflamable neighborhoods (Alarcón et al. 1999). Residents were concerned about the potential health effects of large electro-magnetic fields that these towers would create, and there were numerous protests calling on the company to change the cabling route. Argentina's electricity regulator ENRE granted the authorizations relevant for the project's implementation, but the company's engagement and communication with the nearby communities regarding the environmental and health effects of the power lines was heavily criticized (Residents of Villa Inflamable 2008).

Despite the community's protests and filing of a lawsuit, Endesa went ahead with the construction of the high-tension towers and the route it had planned, claiming it had been 
granted authorization by the relevant public authorities and was complying with relevant regulations. When the construction of the power lines was finished, the only seemingly possible option to solve the difficulties experienced by Villa Inflamable's residents was limited to resettling the families (MatanzaRiachuelo Basin Authority 2011). The residents felt like their rights and interests had been sacrificed without properly or thoroughly consulting them simply because the project was deemed "essential" for supplying Endesa's industrial and commercial customers with more electricity (Tinnirello 2006). In total, 25 families were involuntarily relocated. At first, the relocated families were not even consulted about the relocation destination or the types of dwellings they would be offered. This was only done after a CSO, the Civilian Association for Equality and Justice, formally requested that the residents should be involved in their own resettlement process (FARN 2011b).

In Peru, a local Endesa manager explains that the company engages with communities impacted by its operations by "introducing them to improved management tools that can build their capacity to generate their own vision of development and plan a strategy for long-term sustainable development, in which they have adequate education, good basic infrastructure, and dignified and productive jobs" (Prieto 2008). However, these good intentions contrast sharply with claims by local municipal authorities and community residents that Edegel does not seek out meaningful and participatory relationships with the towns and communities adjacent to its generation facilities (Mungi 2008). The company's initiatives to engage stakeholders are perceived by locals as being more focused on philanthropic activities and gift-giving in the communities than sustained engagement with communities and governments on local planning and development issues. Residents of a community adjacent to the Ventanilla power station claim that there is no engagement with their community other than a Christmas present offered by the company to the community's children (Ventanilla Resident 1 2008, Ventanilla Resident 2 2008). Edegel financially supports schools in the Ventanilla and Monobamba regions and promotes the development of productive activities, such as fish farming. However, communities in these areas lack access to basic levels of electric service, and local authorities complain that they are not consulted on the company's plans or decisions, nor is there any community participation in decision-making processes to determine their real needs. One official from the town of Monobamba noted that Edegel's power plants are "closed and fortified spaces [that] are highly differentiated from their surroundings and contrast with the nearest villages and communities" (Mungi 2008).

In terms of engagement with researchers for the present study, a manager at Endesa headquarters in Madrid engaged constructively and provided much information. In Peru, local researchers were able to interview one Endesa manager, but Eskom management in Argentina declined to engage with researchers. 
Assume full value chain responsibility

Endesa embraces what it calls a "trend" in

$\mathrm{CR}$ for corporations to extend their social responsibilities to suppliers and contractors. In order to do so, Endesa employs different strategies to help suppliers and contractors to incorporate CR issues into their management. For example, Endesa's CEO has written a letter to all major suppliers and contractors informing them about the UN Global Compact and encouraging them to sign up to its principles. In addition, Endesa includes CR criteria in contracts with suppliers and contractors and monitors business partners in countries and on issues where there is an elevated risk of non-compliance (Endesa 2008a:68). According to Endesa (2008a:90), no contract has ever been terminated as a result of this monitoring. Due to the importance of $\mathrm{OHS}$ throughout the electricity supply chain, Endesa has implemented a policy of providing all subsidiaries with country-specific OHS operating guidelines to be distributed to all contractors. Endesa has a number of initiatives aimed at guaranteeing the extension of the company's standards to its contractors such as prizes for best practices and communicating Endesa standards directly to the employees of contractors. Endesa's commitment to assuming full value chain responsibility is also evidenced by the fact that it is one of the few electric utilities that reports on $\mathrm{CO}_{2}$ emissions in its supply chain (mainly related to the transport of fuel) (Endesa 2011a).

Endesa has also undertaken a number of initiatives in Argentina and Peru demonstrating commitment to improving conditions among business partners. In Argentina, the company's "General Conditions" for suppliers outlines that contractors applying for contracts will be evaluated on labor, safety, environmental, and human rights aspects, as well as on legal compliance, quality and price (Endesa Dock Sud 2006). Endesa identified business partners involved in high-risk activities, and as of 2010, had audited 85 such business partners to ensure that the company's standards were being observed (Endesa 2011d). Endesa has also sought OHSAS 18001 certification from several of its main contractors (Endesa Costanera 2010a, EDESUR 2011). Despite these measures, the Federation of Energy Workers (FeTRA 2007) attests that contracted workers, who are not unionized and not covered by a collective bargaining agreement, enjoy less favorable working conditions when it comes to wages, working hours, and OHS. The majority of recent work-related accidents among electricity employees in Argentina and Peru have occurred among contracted employees (Endesa Dock Sud 2009).

In Peru, Edegel claims that its contractors are required to comply with all applicable environmental and occupational health and safety legislation. In 2007, the company announced that it was extending the principles of the SA 8000 standard as a requirement for its contractors and suppliers in order to ensure their compliance with legally established labor conditions. The company also reported that the quality management and OHS management systems of the companies it works with are certified under ISO 9001 and OHSAS 18001 (Endesa Edegel 2008). 
Maximize transparency and provision

\section{of information}

Endesa (2011f) claims that "Transparent behaviour is Endesa's most valuable asset in a constantly changing environment". The company provides a high degree of accessibility and visibility of $C R$ policies through various channels including its website, annual sustainability reports, and specific policy documents. The company's annual sustainability reports include a significant amount of information about the company's host country operations. The most recent sustainability report follows the new GRI G3.1 guidelines and the "Electric Utilities Sector Supplement". The report was awarded an A+ rating by GRI and was independently verified by an external entity. The report also includes all the principles of the AA1000 standard (AccountAbility 2008) and the UN Global Compact (Endesa 2011d). The company does not provide detailed information about its business relationships, as encouraged by SEP norms.

In Argentina, Endesa claims that providing information to stakeholders is essential and notes that its primary channel for providing information to stakeholders is its annual sustainability reports, which are in line with GRI standards (Endesa Dock Sud 2009). However, as of January 2012, the most recent sustainability reports for two of Endesa Argentina's most important electricity generation facilities, Dock Sud and El Chocón, were out of date by at least two years. Dock Sud's latest available report is from 2008, El Chocón's from 2009. In addition to the sustainability report, the company lists "specific reports" and its website as its specific channels of communication with social and environmental organizations. For employees, specific channels of communication are the internal communication board, an internal newsletter, and the website. In Peru, Edegel produces an annual sustainability report according to the GRI reporting guidelines (Endesa Edegel 2008). This is complemented by a high level of transparency regarding the technical aspects of its generation operations, with much information available on its website. In both Argentina and Peru, all CR policies and reports are translated into Spanish. This is logical given that the corporate headquarters' language is also Spanish.

\subsubsection{The European mode and Endesa's performance on the SEP benchmarks}

Endesa's performance on the SEP benchmarks indicates that the European mode of business culture conditions how Endesa takes up the internationally-derived standards for SEP and, to a somewhat lesser degree, how it applies them in practice. For example, Endesa's frequent reference to international standards for CR in its CR policies is largely reflective of the European mode's tendency to rely heavily on (voluntary) normative standards for CR. In addition, Endesa's policies and management procedures at the headquarters level exhibit a clear focus on core-business activities as is encouraged by SEP norms. This is as to be expected from a company originating from the European mode. Table 6.5 provides an overview 
of the degree to which Endesa's performance on SEP benchmarks is deemed to reflect the European mode as described in Chapter 4.

Interestingly, there are several areas where Endesa's performance is deemed to be out of line with expectations based on the European mode. For example, despite the fact that environmental issues such as climate change, biodiversity, pollution, and natural resource use are generally given high priority in Europe, Endesa operates and continues to invest in primarily fossil fuel-based electricity generation facilities.

In general, the European mode appears to play a stronger "conditioning" role when it comes to Endesa's SEP-related policies than with regard to its practice in Argentina and Peru. For example, despite having a clear focus on core-business activities in policies at the headquarters level, as is to be expected from a company originating from the European mode, Endesa's practice in Argentina reveals a definite tendency toward philanthropic and charitable projects. A similar trend is revealed by Endesa's performance on the stakeholder engagement benchmark. Despite having policies aimed at engaging stakeholders in the European tradition, Endesa's initiatives in Argentina and Peru are perceived by local stakeholders as being more focused on philanthropic activities and gift-giving in the communities than sustained engagement with communities and governments on local planning and development issues.

Table 6.5: Degree to which Endesa's performance on SEP benchmarks reflects the European mode of business culture

\begin{tabular}{|c|c|c|}
\hline $\begin{array}{l}\text { Is Endesa's policy/practice reflective of the European mode on the } \\
\text { following SEP benchmarks? }\end{array}$ & Policy & Practice \\
\hline Endorse international normative standards for CR & Largely reflective & Moderately reflective \\
\hline $\begin{array}{l}\text { Adopt a commitment to CR in core-business activities and } \\
\text { decision-making }\end{array}$ & Moderately reflective & Moderately unreflective \\
\hline Increase access to affordable electricity & Moderately unreflective & Largely reflective \\
\hline Respect labor rights & Moderately reflective & Largely reflective \\
\hline $\begin{array}{l}\text { Minimize environmental impact, including contribution to climate } \\
\text { change }\end{array}$ & Moderately reflective & Moderately unreflective \\
\hline Prioritize renewable sources of energy for electricity & Moderately unreflective & Moderately unreflective \\
\hline Contribute to local economic development & $\mathrm{n} / \mathrm{a}$ & $\mathrm{n} / \mathrm{a}$ \\
\hline Ensure reliable supply & Moderately reflective & Moderately unreflective \\
\hline $\begin{array}{l}\text { Engage in meaningful stakeholder consultation and participatory } \\
\text { decision-making }\end{array}$ & Moderately reflective & Moderately unreflective \\
\hline $\begin{array}{l}\text { Assume responsibility for impacts throughout all business } \\
\text { relationships }\end{array}$ & Moderately reflective & Largely reflective \\
\hline Maximize transparency and provision of information & Moderately reflective & Largely reflective \\
\hline
\end{tabular}




\section{Chapter 7}

Case study - SN Power
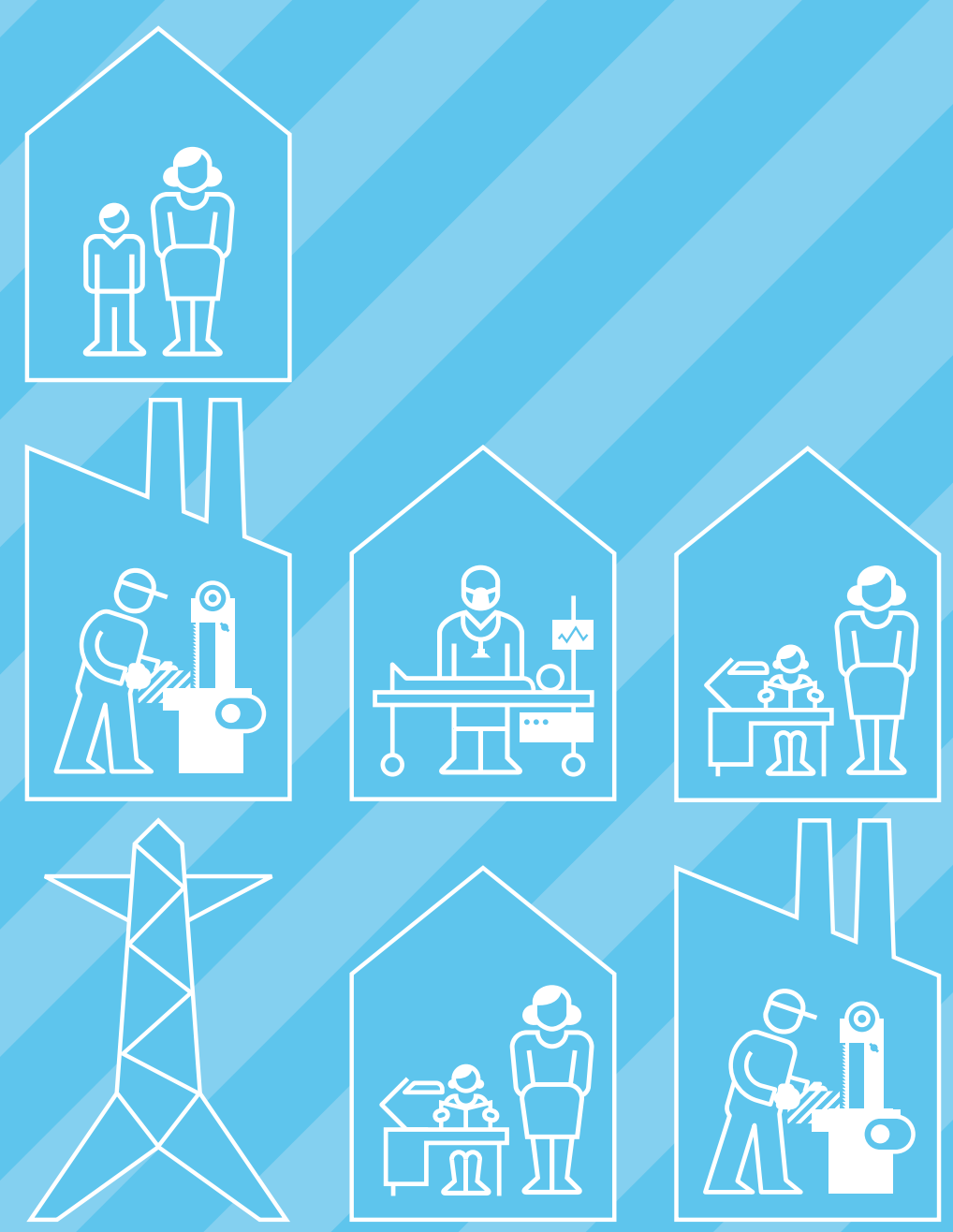


\subsection{SN Power background and operations}

\subsubsection{Basic company information}

SN Power, headquartered in Oslo, Norway, was established in 2002 as a 60/40 joint venture between Statkraft and Norfund. The Statkraft Group is a Norwegian public utility company that specialises in hydro and wind power. It operates 164 hydropower plants in Scandinavia, three wind farms, and three natural gas plants in Norway and Germany. Norfund (2008) is a Norwegian governmentfunded "risk capital" investment agency that "facilitates economic growth and poverty reduction by investing risk capital in profitable businesses in the Global South". It invests by providing equity, quasi-equities, and loans. Its investment in SN Power is in the form of equity. Its other investments include direct investments in hotels, phone companies, fish and food processors, and other companies. It is also involved in a number of investment funds, fund management operations, and loans portfolios.

As a joint venture between Statkraft and Norfund, SN Power is a $100 \%$ public entity, but it operates as a fully commercial enterprise. Although SN Power was created as a profitmaking enterprise, the company was also founded with the aim of making a positive contribution to sustainable development. It is for this reason that the company operates exclusively in countries without universal access to electricity and works solely with renewable energy technologies (Kopstad 2008). In 2010, SN Power operated 1,481 MW of installed (primarily hydroelectric) electricity generation capacity, had an annual output of 5,954 GWh, employed 427 people (including subsidiaries), and generated US \$114 (€87) million in revenue. In terms of fuel mix, 95\% of SN Power's electricity generation capacity is based on large hydro, $3 \%$ on wind and $2 \%$ on small hydro (SN Power 2012a).

\subsubsection{Operations and investments in the Global South}

SN Power specializes in hydropower generation activities in the Global South. The company's current generation capacity is exclusively hydro-based, although it is developing one wind power project in Chile. The company currently owns and operates 16 hydropower plants, with five more in various stages of planning and construction. SN Power is present primarily in Asia and Latin America. Peru hosts the largest number of SN Power's activities, with eight hydropower plants with a combined generating capacity of nearly $300 \mathrm{MW}$. The company has also been seeking to enter the African market, but two of its initial ventures into Uganda and Mozambique were abandoned in 2008 due to profitability concerns (Among 2007). Nevertheless, SN Power is making a new push to enter the African market with its investment in the Lunsemfwa and Mulungushi projects in Zambia (SN Power 2012b). Figure 7.1 illustrates SN Power's global presence for electricity generation operations in 2012. 
SN Power has two major subsidiaries in Peru, Cahua S.A. and ElectroAndes S.A., both of which are involved in the generation of electricity from primarily small-scale hydroelectric facilities. SN Power acquired Cahua S.A. from
American NRG Energy in November 2006 and bought ElectroAndes S.A. from the US group PSEG Global for US $\$ 390$ million (€296 million) in September 2007. In 2008, these two subsidiaries were combined to form SN Power

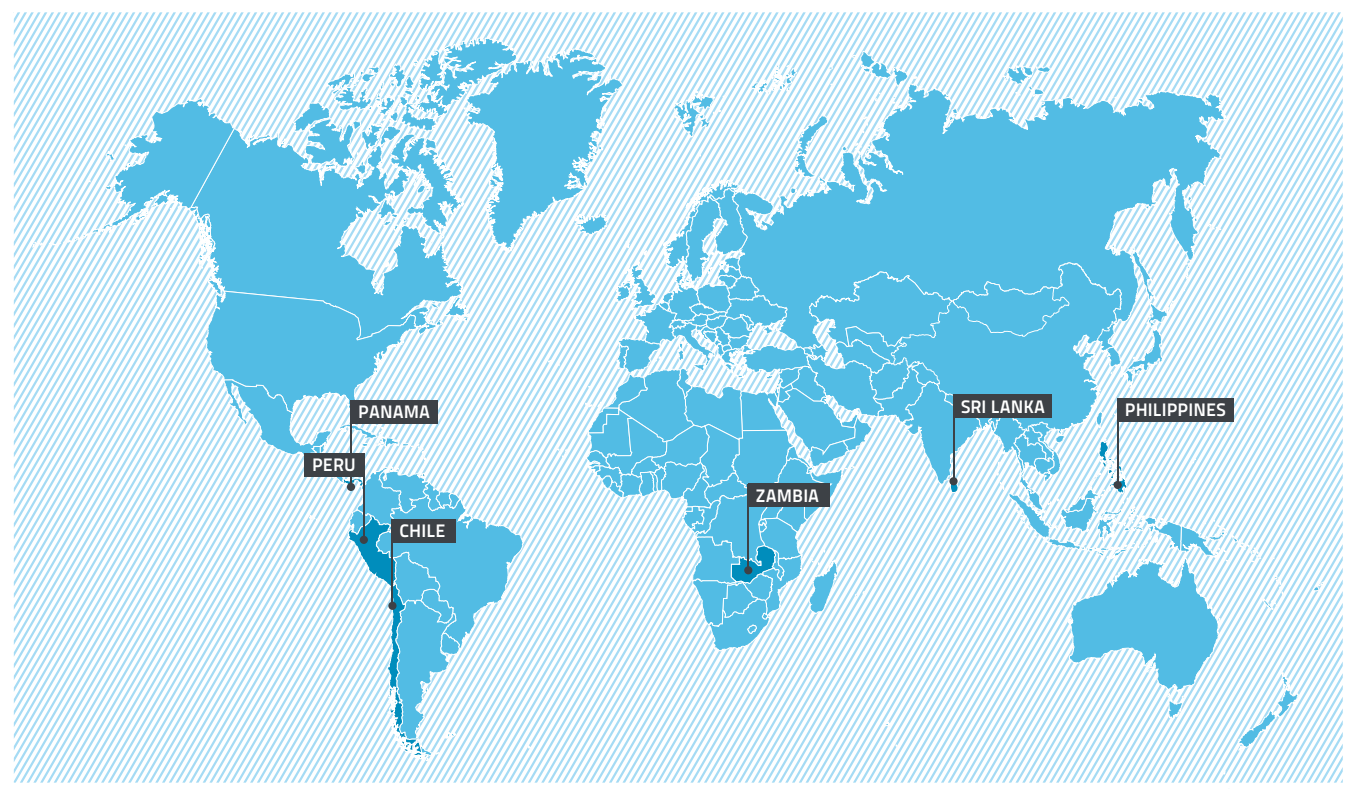

Figure 7.1: SN Power's global presence, 2012

Table 7.1: SN Power's electricity generation units in Peru, 2012

\begin{tabular}{|l|l|l|l|l|l|}
\hline Subsidiary & Project & Capacity (MW) & $\begin{array}{l}\text { Mean annual } \\
\text { output (GWh) }\end{array}$ & Fuel \\
\hline Cahua S.A. & Arcata & Cayarani & 5.4 & 37 & Hydro \\
\hline & Cahua & Manás & 43 & 280 & Hydro \\
\hline & Pariac & Huaraz & 4.9 & 24 & Hydro \\
\hline & Gallito Ciego & Yonan & 37 & 150 & Hydro \\
\hline & Pacasmayo & Pacasmayo & $n / a$ & 0 & Thermal \\
\hline & Malpaso & Paccha & 54.5 & 207 & Hydro \\
\hline & Pachachaca & La Oroya & 9 & 45 & Hydro \\
\hline & La Oroya & La Oroya & 9 & 65 & Hydro \\
\hline & Yaupi & Ulcumayo & 108 & 789 & Hydro \\
\hline
\end{tabular}

Based on: SN Power 2012b 
Peru (SNPP). Together, the companies have a total of $270.8 \mathrm{MW}$ of installed capacity and a mean annual output of $1,597 \mathrm{GWh}$ of electricity. In June 2007, SN Power withdrew the thermal station C.T. de Pacasmayo from the national interconnected system (from commercial operations), and the station is currently no longer generating any electricity. Table 7.1 reveals all of SN Power's installed capacity for electricity generation in Peru.

\subsection{SN Power's performance on the SEP benchmarks}

This section begins with an overview (in table format) of SN Power's performance, in both policy and practice, on the 11 SEP benchmarks. For each SEP benchmark, a reminder of the guiding international normative standards (taken from Chapter 3 ) is provided alongside key indicators of the company's performance and an evaluation of the degree to which the company's policy and practice are in line with the SEP standards on that issue. Following the overview, the results of the empirical research on SN Power's policy and practice in Peru are detailed for each SEP benchmark. These findings form the basis for the overview and evaluation provided in Table 7.2. The chapter concludes with a brief reflection on the relationship between the Nordic mode of home-country business culture (as outlined in Chapter 4) and SN Power's performance on the SEP benchmarks. 
Table 7.2: Summary of SN Power's performance on SEP benchmarks in policy and in practice

\begin{tabular}{l} 
SEP benchmark \\
General approach to corporate responsibility \\
\hline Endorse international normative standards for CR \\
\hline
\end{tabular}

Adopt a commitment to CR in core-business activities and decisionmaking

\section{Guiding international normative SEP standards}

Electric utilities should seek to bring their policies in line with internationally recognized and verified standards for CR (ILO 2001, WBCSD 2002, IFC 2011, ISO 2011, OECD 2011، UNGC 2008, UNHRC 2011).

Electricity companies should adopt a holistic and integrated view of the role and impacts of electricity provision, considering and balancing the essential elements of economic development, environmental quality, and social equity in utility operations. This entails a policy commitment to CR throughout all core-business activities, as opposed to a primary focus on philanthropic activities (WBCSD 2002, MVO Platform 2012). Electric utilities should seek to introduce environmental and social factors and procedures into high-level corporate planning and decision-making and should aim to have a board-level position responsible for CR issues (WBCSD 2002).

\section{Social issues}

Increase access to affordable electricity
Electric utilities should develop policies that promote access to electricity priced at affordable levels for all, paying special attention to disadvantaged communities (ILO/Palast et al. 2000, WBCSD 2002, PSI/Pillinger 2009). In order to do so, companies should undertake initiatives to extend electricity services to unserved and underserved communities, particularly in rural or remote areas, but also to the poor in urban and peri-urban areas (WBCSD 2002, UNDP/Modi et al. 2005). 


\section{Indicators of SN Power's policy}

- Endorses five sets of SEP-relevant normative standards: UNGC, ILO Core Conventions, IFC Performance Standards, IHA Sustainability Guidelines, UN Human Rights Declaration

- Deeply-seated and traditionally Norwegian values such as "long-term thinking" and a "renewable energy tradition" provide a "competitive advantage"

- No mention of standardized procedures and measurements such as ISO or OHSAS at the corporate headquarters level, but many individual subsidiaries do have certifications

- Moderately in line with SEP standards

" CR policy takes a holistic approach and largely focuses on core-business activities

- Highest level of direct responsibility for CR is the board level Executive VP for social and environmental programs

" CR managers in every country in which the company operates have direct contact with CR director, who is in the executive management team

- CR director mandated to spend a significant portion of time on the ground at operations

- Largely in line with SEP standards

\section{Indicators of SN Power's practice in Peru}

- No specific reference to international standards, but does say company adheres to same corporate principles everywhere

- Many generation units have ISO 9001 and 14001 and OHSAS 18001 certification

- Moderately in line with SEP standards

- Aside from core focus on development of renewable energy, CR programs are largely philanthropic activities

- Only 1 of 7 CR activities focus on core business (a rura electrification project)

- Moderately in line with SEP standards
- Realizes that "access to electricity is a right for all communities", but is not specific on increasing access or ensuring affordability

" Has developed a small number of small-scale, off-grid projects to improve rural electrification

- $100 \%$ of company's total 1,481 MW of electricity generation capacity is located in countries without universal electrification; amount of electricity generation capacity very limited compared to other companies

- Moderately in line with SEP standards
- Produces 1,597 GWh of electricity per year

- Many plants located in rural districts with low electrification rates, but majority of electricity generated is sold to industrial (primarily mining) operations in the region or transported to distant population centers

- Electrification rate in the area of the Cahua plant is 53\%; rate in ElectroAndes area of operation also approximately $50 \%$

- No information could be gathered on electricity prices in local areas

- Policy on increasing access is to map rural clients in area of operation to determine energy needs; has helped one community organize a communal distribution company in order to access local energy grid

- Has one rural electrification project

- Local residents satisfied with programs to make electricity affordable for communities (supplies free and reduced electricity to some communities)

- Moderately in line with SEP standards 


\section{Respect labor rights}

\section{Environmental issues}

Minimize environmental impact, including contribution to climate change
Electric utilities should respect workers' full rights and provide workers with fair pay, job security, and decent working conditions, including high levels of health and safety standards in all aspects of operations (WBCSD 2002, PSI/Pillinger 2009, OECD 2011). Furthermore, electric utilities should uphold freedom of association and the right to collective bargaining and pay particular attention to the right to strike (ILO 1998, ILO 2001, UNGC 2008, OECD 2011). Companies should ensure that these rights are respected not only among their own employees, but also among suppliers, contractors, and other business relationships (ISO 2011, OECD 2011, UNHRC 2011).

\section{Guiding international normative SEP standards}

Electric utilities should seek to minimize emissions and discharges to air and water and continually develop and implement low-pollution and low-environmental impact technologies (WBCSD 2002, UNGC 2008, PSI/Pillinger 2009, OECD 2011). Companies should manage their environmental impact in line with internationally recognized and verified standards, such as the ISO 14000 series and should always undertake rigorous and verifiable environmental impact assessments (WCD 2000, WBCSD 2002, IHA 2004, OECD 2011,). Electric utilities should minimize their contribution to climate change and develop strategies for reducing GHG emissions (WBCSD 2002, OECD 2011). As a matter of priority, electric utilities should seek to avoid impacts on biodiversity and ecosystem services, refraining from any activities in areas of critical habitat with high biodiversity value while at the same time initiating or supporting conservation and biodiversity efforts related to impacts on natural habitats from utility operations (WBCSD 2002, IHA 2004, IFC 2011).

Electric utilities should evaluate the renewable energy potential of the host country and prioritize development and exploitation of renewable options over fossil fuel-based technologies (WBCSD 2002, UN/IAEA 2007, PSI/Pillinger 2009). Electric utilities should also prioritize renewables in their research and development programs and should support the diffusion of renewable energy technologies into the local economy (WBCSD 2002, UNGC 2008). 
- Endorses ILO Core Conventions on child labor but not other core conventions

- No public policy on minimum wage, freedom of association, or working hours

- Does the company have policies and structures in place to engage \& consult workers on key operational decisions?

- OHS is a focus of labor policies; aims for zero accident and injury rate in all projects in all phases

- With regard to labor rights among business partners, an "important aspect of the company's project management system" is "the use of qualified contractors with proven records on HSE"

- Moderately in line with SEP standards
- ElectroAndes has a union, but Cahua does not; ElectroAndes union covers some contract workers

- ElectroAndes union indicates that company management respects union rights

- No indication as to whether a CBA is in place

- No strikes in recent years

- No information available on wages

- Cahua has OHSAS 18001:2000 certification and established a Safety Committee with workers and management

- Low accident rate among employees, but two deaths among ElectroAndes contractors in 2007

- Outsourcing and job security is a constant source of worry and concern for workers

- Some contract workers covered by ElectroAndes union, but others experience poor working and OHS conditions, must supply their own safety equipment

- Moderately in line with SEP standards

\section{Indicators of SN Power's practice in Peru}

- Company-wide EMS is not ISO-certified, but based on "international best practices" and Norwegian competence in hydropower

- Has a policy to always conduct ElAs, even when not required by law

- Exclusive focus on hydro and wind means minimal $\mathrm{CO}_{2}$ emissions, but no publicly available policy or methodology to determine GHG emissions from hydroelectric reservoirs

- Mainstay of climate strategy is developing renewable energy projects and actively seeking CDM certification to generate revenue

- No publicly available policy on protecting biodiversity

- Average capacity of company's 17 hydroelectric facilities is $85 \mathrm{MW}$, and the largest is the $381 \mathrm{MW}$ Magat plant in the Philippines

- Does have several small-scale hydro facilities in Peru and Sri Lanka, but build program contains only large-scale hydro, up to $880 \mathrm{MW}$ in Nepal

- Moderately in line with SEP standards

" Generated 5,954 GWh in 2012, 95\% large hydro, 3\% from small hydro, and $2 \%$ from wind

" Generated 1,599 GWh in 2012, 89\% large hydro and 11\% smal hydro

- Currently constructing one $168 \mathrm{MW}$ large hydro plant

- Not promoting other, more sustainable, forms of renewable electricity generation

" Largely in line with SEP standards 


\section{Economic issues}

\section{Contribute to local economic development}

\section{Ensure reliable supply}

\section{Cross-cutting issues}

Engage in meaningful stakeholder consultation and participatory decision-making

\section{Guiding international normative SEP standards}

Electric utilities prioritizing the development of sustainable local energy-related infrastructure and employing local workers to the greatest extent practicable and providing those workers with sustainable jobs and training to improve skill levels (WBCSD 2002, PSI/Pillinger 2009, OECD 2011). In order to maximize local economic development and job creation, electric utilities should support local small and medium-sized enterprises in their procurement practices and contribute to the long-term development prospects of the host country through the transfer and rapid diffusion of technologies, skills and knowledge (WBCSD 2002, OECD 2011). Fair payment of due taxes to local, regional and national governments in the host country is a crucial element of contributing to local economic development (OECD 2011, MVO Platform 2012).

Electric utilities should ensure reliable supply by minimizing generation interruptions, maintaining contingency plans, responding to interruptions in a timely manner, and providing timely information to the public about planned and unplanned interruptions (WBCSD 2002, CEER 2005).
Electric utilities should adopt a participatory approach to the planning/designing, developing, operating, and monitoring/ evaluation of electricity projects and consult and engage stakeholders throughout the entire process in order to provide meaningful opportunities for their views to be taken into account in decision- making (WCD 2000, WBCSD 2002, IFC 2007, UNGC 2008, PSI/Pillinger 2009, OECD 2011). The consultation process should be tailored to local decisionmaking processes and involve the timely provision of all relevant information about activities, impacts, and potential impacts translated into local languages (WBCSD 2002, ISO 2011). The process should take into account the needs of disadvantaged or vulnerable groups, and if Indigenous Peoples are affected by a potential project, electric utilities must gain the free, prior and informed consent of those peoples before proceeding with any part of the project cycle (IFC 2011). 


\section{Indicators of SN Power's policy}

" Aims to "contribute to sustainable economic growth"

- Claims it contributes to local economic development through job creation for local residents, contracts to local suppliers and service providers, tax generation, and active engagement in knowledge and skills transfer to host communities.

- Has a policy to prioritize local workers

- Has a policy to prioritize SME suppliers in host countries

- Largely in line with SEP standards

\section{Indicators of SN Power's practice in Peru}

- Cahua directly employs 30 people, the majority of whom are Peruvian

- No information could be gathered about prioritization of SME suppliers

- No information on total amount of investment, but currently investing \$400 (€303) million in $168 \mathrm{MW}$ Chaves plant

- No information could be gathered on the amount or rate of taxes paid

- Largely in line with SEP standards
- No public policy on ensuring reliability of supply, responding to interruptions in a timely manner, or providing timely information to the public about planned and unplanned interruptions

- Moderately out of line with SEP standards
- Average capacity factor is $68 \%$

- 88 involuntary or forced power cuts in 2006

" Service deemed "regular" by customers and regulators

- Interruptions resolved within a short time span, but little information provided to customers as to causes

- No indication of fines by regulators for inadequacies related to reliability of supply

- Moderately in line with SEP standards
- Aims to "reflect the priorities and concerns of local communities in decision-making processes and to minimize potential negative effects through a combination of careful planning, design adjustments and operational improvements."

- Conducts an SIA and organizes open meetings to inform stakeholders about plans

- Does not reference international standards on stakeholder engagement

- No endorsement or reference to FPIC

- SN Power CR managers were willing to engage with the author in research related to the present study

- Moderately in line with SEP standards
- Has mapped stakeholders and their needs, then sought to engage them to become "key actors in their own sustainable development"

- Some local residents praised engagement practices, others characterized engagement practices as charity and gift-giving

- Local management declined to engage with field informants in their research related to the present study

- Moderately in line with SEP standards 


\section{Cross-cutting issues}

Assume responsibility for impacts throughout all business relationships
Guiding international normative SEP standards

Electric utilities should seek to prevent, avoid and mitigate negative impacts throughout their entire range of business relationships, including suppliers and contractors (ISO 2011, OECD 2011, UNHRC 2011). Companies should seek to leverage their buying power to improve social and environmental conditions among business relations, and monitor performance using third party audits (WBCSD 2002)

Electric utilities should apply a high degree of transparency in operations, including reporting on activities and progress, measurement, business partners and relationships, and interactions with government and the public (UNECE 1998, WBCSD 2002, UNGC 2008, OECD 2011). Electric utilities should regularly report on their activities, progress, and performance against "the triple bottom line" using internationally accepted sustainability reporting guidelines such as the GRI's G3 guidelines and the Electric Utilities Sector Supplement (GRI 2008), have an external party verify the data, and disseminate the report in an appropriate manner (WBCSD 2002, OECD 2011).

\subsubsection{General approach to corporate responsibility}

\section{Endorsement of international normative standards for corporate responsibility}

SN Power believes that normative standards for CR take on added importance for TNCs operating in the Global South. According to Kopstad (2008), because regulatory frameworks are often weaker in the Global South, companies need to play a greater role in ensuring that standards for the protection of human rights and the environment are met. In these situations, companies must have clear ethical standards in both policy and practice. In general, however, internationally recognized normative standards for $\mathrm{CR}$ do not receive a prominent place in SN Power's CR materials. The only standard to be found in the CR section of the company's website is a mention of the company's membership in the UN Global Compact. The company does not mention standardized management certifications such as ISO and OHSAS at the company-wide level, but many of its subsidiaries and power plants in the Global South do have such certifications.

Though not prominently referenced in SN Power's CR materials, international CR norms are integrated into the company's core CR principles. SN Power's (2007a, 2007c) "Business Principles" are based on five sets of international standards for CR:

- UN Universal Declaration on Human Rights

- UN Global Compact (SN Power is a member 


\section{Indicators of SN Power's policy}

" Recognizes responsibility for impacts of business partners

- Seeks to select business partners on social and environmental criteria, but admits price and quality remain key

- Includes sustainability and safety clauses in contracts with business partners

- Monitors performance through internal audits; does not engage external parties for audits but may consider doing so in future

- Largely in line with SEP standards

- Aims for "open reporting, clearly detailing our efforts across all our areas of business operation"

- CR issues are prominent on website but actual CR policy documents are limited

- Operations in the Global South figure prominently in annual report

- Does not report according to the GRI, though majority owner Statkraft does ( $\mathrm{B}+$ rating)

- Does not provide transparency about its business relationships

- Moderately in line with SEP standards
Indicators of SN Power's practice in Peru

- No information could be gathered on this issue
Primary channels of communication used to communicate with stakeholders include website, stakeholder mapping, SIA, information meetings

As of September 2008, no SIAs were available online

Spanish language website contains many, but not all, CR documents

- Some customers indicated they had received no information on the causes of supply interruptions

Moderately in line with SEP standards

\section{of the UNGC)}

- ILO Core Conventions on abolition of child labor (Conventions 138 and 182)

- IHA Sustainability Guidelines

- IFC Policy and Performance Standards on Social and Environmental Sustainability.

\section{SN Power's Lauritzsen (2011) contends that} the IFC standards are the most influential for the company, given that compliance with them is key for securing international financing for projects. However, SN Power (2012a) claims that more deeply-seated and traditionally Norwegian values such as "long-term thinking" and a "renewable energy tradition" provide SN Power with its "competitive advantage". For example, SN Power (2012c) emphasizes how its environmental management system is based on Norway's traditional "expertise and competence in developing hydropower". Kopstad (2008) maintains that commitments to $\mathrm{CR}$ and sustainability are "deeply ingrained in SN Power's business culture".

In Peru, SNPP (2011) makes no reference to international normative standards on its website. The company does note that "All SN Power operations and projects around the world adhere to the same corporate business principles". The principles are listed, but they do not make reference to international standards. Many of the company's generation units in Peru do have ISO 9001 and 14001 as well as OHSAS 18001 certification. 
Adopt a commitment to CR in core-business

\section{activities and decision-making}

SN Power (2007c: 2) claims that it is "committed to social and environmental sustainability throughout [its] business" and that sustainable development is one of its top priorities. The company's CEO has stated that the standards and principles it adheres to "are embedded into [the company's] business model as [it] expand[s] in a socially and environmentally responsible manner" ( $\varnothing$. Andresen quoted in SN Power 2008e).

According to Kopstad (2008), the standards are incorporated into the company's project management system from the idea phase through planning and project operation. She notes that the company seeks to make health, social and environmental considerations an integral part of project planning, operation and reporting by giving importance to these issues throughout the company's management structure, from the field in host countries all the way up to top management at the headquarters in Oslo. CR officers in the field communicate with the CR managers at the headquarters through regular dialogue and meetings. In turn, these CR managers have regular contact, both formal and informal, with top management, and quality issues are incorporated into top management decisions through a board member who is responsible for CR issues and by having the head of the CR department on the company's management team (SN Power 2008e). In mid-2008, the company created a new position entitled Director of Social and Environmental Programmes to work on CR issues. The new director spends a significant portion of his/her time in the field following up on specific projects and issues, and reports to the executive vice-president for CR.

While SN Power claims that it "implement[s] programs alongside all projects to limit the negative impact on local communities and environments", the company's articulation of its values and standards remains rather general and lacks specific indicators and monitoring protocols to measure the degree of implementation of its values in its operations in the Global South (SN Power 2008a). Kopstad (2008) acknowledges that, since the company is so young, the implementation of its values and standards may not yet be fully systematic in all of its projects, but claims to be learning and improving with each new project.

In Peru, Kopstad (2008) claims that SNPPs CR activities are based on the following values, which cover integrated social, environmental and economic issues and interests:

" The generation of clean energy

- Permanent care for the environment and worker safety

- Fluent communication and mutual collaboration with communities in the areas where it operates

- Implementation of CSR policies through the activities that are in the interest of the community and aimed at promoting their sustainable development

- Continuous staff capacity-building.

SN Power operates relatively small hydroelectric generation projects in Peru, giving its core 
business an unmistakable environmentally sustainable tint. An analysis of the social and economic CR activities of SN Power's subsidiaries in Peru also reveals a distinct philanthropic tendency. For example, ElectroAndes (2008) mentions the following CR activities: school campaigns, building infrastructure for risk prevention and social support through donations to institutions. The company also undertakes Christmas campaigns in remote communities, as well as health campaigns, and collaborates with United Way International in supporting social investment projects. In SN Power Peru's (2011) annual CR folder outlining its 2010 CR activities, only one of the seven social projects mentioned had to do with the company's core business of electricity generation (a rural electrification project in Junín Province).

\subsubsection{Social issues}

\section{Increase access to affordable electricity} Kopstad (2008) notes that SN Power was founded with the aim of making a positive contribution to development, which is why its operations are exclusively in the Global South. The company also states that it recognizes that "power generation is a prerequisite for development, particularly in areas where it is unavailable or in acute shortage" (SN Power 2011b). $100 \%$ of the company's total 1,481 MW of electricity generation capacity is located in countries without universal electrification. That said, there is no explicit mention of the issue of increasing access to affordable electricity in the company's CR policies, and only one of the four examples of "successes" that SN Power (2011b) gives on its "Community development" webpage has to do with a (rural) electrification project (in Nepal).

SN Power's decision to withdraw plans to invest in Uganda provides an example of the difficult trade-offs the company has to make when it comes to balancing profitability and commercial viability with its commitment to increasing access to electricity and poverty eradication. In December 2004, SN Power announced that it would make Uganda its entry point for Africa in the energy sector. Despite an abundance of the natural resources necessary for power generation, including ten potential hydropower sites along the Nile alone, Uganda cannot meet its domestic energy needs. Currently, only about $9 \%$ of Uganda's population has access to electricity, and, while power demand is growing at an annual rate of $9 \%$, the growth in supply is $0 \%$.

The Ugandan government had hoped that SN Power's entry into the country would help to improve Uganda's dismal electricity situation by providing power to an additional $15 \%$ of the population. However, in 2006 SN Power decided to withdraw from the Ugandan projects because it deemed them financially unviable. According to Kopstad (2008), the projects "did not fit [SN Power's] corporate strategy and the commercial viability was not strong enough". SN Power (2007b) sold its rights to Norwegian power company Troenderenergi, which agreed to develop the sites. Nevertheless, the transfer of rights and related delay mean that residents 
of western Uganda continue to endure irregular or no power supplies beyond the originallyplanned 24-month construction period.

Kopstad (2008) responded to the situation saying that SN Power's construction as a for-profit commercial enterprise requires it to make a return on investment for its investors and that the company conducts due diligence studies on all potential projects to ensure that they will meet this requirement before making the decision to invest. She added that, although SN Power decided not to invest in the project, it did make considerable efforts to facilitate the transfer of the project to another company so that the project would eventually go ahead.

\section{SN Power Peru claims that the company}

"realizes that the access to electricity is a right for all communities" (Kopstad 2008). In 2012, the company had a total of $271 \mathrm{MW}$ of installed electricity generation capacity in Peru, and generated a total of 1,597 GWh of electricity. Though many of SN Power's plants are located in rural districts with a low electrification rate, the majority of the electricity generated by the company is sold to industrial (primarily mining) operations in the region, or transported to far away population centers (Anonymous municipal official 2008). The Manás district, where SN Power's Cahua hydroelectric plant is located, has an electrification rate of just $53 \%$ (ICEI 2005). The rural areas of the district are not supplied with any basic public services. The situation is similar in the regions where ElectroAndes is active, where most rural households are not connected to the electricity grid. The investments required to increase rural electrification rates are so high that neither the provincial nor the regional governments are willing to invest. According to a government official from $\mathrm{La}$ Oroya, SN Power is also unwilling to take up the costs required for greater rural electrification (Anonymous municipal official 2008). Kopstad (2008) acknowledged this unwillingness, adding "SNPP is a hydroelectric generation company not involved in the distribution business". SN Power Peru (2011) currently has one rural electrification project in Peru (the community of San Pedro el Pari). Instead of directly working on implementing electrification projects, the company maps the rural clients under its area of influence to determine their needs. In one case, the company claims it has helped the communities of Macashca organize themselves into a communal distribution company in order to access the local energy distributor (Kopstad 2008).

While much of the Manás district is not supplied with electricity, the communities that do have access seem satisfied with SN Power's programs to make electricity affordable for the locals. One resident from the Pacasmayo district pointed out that the Cahua power station "supports" the farming community by offering a low price on electricity and the maintenance activities it undertakes (Pacasmayo resident 12008 ). Another resident claimed that the community pays nothing for the electricity service because the company maintains high tension towers on the community's territory (Pacasmayo resident 2 2008). A third resident corroborated this, noting that the company and the farming community have reached an agreement by 
which the company supplies free electricity to the community in exchange for the use of community land as well as for the potential health impacts that the community's inhabitants are exposed to as a result of their proximity to the high tension towers (Pacasmayo resident 3 2008). Kopstad (2008) explains that the agreements to provide the communities with free electricity were made decades ago, before SN Power began its activities there. Although it is generally not the company's policy to honor these agreements, SN Power decided to continue doing so because of the company's belief that surrounding communities have a right to electricity (Kopstad 2008).

\section{Respect labor rights}

SN Power (2008d) refers to ILO Core

Conventions 138 and 182 on the minimum age for employment and the worst forms of child labor as the basis for its policy on labor issues. The company also has policies on nondiscrimination and a commitment to allow employees to continue to develop their skills throughout their employment at SN Power. No mention is made of minimum wage, freedom of association, or working hours in SN Power's CR policies. Occupational health and safety is a particular focus of SN Power's labor policies, and the company aims for a zero accident and injury rate in all projects in all phases. With regard to respect for labor rights among contractors, SN Power's (2011b) policy is only to work with "qualified contractors with proven records on HSE". The company stipulates minimum health and safety standards in contracts with contractors.
In Peru, the Cahua power station employs 30 permanent workers, but they are not organized in a trade union. In fact, none of Cahua S.A.'s employees are members of a trade union. The former trade union was closed when the company was privatized, and no new trade union has been established since then (Cahua employee 1 2008). Employees of ElectroAndes are organized in a union affiliated with the national power sector trade union. Union leaders have indicated that their trade union rights have been respected since SN Power merged in 2007 (Mayo 2008). The union primarily represents workers employed directly by ElectroAndes, but some contract workers are also members.

The union played a strong role during SN Power's acquisition of ElectroAndes and the consequent restructuring, a time when there were worries about lay-offs and redundancies. At one point, ElectroAndes employees were forced to take 15-to-20-day holidays. Upon return, no employees were made redundant, but many were transferred to other positions. However, workers did indicate that they were forced to work overtime hours without compensation during the restructuring process (ElectroAndes employee 1 2008). The threat of future redundancies also still looms, and workers continue to worry about their job safety. One employee stated that workers have been in a constant state of "extreme worry" as to whether or not they would be able to keep their jobs since SN Power bought the company (ElectroAndes employee 2 2008). 
With regard to occupational health and safety, Cahua S.A. achieved OHSAS 18001:2000 certification for all its production facilities and administrative offices in 2007, and the company has implemented new OHS indicators in coordination with SN Power's HSE Management in Norway. The company has established a Safety Committee consisting of both worker and company representatives. In 2007, the accident frequency rate was 0.01 accidents per 226,800 yearly hours worked in its production facilities (SNPP 2009). Workers confirmed that Cahua S.A. follows rigorous OHS methods and regulations (Cahua employee 1 2008).

Workers employed by ElectroAndes indicated that the company provides the required safety equipment and uniforms. ElectroAndes experienced two work-related accidents in 2007, both of which occurred with subcontracted personnel. However, subcontracted workers who perform outsourced tasks for ElectroAndes and who are not members of the union work under different health and safety conditions from directly employed workers. They have to bring their own clothing, tools and materials (Mayo 2008).

\subsubsection{Environmental issues}

\section{Minimize environmental impact, including contribution to climate change}

Environmental management is an integral part of SN Power's Project Management System. The system is not ISO 14001 certified, but SN Power (2012c) claims that the system is based on "international best practices" and Norway's traditional "expertise and competence in developing hydropower". In order to monitor and ensure the implementation of its values by its subsidiaries, SN Power has CR staff on the ground for every project and carries out an EIA and an SIA on all of its projects prior to investment. The assessment documents give information about the project itself; the social and environmental contexts; the company's plans for compliance with environmental legislation; environmental, social, and aesthetic baseline data; and a citizen participation strategy. The findings of these assessments are used to develop social and environmental management plans, which are to be implemented, audited, and followed up on throughout the life cycle of its projects (Kopstad 2008).

As a result of the renewable-only fuel mix, the company claims that its operations do not emit any significant greenhouse gases. However, hydropower can be a significant source of GHG emissions through the submersion and subsequent rotting of $\mathrm{CO}_{2}$-absorbing plants especially if it involves large reservoirs, but also smaller run-off-river projects. SN Power has not sought to measure and manage these emissions.

The company has an active policy on carbon credits. It seeks to comply with the Clean Development Mechanism (CDM) criteria in order to be eligible for receiving these carbon credits. According to SN Power (2008c), the company's "role in combating climate change as a significant supplier of renewable energy was reinforced in 2007 when [its] second project got registered under the Kyoto Protocol's CDM". 
It should be noted that these CDM projects generate significant additional revenues for the company. For example, the La Higuera project in Chile, which is CDM certified, is expected to generate approximately $\$ 9.4$ (€7.1) million dollars in revenue per year. The company also hopes to get its Totoral wind project in Chile CDM certified, and cites the CDM as a significant factor in its decision to invest in renewable energy technologies. SN Power's Allain Duhangan project applied for CDM in September 2006, but construction was already well underway at the end of 2004, casting doubt on the extent to which the CDM certification led to "additional" GHG reductions.

Despite the fact that the exploitation of hydroelectricity has a significant impact on biodiversity, SN Power does not have a publicly available policy on the issue. The average capacity of the company's 17 hydroelectric facilities is $85 \mathrm{MW}$, and the largest is the 381 MW Magat plant in the Philippines. Although SN Power does have several small-scale hydro facilities in Peru and Sri Lanka, the company's current build program contains only large-scale hydro plants, the largest being an $880 \mathrm{MW}$ facility in Nepal (SN Power 2012b). This implies that the company will have an increasing impact on biodiversity.

\section{Other than the fact that SN Power's} environmental management system is not certified by an independent body and the lack of a public policy on biodiversity, the company's policies on minimizing environmental impact are moderately in line with international SEP norms (OECD 2011, WBCSD 2002, UNGC 2008, Pillinger 2009).

In Peru, SNPP (2011) states that it actively monitors air quality, liquid effluents, noise, lighting, and electromagnetic fields for all of its hydroelectric stations. The company also claims all its production facilities and administrative offices are implementing a program for waste control that involves waste transportation and final disposal through authorized contractors. According to Mayo (2008), the company maintains very precise regulations and procedures to ensure environmental protection. With regard to protection of biodiversity, Cahua has implemented measures to improve canal reservoirs and related structures, reforest and maintain green spaces, and conduct environmental training, but little information was available as to exactly where these training programs took place (SNPP 2011). The average capacity of SN Power's eight hydroelectric facilities is $34 \mathrm{MW}$, the largest being the 108 MW Yaupi facility in Ulcumayo. The country is currently developing a $168 \mathrm{MW}$ facility at Cheves. ElectroAndes' Pachachaca and Malpaso plants draw water from Lake Pomacocha and Lake Mantaro, respectively, both in Peru's Junín region. Junín is a mining region where copper and lead are both mined. The La Oroya district, where Pachachaca is located, is one of the world's top ten most polluted places according to the Blacksmith Institute (Blacksmith Institute 2012). This has led to conflicts with local communities and authorities. For example, residents of the farming community of San Pedro de Pari in the Junín region are seeking compensation 
for damages generated by a large number of mining and electricity companies, including ElectroAndes. The main issues at stake involve socio-spatial aspects related to the pollution of Lake Chinchaycocha. The crisis reached its peak when community residents seized the Upamayo dam in September 2008. Pollution caused by ElectroAndes is thus difficult to assess, as its plants are mostly located in areas of large-scale mining, which in itself already creates large-scale environmental pollution. In general terms, the mining operations, not electricity generation, are very likely the major factor in the pollution and related social unrest, but these issues indicate the troublesome context in which SN Power operates in Peru.

\section{Prioritize renewable sources of energy for electricity}

$100 \%$ of the $5,954 \mathrm{GWh}$ of electricity generated by SN Power in 2012 is based on renewable energy sources. 5,649 GWh (95\%) comes from large hydroelectric facilities, $196 \mathrm{GWh}(3 \%)$ from small hydropower stations, and $109 \mathrm{GWh}(2 \%)$ from wind. SN Power's current build program comprises four large hydroelectric facilities totaling 1,258 MW, the largest of which is the 880 MW facility at Tamakoshi, Nepal (SN Power 2012b).

The situation in Peru mirrors the company's global trajectory. In May 2007, Cahua withdrew its only fossil fuel-based generation facility, Central Térmica de Pacasmayo, from the network for commercial electricity distribution. According to SN Power (2008e), the plant was taken out of service because it was no longer profitable. Since this withdrawal, SN Power relies solely on hydroelectric power plants in Peru. Four of these facilities are smaller than $10 \mathrm{MW}$ and thus classify as sustainable "smallscale" hydroelectric facilities. These small-scale facilities total $28.3 \mathrm{MW}$ of capacity and produced 169 GWh of electricity (11\% of the company's total Peru production) in 2012. The company's four other Peruvian hydro stations, which range from $37 \mathrm{MW}$ to $108 \mathrm{MW}$ and are thus considered large scale, comprised $89 \%$ of production in Peru in 2012. The company is planning one new hydroelectric facility in Peru, the 168 MW Chaves plant. This will be the largest plant SN Power operates in Peru (SN Power 2012b).

\subsubsection{Economic issues}

\section{Contribute to sustainable local economic development}

The first of SN Power's two goals is to "contribute to sustainable economic growth". SN Power (2008c: 5) claims that it does business "in a way that adds value in the local communities and the countries in which [it] operate[s]". In order to do so, the company aims to create long-term value for the countries in which it operates by investing in and operating profitable renewable energy projects. To ensure that the local economic development it fosters is sustainable, SN Power (2008e: 1) "includes appraisal of the risks and rewards as well as sustainable development considerations (economic, social and environmental) as key criteria for investment and divestment decisions". As examples of the economic benefits its operations provide to local 
communities, SN Power (2007c) lists job creation for local residents, contracts to local suppliers and service providers, tax generation, and active engagement in knowledge and skills transfer to host communities. This policy is largely in line with SEP norms (WBCSD 2002, OECD 2011, Pillinger 2009).

In Peru, Cahua directly employs 30 people. The company has a two-pronged employment program: a permanent employment program giving preference to workers who live in the areas where the company operates, and a temporary employment program through which it hires qualified and non-qualified personnel for the power station's operation and maintenance. This policy is also applied to the contractors who provide services to the power stations (SNPP 2009). No information could be gathered on how much the company has invested in Peru thus far, but it is currently investing $\$ 400$ (€303) million in the new 168 MW Chaves plant. No information could be gathered on the amount or rate of taxes SN Power pays in Peru.

\section{Ensure reliable supply}

At the headquarters level, no public policy on ensuring reliable supply could be found. International SEP norms encourage electric utilities to ensure reliable supply by minimizing generation interruptions, maintaining contingency plans, responding to interruptions in a timely manner, and providing timely information to the public about planned and unplanned interruptions (CEER 2005, WBCSD 2002).

The average capacity factor of SN Power's eight hydroelectric power plants in Peru is $68 \%$
(SN Power 2012b). The company experienced 88 involuntary or forced power cuts in 2006. Cahua experienced 19, of which thirteen were attributed to failures in the protection system, three to natural or environmental phenomena, and three to human error (COES-SINAC 2007). From the perspective of both local residents and authorities, the electricity service was deemed "regular" for households receiving electricity services. Sudden interruptions were resolved by the company within a short time span, although their causes remained unclear to many residents (Descalzi 2008). ElectroAndes experienced 67 involuntary failures or forced power cuts in 2006. Ten were due to failures in the protection system, 16 to external causes, 17 to environmental or natural phenomena, 1 to human error and 23 to unidentified causes (COES-SINAC 2007). The company's performance in Peru is moderately in line with SEP norms.

\subsubsection{Cross-cutting issues}

Engage in meaningful stakeholder consultation and participatory decision-making

Although not expressly based on any international normative standards, SN Power's policies and procedures related to sustainable development are based two principles:

- Fluent communication and mutual collaboration with communities in the areas where it operates

- Implementation of CR policies through the activities that are in the interest of the community and aimed at promoting their sustainable development. 
According to Kopstad (2008), "During project assessment and construction, SN Power works closely with local communities to understand their needs and help ensure that our projects deliver social benefits. We aim to reflect the priorities and concerns of local communities in our decision-making processes and we try and minimize potential negative effects through a combination of careful planning, design adjustments and operational improvements."

The company seeks to establish a regular and open dialogue on environmental and social performance with host communities and other stakeholders and aims to reflect the priorities and concerns of local communities in decisionmaking processes.

For SN Power, the definition of "stakeholder" and determining which stakeholders it needs to engage with depends on the project (Kopstad 2008). In order to receive feedback from stakeholders, SN Power holds early information meetings to inform stakeholders about its projects and plans. These meetings are held either in collaboration with local public bodies or as stand-alone open meetings. Furthermore, SN Power conducts a social impact assessment before beginning any project. This SIA provides a platform for dialogue with local stakeholders and provision of information and allows the company to develop a citizen participation strategy. The findings of the SIA are used to develop social management plans, which are to be implemented, audited, and followed up on throughout the life cycle of its projects (Kopstad 2008). The company makes no mention of affected stakeholders' right to FPIC, although it should be noted that SN Power decided to abandon plans to develop a hydropower project in Chile after a five-yearlong conflict with Indigenous Peoples there (Mapuche Communities 2008). Managers at SN Power headquarters in Oslo provided input and constructively engaged with researchers for the present study.

In Peru, SNPP has created a conceptual framework to implement the company's policies related to community engagement. The first step is to map stakeholders, the socio-economic context, and the most urgent needs. On the basis of this mapping, a work plan is developed to address issues such as community capacity-building and sustainable development. According to Kopstad (2008), as of 2008 ElectroAndes had mapped 28 relevant communities this way, while Cahua had mapped 21. Kopstad (2008) states that, "With this framework, SNPP develops the idea of 'working together with the communities of our areas of influence' as a facilitator in order to reinforce the strengths of the local stakeholders so that they become key actors of their sustainable development process".

SN Power Peru's engagement policies received some praise from local residents, but others noted that the company's engagement with the community is primarily focused on charitable or philanthropic activities such as Christmas gift-giving (Pacasmayo residents 1, 2, and 3, 2008). The management of SN Power Peru declined to speak to local researchers or provide any input or information for the present study, 
despite repeated requests and ample time, indicating an unwillingness to engage with civil society on critical sustainable development issues at the local level.

\section{Assume responsibility for impacts throughout all business relationships}

Kopstad (2008) asserts that SN Power always includes CR issues in the tenders and evaluations it makes when selecting contractors and suppliers, paying particular attention to the health and safety record of potential business partners. She does acknowledge, however, that price and technical quality remain key criteria for choosing suppliers and contractors. In terms of how the company balances the trade-off between social and environmental criteria on the one hand, and price, which has a more direct effect on profits, on the other, Kopstad (2008) believes that the suppliers and contractors that are most competitive on price and technical quality generally also have a good record on sustainability issues.

Once SN Power decides to do business with a supplier or contractor, the company's policy is to provide copies of its Values and Principles to all partners and key contractors and require that they align themselves with the principles set out in the document (Kopstad 2008). The company asserts that it will not enter into partnerships that are not aligned with its Values and Principles. In order to ensure compliance, SN Power includes sustainability clauses in contracts with its business partners. Examples of the criteria taken up in such clauses include requirements that contractors appoint a community officer, employ a quota of local residents, and contribute to local HIV/ AIDS prevention programs. SN Power monitors compliance with its Values and Principles by maintaining its own direct relations with communities in order to receive feedback on contractor performance. SN Power does conduct periodic audits of some of its business partners. These audits are carried out internally, without the use of independent third parties or involvement of local civil society and labor groups, but this is something SN Power may consider doing in the future (Kopstad 2008). No information could be gathered about the company's performance on this issue in Peru.

\section{Maximize transparency and provision of information}

SN Power (2011a) notes that it aims to "engage with stakeholders in a spirit of open dialogue and open reporting, clearly detailing our efforts across all our areas of business operation". Though CR is featured prominently on the company's website, a limited number of policy documents are actually available on the site. And although its majority owner, Statkraft, does report using the GRI G3 and EUSS sustainability reporting guidelines (B+ rating in 2011), SN Power itself does not. SN Power also does not publish a separate annual sustainability or CR report. The company's 2010 Annual Report contains six pages on environmental, social, and local economic development issues (SN Power 2011a). The company's operations in the Global South feature prominently in the report. SN Power's policy is to publish all of its impact assessments online. However, as of September 2008, only one assessment (for La Higuera, Chile) was available. 
In Peru, the primary channels used to communicate with stakeholders include the company's website, stakeholder mapping, SIA, and information meetings. As of September 2008 , none of the impact assessments for the company's projects in Peru was available online. The company does have a Spanish language website and has translated its values and business principles document into Spanish, although the code of conduct is only available in English. Like the company's principal website, the Peru-specific website contains only a limited number of actual policy documents. In 2010, SN Power Peru began publishing an annual CR folder outlining the CR activities undertaken during the previous year (SN Power Peru 2011). Some customers indicated they had received no information from the company on the causes of supply interruptions (Descalzi 2008).

\subsubsection{The Nordic mode and SN Power's performance on the SEP benchmarks}

An SN Power manager maintains that "deeply rooted" Nordic traditions serve as the basis for the company's competitive advantage (Kopstad 2008). Indeed, SN Power's performance on the SEP benchmarks indicates that the Nordic mode of business culture plays a significant role in conditioning how SN Power takes up the internationally-derived standards for SEP and how it applies them in practice. Table 7.3 provides an overview of the degree to which SN Power's performance on SEP benchmarks is deemed to reflect the Nordic mode, as described in Chapter 4.
With regard to uptake of normative standards, SN Power's policy is largely reflective of the Nordic mode. While it does reference some of the leading international CR standards, these standards are not the primary factor defining SN Power's CR policies. Instead, the company's approach to CR largely draws on the region's deeply ingrained tradition of "leading the way" on business ethics issues, rather than relying solely on international consensus (Norwegian Ministry of Foreign Affairs 2009). This element of the Nordic mode dates back to the beginning of the industrial period (Kuhnle and Ervik 1996). This approach is also replicated in Peru.

SN Power's integration of SEP issues into high-level, core-business decision-making is also largely in line with the Nordic mode, which generally exhibits a holistic approach to CR (Kuhnle and Ervik 1996, Aarhus 2010) and support for ethical standards at a high level within a company (Hohnen 2009, Aarhus 2010). However, SN Power's focus on noncore-business, charity-type projects in its CR activities in Peru is only moderately in line with the Nordic mode and the company's own policies.

The fact that SN Power makes its involvement in electricity projects heavily dependent on their environmental impact (renewable projects only) is largely reflective of the Nordic mode's long history of and experience with hydropower. This means that the company excludes (environmentally unfriendly) projects that could increase access for large numbers of people. In Peru, communities served by SN Power 
Table 7.3: Degree to which SN Power's performance on SEP benchmarks reflects the Nordic mode of business culture

\begin{tabular}{l|l|l}
\hline $\begin{array}{l}\text { Is SN Power's policy/practice reflective of the Nordic mode on the } \\
\text { following SEP benchmarks? }\end{array}$ & Policy & Practice \\
\hline Endorse international normative standards for CR & Largely reflective & Largely reflective \\
\hline $\begin{array}{l}\text { Adopt a commitment to CR in core-business activities and } \\
\text { decision-making }\end{array}$ & Largely reflective & Moderately reflective \\
\hline $\begin{array}{l}\text { Increase access to affordable electricity } \\
\text { Respect labor rights }\end{array}$ & Largely reflective & Largely reflective \\
\hline $\begin{array}{l}\text { Minimize environmental impact, including contribution to climate } \\
\text { change }\end{array}$ & Moderately reflective & Moderately reflective \\
\hline Prioritize renewable sources of energy for electricity & & \\
\hline Contribute to local economic development & Largely reflective \\
\hline Ensure reliable supply & Moderately reflective & Moderately reflective \\
\hline $\begin{array}{l}\text { Engage in meaningful stakeholder consultation and participatory } \\
\text { decision-making }\end{array}$ & Moderately unreflective & Largely reflective \\
\hline $\begin{array}{l}\text { Assume responsibility for impacts throughout all business } \\
\text { relationships }\end{array}$ & Moderately reflective & Moderately reflective \\
\hline Maximize transparency and provision of information & n/a \\
\hline
\end{tabular}

electricity are satisfied with the company's affordability programs. However, in many cases SN Power Peru does not serve the communities closest to its electricity generation facilities, despite a CR policy that purports to support and develop local communities.

With regard to respect for labor rights, SN Power performance is only moderately reflective of the Nordic mode, which has a long tradition of social dialogue between business and labor and generally high respect for labor rights. SN Power does not mention the critical labor rights issues of minimum wage, freedom of association, and working hours in its CR documents, and although working conditions in Peru are generally good, serious concerns remain with regard to job security and the lack of a union presence at SN Power's Cahua station.
SN Power's performance on transparency is only moderately reflective of the Nordic mode, which, with its long tradition of transparency, would seem to expect a higher degree of information to be provided to stakeholders than SN Power does in Peru. 


\section{Chapter 8}

\section{Case study - Datang}
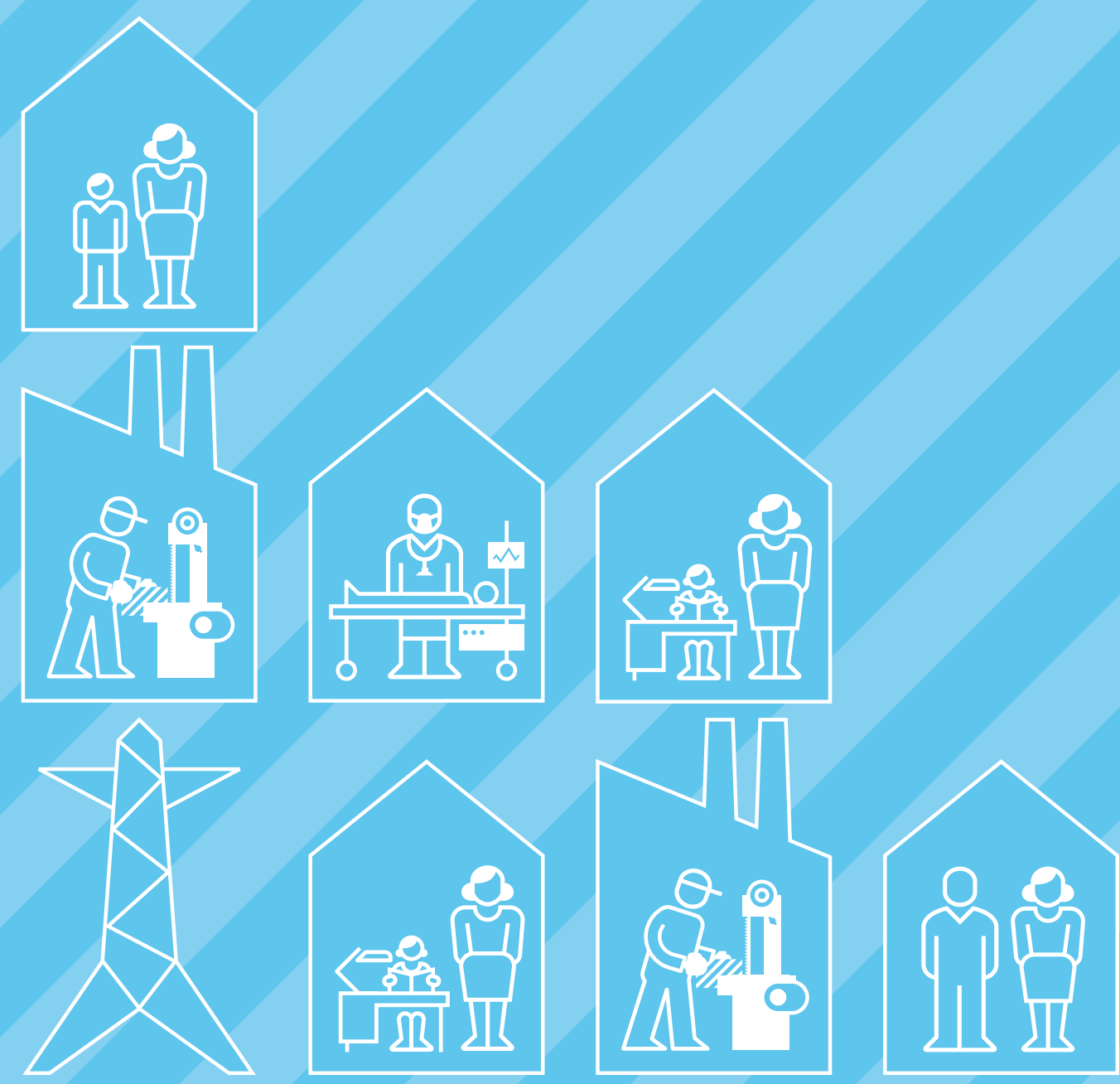


\subsection{Datang background and operations}

\subsubsection{Basic company information \\ State-owned China Datang Corporation} (hereinafter Datang) is China's second largest domestic power producer and the country's largest hydropower developer, though the vast majority $(88 \%)$ of its electricity production is coal-based. Datang's installed electricity generation capacity has been growing rapidly in recent years, reaching over $105,000 \mathrm{MW}$ that produced $472,575 \mathrm{GWh}$ of electricity in 2010 . Datang recorded revenues of more than $¥ 175$ billion ( $\$ 28 / € 21$ billion) and profits of more than $¥ 1.5$ billion (\$241/€183 million) in 2010 (Datang 2011b).

Datang's primary focus has been on coal-fired power, and the company ranks as China's second largest consumer of coal (103 Mt in 2008) and second-largest emitter of carbon dioxide (250 Mt in 2008) (Greenpeace 2009). Datang owns China's largest coal-fired power station, the Inner Mongolia Tuoketuo power station (Datang 2011b). However, higher costs due to rising coal prices have pushed Datang towards other forms of power generation. The company owns the largest wind farm in operation in the world, the Inner Mongolia Chifeng Saihanba Wind Farm. It also owns the second largest hydropower project in operation in China, Longtan Hydropower Project (Datang 2011b).
Since 2008, Datang has been restructuring to reduce the number of subsidiary companies to concentrate on energy generation infrastructure. One of Datang's most important subsidiaries is Datang International Power Generation Company Limited (Datang Power), which is $34 \%$ owned by Datang and is listed on the London and Hong Kong stock exchanges. With $30,000 \mathrm{MW}$ of installed capacity from interests in coal-fired, wind-powered, and hydroelectric facilities, Datang Power accounts for approximately one-quarter of parent Datang's capacity (Datang 2010).

\subsubsection{Operations and investments in the Global South}

Although the vast majority of its electricity generation capacity is located within its home country, Datang is operating or constructing six power plants in five Southern countries outside China: Burma, Cambodia, Kazakhstan, Indonesia, and Lao People's Democratic Republic (PDR). When they are all completed, these power plants will comprise 2,187 MW of electricity generation capacity, 1,917 MW $(88 \%)$ of it from large hydroelectric facilities. As part of the Stung Atay project in Cambodia, Datang is also constructing a $302 \mathrm{~km}$ long, high-voltage transmission line to connect the plant to Phnom Penh, as well as a substation for distribution. Figure 8.1 below maps Datang's global operations, and Table 8.1 lists the details of Datang's power plants abroad. Additional operation specifications for Datang's two mainstream Mekong dams, Pak Beng and Sanakham, both in Lao PDR, are as follows: 
- Pak Beng will have a height of $76 \mathrm{~m}$, a crest length of $943 \mathrm{~m}$, an active storage of 442 million $\mathrm{m}^{3}$, and a maximum reservoir area of $87 \mathrm{~km}^{2}$.
- Sanakham will have a height of $38 \mathrm{~m}$, a crest length of $630 \mathrm{~m}$, an active storage of 106 million $\mathrm{m}^{3}$, and a maximum reservoir area of $81 \mathrm{~km}^{2}$.

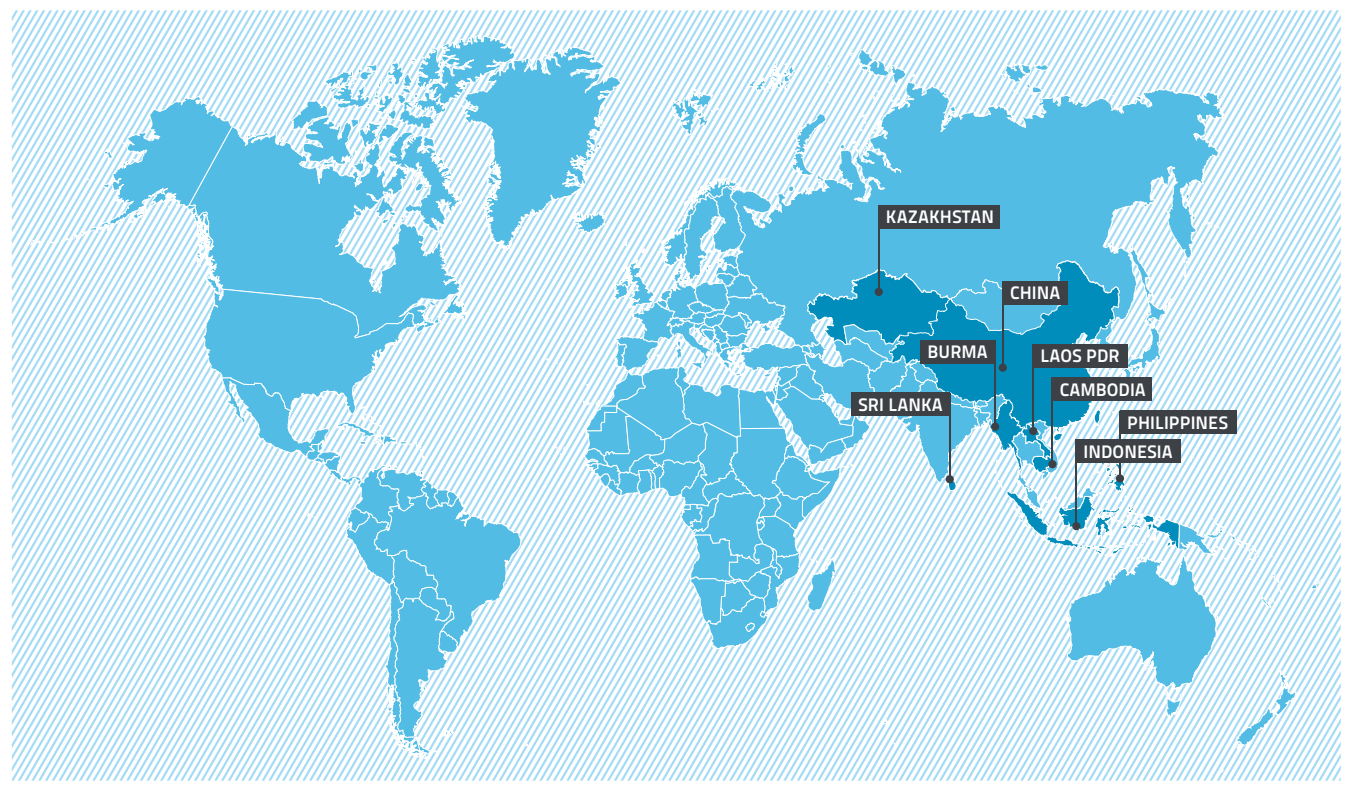

Figure 8.1: Datang's global presence, 2010

Table 8.1: Datang power plants outside China that are operational or under construction, 2010

\begin{tabular}{|c|c|c|c|c|c|}
\hline Country & Facility name (location) & Fuel type & $\begin{array}{l}\text { Capacity } \\
\text { (MW) }\end{array}$ & $\begin{array}{l}\text { Investment } \\
\text { (\$/€ million) }\end{array}$ & Status \\
\hline Burma & Dapein I & Hydro & 240 & $\$ 270 / € 206$ & Operational \\
\hline Cambodia & $\begin{array}{l}\text { Stung Atay (Ou Saom commune, } \\
\text { Veal Veaeng district, Pursat } \\
\text { province) }\end{array}$ & Hydro & 120 & $\$ 360 / € 275$ & $\begin{array}{l}\text { Under construction, } \\
\text { operational in } 2013\end{array}$ \\
\hline Indonesia & Kualatanjung & Coal & 270 & $\$ 300 / € 229$ & Under construction \\
\hline Kazakhstan & Rudnichnyi $1 \& 2$ & Hydro & 42 & $\mathrm{n} / \mathrm{a}$ & $\begin{array}{l}\text { Under construction, } \\
\text { operational in } 2014\end{array}$ \\
\hline \multirow[t]{2}{*}{ Lao PDR } & $\begin{array}{l}\text { Pak Beng (mainstream Mekong } \\
\text { River, Oudomsay) }\end{array}$ & Hydro & 855 & $\mathrm{n} / \mathrm{a}$ & $\begin{array}{l}\text { Under construction, } \\
\text { operational in } 2016\end{array}$ \\
\hline & $\begin{array}{l}\text { Sanakham (mainstream Mekong } \\
\text { River, Vientiane) }\end{array}$ & Hydro & 660 & $\$ 880 / € 673$ & $\begin{array}{l}\text { Under construction, } \\
\text { operational in } 2016\end{array}$ \\
\hline
\end{tabular}


In its 2010 Sustainability Report, Datang (Datang 2011a) notes that it has begun to "accelerate the implementation of [the company's] 'Going Out' strategy". Thus, in addition to the power plants in the operational and advanced construction stages in Table 8.1, Datang $(2011 \mathrm{a}, 2010)$ is planning to build and operate an additional 5,000-7,000 MW of capacity outside China in the medium and long term. Some of the projects for which a Memorandum of Understanding ( $\mathrm{MoU}$ ) has already been signed include wind power projects in Australia and Kazakhstan.

\subsection{Datang's performance on the SEP benchmarks}

This section begins with an overview (in table format) of Datang's performance, in both policy and practice, on the 11 SEP benchmarks. For each SEP benchmark, a reminder of the guiding international normative standards (taken from Chapter 3 ) is provided alongside key indicators of the company's performance and an evaluation of the degree to which the company's policy and practice are line with the SEP standards on that issue. Following the overview, the results of the empirical research on Datang's policy and practice in Cambodia and Lao PDR are detailed for each SEP benchmark. These findings form the basis for the overview and evaluation provided in Table 8.2. The chapter concludes with a brief reflection on the relationship between the Chinese mode of home-country business culture (as outlined in
Chapter 4) and Datang's performance on the SEP benchmarks. 
Table 8.2: Summary of Datang's performance on SEP benchmarks in policy and in practice

\begin{tabular}{l} 
SEP benchmark \\
General approach to corporate responsibility \\
Endorse international normative standards for CR \\
\hline
\end{tabular}

Adopt a commitment to CR in core-business activities and decisionmaking

\section{Guiding international normative SEP standards}

Electric utilities should seek to bring their policies in line with internationally recognized and verified standards for CR (ILO 2001, WBCSD 2002, IFC 2011, ISO 2011, OECD 2011 UNGC 2008, UNHRC 2011).

Electricity companies should adopt a holistic and integrated view of the role and impacts of electricity provision, considering and balancing the essential elements of economic development, environmental quality, and social equity in utility operations. This entails a policy commitment to CR throughout all core-business activities, as opposed to a primary focus on philanthropic activities (WBCSD 2002, MVO Platform 2012). Electric utilities should seek to introduce environmental and social factors and procedures into high-level corporate planning and decision-making and should aim to have a board-level position responsible for CR issues (WBCSD 2002).

\section{Social issues}

Increase access to affordable electricity

\section{Respect labor rights} attention to disadvantaged communities (ILO/Palast et al.

(15)

\section{Electr}

Electric utilities should develop policies that promote access to electricity priced at affordable levels for all, paying special 2000, WBCSD 2002, PSI/Pillinger 2009). In order to do so, companies should undertake initiatives to extend electricity services to unserved and underserved communities, particularly in rural or remote areas, but also to the poor in urban and peri-urban areas (WBCSD 2002, UNDP/Modi et al. 2005).

Electric utilities should respect workers' full rights and provide workers with fair pay, job security, and decent working conditions, including high levels of health and safety standards in all aspects of operations (WBCSD 2002, PSI/Pillinger 2009, OECD 2011). Furthermore, electric utilities should uphold freedom of association and the right to collective bargaining and pay particular attention to the right to strike (ILO 1998, ILO 2001, UNGC 2008, OECD 2011). Companies should ensure that these rights are respected not only among their own employees, but also among suppliers, contractors, and other business relationships (ISO 2011, OECD 2011, UNHRC 2011). 


\section{Indicators of Datang's policy}

- Relies primarily on Chinese CR standards such as those developed by the CASS and the SASAC

- Member of the UNGC since 2008

- No reference to standardized certifications for EMS or OHS.

- Moderately out of line with SEP standards

- General nature and focus of CR at times ambiguous, but does identify core business activities as focus

- Very limited amount of specific issue CR policies on website or referred to in reports

- General manager, not CEO or board-level, appears to be the highest person responsible for CR issues

" Management-level "Leader teams" on various CR issues report to the general manager.

- Moderately out of line with SEP standards

\section{Indicators of Datang's practice in Cambodia and Lao PDR}

No reference to any of the international nor Chinese CR standards

- No reference to standardized certifications for EMS or OHS.

- Largely out of line with SEP standards

- CR activities focused on charity projects

- Donation to Laotian government to build two schools, a hospital, and a supermarket in the vicinity of one dam site.

- Largely out of line with SEP standards
- No publicly available policy commitment to increasing access to affordable electricity

- $100 \%$ of $105,896 \mathrm{MW}$ capacity located in countries without universal electrification, but only $240 \mathrm{MW}(0.2 \%$ of total capacity) outside China

- Clearly favors very large power plants; does not promote off-grid, decentralized electricity provision projects.

- Moderately in line with SEP standards

- No mention of ILO Core Conventions, but does have policies against child labor and discrimination

- No endorsement or mention of workers' right to strike or to freedom of association and collective bargaining. "Employees assembly" instead

- No indication policies/structures to engage \& consult workers on key operational decisions

- OHS policy includes a health \& safety system (though not certified by international bodies), providing workers with safety equipment and training, and regular health checkups for workers

" No indication of a policy aimed at ensuring that the rights of workers at the company's business relationships (e.g. contractors and suppliers) are respected.

- Largely out of line with SEP standards
- Not yet producing electricity but projected to produce $526 \mathrm{GWh} /$ year in Cambodia and 6,640 GWh/year in Lao

- Electrification rates in Cambodia and Lao are $24 \%$ and 55\%, respectively

No indication of programs to ensure electricity reaches underserved and will be affordable

- Large, centralized hydropower stations only; no promotion of offgrid, decentralized access

Moderately out of line with SEP standards

- No union present and no CBA in place

- Piece-rate pay system in effect in both countries; typical wage paid to manual laborers in Cambodia $\$ 5(€ 3.80)$ /day and in Lao $\$ 6.25$ (€4.76)/day; in both cases approximately double local minimum

- No information on OHS systems or policies in either country could be gathered, but working conditions reported to be harsh in both countries; "15-hour days common"

- No assessment of business relations on respect for labor rights.

- Largely out of line with SEP standards 


\section{Environmental issues}

\section{Minimize environmental impact, including contribution to climate} change

\section{Guiding international normative SEP standards}

Electric utilities should seek to minimize emissions and discharges to air and water and continually develop and implement low-pollution and low-environmental impact technologies (WBCSD 2002, UNGC 2008, PSI/Pillinger 2009, OECD 2011). Companies should manage their environmental impact in line with internationally recognized and verified standards, such as the ISO 14000 series and should always undertake rigorous and verifiable environmental impact assessments (WCD 2000, WBCSD 2002, IHA 2004, OECD 2011,). Electric utilities should minimize their contribution to climate change and develop strategies for reducing GHG emissions (WBCSD 2002, OECD 2011). As a matter of priority, electric utilities should seek to avoid impacts on biodiversity and ecosystem services, refraining from any activities in areas of critical habitat with high biodiversity value while at the same time initiating or supporting conservation and biodiversity efforts related to impacts on natural habitats from utility operations (WBCSD 2002, IHA 2004, IFC 2011).

Electric utilities should evaluate the renewable energy potential of the host country and prioritize development and exploitation of renewable options over fossil fuel-based technologies (WBCSD 2002, UN/IAEA 2007, PSI/Pillinger 2009). Electric utilities should also prioritize renewables in their research and development programs and should support the diffusion of renewable energy technologies into the local economy (WBCSD 2002, UNGC 2008).

\section{Economic issues}

Contribute to local economic development
Electric utilities prioritizing the development of sustainable local energy-related infrastructure and employing local workers to the greatest extent practicable and providing those workers with sustainable jobs and training to improve skill levels (WBCSD 2002, PSI/Pillinger 2009, OECD 2011). In order to maximize local economic development and job creation, electric utilities should support local small and medium-sized enterprises in their procurement practices and contribute to the long-term development prospects of the host country through the transfer and rapid diffusion of technologies, skills and knowledge (WBCSD 2002, OECD 2011). Fair payment of due taxes to local, regional and national governments in the host country is a crucial element of contributing to local economic development (OECD 2011, MVO Platform 2012). 


\section{Indicators of Datang's policy}

- Vast coal-fired production means major impact on environment. Two-pronged policy to reduce waste \& pollution: increase efficiency of coal plants and develop more renewables

- Company-wide EMS in place, but not certified by an international body

- Policy to always conduct an EIA at projects in China (as is required by Chinese law), but no indication of policy outside China

- Does not report on global $\mathrm{CO}_{2}$ emissions, but estimated to be at least $250 \mathrm{Mt}$ in 2008

- Set target to reduce $\mathrm{CO}_{2}$ emissions by $45 \mathrm{Mt}$ by replacing coal with hydro, wind, and nuclear. Not clear if targets have been reached.

- Vast hydroelectric production through large dams means major impact on biodiversity. Provides examples of biodiversity protection, but no clear comprehensive policy to do so

" Largely out of line with SEP standards

- 2010 fuel mix for electricity generation includes $88 \%$ nonrenewable (all coal) and $12 \%$ renewable $(10 \%$ large hydro, $2 \%$ wind)

" Believes "companies have a responsibility to advance renewable energy sources" and is vigorously developing hydropower and wind. However, an even greater share of build program is dedicated to non-renewable nuclear and coal.

" Moderately out of line with SEP standards

- Considers its main contribution to society "driving economic development by meeting power demands and paying taxes". Invests billions in electricity infrastructure and pays billions in taxes

- Claims it contributes to local economic development in China through increasing power supply and paying taxes, but most examples given are of philanthropic projects and charitable donations

" "Supports local economic and social development" in operations outside of China, but example provided is "by actively participating in social welfare to support the development of education and to alleviate the hardships of affected residents"

- No mention of a policy to prioritize local workers or SME suppliers

- Moderately in line with SEP standards
Indicators of Datang's practice in Cambodia and Lao PDR

- No information on EMS or EIA in Cambodia or Lao could be gathered

- GHG emissions and other air pollution from three planned hydropower stations will be negligible

- Primary environmental impact is on biodiversity and river ecology

- Datang's average dam size in Cambodia is 120 MW, in Lao 758 MW.

- Moderately out of line with SEP standards

- When construction is complete, $100 \%$ of electricity generated will be from large hydroelectric dams

- Not promoting other, more sustainable electricity generation technologies.

- Moderately in line with SEP standards

- Unclear exactly how many are employed, but Datang claims it has employed up to 1,000 Cambodians

- No indication of percentage of Cambodian and Lao nationals employed, but widespread criticism about failure to provide locals with jobs, using migrant Chinese laborers instead; manager says that only Chinese laborers will accept the harsh working conditions

- No indication of prioritizing SME suppliers

- Investing \$360 million (€265 million) in Cambodian electricity infrastructure and $\$ 880$ million ( $€ 673$ million) plus an undisclosed amount in Lao

- No information could be gathered about the amount or rate of taxes paid.

- Moderately out of line with SEP standards 
Ensure reliable supply

\section{Cross-cutting issues}

Engage in meaningful stakeholder consultation and participatory decision-making
Electric utilities should ensure reliable supply by minimizing generation interruptions, maintaining contingency plans, responding to interruptions in a timely manner, and providing timely information to the public about planned and unplanned interruptions (WBCSD 2002, CEER 2005).

\section{Assume responsibility for impacts throughout all business relationships}

\section{Guiding international normative SEP standards}

Electric utilities should adopt a participatory approach to the planning/designing, developing, operating, and monitoring/ evaluation of electricity projects and consult and engage stakeholders throughout the entire process in order to provide meaningful opportunities for their views to be taken into account in decision- making (WCD 2000, WBCSD 2002, IFC 2007, UNGC 2008, PSI/Pillinger 2009, OECD 2011). The consultation process should be tailored to local decisionmaking processes and involve the timely provision of all relevant information about activities, impacts, and potential impacts translated into local languages (WBCSD 2002, ISO 2011). The process should take into account the needs of disadvantaged or vulnerable groups, and if Indigenous Peoples are affected by a potential project, electric utilities must gain the free, prior and informed consent of those peoples before proceeding with any part of the project cycle (IFC 2011).

Electric utilities should seek to prevent, avoid and mitigate negative impacts throughout their entire range of business relationships, including suppliers and contractors (ISO 2011, OECD 2011, UNHRC 2011). Companies should seek to leverage their buying power to improve social and environmental conditions among business relations, and monitor performance using third party audits (WBCSD 2002).

Electric utilities should apply a high degree of transparency in operations, including reporting on activities and progress, measurement, business partners and relationships, and interactions with government and the public (UNECE 1998, WBCSD 2002, UNGC 2008, OECD 2011). Electric utilities should regularly report on their activities, progress, and performance against "the triple bottom line" using internationally accepted sustainability reporting guidelines such as the GRI's G3 guidelines and the Electric Utilities Sector Supplement (GRI 2008), have an external party verify the data, and disseminate the report in an appropriate manner (WBCSD 2002, OECD 2011). 
- Claims it meets power supply agreements with the government and state grid company

- Invested $\$ 9.6$ billion (€7.3 billion) in electricity infrastructure to ensure reliability of supply in China in 2010.

- Largely in line with SEP standards

\section{Indicators of Datang's policy}

- No evidence of a participatory approach or any meaningful engagement with stakeholders

- No reference to international standards on stakeholder engagement

- No mention of FPIC

- Did not respond to requests for engagement in research related to the present study

- Largely out of line with SEP standards
- Little information gathered because plants are not yet supplying electricity

- No information readily available on contingency plans to respond to generation interruptions in a timely manner.

\section{Indicators of Datang's practice in Cambodia and Lao PDR}

"Way of engaging stakeholders is "different" from China; has engaged to some degree with stakeholders regarding serious issues such as displacement

- Still often rely on local governments to engage communities rather than engaging directly

- Villagers seek "more chances to talk to the company"

- Local managers engaged with researchers and provided information on condition of anonymity

" Moderately out of line with SEP standards
- No mention of responsibility for or seeking to prevent, avoid, and mitigate negative social and environmental impacts throughout business relationships

- Supplier assessment focused on quality, fair competition, and corruption

- Does not assess suppliers on human rights

- Uses a questionnaire to assess suppliers.

- Largely out of line with SEP standards

- Relatively low degree of accessibility \& visibility of the CR policies. Website contains annual sustainability reports, but little information on issue-specific policies available

- Minimal information on operations outside China included in sustainability report

- Uses GRI G3 \& EUSS as a guideline for reporting. Reporting status "undeclared"

- Claims that sustainability report is evaluated by an external third party, but GRI lists reports as "not externally assured"

" Provides transparency about some customers, but not other business relationships

- Moderately out of line with SEP standards
- Monitors contractors on quality and technical details, but not on labor rights or environmental performance.

- Largely out of line with SEP standards

- No information could be gathered on the degree of local-language information about operations and its CR policies provided to stakeholders.

" Largely out of line with SEP standards 


\subsubsection{General approach to CR}

\section{Endorse international normative standards for $C R$}

With regard to normative standards for CR, Datang $(2011 a, 2010)$ primarily relies on Chinese standards such as the China Corporate Social Responsibility Report Guide developed by the CSR Research Center at the Chinese Academy of Social Sciences (CASS 2003) and the State-owned Assets Supervision and Administration Commission of the State Council's (SASAC) (2008) "Guidelines for state-owned enterprises directly under the Central Government on fulfilling corporate social responsibilities". Datang also uses Chinese laws and regulations and international agreements signed by China for normative guidance (2011a). In terms of internationallyrecognized standards, Datang publicly endorses the UN Global Compact, to which it has been a member since 2008. Past Datang social responsibility reports have referred variously to certification standards such as SA8000 (Datang 2008) and AA1000 (Datang 2009b), although the company's 2010 Social Responsibility Report does not mention them.

Local management in Cambodia and Lao PDR make no mention of any of the international or Chinese standards for CR nor do the hydroelectric dam facilities, which are still under construction in those countries, have any standardized environmental management or OHS certifications.
Adopt a commitment to CR in core-business activities and decision-making

The nature and focus of Datang's approach to CR is difficult to pin down, as the company's publicly available CR information and documents are at times ambiguous and contradictory. According to Datang (2011c), the company's primary commitment to society is to "increase economic benefit" while "profiting through conservation". The company's press release for its 2010 Sustainability Report contains a jumble of CR targets, commitments, and achievements, many of them related to core-business aspects. According to Datang (2011c), the 2010 report reveals that:

"Datang was committed to building an intrinsically safe enterprise, comprehensively advanced standardized and refined safe production management, maintained safe and steady production, and made active contributions to power supply to major events such as the 'World Expo' and 'Asian Games'; was committed to building an energy-conserving and environment-friendly enterprise, actively honored its commitments to energy conservation and emission reduction, enormously reduced coal consumption for power supply, accomplished standard discharge of major pollutants, maintained the advanced level of energy conservation and emission reduction, and fulfilled the task of energy conservation and emission reduction in the 'eleventh five-year' period one year earlier; was committed to building a harmonious enterprise, established an assessment system that organically integrates assessment results with staff 
career development, post promotion and salary income, comprehensively repaid the society and actively promoted local social and economic development through job enlargement, public welfare, poverty alleviation and disaster relief, thus accomplishing the common development of the corporation and interested parties."

The company's overwhelming zeal with regard to the core-business aspect of emissions reduction (mentioning the issue four times in the single sentence above) is difficult to reconcile with the fact that Datang ranks among the top in the world in terms of emissions from coal-fired electricity production and that it is vigorously continuing to develop coal-fired capacity. Though the company mentions many core-business social, environmental, and economic aspects in its sustainability report, seemingly in line with international SEP norms (WBCSD 2002, MVO Platform 2012), many of these mentions are made in a superficial way without being backed up by specific policies or more detailed references. There is a distinct lack of issue-specific CR policies and documents available on the company's Chinese and English websites or referred to in the company's other CR materials (though interestingly some of Datang's internationally-oriented subsidiaries (Datang 2010, Datang 2012) have developed a wider range of issue-specific CR policies than the parent company). For example, in the "Message from the President" on the front page of its 2010 Sustainability Report, Datang claims that the primary way it contributes to local economic development is through "meeting power demands and paying taxes"
(Datang 2011a). However, no corporate policy on the issue is provided, and the "Win-win Cooperation" section of the "Common Development" chapter contains primarily examples of charitable donations to community development projects and cultural activities (Datang 2011a). Similarly, Datang claims that it "supports local economic and social development" in its operations outside China, but the example that it provides for how it does so is "by actively participating in social welfare to support the development of education and to alleviate the hardships of affected residents" (Datang 2011).

In terms of management structures for $\mathrm{CR}_{t}$ Datang (2010) indicates it has a "standardized management" system for CR issues that covers the whole company. The system consists of a number of management level "Leader Teams" for CR issues like Occupational Health and Safety (OHS), environmental management, conserving energy, and reducing emissions, all of which report directly to the general manager. There is no indication regarding whether there is any board-level representative responsible for CR issues, as is encouraged by the SEP norms (WBCSD 2002).

In Lao PDR, Datang's CR activities are focused on charity and philanthropy. According to one local manager, the primary CR activity undertaken by Datang has been to donate $\$ 800,000(€ 614,183)$ to the Laotian government to build two schools, one hospital, one supermarket, and residential quarters in the vicinity of Pak Beng. This was done in 
order to "establish a good, cooperative relationship with the locals" (Anonymous manager in Lao PDR 4 2009).

\subsubsection{Social issues}

\section{Increase access to affordable electricity}

The fact that Datang's annual electricity production ranks among the highest of any company in the world, and the fact that it is planning to double its production capacity in the coming years, means that Datang will be providing an enormous amount of electricity. However, though $100 \%$ of Datang's current 105,896 MW of electricity generation capacity is located in countries without universal electrification, the vast majority is in China. Only $240 \mathrm{MW}(0.2 \%$ of total capacity) is located in Southern countries outside China. The majority of its build program is also focused in China, though it is planning to add an additional 1,947 MW beyond its borders. Datang's strategy for increasing electricity production clearly views bigger as better when it comes to power plants, favoring large, centralized grid projects over smaller units. Datang (2011c) boasts that it "leads the industry in China in terms of thermal power plants with a capacity of $600 \mathrm{MW}$ or larger", and it has a prominent graphic on its website highlighting the large size of its power plants. In addition, Datang has touted its recent decommissioning of smaller, less efficient coal-fired power plants in favor of larger coal-fired plants (Datang 2011a). The company does not promote smaller, off-grid electricity projects.
The sheer amount of electricity generation capacity that Datang operates and is developing, both inside China and abroad, means that the company provides and will continue to provide large amounts of electricity. However, it is also not clear to what degree Datang's significant new generation capacity will go to actually increasing access to electricity for poor communities that currently lack access. Much of its electricity production in 2010 went to supplying mega-events such as the World Expo and the Asian Games (Datang 2011a). Neither increasing access to electricity for those lacking it nor making electricity more affordable for the poor are mentioned by Datang in any of its CR documents or policies, despite international SEP norms' suggestion that electricity companies do so (ILO/Palast et al. 2000, PSI/Pillinger 2009, UNDP/Modi et al. 2005, WBCSD 2002). Datang claims that it is not a distributor of electricity (merely a producer), and thus it is not responsible for access or affordability issues. However, international SEP norms suggest that even generation-only companies can support increased access by developing off-grid, decentralized production in underserved areas.

Datang's power plants in Cambodia and Lao PDR, where electrification rates are $24 \%$ and $55 \%$ respectively (IEA 2011), are all large hydroelectric power stations with dams and reservoirs. All three plants are still in the construction phase and are therefore not yet producing electricity. If one assumes a capacity factor of $50 \%$, Datang's $120 \mathrm{MW}$ hydroelectric plant in Cambodia will produce 526 GWh of electricity 
each year, and the company's combined 1,515 MW of hydro capacity in Lao PDR will produce $6,640 \mathrm{GWh}$ each year. There is no indication that the company has any programs in place in Cambodia or Lao PDR to ensure that this electricity reaches underserved areas of the country or that it will be affordable for the poor.

\section{Respect labor rights}

Datang (2011a) does not specifically endorse or mention the ILO Core Conventions in the "Rights of Employees" section of its sustainability report, but it does cover some of the labor rights expressed in the conventions. For example, Datang (2009b) notes that it has a policy on promoting fair and indiscriminative employment practices and prohibits child labor. Datang (2011a) also insists that all workers it employs have a formal contract. However, no mention is made of workers' right to freedom of association, collective bargaining, or to strike despite the fact that these are seen as basic rights of workers by international SEP norms (ILO 1998, ILO 2001, UNGC 2008, PSI/Pillinger 2009, OECD 2011). Instead of an independent union, an "employees' assembly" exists at Datang facilities.

There are no unions at Datang's construction sites in Cambodia or Laos, nor is any collective bargaining agreement (CBA) in place. In both countries, a piece rate pay system is used for most workers at the dam construction sites. A group of Chinese migrant laborers doing tunnel drilling claimed that they were paid only by the metre of tunnel drilled, with no additional pay for overtime, weekends or holidays (Chinese migrant laborers in Cambodia 2010). Other workers said they were paid by the cubic metre of dirt they dug (Cambodian laborer 1 2010). Workers reported that, through this system, they could make approximately KHR 20,000 (\$5.04; €3.80) per day, or approximately $\$ 100$ (€76) per month, doing basic manual labor (Cambodian laborer 1 2010). A manager in Laos explained that the piece-rate system and the wages are similar to those in Cambodia, with local laborers earning approximately 50,000 kip (\$6.25/€4.76) per day (Anonymous manager in Lao PDR 2 2009). In both countries, the monthly wage represents approximately double the national minimum wage (Han 2010). However, the piece-rate pay system ensures that laborers often have to work long days in order to make that wage, and working conditions at the hydroelectric dam construction sites in both countries are reported to be harsh. One worker in Cambodia complained that the searing heat and the heavy workload made the working conditions "exhausting beyond imagination" (Cambodian laborer 1 2010).

A group of Chinese migrant laborers in Cambodia claimed that "15-hour days are common" on the dam construction site (Chinese migrant laborers in Cambodia 2010), which is far more than stipulated in ILO standards (ILO 2001). The harsh conditions and piece-rate pay system mean that many Cambodians and Laotians refuse to work on the sites, and that many migrant Chinese laborers are employed instead. A manager in Laos confided that, "In reality, Chinese companies can only employ Chinese laborers. We don't pay overtime, 
and there is minimal job security or safety protection. These are the conditions under which laborers working for us must work. If you treat Laotian laborers like this, they protest. So Chinese companies have to employ Chinese laborers" (Anonymous manager in Lao PDR 3 2009). As will be seen in the section on "Contribution to local economic development" below, the employment of large numbers of Chinese workers instead of Cambodian and Laotian laborers has angered many locals in both countries.

Datang's sustainability reports (2011a, 2009b) contain a "Safe Development" chapter that details the OHS measures the company has taken. These include establishing a quality, health, safety, and environment system in 2008 (though the system is not publicly available and there is no indication that this system is based on international OHS certification standards). This provides workers with safety equipment and training, and develops a handbook for workers entitled "Safe Production and How to Respond to Emergencies". Datang also arranges regular health check-ups for workers.

There is no indication that Datang has any policies or structures in place to engage and consult with workers on key operational decisions. Nor is there any indication that the company has any policies aimed at ensuring that the rights of workers at the company's business partners (e.g. contractors and suppliers) are also respected, as suggested by international SEP norms (UNHRC 2011, OECD 2011, ISO 2011). No information on the degree of outsourcing or job security at Datang's operations in Cambodia or Lao PDR could be gathered. In terms of monitoring suppliers and contractors for working standards as encouraged by SEP standards (PSI/Pillinger 2009, UNHRC 2011, OECD 2011, ISO 2011), a manager in Cambodia confided, "When we contract out projects to construction companies, the contractors sometimes recruit migrant workers from labor service output companies at home [in China]. We require that the company be responsible for technical administration, evaluation of construction progress and quality. We don't evaluate whether the contractors have respected the migrant workers' rights" (Anonymous manager in Cambodia 12010 ).

\subsubsection{Environmental issues}

\section{Minimize environmental impact, including contribution to climate change}

Datang produced 472.6 TWh of electricity in $2011,88 \%$ of which was based on coal. As a result, Datang's impact on the environment and the climate ranks among the most significant of any company in the world. Datang (2011a, Datang 2008) insists that it does have an Environmental Management System (EMS) in place. However, the details of the system are not publicly available and there is no indication that this system is based on international certification standards such as ISO 14000. The Chinese government requires that all new power plants in China undergo an environmental impact assessment, and Datang (2011a, Datang 2008) claims that it meets this 
requirement. The company does not indicate, however, that its policy is to also always conduct Environmental Impact Assessments (EIAs) at its power plants outside China.

Datang does not report on its $\mathrm{CO}_{2}$ emissions, but a study by Greenpeace (2009) indicated that the company emitted $250 \mathrm{Mt}$ of $\mathrm{CO}_{2}$ in 2008. Datang generated 352,956 GWh of electricity in 2008, which would give it an emission intensity of $0.71 \mathrm{tCO}_{2} / \mathrm{MWh}$. In 2012, Datang committed to report its $\mathrm{CO}_{2}$ emissions according to the CDP. Despite its significant, but unreported $\mathrm{CO}_{2}$ emissions, Datang does make considerable mention of its policy and commitment to reducing those emissions. The company notes that, "Companies have a responsibility to contribute to the development of a low-carbon economy in order to meet environmental standards" (Datang 2010). However, as China's second largest power producer, the company has also signed a binding agreement with the Chinese Ministry of Environmental Protection aimed at reducing its environmental impact and thus has both a legal and a political duty to reduce its emissions and impact.

In order to do so, Datang (2011a) proposes a two-pronged strategy: 1) combustion efficiency (i.e. reduce coal consumption), and improve pollution controls to reduce emissions of $\mathrm{CO}_{2^{\prime}} \mathrm{SO}_{2}$ and $\mathrm{NO}_{\mathrm{x}}$ at existing power plants, and 2) develop "alternative" forms of energy (note that Datang considers large hydro and nuclear power both to be alternative forms of energy). As part of the agreement with the Chinese government, Datang has shut down some of its smaller, less efficient and more polluting coal-fired power plants. The amount of retired coal capacity reached 7,980 MW, with 2,750 MW being shut down in 2010 alone (Datang 2011a). Datang has set clear targets for reducing its $\mathrm{CO}_{2}$ emissions, claiming in 2009 that it aimed to achieve reduction of $45 \mathrm{Mt}$ of $\mathrm{CO}_{2}$ by replacing some of its coal-fired capacity with hydro and wind (Datang 2009b). The Chinese government has also said that it was Datang's "duty" to reduce $\mathrm{CO}_{2}$ emissions by $40 \mathrm{Mt}$ between 2006 and 2010. Neither Datang nor the government indicate whether Datang actually met this target. With regard to pollution control, the company invested $¥ 956$ million (\$152/€115m) in 2010 in new technology to reduce $\mathrm{SO}_{2}$ at all of its power plants and $\mathrm{NO}_{x}$ at $12 \%$ of power plants. The company has also managed to reduce its consumption of coal to some degree by developing more renewablebased capacity (though coal remains its most important fuel source by far), as will be explained in the following section.

Though Datang's electricity production is primarily coal-based, the sheer size of the company means that, although a mere $14 \%$ of the company's electricity generation capacity is based on hydroelectricity, that $14 \%$ results in a total hydro capacity of more than 15,000 MW and makes Datang China's largest hydropower developer. All of Datang's 15,000 MW are situated in large dams, meaning that the company's impact on biodiversity is as significant as its impact on the environment through air emissions and climate change. Datang's sustainability reports do dedicate a significant amount of space to the company's 
biodiversity conservation efforts, although this is primarily just through examples of hydropower stations that undertake actions such as breeding and discharging rare fish species and investing in reforestation (Datang 2011a). No overarching policy for protecting biodiversity is provided, and Datang's Environmental Protection Plan (2006-2010), which focuses primarily on air pollution, contains no information of biodiversity protection. There are no indications of what measures Datang plans to take to protect diversity around the $29,603 \mathrm{MW}$ of new hydroelectric capacity that the company plans to develop in the coming years.

No information on Datang's EMS or EIA in Cambodia or Lao PDR could be gathered. It is clear, however, that given the three large hydroelectric dams that Datang is building in the countries, its most significant environmental impact will not relate to air pollution or $\mathrm{CO}_{2}$ emissions, but to biodiversity and river hydrology and ecology. Datang's Pak Beng (855 MW) and Sanakham (660 $\mathrm{MW}$ ) plants on the mainstream Mekong River in Lao PDR will dam the river and flood a total of $168 \mathrm{~km}^{2}$. According to a report by Halls and Kshatriya (2009) for the Mekong River Commission, Datang's dams will have a significant impact on the migration and spawning of fish, which in turn affects river ecology, biodiversity, and local communities' interests. In addition, the impoundment and flood discharge by the dams will change the natural hydrological conditions of the river. Datang provides almost no information on any policy or EMS it has in place nor any measures it has taken to avoid, mitigate, or manage these impacts. The company does briefly mention that it has installed fish ladders and has enabled confinement feeding in reservoirs to mitigate the negative impact on fish spawning. However, it does not provide any information about how or whether it plans to evaluate the effectiveness of these measures.

\section{Datang should be given credit for setting} targets for and investing in the reduction of $\mathrm{CO}_{2}$ emissions and other air pollutants. However, the sheer volume of Datang's emissions and the magnitude of its environmental impact, along with the fact that Datang continues to vigorously develop coal-fired power plants, means that the company cannot be considered to be in line with international SEP norms stipulating that companies should minimize emissions and prioritize low environmental impact technologies (WBCSD 2002, UNGC 2008, Pillinger 2009, Teske 2010, OECD 2011). Similarly, the company's development of large-scale hydroelectric dams both within and outside China reveal that it does not prioritize avoidance of impacts on biodiversity and ecosystem services (IHA 2004, IFC 2011, WBCSD 2002).

\section{Prioritize renewable sources of energy for electricity}

Though Datang believes that "companies have a responsibility to advance renewable energy sources", Datang's primary focus has been on coal-fired power (Datang 2009b). Datang owns China's largest coal-fired power station, the Inner Mongolia Tuoketuo power station, and 
Table 8.3: Datang's global fuel mix for electricity generation capacity and production, 2010

\begin{tabular}{|l|l|l|l|l|}
\hline Fuel type & $\begin{array}{l}\text { Installed capacity } \\
\text { (MW) }\end{array}$ & \% of total capacity & Production (GWh) & \% of total production \\
\hline Coal & 85,511 & $81 \%$ & 399,030 & $88 \%$ \\
\hline Natural gas & 0 & $0 \%$ & 0 & $0 \%$ \\
\hline Large hydro & 15,313 & $14 \%$ & 47,224 & $10 \%$ \\
\hline Wind & 5,051 & $5 \%$ & 6,237 & $1 \%$ \\
\hline Other renewable & 21 & $0 \%$ & 84 & $0 \%$ \\
\hline TOTAL & $\mathbf{1 0 5 , 8 9 6}$ & $\mathbf{1 0 0 \%}$ & $\mathbf{4 5 2 , 5 7 5}$ & $\mathbf{1 0 0 \%}$ \\
\hline
\end{tabular}

Based on: Datang 2011a

ranks as the country's second-largest consumer of coal (consuming $103 \mathrm{Mt}$ in 2008) and secondlargest emitter of carbon dioxide (emitting 250 Mt in 2008) (Datang 2011b, Greenpeace 2009). However, higher costs due to rising coal prices have pushed Datang towards other forms of power generation. The company owns the largest wind farm in operation in the world, the Inner Mongolia Chifeng Saihanba Wind Farm, and the second largest hydropower project in operation in China, Longtan Hydropower Project (Datang 2011b). Table 8.3 reveals the fuel mix breakdown of Datang's electricity generation capacity and 2010 production.

Other than shutting down some small, inefficient coal-fired plants, Datang does not have a policy to phase out fossil fuel in favor of renewable energy, but its strategy does include more rigorous development of renewables than new coal capacity. In the past decade, Datang has increased the percentage of hydropower in its capacity mix from $11 \%(2,712 \mathrm{MW})$ in 2002 to $14 \%$ (15,313 MW) in 2010. Similarly, the company's wind capacity increased from $0 \%$ in 2002 to $5 \%(5,051 \mathrm{MW})$ in 2010 , and the same period saw the percentage of coal-fired capacity reduced from $89 \%$ to $81 \%$. As can be seen in Table 8.4, Datang's build program for the near future envisions an even greater contribution from renewables, with $27 \%$ of the company's plans for 109,641 MW new capacity going toward large hydro and $16 \%$ toward wind. It must be noted, however, that Datang remains committed to coal, as $26 \%$ of the build program is dedicated to new coal capacity. Interestingly, the largest portion of the build program, $31 \%$, consists of new nuclear powered capacity, which Datang lumps together with wind and hydro in the category "new and renewable energy" (Datang 2011a). Thus while Datang has developed and continues to develop a significant amount of renewable capacity, the simultaneous development of an even greater amount of non-renewable (coal and nuclear) capacity means that the company cannot be considered to be in line with international SEP norms, which suggest that renewable sources should be prioritized (WBCSD 2002, UN/IAEA 2007, UNGC 2008, Pillinger 2009, Teske 2010). Datang's much smaller 1,947 MW build program outside China is mainly based on large-scale 
hydropower development in Burma, Cambodia, Kazakhstan, and Lao PDR for a combined 1,677 MW, $86 \%$ of the ex-China build program. The only thermal station planned is a 270 coal-fired plant in Indonesia, $14 \%$ of the ex-China build program (Datang 2010, Datang 2011a). Despite developing wind power on a large scale in China, Datang is not promoting or developing any other, more sustainable forms of electricity generation technology in these countries. Nevertheless, Datang's more concentrated focus on renewable hydropower in Cambodia and Lao PDR means that it is more in line with international SEP norms on this issue abroad than in its home country.

\subsubsection{Economic issues}

\section{Contribute to local economic development}

Datang (2011a) claims that the primary way it contributes to society is through "driving economic development by meeting power demands and paying taxes". The 472,575 GWh of electricity the company generated in 2010, its investment of $¥ 60.26$ (\$9.6/€7.3) billion in developing electricity infrastructure, and its payment of $¥ 11.5$ (\$1.8/€1.4) billion in taxes in 2010 corroborate that claim. Datang does not have a policy to employ local workers or prioritize SME suppliers in procurement. In terms of promoting economic development at the local level, Datang (2011a) primarily provides examples of charitable donations to community development projects and cultural activities.

Datang says less about promoting local economic development in its operations outside China. The company claims that it "supports local economic development", but the example that it provides for how it does so is "by actively participating in social welfare to support the development of education and to alleviate the hardships of affected residents" (Datang 2011a). There is no indication that Datang employs any sort of mechanism for measuring the overall economic impact of its activities on communities, and nothing is mentioned about prioritizing local workers or SME suppliers of materials at the company's projects either at home or abroad, though such policy measures are recommended by SEP norms (WBCSD 2002, Pillinger 2009, OECD 2011).

In 2007, Huot Pongan, Cambodia's UnderSecretary of State for Industry, Mines and

Table 8.4: Datang's build program as of 2010, by fuel type

\begin{tabular}{|l|l|l|}
\hline Fuel type & Planned capacity (MW) & Share of build program (\%) \\
\hline Nuclear & 33,989 & 31 \\
\hline Large hydro & 29,603 & 27 \\
\hline Coal & 28,507 & 26 \\
\hline Wind & 17,543 & 16 \\
\hline TOTAL & 109,641 & 100 \\
\hline
\end{tabular}


Energy, claimed, "We don't have enough electricity. China is developing that for us. China has been here for a long time and will stay here for a long time" (cited in Brewer 2008). Datang is investing $\$ 360$ million (€265 million) in the Stung Atay power station in Cambodia and $\$ 880$ million (€673 million) in the Sanakham plant in Lao PDR. Datang does not publish information about its investment amount in the Pak Beng dam in Lao PDR (Datang 2012). Datang is clearly contributing to economic development through increasing power supply, but the practice of bringing in a large number of Chinese workers rather than hiring and training locals is a major point of contention with communities adjacent to the dam construction sites. According to Parnell (2010), Cambodian law stipulates that the labor force brought in from a foreign investor's home country should not exceed $10 \%$, and a similar regulation in Laos sets a $25 \%$ ceiling on non-local workers. Datang (2010) claims that it has employed up to 1,000 Cambodian laborers at the peak of construction activities. However, field visits to dam construction sites in Cambodia and Lao PDR revealed a far lower number of Cambodian and Lao nationals at work, and a high percentage of Chinese employed. Company managers in Lao PDR suggested that the harsh working conditions involving long hours, no overtime pay, and minimal safety protection are something that only Chinese migrant workers will accept because they are used to such conditions in China (Anonymous manager in Lao PDR 3 2009). Another manager asserted his opinion that Laotians are "lazy" and "weak" and said, "If we hire large numbers of Laotians, the quality and procedure of the projects will be negatively affected, so we have to recruit more Chinese and use few locals" (Anonymous manager in Lao PDR 2 2009). With regard to the Cambodian and Lao regulations on use of domestic versus foreign labor, a representative of the Chinese Chamber of Commerce in Cambodia clarified that, "In terms of the number of local laborers, Chinese state-owned power enterprises are far from the legal proportion stipulated in the regulations. However, this does not simply or directly mean that they have violated the laws, because, in many cases, if Chinese companies need to introduce large numbers of Chinese, they would submit application to and seek approval from the Congress or the government" (Anonymous representative of the Chinese Chamber of Commerce in Cambodia 2010). It is not clear whether Datang has applied for or received such permission.

Thus both in its corporate policies as well as in practice in Cambodia and Lao PDR, Datang has performed well on developing energyrelated infrastructure to drive economic development (WBCSD 2002). However, it has performed poorly in some of the other areas, such as prioritizing local workers and small and medium-sized enterprise (SME) suppliers, suggested by the SEP norms to drive local economic development (WBCSD 2002, Pillinger 2009, OECD 2011).

\section{Ensure reliable supply}

Datang (Datang 2011c) prides itself on "maintaining a steady supply of electricity". Datang has power supply agreements with the 
Chinese government and state grid company, and notes that "we strictly execute the synchronization and dispatching agreements as well as power (heat) purchase agreements and supply sufficient and qualified power and heat to users" (Datang 2009a).

The company claims that it invested $¥ 60.26$ billion (\$9.6/€7.3b) in electricity infrastructure to ensure reliability of supply in China in 2010 (Datang 2011a). No further information was readily available on whether Datang has a policy on maintaining contingency plans to respond to generation interruptions in a timely manner, as is encouraged by SEP norms (WBCSD 2002, CEER 2005).

Little information could be gathered on this issue in Cambodia and Lao PDR because the hydroelectric facilities are not yet supplying electricity. However, there was no information readily available on whether Datang maintains contingency plans to respond to generation interruptions in a timely manner.

\subsubsection{Cross-cutting issues}

Engage in meaningful stakeholder consultation and participatory decision-making

Despite the high impact on the health and livelihoods of the human population living near Datang's coal-fired and hydroelectric power plants and construction sites, the company does not have a public policy on stakeholder engagement and participation in decisionmaking. The sustainability report mentions various stakeholders, but this is generally done in a superficial manner lacking in content and detail. Though Datang suggests that its projects "should be acknowledged and monitored by the society" (Datang 2008), no mechanism for stakeholder consultation or participatory decision-making is provided, nor are there any references to any of the international normative standards on stakeholder engagement (IFC 2007, OECD 2011, Pillinger 2009, WCD 2000, ISO 2011, IFC 2011, IHA 2004). No mention is made of stakeholders' rights to free, prior, and informed consent. The 2010 report highlights several stakeholders that have praised Datang, but the range of stakeholders is limited to the provincial government of Guangxi, the municipal government of Beijing, the Chinese State Grid Corporation and another business partner, and the China office of the Global Compact (Datang 2011a). There is no evaluation from affected communities, workers, or CSOs. No mention is made of the communities voluntarily and involuntarily relocated from the company's hydropower sites. Instead, "stakeholder engagement" activities organized by Datang include a press conference to launch its annual sustainability report and workshops for journalists aimed at "enhancing the public understanding of China Datang Corporation" (Datang 2008).

Datang headquarters did not respond to repeated requests by e-mail, post, and telephone for information for the present study. Researchers in Cambodia and Laos were able to interview local Datang managers, who insisted on remaining anonymous. 
Datang's damming of the mainstream Mekong River and flooding of $168 \mathrm{~km}^{2}$ in Lao PDR will have a significant impact on the lives and livelihoods of communities and individuals living in the vicinity of the dams (Halls and Kshatriya 2009). The flooding will lead to the displacement of people living in and near the planned reservoir area. A Datang manager in Lao PDR described one particularly problematic resettlement the company is facing involving a traditional Hmong village. The village is located just outside the reservoir area and so above ground will not be flooded, but traditional Hmong houses have wooden foundations that reach down into the ground. The damming of the river will cause the groundwater to rise nearly to the surface for the whole year, when normally this happens for just one month each year. Constant saturation of the wooden foundation in water will cause it to rot (Anonymous manager in Lao PDR 4 2009). In addition to those in the way of the reservoir, many more poor rural communities that depend heavily on the fish and other aquatic flora and fauna for their livelihood will be affected by the dams. Others use the river for transportation or other economic activities (Halls and Kshatriya 2009). For these types of serious impacts, international SEP norms consider extensive meaningful consultation with all affected stakeholders to be essential (IFC 2007, OECD 2011, Pillinger 2009, WCD 2000, ISO 2011, IFC 2011).

Datang claims that its procedures on involuntary displacement in Laos have followed the Lao displacement law with regard to compensation of affected people and guidelines set by the World Bank and the Asian Development Bank on consultation and other issues. However, one manager in Laos asserted, "Actually there is no need for us to follow the high World Bank standards because the Lao regulations and permitting processes ensure that Western standards are applied" (Anonymous manager in Lao PDR 4 2009). The Lao government has organized public hearings encouraging participation from a wide range of stakeholder groups, including local communities and CSOs for a hydroelectric dam being constructed by Sinohydro. However, a similar procedure has not been conducted for Datang's hydropower projects in Laos, because the project is still under the process of prior consultation and agreement. A manager explained that in his opinion, the involuntary displacement problem is not as bad in Lao PDR as it is in China because the area of submerged land and the number of displaced people are relatively small and because the displaced communities in Lao PDR are often so poor that they are willing to be resettled in the hope of a better life (Anonymous manager in Lao PDR 12009 ).

Several managers also indicated that much more was expected of the company with regard to stakeholder consultation in Lao PDR than in China. "When we seek to acquire land in Lao PDR, the government plays an intermediary role, but the agreement is mainly achieved through direct communication and negotiation between the company and the local residents" (Anonymous manager in Lao PDR 1 2009). This is quite different from the 
approach in China where the government is fully responsible for land acquisition, compensation, and resettlement. "It is not necessary for enterprises in China to negotiate with the displaced people or the residents whose interests have been harmed because our agreement is with the government, not the people" (Anonymous manager in Lao PDR 3 2009). In Lao PDR, on the other hand, "we must talk to and negotiate with the local governments, village leaders, and residents face-to-face. All the relationships should be built and compensation standards have to be agreed through negotiation. We are obliged to report to the local government the contracts that have been signed by local residents, such as the size of land to be submerged, the number of displaced people, compensation standards, and so on" (Anonymous manager in Lao PDR 3 2009). However, another manager admitted that Datang has experienced difficulties with this approach and that the company's Chinese culture and customs still sometimes lead it to adopt a more "Chinese" way of doing things (Anonymous manager in Lao PDR 4 2009). He described a situation in which Datang had negotiated a resettlement agreement with some villagers. However, after receiving compensation, the villagers refused to move. He noted that such a situation would be "unlikely to happen in China where the local government will intervene if we have any difficulty". Instead of further direct dialogue and engagement with the community to attempt to resolve the dispute, the manager confided that, "The only way we could think of to resolve the issue was to give the money to the government and ask them to solve the problems" (Anonymous manager in Lao PDR 4 2009).

With regard to Datang's performance on stakeholder engagement in Cambodia, Han (2010) noted that "with the friendly ChinaCambodia relationship, most of the hydropower cooperation projects reflect the will and interests of the governments rather than the public. Therefore, Chinese investments in Cambodia have encountered many problems". In an interview with researchers collecting information for the present study, a Cambodian villager said, "We would like more chances to talk to the company. We wanted to talk to them face-to-face but we did not have the opportunity. I think the company should hire a good interpreter who can truly understand the opinions of the local people and deliver the messages to the company" (Cambodian villager 12010).

\section{Assume responsibility for impacts throughout} all business relationships

Datang (2011a) does not make any mention of having responsibility for or seeking to prevent, avoid, and mitigate negative social and environmental impacts throughout its range of business relationships, as is suggested by international SEP norms (OECD 2011, UNHRC 2011, ISO 2011). Datang does say that it uses a survey to assess suppliers, but this is primarily focused on aspects such as quality, unfair competition, and bribery. On the GRI G3 indicator that deals with screening of human rights among suppliers and contractors (item 97), Datang responded "not covered" in its 2010 
sustainability report (Datang 2011a). A manager in Cambodia confirmed that this is also the practice there, noting "When we contract out projects to construction companies... we require that the company be responsible for technical administration, evaluation of construction progress, and quality. We don't evaluate whether the contractors have respected the migrant workers' rights" (Anonymous manager in Cambodia 12010 ).

\section{Maximize transparency \& provision of information}

Other than its annual sustainability reports, very little is available in the way of $C R$ policies on Datang's website. However, Datang sustainability reports have been praised by the Chinese Academy of Social Sciences and the China office of the Global Compact as being "well-organized, readable, and innovative" (Datang 2011c). Sustainability reports dating back to 2007 can be found on the company's website, and the reports covering the years 2006-2008 have an English and a Chinese version (no English version is available for the 2009 or 2010 report). Datang primarily relies on the CASS "Guidelines on Formulation of Chinese CSR Reports" as a reporting framework for its sustainability reports. Datang also uses elements of the GRI G3 and EUSS reporting guidelines, but it has an "undeclared" rating, which generally means that only a very few of the GRI indicators are actually reported on (GRI 2012). For example, Datang does not report on GRI indicators related to human rights. Some information is provided about Datang's customers (primarily the Chinese
State Grid Company), but no information about suppliers of coal or other materials is included. Datang claims that its sustainability reports have been quality assured by an independent assurer since 2008, but the GRI (2012) lists Datang's report as "not externally assured". The sustainability report provides minimal information on Datang's operations outside China. No information could be gathered on the degree to which Datang provides local language information about its operations and its CR policies to stakeholders in Cambodia and Lao PDR.

\subsubsection{The Chinese mode and Datang's performance on the SEP benchmarks}

Datang's performance on the SEP benchmarks indicates that the Chinese mode of business culture plays a significant role in conditioning how Datang takes up the internationallyderived standards for SEP and, to a lesser degree, how it implements them on the ground in host countries. Table 8.5 provides an overview of the degree to which Datang's performance on SEP benchmarks is deemed to reflect the Chinese mode as described in Chapter 4 and summarized in Table 4.1.

The priority Datang gives to Chinese CR standards is reflective of the Chinese mode, which has sought to define CR on its own terms rather than simply following the "Western model" of CR. Nevertheless, the UN Global Compact is an international standard that is relatively highly-regarded by the Chinese 
Table 8.5: Degree to which Datang's performance on SEP benchmarks reflects the Chinese mode of business culture

\begin{tabular}{|c|c|c|}
\hline $\begin{array}{l}\text { Is Datang's policy/practice reflective of the Chinese mode on the } \\
\text { following SEP benchmarks? }\end{array}$ & Policy & Practice \\
\hline Endorse international normative standards for CR & Largely reflective & Moderately reflective \\
\hline $\begin{array}{l}\text { Adopt a commitment to CR in core-business activities and } \\
\text { decision-making }\end{array}$ & Largely reflective & Moderately reflective \\
\hline Increase access to affordable electricity & Largely reflective & Moderately unreflective \\
\hline Respect labor rights & Largely reflective & Largely reflective \\
\hline $\begin{array}{l}\text { Minimize environmental impact, including contribution to climate } \\
\text { change }\end{array}$ & Largely reflective & Moderately reflective \\
\hline Prioritize renewable sources of energy for electricity & Largely reflective & Moderately unreflective \\
\hline Contribute to local economic development & Largely reflective & Moderately unreflective \\
\hline Ensure reliable supply & Moderately reflective & $\mathrm{n} / \mathrm{a}$ \\
\hline $\begin{array}{l}\text { Engage in meaningful stakeholder consultation and participatory } \\
\text { decision-making }\end{array}$ & Largely reflective & Moderately reflective \\
\hline $\begin{array}{l}\text { Assume responsibility for impacts throughout all business } \\
\text { relationships }\end{array}$ & Moderately reflective & Moderately reflective \\
\hline Maximize transparency and provision of information & Largely reflective & Moderately unreflective \\
\hline
\end{tabular}

mode, so it is no surprise that Datang publicly endorses this standard. It is surprising that Datang has not sought ISO 14000 certification for its environmental management, as this is another international standard that is generally well regarded in the Chinese mode.

Datang's focus on economic development is reflective of the Chinese mode and Chairman Deng Xiaoping's imperative of setting "economic development as the central task". Datang's tendency toward an unstructured CR program whose primary visible contribution is charitable donations - such as those to schools and supermarkets in Lao PDR - is also typical of the Chinese mode, which the Asia-Pacific
Economic Cooperation Secretariat (APEC 2005) characterizes as resting on a long tradition of charity and philanthropy expressed through unstructured, ad hoc donations rather than through a coherent strategic corporate policy. The frenzied approach to mentioning CR targets, commitments, and achievements reflects the Chinese mode's more recent public embrace of the notion of CR as contributing to broader sustainable development.

Datang's attention to large-scale, national economic development and international "prestige" events, rather than the (human) rights of individuals is largely reflective of the Chinese mode. Datang makes no mention 
of workers' right to freedom of association, collective bargaining, or to strike. Datang's lack of respect for labor rights in Cambodia and Lao PDR appears largely conditioned by the Chinese mode.

Datang's policy to focus on developing large coal and hydroelectric plants reflects the Chinese mode, which prioritizes economic development and the generation of as much electricity as possible to supply the growing economy. However, the fact that Datang has not sought ISO certification for its EMS is surprising given the Chinese mode's uptake of technical standards like the ISO 14000 series (Sutherland and Whelan 2009). Datang's focus on hydropower only in Cambodia and Lao PDR is less reflective of the Chinese mode.

Datang's approach of primarily top-down rather than participatory - decision-making and very limited engagement with stakeholders is largely reflective of the paternalistic overtone of the Chinese mode. A Datang manager in Lao PDR admited that though they are trying new and "different" forms of stakeholder engagement, the company often reverts to the "Chinese way" of doing things (Anonymous manager in Lao PDR 4 2009). Datang does check and audit suppliers and contractors, but "doesn't evaluate whether the contractors have respected the migrant workers' rights" (Anonymous manager in Cambodia 1 2010). This approach is in line with the Chinese mode, which does demonstrate considerable interest in monitoring the supply chain (Welford 2005), but places lower priority on social and environmental concerns than on economic interests. This is true both in a company's own operations as well as in its business relationships. 


\section{Chapter 9}

Case study - Eskom
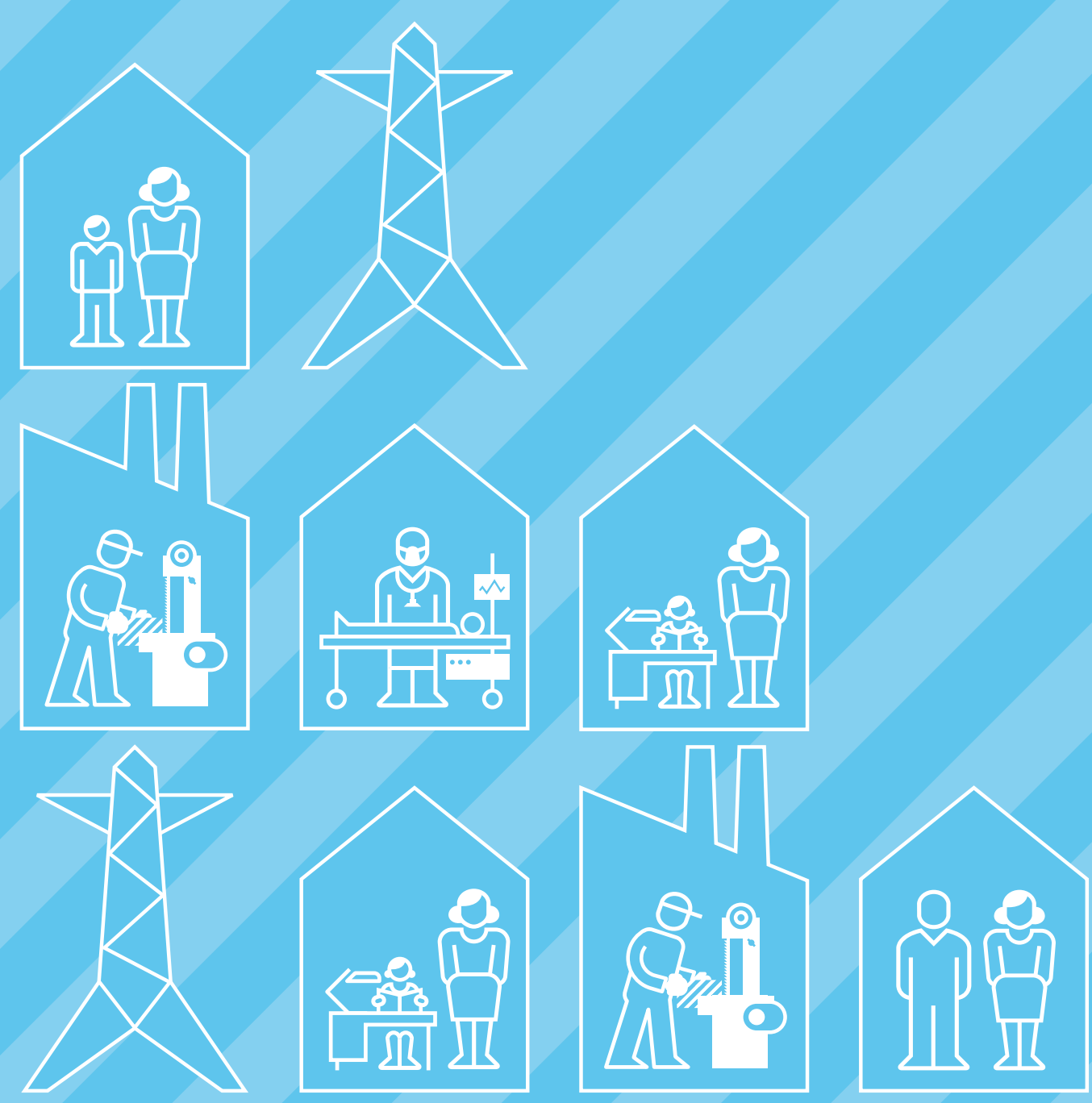


\subsection{Eskom background and operations}

\subsubsection{Basic company information}

Based in Johannesburg, South Africa, Eskom Holdings Limited (Eskom) is a vertically integrated, state-owned electric utility that generates, transmits and distributes electricity to industrial, mining, commercial, agricultural, and residential customers and redistributors in Africa. Since its founding in 1923, the history of Eskom (then called Escom) is deeply intertwined with the development and industrialization of South Africa and the country's mining industry in particular.

Eskom was created as a commission intended to grow the capacity of electricity supply at cost price by acquiring private power plants or building new ones (1984). The cornerstone of South African industrial development at the turn of the century was rich mineral deposits. Eskom was created to supply cheap, reliable electricity to South Africa's globally-competing gold mining industry. By 1948 Eskom was in control of nearly $100 \%$ of electricity provision in South Africa, and $59 \%$ of that electricity was being consumed by gold mining (Gentle 2009). Energy requirements were structured around these needs, and - in what is known as the minerals-energy complex - the dominance of industrial interests (primarily mining) informed the energy supply paradigm and state development policy in South Africa. South Africa's industrialization benefited from the "cheap" power derived from the country's abundant coal supply and non-profit-based production by Eskom for well over half a century (Fine and Rustomjee 1996).

In 1985 and again in 1987, the 1922 Electricity Act was amended to effectively replace the commission nature of Eskom with a parastatal one. This was at a time when the apartheid government was moving towards neoliberal policies and was privatizing a number of state-owned companies (Gentle 2009). The move was intended to create greater financial control and to professionalize the utility after large-scale expansion had dramatically increased the price of electricity (Government of South Africa and De Villiers 1984). The amendments did away with stipulation that "electricity should be supplied in the public interest [...with] operations being carried out neither for profit or loss" (Government of South Africa 1922). This was replaced by "consideration for consumer needs being satisfied in the most cost effective way, subject to resource constraints and the national interest" (Government of South Africa 1985 cited in Gentle 2009).

A national electricity regulator was created in 1995 and Eskom was directly regulated by an external body for the first time since 1923. Eskom therefore reorganized, collecting regulated and unregulated business activities into separate units. The transmission, distribution and generation functions of the utility were ring-fenced in preparation for privatization. Eskom Enterprises became the umbrella for unregulated activities. The objective of the Eskom Enterprises division was to generate large-scale profits from diversified 


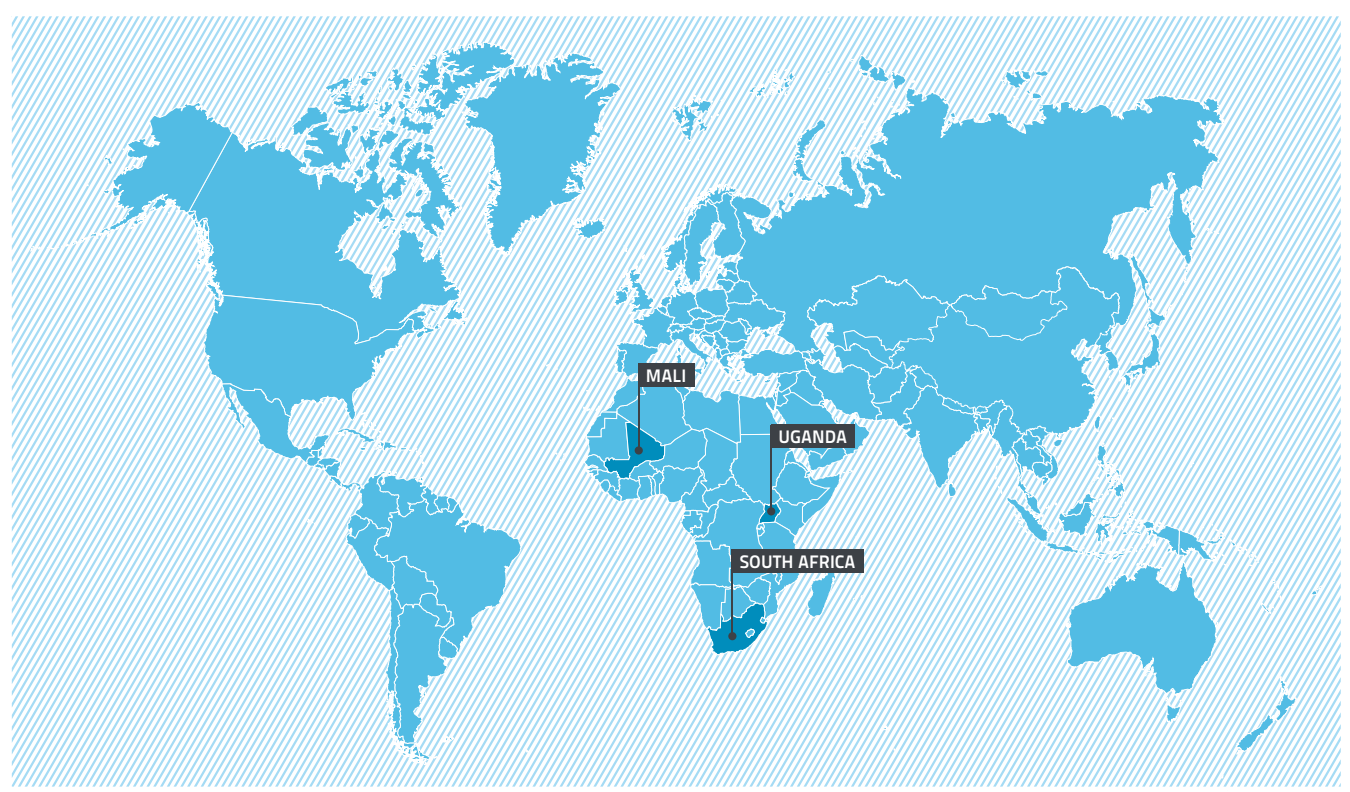

Figure 9.1: Eskom's global presence, 2010

unregulated activities within South Africa and the African region. It was to be run essentially as a transnational corporation that would take advantage of the wave of privatization and commercialization in the electricity and communications sectors that were occurring in Africa at the time (Koen 2010). The plans for privatization, however, have not materialized, largely because the price of electricity was so low that private companies could not compete. Eskom had continually reduced the real price of electricity from 1986 to 2004. Eskom remains $100 \%$ owned by the South African government (Steyn 2006).

Today, Eskom's role continues to transcend the provision of electricity alone and remains inextricably intertwined with South Africa's industrial growth and development aspirations.
Eskom (2011) summarizes the company's pledge to contribute to South Africa's development by providing electricity to all South Africans and having mutually beneficial arrangements with support industries, such as the smelting and coal mining sector. With over $40,000 \mathrm{MW}$ of installed electricity generation capacity and 237,430 GWh of electricity produced in 2011, Eskom is by far the largest producer of electricity in Africa and is among the top ten utilities in the world in terms of generation capacity and sales (Eskom 2012a). The company generates approximately $95 \%$ of the electricity used in South Africa and approximately $45 \%$ of all electricity consumed in Africa. Eskom plans to double its electricity generation capacity by 2025 to over 80,000 MW at an estimated cost of $\$ 170$ (€130) billion (Eskom 2010). Eskom's current production park is heavily reliant on coal-fired power stations 
Table 9.1: Overview of Eskom's key operational indicators, 2011

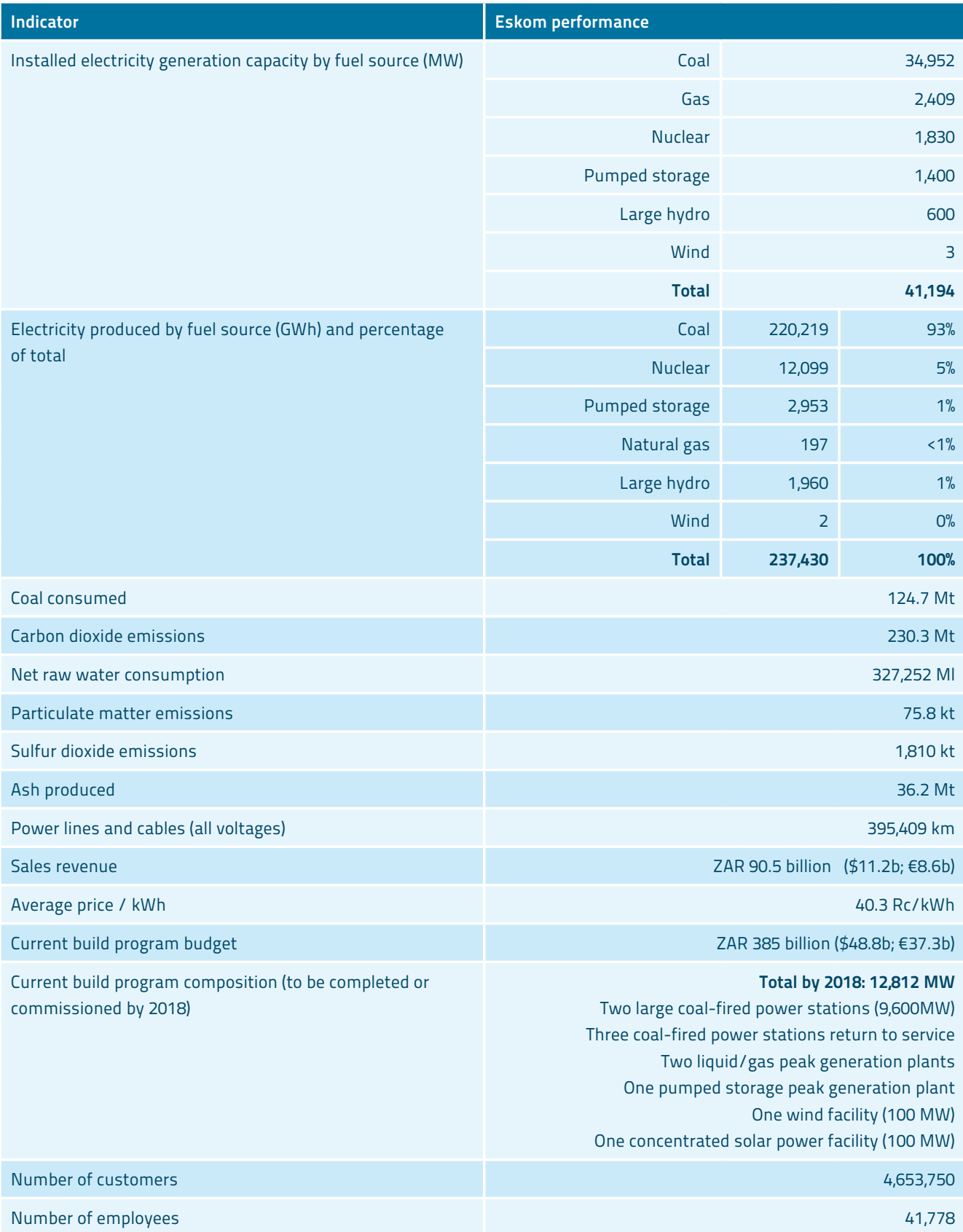


( $93 \%$ of total production is based on coal), and the bulk of Eskom's immediate $\$ 48.8$ (€37.3) billion build program consists of two additional coal-fired power stations. As a result, Eskom is the largest corporate purchaser of coal in the world (Lines 2007) and the second largest emitter of $\mathrm{CO}_{2}$ globally (Grant 2007). Table 9.1 provides an overview of Eskom's performance on some key operational indicators.

\subsubsection{Operations and investments in the Global South}

Eskom began the expansion of its electricity provision operations into other southern African countries with the creation of the Eskom Enterprises division of the company in 1998. Eskom Enterprises was established to pursue all Eskom's non-regulated business, and it immediately began tendering for electricity provision projects in Africa. Eskom's expansion into Africa was touted in its "In Africa" policy as the South African state's tangible infrastructure investment in the African Union's economic development program, the New Partnership for Africa's Development (NEPAD) (Gasa 2005).

At the height of Eskom's involvement on the continent, the company had activities in 30 African countries. However, it soon became apparent that Eskom's electricity provision operations outside of South Africa were not bringing in the returns that were anticipated (Botha 2003). As South Africa experienced power shortages in 2007 and 2008, other African operations came to be seen as a drain on capacity needed in South Africa. Eskom restructured and began to scale back its activities in Africa, selling off a large number of assets and operations in Lesotho, Libya, Uganda, Zambia, and several other African countries between 2007 and 2009. Eskom's 2010 annual integrated report states that the future involvement of Eskom in markets outside South Africa is limited to those projects that have a direct impact on ensuring security of supply for South Africa (Eskom 2011). Eskom remained involved in electricity provision in Mali and Uganda throughout 2012, but had already begun seeking an exit to the Mali concession in 2011 (Eskom 2012a). Figure 9.1 maps Eskom's global presence.

\subsubsection{Eskom in Mali}

In 2001, Eskom Enterprises won a $\$ 75$ million $(€ 57 \mathrm{~m})$ contract to operate and maintain Manantali, a $200 \mathrm{MW}$ hydroelectric power plant on the Bafing River, a tributary of the Senegal River in Mali. The contract has a 15-year term and includes responsibilities for the commercial operation, hydrological planning, management of the reservoir, production planning, power dispatch, power sales and the operation and maintenance of facilities, as well as river flow management. In Mali, Eskom generates approximately $800 \mathrm{GWh}$ per year at the Manantali hydroelectric station and transmits the electricity generated over a 1,500 km high tension transmission line to the Malian capital of Bamako as well as to grids in neighboring Senegal and Mauritania (Business Wire 2001). After 10 years of operation, in early 2012 Eskom announced that it was having "difficulties in executing the demands of the contract" and 
that it had reached an agreement with regiona electricity authorities in Mali to terminate its contract as of 31 July 2012 (Dieme 2012).

Prior to Eskom's entrance as operator of the dam, Manantali had been criticized by international civil society organizations as being "one of the worst dams in the world, putting an end to 1,000 years of successful flood-recession farming; creating major economic impacts for downstream farmers, fishers and herders; harming fisheries, ground water resources and riverine forests; and turning an area with a low incidence of water-borne disease into one of the worst-infected in Africa. Besides all the problems it caused, it also failed to provide promised benefits. The conversion from flood-recession farming (i.e. the cultivation of riverbank areas enriched by silt from retreating annual floods) to irrigated agriculture has been much slower and costlier than expected. In addition, irrigated agriculture has actually been less productive than flood-recession farming, and contributes to water-borne diseases via irrigation canals and water-storage areas" (Pottinger 1997). How Eskom has dealt with these issues since taking over operational control of the dam in 2001 is discussed in this chapter.

\subsubsection{Eskom in Uganda}

In April 2003, Eskom, operating through its subsidiary Eskom Uganda, took over the Uganda Electricity Generation Company Ltd at Jinja, Uganda. In so doing, Eskom assumed control over the operation and maintenance of Nalubaale Dam (previously Owen Falls) and Kiira (previously Owen Falls Extension) hydroelectric plants under a 20-year concession. In 2003, the hydroelectric stations comprised just 300 MW of installed capacity, but since then Eskom has increased capacity by an additional 80 MW. The current total installed capacity of the two facilities is $380 \mathrm{MW}$, but in recent years the plant has only been able to operate at approximately half capacity due to low water levels. In 2010, Eskom Uganda generated a total of 1,253 GWh, which gave it an approximately $51 \%$ share of the entire Ugandan electricity generation market (Mngeni 2011).

\subsection{Eskom's performance on the SEP benchmarks}

This section begins with an overview (in table format) of Eskom's performance, in both policy and practice, on the 11 SEP benchmarks. For each SEP benchmark, a reminder of the guiding international normative standards (taken from Chapter 3 ) is provided alongside key indicators of the company's performance and an evaluation of the degree to which the company's policy and practice are line with the SEP standards on that issue. Following the overview, the results of the empirical research on Eskom's policy and practice in Mali and Uganda are detailed for each SEP benchmark. These findings form the basis for the overview and evaluation provided in Table 9.2. The chapter concludes with a brief reflection on the relationship between the South African mode of home-country business culture (as outlined in Chapter 4) and Eskom's performance on the SEP benchmarks. 
Table 9.2: Summary of Eskom's performance on SEP benchmarks in policy and in practice

\begin{tabular}{l} 
SEP benchmark \\
General approach to corporate responsibility \\
Endorse international normative standards for CR \\
\hline
\end{tabular}

Adopt a commitment to CR in core-business activities and decisionmaking

\section{Guiding international normative SEP standards}

Electric utilities should seek to bring their policies in line with internationally recognized and verified standards for CR (ILO 2001, WBCSD 2002, IFC 2011, ISO 2011, OECD 2011، UNGC 2008, UNHRC 2011).

Electricity companies should adopt a holistic and integrated view of the role and impacts of electricity provision, considering and balancing the essential elements of economic development, environmental quality, and social equity in utility operations. This entails a policy commitment to CR throughout all core-business activities, as opposed to a primary focus on philanthropic activities (WBCSD 2002, MVO Platform 2012). Electric utilities should seek to introduce environmental and social factors and procedures into high-level corporate planning and decision-making and should aim to have a board-level position responsible for CR issues (WBCSD 2002).

\section{Social issues}

Increase access to affordable electricity
Electric utilities should develop policies that promote access to electricity priced at affordable levels for all, paying special attention to disadvantaged communities (ILO/Palast et al. 2000, WBCSD 2002, PSI/Pillinger 2009). In order to do so, companies should undertake initiatives to extend electricity services to unserved and underserved communities, particularly in rural or remote areas, but also to the poor in urban and peri-urban areas (WBCSD 2002, UNDP/Modi et al. 2005). 


\section{Indicators of Eskom's policy}

- Endorses UNGC, ILO Core Conventions, King Code, AA1000, Equator Principles

- Wide use of ISO (in particular 14000) and South African NOSA certification system

- Moderately in line with SEP standards

- Prior to corporatization, CR largely focused on core-business electrification. Since corporatization, philanthropy and charity much more prominent

" "Corporate Social Investment" defines approach to CR

- (BB)BEE give high degree of importance

- A board-level committee chaired by the CEO handles safety, health and environmental issues

- Environmental performance managed as an integral part of Eskom's governance structure

- Moderately in line with SEP standards

\section{Indicators of Eskom's practice in Mali and Uganda}

- Local managers are largely unaware of international normative standards, emphasize "African" values

- Plants have NOSA certification and reference ISO 9000 and 14000

- Moderately out of line with SEP standards

- SHE policy in place, but CR almost wholly focused on philanthropic activities and charitable donations

" Eskom has "no responsibility" for core-business CR aspects like increasing access to affordable electricity, which are "far removed from the company's role in the project"

- Largely out of line with SEP standards
- Before corporatization, Eskom performed remarkably well on increasing access to electricity, which it claimed was its "CR" to do. Since corporatization, Eskom has backed away from this claim and has performed less well on increasing access

- Electricity prices have been prohibitively high for the poorest, resulting in a high number of (self) disconnections. Large (mainly mining) corporations have largely been the prime beneficiaries of Eskom's cheap electricity

- Focus on supplying large corporate customers, especially in electricity exports

- Little to no support for off-grid, decentralized electricity provision projects

- $100 \%$ of Eskom's total installed capacity of 41,194 is located in countries without universal access to electricity, but only $580 \mathrm{MW}(1 \%)$
- Eskom produces $800 \mathrm{GWh} /$ year in Mali and 1,250 GWh/year in Uganda.

- National electrification rate in Mali is $45 \%$, but the rate in the areas adjacent to Eskom's operations is $10 \%$. In Uganda, national rate is $9 \%$, and below $1 \%$ in Eskom's area of operation

- Electricity prices for small domestic consumers in area of operations are $\$ 0.12 / \mathrm{kWh}$ in and Mali and $\$ 0.17$ in Uganda (highest in the region)

- Local managers unaware of electrification rates and electricity prices in adjacent communities. Promoting access to affordable electricity is "neither Eskom's mandate nor responsibility"

- No off-grid, decentralized projects. Operations have been exclusively centralized and grid-based

- In Uganda, one community leader praised Eskom for bringing electricity to his community, but other stakeholders were largely disappointed with lack of progress in increasing access or affordability

- Largely out of line with SEP standards 


\section{Respect labor rights}

\section{Environmental issues}

Minimize environmental impact, including contribution to climate change
Electric utilities should respect workers' full rights and provide workers with fair pay, job security, and decent working conditions, including high levels of health and safety standards in all aspects of operations (WBCSD 2002, PSI/Pillinger 2009, OECD 2011). Furthermore, electric utilities should uphold freedom of association and the right to collective bargaining and pay particular attention to the right to strike (ILO 1998, ILO 2001, UNGC 2008, OECD 2011). Companies should ensure that these rights are respected not only among their own employees, but also among suppliers, contractors, and other business relationships (ISO 2011, OECD 2011, UNHRC 2011).

\section{Guiding international normative SEP standards}

Electric utilities should seek to minimize emissions and discharges to air and water and continually develop and implement low-pollution and low-environmental impact technologies (WBCSD 2002, UNGC 2008, PSI/Pillinger 2009, OECD 2011). Companies should manage their environmental impact in line with internationally recognized and verified standards, such as the ISO 14000 series and should always undertake rigorous and verifiable environmental impact assessments (WCD 2000, WBCSD 2002, IHA 2004, OECD 2011,). Electric utilities should minimize their contribution to climate change and develop strategies for reducing GHG emissions (WBCSD 2002, OECD 2011). As a matter of priority, electric utilities should seek to avoid impacts on biodiversity and ecosystem services, refraining from any activities in areas of critical habitat with high biodiversity value while at the same time initiating or supporting conservation and biodiversity efforts related to impacts on natural habitats from utility operations (WBCSD 2002, IHA 2004, IFC 2011).

Electric utilities should evaluate the renewable energy potential of the host country and prioritize development and exploitation of renewable options over fossil fuel-based technologies (WBCSD 2002, UN/IAEA 2007, PSI/Pillinger 2009). Electric utilities should also prioritize renewables in their research and development programs and should support the diffusion of renewable energy technologies into the local economy (WBCSD 2002, UNGC 2008). 
- Endorses ILO Core Conventions, though the right to strike is not guaranteed

- Public support for freedom of association and collective bargaining, but electricity's status as an "essential service" prohibits workers from striking

- Policies and structures are in place to engage and consult workers on key operational decisions

- SHE policy contains comprehensive framework for dealing with $\mathrm{OHS}$ issues. Zero tolerance policy for injuries, but in 2007-2011 there were 39 fatalities among Eskom employees, and 221 fatalities among contractors working for Eskom

- SHE policy covers the rights of workers at contractors and suppliers. Eskom evaluates potential suppliers and contractors on $\mathrm{OHS}$

- Largely in line with SEP standards

\section{Indicators of Eskom's policy}

- Enormous environmental impact, but does have in place a policy to continually reduce some aspects of its environmental pollution. At the same time, plans for maintaining and even increasing the company's reliance on fossil fuels (primarily coal) indicate a lack of commitment to genuinely minimizing environmental impact

- Company-wide ISO 14000-based EMS in place, though not all divisions are certified

- Global frontrunner on conducting EIAs

- Global $\mathrm{CO}_{2}$ emissions 230.3 Mt in 2011; world's secondlargest corporate emitter of $\mathrm{CO}_{2}$

- Plans to increase GHG emissions in the short and medium term, then peak, plateau, and reduce emissions in the long term

- Policy on biodiversity is largely to align itself with South Africa's commitments under the CBD, a WBCSD pilot program, and partnerships with conservation CSOs

- Largely out of line with SEP standards

- Current annual electricity production is $237,430 \mathrm{GWh}: 93 \%$ coal, $5 \%$ nuclear, $1 \%$ other non-renewable, $1 \%$ hydro

- Though conducting research on renewables, Eskom will continue to prioritize fossil fuels

- R\&D budget for sustainable energy in 2010: $\$ 253,865$

- Current build program consists mainly (75\%) of new coal capacity, some gas, and less than $2 \%$ wind and solar

- Largely out of line with SEP standards
- Unions present in Mali and Uganda - $80 \%$ and $56 \%$ of workers are members, respectively

- Generally good relationship between the union and the management in both countries, though some disputes over wages in Uganda. Eskom Mali manager says workers have the right to strike

- CBA in effect in Mali and Uganda, covering $80 \%$ of workers in Mali

- Lowest paid employees of Eskom Mali earn \$100/month, double the legal minimum wage

- Local management implements SHE policy on OHS. 1 fatality reported in Uganda in 2009. No fatalities, though one poisoning incident in Mali

- In Mali, manager insists all workers have a contract. In Uganda, $50 \%$ of the total number of workers at the plant are contract workers. Eskom Uganda monitors contractors on OHS issues

" Largely in line with SEP standards

\section{Indicators of Eskom's practice in Mali and Uganda}

- EMS based on ISO 14000 in place, but certification not (yet) achieved

- Eskom Mali did conduct an EIA prior at the start of its Manantali operations. Unclear if Eskom Uganda did

- Some reports of Eskom dumping waste into river water in Mali

- Purely hydro-based operations in Mali and Uganda mean negligible emissions of GHGs or other air pollutants

- Manantali dam had devastating impact on biodiversity when it was constructed prior to Eskom's arrival. Though Eskom has little experience or policy guidance on managing aquatic ecosystems or river sedimentation, no major negative impacts on biodiversity have been reported. Eskom Mali relies largely on local environmental authorities for guidance on biodiversity

- Moderately in line with SEP standards

- 800 GWh/year in Mali, to 1,600 GWh/year in Uganda, both $100 \%$ large hydro

- No evidence that the company promotes other, more sustainable, forms of renewable electricity generation

- Largely in line with SEP standards 
Economic issues

\section{Contribute to local economic development}

Ensure reliable supply

\section{Cross-cutting issues}

Engage in meaningful stakeholder consultation and participatory decision-making

\section{Assume responsibility for impacts throughout all business} relationships
Guiding international normative SEP standards

Electric utilities prioritizing the development of sustainable local energy-related infrastructure and employing local workers to the greatest extent practicable and providing those workers with sustainable jobs and training to improve skill levels (WBCSD 2002, PSI/Pillinger 2009, OECD 2011). In order to maximize local economic development and job creation, electric utilities should support local small and medium-sized enterprises in their procurement practices and contribute to the long-term development prospects of the host country through the transfer and rapid diffusion of technologies, skills and knowledge (WBCSD 2002, OECD 2011). Fair payment of due taxes to local, regional and national governments in the host country is a crucial element of contributing to local economic development (OECD 2011, MVO Platform 2012).

Electric utilities should ensure reliable supply by minimizing generation interruptions, maintaining contingency plans, responding to interruptions in a timely manner, and providing timely information to the public about planned and unplanned interruptions (WBCSD 2002, CEER 2005).

Electric utilities should adopt a participatory approach to the planning/designing, developing, operating, and monitoring / evaluation of electricity projects and consult and engage stakeholders throughout the entire process in order to provide meaningful opportunities for their views to be taken into account in decision- making (WCD 2000, WBCSD 2002, IFC 2007, UNGC 2008, PSI/Pillinger 2009, OECD 2011). The consultation process should be tailored to local decisionmaking processes and involve the timely provision of all relevant information about activities, impacts, and potential impacts translated into local languages (WBCSD 2002, ISO 2011). The process should take into account the needs of disadvantaged or vulnerable groups, and if Indigenous Peoples are affected by a potential project, electric utilities must gain the free, prior and informed consent of those peoples before proceeding with any part of the project cycle (IFC 2011).

Electric utilities should seek to prevent, avoid and mitigate negative impacts throughout their entire range of business relationships, including suppliers and contractors (ISO 2011, OECD 2011, UNHRC 2011). Companies should seek to leverage their buying power to improve social and environmental conditions among business relations, and monitor performance using third party audits (WBCSD 2002). 


\section{Indicators of Eskom's policy}

" Aims to "create jobs and new industries through local content associated with Eskom's massive capacity expansion program" yet also has "mutually beneficial arrangements" with large multinational enterprises

- No publicly available policy to contribute to local economic development (through either supporting local workers or suppliers) in countries other than South Africa

Moderately out of line with SEP standards
- Ensuring reliability of supply is key strategic aim

- Issue is an R\&D priority: Invested \$2.8 million to improve availability and reliability of power plants in 2010

- Moderately in line with SEP standards

\section{Indicators of Eskom's practice in Mali and Uganda}

- Eskom Mali employs 160 from Mali, Senegal, and Mauritania, with only 1 (the director) from South Africa; however, employment of locals from the Manantali area has been much more limited

- Employs 108 people in Uganda, only 2 of whom are non-Ugandan and many of whom come from the area where the plant is located. Also employs another 107 as contractors

- Local suppliers are not necessarily prioritized

- Invested \$439 million in electricity infrastructure in Mali, \$10 million in Uganda

- Pays $\$ 2.6$ million per year in taxes to local tax system in Mali, $\$ 180,000$ per year in royalties to local municipalities in Uganda

- Major dispute over Eskom's investment in the maintenance of electricity infrastructure in Mali is causing Eskom to abandon operations there

- Moderately in line with SEP standards

- Electricity supply largely unreliable in Mali and Uganda, load shedding and blackouts frequent

- Capacity factor at plants in Mali and Uganda less than 50\%

- Moderately out of line with SEP standards
" "Addressing the expectations of stakeholders such as employees, shareholders, governing bodies, suppliers, consumers and the wider community is an essential aspect of CR"

- References international standards such as UNGC, AA1000 and King Code

- No endorsement of FPIC

- Managers, willing to engage, provide (some) information, and facilitate local contacts for the present study

- Moderately in line with SEP standards
- Eskom does seek to prevent, avoid and mitigate negative impacts, at least among suppliers and contractors

- Seeks to "drive transformation through its procurement strategy" and evaluates potential suppliers and contractors on SHE issues

- Supplier code of conduct largely focused on avoiding corruption

- No information on corporate policy to audit suppliers and contractors on social and environmental issues

" Moderately in line with SEP standards
" Very limited engagement with stakeholders in Mali and Uganda. Consultations not open to public (participants exclusively selected) and primarily around security issues

- In Mali, Eskom did not engage with land owners when expropriating land, simply paid compensation to local government. Managers had not heard of FPIC

- Various stakeholders (e.g. community members, local government officials) in Mali and Uganda criticized the lack of engagement and participatory decision-making

" Managers in Mali and Uganda very willing to engage, be interviewed, provide information, and facilitate contacts for the present study

- Largely out of line with SEP standards

- Eskom Uganda supplier vetting procedures include safety and environmental requirements, but no mention of respect for labor rights beyond $\mathrm{OHS}$

- Eskom Uganda monitors contractors on compliance with Ugandan labor law

- Moderately in line with SEP standards 


\section{Cross-cutting issues}

Maximize transparency and provision of information
Guiding international normative SEP standards

Electric utilities should apply a high degree of transparency in operations, including reporting on activities and progress, measurement, business partners and relationships, and interactions with government and the public (UNECE 1998, WBCSD 2002, UNGC 2008, OECD 2011). Electric utilities should regularly report on their activities, progress, and performance against "the triple bottom line" using internationally accepted sustainability reporting guidelines such as the GRI's G3 guidelines and the Electric Utilities Sector Supplement (GRI 2008), have an external party verify the data, and disseminate the report in an appropriate manner (WBCSD 2002, OECD 2011).

\subsubsection{General approach to corporate responsibility}

\section{Endorsement of international normative standards for corporate responsibility}

Of the four major internationally recognized standards for CR, Eskom only publicly expresses its endorsement and observance of the UN Global Compact, in which it has been a participant since the initiative was launched in 2000. In terms of other international CR standards, Mavhungu (2010) claims that Eskom's stakeholder engagement practices are aligned with the regionally-important King III code for corporate governance and the AA1000 stakeholder engagement standard principles of materiality, completeness and responsiveness. The company also endorses the ILO Core Conventions, though Eskom's workers' classification as "essential services" limits their right to strike as established under ILO conventions 87 and 98. Eskom has also incorporated the Equator Principles - a global benchmarking tool for managing environmental and social issues in global financing - in order to raise funds for its two new large-scale coal-fired power stations.

The importance the company places on regional standards is also reflected in the approach taken by the management at Eskom's subsidiaries in Mali and Uganda. For example, Eskom Mali's managing director notes that Eskom prides itself on being an "Africa-oriented electricity company" steeped in African values, as opposed to "Western companies" that had failed in electricity provision in Africa (Coulibaly 2012). The degree to which the other, more internationally used, standards have filtered down to Eskom management in host countries, however, is questionable. In their policy documents, Eskom's subsidiaries in Mali and Uganda do not refer to or endorse any of the international standards endorsed by Eskom headquarters. When asked about the standards, senior Eskom managers in Uganda indicated they were not aware of the existence or relevance of the OECD Guidelines or the UN Global Compact (Mngeni 2011). Instead, local management uses national laws as a normative 
Indicators of Eskom's policy

Relatively high degree of information on CR policies available on website and in annual report

- Annual report largely according to the GRI (G3 + EUSS) guidelines, B+ application level

- Little to no transparency or information provided on operations in countries outside of South Africa

No information on business partners such as customers \& suppliers

Moderately in line with SEP standards
Indicators of Eskom's practice in Mali and Uganda

Stakeholders frequently criticize Eskom's lack of transparency and provision of information

No translation of SEP information into local languages

Managers at Eskom Uganda consider operational information "too technical to share with the communities"

Primary channels of communication with stakeholders are the website (though little financial, operational or SEP-related information is available) and community forum (solely focused on security issues)

Largely out of line with SEP standards (and legal) benchmark. A manager of Eskom Uganda explained his view that the Ugandan labor law sufficiently covers any relevant international standards and that the company's safety, health and environment (SHE) policy is based on Ugandan laws (Matarutse 2011). Similarly, in Mali, the managing director expressed his confidence that all relevant ILO standards are incorporated into the Malian labor law, by which the company seeks to abide (Coulibaly 2012).

With regard to making use of standardized procedures and measurements, Eskom has implemented a company-wide EMS based on IS014001. Many divisions of the company are ISO 14000-certified, and those that are not are supposed to work towards the standard (Eskom 2009a). Eskom's electricity generation division falls into the latter category. Although not officially ISO-certified, Eskom (2012a) claims that internal audits reveal the division as being in compliance with the standard. Eskom also uses the South African-developed National Occupational Health and Safety Association
(NOSA) certification system for OHS issues. In addition, Eskom introduced standardized EIAs on new projects in South Africa as early as the 1980s, when national legislation only recommended it (Eskom 2002).

Eskom subsidiaries in Uganda and Mali also follow the parent company's general inclination toward standardization. Eskom Uganda has a four-star rating under the NOSA certification system, and it applied for ISO 9001 and 14001 certification in March 2011 (Kiryowa 2011). Although itself not certified, Eskom Mali references ISO 9000 and 14001-based instructions developed by the regional environmental authorities, to which it is required to abide and upon which it has based its EMS (Kaba 2012).

\section{Adopt a commitment to CR in core-business activities and decision-making}

Prior to commercialization in 2001, Eskom's CR efforts were focused primarily on the corebusiness activity, facilitating connections of new households to the electricity grid and 
reducing the price of electricity (Eberhard 2007). Since then, however, philanthropy and charity, under the banner of corporate social investment (CSI), have been given a much more prominent focus in Eskom's approach to CR. Some stakeholders attribute this shift to an attempt by the company to deflect attention from the company's shortcomings in taking responsibility within its core-business areas, as well as to an investment in "marketing" the company with an expectation of making a financial return on its investment (Klins et al. 2010). Nevertheless, Mavhungu (2010) maintains that the company continues to strive to integrate environmental and sustainability concerns into its core-business systems and operations by "entrenching environmental management objectives and criteria in procurement and investment strategies, governance structures, operational practices, and decision-making processes".

\section{Eskom's approach to CR is also strongly} influenced by black economic empowerment (BEE) and broad-based black economic empowerment (BBBEE) concerns through employment and procurement processes. The company has several policies in place to regulate this core-business aspect of the company's performance, and Eskom has performed well by this yard stick, having met and exceeded targets in this area set down in its shareholder compact. Although Eskom's historical focus on the core business of electrification was clearly in line with international SEP norms (e.g. WBCSD 2002, MVO Platform 2012), the tendency toward philanthropy and charity are a departure from the norms.

Eskom's subsidiaries in Mali and Uganda take the trend toward charity and corporate marketing and away from core-business aspects even further than is the case in policy at the corporate headquarters. Aside from Eskom Uganda's SHE policy with NOSA certification (described above), the company's $\mathrm{CR}$ program is largely oriented toward philanthropy rather than toward core-business operations. For example, Eskom Uganda's (2008) website contains no annual operational or financial reports and no reports on corebusiness environmental and social impacts. The website does contain information on Eskom Uganda's core CR implementation structure, its "Sponsorship Committee", which funds selected project applications from the public. Selected projects include teaching young female victims of war skills like tailoring and housekeeping, sending food and clothes to victims of famine and natural disasters in Eastern Uganda, and planting trees around school premises. Eskom Uganda's managing director explains that these projects have been used to promote and market Eskom to the local community (Mngeni 2011).

The situation is even clearer in Mali, where Eskom managers were unaware of how much locals in the vicinity of Eskom's power plant pay for electricity. When asked, the managers noted that "This element is far removed from Eskom's role in the project" and that "Eskom has no responsibility whatsoever for determining or increasing access to electricity supply in Mali. 
These are the duties of the Malian electricity supply company, EDM" (Coulibaly 2012, Dikgale 2012). Nevertheless, the Eskom Mali managers insist that the company contributes to poverty alleviation, just not through their core business (e.g. programs to extend rural electrification, use of non-grid technologies, etc.), but rather through charitable donations. Eskom Mali funds food aid to the population during times of low agricultural productivity (Coulibaly 2012) and provides financial assistance to entrepreneurs in the community, for example, providing XFA 500,000 (\$998, €762) to the chief of Manantali's son to start a welding workshop (Toure 2012). The company also finances the organization of festivals, cultural events, and TV shows and the building of roads, hospitals, and school (Kaba 2012). Ironically, however, the Mayor of Manantali (Dembele 2012) reported that a majority of the schools in Manantali are without electricity, including schools that were built as part of the "community development" programs implemented by companies operating the Manantali hydroelectric power station.

With regard to management structures to implement CR policy and foster high performance on CR issues, Eskom's corporate policies are largely in line with international normative standards, suggesting that electric utilities have a board level position responsible for CR issues (WBCSD 2002). On some CR issues, Eskom's CEO takes direct responsibility. For example, Eskom's SHE policy places final responsibility with the corporate board and is administered by a board-level subcommittee chaired by the CEO. Mavhungu (2010) explains that "environmental performance is managed as an integral part of Eskom's governance structure, from the board to the executive management committee. Accountable environmental managers and environmental practitioners from the various line divisions ensure the effective implementation of environmental management systems throughout our business."

\subsubsection{Social issues}

\section{Increase access to affordable electricity}

Eskom's (2011) pledge to "provide electricity to all South Africans" is a legacy of its "Electricity For All" initiative, first adopted in 1987. Prior to Eskom's commercialization in 2001, the company made significant inroads in terms of increasing access to electricity in South Africa. Under apartheid, Eskom had limited access to households in urban areas that fell under local authorities, but as apartheid began to fall, Eskom saw an opportunity to gain access due to the segregated nature of local governance and the lack of capacity of Black Local Authorities to supply their citizens with electricity. By 1994, Eskom had taken over the rights of supply in 130 townships (Marquard et al. 2007). Eskom's annual new connections peaked at 313,179 in 1995, and in that year it also began supporting local governments in making additional connections. Though it did not meet its target of bringing electricity to an additional five million people between 1990 and 1997, Eskom's electrification program, one of the largest ever undertaken, made over two million connections in the 1990s (see Figure 9.1). 
These connections were undertaken and financed by Eskom as part of its stated CR (Peters 2011). Eskom thus made considerable progress in connecting previously disadvantaged South Africans to the national grid, an experience that it could apply in other African countries. Indeed, many of the projects initially embarked upon by Eskom subsidiaries in other African countries related to electrification schemes. The company was squarely in line with international norms encouraging electric utilities to develop policies and undertake initiatives to extend electricity access to unserved and underserved communities, particularly in rural or remote areas, but also to the poor in urban and peri-urban areas (Palast et al. 2000, WBCSD 2002, UNDP/Modi et al. 2005, PSI/Pillinger 2009).
Despite Eskom's success in this area, electricity prices and the inability of many poor households to pay those prices led Eskom to take measures that often worked contrary to its diligent provision of new connections. Although Eskom claims that it provides the "cheapest electricity in the world", those cheap prices have primarily been for large industrial consumers, not residential customers, and Eskom has consistently petitioned South Africa's electricity regulator for higher electricity rates (Eskom 2013a). In 2008, the South African average price of electricity was ZAR 0.25 ( $\$ 0.03$, $€ 0.02$ ) per kWh, but rural consumers and in many townships rates reached ZAR 0.48 ( $\$ 0.06$, $€ 0.04$ ) per kWh (McDonald 2002). A 2004 study by Wits University revealed that the average household in Soweto was spending one quarter of its income on its electricity bill (Dugard

Figure 9.1: New electricity connections made by Eskom in South Africa, 1991-2011

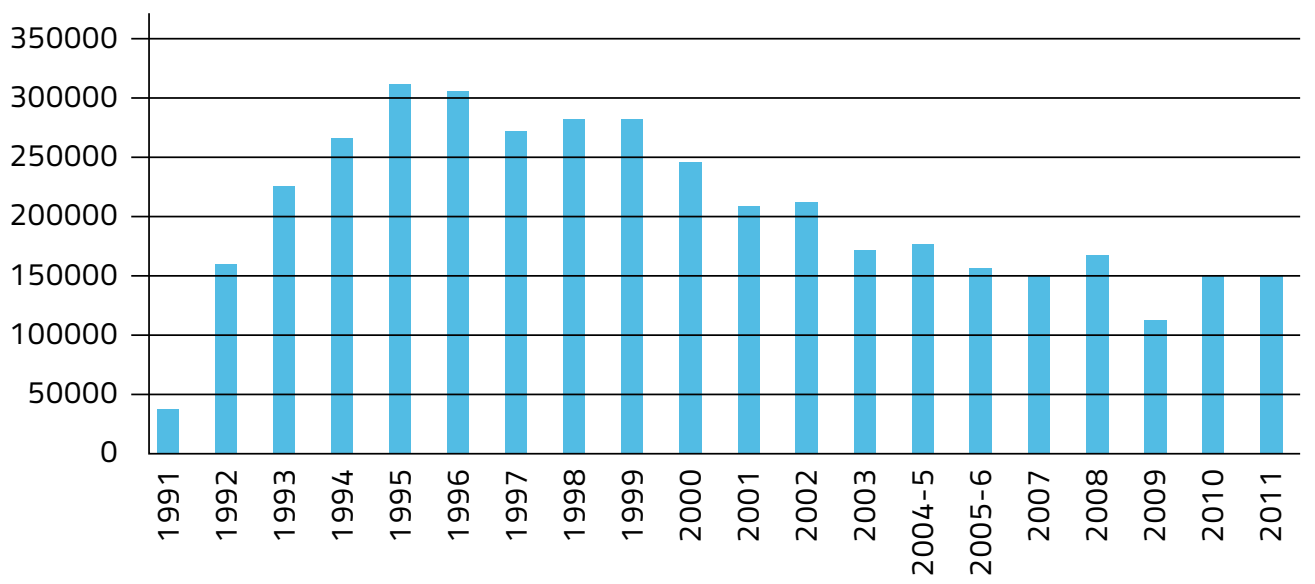

Source: Eskom's 1991-2011 annual reports 
2009). The inability to pay the electricity bill led Eskom to disconnect as significant a number of households as it was connecting each year. $A$ query in STATSSA (2009) revealed that, in 2008, 846,708 South African households (including those supplied by both Eskom and municipal providers) reported having electricity cut off due to non-payment each month. As another strategy to increase cost recovery, Eskom began installing pre-paid meters, as opposed to conventional monthly metering, in newlyconnected low-income households. This created a system of "self-disconnection" as a means to manage non-payment and instill consumer discipline (Van Huesden 2009). A study by PDG (2010) reported one-third of the new prepaid metering connections made by Eskom in 2008/9 recorded zero consumption for the year, an indication of a self-disconnection trend far more widespread than the official number of disconnections suggests. According to the South African Department of Energy (2010), $95 \%$ of households in South Africa use prepaid metering.

In addition, as can be seen in Figure 9.1, Eskom's push for electrification began to lose steam in the late 1990s, and the number of new connections has steadily declined since 1999. After the commercialization of Eskom, the company was no longer willing to play a central role in electrification. Eskom's own new connections decreased, and the company also reduced its support of local governments' electrification programs. PDG (2010) has concluded that "the pace on new electricity connections has slowed significantly in recent years and now no longer keeps pace with new household formation."

One reason for the reversal in progress with electrification was that many of the households connected through the electrification program were simply too poor to pay for the 350kWh/ month that Eskom had projected as the commercially viable consumption level (Clarck 2005). This meant that increasing electrification of poor households was a financial drain rather than an income generator. The company argued that, since under corporatization it paid taxes and dividends, electrification should become a government-financed activity (Hallowes and Munnik 2007, Marquard et al. 2007). With Eskom no longer willing to contribute, the main impetus behind the electrification program shifted to the South African government, which began funding electrification directly from state funds (Marquard et al. 2007). This marked a departure from Eskom's earlier commitment and the international SEP norms' encouragement of electric utilities to actively promote affordable access.

Eskom's venture into Africa revealed a similar pattern. Though many of the projects embarked on by Eskom subsidiaries in other African countries related to electrification schemes, Eskom soon began to experience difficulties recouping connection costs and the limited potential for profit (World Bank 2009). The company quickly lost interest in this aspect of the business, despite its developmental necessity and insistence from international SEP norms. Currently, although $100 \%$ of Eskom's 
total installed capacity of $44,193 \mathrm{MW}$ is located in countries without universal access to electricity, the vast majority of this capacity is in its home market of South Africa. Outside of its home country, the company only has 580MW of capacity ( $1 \%$ of its total capacity). Given Eskom's further withdrawal from Mali in July 2012 (Coulibaly 2012) and the absence of any new capacity outside South Africa in its immediate build plan, the company appears to have all but abandoned its commitment to increasing access to affordable electricity in Africa. This unstated policy is also evident in the company's current operations in Mali and Uganda, where the lack of access to electricity remains acute.

More than 28 million people in Uganda lack access to electricity, giving the country an effective electrification rate of $9 \%$ - the absolute lowest in the world (IEA 2011). The population with access to electricity is heavily concentrated in a few major urban areas, and the electrification rate in rural areas, where Eskom's hydroelectric plant is located, is below $1 \%$ (IEA 2011). At the same time, Ugandans also pay the highest electricity rates in the region (Gore 2008). In 2011, the price of electricity was 870 Ugandan shillings $(\$ 0.35, € 0.27)$ per kWh, but government subsidies reduce the price by approximately $50 \%$ so that small domestic consumers pay 422 Ugandan shillings $(\$ 0.17, € 0.13)$ per kWh. Large industrial consumers pay less - 380 shillings $(\$ 0.15, € 0.12)$ per kWh (Binyina 2011). The high price of electricity in Uganda has resulted in the country also having one of the highest rates of disconnection due to non-payment of any country in the region (Binyina 2011). According to the United Nations Economic Commission for Africa (UNECA 2007: 54), there has been little progress in improving either access to and affordability of electricity since 2001.

Despite this dire situation, managers at Eskom Uganda say that they "do not consider it within [their] mandate nor responsibility to address issues of access to and affordability of electricity", and they are aware neither of the number of customers served by the electricity generated by the company nor the electrification rates or prices paid in areas adjacent to the Eskom plant (Mngeni 2011). The electricity generation capacity of Eskom Uganda's hydroelectric facility is $380 \mathrm{MW}$. However, low water levels allow operation for only approximately half the year (i.e. a capacity factor of $46 \%$ ), meaning that Eskom only generates approximately $1,600 \mathrm{GWh}$ of electricity for the country each year (Mngeni 2011). A community leader from one of the villages adjacent to the plant did note that inhabitants of his village benefit from the Eskom plant because they have electricity (Nicholas 2011), but it is clearly not enough to satisfy local or national need.

Ugandan electricity authorities have publicly called on Eskom and other electricity companies to invest in the country's electricity infrastructure (Kyoko and Wandera 2011). In 2009, Uganda's Electricity Regulatory Authority specifically requested that Eskom invest in the 250MW Bujagali Power Plant currently being planned. Eskom Uganda submitted a bid to Eskom headquarters for consideration (Mngeni 
2011), but company headquarters had already made a decision to give priority to South Africa to meet the electricity needs leading up to and during the World Cup and not to invest in Africa at least until after the event was over (Carlo 2011). Ugandan officials were dismayed that the company would prioritize the World Cup over expanding access to electricity in Africa (Kyoko and Wandera 2011).

In Mali, the national electrification rate is approximately $45 \%$, but in rural areas such as Manantali, where Eskom's activities are located, the rate is just 10\% (Akante 2012). In 2011, electricity prices for small domestic users in rural areas like Manantali were between XOF 59 (\$0.12, €0.09) for consumers of $<50 \mathrm{kWh} /$ month) and XOF $128(\$ 0.26$, €0.20) per kWh (Energie du Mali 2012). At the 200MW Manantali power station, Eskom generates approximately $800 \mathrm{GWh}$ per year, meaning that it operates at less than half $(45 \%)$ of its full capacity (Dikgale 2012). According to the Secretary General of the Manantali Council (Sissoko 2012), apart from the Eskom offices and houses and the houses of locals living along the road, the communities closest to the Manantali dam have no electricity. The majority (70\%) of the schools in Manantali do not have access to electricity. Yet similar to their counterparts in Uganda, Eskom Mali managers are unaware of how much local households pay for electricity and claim that "Eskom has no responsibility whatsoever for increasing access to electricity. All Eskom does is respect the production quotas agreed with the [electricity supply company] EDM" (Dikgale 2012, Coulibaly 2012).
With regard to promoting off-grid, decentralized electricity provision projects to increase access to electricity in rural areas, Eskom's policies have been ambiguous. Though a joint venture between Eskom and Shell was granted a concession to supply off-grid access to electricity in the late 1990s, the two energy giants sold the company before it could make any real progress in expanding access (Prasad 2007). While Eskom's grid-based electrification program achieved over four million connections between 1991 and 2011 (Eskom 2012a), its commitment to facilitating off-grid access has lagged far behind, with only 50,000 connections made during the same 20-year period (South African Department of Energy 2010). The Energy Research Centre (2004) argues that the off-grid market was in fact undermined by Eskom's "Electricity for All" campaign, claiming it led to widespread expectations by rural communities that they would receive grid electricity in the near future and thus did not need off-grid initiatives.

Eskom's technical expertise as applied in the broader African context, including in Mali and Uganda, has also been specifically limited to large, grid-based centralized electricity generation, despite the fact that SEP norms suggest that off-grid, decentralized technologies can be particularly effective in increasing access to electricity in rural and remote areas (Modi et al. 2005).

Eskom's recent ambivalence toward increasing access to affordable electricity for poor households potentially stems from its focus 
on industrial customers. The 2010 Annual Report produced by Eskom (2011) notes that the company "seeks mutually beneficial arrangements with industry, particularly support industries such as the coal mining sector". For many years, Eskom was the cheapest supplier of electricity to industry in the world, by a significant margin (Eskom 2010). In fact, as part of the special "mutually beneficial" deals Eskom makes with some industrial business partners, the company supplies electricity at below cost price. For example, in 2009 Eskom industrial customer BHP Billiton is reported to have paid roughly ZAR 0.15 (\$0.02, €0.01) per kWh for power at a time when Eskom calculated its "average total cost" of producing electricity at ZAR 0.27 (\$0.04, €0.03) per kWh (Carnie 2010b). Fine and Rustomjee (1996) believe that, where people come to enjoy electrification, it is normally as a by-product of Eskom's arrangements with industry, rather than as a result of conscious social development planning by the company. In 2000, four of Eskom's top ten customers in South Africa were large multinational corporations, while six were metropolitan or municipal areas (Eskom 2001). Similarly, most of Eskom's electricity exports to neighboring countries in Southern Africa are not to electrify local homes and small businesses, but rather to supply large industrial players. In $2009,72 \%$ of the 9,123 GWh of the electricity Eskom exported to its powerstarved neighbors in Mozambique and Namibia went to three large multinational customers (Eskom 2010, 228). Eskom does not disclose who these customers are but it is largely suspected that these include BHP Billiton's
Mozal aluminum smelter in Mozambique and Anglo American's Skorpion Zinc mine in Namibia (Carnie 2010a, Eskom 2009a: 48).

\section{Respect labor rights}

Eskom clearly endorses the ILO Core

Conventions, and the company has integrated these into its own corporate policies. Eskom's Employment Relations Policy incorporates the convention on freedom of association, and the company has a Recognition Agreement with its trade unions that regulates collective bargaining processes. The company adheres to Employment Equity legislation and has a policy on Employment Equity. The company strictly prohibits child labor and forced labor (Mavhungu 2010). With regard to the right to strike, as established under ILO conventions 87 and 98, Mavhungu (2010) notes that "the Generation, Transmission and Distribution of power has been designated as 'essential service', and therefore Eskom employees in these divisions are prohibited from engaging in a strike unless there is a Minimum Services Agreement (MSA). There was an MSA which gave the majority of the Eskom employees an opportunity to go on strike in place but it was cancelled by the trade unions. Outside of South Africa, Eskom abides by the local laws, [most of which include a provision prohibiting strikes by electricity sector workers,] but goes no further to protect workers' right to strike."

Neither Eskom Uganda nor Eskom Mali make explicit reference to the ILO conventions, but both claim that the norms are integrated into the labor laws of the countries (Lumbuye 2011، 
Kaba 2012). A union is present at the Eskom Uganda plant, and currently $62(56 \%)$ of the company's 110 employees are members of the union (Lumbuye 2011). In Mali, various unions exist within the Manantali operations, primarily based on the workers' home country. Senegalese, Mauritanian and Malian workers each belong to separate unions. There is also a general umbrella union covering all three country unions at Eskom. Eskom Mali managers insist that the Mali labor law ensures Malian workers' right to strike under certain conditions, but there have been no strikes in Mali in recent years (Coulibaly 2012, Kaba 2012). In general, union leaders in both countries indicated that relations between the unions and company management were largely amicable (Olargin 2011, Job 2012). In Uganda, Olargin (2011) indicated that workers had some outstanding grievances related to wages and salaries, but in Mali, Job (2012) noted that workers are paid satisfactorily. The lowest paid employees of Eskom Mali earn XOF 50,000 $(\$ 100, € 76)$ per month, nearly double the legal minimum wage. Collective bargaining agreements also exist in both countries, covering $80 \%$ of workers in Mali (Lumbuye 2011, Job 2012). In Mali, however, Eskom managers admit the agreement is "not completely adhered to" by Eskom, in particular with regard to dealing with workers' grievances in a timely manner (Coulibaly 2012, Kaba 2012).

In terms of engaging and consulting workers, Mavhungu (2010) explains Eskom has structures at all levels that deal with consultations with workers, both collectively through trade unions as well as individually.
Mavhungu (2010) provides the example of children not being allowed at the workplace due to safety concerns, but that, after consultations with the workers, the policy was changed. Union leaders at Eskom Mali also report that structures are in place to consult workers (Job 2012). A grievance related to wages was reportedly resolved through dialogue between workers and management. And although workers at Eskom in Mali praised the company's managing director for his willingness to engage in dialogues with workers, they lamented that he is often not empowered to take key operational decisions and must refer matters to corporate headquarters in South Africa (Job 2012).

With regard to occupational health and safety, Eskom's Safety Health and Environment (SHE) Policy, introduced in 2007, constitutes a comprehensive framework for dealing with $\mathrm{OHS}$ issues. The policy insists that safety and health issues are an integral part of the company's operations, and explains that final responsibility for implementing the policy lies with a board-level committee chaired by the CEO. The policy also expresses Eskom's target of having zero occupational injuries and illnesses, although in the five years between 2007-2011 there were 39 fatalities among Eskom employees, and 221 fatalities among contractors working for Eskom (Eskom 2012a). The policy also states that Eskom will engage key stakeholders, including employees and organized labor, on all elements of SHE issues (Eskom 2007). Eskom subsidiaries in Mali and Uganda also place a high degree of 
importance on OHS issues. Both companies refer prominently to the corporate SHE policy, as well as the South African NOSA standard for OHS. Relatively few occupational injuries were reported in recent years at the subsidiaries. In Uganda, one fatality occurred in 2009 in the machine room, and one worker suffered a broken a leg after slipping on fluid that had seeped out onto the floor of the plant (Kiryowa 2011). In Mali, a recent accident involving carbon discharge during start-up operations intoxicated some workers. The accident did not result in any deaths or permanent injuries, but the company did have to cover medical expenses amounting to XOF 1 million $(\$ 1,993$, $€ 1,524)$ (Job 2012).

Eskom does not report on GRI indicators related to outsourcing and subcontracting in terms of the GRI, despite the fact that this is a key labor concern. At Eskom Uganda, just under $50 \%$ of the 215 people working at the plant are outsourced contract laborers. Outsourcing figures were not available for Eskom Mali, although managers there insist that job security is high and that all employees have contracts (Kaba 2012). However, it is uncertain what Eskom's departure from Mali will mean for the workers at the dam.

In terms of ensuring that the rights of workers at the company's business relationships (e.g. contractors and suppliers) are also respected. Eskom's SHE policy explicitly covers OHS issues among contract workers, noting that Eskom "will work with supplier and customers to integrate SHE issues into their operations, and contractors working under our supervision, or on Eskom premises, will comply with this policy" and that the policy "will apply during the evaluation of all contracts, projects, and proposals" (Eskom 2007). However, aside from OHS issues, the company does not have a policy aimed at ensuring that other internationally recognized labor standards or decent work provisions are upheld among business partners. Eskom Uganda seeks to ensure that the rights of contract workers are respected by signing an agreement with the service provider stipulating that the contractor must adhere to all Ugandan labor laws, including regarding wages. Eskom Uganda then monitors contractors to make sure they are adhering to the agreement (Lumbuye 2011).

\subsubsection{Environmental issues}

\section{Minimize environmental impact including contribution to climate change}

Generating approximately 232 TWh of electricity each year, the vast majority (93\%) of it by burning low-grade coal, means that Eskom has an enormous impact on the environment (Mavhungu 2010). Eskom buys more coal than any other company in the world (Lines 2007), is the world's second largest corporate emitter of $\mathrm{CO}_{2}$ (Grant 2007), and is South Africa's largest polluter in terms of sulfur dioxide $\left(\mathrm{SO}_{2}\right)$, nitrogen oxide $\left(\mathrm{NO}_{\mathrm{x}}\right.$ ) and particulate matter (PM) (South African Department of Energy 2002, Eskom 2009a).

According to Mavhungu (2010), in order to manage its significant environmental impacts, 
Eskom has adopted a systems approach to environmental management in order to ensure continual improvement in environmental performance. As laid out in the company's SHE policy, environmental management objectives and criteria are entrenched in Eskom's procurement and investment strategies, governance structures, operational practices and decision-making processes. As mentioned above, the SHE policy is overseen by a board-level subcommittee comprised of four independent non-executive directors, the CEO and two board members. The CEO chairs the committee and therefore carries theoretical responsibility for sustainability (Eskom 2007). In line with this objective, the company has developed and implemented environmental management systems throughout all operational divisions. The EMS is based on ISO 14001, although not all divisions are ISOcertified. Notably, the Generation Division is not certified. Through the EMS and the main Eskom environmental procedure EPC 32-96, environmental considerations are taken into account during formal or technical planning processes of the Eskom divisions. "The Environmental Plan forms an integral part of the Technical Plan process. In addition, all projects submitted to the Generation Investment Committee for approval require environmental input in order to provide assurance that legal requirements are met and all risks and liabilities are identified and managed" (Eskom 2006). This then feeds into Eskom's integrated strategic electricity planning framework, which takes environmental life cycle assessments into account. Line management in the generation division is responsible for EIAs and must generate environmental reports, which are then centralized. The World Bank (2010b) reports that Eskom has approximately 120 personnel dedicated to supporting these functions. These environmental management policies are largely in line with the procedural, management aspects (not the actual impact aspects) of the normative standards for SEP.

As Eskom's sixth largest R\&D project, the company spends approximately ZAR 16 $(\$ 2, € 1.5)$ million each year on research and development aimed at managing and reducing environmental impacts (Mavhungu 2010). Two of the most important areas for Eskom are emissions reduction through improvements in operational efficiency and water management (Mavhungu 2010). Eskom's power plants have been designed to burn low-grade coal and therefore have a relatively high emissionintensity. The company thus sets and continuously monitors the operational and conversion efficiency targets of power plants in order to drive combustion efficiency. However, variations in quality of fuel delivered to power stations often has a negative impact on the ability to meet combustion efficiency targets and increases the impact on the environment (Mavhungu 2010). Given the scarcity of water in South Africa, the company places a high degree of importance on managing water, and Eskom is a world leader in the use of dry-cooling technology aimed at saving water. The downside of this technology is that it is more emissions-intensive (Macknick et al. 2011), further exacerbating Eskom's high emissions. 
Water management is important in a different regard at Eskom's hydroelectric operations in Mali and Uganda, which generate minimal air pollution and GHG emissions. Unlike its long experience with coal-fired electricity generation, Eskom historically exhibited limited experience with hydropower and the management of aquatic ecosystems and river sedimentation. Eskom was very new to such issues in 2001 when it assumed control of the plant in Manantali, an area where water resource management is a sensitive priority, one that affects the health and livelihoods of thousands of poor rural inhabitants of the river basin.

Although Eskom $(2004,25)$ at one point criticized Mali for having "scant environmental legislation", the company's SHE policy contains little guidance on aquatic ecosystem and river sedimentation management. The managing director of Eskom Mali (Coulibaly 2012) thus admitted that the company relies heavily on an environmental management manual Manual A10.12 - developed by regional Malian environmental authorities. Although it submits environmental reports to the environmental authorities, Eskom does not report to the local public on its impacts on the aquatic ecosystem and river sedimentation in Manantali. However, Manantali community leaders (Dabor 2012, Diallo 2012, Takoure 2012) claim that Eskom dumps treated waste into the Senegal River, which communities throughout the area use for drinking and cooking purposes. Eskom Uganda implements the company's SHE policy and ISO 14000-based EMS. The company has not yet achieved ISO 14001-certification, but is currently seeking it (Kiryowa 2011). Ugandan electricity regulators indicated that Eskom Uganda had a good track record on environmental issues (Kyoko and Wandera 2011).

Eskom has been a global frontrunner with regard to EIAs, adopting a standard practice - though not an official policy - of conducting EIAs on all new projects as early as the 1980s, when South African legislation only recommended it (World Bank 2010b). ElAs are the responsibility of Eskom line managers, who can draw guidance from the Eskom Environmental Management Programme, related to collecting baseline data on appropriate environmental issues; identifying and predicting environmental impacts and their significance (including the activity or root cause associated with any significant impact); setting objectives and targets to address root causes; actions to be taken to meet objectives and targets; integration of the above actions into project/operational systems, documentation, and contracts; linking performance to existing business performance measures and reporting practices by means of key performance indicators; monitoring; and auditing.

Although it assumed control of the hydroelectric dams in Mali and Uganda after they were already built and operating, Eskom Mali evaluated the EIA that was conducted before the dam was constructed and prepared an additional EIA prior to purchasing the operating rights (Coulibaly 2012). Managers at Eskom Uganda indicated that they were not sure if an EIA was carried out before the dam was 
constructed. Their role, however, is to ensure that they adhere to the National Environmental Act. According to the systems they have put in place, in their opinion it is adhered to.

Eskom's heavy reliance on coal-fired power plants to generate electricity ensures that the company's GHG emissions and contribution to climate change are significant, the second highest in the world (Grant 2007). Although it does not report its GHG emissions in the CDP system, the company's 2011 Annual Report indicates that its $\mathrm{CO}_{2}$ emissions that year amounted to 230.3 Megatons, for an emission intensity of $0.99 \mathrm{tCO}_{2} / \mathrm{MWh}$ (Eskom 2012a).

Despite this significant impact, Eskom is not planning to reduce GHG emissions in the short or medium term. Indeed, Eskom GHG emissions have steadily risen in recent years. Eskom has openly stated that it actually plans to increase its GHG emissions in the short term, peak and stabilize them in the medium term, and eventually begin to reduce them in the long term (Mavhungu 2010). Indicative of the company's relatively low priority on reducing emissions, Eskom is currently building a number of new large, coal-fired power stations, and its R\&D project on mitigating climate change received the third lowest budget (ZAR 7 million, \$889,500, €677,500) of all Eskom R\&D projects in 2010.

Although it is not considering reducing emissions in the near future, Eskom has finally moved away from a least-cost optimization imperative in strategic electricity planning and toward a plan that envisions achieving a low-carbon (distant) future, in what it calls a long-term mitigation scenario (Mavhungu 2010). The company maintains, however, that it cannot achieve the scenario on its own and that it will require significant financial assistance from the government and "socially responsible" investors to do so (Mavhungu 2010).

A key area of Eskom's six-point plan on climate change is focused on developing adaptation strategies to ensure reliability and continuity of supply, primarily approaching the issue from a risk and insurance point of view. Climaterelated risks include water scarcity and extreme weather that could cause infrastructure damage and force relocation of people. The company has also done a scoping survey of what other utilities around the world are doing in terms of adaptation strategies (Mavhungu 2010).

Eskom's policy on biodiversity is to ensure that it is aligned with South Africa's commitments under the Convention on Biological Diversity (CBD) and national biodiversity strategy, which entails "the conservation of biological diversity, the use of the components of biological diversity in a sustainable manner, and the fair and equitable sharing of the benefits of biological diversity" (Mavhungu 2010). In order to achieve this, Eskom is participating in an initiative led by the WBCSD to develop and pilot a corporate guide for ecosystem valuations, and the company is involved in two partnerships with conservation CSOs in South Africa to protect and rehabilitate ecosystems. Although the disastrous impact that the Manantali dam had on biodiversity 
when it was constructed and prior to Eskom's arrival has been documented (Pottinger 1997), little information could be gathered on Eskom's impact on biodiversity in Mali. Eskom Mali clearly relies primarily on local environmental and river management authorities to ensure that biodiversity is protected. The company simply follows the environmental management plan, which includes the protection and conservation of marine fauna and flora, developed by the regional environmental authority SOGEM (Coulibaly 2012).

\section{Prioritize renewable sources of energy for electricity}

In terms of utilizing renewable sources of energy to generate electricity, Eskom is far behind its competitors and largely out of line with international norms on the issue. Despite over a decade of research into renewables, Eskom's truly sustainable capacity consists of a wind test facility of $3 \mathrm{MW}$, a negligible $0.007 \%$ of its total installed capacity. The company does have another $600 \mathrm{MW}$ of installed renewable hydro capacity, but even this only amounts to $1.45 \%$ of total capacity. As can be seen in Table 9.1 above, a full $93 \%$ of electricity generated by Eskom in 2009 was coal-based.

At the World Summit on Sustainable Development in 2002, Eskom pledged to reduce its reliance on coal-fired electricity by $10 \%$ by 2012 (Macleod 2006). This pledge will clearly not be met, as in the near and medium-term future, Eskom clearly does not have a policy prioritizing renewable sources of energy in favor of fossil fuels, as is recommended by international SEP norms (WBCSD 2002, UN/IAEA 2007, Pillinger 2009 , Teske 2010). The vast majority (78\%) of the company's 12,821 MW, ZAR 385 (\$48.8, €37.3 billion) immediate build program consists of two large coal-fired facilities, which will add up to $10,000 \mathrm{MW}$ of capacity. Eskom's ambitions for adding new renewable capacity to its production park are modest. Eskom's research goal for its renewables project is to enable the company to incorporate $200 \mathrm{MW}$ of renewable energy into its generation fuel mix by 2013 (Mavhungu 2010). However, the first projects that were cut out of the build program when the company experienced cash shortages were the 100MW Sere wind facility and the 100MW Upington concentrated solar power (CSP) project (Creamer 2011, Hancock 2011). Even if it is assumed that these two projects are completed by 2018 with the rest of the company's 12,812 MW build program, this gives the company a total installed capacity of over $55,000 \mathrm{MW}$, of which $0.18 \%$ (up from $0.007 \%$ in 2009) will be based on wind. Hydroelectric capacity will actually see a relative decrease from 1.5\% in 2011 to $1.4 \%$ in 2018.

Although the company is currently conducting research into new technologies such as CSP, which has enormous potential in sunny South Africa, Mavhungu (2010) admits that the company has progressed little and that "funding for renewables has been a stumbling block". Yet the company has dedicated what few resources are available for investment in new technologies primarily toward improving carbon-based generation techniques rather than developing renewables. Of the ZAR 11 $(\$ 1.2, € 1)$ million on R\&D for new generation 
technologies in 2009, only approximately ZAR 2 million $(\$ 253,865, € 193,529)$ went to sustainable technologies (a CSP project), while much was spent on coal gasification, carbon capture and storage, carbon sequestration techniques, fine coal utilization, and municipal waste combustion (Mavhungu 2010). This is largely out of line with international SEP norms guidance to electric utilities to prioritize renewables in their research and development programs (WBCSD 2002, UNGC 2008).

Part of Eskom's strategic engagement with Africa is a desire to increase the level of hydropower imported as a means of mitigating this heavy coal reliance. Indeed, in contrast to the clear trend in South Africa, Eskom operates solely large-scale hydroelectric facilities in Mali and Uganda. Additional projects in feasibility and prefeasibility stages in the Democratic Republic of Congo (DRC), Namibia, Angola, Zambia and Zimbabwe are all large hydro projects (SADC 2012). Although its large-scale hydroelectric facilities are renewable sources of energy, there is no evidence that the company is working to promote other, more sustainable, forms of renewable electricity generation in any of these countries as encouraged by international norms.

\subsubsection{Economic issues}

\section{Contribute to sustainable local economic}

\section{development}

Eskom's policy on contributing to local economic development is largely focused on the development of local businesses and skills within South Africa (Mavhungu 2010). Eskom (2011) summarizes the company's pledge to contribute to South Africa's development by "creating jobs and new industries through local content associated with Eskom's massive capacity expansion programme". At the same time, however, the company is also largely engaged in supporting, through "mutually beneficial arrangements," large multinational enterprises (generally in the mining sector), rather than small and medium-sized enterprises (Eskom 2011). Many of Eskom's investments in electricity provision operations in other African countries were also driven by the infrastructure needs of other South African corporations active on the continent, especially in the mining and mineral beneficiation sectors, rather than the aim of improving local energy-related infrastructure per se (McDonald 2009a). The company does not have a policy at the headquarters level related specifically to contributing to local economic development (through either supporting local workers or suppliers) in countries other than South Africa and is thus seemingly out of line with international norms encouraging companies to prioritize the development of sustainable local energy-related infrastructure (WBCSD 2002, PSI/Pillinger 2009, OECD 2011). Instead, Eskom's policy is oriented toward a model of economic development driven by large industry, primarily the mining sector.

Since 2003, Eskom Uganda has invested $\$ 10$ (€7.6) million in its Uganda electricity provision operations (Ossiya 2011), but the company considers its main contribution to 
local economic development in Uganda to be the royalties and fees it pays to the local and national government and its sponsorship of community projects. Eskom Uganda pays $\$ 180,000(€ 137,399)$ each year in royalties to the municipality of Jinja and the Bwikwe/ Njeru Town Council. The royalties, which are required by law, are intended to compensate for the people that were displaced when the dam was constructed (Matarutse 2011). The royalties paid by Eskom Uganda are important for the local governments, comprising $8 \%$ of the annual budget of the municipality of Jinja and $47 \%$ of the annual budget of the Njeru Town Council (Eriab 2011, Kitoke 2011). However, local officials admit that the royalties rarely end up in the hands of the affected communities, and are instead used for projects that directly benefit the municipality or township and that local officials believe will indirectly benefit the communities (Eriab 2011). The Njeru Town Council passed a resolution to ensure that more of the royalties flow directly to the communities, but the resolution has not yet been implemented because the council still needs the money to cover its own expenses (Kitoke 2011). In addition, Eskom Uganda pays $\$ 250,000(€ 190,832)$ per year in license fees to the Ugandan Electricity Regulation Authority (ERA). The company has an annual charity budget of approximately US\$71,000 $(€ 54,266)$ to finance community projects (Ossiya 2011).

In Mali, Eskom has invested significantly more than in Uganda. The company has invested XOF 220 billion (\$439, €335 million) in electricity infrastructure in the country since entering the Malian market in 2001. Eskom currently provides $70 \%$ of Mali's entire electricity supply (Coulibaly 2012). Eskom also pays approximately XOF 1.3 billion (\$2.6, €2.0 million) per year in taxes to the Bafoulabe tax system. These dues are strictly mandatory, but Eskom contributes an extra XOF 20 million $(\$ 39,880, € 30,490)$ to help develop the Manantali community (Kaba 2012).

However, a major dispute has arisen between Eskom and Malian electricity authorities regarding Eskom's investment in and maintenance of local electricity infrastructure. Regional government officials (SOGEM) allege that Eskom has failed to invest enough money to ensure proper maintenance of electricity infrastructure, as is required in the contract (Toure 2012). Eskom claims that the high maintenance costs make its operations there unprofitable and requested that the Malian government also contribute to the maintenance costs (Kaba 2012). When Malian authorities refused, the dispute was initially escalated to the International Court of Arbitration in Paris, but was later withdrawn. This dispute appears to be the reason Eskom has decided to abandon its operations in Mali as of July 2012.

In terms of employment, Eskom has largely performed in line with international normative standards related to prioritizing the employment and training of local workers. Eskom Uganda directly employs 107 people, only 2 of whom are non-Ugandans and many of whom come from the area where the plant is located (Lumbuye 2011). One local community leader considers that employment is the 
primary benefit the people from his village derive from Eskom Uganda (Kamugisha 2011). The company also indirectly employs another 108 workers through contractors. One point of criticism came from Ugandan electricity regulatory authorities, who criticized Eskom for failing to hire and train more local engineers at the plant, relying instead on a small number of overworked engineers (Kyoko and Wandera 2011). In Mali, Eskom employs 160, only one of whom (the South African managing director) in not from either Mali, Senegal or Mauritania (Kaba 2012). Eskom Mali has, however, not been as successful as Eskom Uganda in employing locals from the communities near its power plant. Eskom Mali's managing director admitted that the company "has not provided a large amount of employment opportunities for the local people" (Coulibaly 2012). In both Uganda and Mali, Eskom has conducted frequent training and skill-building programs for local employees, often taking employees to South Africa for capacity-building workshops in order to transfer skills and expertise (Ossiya 2011).

Eskom has not performed as well based on the normative standards' guidance on prioritizing local suppliers. Eskom Uganda insisted that "There are few [local] suppliers that can be relied upon to deliver on time and good quality. Local suppliers also require $50 \%$ up-front payment" (Mngeni 2011).

\section{Ensure reliable supply}

"Ensuring reliable supply of electricity to all South Africans" is one of Eskom's four strategic objectives, and the company sets reliability targets (Eskom 2012a). Nevertheless, load shedding throughout the late 2000s and continued threats of blackouts indicate that Eskom's plans on security of supply are inadequate (Energy Forecast 2011). The company does not have any publiclyavailable policies detailing what response can be expected from Eskom before and during interruptions. The company's 2011 Annual Report reveals that prepaid customers endure more frequent and longer interruptions. The duration of interruptions for prepaid customers is approximately double that of large industrial users and other users on a monthly billing system (Eskom 2012a). Eskom does appear to prioritize the issue in its $R \& D$ program, given that the company's second-largest $R \& D$ program (ZAR 22, \$2.8, €2.1 million in 2010) is dedicated to improving the availability and reliability of power plants (Mavhungu 2010).

In Uganda, load shedding and rolling blackouts are frequent and have increased in recent years due to a shortage of supply (Kyoko and Wandera 2011). After taking over the Nalubaale and Kiira hydroelectric stations in 2003, Eskom expanded the plants' capacity by $80 \mathrm{MW}$ to reach a total of $380 \mathrm{MW}$. Despite this addition and Eskom Uganda's insistence that it is a reliable source of electricity for the Ugandan electricity net, the age of the plant and low water levels have meant that Eskom has operated the plant at less than half $(46 \%)$ of its nominal capacity in recent years (Mngeni 2011). Eskom blames the reliability problems on the Ugandan government's decision to halt dieselbased electricity generation and the resulting 
lack of sufficient supply to meet demand. Yet at the same time, Ugandan electricity officials claim that Eskom could easily do more to ensure reliable electricity supply in Uganda by investing in the Bujagali power project (Kyoko and Wandera 2011).

Electricity supply is also frequently unreliable in Mali. Although Eskom provides 70\% of Mali's entire electricity supply (Coulibaly 2012), its Manantali power station also often operates at less than half $(45 \%)$ of its nominal capacity due to water levels (Dikgale 2012). In addition, Malian electricity officials suggest that Eskom's failure to invest properly in maintenance of electricity infrastructure has negatively affected the reliability of supply (Toure 2012).

\subsubsection{Cross-cutting issues}

\section{Engage in meaningful stakeholder engagement and participatory decision-making}

According to Mavhungu (2010), "Addressing the expectations of stakeholders such as employees, shareholders, governing bodies, suppliers, consumers and the wider community is an essential aspect of CR. We identify stakeholders, build understanding of stakeholder drivers, and engage stakeholders at appropriate levels of the decision-making process." Eskom's stakeholder engagement practices are based on the AA1000 stakeholder engagement standard principles of materiality, completeness, and responsiveness. Eskom's endorsement of the United Nations Global Compact and its alignment with King III also influence the company's stakeholder engagement process (Mavhungu 2010). The main Eskom environmental procedure EPC 32-96 specifies the establishment of a community grievance procedure "through which all complaints shall be reported, recorded, and investigated in compliance with each business unit's procedure" (World Bank 2010b). However, recent years have seen criticism from civil society that consultation processes on price setting and fuel mix determinations tend to be exclusive and inadequate, and in several recent annual reports Eskom has acknowledged a need for better stakeholder engagement (Eskom 2013b). Nevertheless, Eskom's general corporate policy on stakeholder engagement is moderately in line with international SEP norms on this issue (e.g. WCD 2000, WBCSD 2002, IFC 2007, UNGC 2008, Pillinger 2009, OECD 2011).

Eskom's policies and processes for stakeholder engagement received considerable criticism from various stakeholders in Mali and Uganda. Eskom Uganda has what it calls a "Community Forum" that deals with stakeholder consultation. However, the Community Forum focuses exclusively on security issues and is not actually open to the general public. Instead, the Forum, which meets each month, consists of local council chairmen from 10 councils and representatives of the four different organizations that Eskom Uganda relies on to provide security: the Ugandan People's Defense Force (UPDF), the Ugandan National Police, a private security contractor hired by Eskom to guard the facilities gates, and another private security provider. 
Eskom Uganda insists that participation in the Community Forum is strictly by invitation only due to the sensitive nature of the issues discussed (Kiryowa 2011). Through consultations with the Forum, Eskom Uganda made decisions to establish additional security measures such as a fence around the premises, security CCTV in strategic locations, covert security and intelligence-gathering operatives, sometimes in collaboration with the Ugandan government. The Forum has also facilitated the gathering of intelligence on persons entering the communities around the plant each month, where they came from, and any security threats that they might pose (Kiryowa 2011).

Eskom Uganda recruits and pays local community leaders to head up security teams. These teams patrol the area around the Eskom plant and stop locals from doing anything that might affect the security of the plant. For example, one security team in Buiwke District stops people from fishing near a bridge linked to the power plant (Kamugisha 2011). In Nalufenya Village in the Jinja District, the community security team protecting the Eskom power plant has nicknamed itself the "Village Vigilantes" and has received training from the Ugandan Police (Nicholas 2011). Interviews with two chairmen (Kamugisha 2011, Nicholas 2011) representing two councils in the Community Forum revealed that they were happy to participate in the decision-making on security issues and that they felt that the initiative had strengthened the relationship between Eskom Uganda and the surrounding communities. However, on all other issues aside from security and for all other stakeholders aside from the ones in the Community Forum, Eskom Uganda does not engage in any consultation or any form of participatory decision-making. The company simply states that there is sufficient information from Ugandan electricity regulators and authorities on what is happening in electricity generation, transmission and distribution (Matarutse 2011). This approach to stakeholder engagement and participatory decision-making is clearly out of line with international normative standards on the issue and has been criticized by civil society organizations in Uganda, who claim that they are kept completely in the dark with regard to Eskom's policies and performance on social and environmental issues (Binyina 2011).

The situation is only slightly better in Mali, where Eskom Mali does seek to consult stakeholders as to where they find Eskom lacking and how Eskom could better serve the needs of the people (Coulibaly 2012). At a more local level, however, Eskom Manantali management (Kaba 2012) clearly indicated that the local communities and local government officials are not generally consulted in decision-making pertaining to the company's core business of electricity production and distribution. Stakeholders are consulted only on matters relating to philanthropic development projects, such as the awareness campaigns against AIDS and malaria, and cultural events (Cissoko 2012). It was noted that the Chief of Manantali has "free access to Eskom Manantali's managing director for discussions in cases such as when the fishermen may 
have noticed oil in the water or in the event of any misbehavior by Eskom workers" (Kaba 2012). However, other community leaders in Manantali (Dabor 2012, Diallo 2012, Takoure 2012) complain of being blatantly ignored by Eskom in decision-making processes, even those that involve the community. Although Eskom was not responsible for the original construction of the dam and reservoir, one particular instance in which stakeholder engagement and participatory decision-making would have seemed paramount in 2008 when Eskom sought to expand the land used by the power plant by 1,398 ha to increase the plant's capacity. Although the land was not occupied at the time, many locals possessed title deeds to the land. Rather than itself engaging in any consultation of the local communities or land-owning individuals, Eskom simply paid XOF 123 million $(\$ 245,243, € 187,512)$ in compensation money to the government and left it completely up to the local authorities to engage and negotiate with the local land owners (Cissoko 2012, Dembele 2012).

The company does not endorse the principle of free, prior, and informed consent (FPIC) as stipulated in some international norms (IFC 2011). As a result of a recent World Bank loan evaluation process Eskom underwent, the company adopted a procedure akin to World Bank policy on involuntary resettlement that calls for meaningful consultation. Though this does entail consultation with stakeholders that is free and early in the project planning cycle (i.e. "prior"), Eskom's policy does not recognize a right for affected stakeholders to halt a project by withholding consent (World Bank 2010b). Eskom managers in Mali and Uganda had not heard of the principle of FPIC.

Eskom managers at the headquarters level engaged constructively with the author and researchers as part of the present study, completing (most of) the standardized questionnaire as well as facilitating contacts with local managers in Mali and Uganda. Managers in Mali and Uganda were very willing to engage, taking time to be interviewed in person by the researchers and facilitating contact with other appropriate employees.

\section{Assume responsibility for impacts throughout all business relationships}

Although Eskom does not explicitly state that it considers itself responsible for negative impacts throughout its business relationships, its supply chain policies indicate that it does seek to leverage its buying power to improve conditions in the supply chain as encouraged by international SEP norms (e.g. WBCSD 2002, ISO 2011, OECD 2011, UNHRC 2011). According to Mavhungu (2010), "Eskom works with suppliers, customers and contractors to integrate safety, health and environmental issues into their operations. Contractors working under our supervision or in our premises are expected to comply with Eskom's SHE policy." Despite this policy, in the five years between 2007-2011 there were 221 fatalities among contractors working for Eskom, approximately six times more than the fatality rate among Eskom employees (Eskom 2012a). Eskom (2011) states that it seeks to "drive transformation 
through its procurement strategy", and the SHE policy stipulates that safety, health and environmental concerns are taken into account "during the evaluation of all contracts, projects, and proposals" (Eskom 2007).

The main Eskom environmental procedure EPC 32-96 further stipulates that environmental clauses shall be included in all contract documents for all contractors and that only contractors with "proven track records of sound environmental performance shall be engaged for Eskom projects." Black ownership and BEE targets are also included in Eskom's evaluation of potential suppliers and contractors (Eskom 2009b). Eskom (Eskom 2012b) does have a code of conduct for suppliers, but this is principally focused on avoiding corruption and conflicts of interest. No information could be found on whether Eskom conducts audits of its suppliers and contractors to ensure social and environmental standards are being met.

According to Jiga (2011), Eskom Uganda recognizes and acknowledges publicly its responsibility for conditions at suppliers and contractors. The company has outsourced various services such as cleaning, horticulture, water hyacinth clearing, security, restaurant operation, and transport to contractors. Eskom Uganda has a supply chain policy that guides work with suppliers, but like Eskom headquarters' code of conduct for suppliers, the primary focus in procurement is preventing corruption, namely suppliers trying to influence and bribe Eskom staff to award them the contract (Ossiya 2011). In addition to corruption, supplier vetting procedures also have aspects of safety and compliance on legal and environmental requirements, but no mention is made of respect for labor rights beyond OHS. The purchasing and supply chain management strategy is guided by the company values of integrity, excellence, innovation, and customer satisfaction. Before a service provider is appointed, Eskom Uganda signs an agreement with the service provider that stipulates that they shall adhere to the Ugandan labor law including issues of salaries and wages. Eskom Uganda monitors this to make sure that service providers adhere to the agreement (Jiga 2011).

\section{Maximize transparency and provision of} information

The degree of accessibility and visibility of Eskom's CR policies is relatively high, as the company publishes many, though not all, of its SEP-related policies and procedures online. Eskom also produces an integrated annual report (i.e. including financial, operational, and sustainability aspects) in line with the King III Code, AA1000, the Global Reporting Initiative's G3 guidelines and, since 2011, the GRI's Electric Utility Sector Supplement (EUSS). Eskom's 2011 Integrated Annual Report was reported at a B+ GRI application level, and has a "limited assurance" rating by an independent assurance provider (Eskom 2012a). The B+ rating means that Eskom does not report on all indicators in the GRI or EUSS. For example, Eskom's 2011 Annual Report does not include information on indicators related to water withdrawal and recycling, or impacts on water sources. 
Although the company's annual reports are generally compliant with international normative standards on reporting, the quality and thoroughness of information provided by Eskom on some areas has declined since the company was corporatized in 2001 . For example, Eskom's 1999 environmental report contained 61 pages of information, but by 2002 this was reduced to five pages and referenced previous years' reporting. Eskom's annual report used to disclose the company's largest customers, but have not done so since 2001, despite guidance from international SEP norms to do so (OECD 2011, UNHRC 2011). The area that seems to have suffered the greatest decline in terms of degree of transparency and quality of information is Eskom's operations outside of South Africa. Prior to 2004, Eskom Enterprises published a full report in its own capacity providing a large degree of transparency on its activities in Africa and highlighting the company's support for NEPAD, but this stopped in 2003 . Since then, the degree of information on its African operations and commitment steadily declined each year. The 2009 report contains no specific information on activities in countries outside South Africa and mentions NEPAD in the acronyms section of the report only (Eskom 2010). The 2011 report does not mention NEPAD at all, nor does it contain any detailed information on any of the company's many subsidiaries, associations, and joint ventures outside of South Africa (Eskom 2012a).

This policy and trend at the headquarters level is clearly reflected in Eskom's operations in Mali and Uganda, where Eskom's lack of transparency and provision of information is one of the most frequently-cited grievances by a wide range of stakeholders. In Uganda, civil society organizations expressed concern about the low level of information and transparency provided by Eskom, which they claim routinely refuses public requests for information about its operations. Although the groups acknowledge that Eskom is not necessarily always or solely responsible for the blackouts and high prices, they feel that it is Eskom's responsibility to communicate critical information about electricity provision to the public (Binyina 2011). Eskom Uganda claims that its primary means of providing its stakeholders with information is through the Community Forum (Kiryowa 2011). However, the Forum is primarily focused on security issues and is actually not open to the general public, but rather by invitation only to a select group of community leaders and security company managers.

Through the Forum, the company provides some information on security issues, which the chairmen of councils take to the people in their villages. The company insisted that there is no need to translate any information into local languages unless Eskom is interested in communicating something to the community and they want to have a flyer, which they do sometimes pass out in the community (Matarutse 2011). During the Community Forum, the participants are allowed to speak their local language. Aside from the Forum, Eskom Uganda (2008) does have a website, but it contains no annual operational or financial reports and no reports on core-business 
environmental and social impacts. When asked why this is so, Eskom Uganda management insisted such information is too technical to share with the communities (Kiryowa 2011). However, it should again be noted that the CEO of Eskom Uganda and other senior management did exhibit a high degree of transparency when approached by researchers for the current study, largely providing all of the requested information.

A similar situation exists in Mali, where Eskom managers and Manantali community leaders agree that access to information for affected stakeholders is limited and that Eskom does not make its publications directly available to adjacent communities or to the public at large (Dembele 2012, Diallo 2012). Eskom has established a radio station that broadcasts to the communities surrounding the Manantali, but it uses this only to broadcast music and popular culture events rather than information about the operation or activities of the company (Dembele 2012). Eskom Mali managers explain that stakeholders can go through governmental institutions to obtain information about the company's operations and performance (Coulibaly 2012).

\subsubsection{The South African mode and Eskom's performance on the SEP benchmarks}

Eskom's performance on the SEP benchmarks indicates that the South African mode of business culture conditions Eskom's policymaking on most SEP norms. However, despite the fact that Eskom Mali's director insists that Eskom prides itself on being an "Africa-oriented electricity company" (Coulibaly 2012), the South African mode appears to have much less of a conditioning influence on how Eskom operates on the ground in host countries. Table 9.3 provides an overview of the degree to which Eskom's performance on SEP benchmarks is deemed to reflect the South African mode, as described in Chapter 4.

Eskom's incorporation of some, but far from all, international SEP norms is largely in line with the South African mode, which exhibits a history of using and relying on international standards and voluntary codes related to CR such as the UNGC, but which also retains a tendency toward South African and regionally-developed standards such as the King Code (Rossouw et al. 2002, King et al. 2010). In obtaining a high degree of standardized certifications (such as ISO) for its management procedures, Eskom - both at the home office and at subsidiaries in Mali and Uganda - again reflects the South African mode of placing relative importance on such certifications (King et al. 2010).

Eskom's general tendency toward philanthropic CSI and community development projects rather than core-business CR is largely in line with the South African mode, in which companies are often expected to engage in community development charity projects unrelated to their core business (Visser 2007, Swilling 2010). This approach is intensified in Mali and Uganda, where Eskom's CR/CSI activities and programs are almost entirely focused on non- 
core-business activities. This has led to ironic situations in which Eskom builds a school in a village adjacent to its power plant, but does not supply the school or the village with electricity. The importance Eskom places on (BB)BEE in its CR policies is also strongly typical of the South African mode. Eskom even includes black ownership and BEE targets are also included in Eskom's evaluation of potential suppliers and contractors (Eskom 2009b).

Eskom's abandonment of its earlier commitment to electrification - and its lack of electrification projects benefitting underserved communities in Mali and Uganda - contradicts the South African mode's push and need for development, including increased access to affordable electricity. One could argue, however, that Eskom's focus on large corporate customers is consistent with the South African mode's (primarily mining) industry-dominated economic development model.

On labor-related SEP issues, Eskom's performance, both in corporate policy as well as in practice in Mali and Uganda, is largely in line with the South African mode's history of a strong and well-established labor movement, and a strong respect for labor laws and norms. This is likely a reflection of the success of the labor movement in South Africa, which has ensured that the South African mode places a high priority on respect for labor rights.

Eskom's enormous environmental impact primarily resulting from its near-total focus on coal-fired electricity - is reflective of the South African mode, which suggests prioritization of

Table 9.3: Degree to which Eskom's performance on SEP benchmarks reflects the South African mode of business culture

\begin{tabular}{l|l|l}
\hline $\begin{array}{l}\text { Is Eskom's policy/practice reflective of the South African } \\
\text { mode on the following SEP benchmarks? }\end{array}$ & Policy & Practice \\
\hline Endorse international normative standards for CR & Largely reflective & Moderately unreflective \\
\hline $\begin{array}{l}\text { Adopt a commitment to CR in core-business activities and } \\
\text { decision-making }\end{array}$ & Moderately reflective & Moderately reflective \\
\hline \begin{tabular}{l} 
Increase access to affordable electricity \\
\hline Respect labor rights
\end{tabular} & Moderately unreflective & Largely unreflective \\
\hline $\begin{array}{l}\text { Minimize environmental impact, including contribution to } \\
\text { climate change }\end{array}$ & Largely reflective & Moderately reflective \\
\hline Prioritize renewable sources of energy for electricity & Moderately reflective & Moderately unreflective \\
\hline Contribute to local economic development & Moderately unreflective & Largely reflective \\
\hline Ensure reliable supply & Largely reflective & Moderately unreflective \\
\hline $\begin{array}{l}\text { Engage in meaningful stakeholder consultation and } \\
\text { participatory decision-making }\end{array}$ & Moderately reflective & Moderately unreflective \\
\hline Assume responsibility for impacts throughout all business \\
relationships
\end{tabular}


economic and, to a lesser degree, social issues over environmental concerns. Indicative of the company's relatively low priority on renewable energy and reducing emissions, Eskom is currently building a number of new large, coal-fired power stations, and its R\&D project on mitigating climate change received the third lowest budget of all Eskom R\&D projects in 2010. This is consistent with the South African mode, and indeed the South African Department of Environmental Affairs' (2013) plan to continue to increase greenhouse gas emissions in the near and even medium term. A contradictory element in this regard is the fact that, although Eskom operates only marginal renewable capacity in its home market, its facilities in Mali and Uganda are strictly hydro-based. Unlike its long experience with coal-fired electricity generation, Eskom and the South African mode historically exhibited limited experience with hydropower and the management of aquatic ecosystems and river sedimentation.

Eskom's general corporate policy on stakeholder engagement is moderately reflective of the South African mode, which traditionally embraces the concept of stakeholder engagement, and actively encourages an inclusive approach (Rossouw et al. 2002, Carapinha and de Jongh 2008, King et al. 2010). However, the reality in Mali and Uganda is far from inclusive. Eskom's community engagement there is limited to a few select individuals and is focused solely on the topic of security, while many other stakeholders, including local governmental officials and impacted communities, claim that they are "kept completely in the dark" with regard to Eskom's policies and performance on social and environmental issues (Binyina 2011). 


\section{Chapter 10}

Comparison and discussion

of case study results
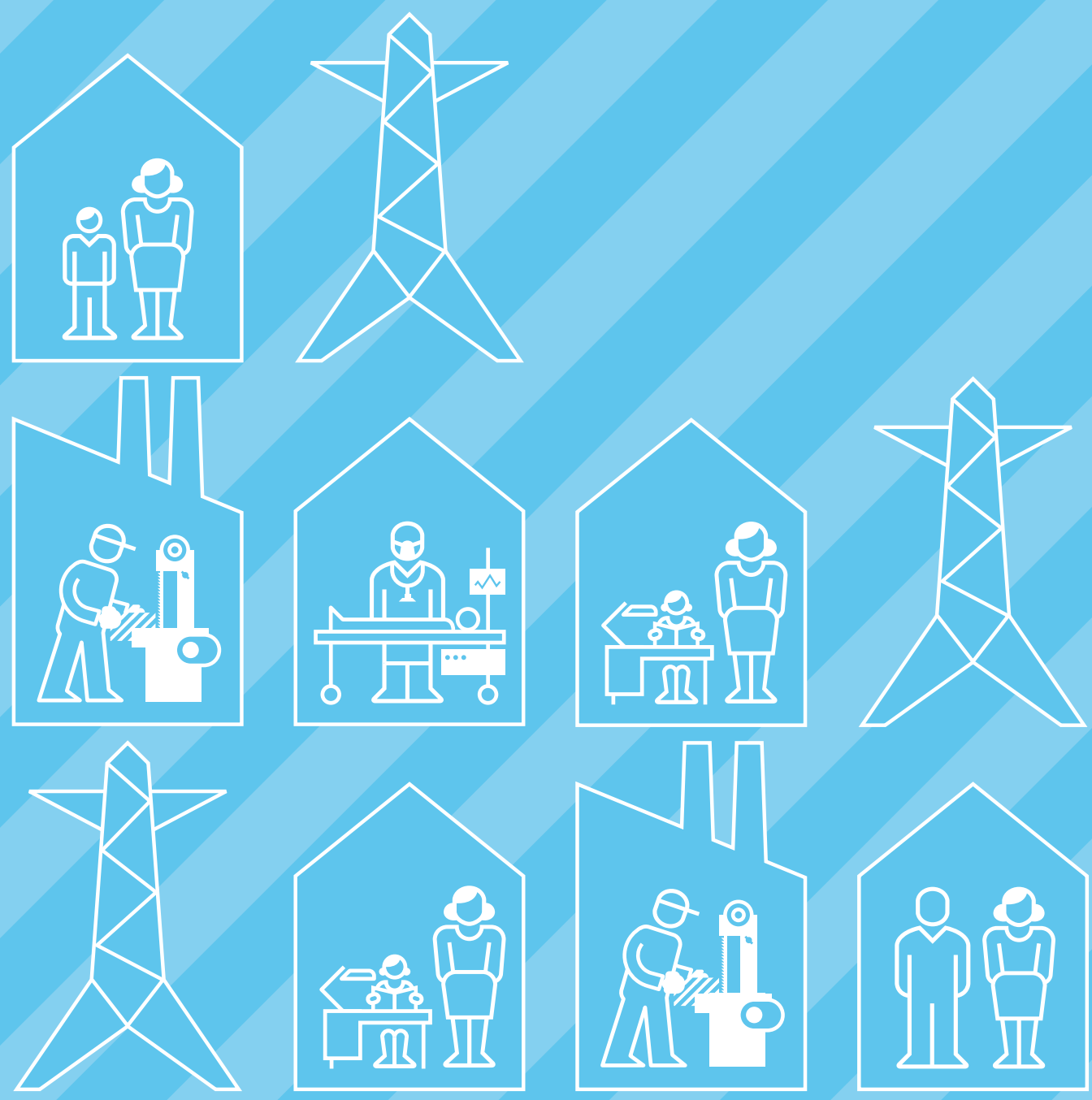
The previous five chapters have detailed the results of the extensive empirical research on the performance of the case study companies with respect to the SEP norms. The presentation covers the two central dimensions of the research design: policy formulation within the corporation and the record of practical implementation in the Global South. A key premise of the approach is that international normative standards for SEP are meant to apply to all electricity TNCs operating in the Global South around the world. As indicated, however, the literature suggests that the mode of business culture in a TNC's home country or region serves as a filter to the SEP norms, and thus "intervenes" to condition the degree to which the TNC adopts the norms; how it "translates" the norms into corporate policy; and how, finally, it works to implement the norms in specific developmental settings.

In order to facilitate the inductive analysis, with the aim of drawing conclusions and determining implications for both corporate decision-making as well as international norm-setting related to SEP, the current chapter provides a systematic comparison and scaling of the five companies in the multidimensional space of SEP policy and practice. For each individual SEP benchmark, a comparison and visualization of how the companies perform vis-à-vis each other on the different normative dimensions is carried out. After an initial analysis of each of the 11 SEP benchmarks, an overall comparison of company performance across all the norms is provided. The comparisons and analysis are based on the findings presented in the individual case study chapters. For more detailed information on company performance, please refer to the relevant chapter. Additionally, based on the findings in the five case studies, an analysis of the relevance of the home-country business culture modes for the overall problematic is presented. Finally, there is a discussion about the potential limitations of the research design and methods, and their potential impact on the results.

\subsection{Comparison of company performance against the individual SEP benchmarks \\ 10.1.1. General approach to corporate responsibility}

\section{Endorse international normative standards for corporate responsibility}

According to international SEP norms, electric utilities should seek to bring their CR policies in line with internationally recognized and verified standards for CR (ILO 2001, WBCSD 2002, UNGC 2008, IFC 2011, ISO 2011, OECD 2011, UNHRC 2011). However, the electricity companies investigated in the present study have vastly different visions on whether and how their CR policies should be based on international $\mathrm{CR}$ standards. The approaches range from a very high degree of explicit incorporation of international standards among SN Power, Eskom, and especially Endesa, to an almost 
total lack of reference to international CR standards in the CR policies of AES.

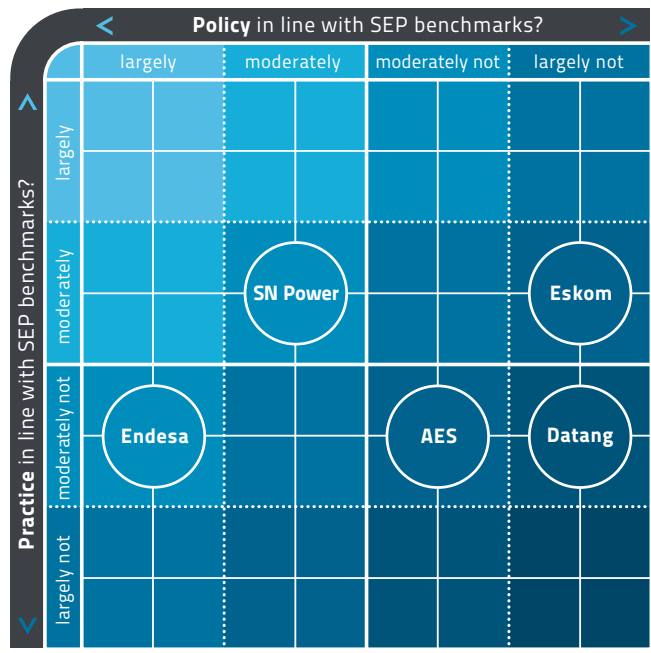

Figure 10.1 Comparison of company performance on the SEP benchmark "Endorse international normative standards for CR".

For AES, having policies that are in line with international standards is clearly less important than policies focused on ensuring strict compliance with legal and regulatory requirements. Beyond legal compliance, profit motives trump voluntary normative guidance on social, environmental, and economic issues. This lack of reference to international normative standards for CR is reflective of the US mode, which exhibits a high degree of government regulation in the electricity industry (Palast et al. 2000) and tends to focus on legal and regulatory requirements over incorporation of norms, suggesting that companies have a voluntary "responsibility" to society (Vogel 1996). Interestingly, AES's subsidiaries in Argentina and Peru make reference to slightly more international CR norms (mainly certification standards) than their parent company.

Uptake of international norms is also weak in Datang's CR policies. The priority Datang gives to Chinese CR standards is reflective of the Chinese mode, which has sought to define CR on its own terms rather than simply following the "Western model" of CR. Nevertheless, the UN Global Compact is an international standard that is relatively highly regarded by the Chinese mode, so it is no surprise that Datang publicly endorses this standard. What is surprising, however, is that Datang has not sought ISO 14000 certification for its environmental management, as this is another international standard that is generally well regarded in the Chinese mode.

In contrast to AES and Datang, Endesa's approach to CR is characterized by a thoroughly developed CR policy and is highly based on international standards and norms. Endesa's heavy reliance on, and reference to, international standards for CR is largely reflective of the European mode's tendency to rely heavily on (voluntary) normative standards for CR. Endesa makes reference in its CR materials to more international standards than any of the other companies analyzed in the present study. Although not as thoroughly norm-infused as Endesa, Eskom also references a large number of international norm sets, while at the same time also relying on several African standards. Eskom's approach in this regard is largely in line with the South African mode, 
which exhibits a history of using and relying on international codes and voluntary certification standards related to CR such as the UNGC and ISO, but which also retains a tendency toward South African standards such as the King Code and the NOSA OHS standard (Rossouw et al. 2002, King et al. 2010).

The importance Eskom places on regional standards is also reflected in the approach taken by the management at the company's subsidiaries in Mali and Uganda. Eskom Mali's managing director notes that Eskom prides itself on being an "Africa-oriented electricity company" steeped in African values as opposed to "Western companies" that had failed in electricity provision in Africa (Coulibaly 2012). The degree to which the other, more internationally used, standards have filtered down to Eskom management in the Global South, however, is questionable. Aside from ISO and NOSA certifications, managers at Eskom subsidiaries in Uganda and Mali had not heard of norms such as the UNGC, AA1000, or the Equator Principles, all of which are explicitly endorsed by headquarters.

SN Power's policies also reference many international CR norms, although the company does not mention these as frequently or as prominently in its CR materials as Endesa. International normative standards are not the primary factor defining SN Power's CR policies as they are with Endesa's. For example, SN Power has not been able to implement international health and safety standards at all of its projects (Kopstad 2008). No international normative standards are referenced in the company's Peru-specific CR materials, but the company claims it adheres to the same standards everywhere it operates. Instead, the company's approach to CR largely draws on the Nordic region's deeply ingrained tradition of "leading the way" on business, rather than relying solely on international consensus (Kuhnle and Ervik 1996). SN Power's (2012a) CR policies are based primarily on "deeply ingrained" Nordic values such as a "long-term investment strategy" and a "Norwegian renewable energy tradition", which the company considers its "competitive advantage". Interestingly, one manager at SN Power indicated that, among all the international CR norms, the IFC standards are the most influential in shaping the company's policies because demonstration of compliance with the IFC standards is often required when seeking loans from banks and international financial institutions.

Looking across all five companies, there is patchy uptake of international CR standards at best. In general, certification standards such as those developed by the ISO (9000 and 14000 series) and OHSAS (18000 series) are taken up more often by the electricity companies than broader CR standards such as the ILO conventions or the OECD Guidelines for Multinational Enterprises. The one international normative standard that does fare well among the electricity companies investigated here is the UN Global Compact. The UNGC was cited in the CR policies of four out of the five companies. There are several reasons that may explain 
why electricity companies are more compelled to endorse the UNGC than other standards. The legitimacy and weight lent to this standard by the official UN status cannot be denied. However, this is likely not to be the only reason, given the fact that other relevant UN standards (such as the UN Universal Declaration of Human Rights and the ILO core conventions) are not equally endorsed. Another reason is that the UNGC, unlike the OECD Guidelines and the ILO conventions, has a corporate "sign-on" system that encourages companies to endorse the principles so they are able to use the UNGC name and logo in corporate communications.

The UNGC also does not have an effective complaints system for stakeholders that believe a participating company is not acting in accordance with the UNGC principles. Also likely playing a role is the fact that the UNGC is the most general and vague of the major international CR norms. The UNGC consists of ten general principles that aim for broad applicability across all industrial sectors and all countries rather than providing specific normative guidance on corporate behavior. The general nature of the UNGC, the corporate sign-on system (which the UN actively promotes), and the lack of an effective complaints mechanism has led a large number of corporations (more than 6,000) to sign up to the UNGC, but has also led to what many have termed "blue-washing" (i.e. using the UN logo purely to improve a corporation's image) (Global Compact Critics 2012). Indeed, Runhaar and Lafferty (2009) conclude that the actual influence that the UNGC has on corporate decision-making and strategy related to CR is limited. They posit that the modest contribution of the UNGC to corporate strategy is a result of the Compact's lack of (industry) specificity and its "lowest common denominator" nature, which particularly reduces its influence among corporate frontrunners in the field of CR.

The findings reveal that role of regional, as opposed to international, CR standards is interesting. In several cases, regional CR standards are as important, if not slightly more important, than international standards. Datang references more Chinese standards than international standards, and SN Power insists that Nordic traditional values shape its policies and give it a competitive advantage. Eskom managers in Mali have never heard of the UN Global Compact, but are highly aware of South African standards. The US mode's "disapproval" of voluntary normative standards in favor of hard legal standards appears to have conditioned AES's approach of basically ignoring the existence of international CR standards.

Overall, the limited uptake of international CR standards by a sample of internationally operating electricity companies should be worrying to norm setters seeking to create a level playing field by having universally agreed standards. The limited uptake is even more concerning in the SEP context because the degree of endorsement of international CR norms is an accurate predictor of whether a company's more detailed policies on specific SEP issue areas will be in line with SEP norms. As can be seen in Table 10.1 below, the present 
analysis indicates that, if a company references multiple international CR standards, that company also develops detailed polices around specific SEP issues that are in line with SEP benchmarks. In other words, companies that go to the trouble of referencing an international norm tend to genuinely incorporate the spirit and letter of the standard into their policies.

\section{Adopt a commitment to CR in core-business activities and decision-making}

According to international SEP norms, electricity companies should adopt a holistic and integrated view of the role and impacts of electricity provision, considering and balancing the essential elements of economic development, environmental quality, and social equity in utility operations. This entails a policy commitment to CR throughout all corebusiness activities, as opposed to a primary focus on philanthropic activities (WBCSD 2002, MVO Platform 2012). Electric utilities should seek to introduce environmental and social factors and procedures into high-level corporate planning and decision-making and should aim to have a board-level position responsible for CR issues (WBCSD 2002). This crucial SEP norm thus contains the essence of a company's vision and approach to $C R$ in general and to the rest of the SEP benchmarks in particular. The framework of home-country modes that guided the selection of case study companies resulted in a significant degree of variance on this benchmark. Figure 10.2 provides a comparison of company performance on the SEP benchmark "Adopt a commitment to CR in core-business activities and decision-making".

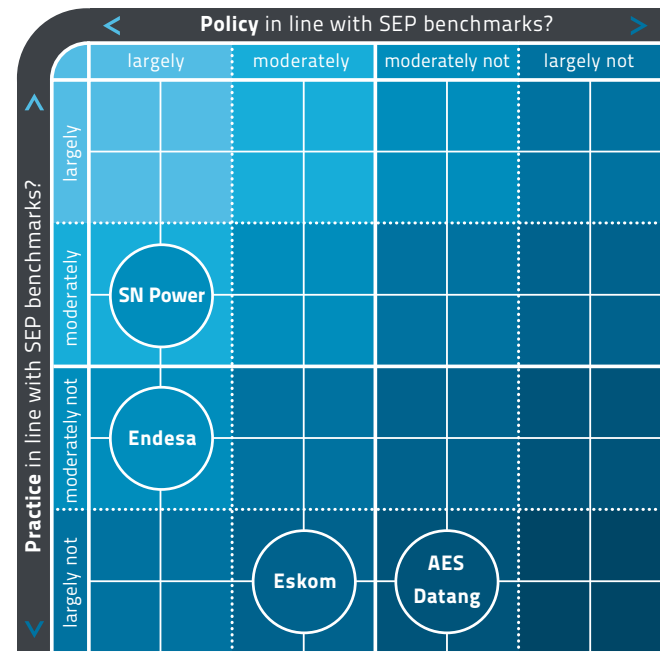

Figure 10.2: Comparison of company performance on the SEP benchmark "Adopt a commitment to CR in core-business activities and decision-making"

AES claims that sustainable development and CR are an integral part of its operations. Yet a thorough analysis of AES's CR-relevant policies and procedures reveals that the overall nature of the company's approach to CR can be characterized primarily as legalistic, philanthropic, and profit-oriented. This is largely out of line with international SEP norms, but is reflective of the US mode's legalistic focus and economic profit orientation. This approach leads the company to implement a CR program in Argentina that is narrowly focused on charity and avoiding legal risk. Argentine officials have openly criticized AES's "lack of commitment to corporate social responsibility" and "pursuit of profit over all else" (Bruera et al. 2008).

\section{Datang's publicly stated focus on economic} development is reflective of the Chinese mode and Chairman Deng Xiaoping's imperative 
of setting economic development as the "central task". Datang's tendency toward an unstructured CR program, where the primary visible contribution is charitable donations, is also typical of the Chinese mode, which rests on a long tradition of charity and philanthropy expressed through unstructured, ad hoc donations rather than through a coherent strategic corporate policy (APEC 2005). Datang's frenzied approach to mentioning CR targets, commitments, and achievements reflects the Chinese mode's more recent public embracing of what was long deemed a "Western" notion of CR as contributing to broader sustainable development. Datang is the only company in the present analysis that does not have a board-level position responsible for CR. Datang's approach is illustrated in Lao PDR, where the primary CR activity involves charitable donations.

In contrast to the policies of AES and Datang, Endesa's policies and management procedures at the headquarters level exhibit a clear focus on core-business activities, as is encouraged by SEP norms. Endesa also has the most extensive $\mathrm{CR}$ management structures in place of any of the companies in the present analysis, including a system of monetary incentives for managers who perform well on CR issues. A core-business CR focus is to be expected from a company that originates from the European mode. However, Endesa is either unable or unwilling to translate this policy approach into a similar core-business approach in its operations in the Global South. In Argentina, Endesa's activities reveal a strong tendency toward philanthropic projects, which results in a CR approach that is more similar to the American and Chinese mode than to the policy at the headquarters level. Local stakeholders claim their relationship with Endesa is based on charity rather than engagement on core-business issues. One felt Endesa's vision of CR is little more than "donating candy bars".

Eskom's historical focus on, and significant success in, the core-business of electrification is clearly in line with international SEP norms. The company is also committed to black economic empowerment, a core-business aspect of labor rights and local economic development and a hallmark of the South African mode. Eskom has performed well in this regard. However, since the company underwent a process of corporatization in the 1990s, its approach has evolved into one that is largely focused on philanthropy and charity. This tendency toward philanthropic and marketing-style "corporate social investment" is a departure from SEP norms. However, it is largely reflective of the South African mode, in which companies are often expected to assist in community development projects outside their core business (Visser 2007, Swilling 2010). Eskom's subsidiaries in Mali and Uganda take the trend toward charity and corporate marketing and away from core-business aspects even further. Eskom managers in these countries openly described the company's CR activities as a marketing tool and insisted Eskom has "no responsibility" for core-business $C R$ aspects like increasing access to affordable electricity, which are "far removed from the company's role in 
the project" (Coulibaly 2012, Dikgale 2012). One local government official relayed the anecdote that, while Eskom has financed the building of a school as part of its CSI, numerous other schools in the same area do not have access to electricity.

SN Power adopts a balanced approach that integrates economic, environmental, and social issues into its core-business activities and high-level corporate planning and decisionmaking. SN Power's policy is also largely in line with the Nordic mode, which generally exhibits a holistic approach to CR (Kuhnle and Ervik 1996, Aarhus 2010) and supports ethical standards at a high level within a company (Hohnen 2009, Aarhus 2010). Although SN Power was created as a profit-making enterprise and operates as a fully commercial entity, the company's core vision and mandate contain a deep commitment to sustainable development, which is reflected by the fact that its operations are limited to providing solely renewable electricity in countries without universal access (Kopstad 2008). The company's focus on hydroelectric generation projects in Peru lends the company's core business a clear tint of environmental sustainability. Apart from this, however, the company's CR programs in Peru are largely philanthropic activities.

This latter observation highlights a trend across all five of the companies in the present analysis. In every single case, the company's CR policies and programs at its operations in the Global South were focused more on philanthropy and charity than on core-business aspects.
This was to be expected from companies such as AES and Datang, whose home-country business culture and corporate headquarter policies encourage such an approach. Eskom's publicly stated focus on corporate marketing and corporate social investment, rather than corporate social responsibility, is probably the most obvious and striking example. However, the philanthropic approach to CR in the Global South is surprising from companies like Endesa and SN Power. Endesa's highly-standardized CR policies do not prevent the company's CR practice in Argentina and Peru from reverting to primarily charitable giving. SN Power, with its "deep rooted" core-business CR values, was the only company in the present analysis that retained a moderately core-business focus in its CR practice in Peru. However, even SN Power's Peruvian CR activities are more focused on philanthropy than is implied by headquarters' policy.

Companies are often expected by communities and local government officials in host countries to provide social goods outside their core business. Many companies describe such charitable giving as their social license to operate. However, according to SEP norms, if companies are to engage in such philanthropic activities, these should come only after ensuring a high standard of performance on all corebusiness SEP areas. Companies should also be aware that charitable projects can actually have negative implications for the long-term sustainable development of a country. Allowing profit-motivated corporations to provide public services other than those for which they are 
specifically contracted and regulated can weaken the role of the government, which is accountable for providing such services in the long term.

\subsubsection{Social issues}

\section{Increase access to affordable electricity}

According to international SEP norms, electricity companies should develop policies that promote access to electricity priced at affordable levels for all, paying special attention to disadvantaged communities (ILO/Palast et al. 2000, WBCSD 2002, PSI/Pillinger 2009). In order to do so, companies should undertake initiatives to extend electricity services to unserved and underserved communities, particularly in rural or remote areas, but also to the poor in urban and peri-urban areas (WBCSD 2002, UNDP/ Modi et al. 2005). This SEP standard is at the heart of the poverty reducing potential and impact of electricity provision and is the core business of all electricity companies. The SEP norms are clear that, while simply generating as much electricity as possible is important, companies must also ensure that the electricity they generate is accessible and affordable for the billions of people who currently lack access. Figure 10.3 provides a comparison of company performance on the SEP benchmark "Increase access to affordable electricity".

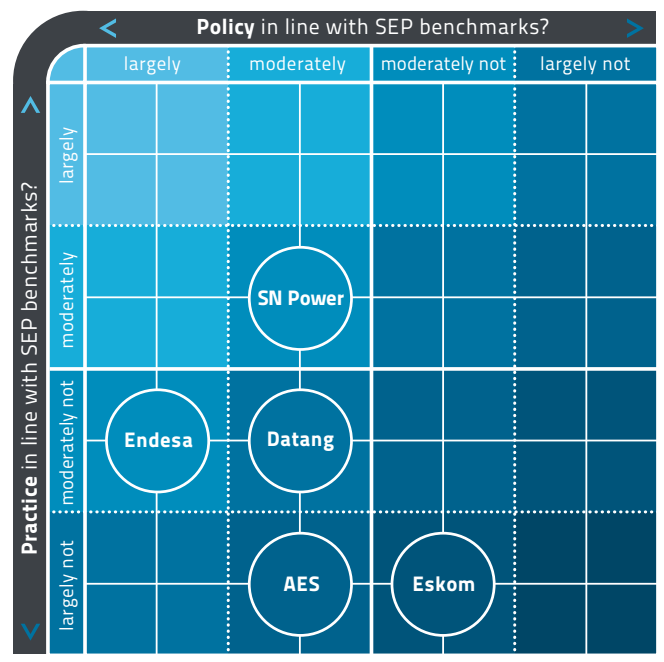

Figure 10.3: Comparison of company performance on the SEP benchmark "Increase access to affordable electricity"

AES produces $36,426 \mathrm{GWh}$ of electricity $(43 \%$ of the company's total global production) in countries without universal access to electricity. AES claims that "Bringing electricity to places that never had it before" is often a "profound outcome" of its business (AES 2012e). The company admits, however, that the main reason for undertaking all of these projects is that they are a "profitable business", a clear vestige of the US mode. The company does not have a publicly available policy on ensuring affordability. In Argentina, AES has lobbied and even sued the Argentine government to raise electricity prices. The company has even been accused of overcharging customers by government officials. The legal approach and the clear prioritization of profitability over ensuring affordability are hallmarks of the US mode.

Datang generates far more electricity $(472,575$ 
GWh in 2010) and is currently developing greater new capacity $(109,641 \mathrm{MW})$ in countries without universal access to electricity than any of the other companies in the present analysis. At the time of writing, less than $1 \%$ of Datang's production is found outside its home market of China. The sheer amount of electricity Datang provides and is developing means the company scores well in terms of increasing overall supply. However, it is not clear to what degree Datang's significant new generation capacity will go to actually increasing access to affordable electricity for poor communities. Neither increasing access to electricity for those lacking it nor making electricity more affordable for the poor are mentioned by Datang in any of its CR documents or policies. Much of its electricity production in $\mathbf{2 0 1 0}$ went to supplying mega-events such as the World Expo and the Asian Games. Datang's focus on large-scale, national economic development, and "prestige" events, rather than increasing access among poor communities, is largely reflective of the Chinese mode.

Endesa generates $47,813 \mathrm{GWh}$ of electricity $(43 \%$ of the company's total global production) each year in countries without universal access to electricity. If production in the respective home countries of Datang (China) and Eskom (South Africa) are not included in the current analysis, Endesa provides the most electricity of all five companies in countries without universal access. Endesa's policies at the headquarters level are largely in line with SEP norms that encourage electric utilities to promote access to electricity priced at affordable levels for all, paying special attention to disadvantaged communities. However, other than the large amounts of electricity it provides to these communities, there is little or no evidence of such programs or initiatives by Endesa in Argentina or Peru, despite the fact that electrification rates are as low as $22 \%$ in some of the areas where Endesa's power plants are located. The majority of Endesa's electricity is transported to large industrial and commercial customers.

Like Datang, Eskom generates an enormous amount of electricity, producing 232,182 GWh in 2011 and currently developing approximately $13,000 \mathrm{MW}$ of new capacity $(109,641 \mathrm{MW})$ in countries without universal access to electricity. Also like Datang, less than 1\% of Eskom's production is found outside its home market of South Africa. In the 1980s and 1990s, Eskom built up an impressive record of increasing access to electricity for disadvantaged South Africans as part of its stated CR. Many of the projects initially embarked upon by Eskom subsidiaries in other African countries were related to electrification schemes, and the company touted its contribution to Africa's development. However, after undergoing corporatization in the late 1990s, Eskom stopped contributing to the electrification program and has largely pulled back from its operations in Africa over the last decade, citing a lack of profitability.

Although a focus on the purely economic aspects of electricity provision is reflective of the South African mode, this marked a 
departure from Eskom's earlier commitment to increasing access. Eskom has touted its cheap electricity, but this has mainly been for its large corporate customers in the mining industry, to whom it has expressed a public commitment. Small domestic consumers have been unable to afford electricity, resulting in a large number of self-disconnections and disconnections by Eskom. There are no programs to increase access or affordability in Mali and Uganda, where Eskom managers were largely unaware of the electricity prices and electrification rates in the areas adjacent to Eskom's power pants. In these areas, electricity prices are among the highest in the region, while electrification rates are among the lowest in the world. Eskom's approach and performance reveals a key dynamic at play within this crucial SEP issue area. Prior to commercialization, social issues clearly took priority and Eskom rapidly increased access to electricity as a part of its CR. After commercialization, profit imperatives clearly outweighed access and affordability, and Eskom abruptly ceased considering the latter as its responsibility, relegating them to matters for the government to address with little or no involvement from the company.

Although $100 \%$ of the electricity generated by SN Power each year is in countries without universal access (and outside its home market), the total amount of electricity produced by the company $(5,954 \mathrm{GWh}$ in 2010) pales in comparison to the other companies in the present study. SN Power's total global production represents just $1 \%$ of Datang's. That said, SN Power is the only company of the five whose performance on increasing access to affordable electricity for communities adjacent to its plants is even moderately in line with SEP norms. The company is engaged in several rural electrification projects and assists other communities in approaching the government to request implementation of such schemes. Communities served by SN Power in Peru are satisfied with the company's affordability programs. Nevertheless, electrification rates in SN Power's areas of operation in Peru remain low, and many adjacent communities remain without access while much of SN Power's electricity is sent to high-density population centers or large industrial users, such as the mining industry. SN Power's policy is generally in line with the Nordic mode, which encourages an egalitarian approach to development that seeks to include all members of society and pays particular attention to the disadvantaged, but that excludes some projects that could increase access for large numbers of people because of environmental concerns (Aarhus 2010).

Comparing approaches and performance across all five companies, Datang is far and away the largest aggregate producer of electricity. Datang currently produces more than twice as much electricity as the second-largest company (Eskom) and is developing 10 times more provision than Eskom. Datang and Eskom provide by far the most electricity in countries without universal access. However, if one considers only countries without universal access outside a company's home country. Datang and Eskom perform very poorly. Both 
companies generate less than $1 \%$ of their total supply in other countries. In this scenario, Endesa provides the most electricity $(47,813$ GWh) and SN Power the highest percentage relative to its total global production (100\%).

Beyond sheer electricity production, however, none of the companies in the present analysis proved to be genuinely committed to increasing access to affordable electricity as conceptualized by the SEP norms. In many cases, the areas in which power plants are located experience low rates of electrification. However, with a few limited exceptions, the TNCs are not serving the communities closest to their electricity generation facilities, despite several having CR policies that proclaim support for local communities. None of the present companies is involved to any significant degree in promoting off-grid, decentralized electricity generation technologies that are widely considered to be key to increasing access to affordable electricity (Modi et al. 2005). Instead, all five companies promote largescale, centralized generation and high-voltage distribution to large users.

Delivering electricity to rural populations is a challenging task because it involves remote areas with dispersed customers whose consumption is low. This means it is generally more expensive (costs of connecting new customers increase exponentially with the customer's distance from a power station), while at the same time the customer base is generally poorer and less able to pay the full cost of service. Cost recovery is thus extremely difficult, and companies that make investment decisions based on a prioritization of financial returns over the needs of the population will generally not pursue such projects (Zerriffi 2007).

The performance of Eskom prior to and postcommercialization is illustrative and reveals clear limits to market-based electrification programs on the scale and at the pace needed to meet the MDGs. SN Power performs relatively well compared to the other five companies, although it too has withdrawn from countries that are desperately in need of investment in electricity infrastructure because of profitability concerns. The amount of electricity that SN Power and the Nordic mode are able to contribute to electrification programs is a drop in the bucket of what is needed to achieve electrification and poverty reduction goals sought by the MDGs.

The present analysis reveals that the current international normative framework for SEP clearly does not effectively encourage electricity companies to invest in sustainable electrification schemes. In the absence of an international normative framework that can truly compel (through sanctions or effective incentives) private capital to invest on a significant scale in sustainable electrification schemes, national governments, individually and collectively, must step in.

If it does not go into electrification schemes, where does all that electricity being generated by the power companies in the present 
study go? The corollary of a general failure to provide affordable electricity to underserved populations is a situation in which the vast majority of the electricity generated by the present companies is supplied to large industrial (e.g. mining operations, refineries, smelters) and commercial (e.g. shopping centers, large sporting events) customers. There is also evidence of a tendency to supply industry with cheaper prices than domestic consumers. This was most clearly revealed by the case of Eskom, but even SN Power, despite its sustainable Nordic mode of doing business, sells a large portion of the electricity it generates in Peru to mining companies.

\section{Respect labor rights}

According to international SEP norms, electric utilities should respect workers' full rights and provide workers with fair pay, job security, and decent working conditions, including high levels of health and safety standards in all aspects of operations (WBCSD 2002, PSI/ Pillinger 2009, OECD 2011). Furthermore, electric utilities should uphold freedom of association and the right to collective bargaining and pay particular attention to the right to strike (ILO 1998, ILO 2001, UNGC 2008, OECD 2011). Companies should ensure that these rights are respected, not only among their own employees, but also among suppliers, contractors and other business relationships (ISO 2011, OECD 2011, UNHRC 2011). Figure 10.4 provides a comparison of company performance on the SEP benchmark "Respect labor rights".

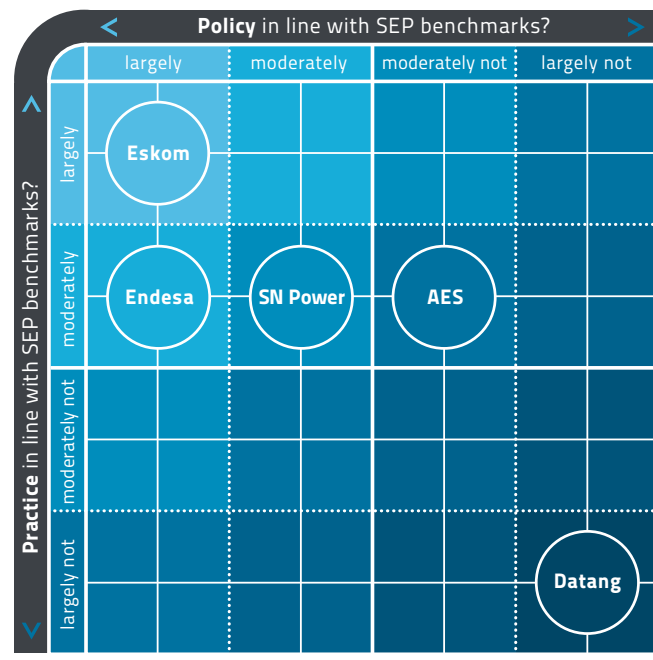

Figure 10.4: Comparison of company performance on the SEP benchmark "Respect labor rights"

With the exception of Datang, the present analysis found company practice in the Global South to be moderately in line with SEP norms encouraging respect for workers' basic rights such as freedom of association, collective bargaining, fair pay, and decent OHS conditions. This was also the case at AES Argentina, despite corporate headquarters' lack of policies (other than on $\mathrm{OHS}$ ) to protect the rights of its workers to decent working conditions and the US mode's relative antipathy towards organized labor. Despite a few reports of companies trying to pressure employees not to join unions, levels of unionization and coverage of collective bargaining agreements was high among all companies except Datang. In many cases, even the lowest paid employees were paid twice the national minimum wage. Although several injuries and fatalities were reported, all of the companies except Datang had in place 
extensive OHS policies and programs to protect employees' safety. The relatively high standards of labor conditions among most of the present electricity companies, regardless of their home country or region, is at least partly attributable to the fact that electricity-sector workers are generally more highly educated and more skilled than their counterparts in the manufacturing and construction industries.

Despite the generally good working conditions, two major concerns for workers of all five case study companies involved the right to strike and job security/outsourcing. Because electricity is considered an "essential service", electricity workers' right to strike is limited in many countries. Eskom Mali was the only company in the present study that insisted its employees have a right to strike. The other companies either do not have a policy or indicate that they simply follow the local law (which means no right, or a restricted right to strike) without taking any additional measures. SEP norms encourage electricity companies to develop contingency plans that allow workers to strike without threatening the reliability of supply.

With regard to job security, the outsourcing of functions previously held by direct employees of the electricity companies has increased markedly in recent years. Outsourcing is particularly common for maintenance tasks, but some companies have also begun to contract out operational duties. This "flexibilization" of the labor market in the electricity industry has meant that many long-term employees of electricity companies have lost their jobs and have been replaced by a less-skilled, lower-paid employee of a company contracted to complete a certain task. Workers at SN Power and Endesa in Peru expressed a constant state of worry that they might be laid off and replaced by a contract laborer. At one Endesa subsidiary in Peru, $80 \%$ of the workforce is contract labor, and only $20 \%$ is employed directly by Endesa. The outsourcing trend is all the more worrying because working conditions among contracted labor are worse than for direct employees, in some cases much worse. Despite pledges by the companies to monitor conditions, contract laborers work longer hours for less pay and are exposed to more occupational health and safety risks. Injury and fatality rates are higher among contract workers than direct employees, and many contract workers are required to supply and pay for their own safety equipment.

Datang is clearly an outlier with regard to respect for labor rights among the five companies. Largely in line with the Chinese mode, Datang does have policies against the use of child labor and discrimination in the workplace, but there is no indication that the company has a policy to respect other core labor rights or provide decent working conditions to its employees. Workers at Datang's dam sites in Cambodia and Lao PDR, many of whom are migrant workers imported from China, work up to 15 hours per day under "harsh" conditions and are paid on a piece-rate basis (Chinese migrant laborers in Cambodia 2010). There is no union or certified $\mathrm{OHS}$ in place. One manager in Cambodia confided, "When we contract out projects to construction companies... 
we don't evaluate whether the contractors have respected the migrant workers' rights" (Anonymous manager in Cambodia 1 2010). It deserves reiterating here that the type of workers and work researched in the Datang case study differs from the other case studies. The Datang case involves workers involved in basic manual labor on hydroelectric dam construction sites, not workers involved in the operations of generating, transmitting, and distributing electricity as was the case with the other companies. Nevertheless, the Datang case study is illustrative of the company's practice and the Chinese mode more broadly.

\subsubsection{Environmental issues}

\section{Minimize environmental impact, including contribution to climate change}

According to international SEP norms, electric utilities should seek to minimize emissions and discharges to air and water and continually develop and implement low-pollution and lowenvironmental impact technologies (WBCSD 2002, UNGC 2008,Pillinger 2009, OECD 2011). Companies should manage their environmental impact in line with internationally recognized and verified standards such as the ISO 14000 series and should always undertake rigorous and verifiable environmental impact assessments (WCD 2000, WBCSD 2002, IHA 2004, OECD 2011). Electric utilities should minimize their contribution to climate change and develop strategies for reducing GHG emissions (WBCSD 2002, OECD 2011). As a matter of priority, electric utilities should seek to avoid impacts on biodiversity and ecosystem services, refraining from any activities in areas of critical habitat with high biodiversity value while at the same time initiating or supporting conservation and biodiversity efforts related to impacts on natural habitats from utility operations (WBCSD 2002, IHA 2004, IFC 2011). Figure 10.5 provides a comparison of company performance on the SEP benchmark "Minimize environmental impact, including contribution to climate change".

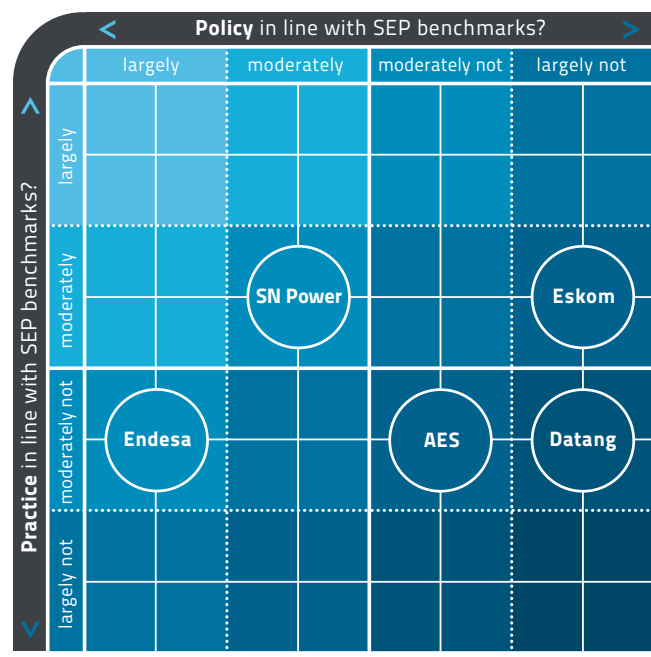

Figure 10.5: Comparison of company performance on SEP benchmark "Minimize environmental impact, including contribution to climate change"

Despite recently achieving ISO 14001 certification of its EMS, AES performs poorly in terms of policy and practice on the environmental standards expressed in SEP norms. The low priority set on achieving a high level of environmental performance is largely reflective of the US mode. The company has the highest total $\mathrm{CO}_{2}$ emissions of the three "Western" companies in the present analysis. It also has the second-highest emission 
intensity - higher than Datang and only slightly lower than Eskom. With $74 \%$ of its new capacity construction based on coal, AES's carbon footprint is set to increase. The company's reporting to the CDP (2011) reveals "a limited ability to measure and manage the company's carbon footprint". Rather than reducing its own emissions, AES's climate strategy is largely focused on profit generating opportunities in emissions trading. Despite operating 23 large dams globally, including a 2,659 MW hydro plant in Brazil, AES has no publicly available policy or document outlining its biodiversity protection policies and measures. AES practice in Argentina is similarly out of line with SEP norms. AES Argentina is among the four largest $\mathrm{CO}_{2}$-emitting companies in Latin America, and it has been censured and fined multiple times by environmental authorities for pollution and environmental management failures.

Datang's massive coal-fired capacity makes it the largest aggregate emitter of $\mathrm{CO}_{2}$ among the five companies in the current study. However, due to the company's similarly massive hydropower capacity and a concerted effort to shut down inefficient plants, Datang's emission intensity is significantly lower than that of Eskom and AES. Datang should be given credit for setting targets for and investing in reduction of emission of $\mathrm{CO}_{2}$ and other air pollutants.

Nevertheless, the sheer volume of its emissions and the magnitude of its environmental impact, along with the fact that Datang continues to vigorously develop coal-fired power plants, mean that the company cannot be considered to be in line with international environmental
SEP norms. Similarly, the company's development of large-scale hydroelectric dams without a comprehensive biodiversity protection policy (both inside and outside China) reveals that it does not prioritize avoidance of impacts on biodiversity and ecosystem services. This approach is, however, firmly in line with the Chinese mode, which openly prioritizes economic development over environmental concerns. The fact that Datang has not sought ISO certification for its EMS is surprising given the Chinese mode's uptake of technical standards like the ISO 14000 series (Sutherland and Whelan 2009).

Of the four "carbon-emitting" companies in the present study (i.e. excluding SN Power), Endesa emits the least $\mathrm{CO}_{2}$ and has by far the lowest emission intensity. The company sets and has met emission reduction targets. Endesa's reporting to the CDP (2011) reveals that it "understands the business issues related to climate change and is building climate-related risks and opportunities into core business". What stands out about Endesa's approach is that, while it is vigorously pursuing emission reduction and clean technology development in its home market, its environmental impact in Latin America is increasing. Two of Argentina's largest $\mathrm{CO}_{2}$-emitting plants belong to Endesa, and $\mathrm{CO}_{2}$ emissions in both Argentina and Peru have risen in recent years. Endesa admits that this situation is because there are stricter emissions regulations in Europe than in the Global South, where the company seeks to follow "voluntary" international norms. Generating approximately 232 TWh of electricity 
each year, the vast majority (93\%) of it by burning low-grade coal, means that Eskom has an enormous impact on the environment. Although it generates only half the amount of electricity that Datang does, Eskom's high emission intensity (the highest of the five companies in the present analysis) means that its total $\mathrm{CO}_{2}$ emissions are comparable to Datang's. Eskom has openly stated that it actually plans to increase its $\mathrm{CO}_{2}$ emissions in the short term, peak and stabilize them in the medium term, and eventually begin to reduce them in the long term. Although this approach is clearly not in line with international normative standards for SEP, it is largely reflective of the South African mode, and indeed the South African government's plan to increase, peak, plateau, and reduce emissions. More broadly, Eskom's enormous environmental impact is reflective of the South African mode, which suggests prioritization of economic and social issues over environmental concerns. Nevertheless the South African mode's tendency toward standardization of procedures has led the company to seek to develop systems to manage its impact. Eskom has a detailed and thoroughly integrated EMS and has been a frontrunner on EIAs.

Other than the fact that SN Power's EMS is not certified by an independent body and the lack of a public policy on biodiversity, the company's policies on minimizing environmental impact are moderately in line with international SEP norms. The prominent role given to environmental issues in SN Power's policies is reflective of the Nordic mode, which places high priority on environmental issues such as minimization of pollution and contribution to climate change (ECOTEC 2007, Aarhus 2010). It should be noted, however, that the company is continuing to develop ever-larger hydroelectric projects, ensuring that the company's impact on aquatic ecosystems and biodiversity is set to increase.

Making comparisons across all five case studies, company policy towards managing environmental impacts follows largely along the lines projected by the home-country modes framework. The two "emerging market" companies, Chinese Datang and South African Eskom, are clearly focused on rapid, large-scale infrastructure development, with environmental issues receiving lower priority. The environmental impact of these companies is concomitantly significant. Interestingly, however, both Datang and Eskom perform slightly better on this SEP standard in their operations outside their home countries. This is due to the fact that both are operating only hydropower in Cambodia/Lao and Mali/Uganda. Nevertheless, impact on aquatic ecosystems and biodiversity remains a concern, especially for Datang. The opposite trend is observed at European Endesa, where a highly standardized and ambitious environmental policy has led to strong performance on emission reduction in its home market. However, this is not reflected in its operations outside China. The company admits that the relative weakness or nonexistence of binding environmental standards in the Global South has resulted in this situation. American AES, on the other hand, is vigorously pursuing business opportunities opened up by climate change such as offset generation and emissions trading markets. 
Prioritize renewable sources of energy

\section{for electricity}

According to international SEP norms, electric utilities should evaluate the renewable energy potential of the host country and prioritize development and exploitation of renewable options over fossil fuel-based technologies (WBCSD 2002, UN/IAEA 2007, Pillinger 2009). Electric utilities should also prioritize renewables in their research and development programs and should support the diffusion of renewable energy technologies into the local economy (WBCSD 2002, UNGC 2008). Figure 10.6 provides a comparison of company performance on the SEP benchmark "Prioritize renewable sources of energy for electricity".

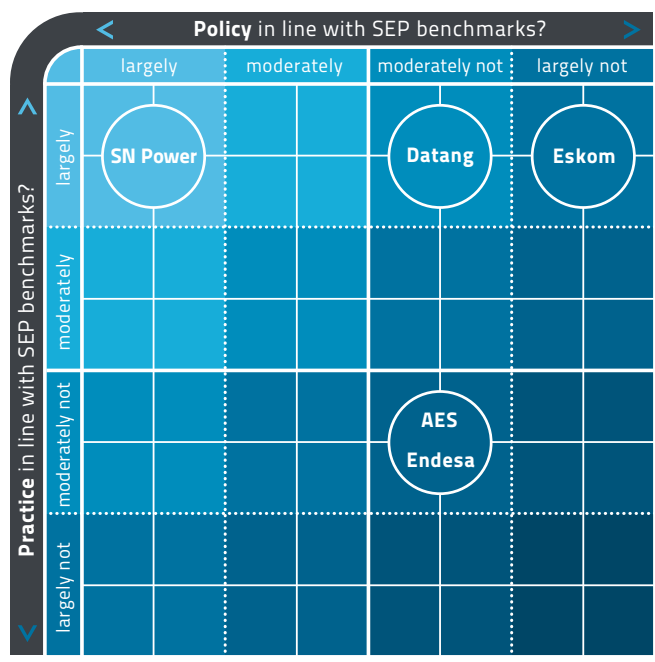

Figure 10.6: Comparison of company performance on the SEP benchmark "Prioritize renewable sources of energy for electricity"

With $78 \%$ of its current production and $85 \%$ of its build program based on non-renewable sources of energy, primarily coal, AES is clearly not prioritizing the development and exploitation of renewable options over fossil fuel-based technologies, as suggested by SEP norms. Its tendency towards the continued development of fossil fuels, which remain more economically attractive from a short-term profit perspective, is largely in line with the US mode. Despite Argentina's high technical potential for renewable sources of energy for electricity, AES has left this potential largely untapped and continues to make widespread use of fossil fuels. The wind and solar power that the company is developing is primarily in developed countries (mainly Europe and the US), while the new coal capacity is planned in the Global South.

The sheer scale of Datang's electricity provision operations again plays a role in analyzing its performance against the SEP benchmark. The company produces more electricity from renewable sources than any of the other companies in the present study. Datang operates the largest wind farm in the world. Although wind power comprises just $2 \%$ if its total production, Datang generates more electricity from wind than SN Power does with its total global capacity. Datang's hydropower production is 10 times the Nordic company's total production. In addition, one-third of Datang's build program is dedicated to renewables, including a significant portion for wind. Datang's much smaller build program outside China is mainly based on large-scale hydropower. However, Datang's development of renewables is overshadowed by the massive amount of coal-fired power it produces and is planning. Nearly $90 \%$ of Datang's current 
production is coal-based, and $58 \%$ of its build program is based on non-renewables, divided equally between coal and nuclear power. Although it is vigorously developing renewable electricity, Datang cannot be considered to be prioritizing renewables in line with SEP norms. Datang's more concentrated focus on renewable hydropower in Cambodia and Lao PDR is largely reflective of the Chinese mode of hydropower development abroad, and means that it is more in line with international SEP norms on this issue abroad than in its home country. Datang's simultaneous development of large amounts of non-renewable and renewable sources of energy reflects the Chinese mode of prioritizing economic development (in this case through increased electricity production) at all costs.

Apart from SN Power, Endesa has the highest percentage (34\%) of renewables in its global fuel mix than any of the other companies in the present study. The company also operates more natural gas capacity than any other company. Although natural gas is a non-renewable fossil fuel, it is cleaner and more efficient than coal. Still, the company continues to invest heavily in non-renewable sources. Only $9 \%$ of the company's 2010 capital expenditures in its home market in $\mathbf{2 0 1 0}$ were in renewable electricity. In Argentina and Peru, Endesa does operate a significant amount of large hydro capacity, but there is no evidence the company is prioritizing other, more sustainable forms of energy. Furthermore, Endesa has recently been shifting some of its natural gas capacity to less efficient, more $\mathrm{CO}_{2}$-intensive fuel oil. Such practices are uncharacteristic of the European mode. Eskom clearly does not have a policy prioritizing renewable sources of energy in favor of fossil fuels. A full $99 \%$ of Eskom's current electricity production is based on non-renewables - $93 \%$ alone on coal - a situation that Eskom is not planning to change in the near or middle term. After recently scrapping a wind power project from its build program due to a lack of funding, Eskom's current build program contains $0 \%$ renewables. Even the company's R\&D expenditures on "new" generation technologies is dominated by coal, with the vast majority of research money flowing into technologies like coal gasification, carbon capture and storage (CCS) for coal plants, carbon sequestration techniques, and fine coal utilization. The company's focus on pushing economic development over environmental concerns is largely indicative of the South African mode (Visser 2008). Part of Eskom's strategic engagement with Africa is a desire to increase the level of hydropower imported as a means of mitigating this heavy coal reliance. Indeed, in contrast to the company's clear predilection for fossil fuels in South Africa, Eskom operates solely large-scale hydroelectric facilities in Mali and Uganda, putting it more in line with SEP norms in practice in the countries.

SN Power is clearly the outlier on this SEP standard. As a matter of policy that the company implements in practice, $100 \%$ of the company's electricity production is based on renewable sources of energy. SN Power's policy and practice are largely in line with the SEP norms and largely reflective of the Nordic 
mode's focus on renewable energy, primarily hydropower. However, it is notable that, although SN Power currently operates a mix of large and small-scale hydro facilities and one wind farm, the company's current build program includes only large hydro.

\section{Making comparisons across all five case} studies, it is clear that, with the obvious exception of SN Power, none of the other companies in the present study is genuinely prioritizing renewable electricity. Chinese Datang is adding massive amounts of new renewable capacity, but the economic development imperative of the Chinese mode is also driving it to develop considerably more non-renewable capacity. A frighteningly impressive $99 \%$ of Eskom's current capacity and $100 \%$ of its build program is based on non-renewable sources, primarily low-grade, high-emission coal. Interestingly, although performing poorly at home, both Datang's and Eskom's model for expansion into other countries in the Global South is largely renewable-based. The converse is the case with AES and Endesa, both of which are developing (some) renewable capacity at home, while non-renewable capacity and technologies are being exported to the Global South. Although totally renewable, SN Power's limited scale makes its overall contribution to global renewable electricity production minor. None of the companies, including SN Power, is prioritizing development of the vast technical potential to generate sustainable electricity from sources like wind, small hydro, solar, geothermal, tidal, or wave.

\subsubsection{Economic issues}

\section{Contribute to local economic development}

According to international SEP norms, electric utilities should prioritize the development of local energy-related infrastructure and employ local workers to the greatest extent practicable, providing those workers with sustainable jobs and training to improve skill levels (WBCSD 2002, PSI/Pillinger 2009, OECD 2011). In order to maximize local economic development and job creation, electric utilities should support local small- and medium-sized enterprises in their procurement practices and contribute to the long-term development prospects of the host country through the transfer and rapid diffusion of technologies, skills and knowledge (WBCSD 2002, OECD 2011). Fair payment of due taxes to local, regional, and national governments in the host country is a crucial element of contributing to local economic development (OECD 2011, MVO Platform 2012). Figure 10.7 provides a comparison of company performance on the SEP benchmark "Contribute to local economic development". 


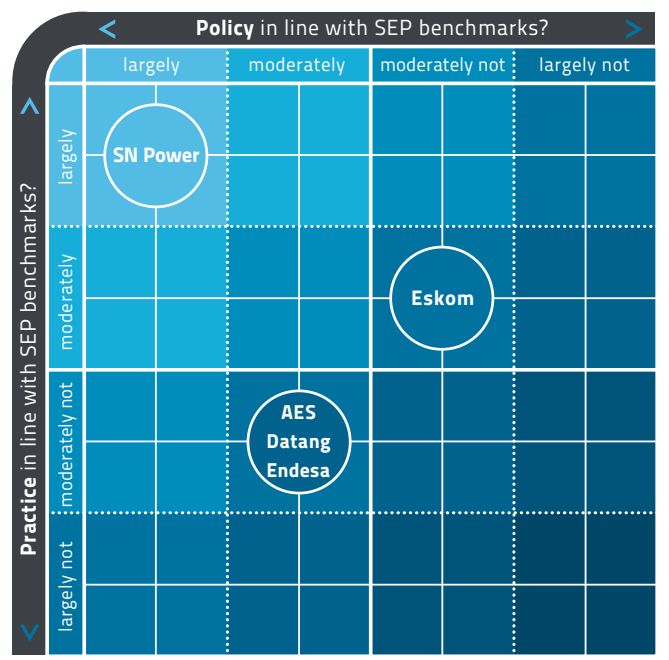

Figure 10.7: Comparison of company performance on the SEP benchmark "Contribute to local economic development"

Though AES (2012e) claims its main contribution to local economic development is "providing a safe and reliable supply of electricity, which is a prerequisite for economic development, security and public welfare", stakeholders in Argentina perceive exactly the opposite. AES has been harshly criticized by regulators and consumers for its perceived lack of commitment to economic development and failure to make sufficient investment in infrastructure. Local residents complain about electricity poles falling down, sub-stations exploding, and transformers short-circuiting. Many claim that AES has had a negative net impact on local economic development, as many businesses suffer financial losses from the sudden and unannounced power cuts. Local authorities blame the situation directly on AES's failure to invest in replacing dangerous, dilapidated infrastructure. Argentina's electricity regulator has fined AES at least 20 times in recent years for this lack of investment and even forced the company (through a law suit) to invest in new electricity poles. AES insists it has invested $\$ 1$ billion (€762 million) in electricity infrastructure, but acknowledges its commitment to investing in infrastructure has "probably not met its clients' expectations" (Anonymous manager in Argentina 1 2008). The company does employ 430 people in Argentina, all Argentine nationals, but it has no publicly available policy for prioritizing local suppliers or workers from the communities in which it operates. It should be noted that the Argentine economic crisis in 2001 and the government's subsequent macroeconomic policies, such as the devaluation of the Argentine currency, made it difficult for TNCs to profit from their investments. AES thus responded largely in line with the American mode, which projects that companies will prioritize profit making and not invest in infrastructure if it is not profitable.

Datang is investing more than $\$ 1.2$ (€1) billion in electricity infrastructure in Cambodia and Lao PDR. This considerable investment is reflective of the Chinese mode's emphasis on economic development. In contrast to this significant contribution to local economic development, the other striking aspect of Datang's approach that was observed in Cambodia and Lao PDR is its failure to prioritize local workers. Datang imports and employs a large number of Chinese migrant workers rather than hiring and training locals. Datang's proclivity for workers who are willing to work under harsh working conditions with few benefits or safety measures stems 
from the Chinese mode's traditions related to a lack of respect for labor rights (Debroux 2009).

With the exception of Datang's construction sites in Cambodia and Lao PDR, Endesa employs the highest number of people in a case study country (3,264 in Argentina). Although its employees are largely Argentine and Peruvian nationals, communities adjacent to Endesa's power plants in Peru complain that the company does not contribute to local economic development by hiring local community members. Endesa proposes to contribute to local economic development through infrastructure development and prioritizing local suppliers. With regard to the latter, in line with SEP norms, Endesa Argentina makes a majority of its purchases for goods and services with local suppliers. Endesa has more difficultly meeting the benchmark set by SEP norms with regard to developing local electricity infrastructure. Although not performing as poorly as AES, the company has been criticized by the electricity regulator in Argentina for "deficiencies in service delivery" and threatened with a cancellation of its contract if it did not increase its level of investment in the electricity infrastructure of the country (ENRE 2011).

Eskom (2011) aims to contribute to economic development by "creating jobs and new industries through local content associated with Eskom's massive capacity expansion program". The company also seeks out "mutually beneficial arrangements" with large multinational enterprises. This approach is largely reflective of the South African mode's penchant for economic development driven by large industry, primarily the mining sector. Eskom's activities in Africa have supported economic development in the electricity sector, but the model of economic development pursued by Eskom and the South African mode is one of supporting large-scale industry. When Eskom initiated its "In Africa" strategy in the 1990s, it emphasized its intention to connect the unconnected and be a major driving force in the New African Partnership for Development (NEPAD). However, within a decade, the company had retreated from these ambitions, stopped mentioning NEPAD in its annual reports, and emphasized its friendly arrangements with mining companies. In 2009, three-quarters of the electricity Eskom exported to its power-hungry neighbors Mozambique and Namibia went to three large multinational mining companies rather than to the $88 \%$ and $66 \%$ of the respective populations that lack access. Again, this approach is consistent with the South African mode's (primarily mining) industry-dominated economic development model.

SN Power proposes to contribute to local economic development through job creation for local residents, contracts to local suppliers and service providers, tax generation, and active engagement in knowledge and skills transfer to host communities. SN Power is the only company in the present study that has a clear policy to prioritize both local workers and local suppliers. 


\section{Ensure reliable supply}

According to international SEP norms, electric utilities should ensure reliable supply by minimizing generation interruptions, maintaining contingency plans, responding to interruptions in a timely manner, and providing timely information to the public about planned and unplanned interruptions (WBCSD 2002, CEER 2005). Figure 10.8 provides a comparison of company performance on the SEP benchmark "Ensure reliable supply".

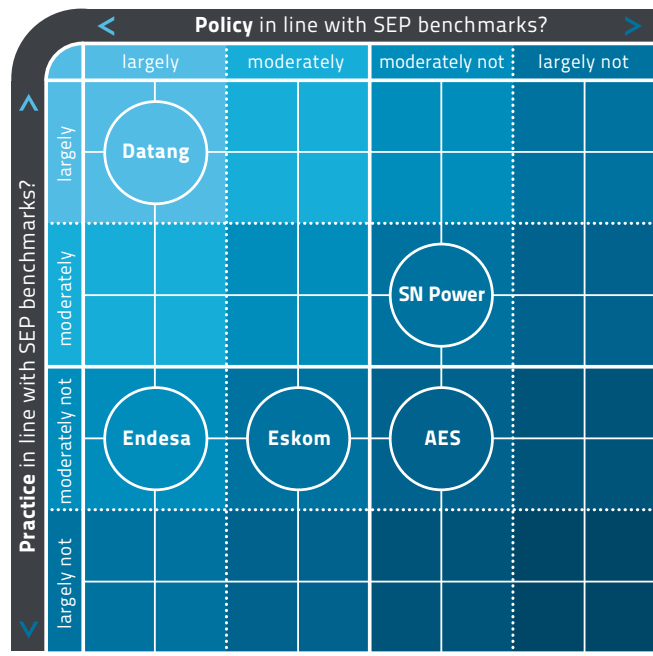

Figure 10.8: Comparison of company performance on the SEP benchmark "Ensure reliable supply"

\section{AES claims that it takes its responsibility} for ensuring a reliable supply of electricity "seriously". However, in Argentina AES has performed poorly on the reliability standards expressed in the SEP norms. The company has been fined and censured numerous times by the electricity regulator for inadequacies related to reliability of supply and a failure to properly maintain electricity infrastructure. Customers and regulatory bodies have also criticized the company's failure to respond to interruptions in a timely manner. The average capacity factor for the company's global production park is just $24 \%$, the lowest among the companies in the present study.

Datang maintains it meets all its power supply agreements with the government and state grid company. The company invests heavily (to the tune of $\$ 9.6$ billion (€7.3b) in 2010) in electricity infrastructure to ensure reliability of supply in China, but is not yet producing any electricity in Cambodia or Lao PDR. At 51\%, the company's average global capacity factor is the second highest among the companies in the present study. The high capacity factor is a result of the company's primary reliance on coal and hydropower, two baseload technologies with high capacity factors.

Endesa (2008b) asserts that "adequate, secure and uninterrupted supply of electrical energy to all customers, wherever they are, must be the [company's] main objective", but acknowledges that its performance on reliability is lower in the Global South than its home market. Though the company's average capacity factor in Argentina is slightly higher than its global average of $37 \%$, Endesa has been fined and censured by regulators for inadequacies related to reliability of supply and for failing to properly maintain electricity infrastructure. Endesa has performed better on this SEP benchmark in Peru, where its average capacity factor is $58 \%$. 
Although no information could be found on Eskom's policies on ensuring reliability of supply, the company does appear to prioritize the issue in its R\&D program. Eskom's secondlargest R\&D program (ZAR 22 million $(\$ 2.8 \mathrm{~m}$, $€ 2.1 \mathrm{~m})$ in 2010) is dedicated to improving the availability and reliability of power plants. Eskom has the highest average global capacity factor $(65 \%)$ among the companies in the present study, a result of its near complete reliance on baseload coal power. However, load shedding has been a nearly constant phenomenon in South Africa in recent decades, with stakeholders claiming that Eskom is ensuring a reliable supply to its industrial customers while domestic consumers experience frequent blackouts and unreliable service. The electricity supply is largely unreliable in Mali and Uganda, where load shedding and blackouts are frequent. The capacity factor of Eskom's hydroelectric plants in both countries is less than $50 \%$.

SN Power also does not have a public policy on ensuring reliability of supply, responding to interruptions in a timely manner, or providing timely information to the public about planned and unplanned interruptions. However, the company has performed relatively well against the SEP benchmark in Peru. The company's average capacity factor in Peru is $68 \%$, well above its global average of $46 \%$. Although the company experienced 88 involuntary or forced power cuts in 2006, the reliability of supply is deemed "regular" by customers and regulators, and there was no indication of fines by regulators for inadequacies related to reliability of supply (Descalzi 2008). Stakeholders indicate that interruptions are resolved within a short time span, but little information is provided to customers as to the causes of the supply interruptions.

\subsubsection{Cross-cutting issues}

\section{Engage in meaningful stakeholder consultation and participatory decision-making}

According to international SEP norms, electric utilities should adopt a participatory approach to the planning/designing, developing, operating, and monitoring/evaluation of electricity projects and consult and engage stakeholders throughout the entire process in order to provide meaningful opportunities for their views to be taken into account in decision-making (WCD 2000, WBCSD 2002, UNGC 2008, IFC 2007, Pillinger 2009, OECD 2011). The consultation process should be tailored to local decision-making processes and involve the timely provision of all relevant information about activities, impacts, and potential impacts translated into local languages (WBCSD 2002, ISO 2011). The process should take the needs of disadvantaged or vulnerable groups into account, and if Indigenous Peoples are affected by a potential project, electric utilities must gain the free, prior and informed consent of those peoples before proceeding with any part of the project (IFC 2011). Figure 10.9 provides a comparison of the company performance on the SEP benchmark "Engage in meaningful stakeholder consultation and participatory decision-making". 


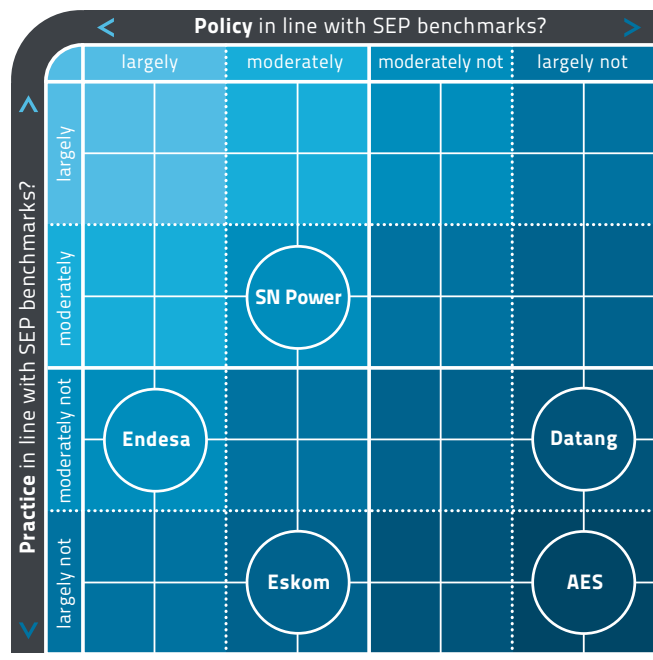

Figure 10.9: Comparison of company performance on the SEP benchmark "Engage in meaningful stakeholder consultation and participatory decision-making"

A thorough analysis of AES's policies reveals that there is no indication of a willingness to engage in meaningful stakeholder consultation and participatory decision-making. The company does not make any reference to international standards on stakeholder engagement. In Argentina, AES's primary mechanism for engaging stakeholders is a customer service hotline for reporting complaints. Although largely out of line with SEP norms, AES's failure to engage stakeholders in a meaningful way is reflective of the US mode, which tends to treat ethical and $\mathrm{CR}$ decisions as the responsibility of individual managers rather than a multilateral process involving various other stakeholder groups (Taka and Foglia 1994).

Although Datang (2008) suggests that its projects "should be acknowledged and monitored by the society", no mechanism for stakeholder consultation or participatory decision-making is provided, nor are there any references to any of the international normative standards on stakeholder engagement. Like American AES, Datang's approach of primarily top-down decision-making, rather than a participatory approach, and very limited engagement with stakeholders is largely reflective of the paternalistic overtone of the Chinese mode, in which "CSR emanates from the personal responsibility of organizational leaders (often also owners) rather than organizational responsibility" and is generally "imposed from the top" (Debroux 2009, Alfonso et al. 2010). That said, the company performed slightly better on this SEP standard in its operations in Cambodia and Lao PDR, where the company appeared to at least be making an effort to engage stakeholders on some of the most serious negative impacts of its operations (e.g. displacement). A Datang manager in Lao PDR said that the company needed to adopt a "different" way of engaging stakeholders than in China. However, he admitted that, in many cases, the company reverts to the Chinese mode of simply dealing with government officials and not engaging in any consultations with affected stakeholders (Anonymous manager in Lao PDR 4 2009).

Endesa references international standards such as the UN Global Compact in its stakeholder engagement policy. This policy entails that Endesa aims to adopt a participatory approach in all phases of electricity projects, consulting and engaging stakeholders throughout the 
entire process in order to provide meaningful opportunities for their views to be taken into account in decision-making. This approach reflects the European mode, which places high value on normative standards and dialogue among stakeholders. However, Endesa's SEP complaint policy contrasts sharply with its practice in Argentina and Peru. Endesa's stakeholder engagement in Argentina and Peru is perceived as being more focused on philanthropic activities and gift-giving than on a relationship based on meaningful engagement and participatory decision-making on issues affecting stakeholders. Residents of a community adjacent to an Endesa power station in Peru claim that there is no engagement with their community other than a Christmas present offered by the company to the children in the community. Furthermore, municipal and regional government officials complain that they are not consulted on the company's plans or decisions, nor is there any community participation in decisionmaking processes. In Argentina, members of a community were involuntarily resettled to make way for an Endesa high-tension transmission. The resettled families claim they were not meaningfully consulted on the decision but were simply told they had to leave and where their new houses would be located.

Eskom's general corporate policy on stakeholder engagement makes reference to several international standards such as UNGC, AA1000, and King Code. The policy explains Eskom's vision that, "Addressing the expectations of stakeholders such as employees, shareholders, governing bodies, suppliers, consumers and the wider community is an essential aspect of corporate responsibility" (Eskom 2011). This policy is moderately in line with international SEP norms and largely reflective of the South African mode, which traditionally embraces the concept of stakeholder engagement, and actively encourages an inclusive approach (Rossouw et al. 2002, Carapinha and de Jongh 2008, King et al. 2010). However, Eskom's policies and processes for stakeholder engagement in Mali and Uganda are exclusive (i.e. limited to village chiefs and local security personnel) and narrowly focused on security issues. Local Eskom managers variously claimed that information about the operations and impacts of its operations was either too complex for local stakeholders to understand, or that stakeholders could approach governmental bodies for such information.

SN Power aims to "reflect the priorities and concerns of local communities in decisionmaking processes and to minimize potential negative effects through a combination of careful planning, design adjustments and operational improvements" (Kopstad 2008). SN Power is the only company that conducts a social impact assessment (SIA) in addition to an EIA prior to beginning all projects. The SIA and EIA processes serve as a platform for the company's engagement with stakeholders. This policy is largely in line with SEP norms and reflective of the Nordic mode, which expects a high degree of engagement with civil society and other stakeholder groups (Kuhnle and Ervik 1996, Aarhus 2010). In Peru, SN 
Power's engagement was also evaluated more positively by stakeholders than that of the other companies in the present study. However, as is the case with the other companies, several stakeholders in Peru reported that much of SN Power's "engagement" with them consists primarily of philanthropic activities and charitable donations (Pacasmayo residents 1, 2, and 3 2008).

It is worrying that all of the companies in the present analysis perform so poorly on this critical issue. Electricity provision is meant to serve the needs of, and contribute to the development of, human populations. However, without meaningful engagement and consultation with stakeholders, most companies in the present study remain unaware of the needs of the affected populations. In some cases, they are not even aware who those populations are. Even Nordic SN Power often reverts to mere gift-giving as its primary form of engagement. Although philanthropic and charitable activities may be expected by affected communities and other stakeholders, these activities all too often take the place of, or distract from, meaningful stakeholder engagement and public involvement in decisionmaking when critical issues are at stake. SEP norms insist that this should not be the case.

Despite the fact that several of the companies in the present study have operations that impact Indigenous Peoples, none of the companies endorse, or even mention, the principle of obtaining free, prior, and informed consent (FPIC) from affected stakeholders before proceeding with proposed projects. Although this principle is still being heavily debated, it has been taken up by normative standards such as the United Nations Declaration on the Rights of Indigenous Peoples (UN 2007) and the IFC (2011) Performance Standards, which clearly establish the rights of project-affected Indigenous Peoples.

Finally, the willingness of the companies to respond to requests for information from the researchers involved in the present study also provides an indication of their commitment to stakeholder engagement. Appropriate CR or sustainability managers were asked to provide input by completing a questionnaire early in the research process, as well as by reviewing and providing comments on a draft version of their respective corporate profile. This process was repeated at both the headquarters level, as well as with local management in the host countries. Managers who did not respond immediately were given multiple subsequent opportunities through follow-up emails, letters, and telephone calls (see Chapter 2 for more on the methods for requesting corporate input). At the headquarters level, managers for Endesa, Eskom, and SN Power provided input, while those at AES and Datang did not. This is largely reflective of the companies' own stakeholder engagement policies, and is also in line with projections by the modes framework. In the host countries, however, the situation was different. Managers for AES, Datang, Endesa, and Eskom responded (although some requested anonymity), while SN Power managers declined to provide input. 
Assume responsibility for impacts throughout all business relationships

According to international SEP norms, electric utilities should seek to prevent, avoid, and mitigate negative impacts throughout their entire range of business relationships, including suppliers and contractors (ISO 2011, OECD 2011, UNHRC 2011). Companies should seek to leverage their buying power to improve social and environmental conditions among business relations, and monitor performance using third party audits (WBCSD 2002). Figure 10.10 provides a comparison of company performance on the SEP benchmark "Assume responsibility for impacts throughout all business relationships".

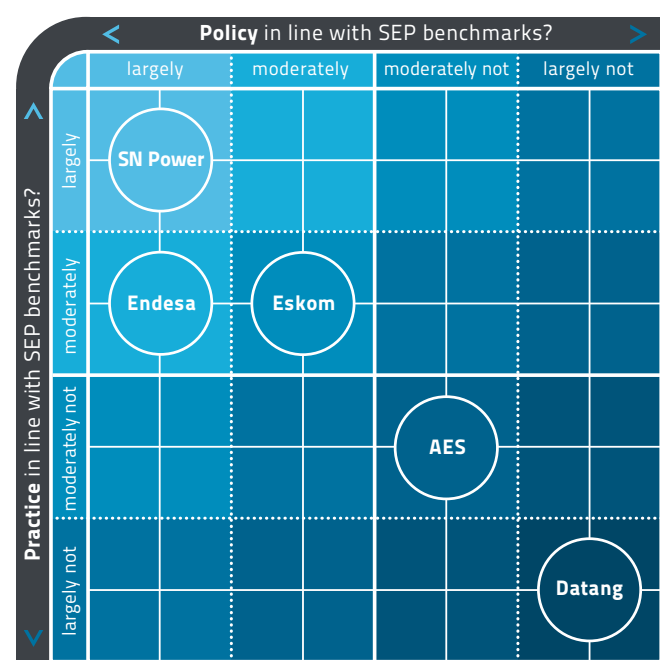

Figure 10.10: Comparison of company performance on the SEP benchmark "Assume responsibility for impacts throughout all business relationships"

AES (2007b: 9) explains that, "We will make purchasing and procurement decisions that achieve the best value for AES, including price, quality, performance, and suitability". Notably, social and environmental considerations are not among AES's criteria for evaluating suppliers. There is also no indication that AES monitors business relations for social and environmental conditions other than inviting contractors to periodic meetings to discuss safety issues. The focus on economic concerns and "achieving the best value" over social and environmental interests, in both its own operations and among its business relations, is largely indicative of the US mode.

Datang (2011a) does not make any mention of being responsible for, or seeking to prevent, avoid, and mitigate negative social and environmental impacts throughout its range of business relationships, as is suggested by SEP norms. A manager in Cambodia confirmed that Datang does monitor contractors' performance on quality and price, but noted, "We do not evaluate whether the contractors have respected the migrant workers' rights". This approach is in line with the Chinese mode, which does demonstrate considerable interest in monitoring the supply chain (Welford 2005), but places lower priority on social and environmental concerns than on economic interests, in its own operations as well as among business relationships.

Endesa embraces what it calls a "trend" in corporate social responsibility for corporations to extend their responsibilities to suppliers and contractors. In order to do so, Endesa employs different strategies to help suppliers and contractors incorporate the company's 
standards on CR issues into their own policies and practices. In addition, Endesa includes CR criteria when contracting suppliers and contractors and monitors business partners in countries and on issues where there is an elevated risk of non-compliance. Due to the importance of OHS throughout the electricity supply chain, Endesa has implemented a policy of providing all subsidiaries with country-specific OHS operating guidelines to be distributed to all contractors. This policy was confirmed in Peru, where the company recently announced that it was extending the principles of the SA 8000, ISO 9001, and OHSAS 18001 standards as requirements for its contractors and suppliers in order to ensure their compliance with the company's policies on working conditions, quality management, and OHS.

Eskom's supply chain policies indicate that it does seek to leverage its buying power to improve conditions in the supply chain, particularly with regard to occupational health and safety issues. In line with the South African mode, black ownership and BEE targets are also included in Eskom's evaluation of potential suppliers and contractors. No information could be found on how Eskom monitors suppliers and contractors to ensure standards are being met.

SN Power also acknowledges the importance of addressing CR issues when selecting contractors and suppliers, and the company claims to pay particular attention to the health and safety record of potential business partners. The company admits, however, that price and technical quality remain key criteria for choosing suppliers and contractors. SN Power includes sustainability clauses in contracts with its business partners and conducts periodic internal audits of some of its business partners.

Making comparisons across all five case studies, the companies in the present study, except for Datang, acknowledge and accept, at least to some degree, their responsibility for ensuring good environmental and labor practices among their direct contractors. Endesa and SN Power have the most thoroughly developed policies on this issue and include SEP-related clauses in contracts with contractors, a practice that SEP norms consider crucial for ensuring respect for standards. This is a positive development given the increase in outsourcing and use of contract labor in the industry. However, in many cases the concern for labor rights among contractors is limited to OHS issues and does not include other areas of decent work, such as job security and payment of a living wage.

Furthermore, little evidence was found to indicate that any of the acceptance of responsibility extends beyond the first tier of direct suppliers and contractors to a more thorough evaluation of the impacts of business partners throughout the full electricity value chain. As mentioned in Chapter 3, the impacts of electricity production and consumption extend far beyond the power plant, both upstream and downstream. Although this too is a rapidly developing field of international law and normative standards, several recently 
adopted standards at the international level including the UN Guiding Principles for Human Rights (UNHRC 2011), the OECD Guidelines (OECD 2011), and the ISO 26000 Guidance on Social Responsibility (ISO 2011) - insist that companies must identify and seek to prevent impacts among their full range of business relationships. However, none of the companies has policies in place to address the potential environmental and social impacts of upstream product chain activities such as sourcing (e.g. mining and extraction) and transport of fuels and construction materials. Similarly, none of the companies in the present study indicates even an acknowledgement of the activities and impacts on the customers to whom they supply electricity. Research on the five case studies reveals that many of the present companies supply large-scale mining operations, which have significant impacts on the environment and adjacent communities. Even Nordic SN Power appears to be supplying, and thus facilitating, the operations of irresponsible mining companies that have caused significant environmental destruction in Peru.

\section{Maximize transparency and provision of information}

Electric utilities should apply a high degree of transparency in operations, including reporting on activities and progress, measurement, business partners and relationships, and interactions with government and the public (UNECE 1998, WBCSD 2002, UNGC 2008, OECD 2011). Electric utilities should report regularly on their activities, progress, and performance against "the triple bottom line", using internationally accepted sustainability reporting guidelines such as the GRI's G3 guidelines and the Electric Utilities Sector Supplement (GRI 2008), have an external party verify the data, and disseminate the report in an appropriate manner (OECD 2011, WBCSD 2002). Based on the findings presented in each of the company case study chapters, Figure 10.11 provides a comparison of company performance on the SEP benchmark "Maximize transparency and provision of information".

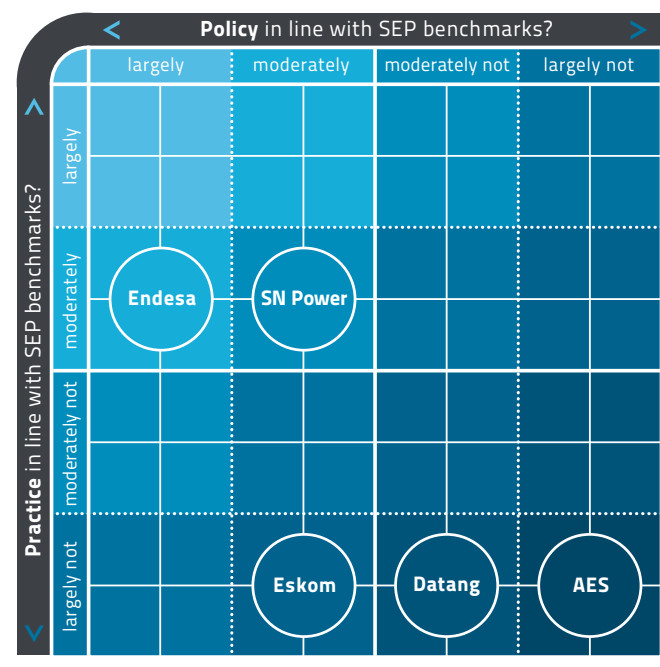

Figure 10.11: Comparison of company performance on the SEP benchmark "Maximize transparency and provision of information"

Indicative of the US mode, AES's policies on disclosure and provision of information are largely designed to comply with legal obligations rather than following international normative guidance on sustainability reporting. For example, AES's disclosure on climate change demonstrates "a reluctance to disclose certain requested information due to commercial 
sensitivity" (CDP 2011). AES is the only one of the five case study companies that does not produce an annual CR report. This is rare for a company of its size and impact.

Although Datang claims to use the GRI as a guideline for reporting, the limited information provided by the company on CR policies and SEP-related issues is largely out of line with international normative standards. However, the relative lack of transparency is generally consistent with the Chinese mode (Chan et al. 2008). The company's incomplete attempt to report using the GRI's guidelines reflects the Chinese mode's uptake of technical reporting standards, while refraining from disclosure on labor and human rights issues (Sutherland and Whelan 2009).

Endesa provides significantly more information on its CR policies and practices than the other companies in the current study. Endesa reports receive the highest reporting rating from both the GRI and the CDP. Many of its subsidiaries around the world, including those in Argentina and Peru, produce their own GRI-compliant sustainability reports (although many are outdated). One area where Endesa is not fully in line with SEP norms is the lack of transparency the company provides with regard to its business relationships with suppliers and customers.

Eskom produces an integrated annual report (i.e. including financial, operational, and sustainability aspects) in line with the King (III) Code, AA1000, the GRI's G3 guidelines, and, since
2011, the "Electric Utility Sector Supplement". Eskom's 2011 report received a B+ application rating from the GRI, and has a "limited assurance" rating by an independent assurance provider (Eskom 2012a). The $\mathrm{B}+$ rating means that Eskom does not report on all indicators requested by the GRI. One such indicator is the number of disconnections the company affects each year. The use of such standardized reporting formats is indicative of the South African mode, which also expects a high degree of transparency and information provision from companies. However, the quality and thoroughness of reporting information provided by Eskom on some SEP issues has declined markedly in recent years. Eskom headquarters no longer reports on any of its operations outside of South Africa. In Mali and Uganda, Eskom's lack of transparency and provision of information is one of the most frequently-cited grievances by a wide range of stakeholders. This is a clear departure from both the SEP norms and the South African mode.

SN Power (2011a) aims to "clearly detail our efforts across all our areas of business operation" in a "spirit of open dialogue and open reporting". Despite being a relatively young and small company, SN Power does publish a significant degree of CR information in annual reports and on its website. Nevertheless, SN Power's policy and practice in Peru are not fully in line with international SEP norms. The Nordic mode, with its long tradition of transparency, also expects the highest degree of transparency, higher than that provided by SN Power (Kuhnle and Ervik 1996, ECOTEC 2007). 
Making comparisons across all five case studies, company policy at the headquarters level largely follows along the lines projected by the modes framework. However, none of the companies provides a sufficient degree of transparency to stakeholders in the Global South. Another interesting finding is that none of the companies provides transparency about important business relations such as customers and suppliers. Transparency standards by norm-setting bodies such as the UN (UNHRC 2011), OECD (2011), and European Commission (EC 2011) insist that companies should identify, prevent, and mitigate negative impacts among their business relationships and encourage companies to disclose information about their relations with business partners such as suppliers, contractors, and customers. information could be gathered, leaving a total of 108 ( 55 policy, 53 practice) evaluated company performance instances. The matrix is depicted in Table 10.1.

Each of the five companies in the current study claims that it is a responsible corporation committed to promoting the sustainable development of host countries in the Global South. However, the analysis of the TNCs' performance on the SEP standards in policy and practice reveals that the uptake of SEP norms by the companies varies widely. The SEP norms are clearly not yet accepted, adopted, implemented, or even interpreted equally by the present case study companies.

\subsection{Overall comparison of company policy and practice relative to the SEP standards}

In addition to the comparisons among the case study companies on individual issues, an overall comparison of their policy and practice on all SEP standards also produces results that contain lessons and implications for policy-makers, company managers, and other stakeholders. Comparing the policy and practice of all five companies on all 11 SEP norms creates a matrix of 110 instances of evaluated performance. In two of the practice fields, no 
Table 10.1: Company performance on all 11 SEP benchmarks in policy and practice

\begin{tabular}{|c|c|c|c|c|}
\hline \multirow{2}{*}{$\begin{array}{l}\text { Are the company's policy and } \\
\text { practice in line with the following } \\
\text { SEP benchmark? }\end{array}$} & \multicolumn{2}{|l|}{ AES } & \multicolumn{2}{|l|}{ Endesa } \\
\hline & policy & practice & policy & practice \\
\hline $\begin{array}{l}\text { Endorse international normative } \\
\text { standards for CR }\end{array}$ & Largely out of line & $\begin{array}{l}\text { Moderately out } \\
\text { of line }\end{array}$ & Largely in line & $\begin{array}{l}\text { Moderately } \\
\text { in line }\end{array}$ \\
\hline $\begin{array}{l}\text { Adopt a commitment to CR } \\
\text { in core-business activities and } \\
\text { decision-making }\end{array}$ & $\begin{array}{l}\text { Moderately out } \\
\text { of line }\end{array}$ & Largely out of line & Largely in line & $\begin{array}{l}\text { Moderately } \\
\text { out of line }\end{array}$ \\
\hline $\begin{array}{l}\text { Increase access to affordable } \\
\text { electricity }\end{array}$ & Moderately in line & Largely out of line & Largely in line & $\begin{array}{l}\text { Moderately } \\
\text { out of line }\end{array}$ \\
\hline Respect labor rights & $\begin{array}{l}\text { Moderately out } \\
\text { of line }\end{array}$ & Moderately in line & Largely in line & $\begin{array}{l}\text { Moderately } \\
\text { in line }\end{array}$ \\
\hline $\begin{array}{l}\text { Minimize environmental impact, } \\
\text { including contribution to climate } \\
\text { change }\end{array}$ & $\begin{array}{l}\text { Moderately out } \\
\text { of line }\end{array}$ & $\begin{array}{l}\text { Moderately out } \\
\text { of line }\end{array}$ & Largely in line & $\begin{array}{l}\text { Moderately } \\
\text { out of line }\end{array}$ \\
\hline $\begin{array}{l}\text { Prioritize renewable sources of } \\
\text { energy for electricity }\end{array}$ & $\begin{array}{l}\text { Moderately out } \\
\text { of line }\end{array}$ & $\begin{array}{l}\text { Moderately out } \\
\text { of line }\end{array}$ & $\begin{array}{l}\text { Moderately out } \\
\text { of line }\end{array}$ & $\begin{array}{l}\text { Moderately } \\
\text { out of line }\end{array}$ \\
\hline $\begin{array}{l}\text { Contribute to local economic } \\
\text { development }\end{array}$ & Moderately in line & $\begin{array}{l}\text { Moderately out } \\
\text { of line }\end{array}$ & Moderately in line & $\begin{array}{l}\text { Moderately } \\
\text { out of line }\end{array}$ \\
\hline Ensure reliable supply & $\begin{array}{l}\text { Moderately out } \\
\text { of line }\end{array}$ & $\begin{array}{l}\text { Moderately out } \\
\text { of line }\end{array}$ & Largely in line & $\begin{array}{l}\text { Moderately } \\
\text { out of line }\end{array}$ \\
\hline $\begin{array}{l}\text { Engage in meaningful stakeholder } \\
\text { consultation and participatory } \\
\text { decision-making }\end{array}$ & Largely out of line & Largely out of line & Largely in line & $\begin{array}{l}\text { Moderately } \\
\text { out of line }\end{array}$ \\
\hline $\begin{array}{l}\text { Assume responsibility for impacts } \\
\text { throughout all business relationships }\end{array}$ & $\begin{array}{l}\text { Moderately out } \\
\text { of line }\end{array}$ & $\begin{array}{l}\text { Moderately out } \\
\text { of line }\end{array}$ & Largely in line & $\begin{array}{l}\text { Moderately } \\
\text { in line }\end{array}$ \\
\hline $\begin{array}{l}\text { Maximize transparency and provision } \\
\text { of information }\end{array}$ & Largely out of line & Largely out of line & Largely in line & $\begin{array}{l}\text { Moderately } \\
\text { in line }\end{array}$ \\
\hline
\end{tabular}




\begin{tabular}{|c|c|c|c|c|c|}
\hline \multicolumn{2}{|l|}{ SN Power } & \multicolumn{2}{|l|}{ Datang } & \multicolumn{2}{|l|}{ Eskom } \\
\hline policy & practice & policy & practice & policy & practice \\
\hline $\begin{array}{l}\text { Moderately } \\
\text { in line }\end{array}$ & Moderately in line & $\begin{array}{l}\text { Moderately out } \\
\text { of line }\end{array}$ & Largely out of line & Moderately in line & $\begin{array}{l}\text { Moderately out } \\
\text { of line }\end{array}$ \\
\hline $\begin{array}{l}\text { Largely in } \\
\text { line }\end{array}$ & Moderately in line & $\begin{array}{l}\text { Moderately out } \\
\text { of line }\end{array}$ & Largely out of line & Moderately in line & Largely out of line \\
\hline $\begin{array}{l}\text { Moderately } \\
\text { in line }\end{array}$ & Moderately in line & Moderately in line & $\begin{array}{l}\text { Moderately out } \\
\text { of line }\end{array}$ & $\begin{array}{l}\text { Moderately out } \\
\text { of line }\end{array}$ & Largely out of line \\
\hline $\begin{array}{l}\text { Moderately } \\
\text { in line }\end{array}$ & Moderately in line & Largely out of line & Largely out of line & Largely in line & Largely in line \\
\hline $\begin{array}{l}\text { Moderately } \\
\text { in line }\end{array}$ & Moderately in line & Largely out of line & $\begin{array}{l}\text { Moderately out } \\
\text { of line }\end{array}$ & Largely out of line & Moderately in line \\
\hline $\begin{array}{l}\text { Largely in } \\
\text { line }\end{array}$ & Largely in line & $\begin{array}{l}\text { Moderately out } \\
\text { of line }\end{array}$ & Largely in line & Largely out of line & Largely in line \\
\hline $\begin{array}{l}\text { Largely in } \\
\text { line }\end{array}$ & Largely in line & Moderately in line & $\begin{array}{l}\text { Moderately out } \\
\text { of line }\end{array}$ & $\begin{array}{l}\text { Moderately out } \\
\text { of line }\end{array}$ & Moderately in line \\
\hline $\begin{array}{l}\text { Moderately } \\
\text { out of line }\end{array}$ & Moderately in line & Largely in line & $\mathrm{n} / \mathrm{a}$ & Moderately in line & $\begin{array}{l}\text { Moderately out } \\
\text { of line }\end{array}$ \\
\hline $\begin{array}{l}\text { Moderately } \\
\text { in line }\end{array}$ & Moderately in line & Largely out of line & $\begin{array}{l}\text { Moderately out } \\
\text { of line }\end{array}$ & Moderately in line & Largely out of line \\
\hline $\begin{array}{l}\text { Largely in } \\
\text { line }\end{array}$ & $\mathrm{n} / \mathrm{a}$ & Largely out of line & Largely out of line & Moderately in line & Moderately in line \\
\hline $\begin{array}{l}\text { Moderately } \\
\text { in line }\end{array}$ & Moderately in line & $\begin{array}{l}\text { Moderately out } \\
\text { of line }\end{array}$ & Largely out of line & Moderately in line & Largely out of line \\
\hline
\end{tabular}




\section{Aggregate company policy}

Of the 55 evaluated company policy instances, combined company performance is considered moderately or largely out of line with internationally-agreed normative standards in $23(42 \%)$ of the instances. In nine $(16 \%)$ of the instances, company policy is largely out of line with the norms. In $15(27 \%)$ of the instances, company policy is considered largely in line with SEP norms. Interestingly, nearly two-thirds of these high policy performances among all five companies are attributable to one company: Endesa. At the other end of the spectrum, AES's policies are not considered largely in line with SEP norms on any of the 11 benchmarks.

Overall, the CR policies of European Endesa and Nordic SN Power are most aligned with international SEP norms of the five case study companies studied. For both of these companies, corporate policy is deemed "largely" or "moderately" in line with the SEP norms on 10 out of the 11 benchmarks. Endesa's policies are considered largely in line with SEP norms on an impressive nine out of the 11 issues. The policies of American AES and Chinese Datang are the most out of line with the SEP norms. Datang's policies are moderately or largely in line with the norms on only three of the benchmarks and are largely out of line with the norms on four benchmarks. AES's policies are moderately in line with the norms on only two of the SEP standards. Eskom falls in the middle, since its policies are moderately in line with SEP norms on just over half of the SEP benchmarks.

The policy comparison across all companies and all SEP benchmarks reveals that a company's degree of endorsement of international CR standards (the first SEP standard) provides a good indication of whether the company's more detailed policies on specific SEP issue areas will be in line with SEP norms. The present analysis indicates that, if a company references multiple international CR standards, that company also develops detailed polices around specific SEP issues that are in line with SEP standards. In other words, companies that go to the trouble of referencing an international norm tend to incorporate the spirit and letter of the standard into their policies. Whether the company then translates that policy into practice on the ground in the Global South is another matter.

\section{Aggregate company practice}

Of the 53 total company practice instances evaluated, the combined company performance is considered out of line with international SEP norms 32 times. This means that, on whole, the case study companies are moderately or largely out of line with internationally-agreed normative standards more than $60 \%$ of the time. In $13(25 \%)$ of the instances, company practice is largely out of line with the norms. In only five $(9 \%)$ of the instances can the companies be considered largely in line with the SEP norms in their practice in the Global South.

AES (in Argentina) and Datang (in Cambodia and Lao PDR) have the worst performance against the SEP benchmark. Their practice is moderately or largely out of line on all but one SEP standard. Datang's practice in Cambodia and Lao PDR is largely out of line with the norms 
on $50 \%$ (five out of 10) of the benchmarks. On the other hand, SN Power's practice in Peru is either largely or moderately in line with SEP norms on all areas measured. Eskom again falls in the middle with its performance in practice moderately in line with SEP norms on just less than half of the SEP benchmarks. Surprisingly, Endesa's performance in practice was only in line with SEP norms on just one-third (four of 11) of the benchmarks.

However, Endesa is not the only company in the present study that demonstrated difficulties or unwillingness to translate its policies into practice. Although it was assumed that corporate practices on the ground in the Global South would largely reflect the corporate policies at the headquarters level, the present analysis reveals the relationship between corporate policy and practice to be much more tenuous. Of the 53 total possible pairings between policy and practice that can be observed in Table 10.1, only 19 (36\%) reflect a strict correlation (i.e. policy largely in line $=$ practice largely in line; policy moderately in line = practice moderately in line, etc.). This means that nearly two-thirds $(64 \%)$ of the time company practice on the ground in host countries does not reflect policy at the headquarters level. If one moves beyond strict correlations and takes general tendency into account (i.e. policy largely or moderately in line = practice largely or moderately in line; policy largely or moderately out of line = practice largely or moderately out of line), the correlation rate rises to $60 \%$. However, this still implies that $40 \%$ of the time company practice does not even reflect the same tendency as policy regarding alignment with SEP norms.

The five case study companies exhibit significant variation in translating policy into practice. SN Power is largely effective in turning its policies into practice on the ground. In eight out of $10(80 \%)$ instances, the company's practice in Peru has the same relationship with the SEP norms as its policy. In contrast, Datang, and particularly Endesa and Eskom, are much less successful in implementing their policies. Endesa's practice in Argentina and Peru has the same relationship with SEP norms as its policy in just one out of 11 instances $(9 \%)$. This means that Endesa's impressive performance against the SEP norms on policy is negated by its inability or unwillingness to see its policies implemented on the ground in a developmental setting. Eskom's practice in Mali and Uganda only has the same tendency toward the SEP norms in three instances. This means Eskom is unsuccessful in implementing its policy a troubling $73 \%$ of the time.

\section{Relative company performance across all 11 SEP norms}

In addition to analyzing the aggregate performance of all five companies, it is interesting explore company performance on the SEP norms relative to each other. If we assign a numeric value to each of the four degrees of congruence with the SEP norms (largely out of line with SEP norms = one point; moderately out of line = two points; moderately in line = three points; largely in line = four points), we can calculate an average relative position for each company 


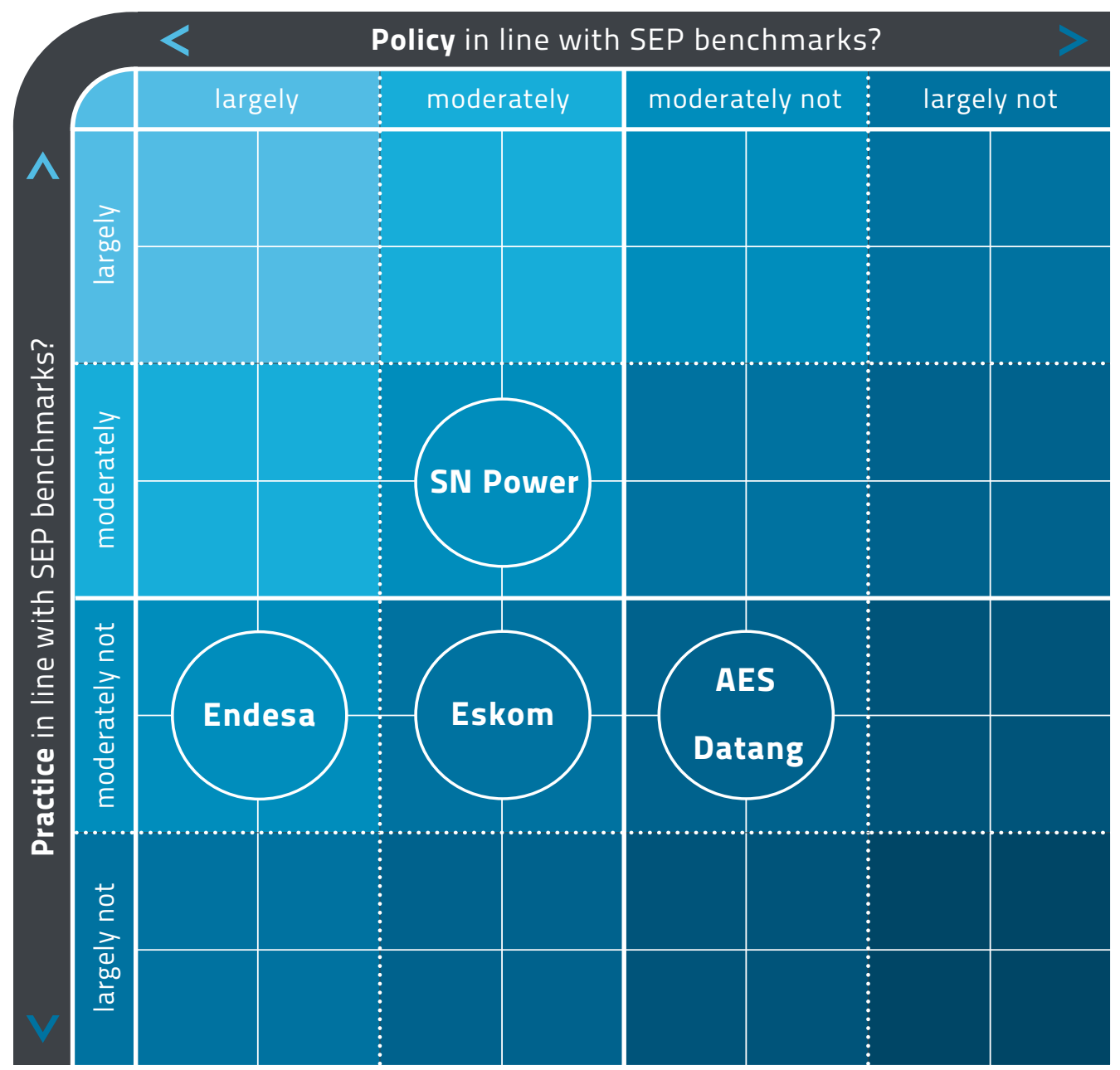

Figure 10.12: Aggregated company performance across all 11 SEP benchmarks

Taking the results provided in Table 10.1 above, we see that AES's policies are largely out of line with SEP norms in three areas (three points), moderately out of line in six areas (12 points), and moderately in line in two areas (six points). A total of 21 points, divided by 11 benchmarks, equals an average of 1.9. This in turns corresponds to an average result that AES policy is moderately out of line with SEP norms.

A similar exercise can be conducted for AES's practice for a score of 1.8 points, or moderately out of line. For the other companies, the results are as follows: Endesa's policy scores an average of 3.7 (largely in line), and its practice scores an average of 2.3 (moderately out of 
line). SN Power policy scores 3.3 (moderately in line), and practice 3.2 (moderately in line). Datang's policy scores an average of 2 (moderately out of line), while Datang practice receives an average of 1.7 (moderately out of line). Eskom's policy scores 2.6 (moderately in line), and practice 2.3 (moderately out of line).

In Figure 10.12, the relative positions of the companies are plotted on the two-dimensional SEP field. The visualization reveals that, overall, American AES and Chinese Datang both average "moderately out of line with SEP norms" on both policy and practice. The practice of four out of the five companies is on average "moderately out of line with SEP norms". Only SN Power averages "moderately in line with SEP norms" on both policy and practice. Strikingly, none of the five companies averages "largely in line with SEP norms" on either policy or practice.

\subsection{Relevance of home- country business culture}

The "modes framework" employed in the present analysis was designed to capture and structure the intervening variable of home-country business culture (see Figure 2.1). This was done to guide a systematized selection of case study companies with the aim of generating variance on corporate approaches to SEP in policy and practice. The modes framework has served this purpose well. Nearly all SEP benchmarks exhibit significant variance among the five case study companies. In addition to its capacity as a systematized generator of variance, it is also instructive to examine in more detail how home-country culture "intervenes" between the internationally-derived standards for SEP and individual company performance to condition the way companies take up the norms and apply them in practice.

The relevance of a framework based on homecountry business culture was confirmed anecdotally during the research by several of the corporate managers interviewed for the present study (both at the headquarters level and at subsidiaries in host countries). The managers from almost all companies indicate that their company's way of doing business is highly influenced by the national/regionalcultural origin of the company. An SN Power manager maintains that the Nordic traditions in which the company is "deeply rooted" serve as the basis for the company's competitive advantage (Kopstad 2008). An AES manager in Argentina confirmed that his company follows the American parent company's tradition of not publishing a high degree of information about SEP issues (Anonymous manager in Argentina 1 2008). Eskom Mali's director insists that Eskom prides itself on being an "Africa-oriented electricity company" seeped in African values, as opposed to "Western companies" that had failed in electricity provision in Africa (Coulibaly 2012). A Datang manager in Lao PDR admits that, although they are trying new and "different" forms of stakeholder engagement, the company often reverts to the "Chinese way" of doing things (Anonymous manager in Lao PDR 4 2009). 
Beyond these anecdotal examples, the homecountry business modes framework proved to be a useful analytic tool for projecting how company policy would match up against the SEP normative benchmark. Table 4.1 in Chapter 4 summarized the expectations - generalized from the literature - as to what the basic features of each mode imply for the uptake of international normative standards related to SEP. Similar to the exercise above, comparing how "reflective" company policy and practice are of the home-country mode on all 11 SEP benchmarks creates a matrix of 110 instances of evaluated performance. No information could be gathered for two of the practice fields, and there was "no clear prediction" by the mode in two cases (see Table 4.1). This leaves a total of 104 evaluated company performance instances (53 policy, 51 practice). The matrix for "reflection of home-country mode" is shown in Table 10.2

In $74 \%$ of the instances, company performance (in both policy and practice) on the SEP norms is considered to be "largely" or "moderately" reflective of the mode exhibited by their home country or region. The modes framework is more accurate when it comes to understanding company policy than with regard to company practice. In $85 \%$ of the instances of company policy, company performance on the SEP benchmarks was determined to be "largely" or "moderately" reflective of the homecountry mode. In terms of corporate practice on the ground in the Global South, the modes framework successfully provides an indication of the general tendency of the company's performance in just $63 \%$ of cases. The modes framework is most relevant in understanding how American AES, Chinese Datang, and Nordic SN Power perform on the SEP benchmarks.

Analysis of the empirical research findings also reveals a number of interesting "inconsistencies" between the corporate behavior expected by the modes and that actually observed in a number of specific instances. The modes framework is least accurate in projecting how European Endesa and South African Eskom perform on the SEP benchmarks, particularly on the ground in host countries. Eskom's practice in Uganda and Mali is deemed "moderately" or "largely" unreflective of the South African mode on $73 \%$ of the benchmarks.

For example, Eskom's abandonment of its earlier commitment to electrification - and its lack of electrification projects benefitting underserved communities in Mali and Uganda - contradicts the South African mode's push and need for development, including increased access to affordable electricity. Another discrepancy is that although Eskom operates only marginal renewable capacity in its home market, its facilities in Mali and Uganda are strictly hydro-based. Unlike its long experience with coal-fired electricity generation, Eskom and the South African mode historically exhibited limited experience with hydropower. Eskom's limited engagement with affected communities in Mali and Uganda also contrasts with the South African mode, which traditionally embraces the concept of stakeholder engagement, and actively encourages an inclusive approach. 
Endesa's practice in Argentina and Peru is also deemed "moderately unreflective" of the European mode on $50 \%$ of the SEP benchmarks. For example, despite the fact that environmental issues such as climate change, biodiversity, pollution, and natural resource use are generally given high priority in Europe, Endesa operates and continues to invest in unsustainable and inefficient fossil fuel-based facilities in Argentina and Peru. Similarly, although the European mode expects a focus on core-business activities in CR programs and a sophisticated degree of stakeholder engagement, Endesa's CR initiatives in Argentina and Peru are clearly more focused on philanthropic activities and gift-giving in the communities than sustained engagement with communities and governments on local planning and development issues.

SN Power's focus on non-core-business, charity-type projects in its CR activities in Peru, and the lack of publicly available policy documents, is only moderately in line with the Nordic mode. Similarly, although the Nordic mode projects the highest degree of transparency from companies, the level of transparency provided by SN Power is only moderately in line with SEP norms.

Despite these anomalies, the national and regional business cultural modes do appear to provide relevant and systematic information for the overall problematic. Certain features of the various modes of home-country business culture "intervene" to condition - but do not completely determine - the dependent variables, and thus contribute to the general understanding of why the "outcomes" of company policy and practice related to the SEP norms are as they are. As will be discussed further in the following chapter, the conditioning role of the modes has important implications regarding the impact of regional and national norms in either enhancing or restricting the potential for better implementation of the general expectations of the UN-based norm set.

\subsection{Discussion of limitations of the analytical framework and research methods and their potential impact on results}

Addressing the urgent nature of the multiple, intertwined issues associated with sustainable electricity provision (OECD/IEA 2010) is what Hisschemöller and Gupta (1999) term a "complex problem". Such complex problems require a big picture approach that uses applied science to produce knowledge and generate inductively-drawn lessons that are concretely and directly relevant for multiple target audiences (Pawson and Tilley 1995:20). Although a "big picture" approach is necessary to address the nature of the problem, there are a number of analytical and methodological limitations associated with such an approach. These limitations may impact the results of the analysis, and they deserve to be discussed here. 
Table 10.2: Degree to which company performance on SEP benchmarks reflects the mode of home-country business culture

\begin{tabular}{|c|c|c|c|c|}
\hline \multirow{2}{*}{$\begin{array}{l}\text { Are the company's policy and practice } \\
\text { reflective of its home-country mode on } \\
\text { the following SEP benchmark? }\end{array}$} & \multicolumn{2}{|l|}{ AES } & \multicolumn{2}{|l|}{ Endesa } \\
\hline & policy & practice & policy & practice \\
\hline $\begin{array}{l}\text { Endorse international normative } \\
\text { standards for CR }\end{array}$ & $\begin{array}{l}\text { Moderately } \\
\text { reflective }\end{array}$ & $\begin{array}{l}\text { Largely } \\
\text { reflective }\end{array}$ & $\begin{array}{l}\text { Largely } \\
\text { reflective }\end{array}$ & $\begin{array}{l}\text { Moderately } \\
\text { reflective }\end{array}$ \\
\hline $\begin{array}{l}\text { Adopt a commitment to CR } \\
\text { in core-business activities and } \\
\text { decision-making }\end{array}$ & $\begin{array}{l}\text { Largely } \\
\text { reflective }\end{array}$ & $\begin{array}{l}\text { Moderately } \\
\text { reflective }\end{array}$ & $\begin{array}{l}\text { Moderately } \\
\text { reflective }\end{array}$ & $\begin{array}{l}\text { Moderately } \\
\text { unreflective }\end{array}$ \\
\hline $\begin{array}{l}\text { Increase access to affordable } \\
\text { electricity }\end{array}$ & $\begin{array}{l}\text { Moderately } \\
\text { unreflective }\end{array}$ & $\begin{array}{l}\text { Moderately } \\
\text { reflective }\end{array}$ & $\begin{array}{l}\text { Moderately } \\
\text { unreflective }\end{array}$ & $\begin{array}{l}\text { Largely } \\
\text { reflective }\end{array}$ \\
\hline Respect labor rights & $\begin{array}{l}\text { Largely } \\
\text { reflective }\end{array}$ & $\begin{array}{l}\text { Moderately } \\
\text { unreflective }\end{array}$ & $\begin{array}{l}\text { Moderately } \\
\text { reflective }\end{array}$ & $\begin{array}{l}\text { Largely } \\
\text { reflective }\end{array}$ \\
\hline $\begin{array}{l}\text { Minimize environmental impact, } \\
\text { including contribution to climate } \\
\text { change }\end{array}$ & $\begin{array}{l}\text { Largely } \\
\text { reflective }\end{array}$ & $\begin{array}{l}\text { Largely } \\
\text { reflective }\end{array}$ & $\begin{array}{l}\text { Moderately } \\
\text { reflective }\end{array}$ & $\begin{array}{l}\text { Moderately } \\
\text { unreflective }\end{array}$ \\
\hline $\begin{array}{l}\text { Prioritize renewable sources of } \\
\text { energy for electricity }\end{array}$ & $\begin{array}{l}\text { Largely } \\
\text { reflective }\end{array}$ & $\begin{array}{l}\text { Largely } \\
\text { reflective }\end{array}$ & $\begin{array}{l}\text { Moderately } \\
\text { unreflective }\end{array}$ & $\begin{array}{l}\text { Moderately } \\
\text { unreflective }\end{array}$ \\
\hline $\begin{array}{l}\text { Contribute to local economic } \\
\text { development }\end{array}$ & $\begin{array}{l}\text { Largely } \\
\text { reflective }\end{array}$ & $\begin{array}{l}\text { Moderately } \\
\text { unreflective }\end{array}$ & $\mathrm{n} / \mathrm{a}$ & $\mathrm{n} / \mathrm{a}$ \\
\hline Ensure reliable supply & $\mathrm{n} / \mathrm{a}$ & $\mathrm{n} / \mathrm{a}$ & $\begin{array}{l}\text { Moderately } \\
\text { reflective }\end{array}$ & $\begin{array}{l}\text { Moderately } \\
\text { unreflective }\end{array}$ \\
\hline $\begin{array}{l}\text { Engage in meaningful stakeholder } \\
\text { consultation and participatory } \\
\text { decision-making }\end{array}$ & $\begin{array}{l}\text { Moderately } \\
\text { reflective }\end{array}$ & $\begin{array}{l}\text { Moderately } \\
\text { reflective }\end{array}$ & $\begin{array}{l}\text { Moderately } \\
\text { reflective }\end{array}$ & $\begin{array}{l}\text { Moderately } \\
\text { unreflective }\end{array}$ \\
\hline $\begin{array}{l}\text { Assume responsibility for impacts } \\
\text { throughout all business relationships }\end{array}$ & $\begin{array}{l}\text { Moderately } \\
\text { unreflective }\end{array}$ & $\begin{array}{l}\text { Largely } \\
\text { reflective }\end{array}$ & $\begin{array}{l}\text { Moderately } \\
\text { reflective }\end{array}$ & $\begin{array}{l}\text { Largely } \\
\text { reflective }\end{array}$ \\
\hline $\begin{array}{l}\text { Maximize transparency and provision } \\
\text { of information }\end{array}$ & $\begin{array}{l}\text { Moderately } \\
\text { reflective }\end{array}$ & $\begin{array}{l}\text { Moderately } \\
\text { reflective }\end{array}$ & $\begin{array}{l}\text { Moderately } \\
\text { reflective }\end{array}$ & $\begin{array}{l}\text { Largely } \\
\text { reflective }\end{array}$ \\
\hline
\end{tabular}




\begin{tabular}{|c|c|c|c|c|c|}
\hline \multicolumn{2}{|l|}{ SN Power } & \multicolumn{2}{|l|}{ Datang } & \multicolumn{2}{|l|}{ Eskom } \\
\hline policy & practice & policy & practice & policy & practice \\
\hline $\begin{array}{l}\text { Largely } \\
\text { reflective }\end{array}$ & $\begin{array}{l}\text { Largely } \\
\text { reflective }\end{array}$ & $\begin{array}{l}\text { Largely } \\
\text { reflective }\end{array}$ & $\begin{array}{l}\text { Moderately } \\
\text { reflective }\end{array}$ & $\begin{array}{l}\text { Largely } \\
\text { reflective }\end{array}$ & $\begin{array}{l}\text { Moderately } \\
\text { unreflective }\end{array}$ \\
\hline $\begin{array}{l}\text { Largely } \\
\text { reflective }\end{array}$ & $\begin{array}{l}\text { Moderately } \\
\text { reflective }\end{array}$ & $\begin{array}{l}\text { Largely } \\
\text { reflective }\end{array}$ & $\begin{array}{l}\text { Moderately } \\
\text { reflective }\end{array}$ & $\begin{array}{l}\text { Moderately } \\
\text { reflective }\end{array}$ & $\begin{array}{l}\text { Moderately } \\
\text { reflective }\end{array}$ \\
\hline $\begin{array}{l}\text { Largely } \\
\text { reflective }\end{array}$ & $\begin{array}{l}\text { Moderately } \\
\text { reflective }\end{array}$ & $\begin{array}{l}\text { Largely } \\
\text { reflective }\end{array}$ & $\begin{array}{l}\text { Moderately } \\
\text { unreflective }\end{array}$ & $\begin{array}{l}\text { Moderately } \\
\text { unreflective }\end{array}$ & $\begin{array}{l}\text { Largely } \\
\text { unreflective }\end{array}$ \\
\hline $\begin{array}{l}\text { Moderately } \\
\text { reflective }\end{array}$ & $\begin{array}{l}\text { Largely } \\
\text { reflective }\end{array}$ & $\begin{array}{l}\text { Largely } \\
\text { reflective }\end{array}$ & Largely reflective & $\begin{array}{l}\text { Moderately } \\
\text { reflective }\end{array}$ & $\begin{array}{l}\text { Moderately } \\
\text { reflective }\end{array}$ \\
\hline $\begin{array}{l}\text { Moderately } \\
\text { reflective }\end{array}$ & $\begin{array}{l}\text { Moderately } \\
\text { reflective }\end{array}$ & $\begin{array}{l}\text { Largely } \\
\text { reflective }\end{array}$ & $\begin{array}{l}\text { Moderately } \\
\text { reflective }\end{array}$ & $\begin{array}{l}\text { Largely } \\
\text { reflective }\end{array}$ & $\begin{array}{l}\text { Moderately } \\
\text { unreflective }\end{array}$ \\
\hline $\begin{array}{l}\text { Largely } \\
\text { reflective }\end{array}$ & $\begin{array}{l}\text { Largely } \\
\text { reflective }\end{array}$ & $\begin{array}{l}\text { Largely } \\
\text { reflective }\end{array}$ & $\begin{array}{l}\text { Moderately } \\
\text { unreflective }\end{array}$ & $\begin{array}{l}\text { Moderately } \\
\text { reflective }\end{array}$ & $\begin{array}{l}\text { Moderately } \\
\text { unreflective }\end{array}$ \\
\hline $\begin{array}{l}\text { Moderately } \\
\text { reflective }\end{array}$ & $\begin{array}{l}\text { Largely } \\
\text { reflective }\end{array}$ & $\begin{array}{l}\text { Largely } \\
\text { reflective }\end{array}$ & $\begin{array}{l}\text { Moderately } \\
\text { unreflective }\end{array}$ & $\begin{array}{l}\text { Moderately } \\
\text { unreflective }\end{array}$ & $\begin{array}{l}\text { Largely } \\
\text { reflective }\end{array}$ \\
\hline $\begin{array}{l}\text { Moderately } \\
\text { unreflective }\end{array}$ & $\begin{array}{l}\text { Largely } \\
\text { reflective }\end{array}$ & $\begin{array}{l}\text { Moderately } \\
\text { reflective }\end{array}$ & $\mathrm{n} / \mathrm{a}$ & $\begin{array}{l}\text { Largely } \\
\text { reflective }\end{array}$ & $\begin{array}{l}\text { Moderately } \\
\text { unreflective }\end{array}$ \\
\hline $\begin{array}{l}\text { Moderately } \\
\text { reflective }\end{array}$ & $\begin{array}{l}\text { Moderately } \\
\text { reflective }\end{array}$ & $\begin{array}{l}\text { Largely } \\
\text { reflective }\end{array}$ & $\begin{array}{l}\text { Moderately } \\
\text { reflective }\end{array}$ & $\begin{array}{l}\text { Moderately } \\
\text { reflective }\end{array}$ & $\begin{array}{l}\text { Moderately } \\
\text { unreflective }\end{array}$ \\
\hline $\begin{array}{l}\text { Moderately } \\
\text { reflective }\end{array}$ & $\mathrm{n} / \mathrm{a}$ & $\begin{array}{l}\text { Moderately } \\
\text { reflective }\end{array}$ & $\begin{array}{l}\text { Moderately } \\
\text { reflective }\end{array}$ & $\begin{array}{l}\text { Moderately } \\
\text { unreflective }\end{array}$ & $\begin{array}{l}\text { Moderately } \\
\text { unreflective }\end{array}$ \\
\hline $\begin{array}{l}\text { Moderately } \\
\text { reflective }\end{array}$ & $\begin{array}{l}\text { Moderately } \\
\text { reflective }\end{array}$ & $\begin{array}{l}\text { Largely } \\
\text { reflective }\end{array}$ & $\begin{array}{l}\text { Moderately } \\
\text { unreflective }\end{array}$ & $\begin{array}{l}\text { Largely } \\
\text { reflective }\end{array}$ & $\begin{array}{l}\text { Moderately } \\
\text { unreflective }\end{array}$ \\
\hline
\end{tabular}


Limitations related to the host-country setting Beyond operational differences between the firms, there are also variations in the local setting in which company performance against the SEP benchmarks was evaluated. The finding that company practice in the Global South frequently deviated from both the stated policy of the TNCs as well as the projection made by the modes framework suggests an important role for the institutional and cultural setting of the host country in shaping company practice on the ground. This situation was recognized and conceptualized by the analytical approach of the present study as a "contextual variable", but the full implications of this variable could not be investigated in detail. It was also not possible to fully control for the contextual variable in the present study, given that there is no single country in which all five case study companies have operations. The closest the present study could come was having two of the case study companies operating in two of the same host countries (AES and Endesa in Argentina, Endesa and SN Power in Peru). This was one of the main motivating factors for choosing Argentina and Peru as the sites for gathering information on those companies.

The methodological approach also attempted to control somewhat for host country setting by investing a single TNC's practice in multiple host countries. For three of the companies (Endesa, Eskom, and Datang) empirical field research on the company's practice was conducted in two host countries. However, for two of the case study companies (AES and SN Power) it was only possible to gather information on the company's practice in one host country. Finally, the methodological approach attempted to control for host country institutional/cultural setting by only gathering information on company performance in the Global South (i.e. Argentina, Cambodia, Lao PDR, Mali, Peru, and Uganda) and selecting two host countries per continent (i.e. Africa, Asia, Latin America). That said, there are obvious institutional, cultural, and socio-economic differences between, for example, Argentina and Mali or Uganda, which undoubtedly have an impact on the company's performance in practice on the SEP benchmarks.

Limitations related to data collection The fact that AES and Datang management at the headquarters did not respond to repeated requests for participation in the present study means that no information on those two companies could be gathered through the questionnaire method. It also means that the overall company profiles of AES and Datang were not reviewed by a manager of the company. In the other three cases (i.e. Endesa, SN Power, and Eskom), the review procedure served to clarify the company's approach and policies on several issues, and to eliminate minor factual mistakes. Fortunately, field informants were able to interview AES and Datang managers in the host countries in order to gather some aspect of the company management's perspective to supplement the information derived from official company publications and communications through the website. However, the AES and Datang managers who spoke to the field informants did not receive official authorization to speak 
on behalf of the company. These managers spoke to the researchers strictly on the condition of having their identity anonymized in any publication. Although this is an accepted methodological practice, and may actually serve to increase the accuracy of the results, it reduces the results' verifiability.

In addition to the lack of engagement from Datang headquarters, the Datang case study presented several other methodological challenges. While all other case study companies were evaluated on their performance on electricity provision (i.e. generation, transmission, and distribution), Datang is thus far only involved in the construction of electricity infrastructure (i.e. power plants and transmission lines) in Cambodia and Lao PDR. Although the logical assumption is that the company's approach during the construction phase will be reflective of how it will behave during the operational phase, it complicates the comparison of Datang's performance with the other companies on some SEP benchmarks such as labor rights (with a far different type of labor and worker being required for the construction phase than the operation phase), and reliability of supply.

For most of the case study companies, there is at least one (in some cases several) SEP issues on which the company does not have a publicly available policy. Although it is recognized that companies often do maintain internal policies on certain issues that they wish to keep confidential, if no policy was publicly available on a certain issue and the company did not indicate through interviews or in response to the questionnaire that an internal policy exists, it was generally assumed that no policy exists. However, in cases where a policy could not be identified, this was often compensated through other measures/questions built into the operationalization of the SEP benchmarks. For example, several policy indicators include operationalizing questions about the company's practice at the headquarters level as a proxy for a company's policy. An example of this is including an evaluation of the company's global fuel mix for electricity generation and its build program/plans in addition to evaluating the company's stated policies on prioritizing renewable sources of energy. Furthermore, in several cases, other documents or events were identified as providing an indication of a company's approach on a certain issue and were included in the analysis as a partial proxy for corporate policy.

The dissertation thus comes with a number of "warts", i.e. recognized limitations in research design and methodological approach. Nevertheless, the "proof of the [dissertational] pudding" lies in the scientific and practical value it holds for addressing the urgent task of making gravely unsustainable patterns of electricity provision more sustainable and more effective in reducing poverty. With "warts and all", the present dissertation contributes directly to efforts to understand and improve the quality, poverty-reducing capability, and contribution to sustainable development that electricity provision can make in the Global South. 


\section{Chapter 11}

Summary and conclusions
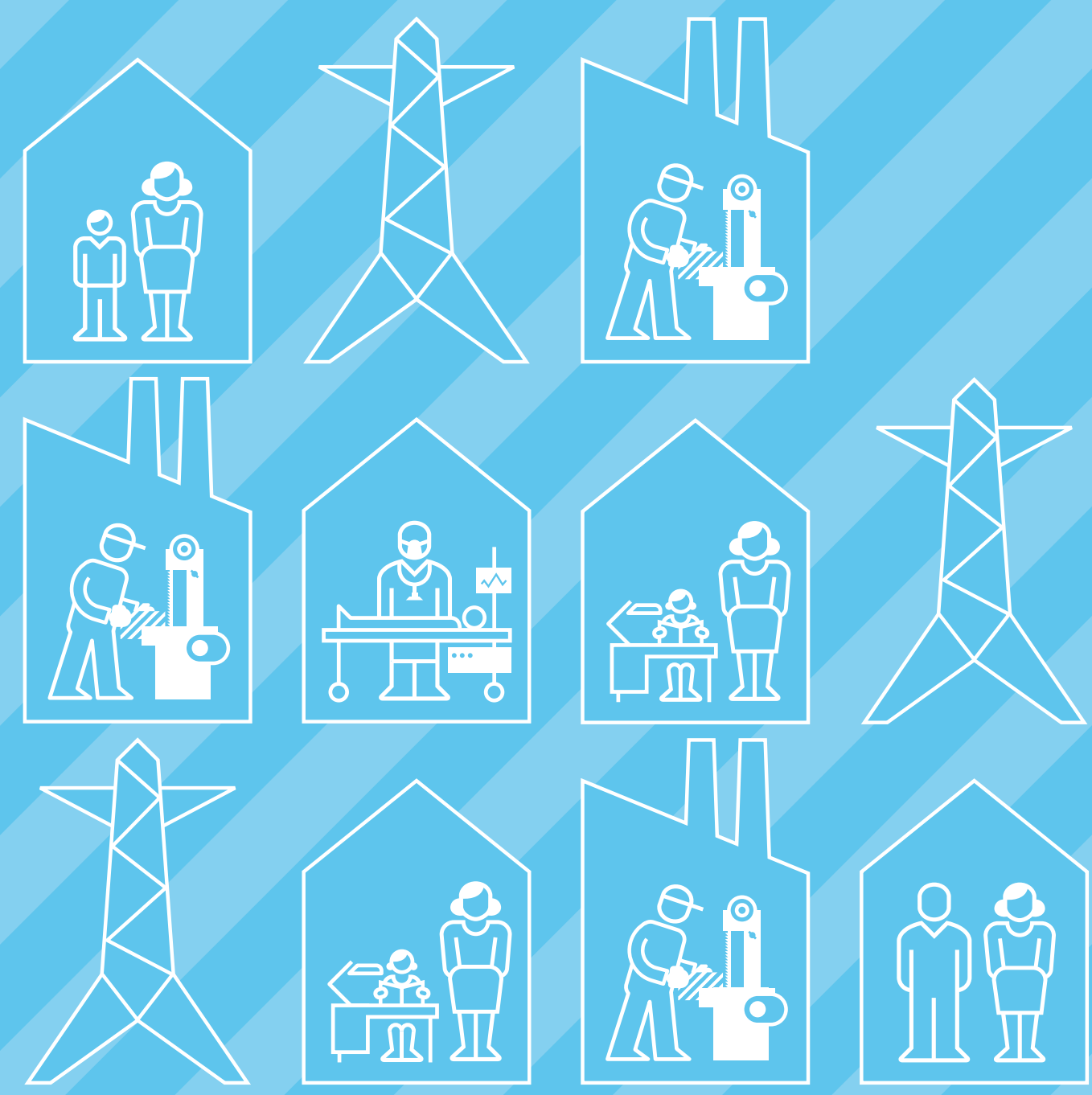


\subsection{Summary of the main contributions}

The issue of sustainable development in electricity provision has never been more relevant. Billions of people around the world live without access to basic electricity services, while the insatiable demand for fossil fuel-based power generation in industrialized nations and emerging economies contributes to a rapidly warming climate. Sustainable electricity provision is set to be a key developmental issue of the $21^{\text {st }}$ century. The United Nations declared 2012 the "Year of Sustainable Energy for All", and UN SecretaryGeneral Ban Ki-moon called on governments, businesses, and civil society to commit to taking concrete actions that will help to achieve sustainable energy for all by 2030 (UN 2012). Recent years have also seen a sharp increase in interest and demands from civil society that electricity be provided in a manner that contributes to sustainable development.

Despite the urgency and increased interest, no clearly and concisely-formulated normative standards for SEP currently exist. There is also relatively little empirical knowledge as to the degree to which transnational electricity companies rely on international norms in developing policies related to SEP and implementing those policies in host countries in the Global South. Given the variable impact TNCs have had on sustainable development, it is important to investigate how such variation comes into play for the electricity sector. This knowledge is relevant for both governments and stakeholders in efforts to improve the quality of electricity services, especially given that governmental protection for electricity consumers and workers in the Global South remains weak and poorly enforced. By improving understanding of how electricity TNCs take up and implement international normative standards for SEP - and by drawing lessons that can improve normative and regulatory frameworks - the dissertation has aimed to improve the quality and contribution to sustainable development of electricity provision in the Global South.

With this context and aim as a point of departure, the central problematic of the present dissertation has involved exploring how individual transnational electricity companies conceptualize, operationalize, and implement norms for SEP - in other words, how "quality kilowatts" are being conceived and implemented. This task called for an appliedscience approach that employs "strategic research" to produce knowledge that can be used for positive change by norm-setting bodies to promote sustainable and equitable energy systems.

The study employed an analytic approach based on normative-empirical analysis. This approach involves the construction of a set of benchmarks anchored in internationally-agreed normative standards applicable to SEP (i.e. the "SEP benchmarks"). Through an empirical methodology centered around qualitative interviews with corporate managers and a wide range of stakeholders affected by electricity 
provision operations, the study has documented and evaluated how "quality kilowatts" are conceived and implemented - and the impact on stakeholders - in five case studies of TNCs from different national/regional "modes" of business culture: AES (US mode), Endesa (European mode), SN Power (Nordic mode), Datang (Chinese mode), and Eskom (South African mode). The inclusion of the Chinese and South African TNCs/modes in the study is seen as a unique and particularly pertinent contribution given the rapidly increasing influence of these fast-growing economies in global political and economic systems.

The approach and findings of the study are deemed relevant for policy makers and normsetters, as well as for a range of stakeholder groups involved in and impacted by current unsustainable patterns of electricity provision. In a structured and systematic way, the study has employed strategic research within the applied-science realm to build knowledge that can be used to promote sustainable and equitable energy systems. Sedlacko and Martinuzzi (2012:6) insist that the type of evaluative methods employed by the present study can "make policies more effective, help manage risks, support transparency, strengthen accountability, and support learning". The knowledge produced by this type of actionoriented research is thus concretely and directly relevant for multiple target audiences. Recognizing the urgency of addressing the increasingly complex and precarious global problems associated with (un)sustainable electricity provision (e.g. climate change, persistent poverty, increasing and structural inequality in power and living standards, failure to make significant progress towards development goals such as the MDGs), the study has employed a broad-brush, big-picture approach (Gupta and Hisschemöller 1997). The research has identified crucial issues and nuances in an unstructured debate and documents how difficult it is to tackle this complex issue. As such, it contributes to the "problem finding" and "problem-structuring" aspect of applied science. Hisschemöller and Gupta (1999:156) view this aspect as necessary for addressing the "unstructured problem" of SEP at an international level.

The compilation and categorization of critical issues and the development of a framework of normative standards related to SEP in Chapter 3 represents one of the dissertation's key practical contributions to the field. It lays a foundation for improved normative guidance and, potentially, for the development of an improved code of CR conduct for the electricity sector. The implications are directly relevant for both norm-setting regimes at the international level and the integration and application tasks of TNCs. This applies particularly to the issue of trade-offs among the economic, social, and environmental pillars of sustainable development.

The study also contributes to academic debates on cross-cultural business management and on CR and corporate strategy in an increasingly globalized world. The exploratory and probing nature of the study has aimed to 
generate new insights and to offer a broad and empirically-based reference point for future studies in the area. The detailed examination of five case-study companies in six different host country settings provides a comparative snapshot of corporate performance within the realm of SEP and is an important part of the dissertation's informational contribution. It provides significant material for open reflection and critical assessment by others, including those who might be interested in different aspects of the general problematic than those highlighted by the dissertation. The extensive gathering of empirical data "on the ground" is thought to contribute significantly to the robustness of the analysis, which has important implications for both current levels of knowledge and the formulation of further more comprehensive research projects and those controlling for other variables. The inductive empirical analysis is unique in that it offers a broad comparison of corporate actors across 11 specific, internationally-endorsed SEP benchmarks in very different developmental settings. The study is thus one of the few analyses that document - through systematic and scientifically-grounded analysis - a significant, globally-observable discrepancy between what TNCs pledge to do in policy and what they actually do on the ground in host country contexts. This failure of TNCS to "translate" policy into practice is a major weakness of international voluntary normative standards addressing CR for sustainable development and SEP.

\subsection{Major conclusions on the research questions}

The dissertation undertook to answer five core research questions:

1. Are there standards for sustainable electricity provision available within the UN-anchored sustainable development framework and other international normative discourses that can be systematized into a common set of benchmarks for evaluating the performance of TNCs involved in electricity provision in the Global South?

2. Given the prospect that a TNC's homecountry business culture conditions its behavior - and assuming that differences in such "modes" of home-country business culture are increasingly important in an era of growing competitive globalization of electricity provision - how are SEP standards generally taken up, interpreted, and valued within various national modes? Can these different modes of business culture be used to structure and analyze variation in TNC uptake of SEP standards?

3. Using an applied-science, "normativeempirical" approach to guide a systematic gathering of information about the operations of electricity TNCs operating in the Global South, how do selected TNCs perform - in both policy and practice vis-à-vis normative benchmarks for SEP and relative to each other?

4. Does an empirical analysis of the performance of electricity TNCs from varying home-country "modes" of business culture 
on the SEP benchmarks contribute to theoretical notions of international business strategy?

5. What general lessons can be drawn from a comparative analysis of TNC performance on SEP benchmarks that are of direct practical relevance for improving the effectiveness of normative frameworks, particularly with regard to increasing uptake and implementation of SEP norms by TNCS and creating a more "level playing field" for sustainable development?

As carried out within the dissertation's analytic design and methodological approach, the study leads to the following conclusions in answer to the research questions.

\subsubsection{Conclusions on research question 1}

Are there standards for sustainable electricity provision available within the UN-anchored sustainable development framework and other international normative discourses that can be systematized into a common set of benchmarks for evaluating the performance of TNCs involved in electricity provision in the Global South?

There is currently no universally-accepted set of normative standards for SEP. Many different organizations, governments, agencies, universities, and initiatives have worked on various elements of sustainable development and electricity provision, but none has yet developed a comprehensive structural framework with sector-specific normative guidelines for electricity companies. Despite the fact that no electricity sector code of conduct exists, there are numerous internationallyaccepted normative standards and initiatives for CR within the UN system and in related international discourse. These normative standards can be condensed into a normative framework for SEP that can be used to benchmark the performance of TNCs involved in electricity provision in the Global South.

In order to construct the SEP benchmarks, a broad survey of relevant literature (e.g. ILO 1998, UNECE 1998, Palast et al. 2000, WCD 2000, ILO 2001, WBCSD 2002, IHA 2004, CEER 2005, Modi et al. 2005, ILO 2006, BSI 2007, IFC 2007, OECD 2007, UN/IAEA 2007, UN Global Compact 2008, Pillinger 2009, Teske 2010, IFC 2011, OECD 2011, UNHRC 2011, GRI 2012, MVO Platform 2012, UN 2012) has been conducted to identify the "critical issues" for SEP on which normative standards must give clear and specific guidance to companies providing electricity (see Chapter 3). These "critical issues" are areas in which the impacts - both positive and negative - of electricity provision on sustainable development are felt most strongly. The 31 critical SEP issues identified could be specifically related to the three classical pillars of sustainable development - social, environmental, and economic - as well as to a category of "crosscutting" issues and one related to governance issues associated with $\mathrm{CR}$ - here viewed as "general approach to CR". Based on a thorough analysis of the most widely-accepted norms related to the 31 critical issues, the multitude of standards, guidelines, and recommendations have been collated and "condensed down" into 
a framework of 11 SEP benchmarks, which the study puts forward as bottom-line standards for "quality kilowatts":

- Endorse international normative standards for CR

- Adopt a commitment to CR in core-business activities and decision-making

- Increase access to affordable electricity

- Respect labor rights

- Minimize environmental impact, including contribution to climate change

- Prioritize renewable sources of energy for electricity

- Contribute to local economic development

- Ensure reliable supply

- Engage in meaningful stakeholder consultation and participatory decision-making

- Assume responsibility for impacts throughout all business relationships

- Maximize transparency and provision of information.

As summarized and operationalized in Table 3.2, the SEP benchmarks have been used to evaluate the performance of electricity companies. The benchmarks represent the first systematization of normative-practical SEP standards for TNCs involved in electricity provision in the Global South. The framework developed here lays a foundation for further debating and developing a sector-wide set of standards that - if effectively implemented - could help to improve the quality, povertyreducing capacity, and contribution to sustainable development of electricity provision in the Global South. Adoption and enforcement of the standards would also contribute to a broader and more effective "leveling of the playing field" for actors involved in providing electricity.

Finally, the results should also have significant implications for scholarly analysis of corporate responsibility for sustainable development. Previous academic efforts to define and standardize the notion of CR for sustainable development have failed to generate consensus on an operational set of standards that can be used as a benchmark for empirically-based evaluative studies of TNC performance. Within the field of electricity provision the study has aimed to go beyond the existing literature to bring together and systematize the relevant standards. This provides a foundation for the normative-empirical approach and hopefully provides a useful point of departure for continued efforts to gain international consensus on standards for SEP and extrapolation to other industrial sectors.

\subsubsection{Conclusions on research question 2}

Given the prospect that a TNC's home-country business culture conditions its behavior - and assuming that differences in such "modes" of home-country business culture are increasingly important in an era of growing competitive globalization of electricity provision - how are SEP standards generally taken up, interpreted, and valued within various national modes? Can these different modes of business culture be used to structure and analyze variation in TNC uptake of SEP standards? 
It was hypothesized that the intervening role of home-country business culture could be employed to guide a systematized selection of case study companies with the aim of generating variance on the study's dependent variables: corporate uptake and implementation of SEP norms in policy and practice. Five national/regional "modes" - US, European, Nordic, Chinese, and South African - have been selected based on their importance as the origin of capital and TNCs involved in electricity provision in the Global South. Drawing on the broad, literature-based portraits of the national/ regional modes of business culture presented in Chapter 4, a general picture emerges regarding the value and interpretation given to international SEP norms by each of the modes. The portraits reveal clear differences among the modes on general qualitative indicators such as evidence of shared values related to sustainable corporate practice, formalization of the a national/regional interpretation of the concepts of sustainable development and $\mathrm{CR}$, regulatory style in the area of sustainable corporate practice, and evidence of prioritization of certain SEP issue areas over others.

The US mode tends to downplay the importance of (voluntary) international SEP standards, exhibiting instead a high degree of strict legalism, individualism, and a focus on short-term profits. Those SEP issues that are regulated and have a clear degree of legality - such as anti-corruption - are an essential part of core-business operations. Unregulated and voluntary SEP issues are relegated to the realm of corporate philanthropy. In contrast, the
European mode regards international standards for SEP as highly important and relies heavily on cooperative initiatives at the UN, EU, OECD, and other international intergovernmental bodies. The Nordic mode also highlights the importance of international SEP norms, and the Nordic region is widely considered to provide global leadership in implementing ethical standards. The Nordic mode encourages companies to view $\mathrm{CR}$ as an integral part of their core business, rather than an external charitable project.

Until very recently, the Chinese mode exhibited disdain - if not outright hostility - towards what it has maintained are "Western" standards for SEP. Although this stance has softened somewhat in recent years, the Chinese mode continues to indicate a clear preference for its own homegrown Chinese standards and norms such as the "Guidelines for state-owned enterprises directly under the Central Government on fulfilling corporate social responsibilities".

Like the Chinese mode, the South African mode also tends to rely heavily on the numerous standards for CR developed in South Africa such as the King Code - but many of the South African codes also reference international standards relevant for SEP. Rather than being a part of core business, however, the South African mode treats SEP issues as separate "corporate social investments" that serve the interests of the corporation rather than the real needs of the community and that "are generally not integrated or aligned to core businesses" (King et al. 2010:364). 
The evaluative analysis indicates that five case study companies exhibit significant variation in the key "uptake" benchmarks: "Endorse international normative standards for CR" and "Adopt a commitment to CR in core-business activities and decision-making". In addition to its capacity as a systematized generator of variance, the relevance and "intervening" nature of the mode of home-country business culture was largely confirmed by the empirical findings and analysis of the case study TNCs' policy and practice on other SEP benchmarks. Several corporate managers interviewed for the present study - both at the headquarters level and the level of subsidiaries in host countries - indicate the relevance of the firm's home-country business culture for its approach to SEP. Beyond such anecdotal examples, the framework of home-country business modes proved to be a useful analytic tool for projecting how company policy matches up against the SEP normative benchmarks.

For AES, international standards are significantly less influential for developing corporate policy than ensuring strict legal and regulatory compliance. Beyond legal compliance, profit motives trump voluntary normative guidance on social, environmental, and economic issues. AES's lack of reference to - and poor performance on - international SEP norms is reflective of the US mode of shareholder capitalism in which return on investment is paramount (Vogel 1996) and CR is primarily a question of strict legal compliance. Both the US mode and AES's focus on legal and regulatory requirements over the incorporation of norms suggest that companies operating within this mode view their "responsibility" to society as relatively unimportant.

Endesa's approach - on the other hand - is characterized by a thoroughly developed CR policy that is firmly rooted in international SEP standards and norms. Endesa's policies are considered largely in line with SEP norms for nine out of the 11 benchmarks. This strongly "standard-based" approach to SEP accurately reflects the European pattern of public-interest focused stakeholder capitalism and a tendency to rely heavily on (voluntary) normative standards for CR.

SN Power has a well-developed CR policy that references several of the SEP norms. However, SN Power's conceptualization and implementation of SEP is less defined by these standards than the Endesa approach. Instead, SN Power's approach to SEP is based more on a "deeply-ingrained" Nordic conception of (sustainable) development that has gradually become embedded in the traditional Scandinavian welfare state model (Kopstad 2008). SN Power (2012) cites the traditional Nordic values of "long-term thinking" and "prioritizing renewable sources energy" as its "competitive advantage". As such, SN Power's approach to SEP norms largely reflects the Nordic mode, which prioritizes adherence to human rights and sustainability standards over short-term socio-economic development and unencumbered provision of as much electricity as possible. 
Datang's approach is clearly less related to international SEP norms than is the case with Endesa and SN power. Uptake of the SEP norms is weak in Datang's CR policies, with the company tending to prioritize Chinese CR standards. As a clear reflection of the Chinese mode, Datang's profile is singularly focused on driving economic development by producing large amounts of electricity in any manner possible. This is done largely without respect for the environment or the rights of its workers and affected communities.

As for Eskom, the company references a significant number of international norms, while at the same time also relying on several African standards. In line with the South African mode, Eskom's approach is focused on providing large quantities of cheap, coal-based electricity primarily to large industrial users to drive economic development. In the past, increasing access to electricity among households was a hallmark of Eskom's approach to CR. However, in recent years the focus has reverted to supplying industry, primarily mining companies. Although largely out of line with SEP norms on environmental issues, Eskom's approach and the South African mode exhibit greater respect for norms related to the human rights of workers and affected communities than Datang and the Chinese mode.

\subsubsection{Conclusions on research question 3}

Using an applied-science, "normativeempirical" approach to guide a systematic gathering of information about the operations of electricity TNCs operating in the Global South, how do selected TNCs perform - in both policy and practice - vis-à-vis normative benchmarks for SEP and relative to each other?

The results of the analysis of TNC performance vis-à-vis the SEP benchmarks are presented in Table 10.1 and summarized graphically in Figure 10.12 in the previous chapter. Overall, the present study concludes that internationallyagreed normative standards are largely not respected by the electricity companies in their operations in the Global South. Of the 53 instances of company practice instances evaluated - with one "instance" representing one company's performance on one benchmark - the aggregate performance is considered out of line with international SEP norms in $60 \%$ of the instances. In $25 \%$ of the instances, company practice is largely out of line with the norms, while in only $9 \%$ of the instances were companies largely in line with the SEP norms.

If one only considers company policy and not actual practice on the ground, the companies perform better against the SEP benchmarks. Performance is considered in line with the SEP norms in $58 \%$ of the 55 policy instances evaluated. In $16 \%$ of the instances, company policy is largely out of line with the norms. Interestingly, nearly two-thirds of these "high" policy performances are attributable to one company: Endesa. At the other end of the 
spectrum, the policies of AES are not considered to be largely in line with any of the 11 SEP benchmarks.

This finding highlights two of the primary conclusions of the analysis: 1) Significant differences exist in performance of the five TNC case studies with respect to the SEP benchmarks. 2) Nearly all companies are unable or unwilling to translate their headquarterslevel policies into practice on the ground, with some clearly less able or less willing to do so than others.

Of the five case-study companies examined in the present study, the CR policies of Endesa and SN Power are most aligned with international SEP norms. The policies of both of these companies are deemed in line with the SEP norms on 10 out of the 11 benchmarks. Endesa's policies are considered largely in line with SEP norms on an impressive nine benchmarks. The policies of AES and Datang are the most out of line with the SEP norms. Datang's policies are in line with the norms on only three of the benchmarks and are largely out of line with four benchmarks. AES's policies are in line with the norms on only two of the SEP benchmarks. South African Eskom falls in the middle with its policies in line with SEP norms on just over half of the benchmarks.

With respect to actual practice, AES (in Argentina) and Datang (in Cambodia and Lao PDR) perform worst on the SEP benchmarks. The practice of both of these companies is out of line with the SEP norms on all but one benchmark. Datang's practice in Cambodia and Lao PDR is largely out of line with the norms on $50 \%$ of the benchmarks. In contrast, SN Power's practice in Peru is moderately in line with SEP norms on eight out of the ten benchmarks evaluated, and largely in line with the norms on two benchmarks. Eskom (in Mali and Uganda) again falls in the middle, with its performance in practice moderately in line with SEP norms on just less than half of the SEP benchmarks. Surprisingly, Endesa's practice in Argentina and Peru is judged to be in line with SEP norms on only four of the benchmarks.

Endesa is, however, not the only company to demonstrate inability or unwillingness to translate its policies into practice. Although it was postulated that corporate practices on the ground in host countries should largely reflect the corporate policies at the headquarters, the analysis reveals the relationship between corporate policy and actual implementation to be much more tenuous. Overall, for nearly two-thirds $(64 \%)$ of the benchmarks, company practice on the ground in host countries does not reflect policy at the headquarters level. In $40 \%$ of the 53 instances measured, company practice does not even carry the same tendency as corporate policy regarding alignment with SEP norms.

Finally, there are significant differences among the five TNC case studies with respect to translating policy into practice. SN Power's practice in Peru has the same relationship with the SEP norms as its policy in $80 \%$ of the instances measured. In contrast, Datang, and 
particularly Endesa and Eskom, are much less successful in implementing their policies on the ground. Endesa's practice in Argentina and Peru has the same relationship with SEP norms as its policy in just $10 \%$ of the instances evaluated. Eskom's practice in Mali and Uganda shows a similar tendency towards the SEP norms in only three instances. This means that Eskom is largely or wholly unsuccessful in implementing its policy in $73 \%$ of the normative dimensions analyzed. The poorer performance on SEP issues in practice than in policy is reflective of a trend across all companies, including SN Power, to focus CR activities in the Global South more on philanthropy and charitable giving than on corebusiness aspects.

\subsubsection{Conclusions on research question 4}

Does an empirical analysis of the performance of electricity TNCs from varying home-country "modes" of business culture on the SEP benchmarks contribute to theoretical notions of international business strategy?

The results of the analysis of TNC performance vis-à-vis the projections made by the homecountry modes are presented in Table 10.2 in the previous chapter. The analysis reveals that the policies of the case study companies are largely reflective of the mode exhibited by their home country or region in two-thirds of the instances. The modes framework is equally relevant across all five case study companies with regard to policy. The framework is less successful in projecting how the companies will perform on SEP benchmarks in practice in the host countries. In less than half of the instances investigated was corporate practice in the Global South deemed largely reflective of home-country mode. The modes framework proved to be most relevant in understanding how American AES, Chinese Datang, and Nordic SN Power perform on SEP benchmarks in practice. The framework is less accurate in projecting how European Endesa and South African Eskom perform in practice. Endesa's practice in Argentina and Peru is not deemed largely reflective of the European mode on any of the SEP benchmarks. Eskom's practice in Uganda and Mali is deemed largely reflective of the mode for only two benchmarks.

Company policy can therefore be considered to be influenced to a significant degree by the home-country business culture. Conceptually, this means that one can speak of a "top-down cultural effect", which can be contrasted with a "top-down normative effect" (i.e. deriving from the established norms of international bodies). The latter - given the wide variation in policy and practice among firms studied - is revealed to be less influential. The existence of "modal" cultural effects means that one can consider the development of a "culturally sensitive lens" that academics, policy-makers, and other stakeholders can use in order to better understand and more effectively regulate and work with electricity-providing TNCs from different countries and cultures. As will be seen below, this finding has implications for improving norm-setting and policy-making related to SEP within varying domains of governance. 
Two other findings lead to more general (and speculative) conclusions as to the role of host-country institutional/cultural factors affecting TNC practice in host countries. First, the modes framework assumes that homecountry business culture has equal impact on policy and practice. However, this was found not to be the case. The modes framework is less able to project how a company actually implements policy on the ground in a host country. Second, the analysis indicates that all companies experience difficulties, and some an outright inability, to put their policies into practice in host countries. This situation is perhaps understandable given the existence of clear institutional/cultural differences between the host countries that were drawn on to analyze and understand company practice on SEP norms. Host-country institutional/cultural factors are conceptualized as a "contextual variable" in the analytic framework of the present analysis (see Figures 2.1 and 4.1). The findings do suggest an important role for the "bottom-up institutional/cultural effect" - an effect shown to be detrimental to the explanatory power of both the international normative standards (i.e. the "top-down normative effect") as well as the company culture explanation (i.e. the posited "top-down cultural effect" of the home-country setting in a company's operations around the world).

With these findings, the study adds to the literature theorizing that firms approach strategic decisions in different ways, reflecting the beliefs and values of the national culture in which they "grew up" (e.g. Hofstede 1983,
Barron 2010). Much is already known about how national culture affects how firms form their own organizational culture and internalize values and norms such as responsibility, equality, innovation, flexibility, and the need to protect the environment. However, the present analysis fills a gap in the literature related to how culturally-grounded approaches to CR for sustainable development condition TNCs' uptake and implementation of international normative standards. This aspect has hitherto been overlooked by scholars interested in the effects of national culture on corporate strategy. The framework and empirical observations made here suggest that corporate strategy related to SEP is demonstrably influenced by the culture in the country or region of origin. The study shows, however, that companies frequently do not translate policy into practice in host countries in the Global South.

\subsubsection{Conclusions on research question 5} What general lessons can be drawn from a
comparative analysis of TNC performance on SEP benchmarks that are of direct practical relevance for improving the effectiveness of normative frameworks, particularly with regard to increasing uptake and implementation of SEP norms by TNCs and creating a more "level playing field" for sustainable development?

Intergovernmental bodies such as the have stressed the need for more effective action to address the current unsustainable nature of electricity provision around the globe (e.g. WCD 2000, IEA 2010a), including more effective rules 
and guidance for international business (e.g. Ruggie 2008, EC 2011). The conclusions drawn from the present analysis contain numerous lessons and implications for norm-setters and policy-makers at the international, regional, and national levels, as well as companies involved in electricity provision and their stakeholders.

\subsubsection{Lesson 1: Progress can be made with regard to problem- structuring by developing an electricity sector "code of conduct" based on SEP norms}

The first lesson is drawn from the conclusion that there is significant variation among the companies regarding their understanding and interpretation of SEP. The inconsistent application of the various social, environmental, and economic normative standards for SEP reveals a widely varying approach to the sustainable development "balance" and considerable divergence in the impact (positive and negative) of TNCs on people and the planet. The lack of consensus as to the values that should be given priority and the lack of shared knowledge on the subject identifies SEP as an "unstructured problem" (Hisschemöller and Gupta 1999). Addressing such problems requires work at a level of abstraction above "technical methods" to focus on problemstructuring and learning.

This implies that a crucial element of any regime or initiative aimed at improving the international framework for SEP, including the UN's "Sustainable Energy for All" initiative and the proposed sustainable development goals, must be to clearly define what is meant by SEP. Doing so involves the development of robust, consensual normative standards for SEP that establish a common normative playing field for electricity provision around the globe. The normative and practical endpoint of the problem-defining exercise would be to develop a common "code of conduct" for the electricity industry as a whole. Existing technical codes of conduct for the electricity sector could be extended to cover CR for SEP issues. Codes for $\mathrm{CR}$ exist in other industrial sectors (e.g. electronics), but are not yet being seriously considered in the electricity sector. The framework of SEP benchmarks developed in the present study provides a solid foundation for developing such an electricity sector code of conduct. The code should be developed through a multi-stakeholder and consultative process among policy-makers electricity companies, electricity sector regulators, electricity sector workers and unions, and other stakeholders such as NGOs and representatives of groups affected by electricity provision operations (Hollaender and Stokman 2012). Although such a code of conduct may initially not be legaly binding (and thus would not address a fundamental shortcoming of the SEP norms described below), it would nevertheless serve to establish a common understanding among companies, policy-makers, and stakeholders as to what is expected from companies providing electricity.

Of crucial importance for developing a common set of norms is the finding that national and 
regional CR standards and values play a significant role in guiding TNC policy. In some cases, national and regional CR standards were more important for the policy and strategy development of TNCs than international norms. Given this finding, it may make sense to consider employing a "culturally sensitive lens" for understanding and addressing different TNCs' reactions to and engagement in the development of a sectoral code of conduct.

It is important to understand that the suggestion of using a "cultural lens" to design and develop norms does not mean that normsetting regimes should settle for the lowest common denominator among the various business cultures. Rather, it implies that norm-setters should, in shaping and "selling" the norms to various stakeholders, consider the differences that national and regional cultures have on how companies from varying backgrounds respond to certain values and standards.

\subsubsection{Lesson 2: Voluntary CR alone is insufficient. It is necessary to work toward a binding regulatory framework to achieve internationally-adopted sustainable development goals and create a more level playing field for SEP}

A second lesson derived from the present study's findings relates to ensuring uptake and implementation of a common normative framework for SEP once one is developed. The present analysis revealed that current voluntary SEP standards are largely not effective in ensuring that electricity TNCS abide by internationally accepted norms. The findings across all five case studies reveal that the international SEP benchmarks are frequently not respected by TNCs. Even in cases where the TNCs proclaimed to have adopted some of the SEP norms, these were often not adequately implemented on the ground in host countries. For example, although Endesa relies heavily on international normative standards for its policies on reducing GHG emissions and its contribution to climate change in its home market, the company's practice at its operations in Argentina and Peru reveals the opposite situation - rising GHG emissions. Endesa admits that the relative weakness or inexistence of binding environmental standards to level the playing field in the Global South has resulted in this situation (Fraile 2008). This implies that even the companies that voluntarily endorse current SEP norms cannot always be assumed to implement them.

A significant, if disquieting, conclusion of the present analysis is that the current international regime of voluntary CR norms and standards for SEP is failing to ensure that electricity provision contributes to sustainable development. Electricity provision by TNCs is all too frequently focused on servicing large industrial users or large population centers to guarantee profit margins rather than expanding access to affordable electricity among poor, underserved communities. The performance of Eskom prior to and post-commercialization is illustrative and reveals clear limits to market- 
based electrification programs on the scale and at the pace needed to meet the MDGs. Even SN Power, which is involved in some rural electrification programs, has neglected local communities in favor of industrial (mining sector) clients in Peru and has withdrawn from countries desperately in need of investment in electricity infrastructure because of profitability concerns. The present analysis thus indicates that the current international normative framework for SEP is unable to effectively encourage electricity companies to invest sufficiently in sustainable electrification schemes or to genuinely respect the right to access affordable electricity.

\section{Voluntary SEP norms are similarly failing} to encourage TNCs to invest sufficiently in renewable sources of energy for electricity and the decentralized electricity provision systems that are the key to SEP and realizing "Sustainable Energy for All". The present analysis of five electricity TNCs reveals that, despite encouragement to the contrary by international SEP norms, most TNCs prefer large-scale, centralized electricity systems based on fossil fuels or large hydroelectric facilities. This is despite the fact that, in all of the host countries examined in the present study, there is an enormous potential for more sustainable and decentralized sources of energy such as wind, small hydro, solar, geothermal, wave, and tidal power (Teske 2010, UN 2012). These are alternative forms of energy that are technologically and economically feasible, but are currently not being exploited (Jacobson and Delucchi 2011).
Even fossil fuel-based electricity generation and large hydroelectric facilities, which are not going to be replaced in the short term, could be brought more into line with principles of sustainable development through more effective SEP regimes. Such regimes can, for example, encourage or compel a rapid transition from coal to natural gas and sustainable hydroelectric development, with transition processes characterized by proper stakeholder engagement and measures for ensuring biodiversity protection.

Despite the fact that designing SEP norms with a "cultural lens" could help to increase the normative uptake by TNCs, a major implication to be drawn from the analysis is that a voluntary framework of SEP norms is not likely to be effective on the scale required to meet (sustainable) development goals. The study thus contributes to a growing body of literature and evidence (e.g. Karnani 2010, Hart 2012) indicating that voluntary CR norms alone are largely ineffective in addressing what Hisschemöller and Gupta (1999) identify as the large, urgent, and complex problems related to sustainable development.

The present study adds to this literature, providing analyses of case examples with lessons and indications as to why voluntary mechanisms are largely ineffective in ensuring that TNCs take up and implement internationally agreed standards (in this case for SEP). Companies can sometimes generate profit while contributing to sustainable development and respecting international norms. 
However, the dynamics surrounding SEP reveal the limits of win-win situations in a world of finite resources and dwindling carrying capacity. The electricity sector - with its crucial role in alleviating poverty, combined with a track record of significant adverse environmental impacts - is the epitome of this situation. Extending access to electricity to all those who need it in a manner that does not degrade the environment is rarely an exercise that generates short-term profits for corporations. Painful trade-offs must be made in order to achieve long-term, complex sustainable solutions. Doing so will likely involve balances and compromises that are perceived as a "loss" rather than a "win" by one or more stakeholder groups.

The present analysis thus indicates that most electricity TNCs will not voluntarily implement normative standards for SEP if doing so means sacrificing profits. Voluntary SEP norms attract only those TNCs that are inclined to a sense of responsibility beyond generating financial returns for shareholders, and only when observing voluntary standards does not conflict with the latter.

There is, in short, a clear need for an international regime that serves to create a level normative playing field for electricity provision and effectively encourages or even compels private capital to increase and improve SEP implementation on a significant scale. At the heart of such a regime lies the development of a binding regulatory framework of minimum SEP standards. This framework would logically be based on an electricity sector code of conduct to be introduced and effectuated at an early stage, and could be supplemented by voluntary initiatives. The SEP framework does not need to be a strictly prescriptive model, dictating exactly which technologies should be employed and how. The framework should be one that lays the groundwork for a balanced and sustainable approach by setting binding minimum standards to avoid the worst abuses by the normative laggards and free-riders.

Corporate responsibility for SEP must thus be taken beyond the realm of voluntary norms through the development of a regulatory regime with appropriate incentives and the ability to enforce sanctions. Setting binding standards at the national level and ensuring that national enforcement bodies have extraterritorial jurisdiction will apparently be necessary to ensure that key social, environmental, and economic standards are sufficiently addressed.

Developing a binding regulatory framework at the international level is no easy task. It will require painful tradeoffs and sacrifices from all sides and stakeholders, as was demonstrated by recent multi-year, multi-stakeholder negotiations that led to the 2011 update of the OECD Guidelines for Multinational Enterprises and the UN Guiding Principles for Business and Human Rights. However, the vision of an electricity system that spurs sustainable development and provides sustainable electricity for all remains the key issue. The SEP benchmarks developed as part of the study contribute to making international goals for sustainable development more reachable by 
staking out clear and consistent norms, along with specific benchmarks and mileposts along the way. The lesson suggesting the use of a culturally-sensitive lens in developing the norms can also help norm-setters in promoting more effective "buy-in" from companies. Although a binding SEP framework is obviously a longer-term goal than the voluntary electricity sector code of conduct, the fast-approaching deadlines for the MDGs imply that policymakers cannot afford to wait to begin putting steps in place.

\subsubsection{Lesson 3: Existing voluntary initiatives can play an important complementary role to a binding framework, but they must be improved and strengthened}

It should be noted here that the conclusion on the need for a binding framework for SEP does not imply that voluntary initiatives are not important and necessary. The analysis indicates that there is a role for voluntary standards to supplement the binding framework. Voluntary initiatives can drive and highlight best practice and encourage innovative front-runners to continually strive to improve. The ideal SEP regime combines mandatory and voluntary approaches to ensure respect for minimum standards and to create a level playing field for all firms operating internationally while at the same time allowing for and encouraging leadership and innovation.

However, the present analysis reveals that current voluntary normative initiatives and frameworks are largely failing to influence corporate behavior adequately. Intergovernmental and regional organizations can strengthen the implementation of current voluntary standards such as the UN Guiding Principles, the OECD Guidelines, and other regional initiatives. Doing so can create competitive pressure on firms to adopt a common standard. Findings from the present analysis reveal a number of lessons and implications for strengthening voluntary normative frameworks. These include:

\section{Sub-lesson 3.1: Strengthening monitoring} and verification. The analysis reveales that standards linked to a certification system, such as those developed by the ISO and OHSAS, are more often taken up by the electricity companies than broader CR standards. This implies that frameworks including a system of certification with monitoring and verification may be more effective than those without compliance monitoring. A complaint mechanism that can effectively address grievances related to violations of the standard can also be one way to increase uptake and compliance with a standard.

\section{- Sub-lesson 3.2: Addressing the issue of} sanctions. In order to increase uptake and influence, frameworks of (non-legally enforceable) normative standards that incorporate consequences for parties breaching the standard. The analysis suggests that standards that are linked to project financing, such as the World 
Bank IFC's Performance Standards, have an advantage in terms of influence over norms that rely solely on the ethical or moral goodwill of companies. Other examples of consequences could include governments withholding state support (e.g. export credit financing, government-backed loans and insurance, tax breaks, public procurement tenders, official development aid contracts, etc.). In order to identify potential breaches of the standards, frameworks should include a grievance mechanism that fulfills the core criteria of legitimacy, accessibility, predictability, equitability, rightscompatibility, and transparency outlined by the UNHCR (2011).

- Sub-lesson 3.3: Promoting multi-level learning and international "up-scaling" and harmonizing. The UN Global Compact was the most frequently cited normative standard among the electricity TNCs in the present analysis. The legitimacy and weight lent to the standard by official UN status was cited by several companies as a reason for referencing the Global Compact in their CR policies. As a UN initiative, the Global Compact has clear advantages over standards such as the OECD Guidelines in terms of geographical applicability and scope, especially with regard to the everincreasing influence and importance of TNCs from emerging economies. These are crucial characteristics of a standard that aims to achieve universal acceptance. At the same time, the importance that companies in the analysis placed on regional standards indicates that a strategy to increase "buy-in" may be to first develop and apply standards regionally before proceeding to harmonize them with initiatives in other regions and scale them up to the international level. An example of such an approach is the UN Convention Against Corruption, which constituted a harmonization of various regionally-developed standards and subsequent "up-scaling" to the UN level.

\subsubsection{Lesson 4: In working towards creating a more level international playing field, there are many smaller steps that various actors from governmental, inter- governmental, civil society, and corporate sectors can take to increase the uptake and implementation of SEP norms by TNCS.}

In addition to pointing to the need to work toward a binding framework for SEP, the present analysis contains concrete lessons for policy-makers at the international level, as well as in home and host countries, as to what else can be done in order to shift currently unsustainable patterns of electricity provision in the direction of sustainable development.

\section{Host countries}

Governments and citizens of host countries in the Global South have the most to gain or lose from the success or failure of a framework for SEP. Although not investigated in detail, 
the present analysis inductively indicates that host-country institutions are influential in structuring corporate practices. Host-country governments could thus play a stronger role in ensuring that companies involved in electricity provision contribute to sustainable development and are held accountable for irresponsible and unsustainable behavior in their countries. Knowing that local business strategies are likely to be embedded in cultural origins can help policy-makers and civil society, respond to and act on the strategies pursued by these firms. Knowledge about a firm's cultural mode can help policy-makers anticipate TNC resistance to new regulations designed to protect the environment or expand access to affordable electricity. At an even earlier stage, the relevance of the modes framework has implications for governments in the Global South as they decide whether to allow investment and participation by TNCs in their electricity market, and which TNCs can be expected to show the most commitment to the sustainable development of host countries.

\section{- Sub-lesson 4.1: Developing local (public)} alternatives. The study reveals that electricity TNCs are largely not respecting minimum SEP standards. In the absence of a binding international framework to ensure adherence to these standards - and given the widespread availability of decentralized sustainable electricity technologies - host country governments may be better advised to keep electricity provision in public hands (i.e. owned and operated by a well-regulated, accountable, domestic public entity) and use the SEP framework to develop sustainable local solutions, rather than depending on profit-seeking TNCs to provide electricity in a sustainable manner. Experiences with nationally and regionally-developed publicsector models in Latin American countries such as Costa Rica and Bolivia reveal the benefits of a well-managed public utility providing a public service (Wilde-Ramsing and Potter 2006, Chavez 2012).

\section{Home countries and intergovernmental bodies}

Countries in the Global South that desperately need investment in electricity infrastructure frequently find themselves in a weak bargaining position vis-à-vis powerful, well-resourced corporations. As a result, governments in such countries are often reluctant to confront big investors about their unsustainable and irresponsible practices. It is therefore crucial that, in the absence of a binding international framework for SEP, TNCs' home countries and intergovernmental organizations act upon their duty to ensure that internationallyoperating electricity firms uphold minimum SEP standards.

Home-country governments can do more to encourage TNCs to abide by existing voluntary normative standards such as the UN Guiding Principles for Business and Human Rights and the OECD Guidelines for Multinational Enterprises. Both of these instruments carry clear duties and responsibilities for home countries related to their interaction with companies based within their territories. The OECD Guidelines contain a dispute 
resolution mechanism (called the "specific instance" mechanism) by which home-country governments can address grievances related to the irresponsible behavior of their companies. Around the world, 43 countries, many of which are home countries to electricity-providing TNCs, are signatory to the OECD Guidelines. These governments should strengthen their procedures for handling complaints alleging breaches to the OECD Guidelines by their TNCS so irresponseble behavior does not go without consequences for companies. This is currently not generally the case due to a lack of political will on the part of home-country governments. Beyond the OECD Guidelines, home countries should clarify their extraterritorial obligations and the jurisdiction of the domestic legal system over internationally operating corporate actors based in their territory.

The present analysis suggests that civil society organizations play a crucial "watchdog" role in discovering and publicizing instances where SEP standards are not being respected by TNCs. This function is particularly important in the absence of an enforceable global SEP framework. Home-country governments should therefore actively support international networks of CSOs in fulfilling the "watchdog" role. CSOs also play a role in giving local communities in the Global South a voice in national, regional, and international decisionmaking forums. Such communities - with their detailed knowledge of the local environment and ecosystems - are particularly well-placed to encourage sustainable management of natural resources and public services. Governments should support efforts to empower CSOs and civil society networks - particularly networks linking groups in the Global South with those in the Global North - to fulfill these roles effectively.

At the international level, there are a multitude of ways in which intergovernmental bodies such as the UN, AU, ASEAN, and the OECD can increase the uptake and implementation of existing SEP norms in addition to working towards a binding SEP framework. Work should be done at the international level to develop policy instruments, recommendations, and mechanisms aimed at encouraging investment from private and public financial institutions in public utilities and strengthening public-sector capacities. In so doing, the quality of service (in the SEP sense) should be prioritized. In this regard, there is a significant opportunity related to the commitment - made by a wide range of governments and other stakeholders at the 2012 UN Conference on Sustainable Development in Rio - to work together to develop a new set of "Sustainable Development Goals" (SDGs). The 61 country delegations from the Global South and North - surveyed by the UN following the conference deemed "sustainable energy / access to energy" to be the "top priority area" for the SDGs (UNDESA 2013). The findings and analysis produced here can contribute directly to the development of a "Sustainable Energy Goal".

International policy-development organizations such as the OECD and UNCTAD should ensure that the policy instruments and 
frameworks they develop include clear advice to governments in the Global South to prioritize the population's needs over return on investment. Policy instruments should contain advice on how to ensure that investor rights (e.g. protecting investment from expropriation) are in balance with investor responsibilities to respect minimum standards and contribute to sustainable development. Economic models and policy advice should also be developed on how to internalize the external costs of electricity provision that are currently being paid by society as a whole through both commandand-control and market-based mechanisms. Policy instruments and advice should also be developed to assist countries in the Global South to improve their own means of publicly financing and managing electricity systems.

\section{Companies and stakeholders}

The findings and conclusions from the present study also contain lessons and implications for managers and practitioners of CR within electricity TNCs. The analysis reveals a need for managers to be sensitized to cultural factors that influence their firm's (and their competitors' firms) approach to corporate responsibility. At a time of growing demands on businesses to act in accordance with the principles of sustainable development, knowing why and how certain practices in their firms came about can help managers respond constructively to pressure from governments and civil society. Understanding what sort of factors influence their company's behavior can facilitate initiatives by pro-CR managers to change unsustainable practices and bring the company in line with SEP norms. Here there are increasing possibilities and business forums (e.g. within the WBCSD) where companies can learn from each other with respect to development of CR policies based on SEP standards and the implementation of those policies on the ground in host countries. The analysis also indicates that the implementation of sustainability and CR policies on the ground in the Global South is more effective, although certainly not guaranteed, if TNCs stress - in their official policies and procedures - that social, environmental, and business excellence are equal and interdependent goals.

Finally, the analysis indicates that civil society plays a crucial role in implementing the SEP framework and ensuring that minimum standards for responsible business behavior are upheld. CSOs can embrace their "watchdog" role by continuing to research and raise awareness about the impacts of electricity companies on SEP. Based on such research and analysis, CSOs can directly engage with decision-makers in government and companies and use grievance mechanisms attached to international normative frameworks such as the OECD Guidelines and the Compliance Advisor Ombudsman at the World Bank. CSOs should strive to build capacity to influence international and regional policy instruments and standards, as well as the capacity to monitor and improve the actual performance of electricity provision activities by TNCs on the ground in host countries. CSOs must also embrace their role in giving underrepresented communities and workers affected by the 
operations of electricity-providing TNCs a voice in international and regional decision-making forums in which normative standards are developed.

\subsection{Reflections on the status quo and suggestions for further research}

It is appropriate to conclude this long and complex journey into the depths of corporate responsibility for sustainable electricity provision with a reflection on 2012 as the "International Year of Sustainable Energy for All". This includes a hard look at the status quo and an assessment of what is still necessary to make sustainable energy and "quality kilowatts" for all a more tangible reality.

One of the most promising outcomes of the 2012 UN Rio +20 Conference on Sustainable Development was the broad, high-level commitment to develop a new set of "Sustainable Development Goals". A survey of countries from around the world identified "sustainable energy / access to energy" to be the "top priority area" for the SDGs (UNDESA 2013). Significantly, countries in the Global North and South alike indicated sustainable energy to be a top priority. 2012 also saw an increase in the number of countries signing up to the UN "Sustainable Energy for All" initiative, as well as new pledges of funding for sustainable energy.
Beyond these two bright spots, however, both Rio +20 and the 2012 UN Climate Change Conference in Doha are widely considered to have failed to make significant progress in developing policy frameworks to address the key drivers of unsustainable patterns of electricity provision and consumption (Jackson 2012). Hopes for a paradigm shift in policies and incentives to ensure more sustainable business practice remain unfulfilled. Governments have declined, for example, to address reducing fossil-fuel subsidies that dissuade electricity companies from shifting investment from coal and other fossil fuels into sustainable, decentralized sources of energy that are more effective in increasing access to electricity.

Thousands of global business leaders attended Rio +20 , and hundreds of new business initiatives were announced (UNGC 2012). Unfortunately, most of these initiatives are piecemeal and unambitious, especially when compared to the urgent challenges of poverty alleviation, natural resource depletion, and climate change. With so many high-profile and influential political and economic decisionmakers in attendance, why have the outcomes been so disappointing? The lessons drawn from the present analysis may offer some insights. Here are three:

1. The difficulty in reaching agreement and taking action on something that - in principle - everyone is in favor of (who is against sustainable development?) reveals that sustainable development, climate change, and poverty eradication continue to be seen 
as vaguely-defined, long-term challenges. More "pressing" and "tangible" problems such as the global financial crisis receive far more attention. This underscores the need - as indicated by Hisschemöller and Gupta (1999) and mentioned above (Lesson 1 under research question 5) - to immediately undertake efforts of "problem structuring" that can make addressing sustainable development and SEP more concrete and tangible and facilitate action in the short term.

2. Moments like Rio +20 - and the general aversion to engaging in problem structuring - reveal that a transition to systems of electricity provision that foster sustainable development will inevitably create winners and losers, especially if that transition takes place at the rapid pace necessary to address the urgent challenges of climate change and poverty eradication. Powerful established interests will resist losing the advantageous position they currently hold and benefits they currently enjoy. As mentioned above, such transitions are rarely "win-win-win" situations - they involve making tough choices and generating costs that someone will have to pay. It is thus essential that political bodies take coordinated action to structure and govern the transition to SEP so that costs are equitably distributed and the big-picture goal of sustainable development remains clearly in sight.

3. The failure of Rio +20 and Doha - and countless global summits before them - indicate that large, international forums may not be the best settings for achieving breakthroughs. At this level, problems have a tendency to remain vague, and decision-makers tend to settle for lowestcommon-denominator results. In contrast, problems addressed at the local and regional level are often better-defined and "closer to home" for those affected and those decision-makers that need to be involved in addressing them. This reinforces the present study's finding that TNCs are often more responsive to national and regional guidelines and standards than those developed at the international level. Using a "bottom-up" approach, national and regional initiatives must thus be strengthened, with government, businesses, and civil society working together as equal partners to solve specific SEP-related problems. Solutions can then be "scaled up" and harmonized with efforts and frameworks at the international level. Public service sectors such as electricity and water provision and healthcare particularly lend themselves to this approach.

These reflections imply that ensuring the SEP framework developed here is effectively implemented and genuinely contributes to furthering sustainable development will require additional "strategic research for sustainable electricity provision".

\section{Suggestions for further research}

The design of the analytic approach and subsequent analysis in the present study have aimed to further normative-empirical 
methodology and advance the scientific debate related to CR for sustainable development, in particular the effectiveness and uptake of international normative standards. This final section provides suggestions for scientists and academics working in similar applied-science areas to develop follow-up research designs and methods that can generate more robust findings to support and further test the general conclusions and implications presented above.

The small-n, comparative case study approach was a deliberate choice for the structured qualitative analysis employed in the present dissertation. The comparative case study method is recognized for its usefulness in addressing complex, multivariate situations, including those related to cross-cultural studies. Nevertheless, the limited number of case studies examined in the present study represents a methodological consideration that impacts the results and potential for generalizing the findings. In future research, the five case studies examined in this report could be subjected to more in-depth analysis, with the number of models and firms expanded to make the findings and conclusions more robust. The present study covered the countries and regions that are currently the source of the vast majority of FDI in the electricity sector, and included an examination of case studies and business cultures from two of the most important emerging economies - China and South Africa. TNCs based in the other four "BRICSAM" countries (i.e. Brazil, Russia, India, and Mexico) could also be included so as to document the increasingly important role that these countries and companies based in their territory are playing in electricity provision in the Global South.

Future studies should also seek to tease out the role of firm size and ownership structure in determining how a company takes up international SEP norms and performs against SEP benchmarks. Although the results of the present analysis did not indicate (operational or financial) firm size to be a significant factor follow-up studies could more systematically address firm size and overall scale of operations in the provision of electricity. Similarly, future analyses could focus on investigating whether domestic foreign policy agendas act as an additional intervening variable in determining the performance of state-owned enterprises on the SEP benchmarks.

Follow-up research is also required to improve on the method employed by the present study to measure and classify company performance on the SEP benchmarks. By expanding the number of Likert-type categories employed to classify performance, additional important nuances in policy and practice could be captured. Studies focusing on a more limited range of SEP benchmarks could concentrate more on making the operational indicators for each benchmark more specific and objectively measurable. This could also provide vital new information on the possible need for more focused prioritization of specific SEP issues at the corporate headquarters level.

Although the present study avoids most of 
the criticism of the use of simplistic typologies of national/regional culture and varieties of capitalism, future research could refine and enhance the study's analytic model and modes framework by incorporating theoretical insights from other cultural categorization studies. Doing so would likely result in a more sophisticated analysis of the effects of culture on approaches to CR and sustainable development.

It was beyond the scope of the present study to systematically investigate the full implications and role played by the institutional and cultural setting of host countries in shaping company practice on the ground. It is plausible that the host country's institutional environment could provide and additional (qualifying) explanation for the dissertation's findings on the inconsistencies and discrepancies between corporate policy and practice. Future studies should focus on how institutional, cultural, and socio-economic differences among host countries impact a company's performance on the SEP benchmarks at the point of implementation in national cultural settings and local communities. Future research designs should make greater efforts to control for - and integrate the effects of - this crucial contextual variable.

Additional research is needed on the SEP norms identified and systematized by the study. In order to develop a broad framework based on the core social, environmental, and economic pillars of sustainable development as expressed by the Rio Declaration, Agenda
21, and the MDGs, multiple criteria and interdependent norms must be considered, weighed, and balanced. It was with this aim that the original 31 critical issue areas for SEP were here condensed down into 11 SEP benchmarks for the case study analysis and evaluation. A more detailed and comprehensive analysis of corporate performance on each of the 11 benchmarks would provide additional insights, nuances, lessons, and implications. Further investigation of the relevance and impact of some of the SEP benchmarks - such as those on stakeholder engagement, nonfinancial disclosure, and responsibility and due diligence throughout all business relations could provide crucial input and feedback for the further development of normative standards such as the UN Guiding Principles, the OECD Guidelines for Multinational Enterprises, and ISO 26000. In addition, other members of the sustainable development research community who believe certain aspects or pillars of SEP are more important or urgent than others should be engaged to assess a possible "weighting" of the different SEP benchmarks. Doing so could potentially result in a different relative comparison of the performance of the selected TNCs. The present study provides raw material and an initial conceptual framework for making such analyses.

Additional research in the field of international law is also necessary to provide policy-makers with further guidance on how to create a more "level playing field" by studying and facilitating the process by which "soft law" instruments such as standards and guidelines evolve into 
the "hard law" necessary for establishing binding, enforceable responsibilities and rights on various stakeholders. Several studies on this topic exist (e.g. Queinnec and Bourdon 2007, Boyle and Chinkin 2007, Langford et al. 2013), but the process and implications are far from clear. Future research will hopefully be able to employ the perspectives and insights put forth here to significantly improve both the "uptake" and implementation of more consensual and "robust" SEP norms.

Finally, in the absence of a binding framework to ensure that electricity companies abide by minimum SEP standards, continued research on alternative models for financing and providing electricity is clearly needed. Recent studies (e.g. Chavez 2012, Hathaway 2012, McDonald and Ruiters 2012) demonstrating the existence and viability of alternative models at the national and regional level should be examined and elaborated upon to see how such models can be "scaled up". 


\section{Samenvatting in het Nederlands}

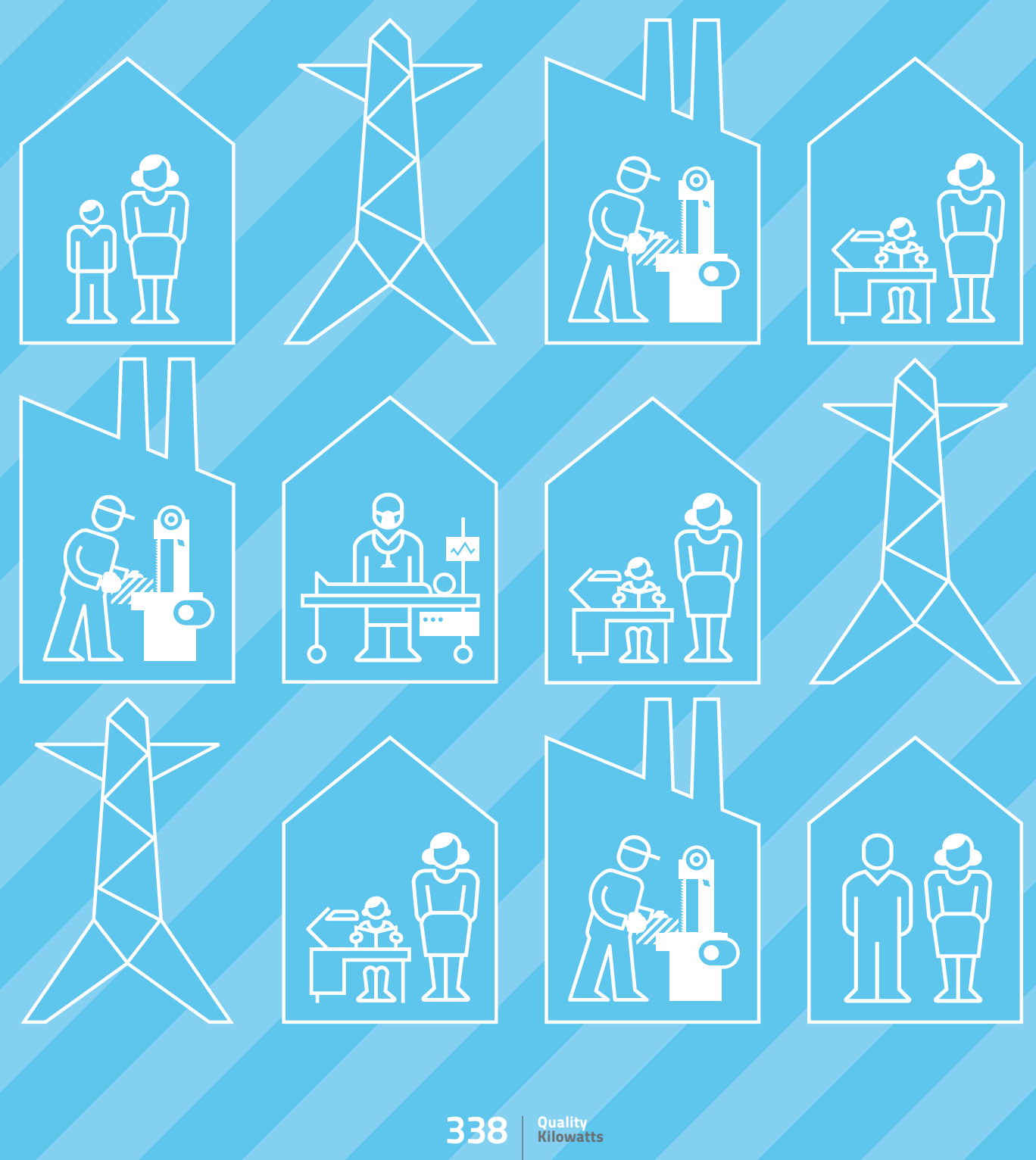




\section{Eerlijke Energie?}

\section{Een normatief-empirische analyse van verantwoord ondernemen voor duurzame elektriciteitsvoorziening in het Zuiden}

Duurzame ontwikkeling in elektriciteitsvoorziening is nog nooit zo'n relevante kwestie geweest. Wereldwijd leven miljarden mensen zonder toegang tot de meest basale elektriciteitsdiensten, terwijl de onverzadigbare vraag naar fossiele brandstoffen in geïndustrialiseerde landen en opkomende economieën bijdraagt aan een snel opwarmende aarde. Duurzame elektriciteitsvoorziening (DEV) is vastgesteld als een zeer belangrijke ontwikkeling in de 21ste eeuw. De Verenigde Naties (VN) verklaarden 2012 als het 'Jaar van de Duurzame Energie voor iedereen'. En VN-secretaris-generaal Ban Ki-moon riep overheden, bedrijven en het maatschappelijk middenveld op concrete acties te ondernemen die bijdragen aan duurzame energie voor iedereen in 2030 (UN 2012). De afgelopen jaren zien we dat het maatschappelijk middenveld meer belangstelling krijgt en steeds nadrukkelijker eisen stelt aan de manier waarop in elektriciteit wordt voorzien, dat die moet bijdragen aan een duurzame ontwikkeling.

Ondanks de noodzaak en de toegenomen belangstelling bestaan er momenteel geen duidelijke en beknopt geformuleerde normatieve DEV-standaarden. Bovendien is er relatief weinig empirische kennis beschikbaar over de mate waarin transnationale elektriciteitsbedrijven gebruik maken van internationale standaarden bij de ontwikkeling van DEV-beleid en de uitvoerging van dit beleid in de 'gastlanden' in het Zuiden. Gezien de wisselende invloed van transnationale ondernemingen (TO's) op duurzame ontwikkeling is het van belang om te onderzoeken of en hoe een dergelijke variatie een rol speelt in de elektriciteitssector. Deze kennis is van belang voor overheden én belanghebbenden bij hun inspanningen om de kwaliteit van de elektriciteitsvoorziening te verbeteren. Vooral omdat overheidsbescherming van elektriciteitsconsumenten en arbeiders in de elektriciteitssector in het Zuiden zwak is en slecht uitgevoerd wordt. Met het vergroten van het inzicht in hoe transnationale elektriciteitsbedrijven internationale normatieve DEV-standaarden benaderen en implementeren - en door lessen te trekken die normatieve en regelgevende kaders kunnen verbeteren - richt dit proefschrift zich op het verbeteren van de kwaliteit en de bijdrage aan duurzame ontwikkeling van de elektriciteitsvoorziening in het Zuiden.

De beschreven context en het geformuleerde doel vormen het uitgangspunt voor de centrale problematiek zoals onderzocht in dit proefschrift: hoe conceptualiseren, operationaliseren en implementeren individuele transnationale elektriciteitsbedrijven de 
standaarden voor DEV? Met andere woorden, hoe worden 'kwaliteits kilowatt' opgezet en uitgevoerd? Dit vraagt om een toegepaste wetenschappelijke benadering die strategisch onderzoek mogelijk maakt. Hiermee wordt kennis gegenereerd die voor positieve veranderingen kan zorgen bij de instellingen die de standaarden ontwikkelen om duurzame en rechtvaardige energiesystemen te promoten.

Het proefschrift zocht antwoord op vijf centrale onderzoeksvragen:

1. Zijn er standaarden voor duurzame elektriciteitsvoorziening binnen het VN-raamwerk van duurzame ontwikkeling en andere internationale normatieve discoursen, die gesystematiseerd kunnen worden in een gemeenschappelijke set van benchmarks om de prestaties van TO's, die betrokken zijn bij de elektriciteitsvoorziening in het Zuiden, te evalueren?

2. Uitgaande van de verwachting dat de bedrijfscultuur van het land waar een TO vandaan komt, het gedrag van het bedrijf sterk beïnvloedt - en aangenomen dat verschillen in zulke bedrijfscultuurmodellen van het 'thuisland' steeds belangrijker worden in een tijdperk van groeiende globalisering en concurrentie van elektriciteitsvoorzieningen - hoe worden DEV-standaarden in het algemeen opgepakt, geïnterpreteerd en beoordeeld binnen die verschillende nationale modellen? Kunnen deze verschillende bedrijfscultuurmodellen gebruikt worden om de variatie waarin TO's deze DEV-standaarden oppakken, te structureren en analyseren?
3. Hoe presteren de geselecteerde TO's - in beleid én in de praktijk - ten opzichte van normatieve DEV-benchmarks en ten opzichte van elkaar? Daarbij gebruikmakend van de toegepaste wetenschap en een normatiefempirische benadering voor de systematische informatieverzameling over de activiteiten van elektriciteits TO's die actief zijn in het Zuiden.

4. Draagt een empirische analyse van de prestaties van elektriciteits TO's uit verschillende thuislanden en met verschillende bedrijfscultuurmodellen op de DEV-benchmark bij tot een theoretisch begrip van internationale bedrijfsstrategieën?

5. Welke algemene lessen kunnen getrokken worden uit een vergelijkende analyse van de prestaties van TO's op de DEV-benchmark, die van direct praktisch belang zijn voor het verbeteren van de effectiviteit van normatieve kaders, vooral voor de toename van de aanpak en implementatie van DEVstandaarden door TO's en het creëren van een grotere 'level playing field' voor duurzame ontwikkeling?

Het onderzoek is uitgevoerd volgens een analytische benadering op basis van normatief-empirische analyses. Deze aanpak omvatte het samenstellen van een reeks benchmarks die verankerd zijn in internationaal overeengekomen normatieve normen voor DEV, ook wel de DEV-benchmarks genoemd. De empirische methodologie is opgebouwd rondom kwalitatieve interviews met managers uit het bedrijfsleven en een breed scala aan stakeholders die een belang 
hebben bij de werkzaamheden van de elektriciteitsvoorziening. Op basis daarvan is in dit onderzoek vastgelegd en geëvalueerd hoe kwaliteits kilowatt worden opgezet en uitgevoerd en wat hun impact is op de stakeholders. Dit is onderzocht aan de hand van vijf casestudies van TO's afkomstig uit verschillende nationale en/of regionale bedrijfscultuurmodellen: AES (VS model), Endesa (Europees model), SN Power (Nordic model), Datang (Chinees model), en Eskom (Zuid-Afrikaanse model). De toevoeging van de Chinese en Zuid-Afrikaanse TO's/modellen in de studie wordt gezien als een unieke en bijzonder relevante bijdrage omdat de invloed van deze snel groeiende economieën in de mondiale politieke en economische systemen snelt toeneemt.

De inductieve empirische analyse is uniek omdat het een brede vergelijking maakt van bedrijfsactoren in elf specifieke, internationaal onderschreven DEV-benchmarks in zeer verschillende ontwikkelings-settingen.

De studie is dus een van de weinige analyses die - door systematische en wetenschappelijk onderbouwde analyse aantoont dat er een belangrijke, wereldwijd waarneembare discrepantie is tussen wat TO's (elektriciteitsbedrijven) in hun beleid beloven te doen, en dat wat ze daadwerkelijk doen in landen waar zij te gast zijn. Het falen van deze multinationals om hun beleid daadwerkelijk te 'vertalen' naar de praktijk is een enorme zwakte van internationale vrijwillige normatieve standaarden die verantwoord ondernemen voor duurzame ontwikkeling en DEV zouden moeten aanpakken.

De aanpak en bevindingen van de studie zijn relevant voor beleidsmakers en de ontwikkelaars van de standaarden, maar ook voor de verschillende stakeholdergroepen die betrokken zijn bij en beïnvloed worden door de patronen van de huidige nietduurzame elektriciteitsvoorziening. Op een gestructureerde en systematische manier heeft dit strategisch onderzoek (binnen de toegepaste wetenschap) kennis vergaard die gebruikt kan worden om duurzame en rechtvaardige energiesystemen te promoten. Sedlacko en Martinuzzi (2012:6) benadrukken dat deze evaluatieve methode in de huidige studie "het beleid doeltreffender maakt, helpt risico's te beheersen, transparantie ondersteunt, verantwoordelijkheid versterkt en het leren ondersteunt". De kennis die voortkomt uit dit soort actiegericht onderzoek is concreet en direct relevant voor meerdere doelgroepen. Door de urgentie om de steeds complexere en precaire mondiale problemen in verband met (niet)duurzame elektriciteitsvoorzieningen aan te pakken - bijvoorbeeld klimaatverandering, aanhoudende armoede, toenemende en structurele ongelijkheid in macht en levensstandaard en het gebrek in vooruitgang van ontwikkelingsdoelen, zoals de Millenniumdoelen - zette de studie in op een 'broad-brush, big-picture' benadering (Gupta en Hisschemöller 1997). Het onderzoek legde essentiële kwesties en nuances in een ongestructureerde discussie bloot en toont aan hoe moeilijk het is om dit complexe vraagstuk aan te pakken. Op die manier draagt het bij aan 
het 'problem finding' en 'problem-structuring' aspect van de toegepaste wetenschap. Volgens Hisschemöller en Gupta (1999:156) is dit aspect noodzakelijk voor de aanpak van het 'ongestructureerde probleem' dat DEV is op internationaal niveau.

De samenstelling en indeling van kritieke kwesties en de ontwikkeling van een raamwerk van normatieve DEV-standaarden in hoofdstuk 3 , zijn de belangrijkste praktische bijdragen van dit proefschrift aan het veld. Het legt de basis voor een betere normatieve begeleiding en mogelijk ook voor de ontwikkeling van een verbeterde gedragscode voor de elektriciteitssector. Het raamwerk is van direct belang voor normbepalende regimes op internationaal niveau én voor de taken van TO's om de standaarden te integreren en te operationaliseren. Dit is vooral van toepassing op de afwegingen die gemaakt worden tussen de economische, sociale en ecologische pijlers van duurzame ontwikkeling.

De studie draagt ook bij aan academische debatten over cross-culturele bedrijfsvoering en over verantwoord ondernemen en bedrijfsstrategieën in een wereld die steeds meer globaliseert. Het verkennende en inductieve karakter van het onderzoek was bedoeld om nieuwe inzichten te verwerven. Maar ook om een breed en empirisch onderbouwd referentiepunt te bieden voor toekomstige studies op dit terrein. Het uitgebreide onderzoek naar vijf casestudie bedrijven in zes verschillende gastlanden biedt een gedetailleerde momentopname voor vergelijking van de bedrijfsprestaties op het gebied van DEV. De casestudies bieden significant materiaal voor open reflectie en een kritische beoordeling door anderen. Ook voor degenen die misschien meer geïnteresseerd zijn in andere elementen van de algemene problematiek dan die die in het proefschrift worden aangehaald. De uitgebreide verzameling van empirische gegevens uit het veld levert een belangrijke bijdrage aan de robuustheid van de analyse. Wat weer belangrijke gevolgen heeft voor het huidige kennisniveau én de formulering van toekomstige, uitgebreidere en op andere variabelen gecontroleerde onderzoeksprojecten. 
\begin{tabular}{l|l} 
Quality & 343 \\
Kilowatts & 343
\end{tabular} 
Refrences

\section{References}


Aarhus, I. (2010), 'Norway'. In The World Guide to CSR: A Country-byCountry Analysis of Corporate Sustainability and Responsibility. W. Visser and N. Tolhurst (Eds.). Sheffield, UK, Greenleaf. AccountAbility (2008), AA1000 AccountAbility Principles Standard 2008. Available at http://www.accountability21.net/default. aspx?id=3074. Accessed 13 Jan 2009.

AEDAS website (2010), 'Q \& A with Richard Welford'. Available at http://www.aedasinthecommunity.com/index.php/q-a-a/ richard-welford. Accessed 20 August 2010.

AES (2006), AES 2006 Fact Book. AES Corporation, Arlington, VA. AES (2007a), 2006 Annual Report. AES Corporation, Arlington, VA. AES (2007b), The AES Values: From Words to Action, AES Code of Conduct. AES, Washington, D.C. Available at http://www.aes. com/pub-sites/sites/AES/content/live/0201391d47b2b011 3038d998a0069dd/1033/AES\%20Code\%20of\%20Conduct.pdf. Accessed 7 September 2008.

AES (2007c), SEC filing, Form 10-K. Filed 23 May 2007.

AES website (2008a), 'Alternative Energy'. Available at http://aes. com/aes/index?page=alternative_energy. Accessed 12 Nov 2008

AES (2008b), Corporate Governance Guidelines of The AES Corporation. Available at http://aes.com/aes/index?page=corporate_ governance. Accessed 6 Nov 2008.

AES website (2008c), 'Global Presence'. Available at http://aes.com/ aes/index?page=global_presence. Accessed 24 October 2008.

AES (2009), 'AES responds to Cultural Survival statement 'Crisis in Panama", Business and Human Rights Resource Centre, Available at http://www.business-humanrights.org/Links / Repository/887061. Accessed 18 April 2012.

AES (2010), CDP 2010 Information Request: AES Corporation. Available at https://www.cdproject.net/Sites/2010/04/304/Investor\%20CDP\%202010/Pages/DisclosureView.aspx. Accessed 8 April 2012.

AES (2011a), 2010 Annual Report AES, Alrington, VA. Available at http://investor.aes.com/phoenix.zhtml?c=76149\&p=irolreportsAnnual. Accessed 4 March 2012.

AES (2011b), Charter of the Nominating, Governance and Corporate
Responsibility Committee of the Board of Directors. AES, Arlington, VA. Available at http://www.aes.com/pub-sites/ sites/AES/content/live/02015810c0f66010f0b98295100 7b78/1033/NomGov\%20Committee\%20Charter\%200ctober\%20 2011.pdf. Accessed 6 April 2012.

AES (2011c), Fact Sheet November 2011. AES, Arlington, VA. Available at http://newsroom.aes.com/phoenix. zhtml?c=202639\&p=irol-factsheet. Accessed 28 January 2012. AES (2012a), 2011 Annual Report AES, Alrington, VA. Available at http://investor.aes.com/phoenix.zhtml?c=76149\&p=irolreportsAnnual. Accessed 4 March 2012.

AES website (2012b), 'About Us: Board Committees'. Available at http://aes.com/aes/index?page=board_commitees. Accessed 5 April 2012.

AES website (2012c), 'About Us: Ethics and Compliance'. Available at http://aes.com/aes/index?page=board_commitees. Accessed 4 April 2012.

AES website (2012d), 'Alternative Energy'. Available at http://aes. com/aes/index?page=alternative_energy. Accessed 5 April 2012.

AES website (2012e), 'Corporate Responsibility'. Available at http:// www.aes.com/aes/index?page=corporate_responsibility. Accessed 4 April 2012.

AES website (2012f), 'Environment'. Available at http://aes.com/ aes/index?page=environment. Accessed 5 April 2012.

AES Argentina (2010), Acerca de nosotros. AES Argentina, Buenos Aires. Available at http://www.aesargentina.com.ar/acerca. htm. Accessed 28 January 2012.

African Press Agency website (2007), 'Cameroon: Residents of Douala protest against pollution'. Available at http://www. apanews.net/apa.php?page=show_article_eng\&id_article=39092. Accessed 14 March 2008.

Akante, I., Board of Directors, Energie Du Mali (2012), personal communication with Y. Tchamba, Bamako, 11 March 2012.

Alarcón et al. (1999), F. Alarcón et al. vs. Central Dock Sud SA et al., lawsuit seeking damages and cessation of pollution and environmental harm. Available at http://www.jose-esain. 
com.ar/index.php?option=com_content\&task=view\&id=105\& temid=2. Accessed 1 June 2011.

Alfonso, F. B., Herrera, M. E. B. and Roman, F. L. (2010), 'Asia'. In The World Guide to CSR: A Country-by-Country Analysis of Corporate Sustainability and Responsibility. W. Visser and N. Tolhurst (Eds.). Sheffield, UK, Greenleaf.

Alimena, A., Deputy Ombudsman for the City of Buenos Aires (2008), personal communication with B. Esteves (FARN), Buenos Aires, 14 August 2008.

Alkin, M. C., Vo, A. T. and Christie, C. A. (2012), 'The evaluator's role in valuing: Who and with whom', New Directions for Evaluation 2012(133): 29-41.

Among, B. (2007), 'Powerless in Uganda', The East African, 10 Dec 2007. Available at http://www.ocnus.net/artman2/publish/ Africa_8/Powerless_in_Uganda.shtml. Accessed 11 Sept 2008 Anonymous manager in Argentina 1, (2008), personal communication with Belén Esteves FARN, Buenos Aires, 5 September 2008

Anonymous manager in Cambodia 1, (2010), personal communication with Prof. Dr. Qin Hui, Phnom Penh, Cambodia, 31 January 2010.

Anonymous manager in Lao PDR 1, (2009), personal communication with Prof. Dr. Qin Hui, Vientiane, Laos, 1 September 2009.

Anonymous manager in Lao PDR 2, (2009), personal communication with Prof. Dr. Qin Hui, Vientiane, Laos, 8 September 2009.

Anonymous manager in Lao PDR 3, (2009), personal communication with Prof. Dr. Qin Hui, Vientiane, Laos, 5 September 2009.

Anonymous manager in Lao PDR 4, (2009), personal communication with Prof. Dr. Qin Hui, Vientiane, Laos, 2 September 2009.

Anonymous municipal official, (2008), personal communication with T. Castellanos (PLADES), La Oroya, Junín, Peru, 14 August 2008. Anonymous representative of the Chinese Chamber of Commerce in Cambodia, (2010), personal communication with Prof. Dr. Qin Hui, Phnom Penh, Cambodia, 28 January 2010.

APEC (2005), Corporate Social Responsibility in the APEC Region: Current Status and Implications. Asia-Pacific Economic Cooperation Secretariat, Singapore. Available at http://www.
apec.org/apec/publications.MedialibDownload.v1.html?url=/ etc/medialib/apec_media_library/downloads/workinggroups/hrdwg/pubs/2006.Par.0006.File.v1.1. Accessed 10 August 2010.

Aragón-Correa, J. A. (1998), 'Strategic Proactivity and Firm Approach to the Natural Environment', The Academy of Management Journal 41(5): 556-567.

Argentine Ministry of Energy (2010), Cálculo del Factor de Emisiones de CO2 de la Red Argentina de Energía Eléctrica. Secretaría de Energía, Buenos Aires. Available at http://www.energia. gov.ar/contenidos/verpagina.php?idpagina=2311. Accessed 1 June 2011.

Argentine Ministry of Environment (2001), Resolution 108/01. Secretaría de Medio Ambiente, Buenos Aires.

Arias, M. and Guillen, M. (1998), 'The transfer of organizational management techniques'. In The Diffusion and Consumption of Business Knowledge. J. Alvarez (Ed.). London, Macmillan: 110-137.

Aupperle, K. E., Carroll, A. B. and Hatfield, J. D. (1985), 'An Empirical Examination of the Relationship between Corporate Social Responsibility and Profitability', The Academy of Management Journal 28(2): 446-463.

Autse, D. (1976), 'Increasing the operating efficiency of hydroelectric plants in mountainous regions', Power Technology and Engineering (formerly Hydrotechnical Construction) 10(6): 581-587.

Baggini, A., Ed. (2008), Handbook of Power Quality. Chichester, England, John Wiley \& Sons.

Bambawale, M. J., D'Agostino, A. L. and Sovacool, B. K. (2011), 'Realizing rural electrification in Southeast Asia: Lessons from Laos', Energy for Sustainable Development 15(1): 41-48.

Barron, A. (2010), 'Exploring national culture's consequences on international business lobbying', Journal of World Business 46(3): 320-327.

Baskin, J. (2005), Corporate Responsibility Practices of Emerging Market Companies - A Fact Finding Study. OECD, Paris. Available at http://www.oecd.org/dataoecd/29/38/35666512.pdf. Accessed 18 April 2012. 
Baxter, P. and Jack, S. (2008), 'Qualitative Case Study Methodology: Study Design and Implementation for Novice Researchers', The Qualitative Report 13(4): 544-559.

Bayliss, K. (2002), Privatisation and Poverty: The Distributional Impact of Utility Privatisation. Centre on Regulation and Competition, Available at http://www.competition-regulation. org.uk/publications/working_papers/wp16.pdf. Accessed 6 Nov 2008.

Bemelmans-Videc, M. L., Rist, R. C. and Vedung, E. O., Eds. (2003), Carrots, Sticks, and Sermons: Policy Instruments and Their Evaluation (Comparative Policy Analysis Series). Piscataway، New Jersey, Transaction Publishers.

Binyina, B. D., Legal Officer, Africa Institute for Energy Governance (AFIEGO) (2011), personal communication with Dr. V. Madziakapita, Kampala, Uganda, 6 October 2011.

Blacksmith Institute website (2012), 'World's most polluted places'. Available at http://www.blacksmithinstitute.org/.

Bosshard, P. (2011), 'Will China Find a New Balance Between the Environment and Economic Growth?', China US Focus, Available at http://chinausfocus.com/slider/will-china-find-a-newbalance-between-the-environment-and-economic-growth/. Accessed 5 October 2011.

Botha, Z. (2003), 'Eskom Enterprises review seeks realignment with parent ', Engineering News, Available at http://www.engineeringnews.co.za/article/eskom-enterprises-review-seeksrealignment-with-parent-2003-07-14. Accessed 1 May 2012.

Boyle, A. and Chinkin, C. (2007), The Making of International Law. Oxford: Oxford University Press.

Brewer, N. (2008), The New Great Walls: A Guide to China's Overseas Dam Industry. International Rivers, Berkley, CA. Available at http://www.internationalrivers.org/files/New\%20Great\%20 Walls\%20report.pdf. Accessed 15 April 2012.

Bruera, P., Secco, M., Slezack, E., Carballo, G., García, C. and Equiza, H. (2008), Gravísimas denuncias contra Edelap en el ENRE. Agencia Comunas website, Available at http://www.agenciacomunas.com.ar/nota.asp?notaid=40410. Accessed 27 January 2012.
BSI (2007), BS OHSAS 18001 Occupational Health and Safety. British Standards Institution, London. Available at http:/ / www. bsigroup.com/en/Assessment-and-certification-services/ management-systems/Standards-and-Schemes/BSOHSAS-18001/. Accessed 29 April 2012.

Burke, P. J. (2010), 'Income, resources, and electricity mix', Energy Economics 32(3): 616-626.

Burton, B. K., Farh, J.-L. and Hegarty, W. H. (2000), 'A Cross-Cultural Comparison of Corporate Social Responsibility Orientation: Hong Kong vs. United States Students', Teaching Business Ethics 4(2): 151-167.

Business News America website (2009), 'Endesa to slash Latin American investments on market conditions'. Available at http://www.bnamericas.com/news/electricpower/Endesa_ to_slash_Latin_American_investments_on_market_conditions. Accessed 30 May 2011.

Business Wire website (2001), 'Eskom Manages Three Country Hydro Power Co-Operation, in an Advisory by Industrialinfo.com'. Available at http://findarticles.com/p/articles/mi_m0EIN/ is_2001_Nov_9/ai_79889067/. Accessed 13 March 2012.

Cahua employee 1, (2008), personal communication with T. Castellanos (PLADES), Manás, Peru, 27 August 2008.

Cambodian laborer 1, (2010), personal communication with Prof. Dr. Qin Hui, Cambodia, 26 January 2010.

Cambodian villager 1, (2010), personal communication with Prof. Dr. Qin Hui, Cambodia, 29 January 2010.

Campbell, D. (1969), 'Reforms as experiments', American Psychologist 24: 409-429.

Carapinha, R. and de Jongh, D. (2008), 'The Meaning and Nature of Corporate Citizenship in South Africa'. In Corporate Citizenship Around the World: How local flavor seasons the global practice Boston, MA, Global Education Research Network and Boston College Center for Corporate Citizenship: 58-79. Available at http://www.ccedeutschland.org/pics/medien/1_1224173416/GERN_for_web.pdf. Accessed 11 August 2010.

Carlo, L., Chairman and Non Executive Director of Eskom Uganda 
(representing Eskom Enterprises) (2011), personal communication with Dr. V. Madziakapita, Johannesburg, South Africa, 24 October 2011.

CARMA website (2007), 'CARMA: Carbon Monitoring for Action'. Available at www.carma.org. Accessed 17 Oct 2008.

Carney, T. (2009), 'AES and GE imitate Enron on coal and climate', Washington Examiner online, 26 May 2009. Available at http://www.washingtonexaminer.com/politics/AES-andGE-imitate-Enron-on-coal-and-climate-46120417.html. Accessed 26 May 2009

Carnie, T. (2010a), 'Eskom in new "sweetheart" deal', IOL News, 8 April 2010. Available at http://www.iol.co.za/news/south-africa/ eskom-in-new-sweetheart-deal-1.479887. Accessed 9 April 2012

Carnie, T. (2010b), 'Sour taste over Eskom's "sweetheart" deals', IOL News, 18 March 2010. Available at http://www.iol.co.za/ news/south-africa/sour-taste-over-eskom-s-sweetheartdeals-1.476767. Accessed 8 April 2012

Carroll, A. B. (1979), 'A Three-Dimensional Conceptual Model of Corporate Performance', The Academy of Management Review 4(4): 497-505.

Caspary, G. (2008), 'Integrating responses to global environmental threats into policies and projects in developing countries', International Journal of Sustainable Development 11(1): 24-44.

CASS website (2003), 'Chinese Academy of Social Sciences China Corporate Social Responsibility Report Guide '. Available at http://bic.cass.cn/english/InfoShow/Arcitle_Show_Cass. asp?BigClassID=1\&Title=CASS. Accessed 11 April 2012.

CDP website (2011), 'What we do'. Available at https:/ / www.cdproject.net/en-US/WhatWeDo/Pages/overview.aspx. Accessed 2 October 2011.

CED (2001), Indicators of Sustainable Development: Guidelines and Methodologies. UN Department of Economic and Social Affairs, Commission on Sustainable Development, New York. Available at http://www.un.org/esa/sustdev/publications/ indisd-mg2001.pdf. Accessed 1 September 2010.

CEER (2005), Third Benchmarking Report on Quality of Electricity
Supply. Council of European Energy Regulators, Brussels. Available at http://www.autorita.energia.it/pubblicazioni/ volume_ceer3.pdf. Accessed 7 Sept 2008.

Central Dock Sud (2007), Informe de Sostenibilidad. Central Dock Sud, S.A. (Endesa), Buenos Aires. Available at http://www. cdssa.com.ar/politica_sostenibilidad.asp. Accessed 30 May 2011.

CEO (2008), Watering down the EU's Climate Policies: A multipronged corporate attack. Corporate Europe Observatory, Amsterdam. Available at http://www.corporateeurope.org/ docs/climatelobby2008.pdf. Accessed 6 Dec 2008.

Chan, L.-H., Lee, P. K. and Chan, G. (2008), 'Rethinking global governance: a China model in the making?', Contemporary Politics 14(1): 3-19.

Chang, S. (2010), Corporate tools and practices for managing supply chain risks: A perspective from China. Presentation to the 10th OECD Corporate Responsibility Roundtable, Paris. Available at http://www.oecd.org/dataoecd/47/48/45634058.pdf. Accessed 10 August 2010.

Chavez, D. (2012), 'Alternatives in the electricity sector in Latin America'. In Alternatives to Privatization: Public Options for Essential Services in the Global South. D.A. McDonald and G. Ruiters (Eds.). London, Routledge - HSRC - LeftWord Books: 453-487.

Chen, C. C. (1995), 'New Trends in Rewards Allocation Preferences: A Sino-U.S. Comparison', The Academy of Management Journal 38(2): 408-428.

Cherni, J. A., Dyner, I., Henao, F., Jaramillo, P., Smith, R. and Font, R. O. (2007), 'Energy supply for sustainable rural livelihoods. A multi-criteria decision-support system', Energy Policy 35(3): 1493-1504.

Chinese migrant laborers in Cambodia, (2010), personal communication with Prof. Dr. Qin Hui, Cambodia, 30 January 2010.

Christie, R. (1984), Electricity, Industry, and Class in South Africa. New York: State University of New York Press.

Christmann, P. (2000), 'Effects of "Best Practices" of Environmental Management on Cost Advantage: The Role of Complementary 
Assets', The Academy of Management Journal 43(4): 663-680.

Cissoko, F., Assistant Legal Director, OMVS-SOGEM (2012), personal communication with Y. Tchamba, Bamako, 6 March 2012.

Ciulla, J. B. (1991), 'Why is business talking about ethics: Reflections on foreign conversations', California Management Review 1991(Fall): 67-86.

Clarck, A. (2005), Resource-based Technology Innovation in South Africa: Innovations in South Africa's Off-grid. Johannesburg: HSRC Press.

COES-SINAC (2007), Estadística de operaciones 2006. Comité de Operación Económica del Sistema Interconectado Nacional, Lima, Peru.

Cordano, M. and Irene Hanson, F. (2000), 'Pollution Reduction Preferences of U.S. Environmental Managers: Applying Ajzen's Theory of Planned Behavior', The Academy of Management Journal 43(4): 627-641.

Coulibaly, O., Managing Director, Eskom Energie Mali (2012), personal communication with Y. Tchamba, Bamako, 5 March 2012.

Cramer Commission (2007), Testing framework for sustainable biomass: Final report from the project group "Sustainable production of biomass". Energy Transition's Interdepartmental Programme Management (IPM), Available at http:// www. swnternovem.nl/mmfiles/Testing\%20framework\%20for\%20 sustainable\%20biomass_tcm24-232796.pdf. Accessed 18 Nov 2008.

Creamer, T. (2011), 'AfDB loan concludes 100-MW Eskom wind farm funding phase', Engineering News, Available at http:// www.engineeringnews.co.za/article/afdb-loan-concludes100-mw-eskom-wind-farm-funding-phase-2011-09-26. Accessed 2 May 2012.

CSC9000 website (2010), 'CSC9000T Management System'. Available at http://www.csc9000.org.cn/en/CSC9000T.asp. Accessed 10 August 2010.

Dabor, B., Mananthali community leader (2012), personal communication with Y. Tchamba, Mananthali, 8 March 2012.

Datang (2008), 2007 Social Responsibility Report - English version. China Datang Corporation, Beijing. Available at http://www. china-cdt.com/uploadfile/media/2007socialresponsibility-e. pdf. Accessed 5 April 2012.

Datang (2009a), 2008 Social Responsibility Report. China Datang Corporation, Beijing. Available at http://www.china-cdt.com/ uploadfile/media/2008socialresponsibility.pdf. Accessed 5 April 2012.

Datang (2009b), 2008 Social Responsibility Report - English version. China Datang Corporation, Beijing. Available at http://www. china-cdt.com/uploadfile/media/2008socialresponsibility-e. pdf. Accessed 5 April 2012.

Datang (2010), 2009 Social Responsibility Report. Datang International Power Generation Co., Beijing. Available at http://www. dtpower.com/upload/news/2010-05-2409-54-01ppic0.pdf. Accessed 15 August 2010.

Datang (2011a), 2010 Social Responsibility Report. China Datang Corporation, Beijing. Available at http://www.china-cdt.com/ uploadfile/media/2010socialresponsibility.pdf. Accessed 5 April 2012.

Datang website (2011b), 'About China Datang Corporation'. Available at http://www.china-cdt.com/en/ABOUTCDT/BRIEFINTRODUCTION/46465.html. Accessed 8 August 2011.

Datang website (2011c), 'CDT releases CSR Report 2010'. Available at http://www.china-cdt.com/en/news/19738612.html. Accessed 8 August 2011.

Datang website (2012), 'China Datang Overseas Investment Co. Projects'. Available at http://www.china-cdto.com//indexAction. ndo?action=showType\&spage=1\&typeid $=8$ ad1 $1850 \mathrm{~d} 2$ bfa06050 12c129342900009\&category=en. Accessed 8 April 2012.

Daugherty, M. and Georgieva, D. (2011), 'Foreign cultures, SarbanesOxley Act and cross-delistingForeign cultures, Sarbanes-0xley Act and cross-delisting', Journal of Multinational Financial Management 21(4): 208-223.

Dean, T. J. and Brown, R. L. (1995), 'Pollution Regulation as a Barrier to New Firm Entry: Initial Evidence and Implications for Future Research', The Academy of Management Journal 38(1): 288-303.

Debesa, F. (2006), 'Le piden al Gobierno nacional que baje el contrato 
con Edelap', Clarín, Available at http://www.clarin.com/ diario/2008/03/26/laciudad/h-03801.htm. Accessed 22 April 2009.

Debroux, P. (2009), 'CSR and Sustainable Development in Asia'. In Asian Business \& Management: Theory, Practice and Perspectives. H. Hasegawa and C. Noronha (Eds.), Palgrave Macmillan: 25-44.

Delmas, M. (2003), In Search of Iso: An Institutional Perspective on the Adoption of International Management Standards. UC Santa Barbara: Institute for Social, Behavioral, and Economic Research, Available at http://escholarship.org/uc/ item/9905b1nv Accessed 12 February 2010.

Delmas, M. and Toffel, M. W. (2004), 'Stakeholders and environmental management practices: An institutional framework', Business Strategy and the Environment 13(4): 209-222.

Delucchi, M. A. and Jacobson, M. Z. (2011), 'Providing all global energy with wind, water, and solar power, Part II: Reliability, system and transmission costs, and policies', Energy Policy 39(2011): 1170-1190.

Dembele, M., Mayor, Manantali District, Mali (2012), personal communication with Y. Tchamba, Manantali, 8 March 2012.

Descalzi, C., Economic Promotion and Social Development division of the Municipality of Pacasmayo (2008), personal communication with T. Castellanos (PLADES), Pacasmayo, Peru, 5 August 2008.

Diallo, T., Manantali community leader (2012), personal communication with Y. Tchamba, Manantali, 8 March 2012.

Dieme, A., Control, Risk and Compliance Director, OMVS-SOGEM (2012), personal communication with Y. Tchamba, Bamako, 6 March 2012.

Dikgale, A., Managing Director, Eskom Manantali (2012), personal communication with Y. Tchamba, Manantali, 10 March 2012.

DiMaggio, P. J. and Powell, W. W. (1983), 'The Iron Cage Revisited: Institutional Isomorphism and Collective Rationality in Organizational Fields', American Sociological Review 48(2): 147-160.

Dimitratos, P., Petrou, A., Plakoyiannaki, E. and Johnson, J. E. (2010), 'Strategic decision-making processes in internationalization:
Does national culture of the focal firm matter?', Journal of World Business 46(2): 194-204.

Dugard, J. (2009), Power to the People? A rights based analysis of South Africa's electricity service. Centre for Applied Legal Studies at Wits University, Johannesburg.

Dunning, J. H. and Narula, R. (2004), Multinationals and industrial competitiveness: A new agenda. Cheltenham: Edward Elgar.

Easson, A. (2001), Tax incentives for foreign direct investment part II: Design considerations', Bulletin for International Fiscal Documentation 55(8): 365-375.

Eawag (2008)، Annual Report 2007. Swiss Federal Institute of Aquatic Science and Technology, Dübendorf, Switzerland. Available at http:// www.eawag.ch/services/publikationen/ jahresbericht/eawag-jb_07e.pdf. Accessed 10 May 2009.

Eberhard, A. (2007), 'The political economy of power sector reform in South Africa'. In Political Economy of Power Sector Reform: The Experiences of Five Major Developing Countries. T.C. Heller and D.G. Victor (Eds.). Cambridge, Cambridge University Press. Available at http://www.gsb.uct.ac.za/files/StanfordCUPBookChapterp215-253_6.pdf. Accessed 2 May 2012.

EC (2001a), Consultation Paper for the Preparation of a European Union Strategy for Sustainable Development: SEC (2001) 517 27. European Commission, Brussels. Available at http://www. eeac-net.org/workgroups/pdf/010424EEACresponsetoSDS pdf. Accessed 1 September 2010.

EC (2001b), Corporate Social Responsibility Green Paper, Promoting a European Framework for Corporate Social Responsibility (COM (2001)366 final of 18.7.2001). European Commission, Brussels. Available at http://eur-lex.europa.eu/LexUriServ/ LexUriServ.do?uri=COM:2001:0366:FIN:EN:PDF. Accessed 5 September 2008.

EC (2002)، A business contribution to sustainable development (COM (2002)347 final of 2.7.2002). European Commission, Brussels. Available at http://eur-lex.europa.eu/LexUriServ/LexUriServ. do?uri=COM:2002:0347:FIN:EN:PDF. Accessed 5 September 2008.

EC (2006), Implementing the Partnership for Growth and Jobs: Mak- 
ing Europe a pole of excellence on CSR (COM(2006)136 final). European Commission, Brussels. Available at http://eur-lex. europa.eu/LexUriServ/LexUriServ.do?uri=COM:2006:0136:FIN: EN:PDF. Accessed 5 September 2008.

EC (2011), A renewed EU strategy 2011-14 for Corporate Social Responsibility. European Commission, Brussels. Available at http://ec.europa.eu/enterprise/newsroom/cf/_getdocument.cfm?doc_id=7010. Accessed 14 December 2011.

ECOTEC (2007), Describing developments in the European electricity sector: Drivers for change: Corporate Social Responsibility - a report to the sectoral social partners. Eurelectric, EPSU and EMCEF, Birmingham. Available at http://www.epsu.org/IMG/ pdf/EN_CSR_European_electricity_sector_FINAL_REPORT. pdf. Accessed 19 May 2008.

Edelap website (no date), 'Nuestra Empresa: Renegociación del contrato de concesión'. Available at http://www.Edelap.com. ar/empresa_contrato.htm. Accessed 19 April 2012.

EDESUR (2011), Informe de Sostenibilidad 2010. EDESUR, Buenos Aires. Available at http://www.edesur.com.ar/documentos/ responsabilidad10.pdf. Accessed 12 February 2012.

Edesur website (2012), 'Servicios al Cliente'. Available at http:// www.edesur.com.ar/servicios_cliente/fr_servicios_cliente. htm.

Egri, C. P. and Herman, S. (2000), 'Leadership in the North American Environmental Sector: Values, Leadership Styles, and Contexts of Environmental Leaders and Their Organizations', The Academy of Management Journal 43(4): 571-604.

El Día website (2005), 'Un golpe de tensión arruinó los equipos de Radio Universidad'. Available at http://www.eldia.com.ar/ catalog01/20051208/laciudad0.asp.

El Sindical website (2010), 'El gremio de Luz y Fuerza se movilizó a Edesur'. Available at http://www.elsindical.com.ar/notas/ el-gremio-de-luz-y-fuerza-se-movilizo-a-edesur/. Accessed 18 April 2012.

ElectroAndes (2008), Memoria Anual 2007. SN Power Peru, Lima. Available at http://www.snpower.com.pe/. Accessed 20 April 2012
ElectroAndes employee 1, (2008), personal communication with T. Castellanos (PLADES), La Oroya, Junín, Peru, 15 August 2008. ElectroAndes employee 2, (2008), personal communication with T. Castellanos (PLADES), La Oroya, Junín, Peru, 15 August 2008. Endesa (2007a), Endesa Annual Report 2006. Endesa, Madrid. Available at http://www.endesa.es/Portal/en/investors/annual_ reports/annual_report/default.htm. Accessed 20 Sept 2008.

Endesa (2007b), Endesa Sustainability Report 2006. Available at http://www.endesa.com/Portal/en/our_commitment/sustainability_2/Informes_publicaciones/default.htm. Accessed 7 Sept 2008.

Endesa (2008a), Endesa Sustainability Report 2007. Endesa, Madrid. Available at http://www.endesa.es/Portal/en/our_commitment/sustainability_3/8_reports_publications/default.htm. Accessed 20 September 2008.

Endesa website (2008b), 'Sustainability Policy'. Available at http:// www.endesa.es/Portal/en/our_commitment/sustainability_3/1_sustainability_policy/default.htm. Accessed 7 Sept 2008.

Endesa (2011a), CDP 2011 CDP Supply Chain 2011 Information Request. Endesa, Madrid. Available at https://www.cdproject. net/Sites/2011/84/5584/CDP\%20Supply\%20Chain\%202011/ Pages/DisclosureView.aspx. Accessed 18 April 2012.

Endesa (2011b), CDP 2011 Investor CDP 2011 Information Request. Endesa, Madrid. Available at https://www.cdproject.net/ Sites/2011/84/5584/Investor\%20CDP\%202011/Pages/DisclosureView.aspx. Accessed 18 April 2012.

Endesa website (2011c), 'Conoce Endesa - Argentina'. Available at http://www.endesa.com/es/conoceendesa/nuestraestrategia/EndesaenelMundo/Latinoamerica/Paginas/Argentina. aspx. Accessed 7 February 2012.

Endesa (2011d), Informe de Sostenibilidad 2010. Endesa, Madrid, Spain. Available at http://www.endesa.com/es/saladeprensa/centrodocumental/Paginas/home.aspx. Accessed 10 February 2012.

Endesa website (2011e), 'Key figures Endesa'. Available at http:// www.endesa.com/en/aboutEndesa/Paginas/keyFfigures. 
aspx. Accessed 4 March 2012.

Endesa website (2011f), 'Our Commitment'. Available at http:// www.endesa.com/en/nuestrocompromiso/Paginas/home. aspx. Accessed 3 April 2012.

Endesa Costanera (2010a), Informe de Sostenibilidad 2009. Endesa Costanera, Buenos Aires. Available at http:// www.endesacostanera.com/sitio/prensaDetalle. asp?id=2011126121944\&tipo=1. Accessed 12 February 2012.

Endesa Costanera website (2010b), 'Nuestro Compromiso'. Available at http://www.endesacostanera.com/sitio/contenido. asp?page=DimAmbiental. Accessed 30 May 2011.

Endesa Costanera (2011), Informe de Sostenibilidad 2010. Endesa Costanera, Buenos Aires. Available at http://www.principio10. org/soporte/76/Informe_de_Sostenibilidad_2010_Costanera. pdf. Accessed 7 February 2012.

Endesa Dock Sud (2006), Condiciones Generales de Contratación. Endesa, Buenos Aires. Available at http://www.cdssa.com.ar/ documentos/Condiciones_Generales_de_Contratacion_CDS. pdf. Accessed 13 February 2012.

Endesa Dock Sud (2007), Informe de Sostenibilidad. Central Dock Sud, S.A. (Endesa), Buenos Aires. Available at http://www. cdssa.com.ar/politica_sostenibilidad.asp. Accessed 30 May 2011.

Endesa Dock Sud (2009), Informe de Sostenibilidad 2008. Central Dock Sud, S.A. (Endesa), Buenos Aires. Available at http:// www.cdssa.com.ar/CDSSA_IDSE_2008.pdf. Accessed 30 May 2011.

Endesa Dock Sud website (2011), 'Corporate values (Valores corporativos)'. Available at http://www.cdssa.com.ar/dimension_ambiental.asp. Accessed 30 May 2011.

Endesa Edegel (2008), Memoria Anual 2007: Informe de Sostenibilidad. Edegel S.A.A. (Endesa), Lima, Peru. Available at http:// www.edegel.com/memoria2007/index.htm. Accessed 30 May 2011.

Endesa Edegel website (2010), 'Responsibilidad Social Empresarial'. Available at http://www.edegel.com/.

Endesa El Chocón (2010), Informe de Sostenibilidad 2009. Enedsa,
Buenos Aires. Available at http:// www.hidroelectricaelchocon.com/noticias/Uploads/20101022145412not.pdf. Accessed 13 February 2012.

Energie du Mali (2012), Tariff Rates as Determined by the Commission for the Regulation of Electricity and Water in Mali-CREE. Energie du Mali, Bamako.

Energy Forecast website (2011), 'Power blackouts'. Available at http://www.energyforecastonline.co.za/index.php/waterand-energy/199-power-blackouts. Accessed 8 May 2012.

Energy Research Centre (2004), Solar electrification by the concession. University of Cape Town, Cape Town.

ENRE (2008), Resoluciones, Central Dock Sud. Ente Nacional Regulador de Electricidad, Buenos Aires. Available at www.enre. gov.ar. Accessed 1 June 2011.

ENRE (2010), Informe Anual 2009. Ente Nacional Regulador de la Electricidad, Buenos Aires. Available at http://www.enre. gov.ar/web/web.nsf/Files/InformeAnual09.pdf/\$FILE/InformeAnual09.pdf. Accessed 12 February 2012.

ENRE (2011), Informe Anual 2010. Ente Nacional Regulador de la Electricidad, Buenos Aires. Available at http://www.enre.gov. ar/web/web.nsf/Files/InformeENRE2010.pdf/\$FILE/InformeENRE2010.pdf. Accessed 12 February 2012.

Enterprise Innovation website (2008), 'China tops CSR survey with HK and Taiwan scoring poorly'. Available at http://www. enterpriseinnovation.net/content/china-tops-csr-survey-hkand-taiwan-scoring-poorly. Accessed 10 August 2010.

EPEN (2011), EPEN informa a sus Clientes los valores del Cuadro Tarifario. Ente Provincial de Energía de Neuquén, Neuquén, Argentina. Available at http://www.epen.gov.ar/cuadro_tarifario/ct_11_2011.pdf. Accessed 18 April 2012.

EPSU, Eurelectric and EMCEF (2004), Corporate Social Responsibility and the European Electricity Sector. EPSU, Eurelectric and EMCEF, Brussels. Available at http://ec.europa.eu/employment_social/dsw/public/actRetrieveText.do;jsessionid=LW1T 1wsWBK18tnDp5KKTjs01QphlyqTXgnYYCz942rGtfBh2FGvT!2 63744025?id=10533. Accessed 5 September 2008.

Eriab, B. N., Deputy Chief Administration Officer, Jinja Municipality 
(2011), personal communication with Dr. V. Madziakapita, Jinja, Uganda, 5 October 2011.

Eskom (2001), Annual Report 2000. Eskom, Johannesburg.

Eskom (2002), Annual Report 2001. Eskom, Johannesburg.

Eskom (2004), Annual Report 2003. Eskom, Johannesburg.

Eskom (2006), Annual Report 2005. Eskom, Johannesburg.

Eskom (2007), Safety, Health, and Environment (SHE) Policy. Eskom, Johannesburg. Available at http://recruitment.eskom.co/ content/Appendix\%205 1.pdf. Accessed 8 April 2012.

Eskom (2009a), Annual Integrated Report 2008. Eskom, Johannesburg. Available at http://www.eskom.co.za/annreport08/. Accessed 2 April 2011.

Eskom (2009b), Implementation of Eskom's Black Economic Empowerment Strategy. Eskom, Johannesburg. Available at http://www.eskom.co.za/content/32-416\%20(0)\%20BEE\%20 Directive\%2015.05.08 1.pdf Accessed 1 May 2012.

Eskom (2010)، Annual Integrated Report 2009. Eskom, Johannesburg. Available at http://www.eskom.co.za/live/monster. php?URL=http \%3A\%2F\%2Fwww.eskom.co.za\%2Fannreport09\% 2F\&Src=Item+443. Accessed 2 May 2010.

Eskom (2011)، Annual Integrated Report 2010. Eskom, Johannesburg. Available at http://financialresults.co.za/2010/ eskom_ar2010/downloads/eskom_ar2010.pdf. Accessed 24 August 2010.

Eskom (2012a), Annual Integrated Report 2011. Eskom, Johannesburg. Available at http://financialresults.co.za/2011/ eskom_ar2011/. Accessed 12 April 2012.

Eskom website (2012b), 'Code of Conduct for Suppliers'. Available at http://www.eskom.co.za/c/article/638/code-of-conduct/. Accessed 8 April 2012.

Eskom website (2013a), 'Commerce \& Industry - Service offering'. Available at http://www.eskom.co.za/c/article/124/serviceoffering/. Accessed 1 January 2013.

Eskom website (2013b), 'Stakeholder Engagement'. Available at http://www.eskom.co.za/c/article/250/stakeholder-engagement/. Accessed 12 January 2013.

Eskom Uganda website (2008), 'Eskom Uganda'. Available at http:// www.eskom.co.ug/. Accessed 7 April 2012.

ESMAP (2005), Securing Energy for Poverty Reduction and Economic Growth: Business Plan 2005-2007. World Bank Energy Sector Management Assistance Programme, Washington, D.C. Available at http://www.esmap.org/filez/pubs/BP200507forWeboptimized.pdf. Accessed 16 March 2009.

FARN website (2011a), 'Caso: Cuenca Matanza-Riachuelo'. Available at http://www.farn.org.ar/participacion/riachuelo/index. html. Accessed 1 June 2011.

FARN (2011b), Informe Ambiental Anual 2011. Fundación Ambiente y Recursos Naturales, Buenos Aires. Available at http://www. farn.org.ar/informe2011.pdf. Accessed 18 April 2012.

Fernández, D., Subsecretary of Culture, Training and Scholarships, Sindicato de Luz y Fuerza de Capital Federal (2008), personal communication with B. Esteves (FARN), Buenos Aires, 15 August 2008.

FeTRA (2007), Ponencia de la Federación de Trabajadores de la Energía de la República Argentina. Buenos Aires. Available at http://www.pazysolidaridad.ccoo.es/imagenes/Informe\%20 ENDESA-FETERA-CTA-ARGENTINA.pdf. Accessed 6 February 2012.

Fig, D. (2002), 'Corporate social and environmental responsibility', South African Labour Bulletin 26, Available at http://www. nedlac.org.za/media/14614/social.pdf. Accessed 6 June 2009.

Fig, D. (2005), 'Manufacturing amnesia: Corporate Social Responsibility in South Africa', International Affairs 81(3): 599-617.

Fine, B. and Rustomjee, Z. (1996), The Political Economy of South Africa: From Minerals-Energy Complex to Industrialisation. Boulder, CO: Westview Press.

FoE UK website (2008), 'Carbon Dinosaurs'. Available at http://www. foe.co.uk/campaigns/climate/press_for_change/carbon_dinosaurs/aes.html. Accessed 14 Mar 2008.

FoEI (2008), 'Colombia Private Energy', Friends of the Earth International website, Available at http://www.foei.org/en/campaigns/finance/ffm/energypriv.html/?searchterm=endesa. Accessed 20 Sept 2008.

Fraile, Á., Sustainable Development Manager at Endesa (2008), per- 
sonal communication with J. Wilde-Ramsing, 28 May 2008.

Friedman, M. (1962), Capitalism and Freedom. Chicago: University of Chicago Press.

Friedman, S. (1987), Building Tomorrow Today: African Workers in Trade Unions, 1970-1984. Johannesburg: Raven Press.

Fthenakis, V. and Kim, H. C. (2008), 'Land use and electricity generation: A life-cycle analysis', Renewable and Sustainable Energy Reviews. 13(6-7): 1465-1474.

Galvin, P. (2009), AES Corporation Participating in the Demise of the Ngobe Tribe of Panama and La Amistad Biosphere Reserve. Center for Biological Diversity, Tuscon, AZ. Available at http:// www.biologicaldiversity.org/news/press_releases/2009/laamistad-04-23-2009.html. Accessed 6 April 2012.

Gasa, B. (2005), 'Department briefing: State-owned Enterprises' Role and Investments in Africa'. Presentation to the Public Enterprises Select Committee of the South African Parliament. 3 August 2005, Pretoria.

Gentle, L. (2009), 'Escom to Eskom: From racial Keynesian capitalism to neo-liberalism (1910-1994)'. In Electric Capitalism: Recolonising Africa on the power grid. D.A. McDonald (Ed.). London, Earthscan and HSRC Press. Available at http://www. hsrcpress.ac.za/product. php?productid=2243. Accessed 28 November 2011.

George, A. L. and Bennet, A. (2004), Case Studies and Theory Development in the Social Sciences. Cambridge, MA: Harvard University Press.

Global Compact website (2011), 'Non-communicating business participants'. Available at http://unglobalcompact.org/COP/ analyzing_progress/non_communicating.html.

Global Compact Critics website (2012), 'Global Compact Critics blog'. Available at http://globalcompactcritics.blogspot.com/. Accessed 24 April 2012.

Gomm, R., Hammersley, M. and Foster, P. (2000), 'Case Study and Generalization'. In Case Study Method: Key Issues, Key Texts. R. Gomm, M. Hammersley and P. Foster (Eds.). London, Sage. Gore, C. (2008), 'Electricity and privatisation in Uganda: The origins of the crisis and problems with the response'. In Electric Capital- ism: Recolonising Africa on the Power Grid. D.A. McDonald (Ed.). London, Earthscan and HSRC Press. Available at http:// www.hsrcpress.ac.za/product.php?productid=2243. Accessed 12 December 2011

Government of South Africa (1922), Statutes of the Union of South Africa, Act no. 42 of 1922. Act to provide for the supply and control of electricity and for other purposes incidental thereto. Government Printers, Cape Town.

Government of South Africa (1985), Statutes of the Republic of South Africa, Electricity Amendment Act, Act No. 50 of 1985. Government Printers, Pretoria.

Government of South Africa and De Villiers, W. J. (1984), Report of the Commission of Inquiry Into the Supply of Electricity in the Republic of South Africa. Government Printer, Pretoria.

Grant, L. (2007), Eskom comes second on world emissions list. Treevolution, Available at http://treevolution.co.za/2007/12/ eskom-comes-second-on-world-emissions-list/. Accessed 27 November 2011.

Graus, W., Voogt, M. and Langeraar, J. W. (2004), Ranking Power: Scorecards Electricity Companies. WWF and Ecofys, London. Available at http://www.worldwildlife.org/climate/Publications/WWFBinaryitem4919.pdf. Accessed 12 November 2009. Greenpeace (2009), Polluting Power: Ranking China's Power Companies. Greenpeace China, Beijing. Available at http:// www.greenpeace.org/raw/content/eastasia/press/reports/ power-ranking-report.pdf. Accessed 7 August 2011.

GRI (2008), Sustainability Reporting Guidelines \& Electric Utilities Sector Supplement: Pilot Version. Global Reporting Initiative, Amsterdam. Available at http://www.globalreporting.org/ ReportingFramework/SectorSupplements/ElectricUtilities/. Accessed 19 May 2008.

GRI website (2012), 'China Datang Corporation (CDT)'. Available at http://database.globalreporting.org/companies/view/1650. Accessed 15 April 2012.

Gunningham, N., Kagan, R. and Thornton, D. (2003), Shades of Green: Business, Regulation, and Environment. Stanford: Stanford University Press. 
Gupta, J. and Hisschemöller, M. (1997), 'Issue Linkages: A Global

Strategy Towards Sustainable Development', International Environmental Affairs 9(4): 298-308.

Ha, P.-H. and Porcaro, J. (2005), 'Energy and the Millennium Development Goals The Impact of Rural Energy Services on Development', Journal of International Affairs 58(2): 193-207.

Haar, L. N. and Jones, T. (2008), 'Misreading liberalisation and privatisation: The case of the US energy utilities in Europe', Energy Policy 36(7): 2610-2619.

Hague, R., Harrop, M. and Breslin, S. (1992), Comparative Government and Politics: An Introduction (3rd Edition). Houndsmill: Macmillan.

Halkier, B. (2011), 'Methodological Practicalities in Analytical Generalization', Qualitative Inquiry 17(9): 787-797.

Hall, D. (2005), Electricity privatisation and restructuring in Latin America and the impact on workers. Public Services International Research Unit, University of Greenwich, London. Available at www.psiru.org. Accessed 19 May 2008.

Hall, P. and Soskice, D. (2001), Varieties of Capitalism: The Institutional Foundations of Comparative Advantage. Oxford: OUP. Hallowes, D. and Munnik, V. (2007), Peak. Poison: The Elite Energy Crisis and Environmental Justice. groundWork, Pietermaritzburg.

Halls, A. S. and Kshatriya, M. (2009), Modelling the cumulative barrier and passage effects of mainstream hydropower dams on migratory fish populations in the Lower Mekong Basin: MRC Technical Paper No. 25. Mekong River Commission, Vientiane, Lao PDR. Available at http://www.mrcmekong.org/assets / Publications/technical/tech-No25-modelling-cumulativebarrier.pdf. Accessed 17 April 2012.

Hamann, R. (2009), 'South Africa: The Role of History, Government and Local Context'. In Global Practices of Corporate Social Responsibility. S.O. Idowu and W.L. Filho (Eds.). Berlin, Springer: 435-459.

Han, Cambodian legal practitioner in the Cambodian Congress (2010), personal communication with Prof. Dr. Qin Hui, Phnom Penh, Cambodia, 30 January 2010.
Hancock, T. (2011), 'Construction on solar power demonstration plant to start in 2013', Engineering News, Available at http:// www.engineeringnews.co.za/article/100-mw-solar-powerplant-for-northern-cape-2011-04-15. Accessed 2 May 2012 Hart, S. (2012), 'The Crash of Cougar Flight 491: A Case Study of Offshore Safety and Corporate Social Responsibility', Journal of Business Ethics: 1-23.

Hathaway, T. (2012), 'Electrifying Africa: Turning a continental challenge into a people's opportunity'. In Alternatives to Privatization: Public Options for Essential Services in the Global South. D.A. McDonald and G. Ruiters (Eds.). London, Routledge - HSRC - LeftWord Books: 353-387.

Heller, T. C., Tjiong, H. and Victor, D. G. (2003), Electricity Restructuring and the Social Contract: PSED Working Paper \#15. Stanford University, Stanford, CA. Available at http://iis-db. stanford.edu/pubs/20203/elect_restruct_social_contract. pdf. Accessed 19 May 2008.

Hidalgo, N. (2009), Tercerización y flexibilización laboral: Trabajadores contratados de EDESUR. Federación de Trabajadores de la Energía de la República Argentina (FeTERA-CTA), Buenos Aires. Available at http://www.izquierda.info/modules.php?n ame=News\&file=print\&sid=7962. Accessed 18 April 2012.

Hirschberg, S., Dones, R., Heck, T., Burgherr, P., Schenler, W. and Bauer, C. (2004), Sustainability of electricity supply technologies under German conditions: A comparative evaluation. Paul Scherrer Institut, Villingen. Available at http://manhaz. cyf.gov.pl/manhaz/Warsztaty_10_2004/wp4/psi_materials/ILK\%20-\%20PSI\%20Report\%2004-15.pdf. Accessed 1 September 2010

Hisschemöller, M. and Gupta, J. (1999), 'Problem-Solving through International Environmental Agreements: The Issue of Regime Effectiveness', International Political Science Review 20(2): $151-174$

Hoel, M. (2011), Norwegian and Nordic industrial policy: Corporate social responsibility, equality and innovation. Norwegian Ministry of Trade and Industry, Oslo. Available at http://www. eierberetningen.no/2010/index.php?seks_id=55809\&path= 
55807| \&element=Kapittel\&lang=english. Accessed 24 May 2011.

Hoffman, A. and Ventresca, M., Eds. (2002), Organizations, Policy and the Natural Environment: Institutional and Strategic Perspectives. Stanford, Stanford University Press.

Hofstede, G. (1983), 'The Cultural Relativity of Organizational Practices and Theories', Journal of International Business Studies 14(2): 75-89.

Hofstede, G. (2001), Culture's Consequences: Comparing Values, Behaviors, Institutions, and Organizations Across Nations, 2nd ed. London: Sage.

Hofstede, G., Neuijen, B., Ohayv, D. D. and Sanders, G. (1990), 'Measuring organizational cultures: a qualitative and quantitative study across twenty cases', Administrative Science Quarterly 35(2): 286-316.

Hohnen, P. (2009), Non-financial reporting: Denmark ups the ante. Ethical Corporation, Available at http://www.ethicalcorp.com/ content.asp?ContentID=6280 Accessed 13 Jan 2009.

Hollaender, K. and Stokman, F. (2012), 'Dynamic decision analysis for monitoring and facilitating the Dutch Costa Due stakeholder dialogue on sustainable energy'. In Governance by Evaluation for Sustainable Development: Institutional Capacities and Learning. M. Sedlacko and A. Martinuzzi (Eds.). Cheltenham, UK and Northampton, MA, USA, Edward Elgar: 151-172.

Holmes, R. and Watts, P. (2000), Corporate social responsibility: Making good business sense. World Business Council for Sustainable Development, Geneva. Available at http://www. wbcsd.org/DocRoot/lunSPdlKvmYH5HjbN4XC/csr2000.pdf. Accessed 8 September 2010.

Hoy website (2011), 'Protesta de empleados de Edelap'. Available at http://www.diariohoy.net/accion-verNota-id-163331-fecha-2011.10.28. Accessed 19 April 2012.

IBM (2008), The Global CEO Study: The Enterprise of the Future. Available at http://www.ibm.com/ibm/ideasfromibm/us/ ceo/20080505/. Accessed 10 August 2010.

ICEI (2005), X Population Census, Section V: Housing. Peruvian National Institute of Statistics and Information, Lima.
ICRC (2006), Business and International Humanitarian Law: an introduction to the rights and obligations of business enterprises under international humanitarian law. International Committee of the Red Cross, Geneva. Available at http:// www.icrc.org/Web/Eng/siteeng0.nsf/htmlall/p0882/\$File/ ICRC_002_0882.PDF!Open. Accessed 6 Dec 2008.

IEA (2009), CO2 Emissions from Fuel Combustion. OECD International Energy Agency, Paris. Available at http://www.iea.org/ co2highlights/co2highlights.pdf. Accessed 24 August 2010.

IEA (2010a), Energy Poverty: How to make modern energy access universal (Special excerpt of the World Energy Outlook 2010). OECD International Energy Agency, Paris. Available at http:// www.worldenergyoutlook.org/docs/we02010/we02010_poverty.pdf. Accessed 1 February 2011.

IEA website (2010b), 'Task 29'. Available at http://www.task29.net/. Accessed 1 September 2010.

IEA (2011), World Energy Outlook: Access to Electricity. International Energy Agency, Paris. Available at http://www.iea.org/weo/ electricity.asp. Accessed 12 December 2011.

IFC (2006), Performance Standards on Social and Environmental Sustainability. International Finance Corporation, Washington, DC. Available at http://www.ifc.org/ifcext/sustainability. nsf/AttachmentsByTitle/pol_PerformanceStandards2006_ full/\$FILE/IFC+Performance+Standards.pdf. Accessed 9 Sept 2008.

IFC (2007), Stakeholder Engagement: A Good Practice Handbook for Companies Doing Business in Emerging Markets. International Finance Corporation, Washington. Available at http:// www.ifc.org/ifcext/enviro.nsf/attachmentsbytitle/p_stakeholderengagement_full/\$file/ifc_stakeholderengagement. pdf. Accessed 4 October 2011.

IFC (2011), IFC Performance Standards - 2012 Edition. International Finance Corporation, World Bank Group, Washington, D.C. Available at http://www1.ifc.org/wps/wcm/connect/topics_ext_content/ifc_external_corporate_site/ifc+sustainab ility+framework/2012+edition/2012-edition\#PerformanceStandards. Accessed 2 January 2012. 
IHA (2004), Sustainability Guidelines. International Hydropower Association, London. Available at http://www.hydropower.org/ downloads/IHA\%20Sustainability\%20Guidelines_Feb04.pdf. Accessed 9 September 2008.

ILO (1989), C169 - Indigenous and Tribal Peoples Convention. International Labor Organization, Geneva. Available at http:// www.ilo.org/dyn/normlex/en/f?p=1000:12100:0::NO::P12100_ INSTRUMENT_ID:312314. Accessed 22 October 2012.

ILO (1998), ILO Declaration on Fundamental Principles and Rights at Work. International Labour Organization, Geneva. Available at http://www.unhcr.org/refworld/docid/425bbdf72.html. Accessed 1 October 2011.

ILO (2001), ILO Tripartite Declaration of Principles concerning Multinational Enterprises and Social Policy (Third edition). International Labour Organization, Geneva. Available at http:// www.ilo.org/wcmsp5/groups/public/@ed_emp/@emp_ent/ documents/publication/wcms_101234.pdf. Accessed 6 October 2011.

ILO (2006), Utilities: Governance \& CSR. Available at http://www.ilo. org/public/english/dialogue/sector/sectors/utilit/gov.htm Accessed 3 September 2008

INDEC website (2002), 'Censo 2001, Resultados Definitivos, Resultados Publicados Provinciales, 24 partidos del Gran Buenos Aires'. Available at http://www.indec.gov.ar/cens02001s2_2/ ampliada_index.asp?mode=04. Accessed 30 May 2011.

INEIP (2005), X Censo de Poblacion, V de Vivienda: Censo 2005 Resultados Definitivos. Instituto Nacional de Estadística e Informática del Perú, Lima, Peru.

International Rivers (2008), Dirty Hydro: Dams and Greenhouse Gas Emissions. International Rivers, Berkeley, CA. Available at http://internationalrivers.org/files/dirtyhydro_final_lorez. pdf. Accessed 10 May 2008.

IODSA (2009), King Report on Corporate Governance for South Africa 2009. Institute of Directors of South Africa, Johannesburg. Available at http://african.ipapercms.dk/IOD/KINGIII/kingiiicode/. Accessed 12 August 2010.

ISO (2011)، ISO 26000 Guidance on Social Responsibility. Interna- tional Organization for Standardization, Available at http:// www.iso.org/iso/social_responsibility. Accessed 30 July 2011. Jackson, T. (2012), 'How fear led world leaders to betray green economy', The Guardian, Available at http://www.guardian. co.uk/sustainable-business/rio-20-tim-jackson-leadersgreen-economy. Accessed 2 January 2013.

Jacobson, M. Z. and Delucchi, M. A. (2011), 'Providing all global energy with wind, water, and solar power, Part I: Technologies, energy resources, quantities and areas of infrastructure, and materials', Energy Policy 39(2911): 1154-1169.

Jennings, P. D. and Zandbergen, P. A. (1995), 'Ecologically Sustainable Organizations: An Institutional Approach', The Academy of Management Review 20(4): 1015-1052.

Jica, D., Edegel worker representative, Planta Santa Rosa (2008), personal communication with T. Castellanos (PLADES), Lima, Peru, 1 August 2008.

Jiga, N., Procurement Manager, Eskom Uganda (2011), personal communication with Dr. V. Madziakapita, Jinja, Uganda, 4 October 2011.

Job, A., President, Eskom Mali Workers' Syndicate (2012), personal communication with Y. Tchamba, Bamako, 6 March 2012.

Jones, A. (2010), 'United States of America'. In The World Guide to CSR: A Country-by-Country Analysis of Corporate Sustainability and Responsibility. W. Visser and N. Tolhurst (Eds.) Sheffield, UK, Greenleaf.

Kaba, A. M., Technical and Procurement Director, Eskom Manantali (2012), personal communication with Y. Tchamba, Bamako, 6 March 2012.

Kamugisha, C., Local Council Chairperson, Naminya South, Buikwe District (2011), personal communication with Dr. V. Madziakapita, Buikwe District, Uganda, 5 October 2011.

Kaplan, A. (1964), The Conduct of Inquiry: Methodology for Behavioral Science. San Francisco, CA: Chandler.

Karnani, A. (2010), 'The Case Against Corporate Social Responsibility', The Wall Street Journal online, Available at http://online.wsj. com/article/SB10001424052748703338004575230112664504 890.html. Accessed 8 September 2010 
Karnani, A. G. (2012), Corporate Social Responsibility Does Not Avert the Tragedy of the Commons - Case Study: Coca-Cola India. Ross School of Business Paper No. 1173. Ross School of Business, Available at http://dx.doi.org/10.2139/ssrn.2030268. Accessed 15 July 2012.

Kerckhoffs, T. and Wilde-Ramsing, J. (2010), European Works Councils and Corporate Social Responsibility in the European Energy Sector. SOMO, Amsterdam. Available at http://somo. nl/publications-en/Publication_3533. Accessed 13 February 2011

KGN (2007), 'Government fines AES for $\$ 200$ over antimonopoly law violations', Kazakhstan General Newswire, 5 Oct 2007.

Khanna, N. and Vidovic, M. (2001), 'Facility Participation in Voluntary Pollution Prevention Programs and the Role of Community Characteristics: Evidence from the 33/50 Program', Binghamton University Economics Department working paper.

Kim, B. and Benson, J. (2007), Institutionalism and Capitalism in Organization Studies. Paper presented at the annual meeting of the American Sociological Association, New York. Available at http://www.allacademic.com/meta/p185144_index.html. Accessed 13 February 2010.

King, M. E., de Jongh, D. and Carapinha, R. (2010), 'South Africa'. In The World Guide to CSR: A Country-by-Country Analysis of Corporate Sustainability and Responsibility. W. Visser and N. Tolhurst (Eds.). Sheffield, UK, Greenleaf.

Kirca, A. H., Cavusgil, S. T. and Hult, G. T. M. (2009), 'The effects of national culture on market orientation: Conceptual framework and research propositions', International Business Review: doi:10.1016/j.ibusrev.2009.02.003.

Kiryowa, P., Risk and Compliance Manager, Eskom Uganda (2011), personal communication with Dr. V. Madziakapita, Jinja, Uganda, 4 October 2011.

Kitoke, D., Head of Finance, Njeru Town Council (2011), personal communication with Dr. V. Madziakapita, Njeru, Uganda, 5 October 2011.

Klins, U., Niekerk, J. H. V. and Smit, A. (2010), 'Africa'. In The World Guide to CSR: A Country-by-Country Analysis of Corporate
Sustainability and Responsibility. W. Visser and N. Tolhurst (Eds.). Sheffield, UK, Greenleaf.

Koen, M. (2010), Eskom: Business as Usual in Africa. SOMO, Amsterdam.

Kooijman - van Dijk, A. L. (2008), The Power to Produce: The role of energy in poverty reduction through small scale enterprises in the Indian Himalayas. Enschede, Netherlands: Universiteit Twente.

Kopstad, M. L., External Affairs Manager, SN Power (2008), personal communication with J. Wilde-Ramsing, Amsterdam/Oslo, 10 September and 3 November 2008.

Kovats, R. S. and Haines, A. (2005), 'Global Climate Change and Health: Recent Findings and Future Steps', Canadian Medical Association Journal 172(4).

KPMG (2008), International survey of corporate responsibility reporting. Available at KPMG International survey of corporate responsibility reporting 2008 Accessed 18 April 2012.

Kuhnle, S. and Ervik, R. (1996), 'The Nordic Welfare Model and the European Union'. In Comparative Welfare Systems: The Scandinavian Model in a Period of Change. B. Greve (Ed.). Basingstoke, Macmillan Press.

Kyoko, P. and Wandera, J., Electricty Regulatory Authority of Uganda (2011), personal communication with Dr. V. Madziakapita, Kampala, Uganda, 6 October 2011.

Lafferty, W. M. (2002a), 'Introduction: Grounding the Evaluation of 'Sustainable Development". In Realizing Rio in Norway: Evaluative Studies of Sustainable Development. W.M. Lafferty, M. Nordskag and H.A. Aakre (Eds.). Oslo, Program for Research and Documentation for a Sustainable Society (ProSus). Available at http://www.prosus.uio.no/publikasjoner/Boeker/ Realizing_Rio.htm. Accessed 5 Mar 2009.

Lafferty, W. M. (2002b), Political Evaluation: Premises, Approaches and Methods. Working Paper No. 2/02. Program for Research and Documentation for a Sustainable Society (ProSus), Oslo.

Lafferty, W. M. (2004), 'Introduction: form and function in governance for sustainable development'. In Governance for Sustainable Development. The Challenge of Adapting Form to Function. 
W.M. Lafferty (Ed.). Cheltenham, UK and Northampton, MA, USA, Edward Elgar: 1-31.

Langford, M., Scheinin, M., Vandenhole, W. and Genugten, W. v., Eds. (2013), Global Justice, State Duties: The Extra-Territorial Scope of Economic, Social and Cultural Rights in International Law. Cambridge, Cambridge University Press.

Langlois, C. C. and Schlegelmilch, B. B. (1990), 'Do Corporate Codes of Ethics Reflect National Character? Evidence from Europe and the United States', Journal of International Business Studies 21(4): 519-539.

Lauritzsen، H. F., Vice President Social and Environmental Programs, SN Power (2011), personal communication with J. WildeRamsing, Oslo, Norway, 18 October 2011.

Lawrence, A. and Morell, D. (1995), 'Leading-edge environmental management: Motivation, opportunity, resources and processes'. In Special Research Volume of Research in Corporate Social Performance and Policy, Sustaining the Natural Environment: Empirical Studies on the Interface Between Nature and Organizations. D. Collins and M. Starik (Eds.). Greenwich, CT, JAl Press: 99-126.

Lee, S. Y.-S. and Wickerham, J. (2010), 'China'. In The World Guide to CSR: A Country-by-Country Analysis of Corporate Sustainability and Responsibility. W. Visser and N. Tolhurst (Eds.). Sheffield, UK, Greenleaf.

Legisa, J. A. and Bohorquez, C. C. (2007), 'Contextualización del sector de la electricidad'. In La Responsabilidad Social de la empresa española en Latinoamérica. El caso del sector eléctrico. M. Gonzalez (Ed.). Barcelona, Media Responsable: 15-42. Available at http://www.observatoriorsc.org/nuevaweb/images/ documentos/publicaciones/informes_estudios/libro_sector_electrico.pdf. Accessed 19 May 2008.

Levy, D. and Rothenberg, S. (2002), 'Heterogeneity and change in environmental strategy: Technological and political responses to climate change in the global automobile industry'. In Organizations, Policy and the Natural Environment: Institutional and Strategic Perspectives. A. Hoffman and M. Ventresca (Eds.). Stanford, Stanford University Press: 173-193.
Lijphart, A. (1971), 'Comparative politics and the comparative method', American Political Science Review 65: 682-693.

Likert, R. (1932), 'A Technique for the Measurement of Attitudes', Archives of Psychology 140: 1-55.

Lines, R. (2007), The future of coal in Eskom's energy mix: Presentation to the South African coal industry. Eskom, Available at http://www.sacollierymanagers.org.za/Publications/ Publications/Presentations/2007/11Jan2007/The_Future_of_coal\%20_in\%20Eskoms_energy_mix.pdf. Accessed 27 November 2011.

Lumbuye, A. J., Human Resources Manager, Eskom Uganda (2011), personal communication with Dr. V. Madziakapita, Jinja, Uganda, 5 October 2011.

Lyons, D. I. and Deutz, P. (2010), 'Regional Sustainable Development: Making Development Work in Politically Contingent Space', Sustainable Development 18(4): 183-186.

Maanavilja, L. (2010), 'Europe'. In The World Guide to CSR: A Country-by-Country Analysis of Corporate Sustainability and Responsibility. W. Visser and N. Tolhurst (Eds.). Sheffield, UK, Greenleaf.

Macknick, J., Newmark, R., Heath, G. and Hallett, K. C. (2011), A Review of Operational Water Consumption and Withdrawal Factors for Electricity Generating Technologies. US National Renewable Energy Laboratory, Available at http://www.nrel. gov/docs/fy110sti/50900.pdf. Accessed 1 June 2012.

Macleod, F. (2006), 'Power plan for a dark age', Mail and Gaurdian Online, Available at http://mg.co.za/article/2006-08-25power-plan-for-a-dark-age. Accessed 1 July 2012.

Mapuche Communities (2008), 'Conflicto SN Power: Desde todos los frentes territoriales rotundo rechazo a proyectos hidroeléctricos en Liquiñe', Mapuexpress, 2 Aug 2008. Available at http:// www. mapuexpress.net/?act=news\&id=3046. Accessed 9 Sept 2008

Marcus, A. A. and Nichols, M. L. (1999), 'On the Edge: Heeding the Warnings of Unusual Events', Organization Science 10(4): 482-499.

Marquard, A., Bekker, B., Eberhard, A. and Gaunt, T. (2007), South 
Africa's Electrification Programme. University of Cape Town, Cape Town. Available at http://www.gsb.uct.ac.za/files/ ok.pdf. Accessed 9 May 2012.

Marzinotto, M., Fiamingo, F., Mazzetti, C. and Piparo, G. B. L. (2011)، 'Quality of the supplied electric service: A tool to evaluate the need of protection against lightning surges', Electric Power Systems Research 85(April 2011): 75-81.

Matanza-Riachuelo Basin Authority (2011), 2011 Management Report. Matanza-Riachuelo's Basin Authority (ACUMAR), Buenos Aires.

Matarutse, N., Technical Director, Eskom Uganda (2011), personal communication with Dr. V. Madziakapita, Jinja, Uganda, 4 October 2011.

Mavhungu, J., Stakeholder Centre Manager, Eskom (2010), personal communication with J. Wilde-Ramsing, Johannesburg, South Africa, 23 August 2010.

Mayo, E., Secretary-General, ElectroAndes Workers' Union (2008), personal communication with T. Castellanos (PLADES), La Oroya, Junín, Peru, 15 August 2008.

McCaughey, S. (2010), 'How to end the theft of poor host countries by tax dodging multinationals', Irish Times, Available at http://www.irishtimes.com/newspaper/ opinion/2010/0924/1224279585699.html. Accessed 24 September 2010.

McDonald, D. A. (2002), The Bell Tolls For Thee: Cost Recovery, Cutoffs, and the Affordability of Municipal Services in South Africa'. In Cost Recovery and the Crisis of Service Delivery in South Africa. D.A. McDonald and J. Pape (Eds.). London, HSRC Press.

McDonald, D. A., Ed. (2009a), Electric Capitalism: Recolonising Africa on the Power Grid. London, Earthscan and HSRC Press.

McDonald, D. A. (2009b), 'Introduction: The importance of being electric'. In Electric Capitalism: Recolonising Africa on the Power Grid. D.A. McDonald (Ed.). London, Earthscan and HSRC Press. Available at http://www.hsrcpress.ac.za/product. php?productid=2243. Accessed 8 September 2010.

McDonald, D. A. and Ruiters, G., Eds. (2012), Alternatives to Pri- vatization: Public Options for Essential Services in the Global South. Cape Town, HSRC Press

McGuigan, C. (2007), Power and poverty: World Bank energy reforms and poor people. Available at http://www.christianaid.org uk/issues/trade/resources/povertyandpower.aspx. Accessed 10 Nov 2007

McKay, D. and Vuuren, A. J. v. (2011), 'SA mining to cough up for crimes - Spoor', Mining Mx, Available at http://www. miningmx.com/news/markets/SA-mining-to-cough-up-forcrimes-Spoor.htm. Accessed 18 April 2012.

McSweeney, B. (2002), 'Hofstede's Model of National Cultural Differences and their Consequences: A Triumph of Faith - a Failure of Analysis', Human Relations 55(89): 89-118.

Meadowcroft, J. (2007), 'Who is in charge here? Governance for sustainable development in a complex world', Journal of Environmental Policy \& Planning 9(3): 299-314.

MEyOSP website (2011), 'Personal Edelap'. Available at http://mepriv.mecon.gov.ar/segba/memybces/personal-edelap.htm.

Milstein, M., Hart, S. and York, A. (2002), 'Coercion breeds variation: The differential impact of isomorphic pressures on environmental strategies'. In Organizations, Policy and the Natural Environment: Institutional and Strategic Perspectives. A. Hoffman and M. Ventresca (Eds.). Stanford, Stanford University Press: $151-172$.

Mngeni, N., Managing Director, Eskom Uganda (2011), personal communication with Dr. V. Madziakapita, Jinja, Uganda, 5 October 2011.

Modi, V., McDade, S., Lallement, D. and Saghir, J. (2005), Energy Services for the Millennium Development Goals. United Nations Development Programme (UNDP), Energy Sector Management Assistance Programme, UN Millennium Project, and World Bank, New York. Available at http://www.unmillenniumproject.org/documents/MP_Energy_Low_Res.pdf. Accessed 8 March 2009.

Moon, J. (2002), 'The Social Responsibility of Business and New Governance', Government \& Opposition 37(3): 385-408. 
Mun, Y.-M. (2003), Lights On: Toward Equitable, Sustainable and Democratic Energy Policies.

Mungi, A., Monobamba municipal official (2008), personal communication with T. Castellanos (PLADES), Monobamba, Junín, Peru, 15 August 2008.

Musango, J. K. and Brent, A. C. (2011), 'A conceptual framework for energy technology sustainability assessment', Energy for Sustainable Development 15(1): 84-91.

Mutti, J. and Grubert, H. (2004), 'Empirical asymmetries in foreign direct investment and taxation', Journal of International Economics 62(2): 337-358.

MVO Platform (2007), MVO Referentiekader. MVO Platform, Amsterdam. Available at http://mvo-platform.tuxic.nl/files/ Publicaties/MV0\%20referentiekader\%20NL_tweede\%20druk. pdf. Accessed 5 September 2008

MVO Platform (2012), CSR Frame of Reference. MVO Platform, Amsterdam. Available at http://mvoplatform.nl/news-en/ the-new-csr-frame-of-reference-now-available-in-english/ view?set_language=en. Accessed 8 May 2012.

Nehrt, C. (1996), 'Timing And Intensity Effects of Environmental Investments', Strategic Management Journal 17(7): 535-547.

Nicholas, E., Local Council Chairperson, Nalufenya Village, Jinja District (2011), personal communication with Dr. V. Madziakapita, Nalufenya Village, Uganda, 5 October 2011.

Nissen, S. (1998), 'The Case of Case Studies: On the Methodological Discussion in Comparative Political Science', Quality \& Quantity 32: 399-418.

Nordic Council (2010), Nordic Co-operation Programme for Innovation and Business Policy. Nordic Council, Copenhagen. Available at http://www.norden.org/da/publikationer/publikationer/2010-787. Accessed 25 November 2011.

Nordic Council (2011), Social responsibility and innovation - a challenge for Nordic companies. Nordic Council, Copenhagen. Available at http://www.nordicinnovation.org/da/nyheder/ social-responsibility-and-innovation-a-challenge-for-nordiccompanies/. Accessed 24 November 2011.

Norfund website (2008), 'Norfund - Home'. Available at http://nor- fund.no/. Accessed 11 Sept 2008.

Norwegian Finance Ministry (2008), 'The Government Pension Fund divests its holdings in mining company', Norwegian Finance Ministry website, 9/9/2008. Available at http://www.regjeringen.no/en/dep/fin/Selected-topics/andre/Ethical-Guidelines-for-the-Government-Pension-Fund---Global-/TheEthical-Guidelines.html?id=434894 Accessed 11 Sept 2008 Norwegian Ministry of Foreign Affairs (2009), Corporate social responsibility in a global economy: Report No. 10 (2008-2009) to the Storting. Oslo. Available at http://www.regjeringen.no/ en/dep/ud/Documents/Propositions-and-reports/Reportsto-the-Storting/2008-2009/report-no-10-2008-2009-to-thestorting.html?id=565907. Accessed 10 August 2010.

Nussbaum, B. (2003), 'Ubuntu: Reflections of a South African on Our Common Humanity', Reflections 4, 21-26 Available at http:// www.barbaranussbaum.com/downloads/reflections.pdf. Accessed 11 August 2010.

OECD-NEA (2000), Nuclear Energy in a Sustainable Development Perspective. OECD Nuclear Energy Agency, Paris. Available at http://www.nea.fr/ndd/docs/2000/nddsustdev.pdf. Accessed 1 September 2010.

OECD (2000a), Indicators to Measure Progress - Towards Sustainable Development. Organization for Economic Cooperation and Development, Paris. Available at http://www.oecd.org/ dataoecd/36/39/33703694.pdf. Accessed 1 September 2010.

OECD (2000b), OECD Guidelines for Multinational Enterprises. OECD, Paris. Available at http://www.oecd.org/dataoecd/56/36/1922428.pdf. Accessed 5 September 2008. OECD (2006), OECD Risk Awareness Tool for Multinational Enterprises in Weak Governance Zones. Organization for Economic Cooperation and Development, Paris. Available at http:// www.oecd.org/dataoecd/26/21/36885821.pdf. Accessed 26 Sept 2008.

OECD (2007), Principles for Private Sector Participation in Infrastructure. Organisation for Economic Co-operation and Development, Paris. Available at http://www.oecd.org/ dataoecd/41/33/38309896.pdf. Accessed 24 April 2012. 
OECD (2009), Tax Co-operation 2009: Towards a Level Playing Field. OECD, Paris. Available at http://www.oecd.org/document/2 3/0,3343,en_2649_33745_43555607_1_1_1_1,00.html. Accessed 26 May 2010.

OECD (2011), OECD Guidelines for Multinational Enterprises: Recommendations for Responsible Business Conduct in a Global Context. OECD, Paris. Available at http://www.oecd.org/ dataoecd/43/29/48004323.pdf. Accessed 5 July 2011.

OECD/IEA (2010), Energy Poverty: How to make modern energy access universal (Special excerpt of the World Energy Outlook 2010). OECD International Energy Agency, Paris. Available at http://www.worldenergyoutlook.org/docs/we02010/ we02010_poverty.pdf. Accessed 1 February 2011.

Olargin, V., Branch Secretary, Uganda Allied Electricity Workers Union (2011), personal communication with Dr. V. Madziakapita, Jinja, Uganda, 5 October 2011.

Olivera, F. (2008), 'Advierte el Gobierno que Edelap habría cometido irregularidades', La Nación online, 3 Dec 2008. Available at http://www.lanacion.com.ar/nota.asp?nota_id=1076834\&pid $=5466471 \&$ toi=6269. Accessed 6 Dec 2008

Olson, B. J., Bao, Y. and Parayitam, S. (2007), 'Strategic decision making within Chinese firms: The effects of cognitive diversity and trust on decision outcomes', Journal of World Business 42(1): 35-46.

Ombudsman of Buenos Aires (2007), Resolution 1140/07. Office of the Ombudsman of the City of Buenos Aires, Buenos Aires. Available at www.defensoria.org.ar/institucional/resoluciones/r1140-07.doc. Accessed 8 May 2009.

Orpen, C. (1987), 'The attitudes of united states and South African managers to corporate social responsibility', Journal of Business Ethics 6(2): 89-96.

Ossiya, J., Finance Director, Eskom Uganda (2011), personal communication with Dr. V. Madziakapita, Jinja, Uganda, 5 October 2011.

Pacasmayo resident 1, (2008), personal communication with T. Castellanos (PLADES), Pacasmayo, Peru, 5 August 2008. Pacasmayo resident 2, (2008), personal communication with T.
Castellanos (PLADES), Pacasmayo, Peru, 5 August 2008.

Pacasmayo resident 3, (2008), personal communication with $T$. Castellanos (PLADES), Pacasmayo, Peru, 5 August 2008.

Palast, G., Oppenheim, J. and MacGregor, T. (2000), Democratic regulation: A guide to the control of privatized public services through social dialogue, Sectoral working paper No. 166. ILO, Geneva. Available at http://www.ilo.org/public/english/ dialogue/sector/papers/pubserv/demreg.pdf. Accessed 3 September 2008.

Parnell, T., East West Management Institute (2010), personal communication with Prof. Dr. Qin Hui, Phnom Penh, Cambodia, 25 January 2010.

Patavalis, D. and Aravossis, K. (2004), 'An Evaluation Approach to Environmental Program and Project Planning', Water, Air, \& Soil Pollution: Focus 4(4): 421-431.

Pawson, R. and Tilley, N. (1995), 'Whither (European) evaluation methodology?', Knowledge \& Policy 8(3): 20-34.

PDG (2010), A study into approaches to minimise the impact of electricity price increases on the poor. Palmer Development Group, Johannesburg. Available at http://www.thedti.gov.za/ industrial_development/docs/fridge/NEDLAC_final_report. pdf. Accessed 5 May 2012.

Pereira, M. G., Sena, J. A., Freitas, M. A. I. V. and Silva, N. F. d. (2011), 'Evaluation of the impact of access to electricity: A comparative analysis of South Africa, China, India and Brazil', Renewable and Sustainable Energy Reviews 15(3): 1427-1441.

Peters, E. D. (2011), 'Riding the green wave, Interview with Elizabeth Dipuo Peters, Minister of Energy, Republic of South Africa', Interview by Wangu Mwangi, Capacity.org, Available at http://www.capacity.org/capacity/opencms/en/topics/ change-facilitation/riding-the-green-wave.html. Accessed 4 May 2012.

Pillinger, J. (2009), Quality Public Services for All: PSI Resource Pack. Public Services International, Paris. Available at http://www. world-psi.org/Content/ContentGroups/English7/Defending_ Quality_Public_Services/Resources2/QPS_Resource_pack/ EN_QPSRESOURCEPACK_July2009.pdf. Accessed 1 September 
2010.

Pinkston, T. S. and Carroll, A. B. (1994), 'Corporate citizenship perspectives and foreign direct investment in the U.S', Journal of Business Ethics 13(3): 157-169.

Pottinger, L. (1997), Manantali Dam Changes Will Make a Bad Situation Worse. International Rivers, Available at http://www. internationalrivers.org/en/manantali-dam-changes-willmake-bad-situation-worse. Accessed 13 March 2012.

Prasad, G. (2007), Electricity from solar home systems in South Africa. Energy Research Centre, University of Cape Town, Cape Town. Available at http://www.erc.uct.ac.za/Research/ publications/07Prasad\%20Electricity\%20from\%20SHSsl.pdf. Accessed 2 May 2012.

Prieto, A., Vice-Manager for Communications, Edegel (2008), personal communication with T. Castellanos (PLADES), Lima, Peru, 7 August 2008.

Procter, T. (2007), Measuring emissions: the search for standards.

Climate Change Corp, Available at http://www.climatechangecorp.com/content.asp?ContentID=4985. Accessed 14 Dec 2008.

Procter, T. (2008), Disclosing carbon emissions - do the numbers add up? Available at http://toreda.spaces.live.com/blog/ cns!66434C39482338AF!624.entry. Accessed 21 Aug 2008.

Qin Hui (2008), 'China Competitiveness Enhanced by Disrespect for Human Rights', Aisixiang, Available at http://www.aisixiang. com/data/detail.php?id=20238. Accessed 7 May 2012.

Qin Hui (2010), 'Is There a 'Chinese Model'?', China Market 24.

Queinnec, Y. and Bourdon, W. (2007), The OECD Guidelines for Multinational Enterprises - An Evolving Legal Status. Sherpa and OECD Watch, Paris and Amsterdam. Available at http:// oecdwatch.org/publications-en/Publication_3064. Accessed 2 February 2013.

Ragin, C. C. (1987), The Comparative Method: Moving Beyond Qualitative and Quantitative Strategies. Berkley: University of California Press.

Ramus, C. A. and Steger, U. (2000), 'The Roles of Supervisory Support Behaviors and Environmental Policy in Employee "Ecoinitia- tives" at Leading-Edge European Companies', The Academy of Management Journal 43(4): 605-626.

Reimann, F., Ehrgott, M., Kaufmann, L. and Carter, C. R. (2011), 'Local stakeholders and local legitimacy: MNEs' social strategies in emerging economies', Journal of International Management 18(1): 1-17.

Residents of Villa Inflamable, (2008), personal communication with B. Esteves (FARN), Dock Sud, Buenos Aires, 22 August 2008. Rochlin, G. I. and Meier, A. V. (1994), 'Nuclear Power Operations: A Cross-Cultural Perspective', Annual Review of Energy and the Environment 19(1): 153-187.

Rocío, Resident and small business owner in La Plata (2008), personal communication with B.E. (FARN), La Plata, 2 September 2008.

Rossouw, G. J., van der Watt, A. and Malan, D. P. (2002), 'Corporate Governance in South Africa', Journal of Business Ethics 37(3): 289-302.

Ruggie, J. (2008), Protect, Respect and Remedy: A Framework for Business and Human Rights. United Nations, New York. Available at http://www.reports-and-materials.org/Ruggiereport-7-Apr-2008.pdf. Accessed 7 September 2008.

Runhaar, H., Dieperink, C. and Driessen, P. (2006), 'Policy analysis for sustainable development. The toolbox for the environmental social scientist', International Journal of Sustainability in Higher Education 7(1): 34-56.

Runhaar, H. and Lafferty, H. (2009), 'Governing Corporate Social Responsibility: An assessment of the contribution of the UN Global Compact to CSR strategies in the telecommunications industry', Journal of Business Ethics 84: 479-495.

Russo, M. V. and Fouts, P. A. (1997), 'A Resource-Based Perspective on Corporate Environmental Performance and Profitability', The Academy of Management Journal 40(3): 534-559.

Ruud, A. (2002), 'Environmental management of transnational corporations in India-are TNCs creating islands of environmental excellence in a sea of dirt?', Business Strategy and the Environment 11(2): 103-118.

SADC website (2012), 'Infrastructure - Energy - Hydropower'. Avail- 
able at http://www.sadc.int/themes/infrastructure/en/ hydropower/. Accessed 4 June 2012.

SASAC website (2008), 'Guidelines to the State-owned Enterprises Directly under the Central Government on Fulfilling Corporate Social Responsibilities'. Available at http://www.sasac.gov. cn/n2963340/n2964712/4891623.html. Accessed 10 August 2010

Scott, W. (1992), Organizations: Rational, Natural, and Open Systems. Englewood Cliffs, NJ: Prentice-Hall.

Seawright, J. and John, G. (2008), 'Case Selection Techniques in Case Study Research: A Menu of Qualitative and Quantitative Options', Political Research Quarterly 61(2): 294-308.

Sedlacko, M. and Martinuzzi, A. (2012), 'Governance for sustainable development, evaluation and learning: an introduction'. In Governance by Evaluation for Sustainable Development: Institutional Capacities and Learning. M. Sedlacko and A. Martinuzzi (Eds.). Cheltenham, UK and Northampton, MA, USA, Edward Elgar: 1-18.

SEW (2006), Indicator Selection and Rationale. Sustainable Energy Watch, Paris. Available at http://www.helio-international. org/energywatch/indicators.cfm. Accessed 13 Jan 2009.

Sharma, S. (2000), 'Managerial Interpretations and Organizational Context as Predictors of Corporate Choice of Environmental Strategy', The Academy of Management Journal 43(4): 681697.

Sharma, S. and Vredenburg, H. (1998), 'Proactive corporate environmental strategy and the development of competitively valuable organizational capabilities', Strategic Management Journal 19(8): 729-753.

Shinondola-Mote, H. (2008), Uranium Mining in Namibia: A Blessing or a Curse? Labour Resource and Research Institute, Windhoek.

Sinclair, A. J., Sims, L. and Spaling, H. (2009), 'Community-based approaches to strategic environmental assessment: Lessons from Costa Rica', Environmental Impact Assessment Review 29(3): 147-156.

Sissoko, M. F., Secretary General, Manantali Council (2012), personal communication with Y. Tchamba, Manantali, 8 March 2012.

SN Power (2007a), Business Principles. SN Power, Oslo. Available at http://www.snpower.no/Corporate_Responsibility/info2/ SNP_Business_Principles_for_the_website.pdf. Accessed 15 Sept 2008.

SN Power (2007b), SN Power Annual Report 2006. SN Power, Oslo. Available at http://www.snpower.no/News_and_events/ Reports/SN_Power_Annual_Report_2006.pdf. Accessed 9 Sept 2008.

SN Power (2007c), Values and Principles. SN Power, Oslo. Available at http://www.snpower.no/Corporate_Responsibility/info2/ Values_and_Principles.pdf. Accessed 9 Sept 2008.

SN Power website (2008a), 'Change to SN Power's ownership structure'. Available at http://www.snpower.no/News_ and_events/Press_releases/29661/index_printText_html. Accessed 11 Nov 2008.

SN Power (2008b), CSR. Available at http://www.snpower.no/ Corporate_Responsibility. Accessed 15 September 2008.

SN Power website (2008c), 'Fatal accidents at hydropower project in India'. Available at http://www.snpower.no/News_and_ events/Press_releases/29664/index_printText_html. Accessed 27 Nov 2008.

SN Power website (2008d), 'Our Business'. Available at http:/ / www. snpower.no/Our_business. Accessed 11 Sept 2008.

SN Power (2008e), SN Power Annual Report 2007. SN Power, Oslo. Available at http://www.snpower.no/News_and_events/ Reports/SN_Power_Annual_Report_2007.pdf. Accessed 9 Sept 2008.

SN Power (2011a), The Power of Water: Annual Report 2010. SN Power, Oslo. Available at http://www.snpower.com/Financials/annual\%2Dreport\%2D2010/. Accessed 9 October 2011.

SN Power website (2011b), 'Sustainability'. Available at http:/ / www. snpower.com/sustainability/. Accessed 6 October 2011.

SN Power website (2012a), 'About SN Power'. Available at http:// www.snpower.com/about-us/. Accessed 3 March 2012.

SN Power website (2012b), 'Projects and Plants'. Available at http:// www.snpower.com/projects-and-plants/projects-under- 
development/bajo-frijo/. Accessed 3 March 2012.

SN Power website (2012c), 'Sustainability - HSE'. Available at http:// www.snpower.com/sustainability/. Accessed 20 April 2012.

SN Power Peru (2011), Energía limpia y desarrollo sostenible. SN Power, Oslo. Available at http://www.snpower.com.pe/images/FOLLETO\%20CSR\%202010_tcm121-17939.pdf. Accessed 9 October 2011.

SNPP website (2009), 'Responsibilidad Social Corporativa'. Available at http://www.cahua.com.pe/responsabilidad-social-corporativa.php. Accessed 9 May 2009.

SNPP (2011)، Energía limpia y desarrollo sostenible. SN Power Peru, Lima. Available at http://www.snpower.com.pe/images/ FOLLETO\%20CSR\%202010_tcm121-17939.pdf. Accessed 9 October 2011.

Søderberg, A.-M. and Holden, N. (2002), 'Rethinking Cross Cultural Management in a Globalizing Business World', International journal of cross cultural management 2(1): 103-121.

South African Deaprtment of Energy (2010), Electrification statistics, 2009. Available at http://www.energy.gov.za/files/media/ explained/statistics_electrification_2009.pdf. Accessed 9 May 2012.

South African Deaprtment of Environmental Affairs website (2013), 'South African Government's position on Climate Change'. Available at http://www.climateaction.org.za/cop17-cmp7/ sa-government-position-on-climate-change.

South African Department of Energy (2002), National Inventory Discard and Duff Coal Survey - 2001. Department of Minerals and Energy, Pretoria. Available at http://www.energy.gov.za/ Coal/coal_discard_report.pdf. Accessed 1 May 2011.

South African Department of Energy (2010), Electrification statistics, 2009. Available at http://www.energy.gov.za/files/media/ explained/statistics_electrification_2009.pdf. Accessed 9 May 2012.

South African Department of Mineral Resources (2009), Mining Charter Impact Assessment Report. Pretoria. Available at http://www.dmr.gov.za/publications/finish/108-mineralsact-charter-and-scorecard/126-miningcharterimpact- oct-2009/0.html. Accessed 16 April 2012.

Sovacool, B. K. and Bulan, L. C. (2011), 'Behind an ambitious megaproject in Asia: The history and implications of the Bakun hydroelectric dam in Borneo', Energy Policy 39(9): 4842-4859.

Stajkovic, A. D. and Luthans, F. (1997), 'Business ethics across cultures: A social cognitive model', Journal of World Business 32(1): 17-34.

STATSA (2009), Statistics South Africa: Electricity statistics. Retrieved 2 May 2009, from http:/ / www.statssa.gov.za/

Stern, N. (2006), The Economics of Climate Change: The Stern Review. Cambridge: Cambridge University Press.

Steyn, G. (2006), Investment and Uncertainty: Historical experience with power sector investment in South Africa and its implications for current challenges. University of Cape Town Graduate School of Business, Cape Town. Available at http:// www.gsb.uct.ac.za/files/Eskom-InvestmentUncertainty.pdf. Accessed 8 May 2012.

Stufflebeam, D. L. (1980), 'An interview with Daniel L. Stufflebeam', Educational Evaluation and Policy Analysis 2(4).

Sullivan Principles website (2010), 'The Global Sullivan Principles'. Available at http://www.globalsullivanprinciples.org/principles.htm. Accessed 12 August 2010.

Sutherland, D. and Whelan, G. (2009), Corporate Social Responsibility in China's Largest TNCs. University of Nottingham China Policy Institute, Nottingham, UK. Available at http://www.nottingham.ac.uk/cpi/documents/discussion-papers/discussionpaper-51-csr-ds.pdf. Accessed 10 August 2010.

Sverjensky, N. (2010), 'Beyond petroleum: Why the CSR community collaborated in creating the BP oil disaster', Ethical Corporation, Available at http://www.ethicalcorp.com/content. asp?ContentID=7003\&utm_source=http\%3a\%2f\%2fcommun icator.ethicalcorp.com\%2flz\%2f\&utm_medium=email\&utm_ campaign $=\mathrm{EC}+$ News $+04+08+10+1$ \&utm_term $=$ Beyond + petrol eum\%3a+Why+the+CR+community+collaborated+in+creating+ the+recent+oil+disaster\&utm_content=162861.

Swilling, M. (2010), 'Sustainability, Poverty and Municipal Services: The Case of Cape Town, South Africa', Sustainable Develop- 
ment 18(4): 194-201.

Taka, I. and Foglia, W. D. (1994), 'Ethical aspects of Japanese leadership style', Journal of Business Ethics 13: 135-148.

Takoure, A., Manantali community leader (2012), personal communication with Y. Tchamba, Manantali, 8 March 2012.

Taras, V., Rowney, J. and Steel, P. (2009), 'Half a century of measuring culture: Review of approaches, challenges, and limitations based on the analysis of 121 instruments for quantifying culture', Journal of International Management 15(4): 357-373.

Teske, S. (2010), Energy [r]evolution: A sustainable world energy outlook. European Renewable Energy Council and Greenpeace International, Brussels and Amsterdam. Available at http:// www.greenpeace.org/usa/Global/usa/report/2010/6/greenpeace-energy-r-evolution.pdf. Accessed 3 May 2010.

Thomas, S. (2007), Electricity Liberalization Experiences in the World. Public Services International Research Unit, University of Greenwich, London. Available at www.psiru.org. Accessed 19 May 2008.

TI (2007), Report on the Transparency International Global Corruption Barometer 2007. Transparency International, Berlin. Available at http://www.transparency.org/policy_research/ surveys_indices/gcb/2007. Accessed 25 Sept 2008.

Tinnirello, C. A. (2006), Proyecto de ley para la erradicación del Polo Petroquímico Dock Sud. Argentine National House of Deputies, Buenos Aires. Available at http://www1.hcdn.gov.ar/ dependencias/ceycombust/proyectos/textos/2006/3411D-06.htm. Accessed 1 June 2011.

Toure, C. M., Technical Director, OMVS-SOGEM (2012), personal communication with Y. Tchamba, Bamako, 6 March 2012.

Trompenaars, F. (1993), Riding the Waves of Cluture: Understanding Diversity in Global Business. Chicago: Irwin Professional Publishing.

Tully, S. (2006), 'The Human Right to Access Electricity', The Electricity Journal 19(3): 30-39.

UK Committee on Climate Change (2009), Meeting Carbon Budgets - the need for a step change. First progress report to Parliament. Available at http://www.theccc.org.uk/reports/1st- progress-report. Accessed 22 October 2010.

UN (1992)، Agenda 21. United Nations, New York. Available at http://www.un.org/esa/sustdev/documents/agenda21/ english/agenda21toc.htm\#pre. Accessed 5 Mar 2009.

UN (2007), United Nations Declaration on the Rights of Indigenous Peoples. United Nations, New York. Available at http://www. un.org/esa/socdev/unpfii/documents/DRIPS_en.pdf. Accessed 28 April 2012.

UN (2008), United Nations Declaration on the Rights of Indigenous Peoples. United Nations, New York. Available at http://www. un.org/esa/socdev/unpfii/documents/DRIPS_en.pdf. Accessed 18 January 2012.

UN website (2010), 'Millennium Development Goals'. Available at http://www.un.org/millenniumgoals/. Accessed 8 September 2010.

UN (2012), Sustainable Energy for All. United Nations, New York. Available at http://www.sustainableenergyforall.org/. Accessed 29 April 2012

UN Energy (2005), The Energy Challenge for Achieving the Millennium Development Goals. United Nations, New York. Available at http://www.unhabitat.org/downloads/docs/920_88725_ The $\% 20$ Energy $\% 20$ challenge $\% 20$ for $\% 20$ achieving $\% 20$ the $\% 20$ millenium\%20development\%20goals.pdf. Accessed 7 September 2010.

UN Global Compact (2008), The Ten Principles. United Nations, New York. Available at http://www.unglobalcompact.org/ AboutTheGC/TheTenPrinciples/index.html.

UN/IAEA (1994), Classification of Radioactive Waste: A Safety Guide. United Nations International Atomic Energy Agency, Vienna. Available at http://www-pub.iaea.org/MTCD/publications/ PDF/Pub950e_web.pdf. Accessed 7 May 2007.

UN/IAEA (2007), Energy Indicators for Sustainable Development: Country Studies on Brazil, Cuba, Lithuania, Mexico, Russian Federation, Slovakia and Thailand. United Nations International Atomic Energy Agency, Vienna. Available at http:// www.un.org/esa/sustdev/publications/energy_indicators/ full_report.pdf. Accessed 19 May 2008. 
UNCSD (2001), Indicators of Sustainable Development: Guidelines and Methodologies. United Nations Commission on Sustainable Development, New York. Available at http:// www.un.org/esa/sustdev/publications/indisd-mg2001.pdf Accessed 1 September 2010.

UNCSD website (2010), 'About the UN Commission on Sustainable Development'. Available at http://www.un.org/esa/dsd/csd/ csd_aboucsd.shtml. Accessed 8 September 2010.

UNCTAD (1992), World Investment Report 1992: Transnational Corporations as Engines of Growth. United Nations Conference on Trade and Development, New York.

UNDESA (2013), Survey on the Sustainable Development Goals Synthesis of responses from UN Member State Missions. UN Department of Economic and Social Affairs, New York. Available at http://www.stakeholderforum.org/fileadmin/files/ Analysis\%20SDG\%20UNDESA\%20Survey_Final.pdf. Accessed 2 February 2013.

UNECA (2007), Energy for Sustainable Development: Policy Options for Africa. United Nations Economic Commission for Africa, Addis Ababa. Available at www.uneca.org/eca_resources/ Publications/UNEA-Publication-toCSD15.pdf.

UNECE (1998), Convention on Access to Information, Public Participation in Decision-Making and Access to Justice in Environmental Matters. United Nations Economic Commission for Europe, Århus. Available at http://ec.europa.eu/environment/aarhus/. Accessed 4 October 2011.

UNEP (2009), Energy Technology Factsheet: Small Scale Hydro (SSH). United Nations Environment Programme, Nairobi, Kenya. Available at http://www.uneptie.org/ENERGY/information/ publications/factsheets/pdf/hydro.PDF. Accessed 16 May 2009.

UNEP (2011), Environmental Assessment of Ogoniland. United Nations Environmental Programme, Nairobi, Kenya. Available at http://www.unep.org/newscentre/default.aspx?DocumentID =2649\&ArticlelD=8827. Accessed 22 October 2012.

UNGC (2008), The Ten Principles. United Nations, New York. Available at http://www.unglobalcompact.org/AboutTheGC/
TheTenPrinciples/index.html.

UNGC (2012), Rio+20 Corporate Sustainability Forum: Overview and Outcomes. UN Global Compact, New York. Available at http:/ / www.unglobalcompact.org/docs/news_events/2012_CSF/ Rio_CSF_Overview_Outcomes.pdf. Accessed 2 January 2013. UNHRC (2011), Guiding Principles for Business and Human Rights. United Nations Human Rights Council, Available at http:// www.ohchr.org/documents/issues/business/A.HRC.17.31. pdf. Accessed 8 July 2011.

UNRISD website (2005), 'Business Responsibility for Sustainable Development: Project from 2000-2005'. Available at http:// www.unrisd.org/unrisd/website/projects.nsf/\%28httpProj ectsForProgrammeArea-en\%29/E7F3F4A25DFB0AE980256 B6100514A19?0penDocument\&startyear=2005. Accessed 9 September 2010.

Utting, P. (2002), The Greening of Business in Developing Countries: Rhetoric, Reality and Prospects. London: Zed Books.

Van Huesden, P. (2009), 'Discipline and the new "logic of delivery": Prepaid electricity in South Africa and beyond'. In Electric Capitalism: Recolonising Africa on the Power Grid. D. McDonald (Ed.). Johannesburg, HSRC Press.

van Huijstee, M. and Glasbergen, P. (2010), 'NGOs Moving Business: an Analysis of Contrasting Strategies', Business \& Society. Ventanilla Resident 1, (2008), personal communication with T. Castellanos (PLADES), Ventanilla, Peru, 25 August 2008.

Ventanilla Resident 2, (2008), personal communication with T. Castellanos (PLADES), Ventanilla, 25 August 2008.

Villar, F., FOCO (2008), personal communication with B. Esteves (FARN), Buenos Aires, 15 August 2008.

Visser, W. (2007), 'Revisiting Caroll's CSR Pyramid: An African Perspective'. In Corporate Social Responsibility: Three Volume Set. A. Crane and D. Matten (Eds.). London, Sage: 195-212. Available at http://www.waynevisser.com/chapter_wvisser_africa_csr_pyramid.pdf. Accessed 12 August 2010. Visser, W. (2008), 'Corporate Social Responsibility in Developing Countries'. In The Oxford Handbook of Corporate Social Responsibility. A. Crane, A. McWilliams, D. Matten, J. Moon 
and D. Siegel (Eds.). Oxford, Oxford University Press: 473-479. Available at http://www.waynevisser.com/chapter_wvisser_csr_dev_countries.pdf. Accessed 19 August 2010.

Vogel, S. K. (1996), Freer Markets, More Rules: Regulatory Reform in Advanced Industrial Countries. Ithaca, NY and London: Cornell University Press.

Voß, A. (2005)، A Comparative Assessment of Electricity Generation Options from an ecological and sustainability viewpoint. Universität Stuttgart, Institut für Energiewirtschaft und Rationelle Energieanwendung, Stuttgart. Available at http:// apw.ee.pw.edu.pl/tresc/-eng/09-ElectricityGeneration0ptions.pdf. Accessed 24 August 2010.

Voß, A., Rath-Nagel, S. and Ellersdorfer, I. (2005), A Conceptual Framework for Sustainable Electricity Supply: EUSUSTEL - WP 8.2. Universität Stuttgart, Institut für Energiewirtschaft und Rationelle Energieanwendung, Stuttgart. Available at http:// www.ier.uni-stuttgart.de/publikationen/pb_pdf/04_EUSUSTEL_Final_Report_WP8.pdf. Accessed 24 August 2010.

Waste Management World website (2008), 'UN rewards waste collection project in Brazil'. Available at http://www.wastemanagement-world.com/display_article/341474/123/ART$\mathrm{CL} /$ none/COLTR/1/UN-rewards-waste-collection-project-inBrazil/. Accessed 3 Oct 2008.

WBCSD (2002), Sustainability in the Electricity Utility Sector. World Business Council for Sustainable Development, Geneva. Available at http://www.wbcsd.org/DocRoot/RBiSulvtwIJFFpeANshg/20020930_electricity.pdf. Accessed 15 May 2010.

WCD (2000), Dams \& Development: A New Framework for DecisionMaking. World Commission on Dams, London and Sterling, VA. Available at http://www.dams.org//docs/report/wcdreport. pdf. Accessed 6 September 2008

WCED (1987), Our Common Future: Report of the World Commission on Environment and Development. Oxford University Press, Oxford. Available at http://www.un-documents.net/wcedocf.htm. Accessed 5 March 2009.

Webster, E. (1985), Cast in a racial mould. Johannesburg: Raven Press.
Welford, R. (2004), 'Corporate Social Responsibility in Europe and Asia: Critical Elements and Best Practice', Journal of Corporate Citizenship 13(Spring): 31-47.

Welford, R. (2005), 'Corporate Social Responsibility in Europe, North America and Asia', Journal of Corporate Citizenship 17(spring): 33-52.

Welford, R. (2010), 'CSR and sustainable development', CSR Asia, Available at http://www.csr-asia.com/weekly_detail. php?id=11893. Accessed 20 August 2010.

Welzel, C., Peters, A., Höcker, U. and Scholz, V., Eds. (2007), The CSR Navigator: Public Policies in Africa, the Americas, Asia and Europe. Gütersloh, Germany, Bertelsmann Stiftung.

Wicke, B., Dornburg, V., Junginger, M. and Faaij, A. (2008), 'Different palm oil production systems for energy purposes and their greenhouse gas implications', Biomass and Bioenergy 32(12): 1322-1337.

Wilde-Ramsing, J. and Potter, B. (2006), 'Blazing the Green Path: Renewable Energy and State-Society Relations in Costa Rica', The Journal of Energy and Development 32(1): 69-92.

Witte, B. (2008), 'Chilean Bishop Sounds off on Patagonia Dam Plan', The Patagonia Times, 1 Sept 2008. Available at http://www. patagoniatimes.cl/content/view/633/26/. Accessed $210 c t$ 2008

Wolpe, H. (1972), 'Capitalism and Cheap Labour-Power in SA: From Segregation to Apartheid', Economy and Society 1(4): 425-456. Woodhouse, E. J. (2006), 'The Obsolescing Bargain Redux: Foreign Investment in the Electric Power Sector in Developing Countries', N.Y.U. Journal of International Law and Politics 38(121): 121-219.

World Bank (2008), PPI data update note 11: Investment commitments to energy doubled in 2007. Available at http://ppi. worldbank.org/features/Oct2008/2007EnergyDataLaunch. pdf. Accessed 6 December 2008.

World Bank (2009), Africa Electrification Initiative workshop report, Maputo, Mozambique. International Bank for Reconstruction and Development/World Bank Group, Washington DC. Available at http://siteresources. 
worldbank.org/EXTAFRREGTOPENERGY/Resourc-

es/717305-1264695610003/6743444-1268073052994/

AEI_Full_Proceedings.pdf. Accessed 3 May 2012.

World Bank website (2010a), 'Private Participation in Infrastructure

Project Database: Electricity Sector Highlights'. Available at

http://ppi.worldbank.org/. Accessed 1 February 2011.

World Bank (2010b), Safeguards Diagnostic Review for South Africa

Eskom Investment Support Project. World Bank, Washington

DC. Available at http://siteresources.worldbank.org/INT-

SOUTHAFRICA/Resources/Final-SDR-EISP.pdf. Accessed 3

May 2011.

World Bank (2011), Private Participation in Infrastructure Database.

Retrieved 13 November 2011, from http://ppi.worldbank.org/ index.aspx

World Commission on Dams (2000), Dams \& Development: A New

Framework for Decision-Making. Earthscan Publications Ltd, London and Sterling, VA. Available at http://www.dams.org// docs/report/wcdreport.pdf. Accessed 6 September 2008.

Xinhua (2007), 'Impugnan ambientalistas dominicanos acuerdo con EEUU', Xinhua News Agency, 4 March 2007.

Yale University website (2010), 'Environmental Performance Index 2010'. Available at http://epi.yale.edu/. Accessed 6 August 2010.

Yamin, M. and Sinkovics, R. R. (2008), 'Infrastructure or foreign direct investment? An examination of the implications of MNE Strategy for economic development', Journal of World Business 44(2): 144-157.

Yin, R. K. (2003), Case Study Research: Design and Methods. Newbury Park, CA: Sage.

Zerriffi, H. (2007), Making Small Work: Business Models for Electrifying the World, PSED Working Paper 63. Stanford University, Stanford, CA. Available at http://iis-db.stanford.edu/ pubs/21983/WP63,_Zerriffi,_Making_Small_Work_20070926. pdf. Accessed 19 May 2008.

Zvoleff, A., Kocaman, A. S., Huh, W. T. and Modi, V. (2009), 'The impact of geography on energy infrastructure costs', Energy Policy 37: 4066-4078. 


\section{Acknowledgements}

Writing this dissertation has been a journey that I would not have been able to make were it not for the help and support of many special individuals along the way. I would like to take a few lines here to express my gratitude to them.

First and foremost, my three supervisors deserve a heap of thanks. Hans, thank you for supporting me in this endeavor. Bill and Maarten, you have been so much more than dissertation supervisors to me over the past several years. Maarten, I remember well our first cup of coffee together near the Arnhem train station, where you reacted encouragingly to my proposals and ideas, shared your own thoughts and experiences, and proposed we get in touch with Bill to further develop our ideas. That was the genesis of what would develop into the Bill-Maarten-Joseph alliance: an interesting, fruitful, and enjoyable collaboration that had its share of twists and turns, but throughout which good spirits always prevailed. Our working pattern of periodic intense working sessions followed by dinner together where the conversation meandered between politics, philosophy, music, and history was as enjoyable as it was productive and intellectually stimulating. I always came away from our meetings inspired and energized. Thank you both for sticking with me through all these years and guiding me in developing my own thoughts, vision, and critical analytical capacity.

This dissertation could not have come to fruition without input, information-gathering, and analysis from several individual researchers at partner organizations of SOMO around the globe. Thanks goes to Belén Esteves, María Eugenia di Paola, María Marta di Paola, and Bernardo Veloj from FARN in Argentina; Themis Castellanos and Juan Carlos Vargas from PLADES in Peru; Michael Koen and Aisha Badur from CSRSC in South Africa; Tagbo Agbazue, Yanick Tchamba, and Victor Madziakapita from Influence Africa in South Africa; and Guo Yushan, Liu Zhi, Qin Hui, and Wu Aoqi from the Transition Institute in China.

And to my SOMO colleagues, what can I say? 30 critical, action-oriented researchers, networkers, communicators, managers, controllers, and administrators in one building...it's like a wasps' nest of beautifully-constructed chaos. Your dedication to making the world a better, more just, more sustainable place is inspiring. Your energy gives me strength. Your commitment to structured, fact-based, grounded research, analysis, and communication instills in me confidence that we can and will make a difference. Your light-heartedness and gezelligheid reminds me (daily!) that all work and no play is not good for the soul. All in all, you guys make it a pleasure to come to work every day. Thanks especially to Tim (a co-author of parts of the work), Mariette, Roos, Esther, Ronald, and Joris for your contributions, advice, comments, thoughts, reflections, and support at various phases throughout the process.

Colleagues at CSTM also deserve thanks, especially Frans, for your feedback on presentations of my work, and Ada, for your 
administrative support and advice. Audun at SINTEF Energy Research, thanks to you for your comments, advice, and feedback at the critical early stages of the endeavor.

Ed, thanks for your creative genius that touches every page of this book and so appropriately visually complements and emphasizes the key elements.

Willem and Vasilis, thanks for getting my back! And for the good times and necessary distractions from the heavy $\mathrm{PhD}$ work over the past 4 years.

Mom and Dad, thanks for your unwavering support and encouragement throughout and for all your helpful suggestions on the near-final text.

Mayla, thank you for understanding on all those weekends I was glued to the computer at the kitchen table. You would come to me, nod with resigned understanding and say "Daddy's working" (and only every once in a while try to erase the entire dissertation by banging on the delete button).

Birka, you're my bedrock. You helped me with this endeavor every day, every step of the way. The certainty of your unflagging love and your emotional, mental, and physical presence and support gives me the strength and confidence to love you back with a fury and to extend that passion to all else that I do. You make me beam as I wake each morning, and smile every night as I turn off the light. You're my most attentive and constructive critic, and my most accepting supporter. You kept my spirits up when I was going through rough patches and helped me think through the dissertation's toughest analytical and methodological puzzles. Thanks for your understanding and for stoically doing double, triple, (even quadruple!) duty with Mayla and at home during my intensive writing periods. I love you. 


\section{Curriculum Vitae}


Joseph Wilde-Ramsing was born on the 18th of November, 1978, in Wilmington, North Carolina (USA), where he also completed primary and secondary school. In 2001, Joseph graduated from the University of North Carolina at Asheville with a Bachelor of Arts degree in political science and a Bachelor of Arts degree in Spanish, both with honors. His undergraduate thesis, based on his own field research in Ecuador, examined political graffiti as an alternative form of political participation in the developing democracy of Ecuador. After completing an internship at the United States embassy in Madrid, Spain, and spending a year working on various farms in Spain, Joseph returned to the US to attend a Master's program at Tulane University in New Orleans. In 2004, he graduated from Tulane University with a Master of Arts degree in political science, with a specialization in comparative politics and energy policy. His Master's thesis, based on fieldwork in Costa Rica, explored the relationship between the structure and sustainability of energy systems and institutions in various Central American countries. During his studies, Joseph worked as an English-language proofreader and a Spanish-English and German-English translator for various scientific and academic institutions. In 2005, Joseph began work as a researcher at the Amsterdam-based Centre for Research on Multinational Corporations (SOMO). In 2008, he joined the University of Twente's Centre for Studies in Technology and Sustainable Development (CSTM) as an off-site researcher and Ph.D. candidate. Currently, Joseph is a Senior Researcher at SOMO and Coordinator of the OECD Watch network (a recognized civil society stakeholder at the OECD). He leads SOMO's research and policy advocacy on energy and public services and coordinates SOMO's broader research into various industrial sectors and supply chains. He also serves as an Independent Advisor to the International Corporate Responsibility Committee of the Social and Economic Council (SER) of the Netherlands and a Technical Advisor to the Silicon Valley Toxics Coalition.

Selected publications in peer-reviewed journals:

- J. Wilde-Ramsing (2013). "Quality Kilowatts: A normative-empirical framework for assessing TNC performance on sustainable electricity provision in the Global South". Progress in Development Studies. Forthcoming.

- J. Wilde-Ramsing and B. Potter (2006), "An Unlikely Leader: Energy Policy and Renewable Energy in Costa Rica", Journal of Environment and Development, Vol 32, 1.

" J. Wilde-Ramsing (2001), "Alternative Forms of Political Participation in Developing Democracies: Political Graffiti in Quito, Ecuador" UNC-Asheville Journal of Undergraduate Research, Vol. 14.

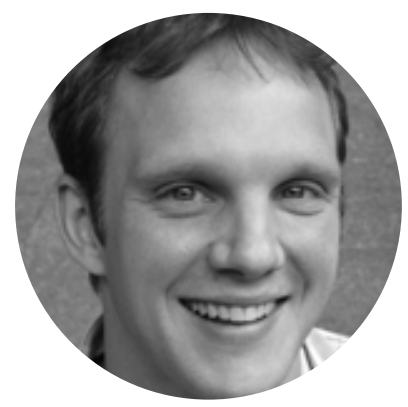

